LBNL-43182

\title{
Acoustic Resonance Characteristics OF Rock AND CONCRETE Containing Fractures
}

\author{
by
}

\section{SEIJI NAKAGAWA}

B.E. (Kyoto University, Japan) 1991

M.E. (Kyoto University, Japan) 1993

A dissertation submitted in partial satisfaction of the

requirements for the degree of

Doctor of Philosophy

in

Engineering-Materials Science and Mineral Engineering

in the

GRADUATE DIVISION

of the

UNIVERSITY of CALIFORNIA at BERKELEY

comittee in charge:

Professor Ronald Gronsky, Chair

Professor Paulo Monteiro

Dr. Kurt Nihei

Fall 1998

This work was supported by the National Science Foundation under Contract No. MSS9212737 and Director, Office of Energy Research, Office of Basic Energy Sciences under U.S. Department of Energy Contract No. DE-AC03-76SF00098. 


\section{DISCLAIMER}

This report was prepared as an account of work sponsored by an agency of the United States Government. Neither the United States Government nor any agency thereof, nor any of their employees, make any warranty, express or implied, or assumes any legal liability or responsibility for the accuracy, completeness, or usefulness of any information, apparatus, product, or process disclosed, or represents that its use would not infringe privately owned rights. Reference herein to any specific commercial product, process, or service by trade name, trademark, manufacturer, or otherwise does not necessarily constitute or imply its endorsement, recommendation, or favoring by the United States Government or any agency thereof. The views and opinions of authors expressed herein do not necessarily state or reflect those of the United States Government or any agency thereof. 


\section{DISCLAIMER}

Portions of this document may be illegible in electronic image products. Images are produced from the best available original document. 


\title{
Acoustic Resonance Characteristics of Rock and Concrete Containing Fractures
}

\author{
Copyright $\odot 1999$
}

by

Seiji Nakagawa

The U.S. Department of Energy has the right to use this document for any purpose whatsoever including the right to reproduce all or any part thereof. 


\begin{abstract}
Acoustic Resonance Characteristics of Rock and Concrete

Containing Fractures
\end{abstract}

by

Seiji Nakagawa

Doctor of Philosophy in Materials Science and Mineral Engineering

University of California, Berkeley

Professor Ronald Gronsky, Chair

In recent years, acoustic resonance has drawn great attention as a quantitative tool for characterizing properties of materials and detecting defects in both engineering and geological materials. In quasi-brittle materials such as rock and concrete, inherent fractures have a significant influence on their mechanical and hydraulic properties. Most of these fractures are partially open, providing internal boundaries that are visible to propagating seismic waves. Acoustic resonance occurs as a result of constructive and destructive interferences of propagating waves. Therefore the geometrical and mechanical properties of the fracture are also interrogated by the acoustic resonance characteristics of materials.

The objective of this dissertation is to understand the acoustic resonance characteristics of fractured rock and concrete. Chapter 2 and 3 show that the spatial distribution and the elastic and viscoelastic properties of fractures in one-dimensional systems have a significant effect on their resonance frequencies and the attenuation. A numerical code that simulates the resonance of three-dimensional bodies containing fractures is developed in Chapter 4 and used to determine the anisotropic elastic moduli of rocks (Chapter 5) and the stiffness 
of a fracture in concrete (Chapter 6) from measured resonance frequencies. In Chapter 7, the dynamic stiffnesses of concrete bridge columns are determined from their resonance frequencies and good agreement with the changes in the static stiffness is found. Chapter 8 shows that a sheared fracture converts a part of normally incident P-waves to polarized Swaves and vise versa, which can be used as a powerful tool for detecting and measuring shear stress on fractures. A sheared fracture is also shown to have a significant effect on the velocity and particle motion of the waves propagating along the fracture (Chapter 9). Chapter 10 unifies the effects of dynamic coupling (dilation) of a sheared fracture and wave propagation and resonance in an infinite series of multiple parallel fractures in a dispersion equation for anisotropic frequency-dependent wave propagation. This equation provides complete solutions for elastic wave propagation in the media which includes such wave phenomena as generalized Rayleigh-Lamb plate waves, fracture interface waves, and acoustic resonances.

Ronald Gronsky, Professor, Department of Materials Science and Mineral Engineering 


\section{Dedication}

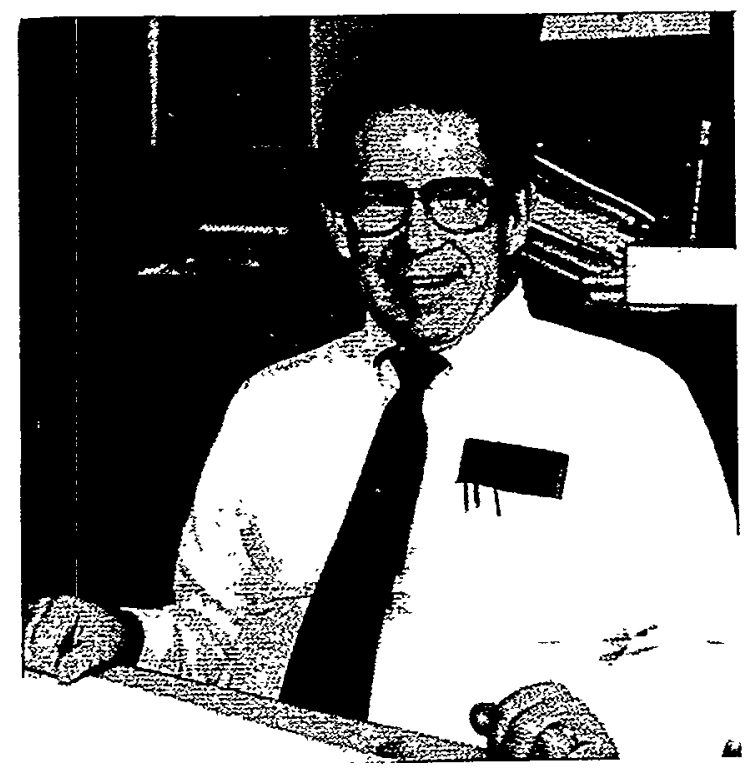

Professor Neville G. W. Cook, 1938-1998

This dissertation is dedicated to the late Professor Neville G. W. Cook, who gave me the opportunity to come to this country, and his wife Jennifer who had supported him with a strong will during years of his struggle against his illness. Dr. Cook always offered me unconditional support both in research and in daily life as a foreign student in this country. I am truly sorry that he did not live to see the completion of our work. 


\section{Table of Contents}

Page

List' of Figures............................................................

List of Tables............................................................ xxi

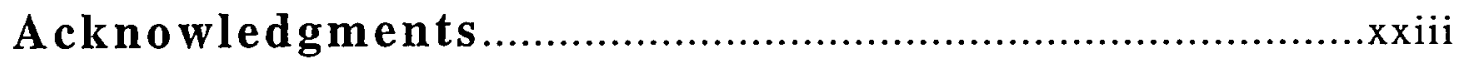

\section{Chapter 1 General Introduction}

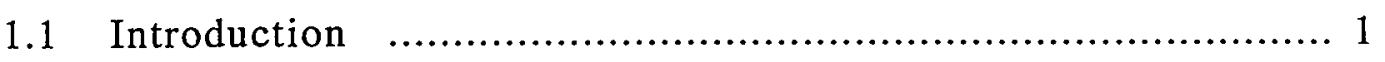

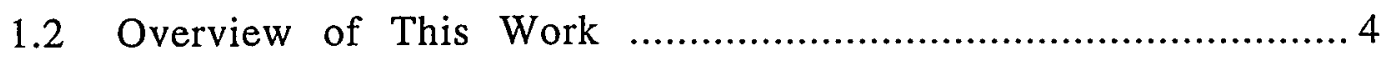

Chapter 2 Analytical Study on the Effects of Fracture Properties on One-Dimensional Resonance

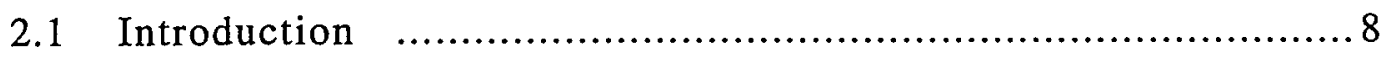

2.2 Finite System Including Single Fracture …….............................. 9

2.2.1 Resonance frequency shift.......................................... 9

2.2.2 Attenuation due to material damping and fracture viscosity.......... 14

2.3 Finite System Including Multiple Fractures ................................ 20

2.3.1 Resonance frequency shift and mode localization.................... 20

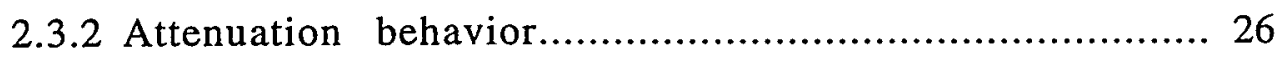

2.4 Resonance of a Delaminated Half Space ...................................... 28

2.5 Infinite System Including Finite Number of Fractures ...................... 34

2.5.1 Transmission and reflection coefficients............................... 34

2.5.2 Spectrum of the first-arriving pulse................................. 37

2.5.3 Coda spectrum.................................................. 40 
2.6 Infinite System Including Infinite Number of Regularly Spaced Fractures 42

2.6.1 Derivation of dispersion equation.............................. 42

2.6.2 Limiting behaviors of velocities.................................. 44

2.6.3 Phase and group velocities.................................... 45

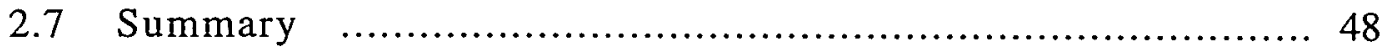

\section{Chapter 3 Resonant Bar Test on Fractured Rock}

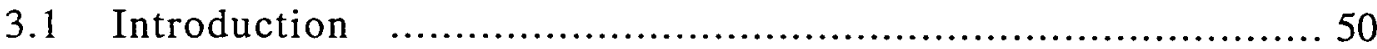

3.2 Experimental Setup .............................................. 52

3.2.1 Sample description........................................... 52

3.2.2 Free vibration test seup....................................... 53

3.2.3 Axially confined vibration test seup.................................53

3.2.4 Measurement of frequency responses...............................53

3.3 Free Vibration of Rod Specimens ....................................... 55

3.4 Resonance of Axially Confined Rod Specimens ............................ 59

3.4.1 Effect of end coupling on resonance............................. 59

3.4.2 Resonance of intact rock specimens................................62

3.4.3 Resonance of fractured rock specimen...........................65

3.4.4 Effect of fluid inside the fracture.....................................67

3.4.5 Effect of attenuative filling.................................... 71

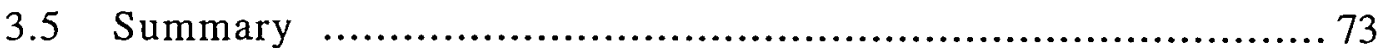

\section{Chapter 4 Numerical Model for Resonance of 3-D Anisotropic Body Containing Fractures}

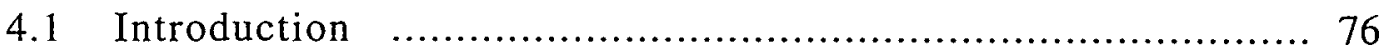

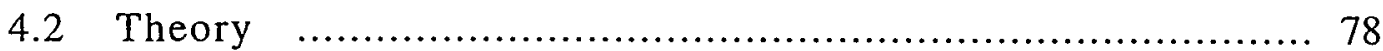

4.2.1 Hamilton's principle......................................... 78

4.2.2 Rayleigh-Ritz method..................................... 80

4.2.3 Rigid and finite stiffness boundary conditions..................... 83

4.2.4 Symmetry of the problem........................................ 83

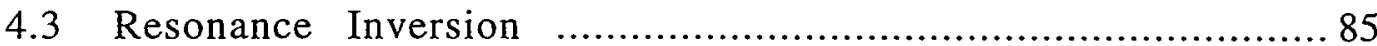

4.3.1 Perturbation of stiffness - Rayleigh's Quotient...................... 85

4.3.2 Determination of elastic moduli for anisotropic material...............86

4.3.3 Determination of anisotropic fracture stiffness.......................88

4.4 Accuracy of Computed Resonance Frequency ...............................8 89 


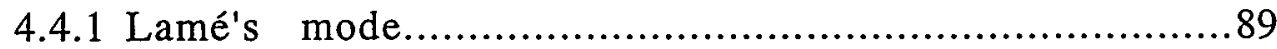

4.4.2 Accuracy of solution for an intact cube.................................. 90

4.4.3 Accuracy of solution for a fractured cube................................91

4.5 Accuracy of Resonance Inversion ............................................. 92

4.5.1 Inversion for isotropic elastic moduli................................... 92

4.5.2 Inversion for isotropic fracture stiffness.................................95

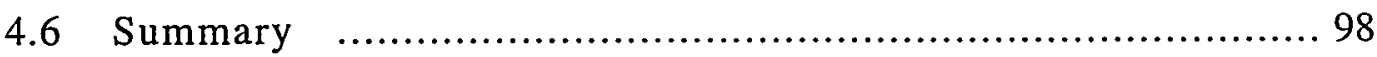

\section{Chapter 5 Resonance of Anisotropic Rock}

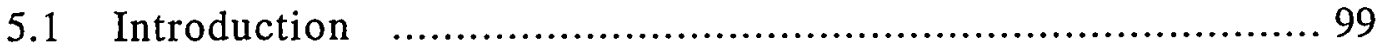

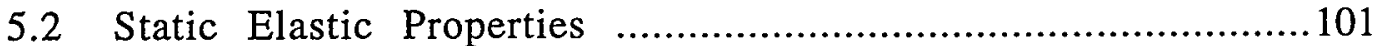

5.2.1 Description of specimens...............................................101

5.2.2 Optical microscope observation for granite............................ 101

5.2.3 Capillary imbibition for granite...........................................107

5.2.4 Static behavior for granite............................................107

5.3 Ultrasonic Transmission Test .............................................116

5.3.1 P-wave anisotropy for granite......................................... 116

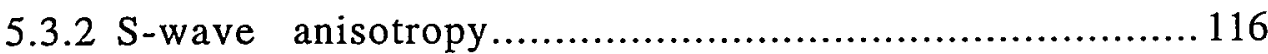

5.3.3 Velocity anisotropy and axial load...................................... 116

5.3.4 High-frequency dynamic elastic moduli................................. 122

5.4 Resonance of Anisotropic Rocks ............................................126

5.4.1 Experimental setup................................................126

5.4.2 Frequency response of an isotropic cube................................126

5.4.3 Frequency response of anisotropic rocks.............................. 131

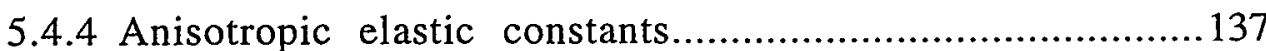

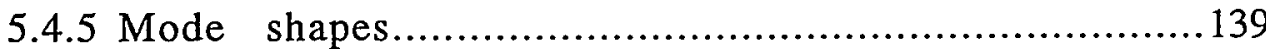

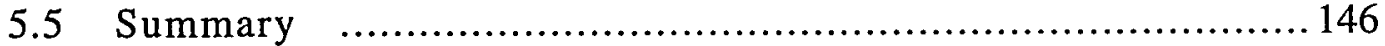

\section{Chapter 6 Resonance of a Fractured Three-Dimensional Body}

6.1 Introduction .......................................................... 148

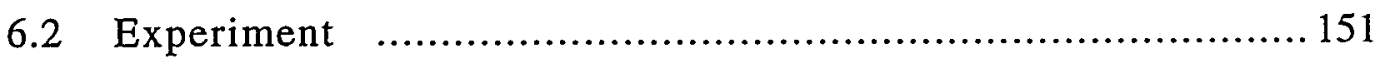

6.2.1 Properties of the intact specimen......................................... 151

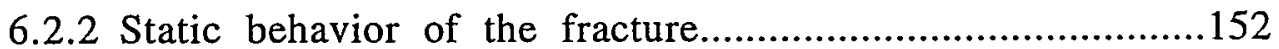

6.2.3 Experimental setup for the resonance tests..............................155

6.2.4 Effect of axial stress on intact specimen................................156

6.2.5 Resonance of the fractured specimen.................................... 156 


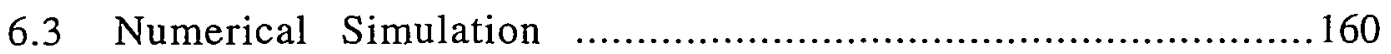

6.3.1 Computed FRF's................................................... 160

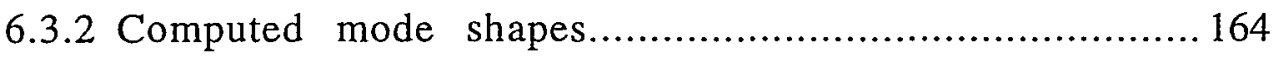

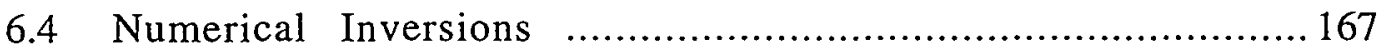

6.4.1 Inversion for elastic moduli..........................................167

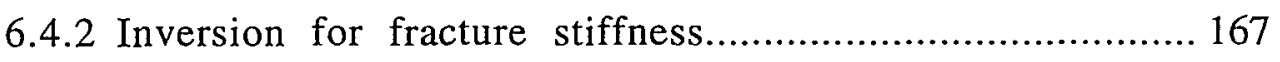

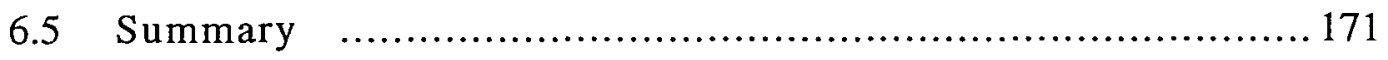

\section{Chapter 7 Resonance of Fractured Concrete Infrastructure}

7.1 Introduction ......................................................... 172

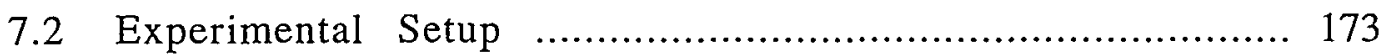

7.2.1 Description of specimens......................................... 173

7.2.2 Seismic wave transmission test...................................... 176

7.2.3 Resonance test.............................................. 179

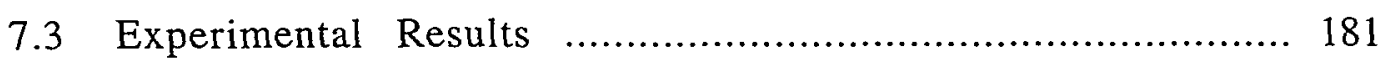

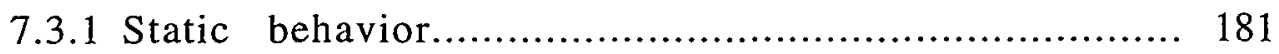

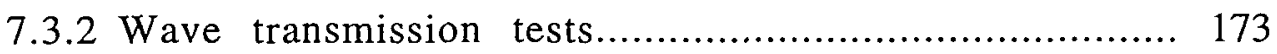

7.3.3 Resonance tests............................................. 190

7.4 Static stiffnesses and Resonance Frequencies ............................. 197

7.4.1 Displacement-discontinuity B.C. for column containing

a compliant interface................................................ 197

7.4.2 Dynamic response of the structure................................... 199

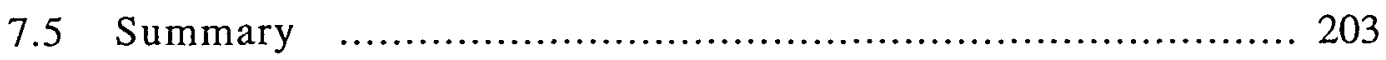

Chapter 8 Dynamic Behavior of a Sheared Fracture and the Extension of Displacement-Discontinuity Theory

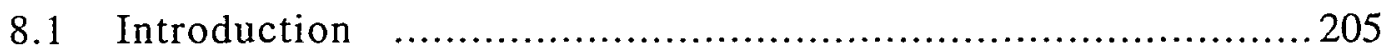

8.2 Mechanism of Shear-induced Wave Conversion ...........................207

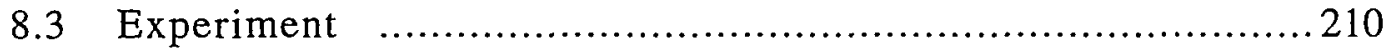

8.3.1 Wave transmission tests across a fracture in rock......................210

8.3.2 Transmission tests across a regular saw-tooth fracture................217

8.4 Extension of Displacement-Discontinuity Theory ………............... 228

8.4.1 Applicability of displacement-discontinuity model

for dynamic problems................................................. 228

8.4.2 Static behavior of an idealized sheared fracture....................... 229

8.4.3 Some basic properties of fracture stiffness 
and compliance matricies. 234

8.4.4 General scattering matrix for plane wavés incident on fracture..... 236

8.4.5 Normal incident waves with cross-coupling fracture stiffnesses... 240

8.4.6 P-SV cross coupling for obliquely incident waves.................... 244

8.5 Numerical Simulation ................................................... 248

8.5.1 Frequency-domain boundary element code (BIEMPS).............. 248

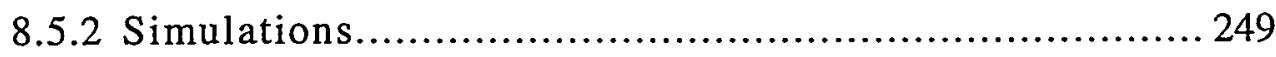

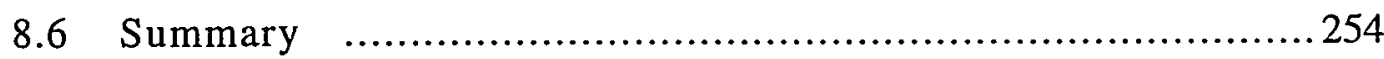

\section{Chapter 9 Wave Propagation along Sheared Fracture}

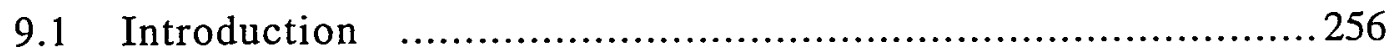

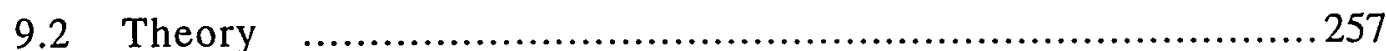

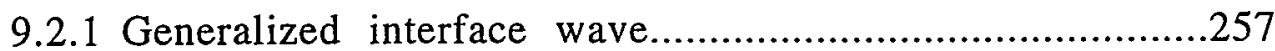

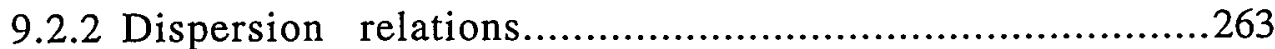

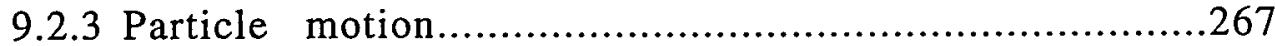

9.3 Numerical Simulation Using Boundary Element Method .................. 273

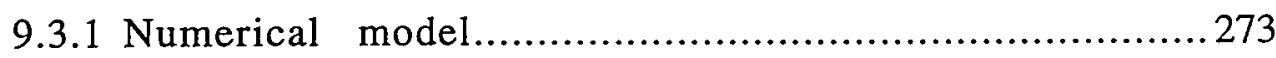

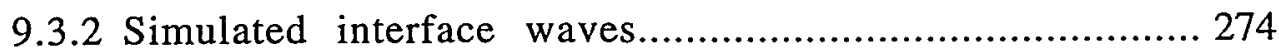

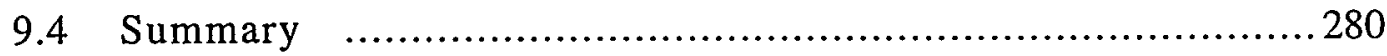

\section{Chapter 10 Wave Propagation and Resonance in Media} Containing Regularly Spaced Parallel Fractures

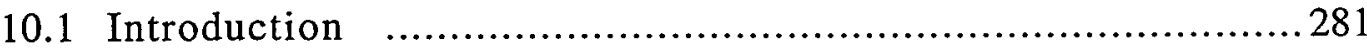

10.2 Derivation of General Dispersion Equation ……............................ 283

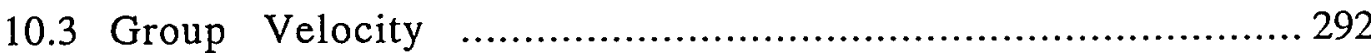

10.4 Decoupled SH-wave in Infinite Series of Regular Fractures ............... 293

10.4.1 Decoupled SH-dispersion equation................................ 293

10.4.2 SH-wave with oblique incidence................................... 294

10.4.3 SH-wave propagation along fractures............................... 296

10.4.4 Particle motions of SH-waves...................................... 301

10.5 Decoupled P-SV Waves in Infinite Series of Regularly Spaced

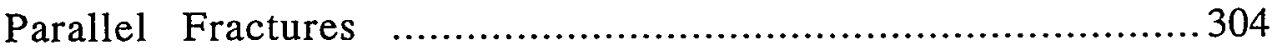

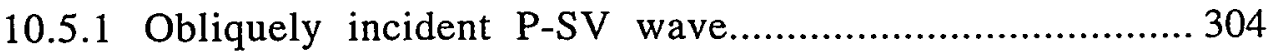

10.5.2 Generalized Rayleigh-Lamb plate waves........................... 308

10.5.3 Particle motions of P-SV waves..................................... 312

10.6 Coupled P-SV-SH Wave Propagation .................................... 318 
10.6.1 General fracture stiffness matrix................................3 318

10.6.2 Wave propagation along $\mathrm{x}-\mathrm{z}$ plane................................3 318

10.6.3 Fully coupled P-SV-SH wave propagation........................318

10.7 Generalized Fracture Interface Wave ..................................... 323

10.7.1 Dispersion equation.......................................... 323

10.7.2 Anisotropic group velocity............................... 324

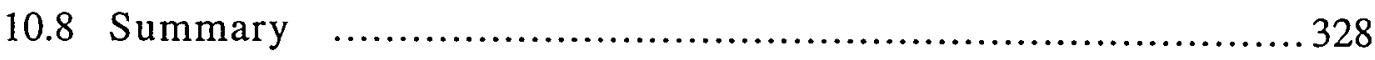

\section{Chapter 11 General Summary and Conclusions}

11.1 Summary and Conclusions ........................................... 330

11.2 Remaining Problems and Future Research .............................. 334

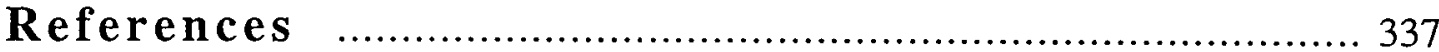

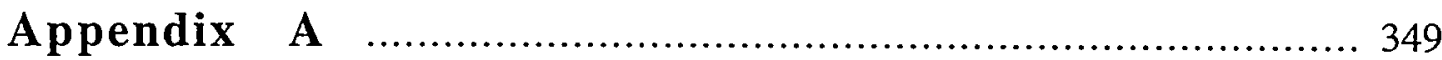

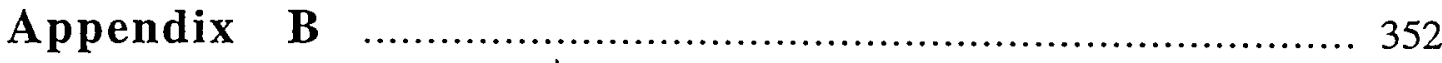




\section{List of Figures}

Page

Figure 2.1 Waterfall plot of FRF(Mobility)'s for a finite bar with a single fracture.

$\begin{array}{lll}\text { Figure } 2.2 & \text { The center resonance frequency } & 12\end{array}$

Figure 2.3 The (a) center fracture stiffness $\kappa_{\mathcal{C}}$ and (b) corresponding impedance ratios $\beta_{\mathrm{c}}$ for the resonances of a fractured bar.

Figure 2.4 Computed mobility distribution in a finite bar with a single fracture at the middle.

Figure 2.5 Computed mobility distribution in a finite bar with a single fracture at the middle.

Figure 2.6 Mechanism for the selective attenuation of odd modes.

Figure 2.7 Effect of viscosity on the resonance frequency and attenuation of the 1st and 2nd odd modes.

Figure 2.8 Computed mobility distribution in a finite bar with nine fractures. 21

Figure 2.9 Resonance frequency shifts for finite bars with regularly and irregularly spaced fractures.

Figure 2.10 Mode shapes for resonances in a bar with regularly and irregularly . spaced fractures with a normalized fracture stiffness $b=10$.

Figure 2.11 Mode shapes for resonances in a bar with regularly and irregularly spaced fractures.

Figure 2.12 Effect of fracture viscosity on the resonance of a multiple fracture system. 
Figure 2.13 FRF's of a delaminated layer on a halfspace for different fracture stiffnesses.

Figure 2.14 The (a) center fracture stiffness $\kappa_{c}$ and (b) corresponding impedance ratios $\beta_{c}$ for the resonances of the delaminated half space.

Figure 2.15 Decomposition of vibration to upgoing and downgoing wave components.

Figure 2.16 Comparison of analytically and numerically (using the half-power method) determined attenuation of vibration in a delamination.

Figure 2.17 Examples of multiple parallel fractures observed in the field.

Figure 2.18 Schematics for regularly and irregularly spaced fractures embedded in an infinite medium.

Figure 2.19 Transmission (T) and reflection (R) coefficients of (a) regularly and (b) irregularly spaced fractures.

Figure 2.20 Comparison of FRF's (power of mobility for finite system and power of transmission coefficient for an infinite system) for finite and infinite systems with 11 fractures.

Figure 2.21 Transmission coefficient for the first-arriving pulse through regularly and irregularly spaced fractures.

Figure 2.22 Phase and group velocities for the first-arriving pulse through regularly and irregularly spaced fractures.

Figure 2.23 Time domain impulse response and spectra of the coda (as indicated in the diagrams) for (a) regularly and (b) irregularly spaced fractures.

Figure 2.24 Dispersion in a regularly spaced infinite series of fractures. $\quad 46$

$\begin{array}{lll}\text { Figure 2.25 Dispersion in regularly and irregularly spaced finite fractures. } & 47\end{array}$

Figure $3.1 \quad$ Experimental setup used for vibration tests on rod specimens. 54

Figure 3.2 Acceleration spectra measured on a source piezoelectric crystal suspended in the air. $\quad 55$

Figure 3.3 Young's modulus determined from resonant bar tests. 57

Figure 3.4 Attenuation of the specimens determined by the half-power method.

Figure $3.5 \quad$ Frequency response spectra of uniaxially confined aluminum and plexiglass rods. 
Figure 3.6 Effect of axial confinement on the resonance frequency (or phase velocity) of aluminum and plexiglass specimens.

Figure 3.7 Effect of axial confinement on the attenuation of aluminum and plexiglass specimens.

Figure 3.8 Measured FRF's of intact rock bars.

Figure 3.9 Increase in phase velocity of rock specimens with increasing axial stress determined from resonance frequencies.

Figure 3.10 Comparison of phase velocities determined by static tests, ultrasonic transmission tests, and resonant bar tests.

Figure 3.11 FRF's of granite bar specimen containing a single through-going fracture at the middle of the bar.

- Figure 3.12 Dynamic fracture stiffness computed from the shift in the odd mode resonance frequencies.

Figure 3.13 FRF's of fractured granite bar showing the effect of fluid in the fracture.

Figure 3.14 Shift of resonance frequencies for a fracture injected with water. 69

$\begin{array}{lll}\text { Figure 3.15 Change in attenuation for a fracture injected with water. } & 69\end{array}$

Figure 3.16 Resonance frequency shifts due to increase in fracture viscosity. 70

Figure 3.17 Attenuation of odd mode resonances by latex membrane in the fracture.

Figure 3.18 Attenuation in a fractured granite specimen with a inserted latex membrane.

Figure 3.19 Possible mechanism for non-monotonic attenuation behavior of odd modes.

Figure 4.1 Modeling fractured elastic material with displacement-discontinuity boundary conditions.

Figure 4.2 Structure of mass (M) and stiffness (G) matrices for a 3-block, 2fracture system.

Figure 4.3 Structure of stiffness matrix for stiffness boundary (connected to rigid foundation).

Figure 4.4 Examples of structure for global base functions for fracture(s) introduced symmetrically in a symmetric body.

Figure 4.5 Convergence of computed resonance frequencies for an isotropic cube 
Figure 4.6 Convergence of the 1st and 2nd Lamé mode resonance frequencies for an isotropic cube.

Figure 4.7 Convergence of computed resonance frequencies for a fractured block

Figure 4.8 Source and receiver locations for observed resonance frequencies of an isotropic cube.

Figure 4.9 Computed FRF(compliance) for forced excitation of an isotropic cube.

Figure 4.10 Convergence of the solution (intact cube).

Figure 4.11 Source and receiver locations for observed resonance frequencies of an isotropic cube including a single through-crack.

Figure 4.12 Computed FRF(compliance) for forced excitation of isotropic cube containing a fracture.

Figure 4.13 Convergence of solution (fractured cube).

Figure 5.1 Rock cube specimens used for resonance spectroscopy.

Figure 5.2 Core orientation of specimens used for optical microscope observation, imbibition tests, and static uniaxial load-displacement tests.

Figure 5.3 Thin section optical-micrographs of granite in three perpendicular directions.

Figure 5.4 Higher magnification optical micrographs of microcracks in mineral grains.

Figure 5.5 Shape and alignment of mineral grains in granite specimen. 106

Figure 5.6 Oven-dried core A, B, and C placed in glass jars with their bases immersed in distilled water.

Figure 5.7 Time history of capillary imbibition into granite cores.

Figure 5.8 Idealized microcrack geometry and orientations from microscope observation and capillary imbibition tests.

Figure 5.9 Experimental setup for static load-displacement test.

Figure 5.10 Uniaxial compression tests on granite specimens cored in three mutually perpendicular directions.

Figure 5.11 Poisson's expansion of specimens around the core axis. 
Figure 5.12 (a) Young's modulus determined from small excursion loops in Figure 5.10(a) and ((b)-(d)) low-axial stress load-displacement tests.

Figure 5.13 Experimental setup for ultrasonic wave transmission tests.

Figure 5.14 P-wave anisotropy of granite core specimens (vacuum saturated with water).

Figure 5.15 S-wave anisotropy of granite core specimens and sandstone and dolomite cubes (room-dry).

Figure 5.16 Stress dependency and anisotropy of P-wave velocity. 120

$\begin{array}{lll}\text { Figure 5.17 Stress dependency and anisotropy of S-wave velocity. } & 121\end{array}$

Figure 5.18 Directions of propagation and polarization (particle motion) of $\mathrm{P}$ and S-waves measured in the cube specimens. 123

Figure 5.19 Experimental setup used for free vibration tests of rock cubes. 127

Figure 5.20 Measured and computed (from measured ultrasonic velocities) frequency response functions. 128

Figure 5.21 Measured and computed FRF's of a glass cube. 130

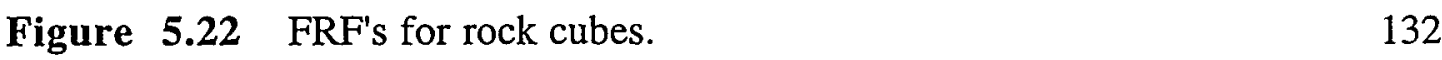

Figure 5.23 Measured and computed FRF's for the granite cube. 133

Figure 5.24 Measured and computed FRF's for the sandstone cube. 134

Figure 5.25 Experimentally determined elastic moduli for granite and sandstone cubes.

Figure 5.26 Experimental setup for measuring mode shapes of rock cubes. 141

Figure 5.27 Several of the observed and computed mode shapes for the lead glass cube.

Figure 5.28 (a) Experimental and computed mode shapes for the granite cube. 143

Figure 5.28 (b) Experimental and computed mode shapes for the granite cube. 144

Figure 5.28 (c) Experimental and computed mode shapes for the granite cube. 145

Figure 6.1 Uniaxial compression of an intact concrete cylinder. 151

Figure. 6.2 Experimental setup used for resonance test of a concrete cylinder containing a single through-going fracture. 
Figure 6.3 Examples of closing displacement of fracture measured by LVDT's on both sides of specimen.

Figure 6.4 Measured FRF for an intact specimen (concrete cylinder). 157

Figure 6.5 (a) Measured FRF's of fractured concrete cylinder (at bottom). 158

$\begin{array}{lll}\text { Figure } 6.5 & \text { (b) Measured FRF's of fractured concrete cylinder (at top). } & 159\end{array}$

Figure 6.6 Comparison of computed FRF's with different degree of approximations.

Figure 6.7 (a) Computed FRF's of a fractured concrete cylinder (at bottom). 162

Figure 6.7 (b) Computed FRF's of a fractured concrete cylinder (at top). 163

Figure 6.8 Comparison between experimentally measured and computed resonance frequencies for a range of axial stresses and fracture stiffnesses, respectively.

Figure 6.9 Effects of fracture stiffness on the mode shapes of a fractured concrete cylinder.

Figure 6.10 Comparison between experimentally measured and computed FRF's.

Figure 6.11 Comparison between static normal fracture stiffnesses from loading tests and dynamic fracture stiffnesses determined using the resonance inversion technique.

Figure 6.12 Comparison between static normal fracture stiffnesses from loading tests and dynamic fracture stiffnesses from the resonance inversion technique.

Figure 6.13 Comparison between experimentally measured and numerically computed FRF's using the inverted fracture stiffnesses.

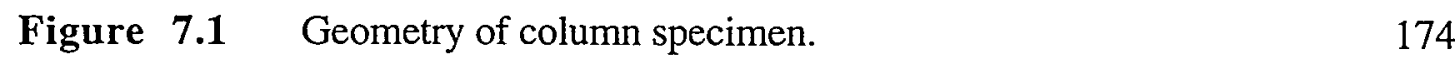

Figure 7.2 Experimental setup for cyclic loading tests for model columns 175

Figure 7.3 Damage introduced by cyclic loading of the column. 176

$\begin{array}{lll}\text { Figure } 7.4 & \text { Repair of the damaged columns. } & 177\end{array}$

Figure 7.5 Source and receiver configuration for seismic transmission tests. 178

Figure 7.6 Response of calibrated impact hammer hit on the surface of an intact column.

$\begin{array}{lll}\text { Figure } & 7.7 & \text { Experimental setup for vibration tests. }\end{array}$ 
Figure $7.8 \quad$ Load-displacement behavior of intact and repaired columns. 182

Figure 7.9 Stiffness of intact, damaged, and repaired columns. 183

Figure 7.10 Examples of waveforms measured at the middle section of an intact column. 184

Figure 7.11 Vertical profiles of P-wave travel time along the diameter of column specimens.

Figure 7.12 Seismograms for specimens before and after damage introduction and repair.

Figure 7.13 Example of measured waveforms for column $415 \mathrm{~S}$.

Figure 7.14 Explanation for different wave behaviors in directions normal and perpendicular to the lateral displacement applied to the columns to introduce damage.

Figure 7.15 Comparison between resonances introduced by different types of sources.

Figure 7.16 Normalized spectra measured using a sledge-hammer source. 192

Figure 7.17 Normalized amplitude of mode shapes measured for the observed resonances.

Figure 7.18 Fitting of a function with an unknown frequency and attenuation parameters for measured transient vibration introduced by an impact from a sledge hammer.

Figure 7.19 Resonance frequencies and attenuation (damping coefficients) for measured vibration of columns hit by a sledge hammer.

Figure 7.20 Idealization of the damaged column.

Figure 7.21 Computed resonance frequencies of the first flexural mode as a function of interface stiffness $\kappa$.

Figure 7.22 Comparison between measured static bending stiffnesses and determined bending stiffnesses from measured resonance frequencies.

Figure 8.1 Mechanism generating cross-coupled waves for normal-incident plane waves.

Figure 8.2 Experimental setup for seismic wave conversion tests across a fracture in a rock specimen.

Figure 8.3 Changes in transmitted and converted waves through a granite specimen before and after a fracture is introduced. 
Figure 8.4 (a) (b) Waves transmitted across a single fracture in a granite specimen.

$\begin{array}{lll}\text { Figure } 8.4 & \text { (c) (d) Waves converted on a single fracture in a granite specimen. } 215\end{array}$

Figure 8.5 Changes in directly transmitted (a) $\mathrm{S}$ and (b) P-waves for different shear stresses.

Figure 8.6 Measured waves along the axis of a fractured rock specimen for a range of shear angles.

Figure 8.7 Steel blocks with regular sawtooth surface.

Figure 8.8 Experimental setup for two steel blocks with a regular sawtooth surface

Figure $8.9 \quad$ (a)(b) Comparison of directly transmitted waves through intact and fractured steel specimens.

Figure $8.9 \quad$ (c)(d) Comparison of converted waves through intact and fractured steel specimens.

Figure 8.10 (a)(b) Directly transmitted waves through a fracture containing dry glass beads.

Figure 8.10 (c) (d) Converted waves through a fracture containing dry glass beads.

Figure 8.11 (a)(b) Directly transmitted waves transmitted through a fracture containing water saturated glass beads.

Figure 8.11 (c)(d) Converted waves transmitted through a fracture containing water saturated glass beads.

$\begin{array}{lll}\text { Figure } & \text { 8.12 Interface consists of a periodic array of flat open microcracks. } 228\end{array}$

Figure 8.13 Boundary element mesh used for computing displacement around a periodic array of inclined flat cracks subjected to far-field stress. 231

Figure 8.14 Comparison between numerical and analytical solutions for cracksurface displacement for the model in Figure 8.11.

Figure 8.15 Computed displacement field for cracks with crack size to interval ratio of $2 \mathrm{a} / \mathrm{s}=0.5$.

Figure 8.16 Computed compliances and stiffnesses of a fracture consisting of a periodic array of inclined cracks.

Figure 8.17 Scattering of waves incident upon a compliant interface (fracture). 238 
Figure 8.18 Matrix equation for the general scattering problem of a fracture including coupling between all displacement discontinuity and traction components.

Figure 8.19 Transmission and reflection coefficients for a P-wave normally incident on a fracture.

Figure 8.20 Transmission and reflection coefficients for an obliquely incident P-wave.

Figure 8.21 Transmission and reflection coefficients for obliquely incident Swave.

Figure 8.22 A numerical model for simulating scattering of waves incident on a compliant fracture.

Figure 8.23 Snapshots of the wavefield interacting with a compliant fracture. Waves are generated by an explosive source.

Figure 8.24 Snapshots of the wavefield interacting with a compliant fracture. Waves are generated by a vertical source.

Figure 8.25 Snapshots of the wavefield interacting with a compliant fracture. Waves are generated by a horizontal source.

$\begin{array}{lll}\text { Figure } & \text { 9.1 Fracture interface wave propagating along a sheared fracture. } 258\end{array}$

Figure 9.2 Graphic representation of individual terms in the dispersion equation for the fracture interface wave.

Figure 9.3 Computed dispersion of phase velocities for interface waves with a coupling fracture stiffness.

Figure 9.4 Particle motion of a single point on the surface of a fracture. 270

Figure 9.5 (a) Particle motions of (quasi-) symmetric Rayleigh interface waves.

Figure 9.5 (b) Particle motions of (quasi-) antisymmetric Rayleigh interface waves.

Figure 9.6 A numerical model for simulating a fracture interface wave along a fracture with coupling fracture stiffness.

Figure 9.7 (a) Snapshots of symmetric and quasi-symmetric fracture interface waves with and without coupling fracture stiffness

Figure 9.7 (b) Snapshots of antisymmetric and quasi-antisymmetric fracture interface waves with and without coupling fracture stiffness 275

Figure $\quad 9.8$ Seismograms for waves generated by a symmetric source. 276 
Figure $\quad 9.9$ Seismograms for waves generated by an anti-symmetric source. 277

Figure 9.10 Particle motions of interface wave on the fracture surface.

Figure 10.1 P-SV-SH wave propagation in a medium including regularly spaced parallel fractures.

Figure 10.2 Matrix equation with dimensionless parameters.

Figure 10.3 Simplified matrix equation with dimensionless parameters.

$\begin{array}{llll}\text { Figure } & 10.4 & \text { Velocity surfaces (group velocity) for decoupled SH-waves. } 295\end{array}$

Figure 10.5 Velocity surfaces for SH-wave propagation in an infinite series of regularly spaced parallel fractures.

Figure 10.6 Behavior of the SH-dispersion equation.

Figure 10.7 Group-velocity dispersion curves for generalized SH-plate waves. 300

Figure 10.8 (a) Snapshots for obliquely incident SH-waves in a medium containing periodic parallel fractures.

Figure 10.8 (b) Views from the top of Figure 10.8(a).

Figure 10.9 (a) Velocity surfaces of $\mathrm{P}$ and SV-waves computed for static effective medium models (left) and exact solutions of the dispersion equation for fractured media (right).

Figure 10.9 (b) Velocity surfaces of $\mathrm{P}$ and SV-waves computed for static effective medium models (left) and exact solutions of the dispersion equation for fractured media (right).

Figure 10.10 Velocity surfaces of $\mathrm{P}$ and SV-waves computed for a constant fracture stiffness $(b=10)$ and a range of wave frequencies.

Figure 10.11 (a) Group velocities of generalized Rayleigh-Lamb waves for low to medium fracture stiffnesses.

Figure 10.11 (b) Group velocities of generalized Rayleigh-Lamb waves for medium to high fracture stiffnesses.

Figure 10.12 Wavenumber (horizontal)-frequency dispersion curves for the generalized Rayleigh-Lamb plate waves.

Figure 10.13 (a) Particle motions in the first pass band for a static fracture stiffness of $b=10$ and wave frequency of $\alpha=4$.

Figure 10.13 (b) Particle motions in the second pass band for a static fracture stiffness of $b=10$ and wave frequency of $\alpha=4$. 
$\mathbf{X X}$ List of Figures

Figure 10.13 (c) Particle motions in the third pass band for a static fracture stiffness of $b=10$ and wave frequency of $\alpha=4$.

Figure 10.14 Velocity surfaces computed from static equivalent medium model (left) and exact dispersion equation (right) for a fractured medium. 319

Figure 10.15 Velocity surfaces computed from an exact dispersion equation for fractured medium.

Figure 10.16 Velocity surfaces for media containing an infinite series of regularly spaced parallel fractures.

Figure 10.17 Anisotropic group velocities of fracture interface wave with a range of $\mathrm{x}-\mathrm{Z}$ coupling fracture stiffness.

Figure 10.18 Particle motions of fracture interface waves on the surface of the lower halfspace. 


\section{List of Tables}

Table 2.1 Computation of center resonance frequency 13

Table 2.2 Fracture Spacings in Finite One-dimensional Systems 22

Table 3.1 Static and Dynamic Properties of Specimens 52

Table 5.1 P and S-wave velocities for cube specimens 122

Table 5.2 Dynamic Elastic Moduli (250kHz 1MHz) 124

Table 5.3 Velocities Determined for Granite Octagons at $1 \mathrm{MHz}$ Source Frequency 125

$\begin{array}{lll}\text { Table 5.4 Resonance Frequencies for Lead Glass Cube } & 129\end{array}$

Table 5.5 Resonance Frequencies for the Granite Cube 135

Table 5.6 Resonance Frequencies for Sandstone Cube 136

Table 5.7 Inverted Elastic Moduli (10kHz 30kHz) 137

Table 6.1 Comparison of computed resonance frequencies (in $\mathrm{Hz}$ ) for singlet modes.

Table 6.2 Comparison of computed resonance frequencies (in $\mathrm{Hz}$ ) for doublet modes.

Table 7.1 Resonance frequency and attenuation of column specimens 195

$\begin{array}{lll}\text { Table 7.2 Measured static bending stiffness of columns } & 197\end{array}$

Table 7.3 Bending stiffness of columns determined from resonance frequency 201 



\section{Acknowledgments}

Among many people who helped me to complete this thesis, first I would like to thank the late Dr. Neville G. W. Cook, who had been my advisor, mentor, and the "father" of our research group until he was gone after years of illness. Although he was physically losing the battle on my last visit to him two weeks before he passed away, his mind was still bright and full of inspiration. As one of his students, it is both my duty and honor to carry and grow what I was given by him.

I cannot thank Dr. Ronald Gronsky enough for looking after me when I was having a hard time with my qualifying examination and for the preparation of my dissertation. Although he is one of the busiest professors in the department, he always cut out some time for me to give a word of advice and to tell many stories about "life as a professor" (which I enjoyed too much and sometimes forgot that he had many other things to do besides chatting with me).

I thank Dr. Paulo Monteiro for his encouragement and many inspirations. Dr. Monteiro gave me the concrete specimen in Chapter 6 in my first semester here in Berkeley. He also gave me an opportunity to participate in the NDE project discussed in Chapter 7. Our research group has a long history of collaboration with his group and I hope this friendship will last and grow in the future.

Dr. Kurt T. Nihei has been my "unofficial" research advisor for years. I do not know how I can thank him enough. Since the time I joined the group, he has been my mentor, 
unofficial advisor, and friend. He is credited for many ideas presented in this thesis. $\mathrm{He}$ and his wife Leigh treated me as if I was their younger brother when we were off work. Skiing, camping, Christmas,... there are countless fun memories with them.

The "boss", Dr. Larry Myer, has been supporting me since I joined the LBL as a graduate student research assistant. He has been traveling around the US (and sometimes around the world) all the year to keep us going. In our regular group meetings, Dr. Myer has always given me very encouraging comments as well as observations that reveal flaws in my results.

I have been fortunate to be able to work with such bright and nice people during the years of my graduate study. Dr. Galen Hesler has given me many insights to the analysis of the seismic wave propagation discussed in this thesis. He is also the only person among the group who speaks in Japanese, which is my own language. The number of glasses of beer I had must be the largest with him. I should also thank his mother, Michiko Hesler, for her great home-made dishes and cookies we enjoy every time he goes to see her during the weekend. Dr. Deborah Hopkins, who I recently found shares a lot of research interests in common with me, has always encouraged me when I have been working on the dissertation. Her "care pack" during the Christmas and New Year days helped me a lot to stay in the office and make progress in writing. We have a great tradition of celebrating each member's birthday in our group (This tradition, as long as I know, was started by Dr. Cook). We always saw Dr. Peter Persoff, Dr. Jill Geller, Dr. Tim Kneafsey, Dr. Sharon Borglin, Dr. Qinhong (Max) Hu among the crowd, singing the birthday song and enjoying the cake cut by a "birthday boy" or a "birthday girl". Dr. Patty Seifert sometimes cooked and brought a cake by herself. Weidong Yi and Jianli Fang joined the group a little before me. I have learned many new things about the computations for seismic wave propagation through their presentations in the group meetings. Brad Bessinger, who probably is the only student doing the rock mechanics in a classical sense, always gave me advice enlightened by his wide knowledge of geology. In the office, our "natural born entertainer" Pascual Benito often came up with a great joke and the other office mates Pingan Huang, Grace Su and I laughed so loud that everybody in the building could hear us. Mike Kowalsky joined us a year ago. Driven by his young active mind, he keeps himself busy both at work and play. His name should be on the list of the thesis committee members since he did the proof-reading for me as well.

Among those who have left our group, I would like to offer special thanks to Dr. 
Roberto Suárez-Rivera for his friendship and valuable insights to research. I am sorry that he moved out of the state to work for a rock mechanics testing company (luckily for them). Dr. Brun Hilbert ("Dr. Sardonic") inspired me with his wealth of knowledge in computational mechanics and with witty conversations during our daily coffee breaks. Taylor Hwong, who now is serving in the Army in Katal, showed me how to do the laboratory rock tests. During many weekends, we worked in the lab together coring rock samples and crushing them in the load frame (and played computer games!).

As a graduate student, financial support has always been a major concern. Especially because, being an international student, I had to pay an incredibly expensive out-of-state tuition fee each semester. For the first two years, the Ishizaka Foundation Fellowship in Japan generously supported me, which also gave me an opportunity to apply for the graduate school here in Berkeley. Later, with Dr. Cook's recommendation, I was supported by the Jane Lewis Fellowship for one year.

In the Mineral Engineering Department on campus, Wanda Capice has always been kind to me to figure out solutions for my financial problems and our research group's management problems since the illness kept Dr. Cook away from the campus. Carol James went through a lot of trouble when I once applied for the filing fee status and then withdrew it.

Back in the country I came from, I want to thank Dr. Ohnishi for his personal support for me and for recommending me to the graduate school in Berkeley. In a sense I have been following his steps since he also graduated from Berkeley 25 years ago after studying rock mechanics. Although he is back in the U.S. now, Dr. Hans Herda helped me so much when I was applying for graduate school in Japan. His optimism and encouragement were what I really needed at the time to soothe my anxiety for the future. I also give thanks to my brother Kenji, to parents Kohji and Sadako and grandparents for showing an understanding for my leaving the country and pursuing my graduate study overseas.

Finally, I want to thank my long-time friend Keizo Kitagawa. He is the person who inspired me to have my graduate study in this country. It has turned out to be the best decision that I ever made but was a hard decision at the time. However, he made it easier by showing me an example: he himself completed four years of study and training in the U.S. Naval Academy immediately before I started graduate studies here.

I am so grateful that I have come to know and make friends with so many wonderful 
people during the five years of my graduate study in Berkeley. Perhaps, they are the most precious things I have achieved since I came to this country, even more valuable than the knowledge and skill I have obtained in my field of research, and this dissertation itself.

Members of Rock Mechanics Lab (most of them)
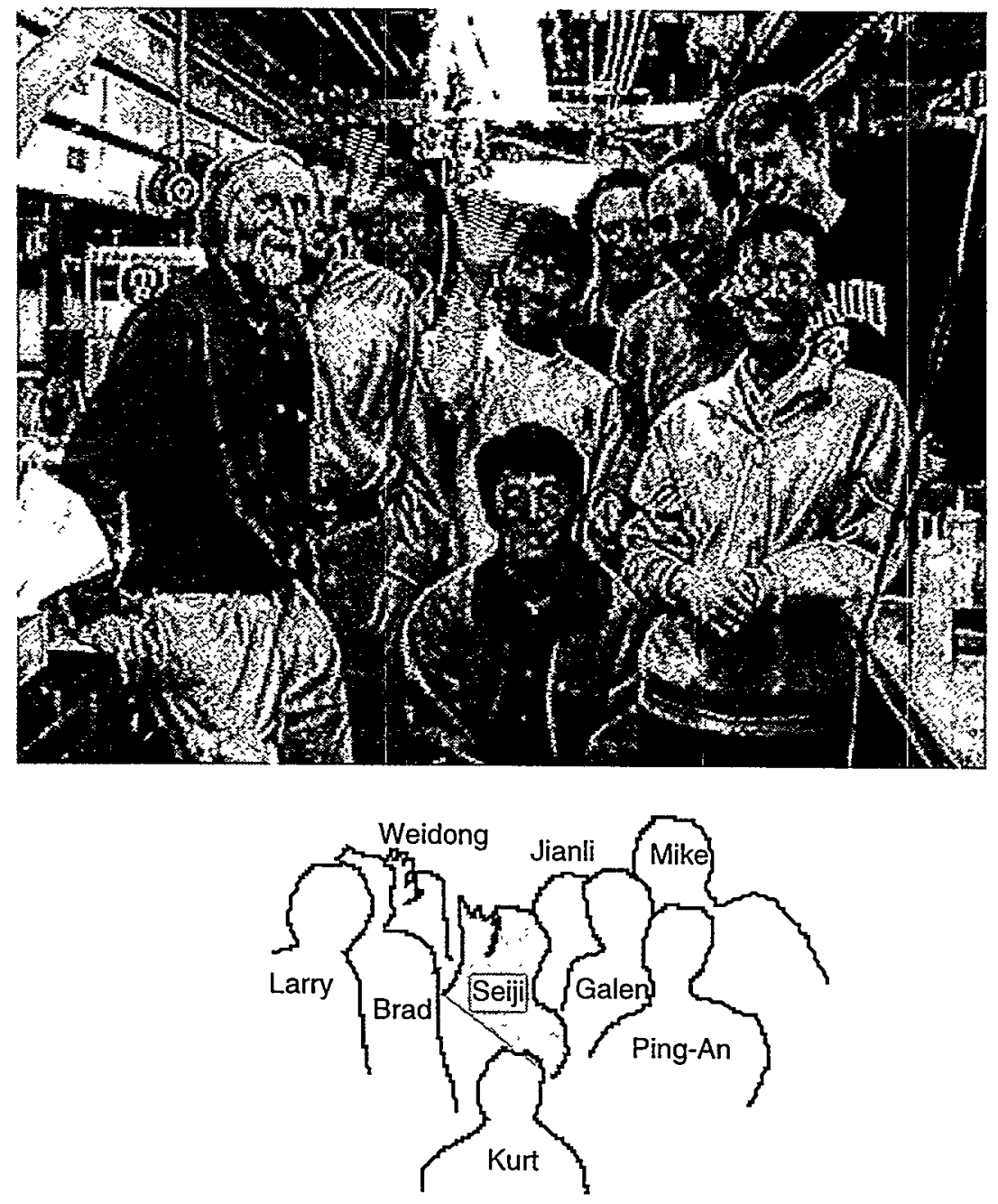


\section{General Introduction}

\subsection{Introduction}

The mechanical and hydraulic properties of rock and concrete are significantly influenced by fractures that range in size from millimeters in a single crystal and mineral grains in rock to meters in infrastructure such as tunnels, buildings, and bridges. Fractured materials typically exhibit decreased stiffness and strength. Slip along a fracture may cause a catastrophic failure of a structure. A fracture also serves as a major conduit of fluid. A large number of fractures in a reservoir rock can be a source of hydrocarbon production. Cracking in a reinforced concrete structure may result in a short service life as corrosive materials (water, oxygen, chloride ion, etc.) can easily access the steel reinforcement.

For a variety of reasons mentioned above, there is a great deal of interest in detecting and characterizing fractures in rock and concrete. Most of the fractures that affect the mechanical and hydraulic properties of materials are fully or partially open. Such fractures are seismically visible depending on their compliance that arises from locally enhanced deformation of the medium around the fracture (e.g., Pyrak-Nolte et al., 1990a). 
Conventionally, propagating seismic waves have been used for detecting and characterizing fractures. A propagating wave interacting with a fracture exhibits changes in velocity, amplitude, spectral content, and direction of propagation. In most cases, the firstarriving part of the observed waves is of interest, as relatively straightforward interpretation can be made on the effect of the fracture on the characteristics of the wave. However in many cases a wave measured in a medium including fractures contains complicated laterarriving waves (seismic coda) that are expected to carry additional information about the internal structure of the medium. This coda is essentially reverberation generated by acoustic resonances within the medium due to waves being multiply reflected by fractures. Therefore, an understanding of the acoustic resonance behavior of media containing fractures is of great value for detection and characterization of fractures.

Acoustic resonance is a phenomenon wherein the dynamic response of a material becomes significantly amplified under certain physical conditions. The amplified response occurs for discrete frequencies (resonance frequencies) that constitute the "sound" or "tone" of the medium if they are in audible range. Acoustic resonance is used for examining the properties of materials and structures in daily life. For example, a physician taps on a patient's chest to examine his or her physical condition from the sound in the chest cavity. A worker at a ceramics factory tries to determine if a piece of china contains any invisible flaws from the tone it makes. These practices are based on the knowledge that changes in material properties and structure result in different resonant characteristics. Qualitative diagnoses are made by "hearing" the changes in the sound.

In recent years, many attempts have been made to use acoustic resonance as a quantitative tool for determining material properties and diagnosing defects in both engineering and geologic structures. A large number of infrastructures such as buildings, bridges, and dams are suffering structural damage due to fractures caused by their extended service time and natural seismic activities. For example, according to the statistics released by the Federal Highway Administration, $24 \%$ of the nation's bridges ( $7.5 \%$ in California) were structurally deficient in the year of 1989 (Tarricone, 1990). Although the statistics are now becoming old, the ratio of deficient structures is expected to have risen rather than dropped during the past decade. Techniques based on acoustic resonance are among the most promising as a cost effective non-invasive inspection method for assessing damage and performance of the infrastructure. Resonant characteristics of structures have been examined for deterioration of bridges (e.g., Mazurek et al., 1990) and dams (e.g., Olson et al., 1990). On the other hand, locating and characterizing fractures in rock mass are also 
important in petroleum engineering, groundwater hydrology, and civil engineering. Geologic structures often manifest themselves as thin slabs or blocks compartmentalized by subparallel and intersecting fractures (Chernyshev and Dearman, 1991). Resonance of such structures potentially provides valuable information about the geometry and mechanical properties of these blocky rock masses.

The primary objective of this research is to understand the acoustic resonance characteristics of fractured rock and concrete using a combination of laboratory testing and numerical modeling. A fracture introduces an internal boundary that scatters and distorts propagating waves. This distortion results in altered resonance frequencies and mode shapes of the fractured rock mass.

For propagating waves, a fracture acts as a frequency-dependent filter that preferentially passes low-frequency waves and reflects high-frequency waves (Schoenberg, 1980; Pyrak-Nolte et al., 1990a). Many researchers have shown that a simple set of boundary conditions called the displacement-discontinuity boundary conditions can be used for modeling the dynamic behavior of a fracture. The applicability of the model was demonstrated numerically by Angel and Achenbach (1985) using a dynamic boundary element method and experimentally by Pyrak-Nolte et al. (1990a). The model has been used extensively for simulating a variety of wave phenomena. Buck et al. (1982) used the model for examining the contact stiffness of fatigue cracks from transmitted and refracted ultrasonic waves. Rehbein et al. (1982) used similar techniques to estimate contact stiffness of the interface between metal couplers. Gu et al. (1996a, b) used the model for examining the waves propagating along a fracture analytically and numerically using a boundary element method. Nihei et al. (1998) examined the behavior of channel waves propagating between parallel compliant fractures analytically and experimentally. PyrakNolte et al. (1990b) examined wave propagation in regularly spaced, multiple, parallel fractures experimentally and Yi et al.(1997) simulated the anisotropic wave propagation due to the fractures using the finite difference method. For the above examples, both the stiffness of the fracture and the wave frequency showed a large effect on the behavior of propagating waves.

Hesler (1995) showed the effect of fracture stiffness on the resonances of a fractured one-dimensional system using the displacement-discontinuity model for the fracture. Hesler's results are analogous to the results obtained by other researchers including Gudmundson (1982), Kam and Lee (1992), and Man et al.(1994) for cantilever beams 
including cracks and notches: an increase in the compliance of a structure due to defects decreases the resonance frequencies and alters the mode shapes. Hesler's results are significant because they show that the localized compliance that arises from the imperfect contact between mating fracture surfaces (unlike open voids or notches like other researchers) has a large effect on the resonances of fractured media that are similar to their effect on the propagating waves.

This research extends Hesler's work to the resonance of more general systems including multiple fractures, elastic anisotropy, and three-dimensional geometry. The research includes a determination of material properties such as elastic moduli and fracture stiffness from experimentally measured resonances. Acoustic resonance is also used to assess the damage due to fractures in concrete sub-structures.

\subsection{Overview of this Work}

This research examines the resonance characteristics of media such as rock and concrete containing fractures. Following this chapter, Chapter 2 discusses the resonance characteristics of fractured one-dimensional systems. Analytical and numerical methods are employed to examine quantitatively the resonance behavior of a system including single and multiple fractures. Attenuation due to fracture is found to have different effects from dissipation of energy in an intact medium. Resonance behavior of finite and infinite, and regularly and irregularly fractured systems are compared. An infinite system shows similar resonance behavior as an equivalent finite system but the attenuation is larger due to radiation of energy into the surroundings. Resonance of an irregular system is characterized by spatial localization of vibration motion, especially for small fracture stiffnesses.

Part of the results from Chapter 2 is demonstrated by laboratory experiments on fractured rock bars in Chapter 3. Stiffness of the fracture is altered by applying axial stress to the specimens through compliant plastic rings. Application of axial stress to the fracture results in an increase in fracture stiffness, leading to an increase in resonance frequencies. A specimen with a fracture injected with water and filled with attenuative material shows an increase in attenuation for predicted resonance modes.

In Chapter 4, a numerical code that can simulate resonance of a three-dimensional object including fractures and material anisotropy is developed based on the Rayleigh-Ritz method. Spectral response of the mode (frequency response function, FRF) and mode 
shape of vibration for specified locations of the source and receiver are computed. The code can also be used for determining anisotropic elastic moduli and fracture stiffnesses from experimentally measured resonance frequencies. In subsequent chapters, the numerical techniques developed in this chapter is used to simulate experimentally observed resonances and to determine elastic properties of specimens.

One of the common characteristics of rock are its anisotropic elastic properties. Chapter 5 discusses the resonance of anisotropic rocks whose anisotropy arises from different types of rock microstructures. Both static and high-frequency dynamic behavior of the rocks are measured to characterize the specimens. The numerical code developed in Chapter 4 is used to determine the anisotropic elastic moduli of approximately transversely isotropic rocks. The resulting elastic moduli are found to range between moduli from static and ultrasonic measurements. The result may suggest a frequency dependent behavior of the stiffness of the rocks due to compliant microcracks and grain contact. Mode shapes of the resonances are also measured using a scanning laser Doppler vibrometer and their basic agreement with the normalized simulations is confirmed.

Anisotropy in granite specimens in Chapter 5 is due to uniformly distributed microcracks in rock. For this microstructure, resonance of the medium could be examined by approximating the compliance introduced by the microcracks as a reduced bulk elastic moduli. However, such an approach cannot be used if the medium contains a distinct fracture. In Chapter 6, resonance of a concrete cylinder including a single through-going fracture is examined experimentally and numerically. The stiffness of the fracture is changed to see its effects on the resonance of the specimen. Measured and simulated resonances for the fractured specimen show shifts in the resonance frequencies and localized resonances as predicted in the analyses of Chapter 2. The numerical code is used to determine the dynamic stiffness of the fracture from measured changes in resonance frequencies. The inverted dynamic normal stiffnesses of the fracture show good agreement with statically measured stiffnesses.

To apply the acoustic resonance technique to assess damage and the effect of repair of civil infrastructure, field resonance measurements on semi-site scale concrete bridge columns are performed in Chapter 7. High-frequency stress wave measurements reveal the distribution and difference in orientation of fractures in the directions normal and perpendicular to the loading direction. However, due to the strong sensitivity of the wave to compliant fractures, the repaired structures do not always show changes in their seismic 
signatures even though the stiffness and ultimate strength of the structures are significantly improved. Measured resonance frequencies and attenuation show good correlations with the measured changes in the static stiffness before and after the destruction and repair of the structures. Dynamic stiffnesses of the structures are determined from measured resonance frequencies and show good agreement with the statically measured stiffnesses. The results demonstrate that the resonance of a fractured structure is a good indicator for assessing the degree of damage and the effect of repair.

Throughout this research, the stiffness of a fracture plays an important role in determining the dynamic behavior of materials and structures. Therefore it is important to understand the fundamental properties for the stiffness of a fracture. In Chapter 7, the dynamic behavior of a fracture subjected to shear stress is examined using propagating seismic waves. Although the discussion is focused on propagating waves, these results are equally important for the resonance of a medium including such fractures. A novel discovery is made for conversion between $\mathrm{P}$ - and S-waves normally incident on a sheared fracture during laboratory experiments. The observed conversions of waves are due to dynamic dilation of fracture whose local contact stiffness is systematically redistributed by an applied shear stress. The dilation behavior can be modeled by cross-coupling components of fracture stiffness that are used for the displacement-discontinuity boundary conditions. Transmission and reflection of seismic waves across a sheared fracture are examined analytically and numerically using a dynamic two-dimensional boundary element method. The results demonstrate distinct changes in the waves interacting with a sheared fracture that can be used for detecting and potentially measuring shear stress on a fracture.

Beyond the seismic waves incident on a sheared fracture in Chapter 8, Chapter 9 discusses the behavior of interface wave propagation along a sheared fracture. A generalized dispersion equation is derived for fracture stiffness with dilation components (coupling fracture stiffnesses). The introduction of the coupling fracture stiffness changes the phase velocities and particle motions for two possible types of fracture interface wave. The changes in the particle motion are clearly visible as a phase lag between waves on opposite sides of the fracture. The observed behavior of the fracture interface wave can be used for detecting and characterizing shear stress on a fracture.

Chapter 10 discusses wave propagation in media that contain parallel, regularly-spaced fractures. Unlike the discussion in Chapter 1 where only the one-dimensional case is treated, a general dispersion equation for fully coupled P-SV-SH waves is presented. 
Waves propagating in the fractured system exhibit transversely isotropic behaviors due to a large compliance in the direction normal to the fractures as is seen from the experimental results in Chapter 5. Fractures in the system can have coupling stiffnesses as discussed in Chapter 8. When the wavelength of the wave propagating in the system is comparable to or shorter than the fracture spacing, both velocity surface and spectrum of the wave exhibit distinct pass and stop band structures for the propagation direction normal to the fractures. Such behavior is analogous to the results for one-dimensional systems (Chapter 1). Within a stop band, the wave amplitude decays exponentially away from a source. As a result, waves propagating parallel to the fractures localize near the source. At boundaries between pass and stop bands, group velocity in the normal direction approaches zero, indicating that the system is at resonance. It is shown that the general dispersion equation degenerates to the SH-plate wave equation and Rayleigh-Lamb plate wave equations (Graff, 1975) for vanishing fracture stiffness, and to a generalized interface wave equation that includes results in Chapter 9 if the layer thickness is much greater than wavelength.

The final chapter summarizes important findings and conclusions in this thesis. Suggestions for possible future research are provided as well. 


\section{Analytical Study on the Effects of Fracture Properties on One-Dimensional Resonance}

\subsection{Introduction}

This chapter examines the acoustic resonance of one-dimensional systems containing single and multiple fractures using analytic and semi-analytic methods. One-dimensional resonance is often encountered in real situations. Some of the examples are the resonant bar tests used for testing materials in laboratory, vertical vibration of structural beams, reflections of seismic pulses in the impact-echo tests (Carino et al., 1986), and multiple reflection of seismic waves normally incident on layered geological structures (Banik et al., 1985a,b; Burridge et al., 1988). Understanding the resonance behavior of fractured onedimensional systems is not only beneficial for interpreting the resonance of the above examples but also provides valuable insights to the resonance of more complicated multidimensional systems.

The objective of this chapter is to develop basic understandings of the effects of single and multiple fractures on the resonances of one-dimensional systems. The primary effects are the shift of resonance frequencies, change in attenuation, and change in mode shapes 
that can lead to localization of vibration energy. These effects are related to boundary conditions and mechanical and geometric properties of fractures such as stiffness, viscosity (due to fluid and filling), number, and locations.

Dynamic behavior of a fracture has been modeled by the displacement-discontinuity boundary conditions (Kendall and Tabor, 1971; Schoenberg, 1980; Pyrak-Nolte et al., 1990a). The model has been successfully used for analyzing wave propagation across and along a compliant interface between solid halfspaces. Frequency response functions (FRF's) of fractured systems were derived by a one-dimensional version of the propagator matrix method (Kennett, 1983). Resonance in a one-dimensional system can be treated in a relatively straightforward manner due to lack of mode conversions between propagating waves (e.g., P-wave, S-wave, and surface wave). A concise summary of the propagator method is given in Appendix A.

In the following discussion, the resonance of a finite system including fracture(s) is first examined, followed by that of an infinite system. The relation between spectral characteristics of waves propagating in the fractured infinite systems and resonance characteristics of their finite counterparts are compared.

\subsection{Finite System Including Single Fracture}

\subsubsection{Resonance frequency shift}

The type of frequency response functions (FRF's) used in this chapter is the mobility, defined as the velocity response of a system against unit force excitation (e.g., Newland, 1989). A dimensionless mobility is defined by multiplying the mobility by the acoustic impedance of the system. For a slender bar including a single fracture (Figure 2.1), an analytic expression for the power of dimensionless source mobility (both source and receiver located at the top) is derived as

$$
M M^{*}=\frac{\left[2 \cos \phi_{0} \cdot \sin \phi_{1}-\beta \cos \left(\phi_{0}+\phi_{1}\right)\right]^{2}}{\left[2 \sin \phi_{0} \cdot \sin \phi_{1}-\beta \sin \left(\phi_{0}+\phi_{1}\right)\right]^{2}},
$$

where $\omega$ is the angular frequency. The derivation of Eq.(2.1) is shown in Appendix B. $\beta$ is the dimensionless acoustic impedance of a fracture defined by

$$
\beta=\frac{2 \kappa / \omega}{Z}
$$




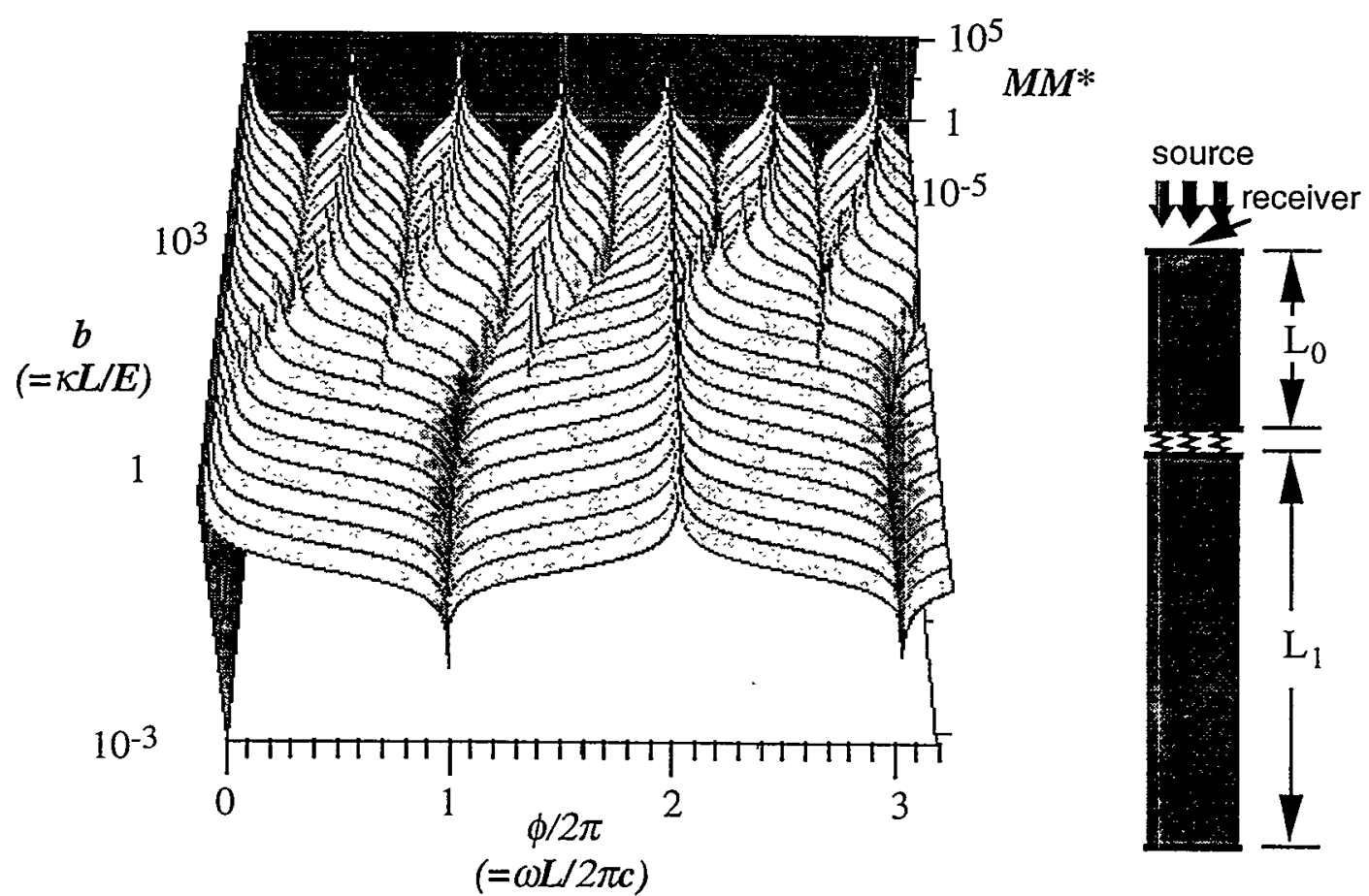

(a) FRF on the 1st segment (source-side)

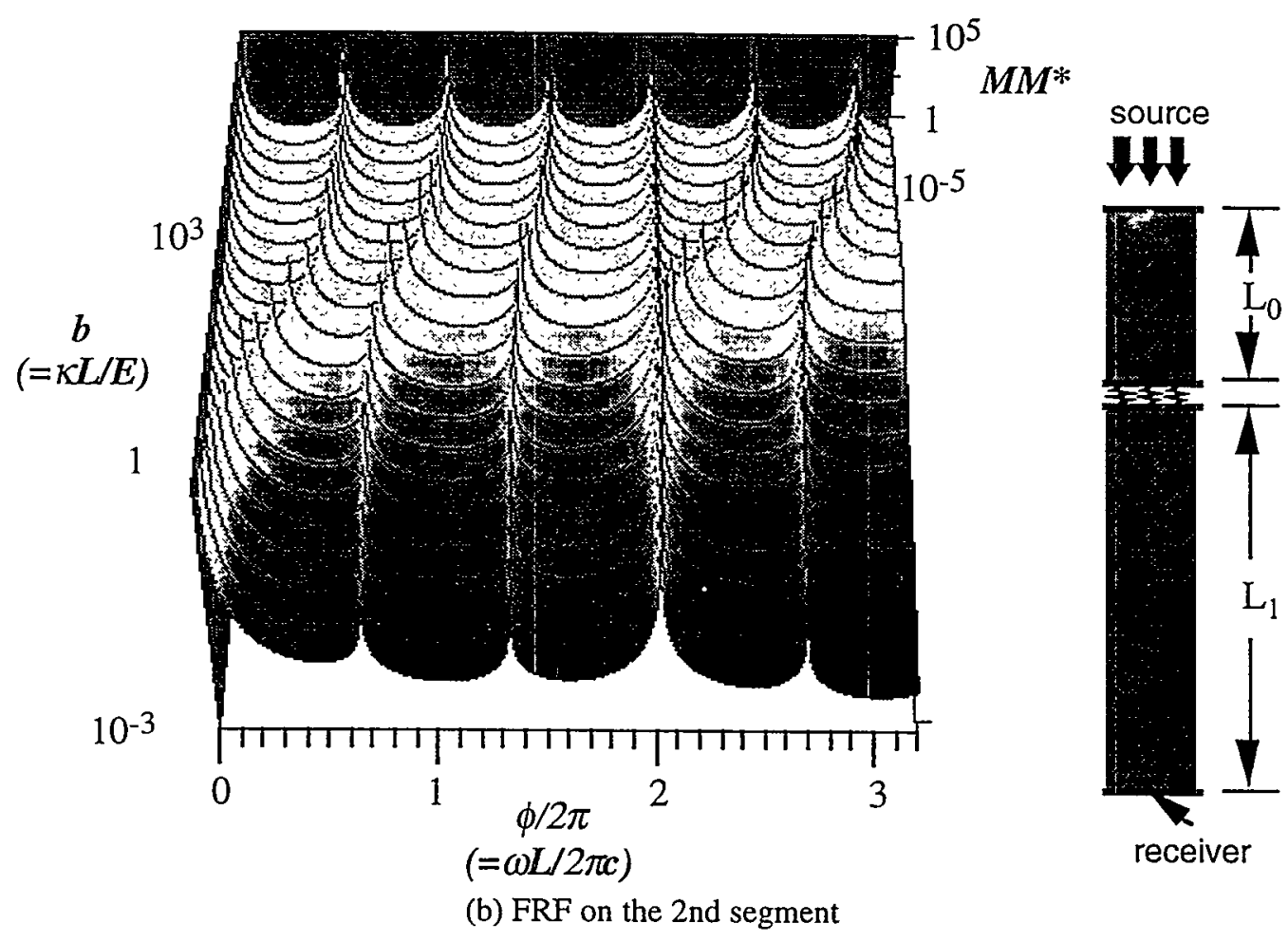

Figure 2.1 Waterfall plot of FRF(Mobility)'s for a finite bar with a single fracture. The fracture separates the bar by a ratio of 1:3. Both frequency and stiffness are dimensionless in the plots. (a) In the first segment $\left(\mathrm{L}_{0} / \mathrm{L}=0.25\right)$, several of the resonance peaks disappear as the fracture stiffness is decreased. (b) There is no vanishing of resonance peaks in the second segment $\left(L_{1} / L=0.75\right)$. 
where $K$ is the specific fracture stiffness and $Z$ is the acoustic impedance of the intact medium, and $\phi_{0}$ and $\phi_{1}$ are the phase angles defined by

$$
\phi_{0}=\omega L_{0} / c, \phi_{1}=\omega L_{1} / c,
$$

where $L_{0}$ and $L_{1}$ are the lengths of the first and second segments in the bar, and $c$ is the velocity of the wave in the intact medium. Mobility at the bottom of the bar (receiver located at the bottom) is given by (derivation shown in Appendix B)

$$
M M^{*}=\frac{\beta^{2}}{\left[2 \sin \phi_{0} \cdot \sin \phi_{1}-\beta \sin \left(\phi_{0}+\phi_{1}\right)\right]^{2}} .
$$

For a high fracture stiffness, $\beta$ in Eq.(2.1) approaches infinity. Resonance occurs at frequencies that satisfy $\sin \left(\phi_{0}+\phi_{1}\right)=0$. Therefore,

$$
\omega_{\text {high }}=\frac{c}{L} \cdot n \pi \quad \mathrm{n}=0,1,2, \ldots
$$

where $L=L_{0}+L_{1}$. If the stiffness of the fracture is low, resonances of the first segment occur for $\sin \left(\phi_{0}\right)=0$, while the second segment resonates both for $\sin \left(\phi_{0}\right)=0$ and $\sin \left(\phi_{1}\right)=0$. Corresponding resonance frequencies are

$$
\begin{array}{ll}
\omega_{\text {low }}=\frac{c}{L_{0}} \cdot n_{0} \pi, & \mathrm{n}_{0}=0,1,2, \ldots \text { (1st segment) } \\
\omega_{\text {low }}=\frac{c}{L_{0}} \cdot n_{1} \pi \text { or } \omega_{\text {low }}=\frac{c}{L_{1}} \cdot n_{2} \pi . & \mathrm{n}_{1}, \mathrm{n}_{2}=0,1,2, \ldots \text { (2nd segment) }(2.6 \mathrm{~b})
\end{array}
$$

This result is particularly interesting because the second sets of solutions in (2.6b) indicate that these modes are localized in the second segment of the bar. Figure 2.1 illustrates the resonance behavior of a system consists of two segments separated by a fracture with length ratio of $L_{0}: L_{1}=1: 3$. The plot shows several modes that vanish in the first segment for small fracture stiffnesses.

From Figure 2.1 it can be seen that the shift in the resonance frequency of each mode occurs for a limited range of fracture stiffnesses. The center of the shift can be examined by a "center resonance frequency" $\omega_{c}$ defined as follows 


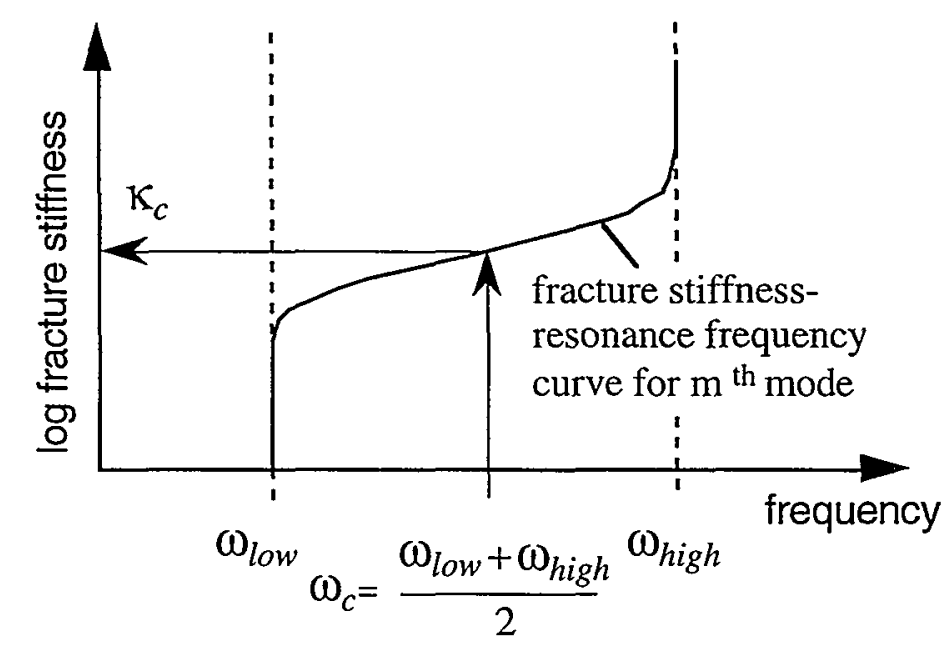

Figure 2.2 The center resonance frequency, $\omega_{c}$, is defined by an average of resonance frequencies for an infinitely stiff fracture and a zero stiffness fracture. The center fracture stiffness, $\kappa_{c}$, is defined as a corresponding stiffness of the fracture stiffness-resonance frequency type curve of the mode.

$$
\omega_{c}=\frac{\omega_{\text {high }}+\omega_{\text {low }}}{2}, \omega_{\text {high }}>\omega_{\text {low }},
$$

where $\omega_{\text {high }}$ and $\omega_{\text {low }}$ are the resonance frequencies for infinitely high and low fracture stiffnesses, respectively. The corresponding "center fracture stiffness" $\kappa_{c}$ and impedance ratio $\beta_{c}$ can be determined from a fracture stiffness-resonance frequency type curve for each mode (Figure 2.2).

To understand the effect of the location of a fracture on resonance frequencies, a finite 1-D system of length $L$ divided by a fracture into two segments is examined. The length ratio between the segments is $L_{0}: L_{1}=\mathrm{m}_{0}: \mathrm{m}_{1}$ where $\mathrm{m}_{0} / \mathrm{m}_{1}$ is assumed to be a rational number. An intact bar of length $L$ and the separate segments (length $L_{0}$ and $L_{1}$ ) have common resonance frequencies only at $\omega_{\text {low }}=c / L M \pi \cdot n \quad\left(\mathrm{n}=0,1,2, \ldots ; \quad M=\mathrm{m}_{0}+\mathrm{m}_{1}\right)$ corresponding to every $\mathrm{M}^{\text {th }}, \mathrm{m}_{0}{ }^{\text {th }}$, and $\mathrm{m}_{1}$ th modes, respectively. As the stiffness of the fracture decreases, the first $M$ resonance frequencies of the initially intact bar (mode 0 to mode $\mathrm{M}-1$ ) decrease and become the first $\mathrm{m}_{0}$ and $\mathrm{m}_{1}$ modes of the separated segments. These frequency shifts repeat every $\mathrm{M}^{\text {th }}$ mode: all the $n=i+M \cdot j(i, j=0,1,2, \ldots .)^{\text {th }}$ modes shift by an equal amounts as the stiffness of the fracture decreases from infinity to zero. For example, if a fracture is introduced in a bar such that $L_{0}: L_{1}=3: 7$, the resulting center frequencies are obtained as in Table 2.1. In this table, only $\omega_{c}$ 's for $j=0$ are shown because the shift of resonance frequencies repeat cyclically for larger $j$ 's. The last column 
in the table yields different $\beta_{c}$ 's. Therefore, there are 10 different types of modes that are distinguished by characteristic $\beta_{c}$ 's. The resulting $\kappa_{c}$ 's (dimensionless) and $\beta_{c}$ 's are plotted against dimensionless $\omega_{c}$ in Figure 2.3. This result also implies that if a fracture separates a bar into segment lengths with a ratio that is not a rational number, an infinite number of $\beta_{0}$ 's result for the resonances of the system.

Table 2.1 Computation of Center Resonance Frequency

\begin{tabular}{|c|c|c|c|c|}
\hline $\mathrm{m}$ & $\begin{array}{c}\text { Intact bar } \\
\phi=\omega_{\text {high }} L / c\end{array}$ & $\begin{array}{c}\text { Block No.1 } \\
\omega_{\text {low }} \cdot L / c\end{array}$ & $\begin{array}{c}\text { Block No.2 } \\
\omega_{\text {low }} \cdot L / c\end{array}$ & $\omega_{c} \cdot L / c$ \\
\hline 0 & 0 & $*$ & $*$ & $*$ \\
\hline 1 & $\pi$ & $(0)$ & $(0)$ & $\pi / 2$ \\
\hline 2 & $2 \pi$ & $10 \pi / 7$ & $*$ & $12 \pi / 7$ \\
\hline 3 & $3 \pi$ & $20 \pi / 7$ & $*$ & $2 \pi+13 \pi / 14$ \\
\hline 4 & $4 \pi$ & $*$ & $10 \pi / 3$ & $2 \pi+5 \pi / 3$ \\
\hline 5 & $5 \pi$ & $30 \pi / 7$ & $*$ & $4 \pi+9 \pi / 14$ \\
\hline 6 & $6 \pi$ & $40 \pi / 7$ & $*$ & $4 \pi+13 \pi / 7$ \\
\hline 7 & $7 \pi$ & $*$ & $20 \pi / 3$ & $6 \pi+5 \pi / 6$ \\
\hline 8 & $8 \pi$ & $50 \pi / 7$ & $*$ & $6 \pi+11 \pi / 7$ \\
\hline 9 & $9 \pi$ & $60 \pi / 7$ & $*$ & $8 \pi+11 \pi / 14$ \\
\hline
\end{tabular}

Note: The fundamental mode for an intact bar becomes one of the rigid body modes $\left(\omega_{\text {low }}=0\right)$ for separate segments. Center resonance frequencies cannot be obtained for $m=0$ (or $n=10 \cdot j, j=0,1,2, \ldots$ ) th modes as they do not show a resonance frequency shift.

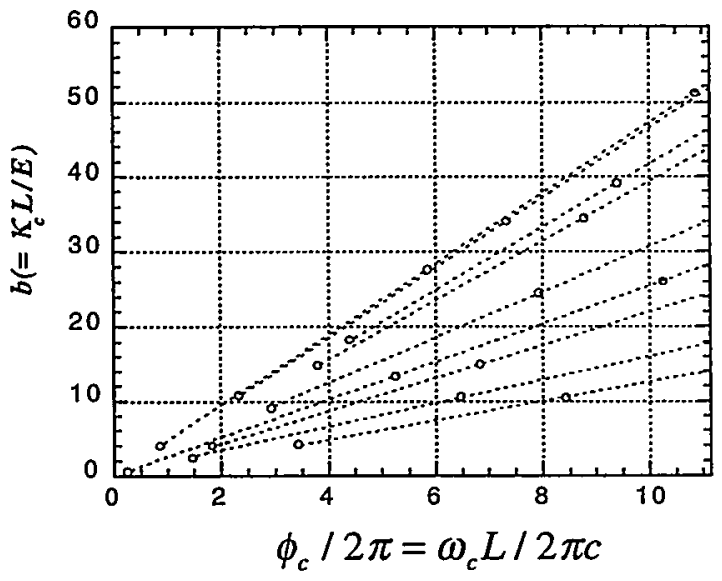

(a) $\kappa_{\mathrm{c}}-\omega_{\mathrm{c}}$

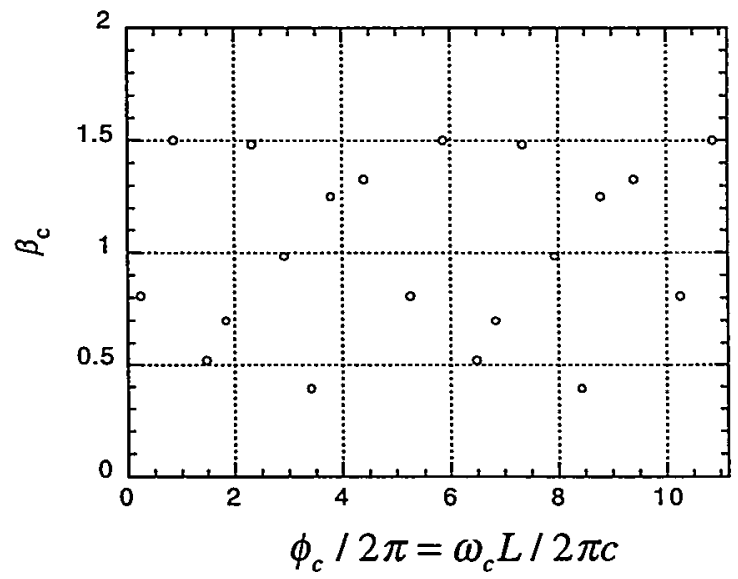

(b) $\beta_{c}-\omega_{c}$

Figure 2.3 The (a) center fracture stiffness $\kappa_{\mathrm{c}}$ and (b) corresponding impedance ratios $\beta_{c}$ for the resonances of a fractured bar. A fracture separates the bar by a ratio of 3:7. There are 9 different $\beta_{\mathrm{c}}$ 's that yield 9 groups of modes which are distinguished by the slope of $\kappa_{c}-\omega_{c}$ plots. 
If a fracture is at the middle of the bar such that $L_{0}=L_{1}=0.5 L$, all the odd modes of an intact bar have decreasing resonance frequency with decreasing fracture stiffness and form pairs with even mode resonances. The even modes are not affected by the fracture. Mobilities for the system are plotted for a range of frequencies and locations along the bar in Figure 2.4. The dark lines in the plot show resonance peaks and the light curves show nodes. Stiffness of the fracture is made dimensionless by the length $L$ and the Young's modulus of the system $E$ to yield a stiffness parameter $b=\kappa L / N E$, where $N$ is the number of fractures in the system (here, $N=1$ ). Center resonance frequencies can be obtained only for the odd modes as

$$
\omega_{c}=\frac{c}{L} \cdot\left(2 k+\frac{1}{2}\right) \pi \quad(\mathrm{k}=0,1,2 \ldots) .
$$

From Eq.(2.4) and Hesler's (1995) work, the following equation must be satisfied at resonance

$$
\beta=\frac{2 \sin \phi_{0} \cdot \sin \phi_{1}}{\sin \left(\phi_{0}+\phi_{1}\right)}, \quad \beta \geq 0 .
$$

For the case considered here, $\phi_{0}=\phi_{1}$ from Eq.(2.3). By introducing Eq.(2.8) into (2.9), it is found that the resulting $\beta$ 's are identical for all modes $\left(\beta_{c}=1.0\right)$. Therefore the corresponding $\kappa_{c}{ }^{\prime}$ s are exactly proportional to the $\omega_{c}{ }^{\prime} s$.

\subsubsection{Attenuation due to material damping and fracture viscosity}

If a medium (intact part of the bar) is attenuative, it has a finite seismic quality factor $Q$. For a finite $\mathrm{Q}$, a phase term used in the propagator matrix (Appendix $\mathrm{A}$ ) becomes complex

$$
\phi=\frac{\omega z}{c}\left(1+i \frac{1}{2 Q}\right)=\frac{\omega z}{c}(1+i \zeta) .
$$

Where $\zeta$ is the vibration damping ratio (e.g., Newland, 1989). Q can be either a constant or a function of frequency. For a bar with a single fracture at the middle, the mobility distribution in the bar is computed for a variety of Q's (Figure 2.5, left half). The plot shows that both even and odd mode resonances attenuate (width of the peak broadens) as the attenuation of the system increases. 


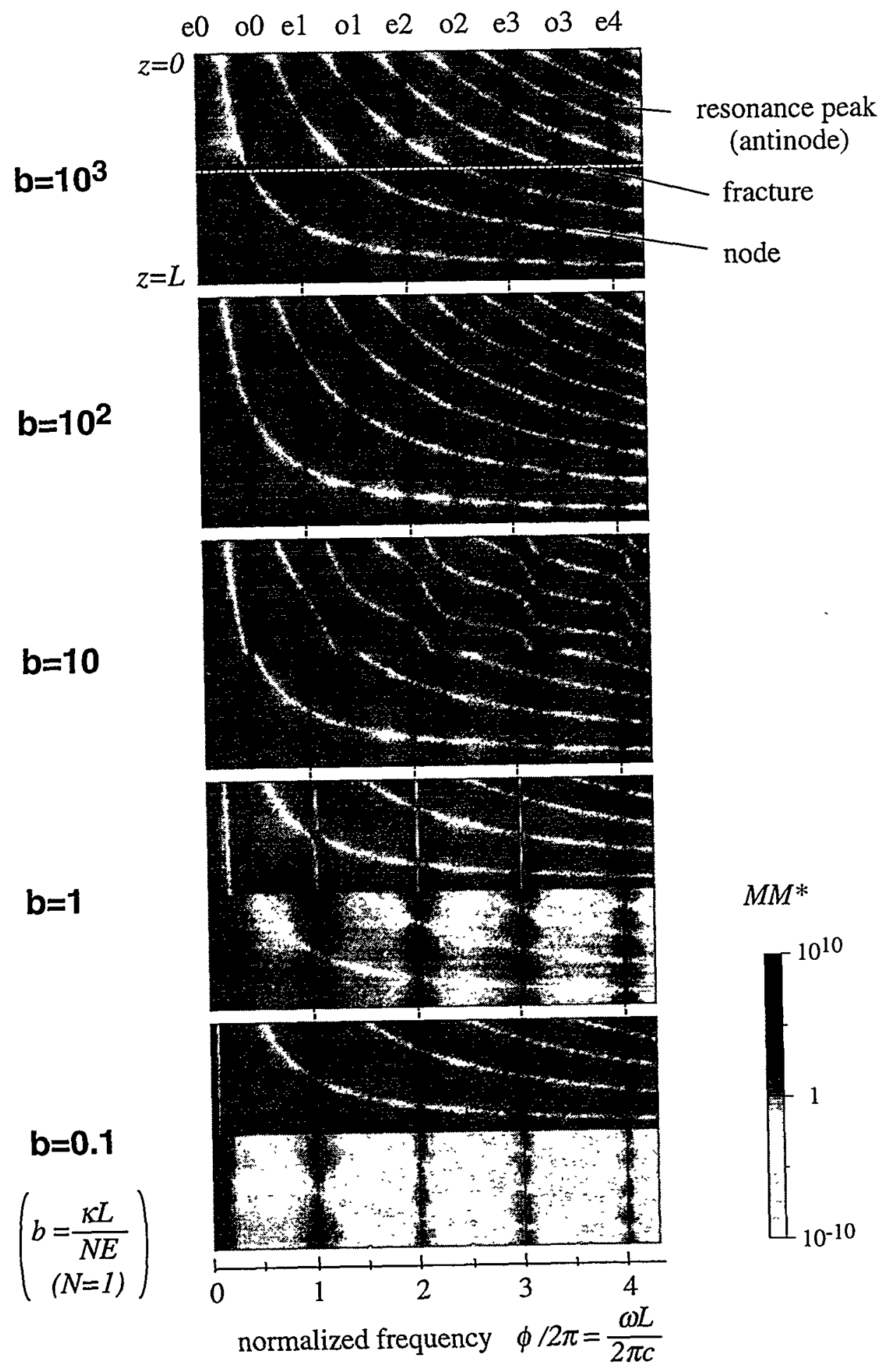

Figure 2.4 Computed mobility distribution in a finite bar with a single fracture at the middle. Dark lines show high amplitude (resonance) and the light curves show low amplitudes (nodes). As the fracture stiffness decreases, the resonance frequencies of the odd modes decrease while the even modes do not shift. 
If fluid is present in the fracture, attenuation of resonance occurs as a result of viscous shear within the fracture. For a clean fracture with partial surface contact surrounded by fluid, an adequate rheological model would be the Kelvin-Voigt model (parallel spring and dashpot model). Pyrak-Nolte (1990a) used the model to explain the viscoelastic effect of fluid-filled fractures on transmitted seismic waves. The effect can be examined by defining a complex specific fracture stiffness (Rokhlin and Wang, 1991)

$$
\kappa=\kappa_{r}+i \kappa_{i}=\kappa_{r}+i \omega \eta
$$

where $\eta$ is the specific viscosity of fluid. In many cases, however, $\eta$ may not correspond to the intrinsic viscosity of the fluid. This is because the resonance loss mechanism of a fracture may have many causes such as damping due to a small amount of clay at the contact surface (Suárez-Rivera, 1992) and local squirting of fluid out of contacting asperities (Mavko and Nur, 1979; Dvorkin et al., 1995). Therefore, the $\eta$ in Eq.(2.11) should be considered as a structural parameter of a fracture rather than the intrinsic viscosity of the fluid. The effect of an increasing viscous component in fracture stiffness on a single fracture system is shown in Figure 2.5 (right column). Viscosity of the models is represented by the viscous relaxation time defined by a specific viscosity normalized by the real part (elastic component) of the fracture stiffness. The fundamental mode's resonance frequency in an intact system $(b=\infty)$ for these examples is assumed to be $f_{0}=\omega_{0} / 2 \pi=11.6 \mathrm{kHz}$. $\left(L=0.203 \mathrm{~m}, \rho=2470 \mathrm{~kg} / \mathrm{m}^{3}\right.$, and $\left.c=4710 \mathrm{~m} / \mathrm{sec}\right)$. Unlike an intrinsic attenuation in the matrix ( $Q$ or $\zeta$ ), a viscosity of the fracture only increases the attenuation of the odd modes. This effect can be explained by the difference in the accompanying mode shapes of the resonances (Figure 2.6). For even modes, the fracture is located on an anti-node of vibration. As the motions of the surfaces on the opposite sides of the fracture are in-phase, the fracture has no effect on the attenuation of resonance. Odd modes, on the other hand, experience the maximum effect as the fracture is located on a node where the amplitude of the opening and closing motion of the fracture is the largest.

A close examination of Figure 2.5 reveals increases in the resonance frequencies of odd modes with increasing viscosity. The changes in resonance frequencies and attenuation with increasing viscosity are examined for a range of fracture stiffnesses. For this purpose, the first and second odd modes (o1 and o2 modes, respectively) were used. In Figure 2.7(a), each type curve represents a different fracture stiffness shown by the dimensionless stiffness parameter $b$. When the stiffness of the fracture is high, an increase in viscosity increases the resonance frequencies of a system with a large fracture stiffness. 


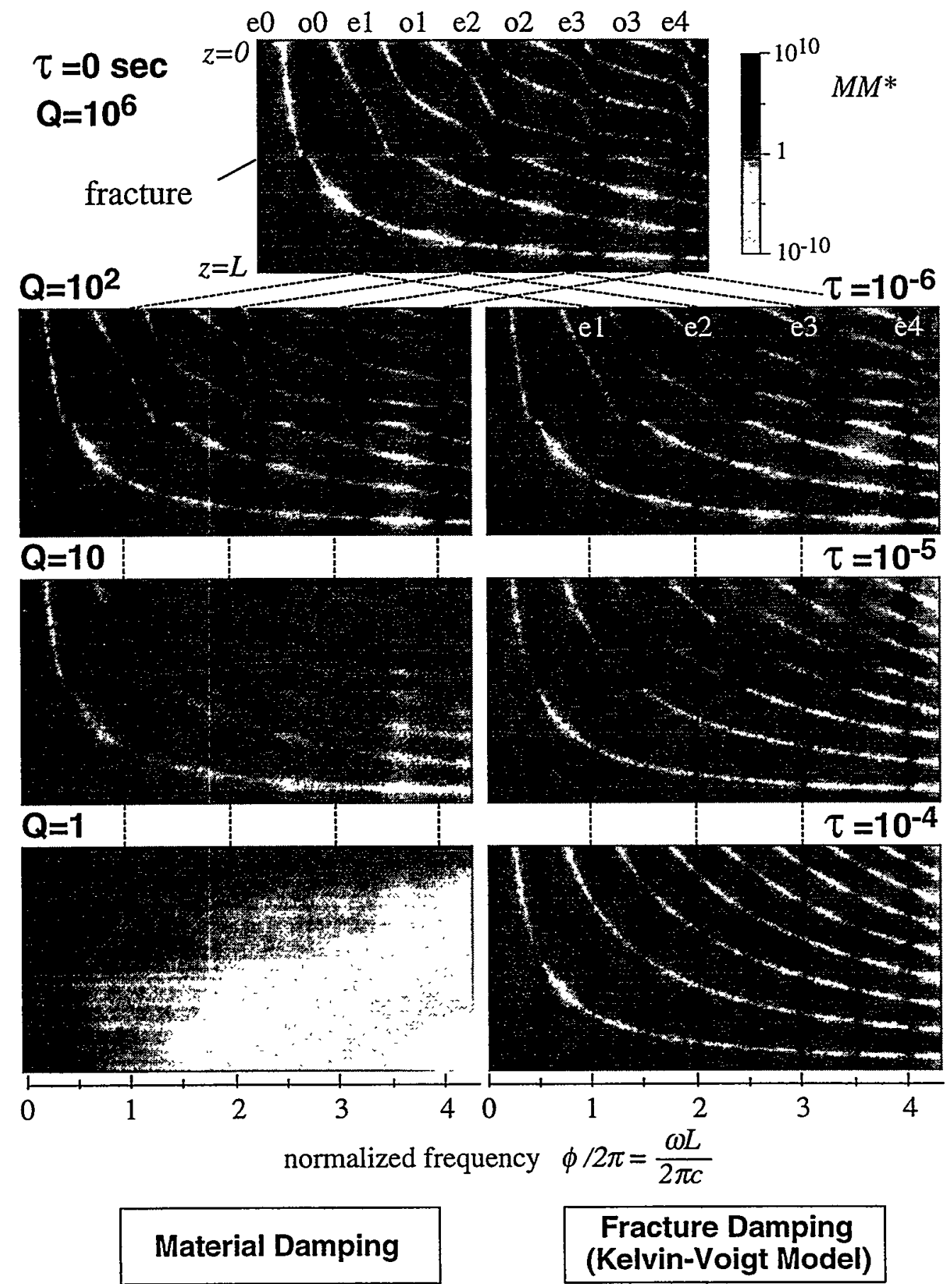

Figure 2.5 Computed mobility distribution in a finite bar with a single fracture at the middle. Fracture stiffness is constant $(b=10)$. Increase in material damping $(Q-1)$ attenuates both even and odd modes while fracture damping (Kelvin-Voigt model, viscous relaxation time $\tau=h / K$ ) attenuates only odd modes. 


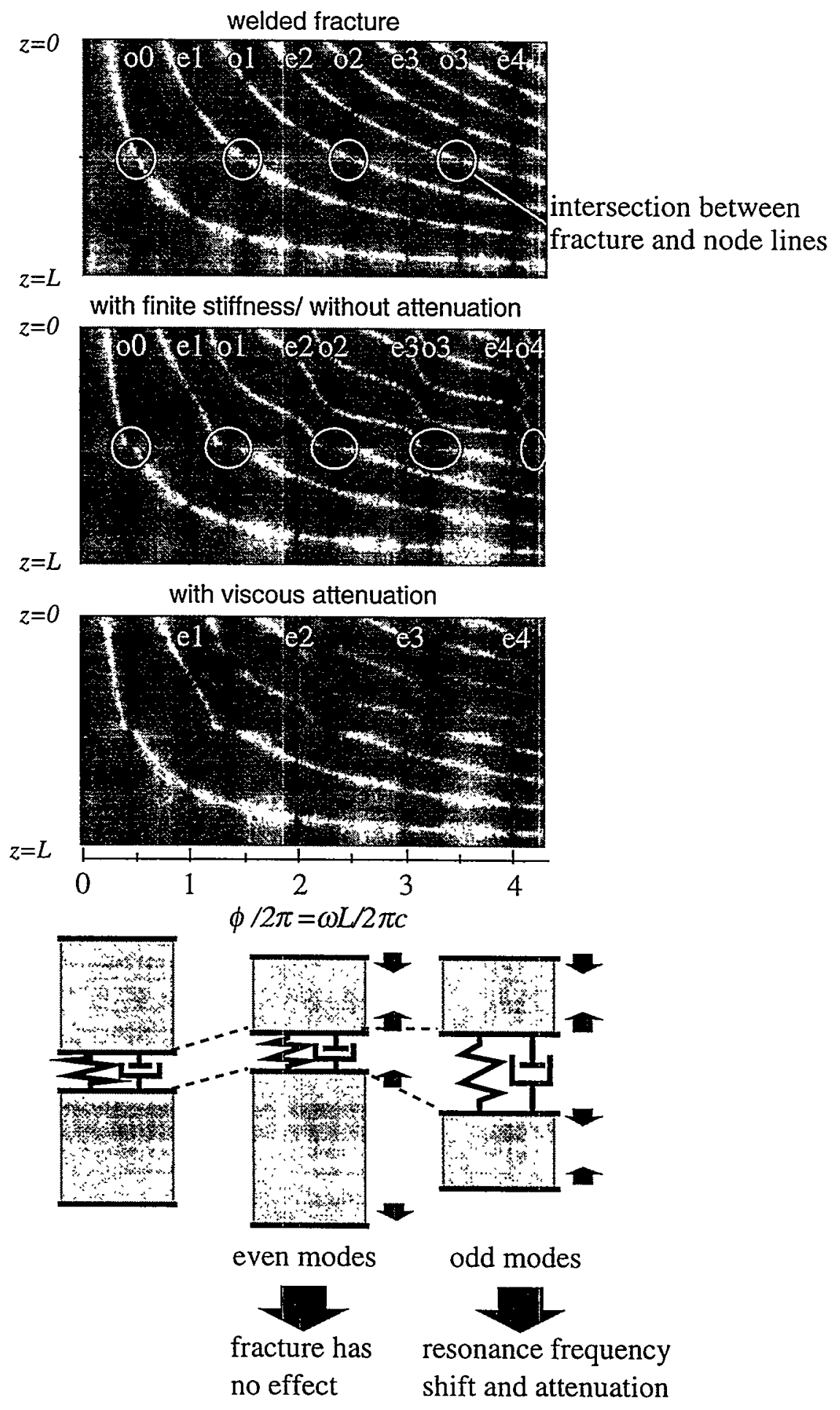

Figure 2.6 Mechanism for the selective attenuation of odd modes. For each mode pair, the odd mode attenuates as it invokes a large relative motion between the surfaces of a fracture. The even mode, on the other hand, does not cause any relative motion across the fracture as the fracture is located on the anti-node of the resonance. 

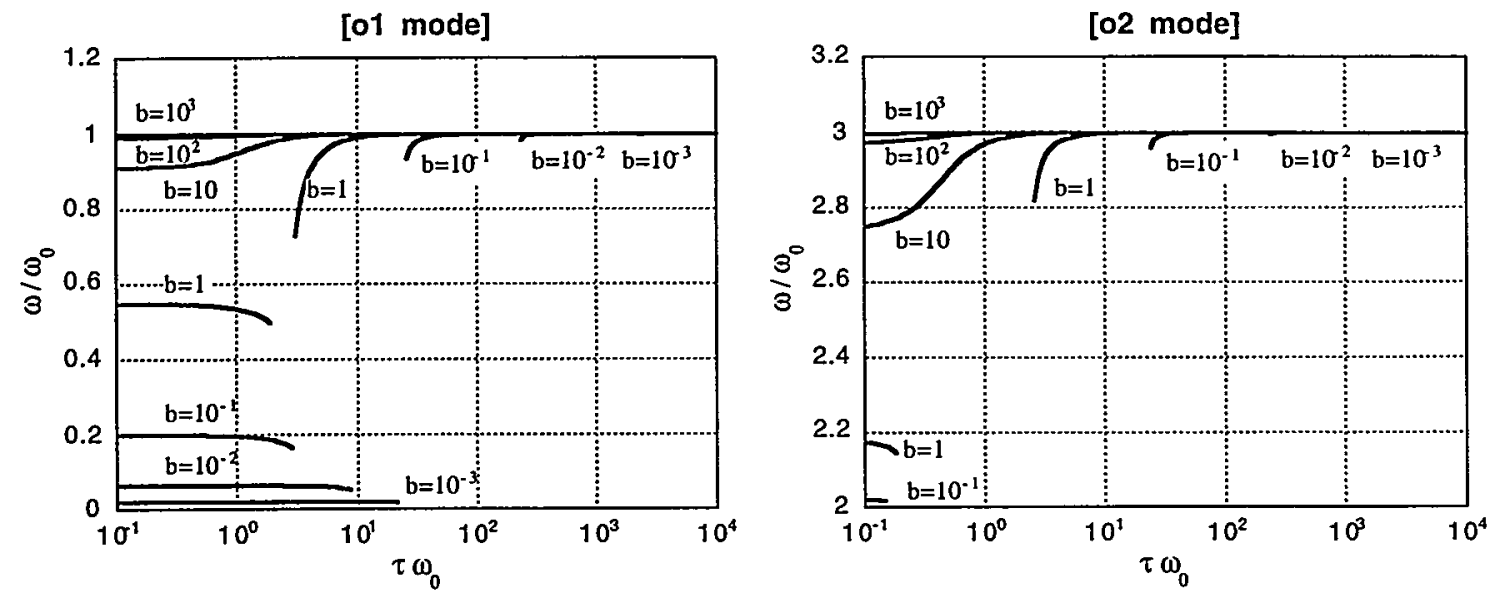

(a) Resonance frequency shift due to increase in viscosity
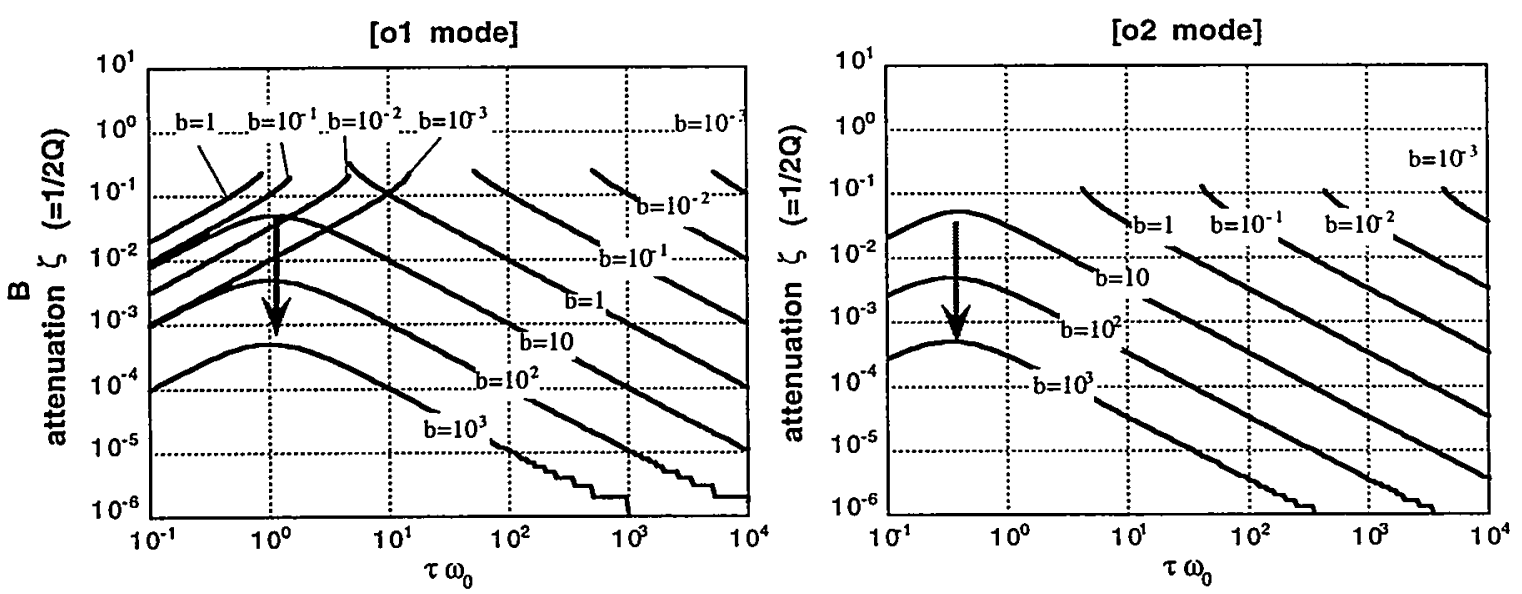

(b) Attenuation due to increase in viscosity

Figure 2.7 Effect of viscosity on the resonance frequency and attenuation of the 1st and 2nd odd modes. Resonance frequency and viscous relaxation time are normalized by the resonance frequency of the 1st mode for an intact system $\left(\omega_{0}\right)$. (a) Increase in viscosity for the high-fracture stiffness system increases the resonance frequency while it decreases the resonance frequency for the low-fracture stiffness system. (b) Attenuation maximizes at an intermediate viscosity. The location of the peaks shifts from high viscosity (large relaxation time) to low viscosity for low fracture stiffnesses. At high fracture stiffnesses, attenuation decreases without changing the peak relaxation time. 
On the contrary, resonance frequencies decrease for a system with a small fracturestiffness. Further increase in viscosity in the low fracture stiffness system results in a vanishing resonance peaks. When the viscosity becomes even larger, the resonance peak recovers. The recovered resonance shows a higher frequency than the original system. Once the peak is recovered, the resonance frequency monotonically increases with increasing viscosity.

Attenuation behavior of the single-fracture system is also shown in Figure 2.7(b). Quality factor $\mathrm{Q}$ and damping coefficient $\zeta$ are computed by the half-power method : $\mathrm{Q}$ is computed from a ratio between the resonance frequency and the bandwidth of the resonance peak with amplitude (power) greater than half-power of the peak (e.g., Newland, 1989). Each type curve shows a single peak. The slopes on both sides of the peaks are 1 and -1 for the low-viscosity and high-viscosity sides, respectively. It should be noted that the half-power method is not accurate for determining the damping coefficient when the attenuation is large (typically, $\zeta>0.1$ ) (Boubié et al., 1987).

\subsection{Finite System Including Multiple Fractures}

\subsubsection{Resonance frequency shift and mode localization}

The results obtained in the previous section are extended to a system including periodic multiple fractures. The mobility distribution in Figure 2.8 shows changes in the resonance frequencies and mode shapes in a system containing 9 fractures. Plots on the left side represent constant fracture spacing and fracture stiffness. The resonance frequency shifts in the periodic system (constant fracture spacing) with increasing fracture stiffness are shown in Figure 2.9(a). From the discussion in the previous section, the difference in the frequency shifts among the modes can be related to how close the fractures are located to the nodes of each mode. Similar to the single fracture case, each 10 modes comprise a single mode group. In these mode groups, the lowest order mode does not change resonance frequency as its anti-nodes are located exactly on the fractures (i.e., they are insensitive to changes in fracture stiffness). A group of resonance peaks is called a "pass band" and a frequency range absent of resonance between adjacent pass bands is called a "stop band" (Ziman, 1964; Hodges and Woodhouse, 1983). In a pass band, vibration can propagate far from a source without attenuating. On the other hand, vibration attenuates exponentially away from a source in a stop band. Such behavior is well known for waves propagating in a regularly stratified heterogeneous media (Bedford and Drumheller, 1994). 

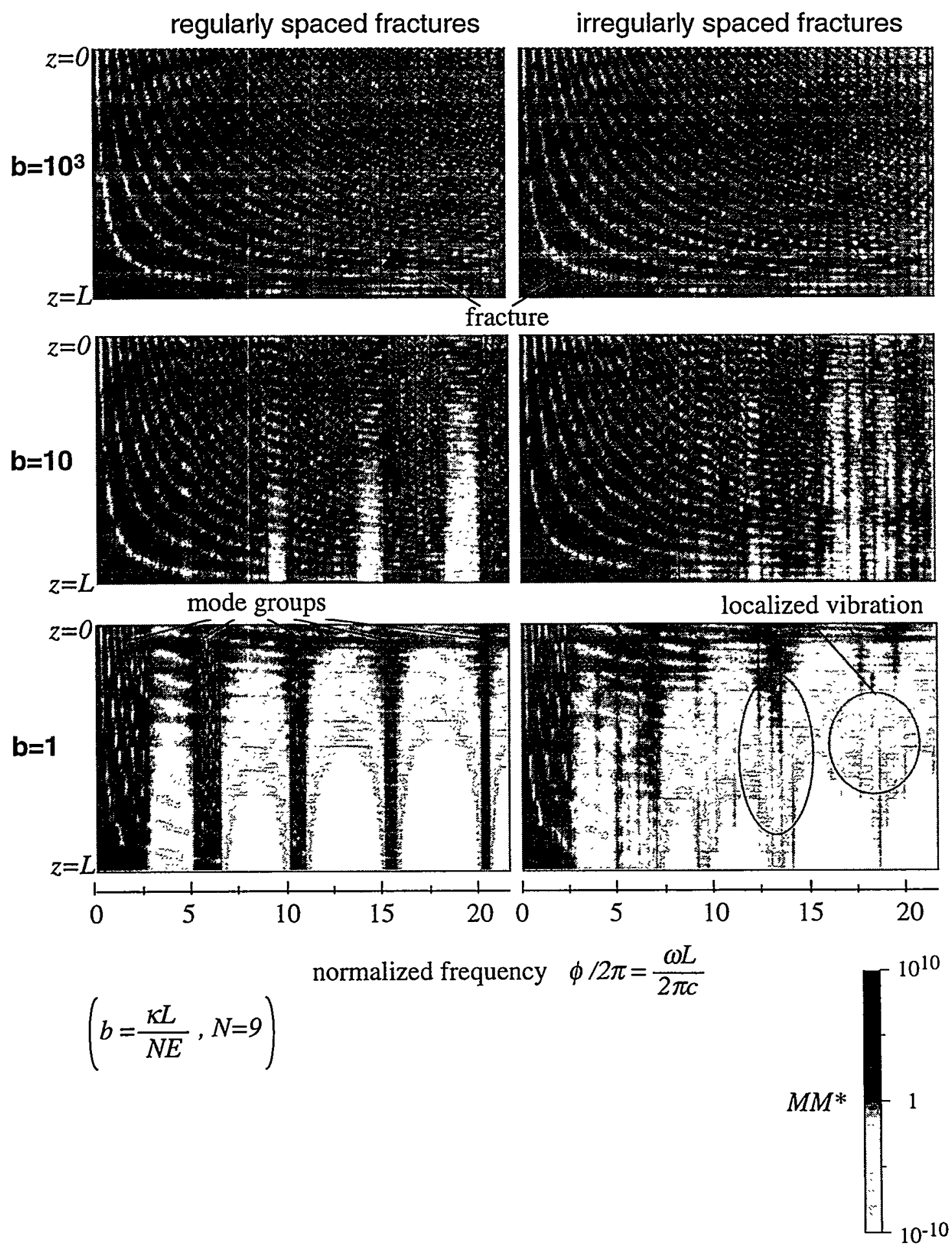

Figure 2.8 Computed mobility distribution in a finite bar with nine fractures. Dark lines show high amplitude (resonance) and the light curves show low amplitude (node). As the fracture stiffness decreases, the odd modes exhibit a decrease in resonance frequencies while the even modes do not shift. 
Table 2.2 Fracture Spacings in Finite One-dimensional Systems

\begin{tabular}{|l|c|c|c|c|c|c|c|c|c|c|}
\hline $\begin{array}{l}\text { layer } \\
\text { number }\end{array}$ & 1 & 2 & 3 & 4 & 5 & 6 & 7 & 8 & 9 & 10 \\
\hline $\begin{array}{l}\text { Regular } \\
\text { system }\end{array}$ & 0.1 & 0.1 & 0.1 & 0.1 & 0.1 & 0.1 & 0.1 & 0.1 & 0.1 & 0.1 \\
\hline $\begin{array}{l}\text { Irregular } \\
\text { system }\end{array}$ & 0.081 & 0.119 & 0.087 & 0.158 & 0.098 & 0.113 & 0.084 & 0.113 & 0.117 & 0.032 \\
\hline
\end{tabular}

When fractures are not regularly spaced, the resonance behavior of the system changes dramatically. The right half of the Figure 2.8 shows the resonance of a system containing 9 fractures with simulated random layer thicknesses. The average fracture spacing of the system is identical to the regularly spaced fractures. The thickness of the layers normalized by the total length of the system is shown in Table 2.2 .

For a system with intermediate fracture stiffness ( $b=\kappa L / N E=10, \mathrm{~N}=9$ ), the pass and stop band structure is not evident. As can be seen from Figure 2.9(b), all the modes experience changes in their resonance frequencies as the fracture stiffness is varied for the irregular system in contrast to the regular system. However, modes that do not show changes in resonance frequency for the regular system show relatively small frequency shifts. When the fracture stiffness is further decreased $(b=1)$, the internal layers exhibit locally enhanced resonances (Figure 2.8). This is the same phenomenon observed for a single fracture system with different segment lengths.

The observed localization of resonance is due to perturbation of acoustic impedance in a nearly periodic system, and is known as Anderson's localization (Anderson, 1958). For example, Hodges and Woodhouse (1983) showed that a system of a stretched spring with masses attached to it exhibits strongly localized modes when a small perturbation in the spacing between the masses is introduced into a regular system. Luongo (1992) also showed that the axial vibration of a continuous beam with distributed restraining longitudinal elastic springs exhibits localized mode shapes when a small perturbation in the stiffness of the beam is introduced.

Mode shapes of resonances for regularly and irregularly spaced fractures are shown for intermediate (Figure 2.10) and low (Figure 2.11) fracture stiffnesses ( $b=10$ and $b=1$, respectively). The amplitudes of the mode shapes are normalized by the maximum amplitude. Both regular and irregular systems' modes exhibit discontinuities in mode 


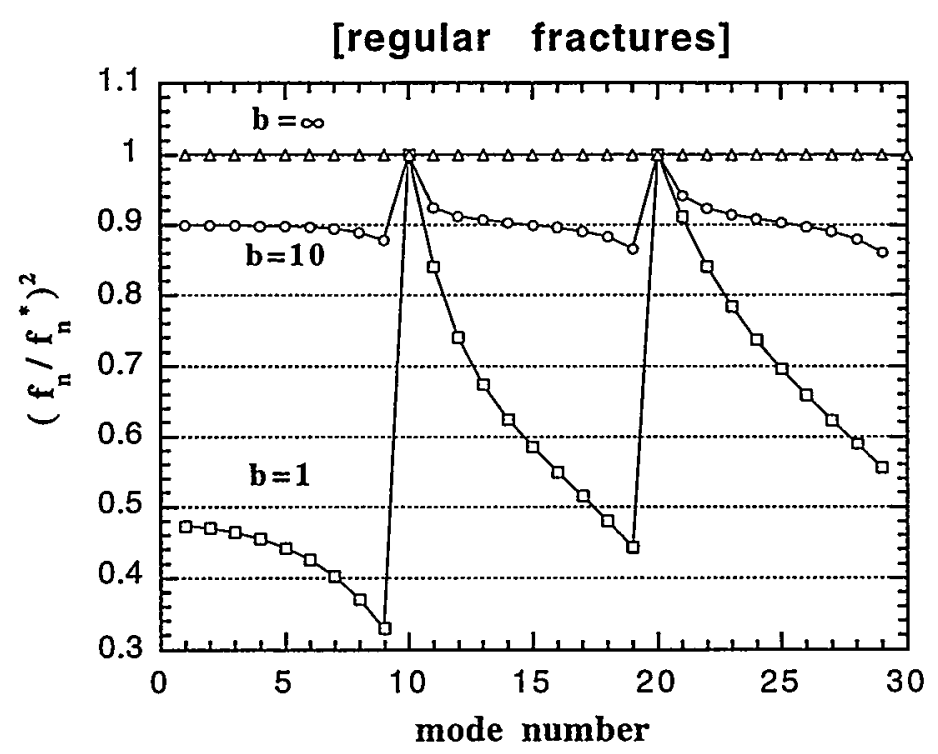

(a) Regularly spaced fractures

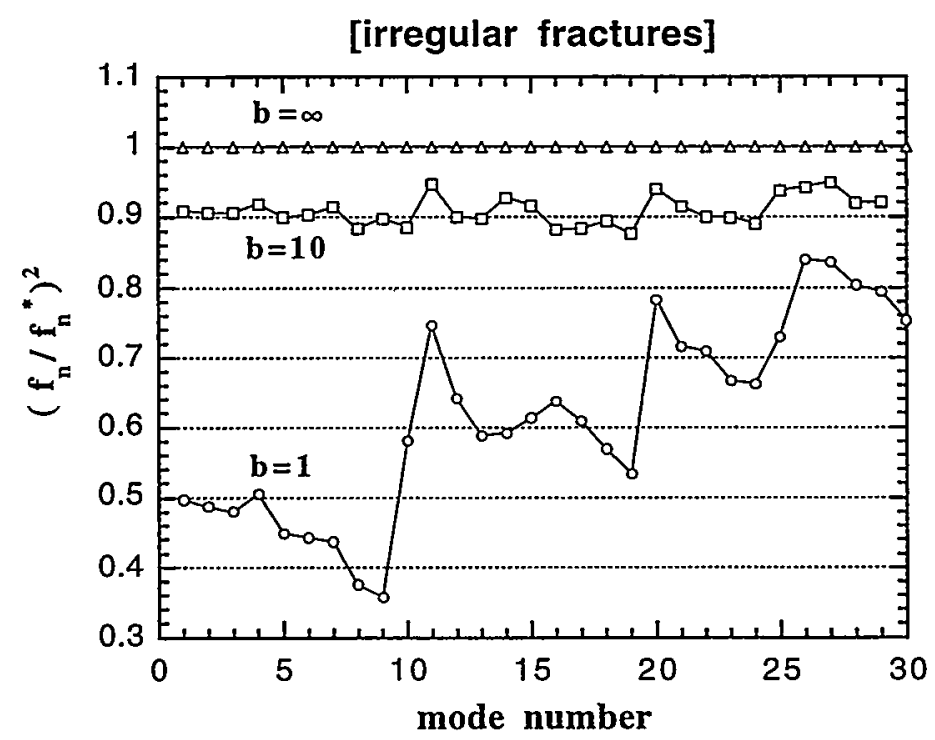

(b) Irregularly spaced fractures

Figure 2.9 Resonance frequency shifts for finite bars with regularly and irregularly spaced fractures. Each resonance frequency $f_{n}$ is normalized by a corresponding intact bar's resonance frequency $f_{n}{ }^{*}$. (a) For regularly spaced fractures, systematic decreases in the resonance frequencies can be seen. Multiples of the 10 th mode do not change the resonoance frequency. (b) Although a systematic shift is present, the system with irregularly spaced fractures does not show the modes that do not change the resonance frequency. 


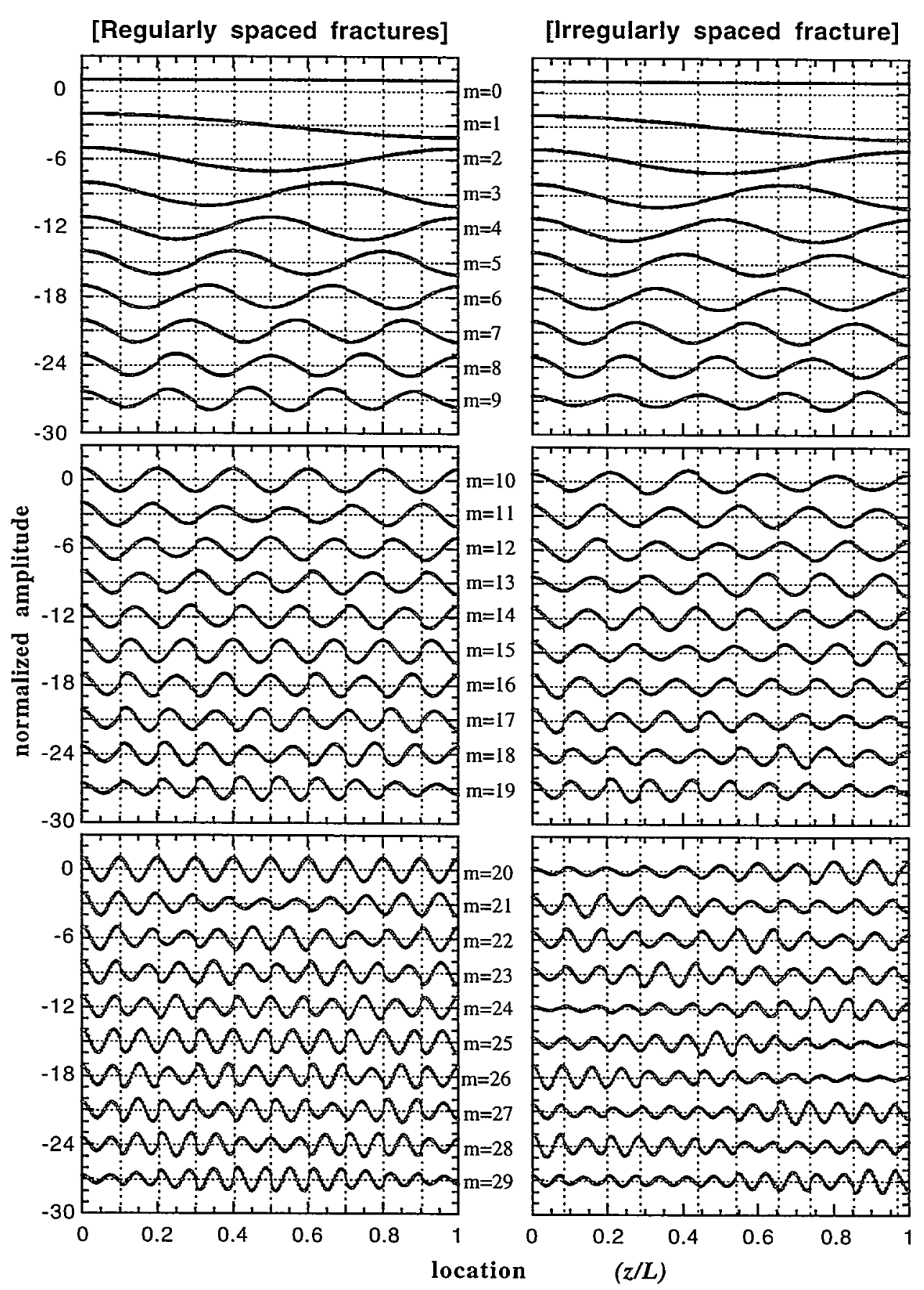

Figure 2.10 Mode shapes for resonances in a bar with regularly and irregularly spaced fractures with a normalized fracture stiffness $b=10$. For finite fracture stiffness, mode shapes become discontinuous across the fractures. For the irregular case, mode shapes are weakly localized for high frequency modes (3rd mode group ( $\mathrm{m}=10$ to $29^{\text {th }}$ modes) 
[Regularly spaced fractures]

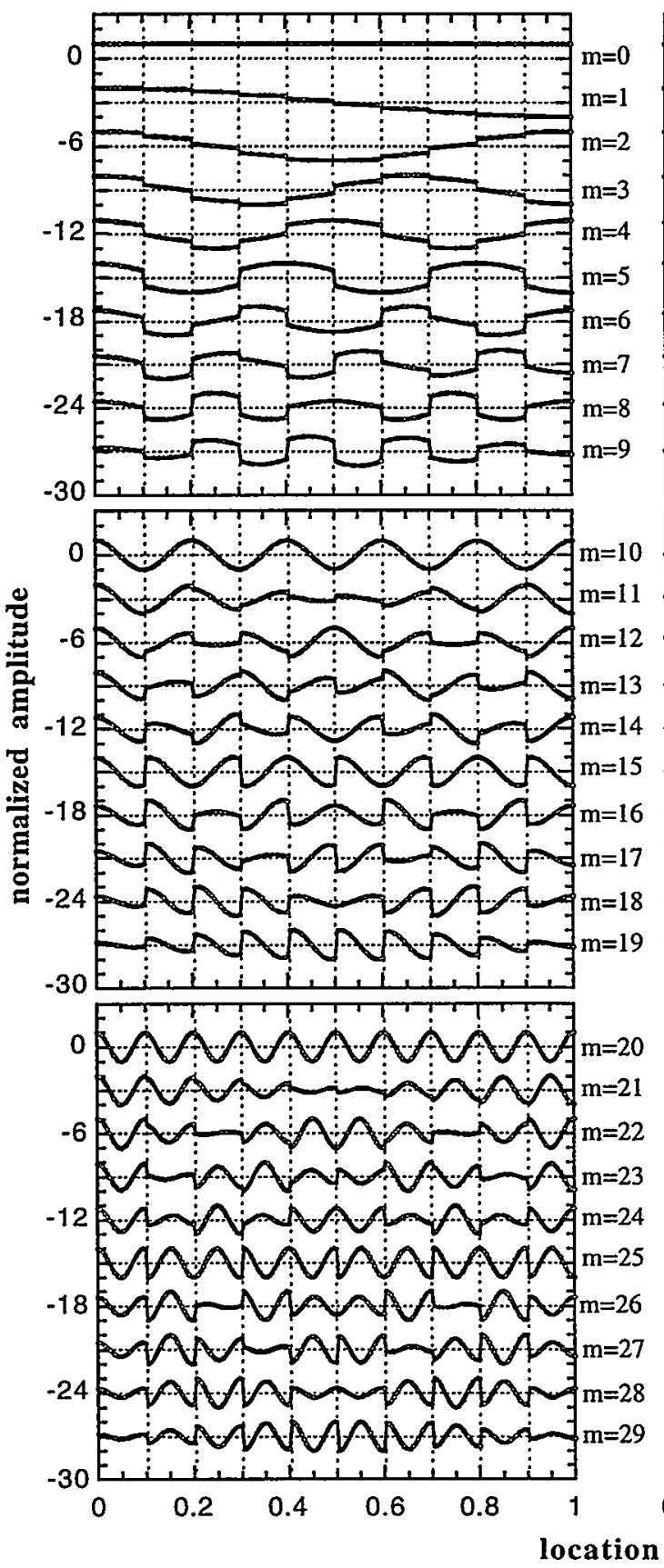

[Irregularly space fractures]
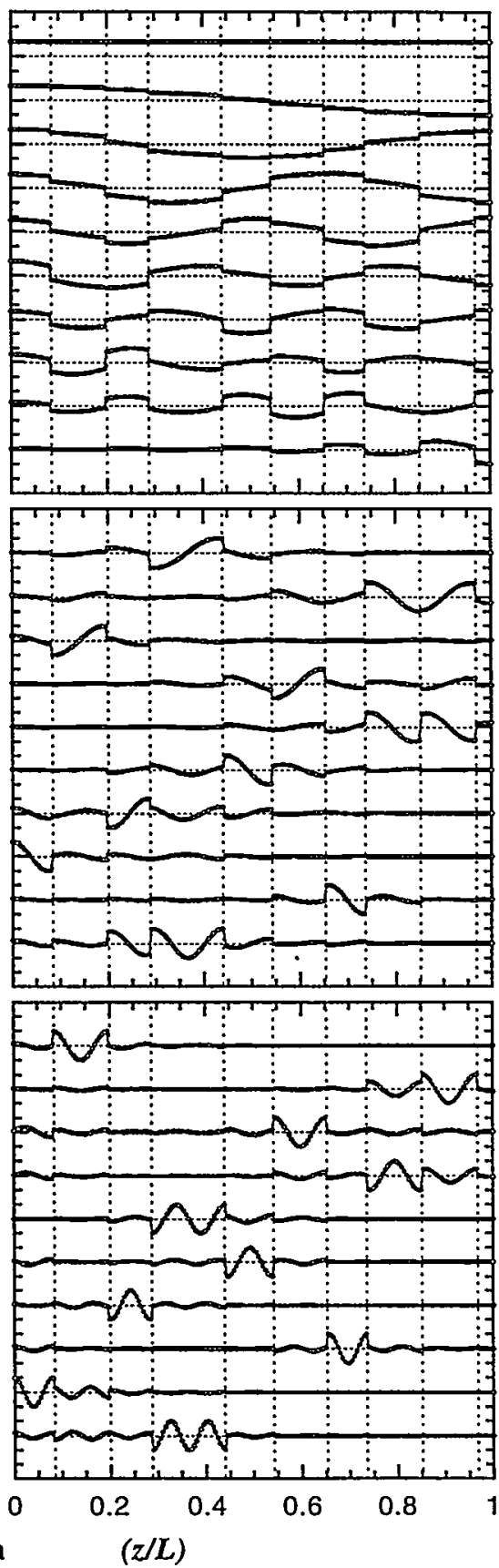

Figure 2.11 Mode shapes for resonances in a bar with regularly and irregularly spaced fractures. With a normalized fracture stiffness $b=1$. For the regular case, the amplitude of vibration is more or less even for the entire bar. The irregular case, on the other hand, except for the modes that degenerate to the rigid body motion of each block ( 0 to $9^{\text {th }}$ mode), causes strongly localized mode shapes to result. 
shapes across fractures (locations of fractures are shown by vertical dotted lines) except for the $0^{\text {th }}, 10^{\text {th }}$, and $20^{\text {th }}$ modes of the regular system. The continuous mode shape across the fractures is realized as the locations of fractures coincide with anti-nodes of the modes where no stress is introduced at resonance. As the mode shape of these modes is identical to that of an intact system, resonance frequencies are not changed by the fractures.

For an intermediate fracture stiffness ( $b=10$, Figure 2.10), mode shapes of the irregular system become asymmetric and start to show locally enhanced amplitude for higher order modes. For a very small fracture stiffness $(b=1$, Figure 2.11 ), the higher order modes in the irregular system are strongly localized. In the regular system, in contrast, amplitude of mode shape is more or less uniform throughout the system.

\subsubsection{Attenuation behavior}

Attenuation due to the viscous behavior of a fracture in a multiply-fractured system is shown in Figure 2.12. For the regularly fractured system, modes with a resonance frequency that is insensitive to a change in the fracture stiffness are not affected by the introduced viscosity. This behavior is analogous to the result in Section 2.2.2. In each mode group, modes that exhibited a large frequency shift tend to show large attenuation. This is because such modes involve a large opening and closing displacement across the fracture surface that leads to large energy dissipation. For an irregular system, in contrast, the systematic grouping and attenuation behavior of the modes are not evident. This is because irregularly spaced fractures sample the nodes and anti-nodes of the mode shape randomly, which makes their contribution to the attenuation approximately equal for all modes. 

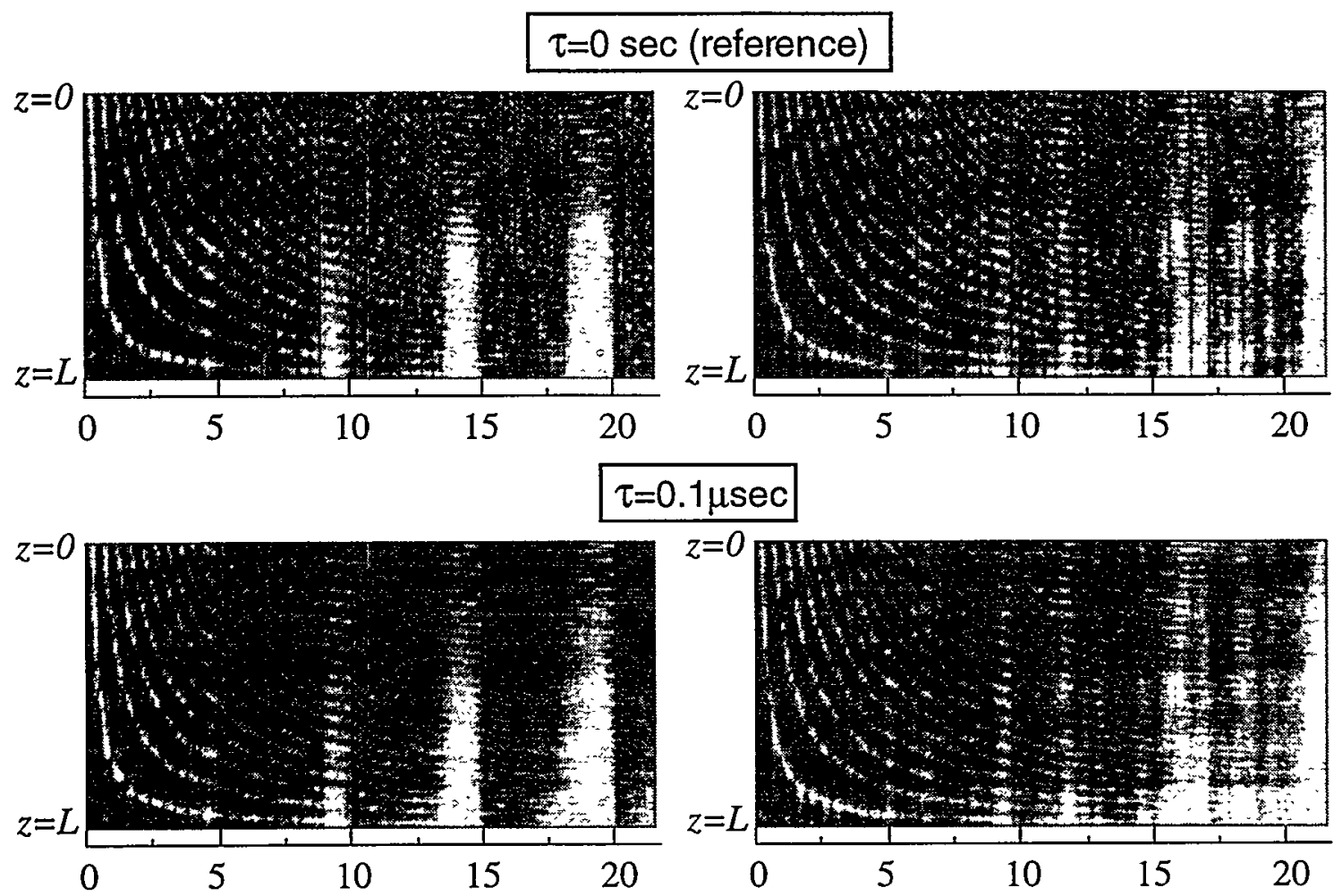

normalized frequency $\phi / 2 \pi=\frac{\omega L}{2 \pi c}$
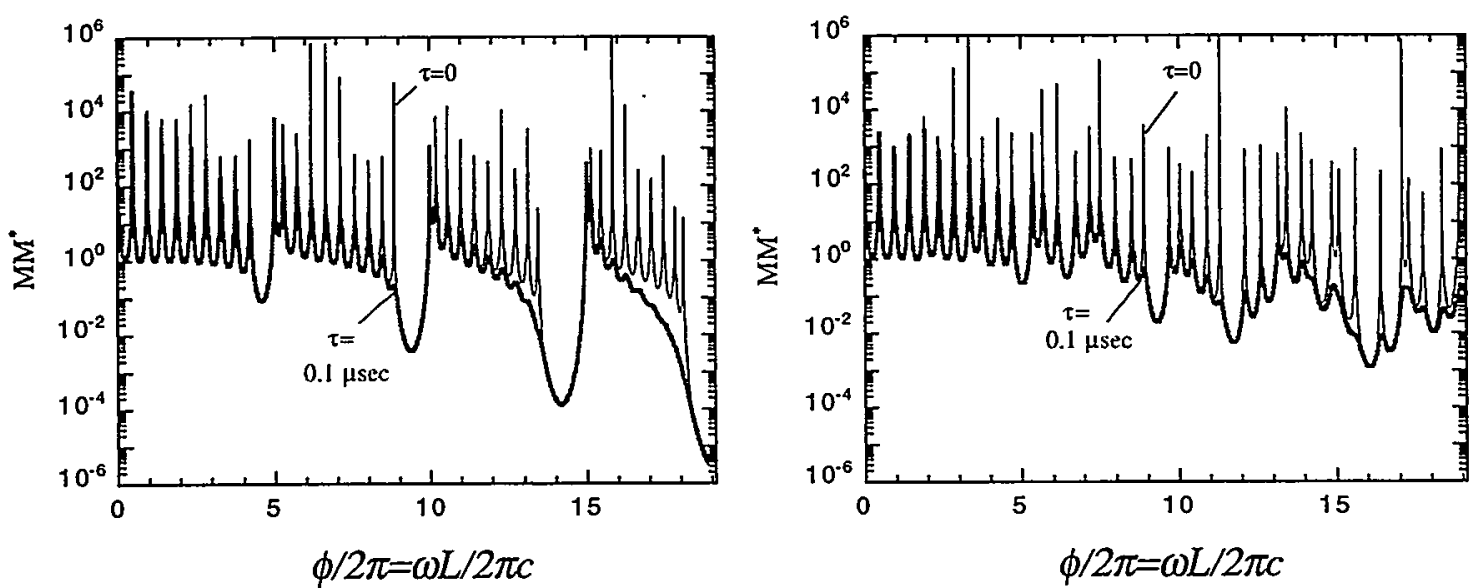

Figure 2.12 Effect of fracture viscosity on the resonance of a multiple fracture system. The FRF plots at the bottom are computed for a receiver located at the bottom of the fractured bar. For a regularly-spaced fracture system, the first mode in each pass band does not attenuate while the other modes attenuate in increasing order with increasing resonance frequency. An irregularly-spaced fracture system shows an increasing trend in attenuation for higher order modes, but does not show the systematic attenuation seen for the regular system. 


\subsection{Resonance of a Delaminated Halfspace}

Before discussing resonance of fractures in an infinite system, the effect of an infinite boundary (halfspace) is examined by studying resonance of a delaminated layer on a halfspace. When the surface of a half-space is detached by a compliant interface (e.g., a fracture), this system can resonate by a uniform dynamic load applied on the surface. For a source and a receiver located on the surface of the delaminated layer (Figure 2.13), the power of the dimensionless mobility is

$$
M M^{*}=\frac{2(1+\cos (2 \phi))+\beta^{2}+2 \beta \sin (2 \phi)}{2(1-\cos (2 \phi))+\beta^{2}-2 \beta \sin (2 \phi)}
$$

where $\mathrm{M}$ is the dimensionless mobility (complex FRF) and the superscript * represents its complex conjugate. The derivation of Eq.(2.12) is given in Appendix B. For the delaminated layer of thickness $L_{0}$, a phase angle $\phi$ is defined by

$$
\phi=\frac{\omega L_{0}}{c}
$$

From Figure 2.13, it can be seen that an increase in the fracture stiffness increases both the resonance frequencies and attenuation. It is also noticed that the increases in the lower order modes' resonance frequencies and attenuation are greater than the higher order modes'. To examine the mode dependent behavior of the resonance frequency shift, the center resonance frequency $\omega_{c}$ (Figure 2.2) and corresponding $\kappa_{c}$ and $\beta_{c}$ are examined. When the stiffness of the fracture is very small, $\beta$ approaches zero and Eq.(2.12) becomes

$$
M M^{*}(\kappa \rightarrow 0)=\frac{1+\cos \left(2 \phi_{0}\right)}{1-\cos \left(2 \phi_{0}\right)} .
$$

This equation shows that peaks of the FRF (resonances) appear periodically in frequency satisfying $\cos \left(2 \phi_{0}\right)=1$, or $\sin \left(\phi_{0}\right)=0$. This is a classical solution for the resonance of a continuous slender bar with finite length (Graff, 1975). As the fracture stiffness increases, the resonance frequencies increase and asymptote to frequencies that satisfy $\tan \left(2 \phi_{0}\right)=-2 \phi_{0}$. This condition is found by searching for local maxima (resonances) of Eq.(2.13) with a large $\beta$. Simultaneously, attenuation of resonance increases and, for an infinitely high fracture stiffness, the mobility, becomes constant $(=1)$ for all frequencies. 

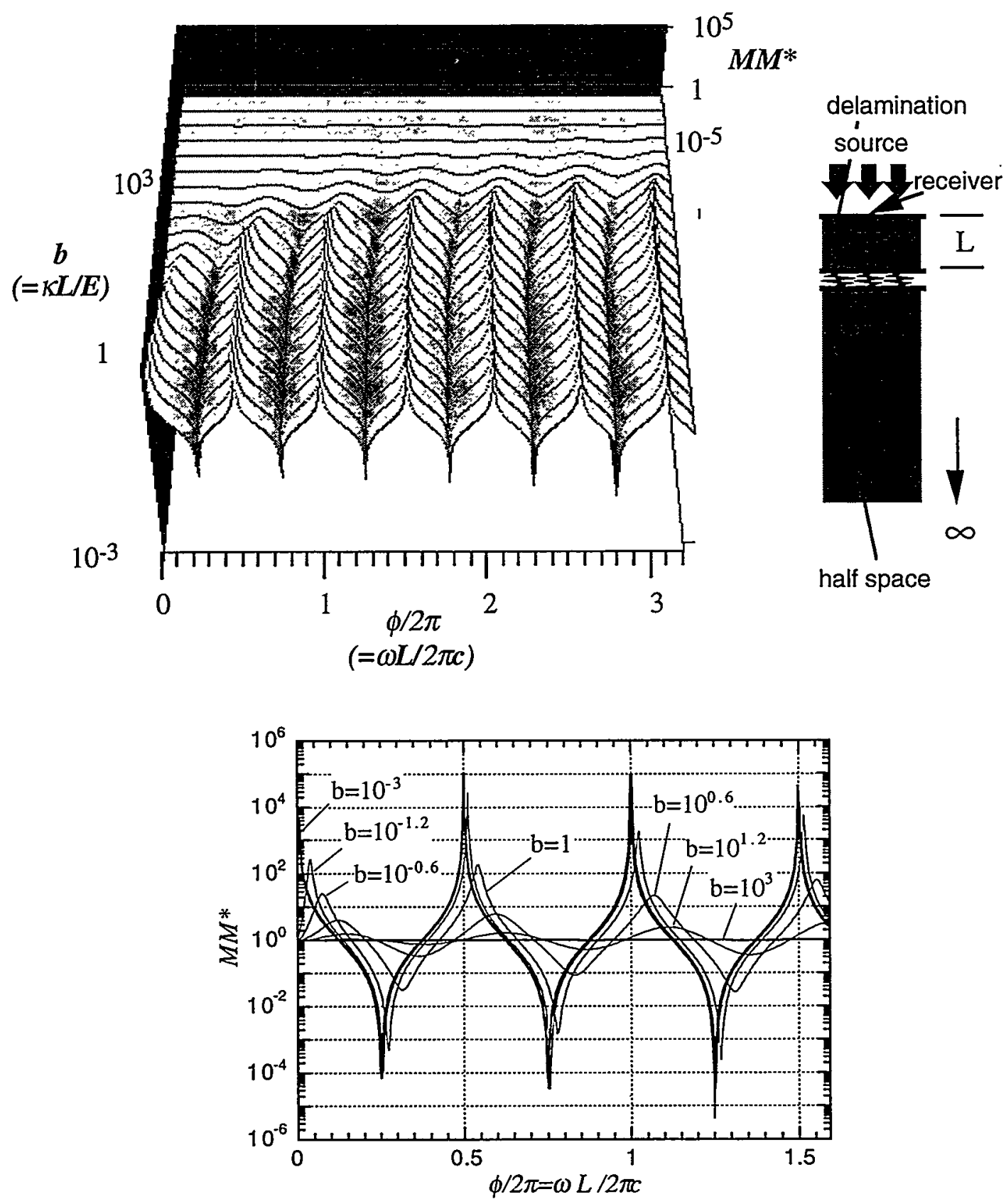

Figure 2.13 FRF's of a delaminated layer on a half space for different fracture stiffnesses. Both frequency and the fracture stiffness are dimensionless. An increase in the stiffness of the fracture introduces an upward frequency shift and a broadening (i.e., attenuation resulting from radiation damping) of the resonance peaks. The shifting and attenuating behavior of the resonance is the largest for the lower order modes. 


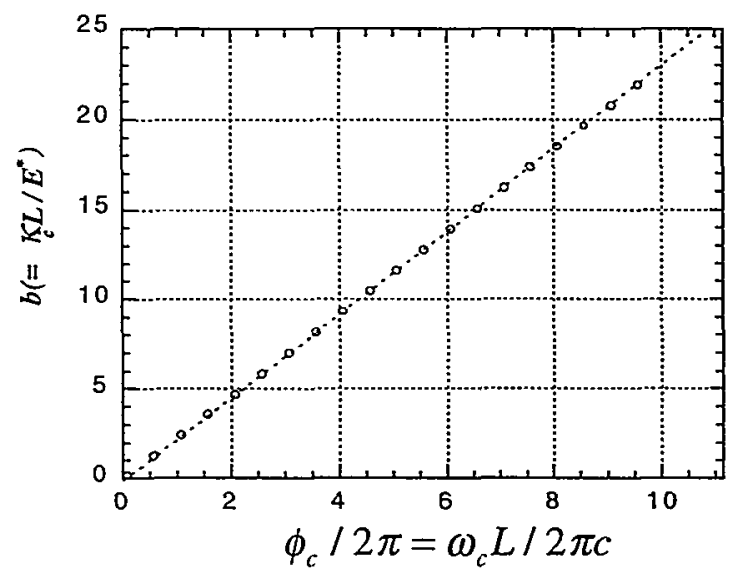

(a) $\kappa_{c}-\omega_{c}$

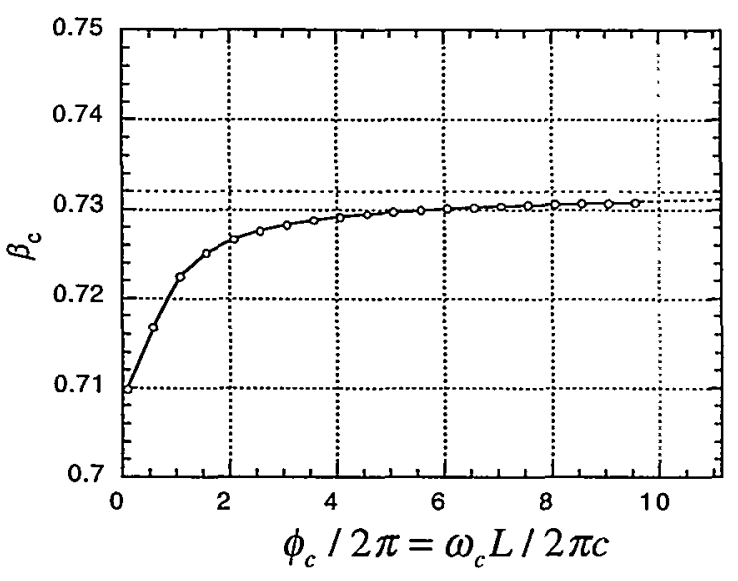

(b) $\beta_{c}-\omega_{c}$

Figure 2.14 The (a) center fracture stiffness $\kappa_{c}$ and (b) corresponding impedance ratios $\beta_{c}$ for the resonances of the delaminated halfspace. $\kappa_{C}$ increases in proportion to the $\omega_{C}$ (or normalized frequency $\phi_{C}$ ), which results in approximately constant $\beta_{c}$ for all modes. $\beta_{c}$ asymptotes to 0.732 for the high order modes.

Figure 2.14(a) and (b) show the center resonance frequencies $\omega_{c}$ 's and corresponding $\kappa_{c}$ 's and $\beta_{c}$ 's. It can be seen that the shift of resonance frequencies occurs for similar impedance ratios that asymptote to a constant $(=0.732)$ for higher resonance frequencies. This indicates that the shift of a higher order mode resonance occurs for proportionally high fracture stiffness.

Attenuation of the resonances in the delaminated layer is due to the radiation of wave energy across the fracture. Therefore, the magnitude of attenuation is determined by the transmission coefficient of the fracture. The seismic quality factor $Q$ and the damping coefficient $\zeta$ are defined as a ratio between energy dissipated in a single cycle of vibration $(\Delta E$ ) and the total energy ( $E$ : sum of strain energy and kinetic energy) in the system as

$$
Q^{-1}=2 \zeta=\frac{1}{2 \pi} \frac{-\Delta E}{E} .
$$

To derive an analytic expression for the damping parameters, vibration in the system is decomposed to waves traveling downward and upward in the delamination and halfspace (Figure 2.15). A time-averaged flux of wave energy escaping from the system is

$$
F_{0}=\frac{1}{2} \rho c \omega^{2}\left|u_{d}^{(h s)}\right|^{2},
$$



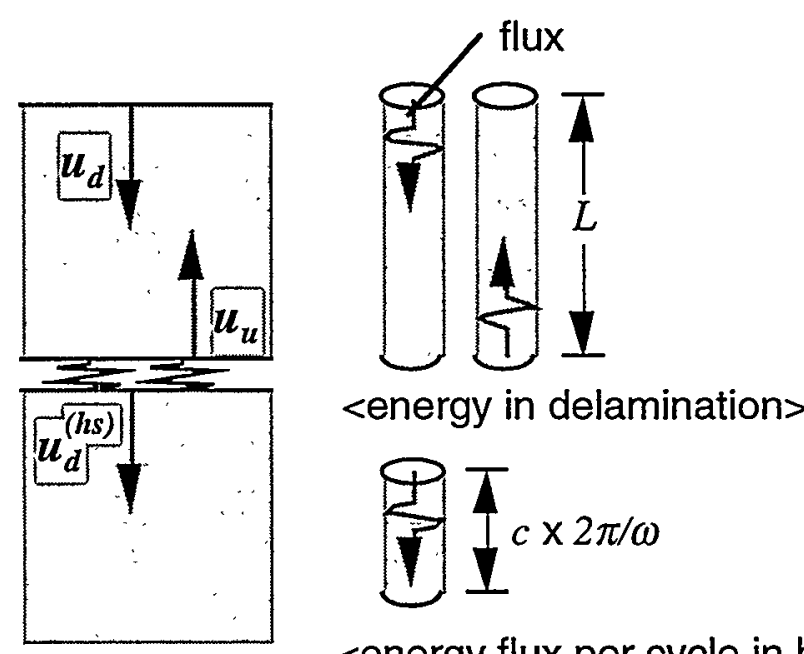

<energy flux per cycle in half spaces

Figure 2.15 Decomposition of vibration to upgoing and downgoing wave components. Amplitudes of the decomposed waves are used to derive expressions for time-averaged wave fluxes. The total energy of vibration is computed by integrating the fluxes of upgoing and downgoing waves in the delamination over travel time $\mathrm{t}=\mathrm{L} / \mathrm{c}$ and the energy loss across the fracture is computed from the downgoing flux integrated over a single period $(2 \pi / \omega)$.

where $u_{d}^{(h)}$ is the displacement of the downgoing wave in the halfspace. During a single cycle of vibration, the amount of the escaping energy is

$$
-\Delta E=F_{0} \cdot \frac{2 \pi}{\omega}=\frac{1}{2} \rho c \omega^{2}\left|u_{d}^{(h s)}\right|^{2} \cdot \frac{2 \pi}{\omega} .
$$

The total energy of vibration in the delaminated layer is also obtained from the fluxes of the waves in the system. The fluxes of the downgoing and upgoing waves are

$$
F_{d}=\frac{1}{2} \rho c \omega^{2}\left|u_{d}\right|^{2}, \quad F_{u}=\frac{1}{2} \rho c \omega^{2}\left|u_{u}\right|^{2},
$$

where $u_{d}$ and $u_{u}$ are the displacements of upgoing and downgoing waves, respectively. The energy of vibration in the layer of length $L$ equals the total energy by a flux for a period of $L / c$ ( $c$ is the phase velocity of the waves). Therefore, the total vibration energy is

$$
E=\left(F_{d}+F_{u}\right) \cdot \frac{L}{c}=\frac{1}{2} \rho c \omega^{2}\left(\left|u_{d}\right|^{2}+\left|u_{u}\right|^{2}\right) \cdot \frac{L}{c} .
$$

Displacements are related to each other by

$$
\left|u_{u}\right|=|R|\left|u_{d}\right|,\left|u_{d}^{(h s)}\right|=|T|\left|u_{d}\right|,
$$




\section{Analytical Study on 1-D Resonance}

where $\mathrm{T}$ and $\mathrm{R}$ are the transmission and reflection coefficients. By introducing the expression (2.20) into (2.17) and (2.19) with a relation $|R|^{2}=1-|T|^{2}$, the $\mathrm{Q}$ and $\zeta$ are computed by

$$
Q=\frac{1}{2 \zeta}=\frac{2-|T|^{2}}{|T|^{2}} \frac{\omega L}{c}=\left(1+2 \beta^{-2}\right) \phi
$$

In the above expression, the phase angle $\phi=\omega L / c . \mathrm{T}$ is the transmission coefficient of a fracture for a normally incident wave given by (e.g., Schoenberg, 1980)

$$
T=\frac{i \beta}{1+i \beta}
$$

where $\beta$ is the impedance ratio of a fracture defined by Eq.(2.2). Eq.(2.21) can be rewritten as

$$
Q=\frac{1}{2 \zeta}=\left[1+\frac{1}{2}\left(\frac{\omega Z}{\kappa}\right)^{2}\right] \frac{\omega L}{c}
$$

The above expression reveals that attenuation decreases monotonically with increasing frequency.

Comparison between analytically determined Q's using Eq(2.23) and Q's measured from the source mobility (Eq.(2.12)) using the half-power method is shown in Figure 2.16. The slight difference between the analytic and measured Q's for large attenuation is due to the inaccuracy of the half-power method. 


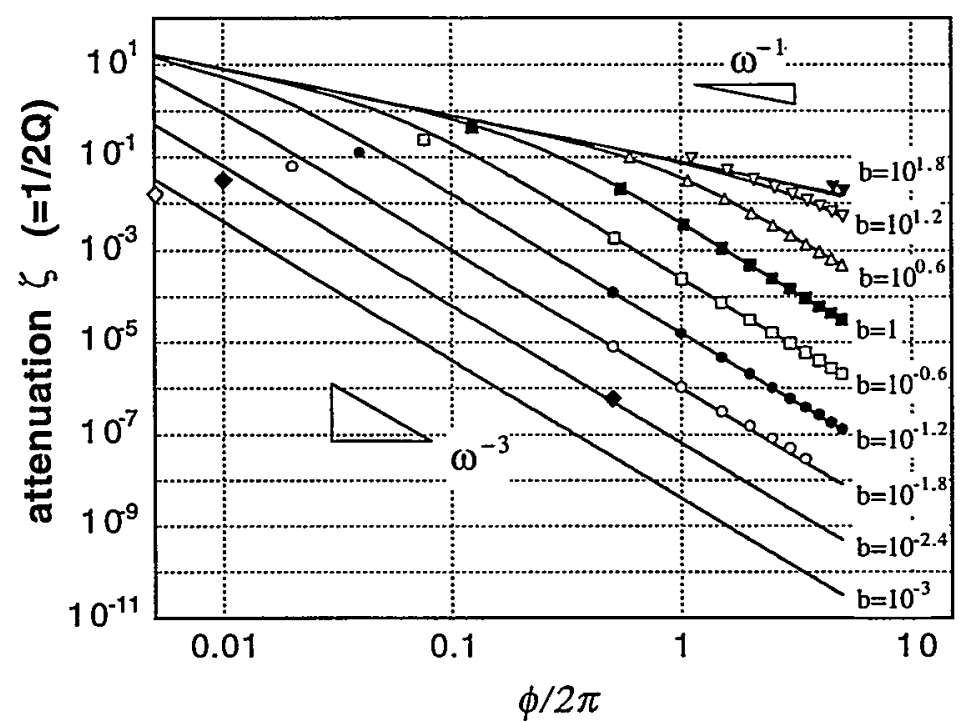

Figure 2.16 Comparison of analytically and numerically (using the half-power method) determined attenuation of vibration in a delamination. Horizontal axis is normalized frequency $(\phi=\omega L c)$. Both results show very close agreement. A slight difference in attenuation for large $\zeta$ (or small $Q$ ) is due to the inaccuracy of the half-power method. 


\subsection{Infinite System Including Finite Number of Fractures}

\subsubsection{Transmission and reflection coefficients}

When the finite fractured system discussed in the Section 2.3 is embedded in an infinite one-dimensional medium, vibration inside the system attenuates even in the absence of material and fracture damping because of the radiation of vibration energy into the surrounding halfspaces. The seismic behavior of such a system is particularly interesting for characterizing a zone of parallel fractures in a geologic unit. Multiple parallel fractures are common features found in almost all rock types, including igneous, sedimentary, and metamorphic rocks. For example, Figure 2.17a shows multiple parallel fractures observed in a sandstone basin by Laubach (1991). Figure $2.17 \mathrm{~b}$ is a sketch of parallel fractures in a basalt unit by Peterson et al. (1993). Peterson et al. examined characteristics of seismic waves transmitting through fractures between boreholes and demonstrated that changes in the waveform can be modeled by the displacement-discontinuity boundary conditions with good agreement.

A wave normally incident on a zone of parallel fractures is multiply scattered within the fractured zone, changing its velocity, amplitude, and spectral characteristics. These changes are expected to have a close relation with the resonance of a finite system. To study this effect, the finite fractured systems examined in Section 2.3 are embedded in an infinite medium (Figure 2.18) and responses of the systems to steady state waves were examined using the displacement transfer function of the system. A displacement transfer function is defined by a displacement spectrum of transmitted or reflected waves normalized by a displacement spectrum of incident waves. The propagator matrix method was used to compute the transfer function. Computed transfer functions for transmitting and reflected waves are essentially the transmission and reflection coefficients of the fractured zone, respectively. These, in turn, are the spectra of the transmitted and reflected waves for an incident impulse.

Transmission and reflection coefficients of regularly and irregularly fractured zones are shown in Figure 2.19. The stiffness of the fractures is identical to the medium stiffness case $(b=10)$ for a finite fractured system used in the previous section. The transmission and reflection coefficients have a complementary relation $\left(\mathrm{T}^{2}+\mathrm{R}^{2}=1\right)$ as the total energy of the waves has to be conserved. As can be seen from the plot, the regular system exhibits 


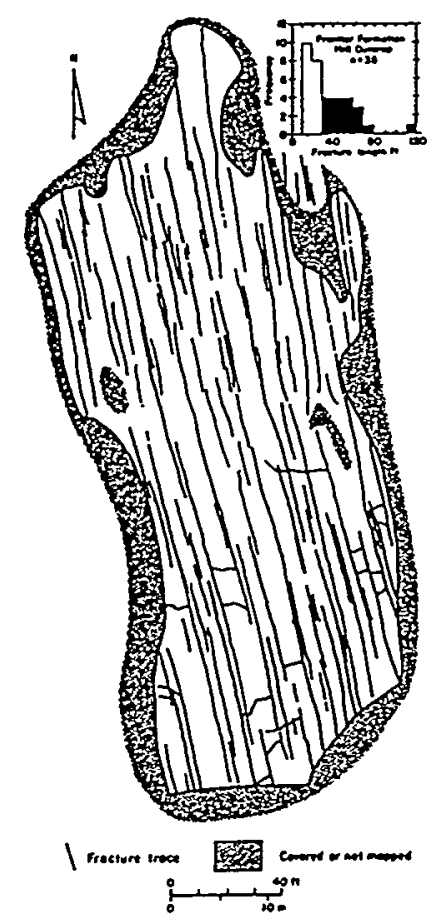

(a) Multiple fractures in a sandstone unit. Little Coal Creek (Hill) outcrop, Wyoming. (After Laubach, 1991)

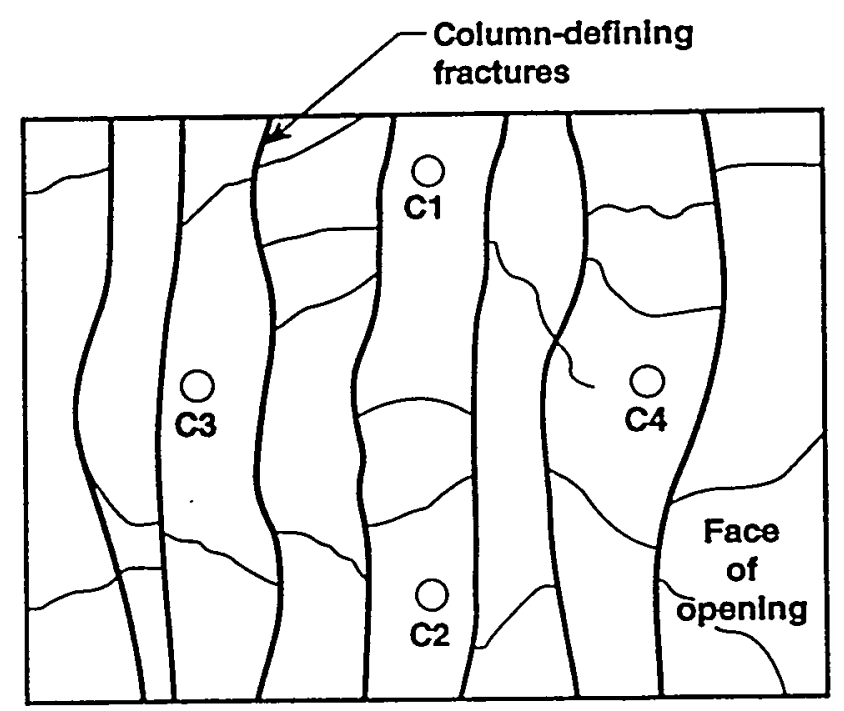

(b) Multiple fractures in a basalt unit. Boreholes used for the cross-hole seismic measurements are also shown. (After Peterson et al., 1993)

Figure 2.17 Examples of multiple parallel fractures observed in the field. 


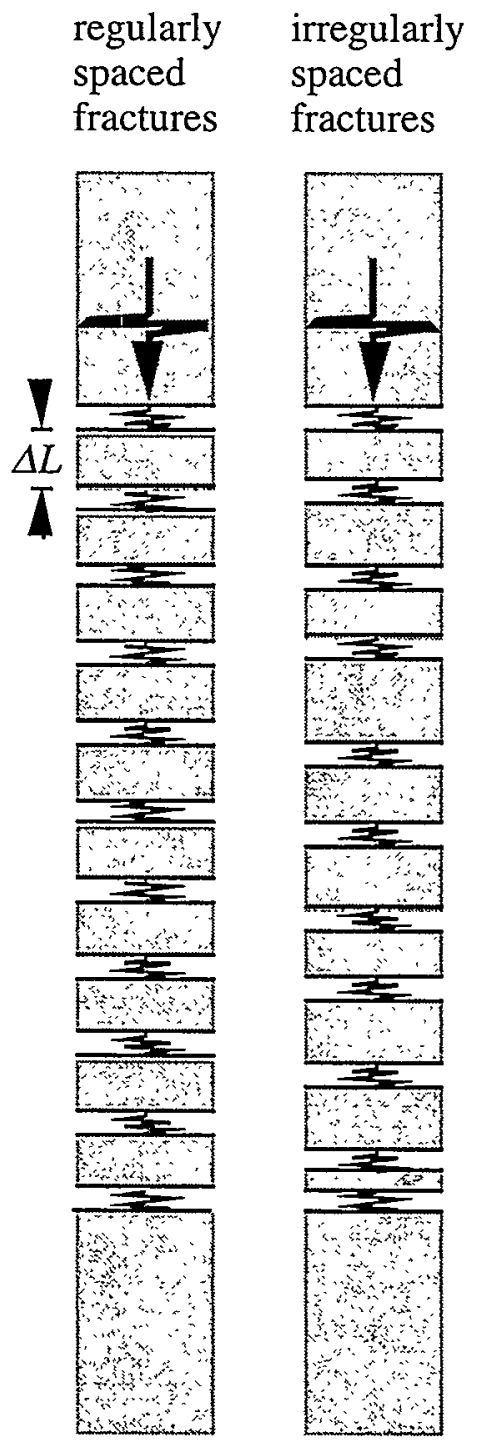

Figure 2.18 Schematics for regularly and irregularly spaced fractures embedded in an infinite medium. A wave is incident on the fractured zone from infinite distance. Transmitting waves and reflected waves are computed and examined for their velocities and spectral characteristics. 

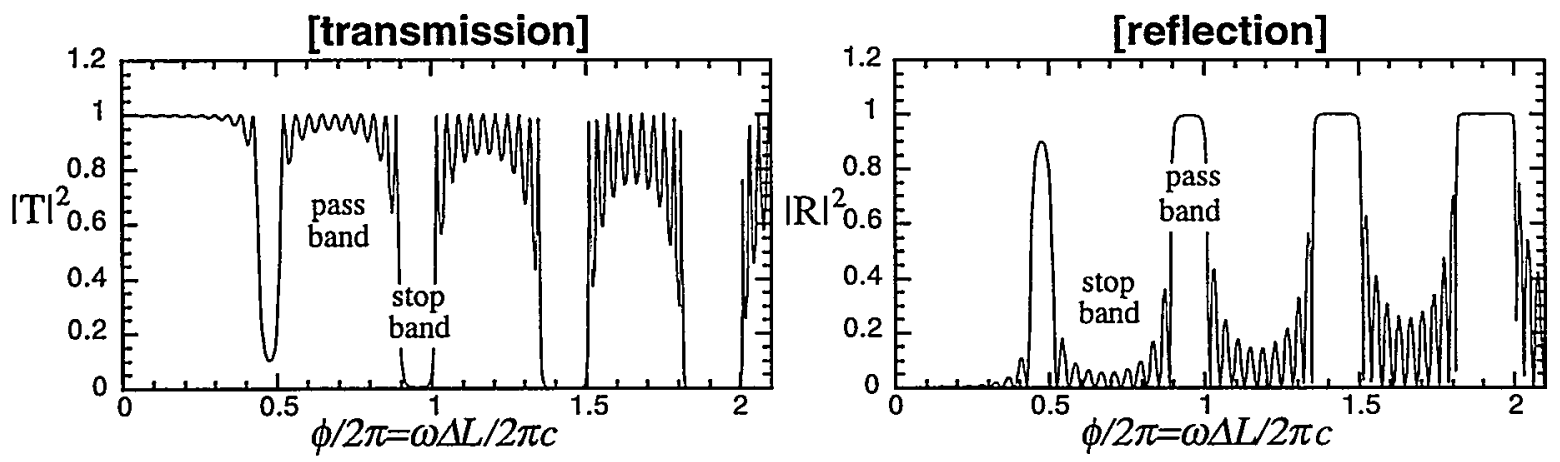

(a) Regularly spaced fractures
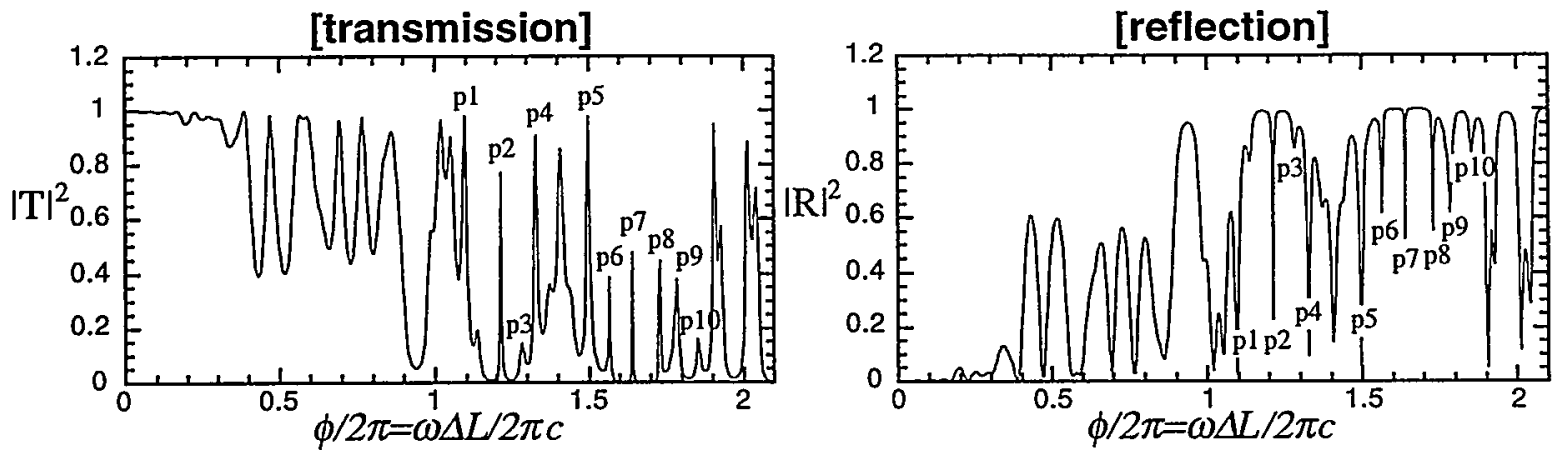

(b) Irregularly spaced fractures

Figure 2.19 Transmission (T) and reflection (R) coefficients of (a) regularly and (b) irregularly spaced fractures. $|T|^{2}$ and $|R|^{2}$ are complementary $\left(|T|^{2}+|R|^{2}=1\right)$ as the energy of the wave has to be conserved. Regularly spaced fractures show distinct pass and stop bands. The band structures are not clear for the irregularly spaced fractures.

distinct pass and stop bands that are not evident in the irregular system. As shown in Figure 2.20, the transmission coefficients of the fractured zone have a close relation to the FRF of the finite systems. The most significant feature is that the structure of the pass and stop bands for the fractured zone is identical to those for the finite system. This indicates that changes in the spectral characteristics of a wave propagating through a zone of fractures reflect resonance characteristics of the internal fractures.

\subsubsection{Spectrum of the first-arriving pulse}

The power spectra of the first-arriving part of the transmitting pulses are shown in Figure 2.21 along with their phase and group velocities in Figure 2.22. The phase and group velocities are computed from the phase spectra of the wave as follows

$$
c_{\text {phuse }}=L / t_{\text {phase }}, \quad c_{\text {group }}=L / t_{\text {group }},
$$




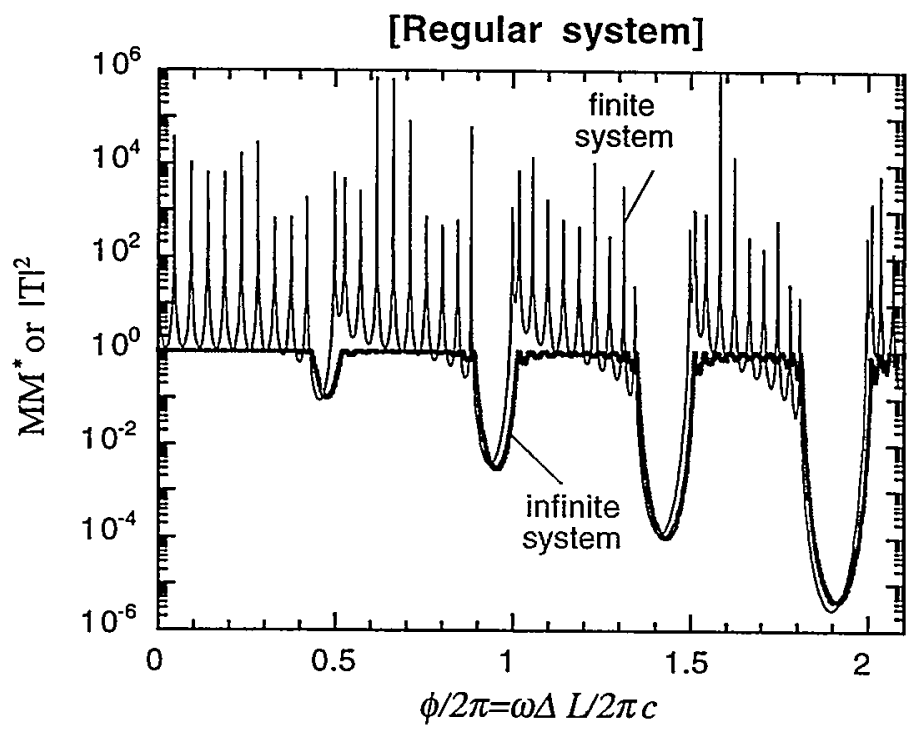

(a) Regularly spaced fractures

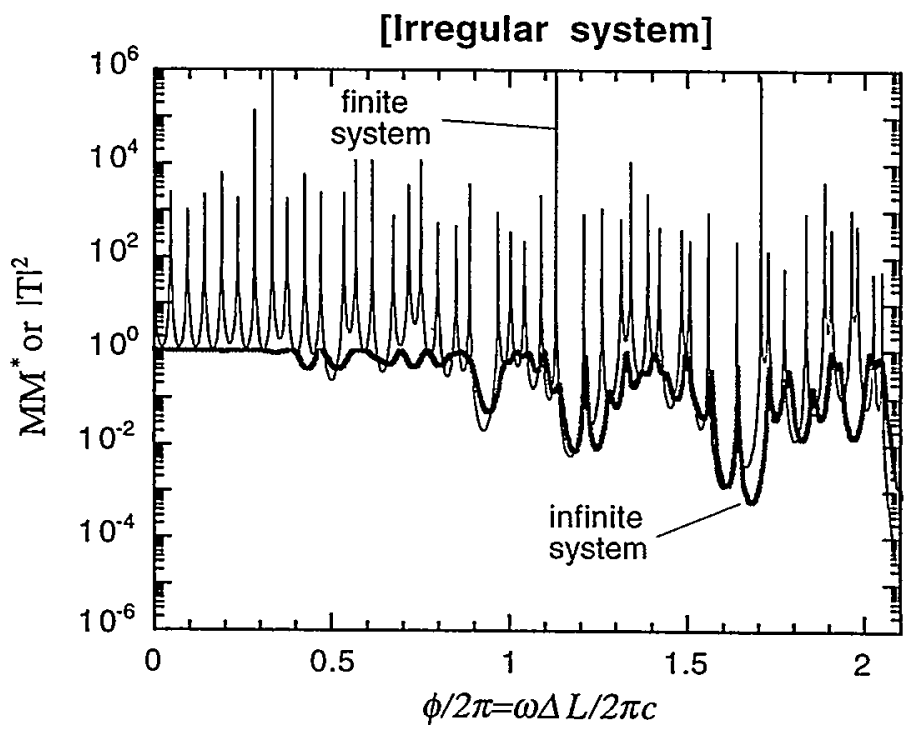

(b) Irregularly spaced fractures

Figure 2.20 Comparison of FRF's (power of mobility for finite system and power of transmission coefficient for an infinite system) for finite and infinite systems with 11 fractures. Frequency is normalized with an average fracture spacing $\Delta \mathrm{L}=0.1 \mathrm{~L}$ ( $\mathrm{L}=$ thickness of the fractured zone) and an intact material velocity. The stiffness of the fractures is constant $(b=10)$. Both regular and irregular systems show strong correlation between the pass and stop band structures for the finite and infinite systems. 


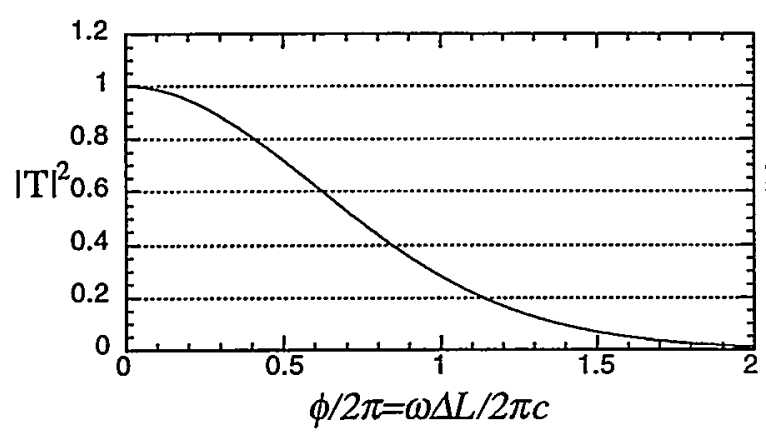

(a) Regular system

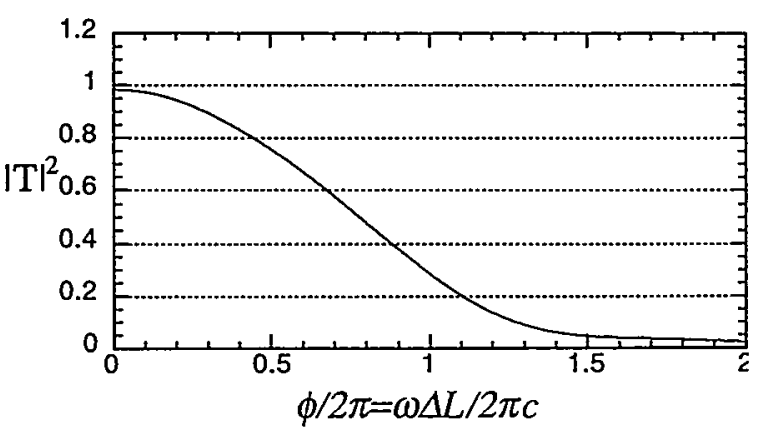

(b) Irregular system

Figure 2.21 Transmission coefficient for the first-arriving pulse through regularly and irregularly spaced fractures. Due to the frequency-dependent low-pass filtering of the fractures, transmission coefficients become small with increasing frequency.

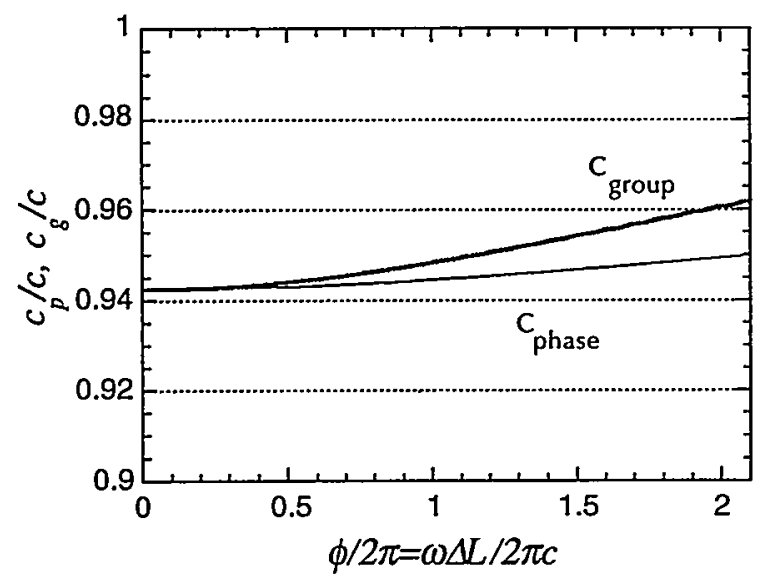

(a) Regular system

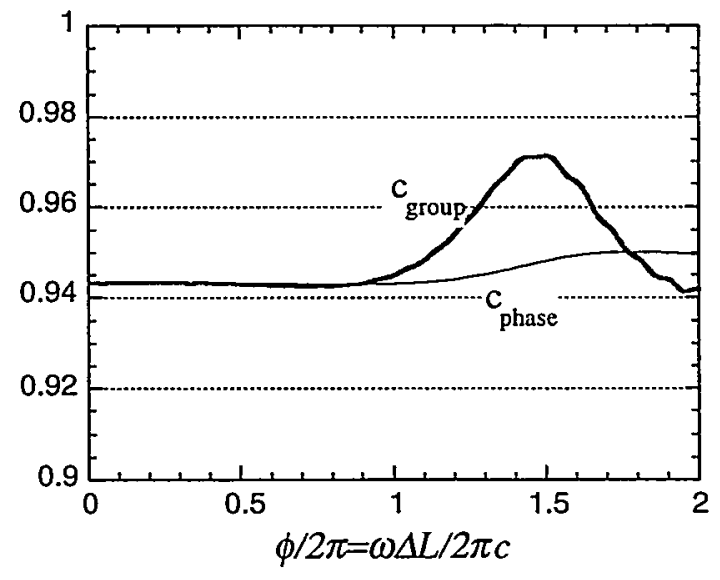

(b) Irregular system

Figure 2.22 Phase and group velocities for the first-arriving pulse through regularly and irregularly spaced fractures. The velocites were computed from phase spectra of the pulses. For both cases, velocities increase with increasing frequency. Such behavior is predicted by the seismic displacement-discontinuity model for transmission across a single fracture (Pyrak-Nolte et al., 1990a). Decrease in group velocity for the irregular system at high frequency is due to the distortion of the pulse by scattered waves within a thin layer. Due to the frequency-dependent low-pass filtering of the fractures, transmission coefficients become small with increasing frequency. 
where $\mathrm{L}$ is the thickness of the fractured zone, and $t_{\text {phase }}$ and $t_{\text {group }}$ are the phase and group time delays of the pulse between a source and a receiver computed from

$$
t_{\text {phase }}=\frac{\theta}{\omega}, t_{\text {group }}=\frac{d \theta}{d \omega}
$$

where $\theta$ is a phase spectrum of the transmitting pulse.

As can be seen from the plot, the spectral amplitude of the transmitting pulse decreases monotonically with increasing frequency. Phase and group velocities, on the other hand, are the slowest at zero frequency and increase with increasing frequency. This is caused by the accumulation of the frequency-dependent time delay across each fracture (Pyrak-Nolte et al., 1990b). Due to the filtering effect of the fractures, amplitude spectra for both regular and irregular systems decrease monotonically with increasing frequency.

\subsubsection{Coda spectrum}

Strong multiple reflections within a fractured zone in an infinite medium can be studied by examining the spectrum of seismic coda for a time-domain impulse response. Figure 2.23 shows impulse responses and coda spectra for the regular and irregular systems. A transmitted pulse for the regular system exhibits much larger energy than the irregular system while the reflected pulse for the irregular system shows more energy than the regular system. This can be interpreted as a time-domain realization of Anderson's localization; perturbation of the acoustic impedance in an irregular system prohibits the propagation of the waves. It can be seen that for the regularly spaced fractures, both codas for transmitted and reflected waves have similar band structures as those for the entire wave train of the transmitted waves. Although the irregularly spaced fractures do not show clear band structures, locations of peaks in the coda spectra are identical to those observed for the transmitted waves. These results indicate that the coda part of the waves are generated by resonances within the fractured zone that radiate the vibration energy to the surrounding halfspaces. One marked characteristic of the coda spectra for the regular system is that there are distinct peaks (resonances) in the spectra at boundaries between pass and stop bands. This phenomenon is further examined in the following section. 

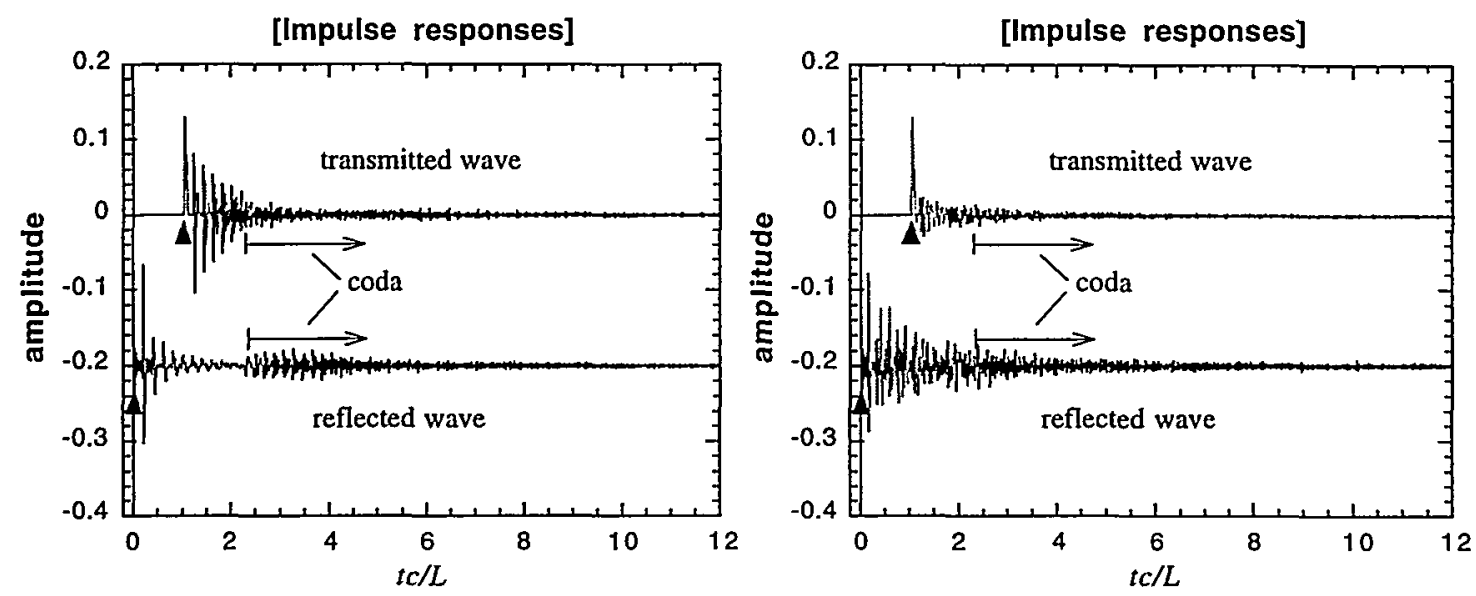

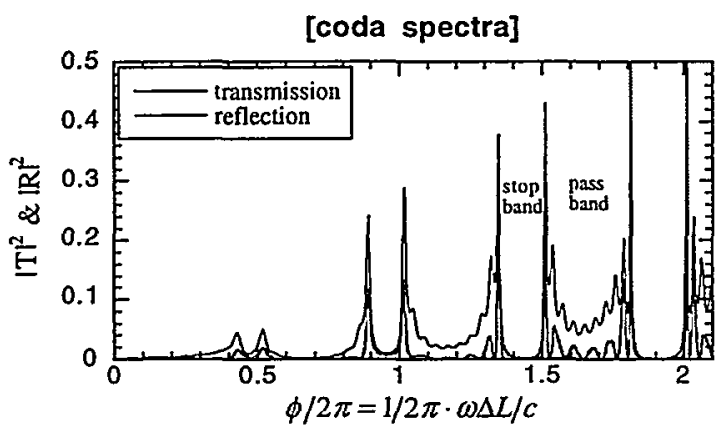

(a) Regularly spaced fractures

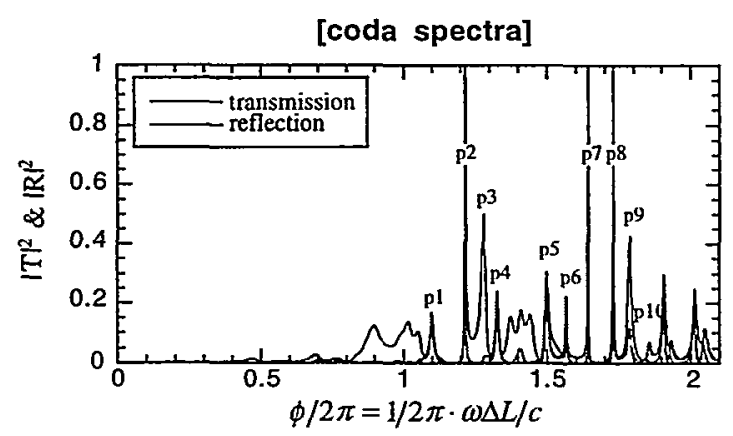

(b) Irregularly spaced fractures

Figure 2.23 Time domain impulse response and spectra of the coda (as indicated in the diagrams) for (a) regularly and (b) irregularly spaced fractures. From the waveforms, it can be seen that the irregular system reflects more wave energy than the regular system. (a) The spectra of the transmitted and reflected wave codas for the regular system have pass and stop bands that are similar especially for high frequencies. (b) Although the band structure is not clear, coda spectra are similar for transmitted and reflected wave codas. These results indicate that the coda (for both reflected and transmitted waves) is the resonances of the fractured zone that radiates the vibration energy into surrounding half spaces. Some of the corresponding peaks (and valleys) for the irregular system are labeled for comparison with Figure 2.19(b). 


\subsection{Infinite System Including Infinite Number of Regularly Spaced Fractures}

\subsubsection{Derivation of dispersion relation}

In this section, one-dimensional wave propagation in a medium with an infinite number of regularly spaced fractures is examined. This is a limiting case of wave propagation in a multiply fractured system. Wave propagation in an infinite system with welded alternating layers has been studied by many researchers including Brillouin (1953), Rytov (1956), and Helbeig (1984). By taking advantage of structural periodicity, displacement and traction, an infinite system can be analyzed using a unit cell that consists of a minimum unique sequence of alternating layers with different acoustic impedances (e.g., Bedford and Drumheller, 1994). The method used for analyzing the wave propagation in impedancecontrast periodic structures is applied for fractured periodic systems to derive a dispersion relation of waves.

Using the propagator method and the displacement-discontinuity boundary conditions for a compliant interface, particle displacement of a steady state wave in an infinite medium with regularly spaced fractures is derived as follows.

Displacement of one-dimensional waves propagating in an intact segment is described by

$$
u(z)=A e^{i(k z-\omega t)}+B e^{-i(k z+\omega t)},
$$

where $k$ is the wave number and $\omega$ is the angular frequency of the wave. The stress is obtained from Eq.(2.26) by applying the spatial derivative as

$$
\sigma(z)=i \omega Z\left(A e^{i(k z-\omega t)}-B e^{-i(k z+\omega t)}\right)
$$

where $Z$ is the acoustic impedance of the intact medium. These expressions are valid within each segment but do not describe the global behavior of the wave. Expressing a wave number for the global (or effective) wave propagation in a single direction by $\hat{k}$, the above expression can be rewritten as

$$
\begin{aligned}
& u(z)=U(z) e^{i(\hat{k} z-\omega t)} \\
& \sigma(z)=T(z) e^{i(\hat{k} z-\omega t)},
\end{aligned}
$$


in which,

$$
\begin{aligned}
& U(z)=A e^{i K^{-} z}+B e^{-i K^{+} z} \\
& T(z)=i \omega Z\left(A e^{i K^{-} z}-B e^{-i K^{+} z}\right)
\end{aligned}
$$

where

$$
K^{+}=k+\hat{k}, K^{-}=k-\hat{k}
$$

Eq.(2.30a) and (2.30b) are independent of time. Applying Floquet's theorem (Floquet, 1883; Brillouin, 1953) requires that $\mathrm{U}(\mathrm{z})$ and $\mathrm{T}(\mathrm{z})$ be periodic functions of layer thickness $\Delta L$, so the following relations are satisfied

$$
\begin{aligned}
& U\left(\Delta L+0^{+}\right)=U\left(0^{+}\right) \\
& T\left(\Delta L+0^{+}\right)=T\left(0^{+}\right) .
\end{aligned}
$$

The displacement-discontinuity boundary conditions at $\mathrm{z}=\Delta L$ are

$$
\begin{aligned}
& T\left(\Delta L+0^{+}\right)=\kappa\left(U\left(\Delta L+0^{+}\right)-U\left(\Delta L+0^{-}\right)\right) \\
& T\left(\Delta L+0^{+}\right)=T\left(\Delta L+0^{-}\right) .
\end{aligned}
$$

Using Eq.(2.32a) and (2.32b), Eq.(2.33a) and (2.33b) become

$$
\begin{aligned}
& T\left(0^{+}\right)=\kappa\left(U\left(0^{+}\right)-U\left(\Delta L+0^{-}\right)\right) \\
& T\left(0^{+}\right)=T\left(\Delta L+0^{-}\right),
\end{aligned}
$$

respectively. By introducing the Eq.(2.30a) and (2.30b) into Eq.(2.34a) and (2.34b), the following matrix equation is derived

$$
\left[\begin{array}{cc}
1-e^{i K^{-} \Delta L} & -1+e^{-i K^{+} \Delta L} \\
\beta\left(1-e^{i K^{-} \Delta L}\right)-2 i & \beta\left(1-e^{-i K^{+} \Delta L}\right)+2 i
\end{array}\right]\left\{\begin{array}{l}
A \\
B
\end{array}\right\}=0
$$

where $\beta=2 \kappa / \omega Z$ is the impedance ratio of a single fracture. The condition for a nontrivial solution to exist is satisfied by equating the determinant of the matrix to zero, which yields

$$
\cos (\hat{k} \Delta L)=\cos (k \Delta L)\left\{1-\frac{\tan (k \Delta L)}{\beta}\right\} .
$$


This is a dispersion equation of the waves propagating in a regularly-spaced infinite onedimensional fractured medium.

\subsubsection{Limiting behaviors of velocities}

When stiffness of the fractures approach infinity (high fracture stiffness limit), Eq.(2.30) becomes

$$
\begin{aligned}
& \cos (\hat{k} \Delta L)=\cos (k \Delta L) . \\
& \therefore \hat{c}_{\text {phase }}=\hat{c}_{\text {group }}=c .
\end{aligned}
$$

Therefore, the wave velocity becomes that of an intact medium.

When the frequency of wave approaches zero (static limit), a Tayler series expansion of Eq.(2.30) becomes

$$
1-\frac{(\hat{k} \Delta L)^{2}}{2 !}+\frac{(\hat{k} \Delta L)^{4}}{4 !}-\ldots . .=1-\frac{(k \Delta L)^{2}}{2 !}+\frac{(k \Delta L)^{4}}{4 !}-\ldots .-\frac{k \Delta L}{2 b}\left\{\frac{k \Delta L}{1 !}-\frac{(k \Delta L)^{3}}{3 !}+\ldots . .\right.
$$

where $b$ is defined by

$$
b=\frac{\kappa \Delta L}{E}
$$

The first order terms of the expression are

$$
\begin{aligned}
& -\frac{(\hat{k} \Delta L)^{2}}{2}=-\frac{(k \Delta L)^{2}}{2}-\frac{(k \Delta L)^{2}}{2 b} \\
& \therefore \hat{k} \Delta L=k \Delta L \sqrt{1+\frac{1}{b}} .
\end{aligned}
$$

Therefore, the phase velocity is

$$
\hat{c}_{\text {phase }}=c / \sqrt{1+\frac{1}{b}}
$$

The group velocity is obtained by taking the derivative of Eq.(2.42) with respect to $\omega$ as 


$$
\hat{c}_{\text {group }}=c / \sqrt{1+\frac{1}{b}},
$$

which is identical to the phase velocity. Both velocities are related to a static stiffness of the fractured system as follows

$$
\begin{aligned}
& \frac{1}{\rho \hat{c}^{2}}=\frac{1+1 / b}{\rho c^{2}}=\frac{1}{\rho c^{2}}+\frac{1}{\kappa \Delta L} \\
& \therefore \frac{1}{\hat{M}}=\frac{1}{M}+\frac{1}{\kappa \Delta L},
\end{aligned}
$$

where $\mathrm{M}$ is the elastic modulus. Eq.(2.46) shows that the compliance of the system derived from a zero-frequency limit of velocities is equal to the static compliance that is given by a sum of the compliances in the intact medium and the fractures (i.e., the Backus average).

\subsubsection{Phase and group velocities}

The phase (or the wave number)-frequency relation given by the dispersion equation Eq.(2.36) is shown in Figure 2.24(a). The dimensionless fracture stiffness is assumed to be $\mathrm{b}=\kappa \Delta L / E=10$. Only real solutions are shown as an imaginary term in a complex solution leads to exponential decay of the wave. Therefore, frequency bands devoid of real solutions are the stop bands that prohibit propagation of waves. Figure 2.24(b) shows phase and group velocities of the wave computed from the dispersion relations. Within each pass band of the infinite system, phase velocity shows weak negative dispersion while group velocity shows both positive and negative dispersion that becomes zero at boundaries between the pass and stop bands. As a wave with zero group velocity is a standing wave, this indicates that the system resonates at these frequencies.

For a system with a finite number of fractures, phase and group velocities are computed from the phase spectra of the transmitting waves. By comparing Figure 2.25 with Figure 2.24 , it can be seen that the 10-layer (11-fracture) system has similar dispersion behavior as the system with an infinite number of fractures. For a regular finite system, the group velocity approaches zero at pass and stop band boundaries. This result verifies that the peaks in the coda spectra in Figure 2.23 are indeed resonances caused by the periodicity of the structure in the system. The irregular system also shows sharp decrease in group 
velocites at the boundary between large and small transmission coefficient ranges, which implies that the system is close to a resonant state.

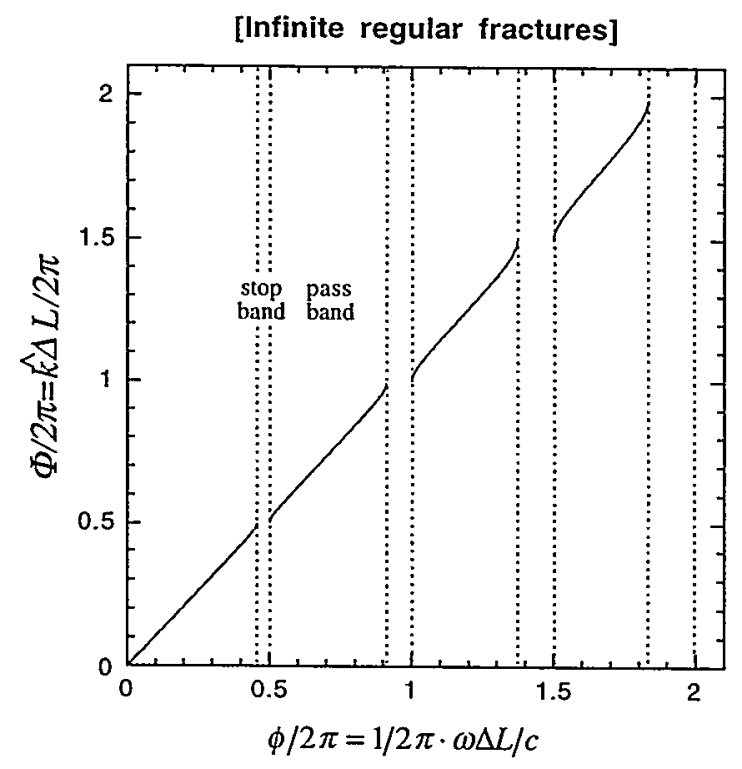

(a) Dispersion relation (wavenumber-frequency)

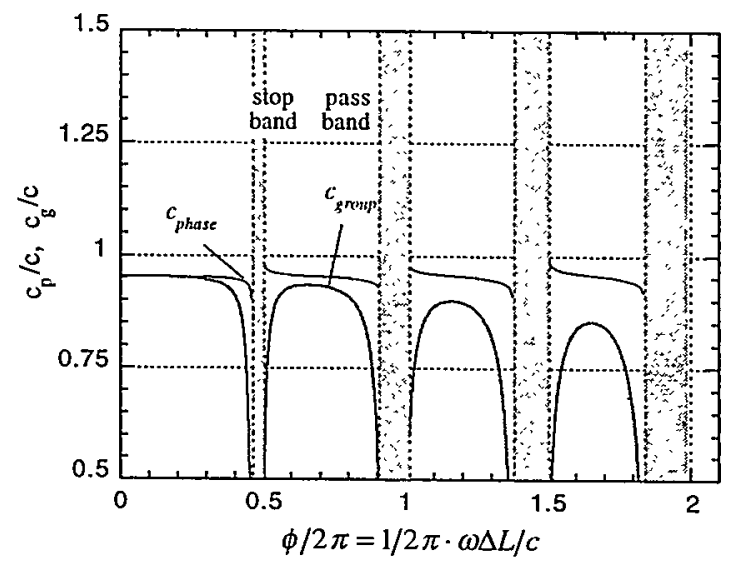

(b) Phase and group velocity

Figure 2.24 Dispersion in a regularly spaced infinite series of fractures. In Figure 2.22(a), only the real solutions of the dispersion equation are shown as a complex solution cannot propagate in an infinite system. Regions where the real solution exists are the pass bands (all energy propagates without attenuating). The shaded regions where no real solution is found are the stop bands. (b) In each pass band, the phase velocity shows a negative dispersion (velocity decreases with increasing frequency) while the group velocity exhibits both positive and negative dispersions, with zero velocity at the boundaries between the pass and the stop bands. 
[Finite regular fractures]

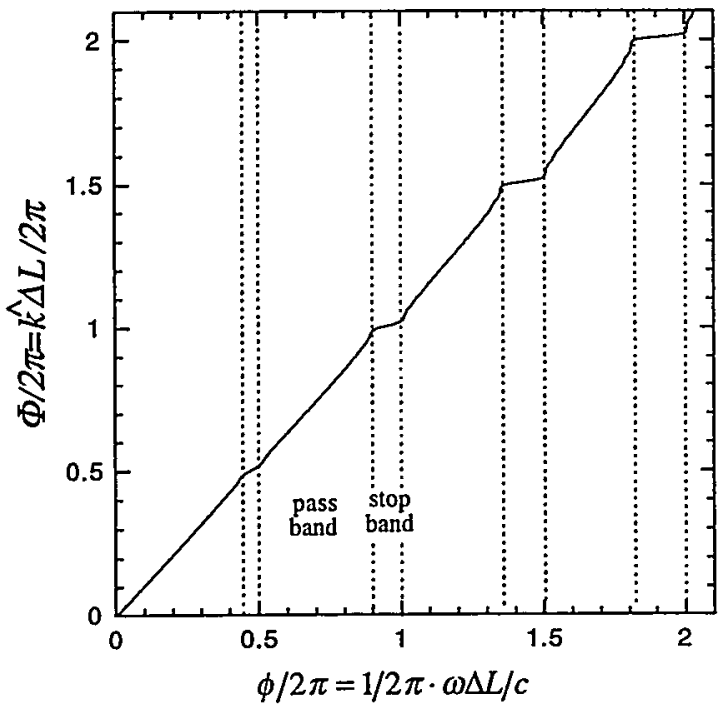

[Finite irregular fractures]

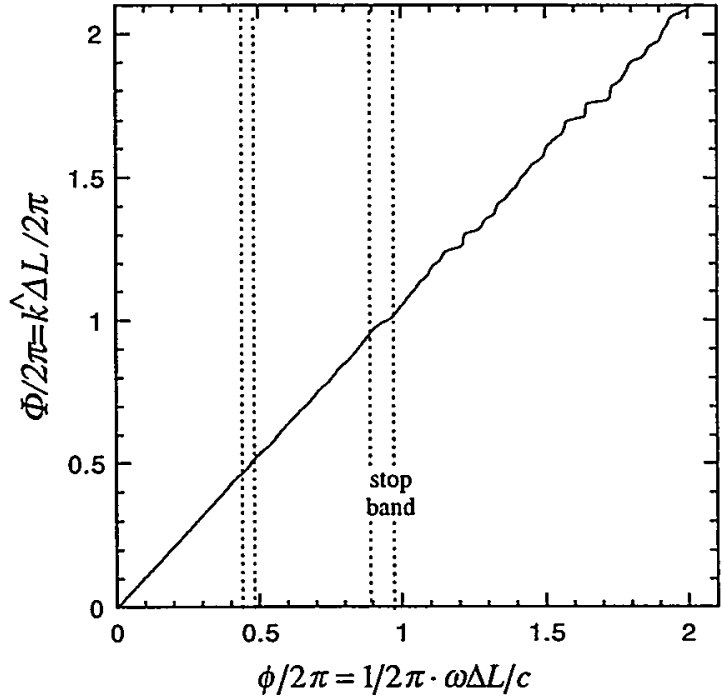

(a) Dispersion relation (wavenumber-frequency)
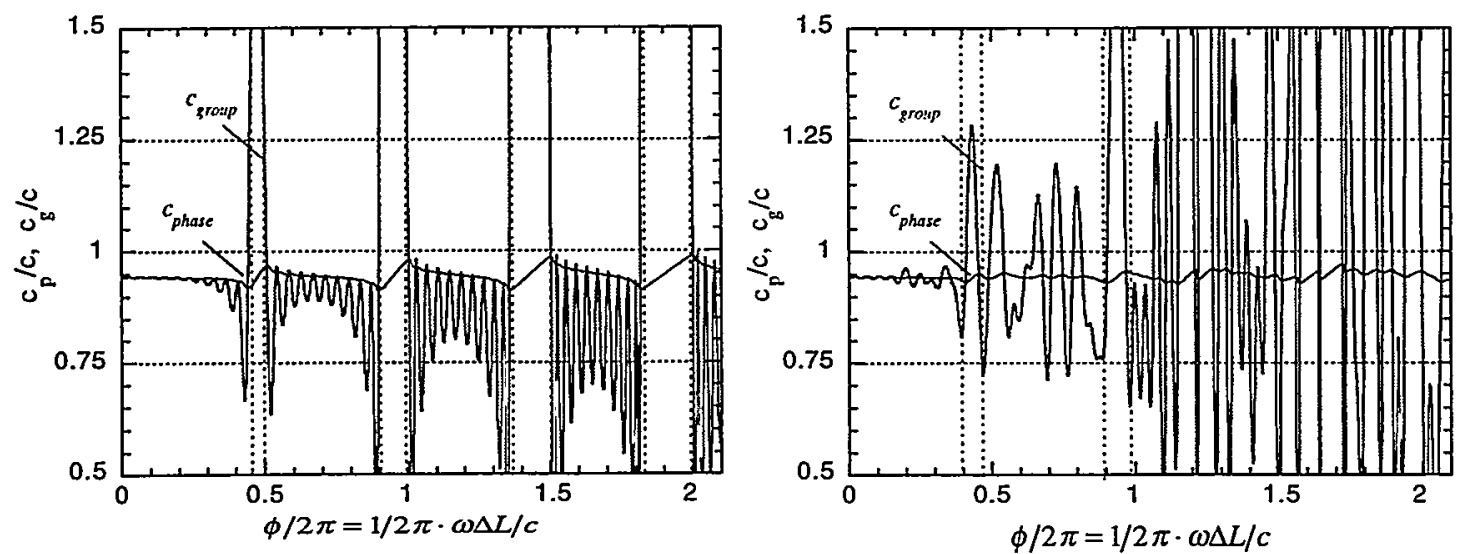

(b) Phase and group velocity

Figure 2.25 Dispersion in regularly and irregularly spaced finite fractures. The regular system exhibit a similar dispersion behavior as the infinite system. The peaks and valleys of group velocity within pass bands are due to the finite number of fractures. The valleys essentially corresponds to the resonances of the finite system. At boundaries between pass and stop bands, there is a sharp decrease in the group velocity. These corresponds to the peaks in the coda spectra observed in Figure 2.23. Small group velocity indicates that the wave in the system is becoming a standing wave (wave with zero group velocity) and the system is near resonance. Although the pass and stop band structures are not clear, the irregular system also shows a sharp decrease in the group velocity at pass and stop band boundaries. 


\subsection{Summary}

This chapter has discussed the effects of fractures on the resonance characteristics of finite and infinite one-dimensional systems.

It has been shown that an impedance ratio between a fracture and intact medium $\beta$ is the controlling parameter for both resonance frequency shifts and attenuation due to rheological and radiation damping. This result provides the link between the resonance characteristics and the mechanical properties of the fracture. For finite fractured systems, the relative locations of the fracture and the nodes of vibration modes are shown to have significant effects on the resonance frequency shift and the attenuation of the resonance. Due to mismatched locations of fractures and vibration nodes and antinodes, resonances of a medium containing irregularly spaced fractures can spatially localize the modal energy between the fractures. These results potentially provide valuable knowledge for detecting and locating fractures in geologic structures built in the subsurface and above ground. Particularly, localized resonance can be a powerful diagnostic tool for detecting motions of a rock mass that is isolated within the structure by fractures.

For interpreting seismic waves propagating through multiple parallel fractures, both regularly and irregularly spaced fractures were embedded in an infinite medium. Computed transmission and reflection coefficients showed that the pass and the stop band structures were identical to those in the FRF of the finite systems. An important implication of this result was that by examining transmission coefficient and reflection coefficient spectra of propagating waves, the resonance characterisitcs of the embedded fractured system can be determined. From the determined resonance characteristics, properties of the fractures (stiffness of fractures, spacings, etc.) might also be determined.

Another important implication is the relation between resonances (standing waves) and propagating waves. In a multiply fractured system, distinction between a propagating wave and a resonance in the system becomes obscure. If a finite system containing fractures is embedded in an infinite medium, perfect resonance (without attenuation) cannot occur due to radiative loss of the energy into surrounding halfspaces. On the other hand, propagating waves show large group time delays and attenuation due to multiply reflected and trapped energy between fractures. Whether wave propagation or resonance dominates the system is determined by the stiffness of the fracture. Perfect wave propagation occurs for infinite fracture stiffness (welded interface) and perfect resonance occurs for zero 
fracture stiffness. For intermediate fracture stiffnesses, resonances of a system can be identified from sharp decreases in group velocities. Therefore, by examining such characteristics in a wave spectrum, the internal structure of a fractured medium can be determined.

This chapter also presented an analytic expression for the dispersion relation associated with an infinite series of regularly spaced fractures. The dispersion relation predicted both pass and stop band structures in the wave spectrum. Within each pass band, a wave propagated at a reduced velocity without attenuating. The group velocity of the wave approached zero at boundaries between pass and stop bands. At these frequencies, the behavior of waves in the system change from that of propagating waves to standing waves.

Many of the above results indicate that acoustic resonance can be used to identify the internal structure of fractured solids. However, for quantitative detection and characterization of fractures, further research should be performed to incorporate the results from this study into techniques such as modal analysis utilizing the finite element method (FEM). The FEM has become a common technique to locate a defect or fracture from resonance with recent advent of computer technology. For example, Cawley and Adams (1979) used the shifts in the resonance frequencies of a structure to determine the location of damage with a finite element model. However, the use of the numerical model without understanding the underlying mechanics may lead to inaccurate or incorrect results. With a basic understanding of the effect of fractures, information contained in the measured resonances can be utilized to their full extent.

In the following chapter, some of the results obtained in this chapter for a finite system including a single fracture will be demonstrated by laboratory experiments using resonant bar tests on thin rods of rock. Resonance and wave propagation in periodically fractured media is revisited at the end of this thesis for the case of multi-dimensional wave propagation in fractured media. 


\section{Resonant Bar Test on Fractured Rock}

\subsection{Introduction}

The resonant bar test has been conducted to measure the elastic and attenuation properties of rock and concrete in laboratory for decades. It is sometimes preferred over ultrasonic transmission tests for several reasons. Among these reasons are the relatively low frequency range employed for the tests $(1 \mathrm{kHz} \sim 100 \mathrm{kHz})$ that is close to the range used for borehole acoustic logging of geological structures and the accuracy of the method in determining attenuation properties (Boubié et al., 1987). Most resonant bar tests, however, are conducted to assess the resonance of intact and homogeneous specimens. As rock and concrete generally contain fractures at many scales, treating a specimen with a fracture as a homogeneous medium may lead to erroneous material properties. Therefore, it is important to understand the effect of fracture properties on the resonance of bar specimens. Furthermore, for detecting and characterizing fractures in situ, dynamic properties of a fracture under a variety of conditions are required. Using a resonant bar test, low-frequency mechanical properties of fractures can be examined in the laboratory. 
Hesler (1995) examined the resonance of rock specimens containing fractures. The specimens used by Hesler were rock cylinders containing healed natural fractures and rods of engineered materials such as plexiglass and aluminum with a notched cross section and a glued interface between the two intact pieces. A similar experiment was conducted by Bamnios and Trochidis (1995) using longitudinal and vertical vibrations of a cantilever beam with a notched cross section. The notched and glued cross sections provided a strong acoustic impedance contrast in the specimens that altered resonance frequencies and mode shapes significantly. By applying the displacement-discontinuity boundary conditions used for modeling wave propagation across and along a compliant interface between two elastic halfspaces (Kendall and Tabor, 1971; Schoenberg, 1980; Pyrak-Nolte and Cook, 1987), Hesler developed a simple equation deriving the effect of fracture stiffness on the resonance frequencies of fractured bars.

It is well known that the stiffness of a fracture increases significantly with application of normal stress due to the increase in the contact between surface asperites (Greenwood and Williamson, 1966; Goodman, 1976; Brown and Scholz, 1985). Therefore a fracture in situ exhibits stiffnesses ranging from zero (open fracture) to infinity (welded fracture). In order to study the effect of fracture stiffnesses on resonance, a range of stresses have to be applied to the fracture. Resonant bar tests are usually conducted on specimens under atmospheric pressure. To test a specimen under high confining pressure similar to field conditions, an elaborate testing setup and corrections of obtained results are required (Lucet et al., 1991), including the use of compressed Helium gas to minimize the acoustic coupling between a specimen and its surrounding media.

The focus of the experiments performed in this chapter is on the effects of fracture properties on the resonances of rock bars. As the stiffness of a fracture created perpendicular to a rod specimen can be changed by applying an axial load, only uniaxial compression is necessary to observe the effects of fracture stiffness on resonances. Vibrations inside the specimen are isolated by compliant plastic rings attached to the ends of the rod. Due to the large acoustic impedance contrast between the specimen and the rings, boundary conditions for the resonance become approximately stress-free. Because the boundary conditions are only approximately stress-free, fracture stiffness on resonance frequencies are not exactly predicted by the theory developed in Chapter 2. However, these results do show basic agreement with the theory presented in the previous chapter. 


\subsection{Experimental Setup}

\subsubsection{Sample description}

The resonance tests were conducted on thin rods of plexiglass, aluminum, and rock (Sierra White Granite and Berea Sandstone). The rod specimens were $32.8 \mathrm{~cm}$ in length and $5.08 \mathrm{~cm}$ in diameter. The granite specimen contained a large number of microcracks that were aligned roughly parallel to a single plane. Detailed measurements on the granite will be shown later (Chapter 5). The sandstone specimen contained bedding structures that were visible as thin, red stained layers, possibly iron oxide formations. These microstructures lead to transversely isotropic elastic properties of the rocks. In preparing the specimens, the rods were cored perpendicular to the plane of anisotropy (microcrack or bedding planes) and oven-dried at $103{ }^{\circ} \mathrm{C}$ for at least 48 hours before the tests. Static and high-frequency dynamic properties of the specimens are shown in Table 3.1.

Table 3.1 Static and Dynamic Properties of Specimens

\begin{tabular}{|c|c|c|c|c|c|}
\hline specimen & density & $\begin{array}{l}\text { dynamic } \\
\text { Young's } \\
\text { modulus }\end{array}$ & $\begin{array}{c}\text { dynamic } \\
\text { Poisson's } \\
\text { ratio }\end{array}$ & $\begin{array}{l}\text { static } \\
\text { Young's } \\
\text { modulus }\end{array}$ & $\begin{array}{c}\text { static } \\
\text { Poisson's } \\
\text { ratio }\end{array}$ \\
\hline aluminum & $2.70 \mathrm{~kg} / \mathrm{m}^{3}$ & $71.7 \mathrm{GPa}$ & 0.34 & $70 \mathrm{GPa}$ & 0.35 \\
\hline plexiglass & $1.18 \mathrm{~kg} / \mathrm{m}^{3}$ & $6.17 \mathrm{GPa}$ & 0.33 & $4.19 \mathrm{GPa}$ & 0.36 \\
\hline $\begin{array}{l}\text { Sierra White } \\
\text { Granite }\end{array}$ & $\begin{array}{l}2.62 \mathrm{~kg} / \mathrm{m}^{3} \\
\text { (ovendried) }\end{array}$ & $19.9 \mathrm{GPa}$ & $\begin{array}{l}\text { assumed } \\
\text { to be } 0\end{array}$ & $21.1 \mathrm{GPa}$ & 0.038 \\
\hline $\begin{array}{l}\text { Berea } \\
\text { Sandstone }\end{array}$ & $\begin{array}{l}2.10 \mathrm{~kg} / \mathrm{m}^{3} \\
\text { (ovendried) }\end{array}$ & $9.22 \mathrm{GPa}$ & 0.066 & $9.36 \mathrm{GPa}$ & 0.055 \\
\hline
\end{tabular}

Note: Static Young's moduli were determined from the initial slope of the stress-strain curves at OMPa axial stress after a single cycle of load was applied to the specimens. Dynamic Young's moduli were determined from ultrasonic transmission tests using $1 \mathrm{MHz}$ sources. For the granite specimen, the dynamic Poisson's ratio was assumed to be zero to obtain the Dynamic Young's modulus from the P-wave modulus. This was done because the elastic properties of the rock could not be properly determined from the $\mathrm{P}$ - and S-wave velocities due to the strong elastic anisotropy. 


\subsubsection{Free vibration test setup}

Conventional free vibration tests were conducted to measure the resonance frequencies and attenuation of rod specimens without confinement. The specimens were held in styrofoam rings to isolate the vibration (Figure 3.1a). A small piezoelectric crystal with a resonance frequency of $1 \mathrm{MHz}$ was attached to one end of the specimen using adhesive wax (Petro Wax, PCB Piezotronics). Either swept sine waves (chirp signal) or continuous sine waves (stationary waves) sweeping a range of frequencies were used to drive the crystal and resonate the specimens after amplification of the signals by a voltage amplifier (KrohnHite 7602). The resulting accelerations were measured by a miniature accelerometer (PCB Piezotronics, 309A, resonance frequency $>120 \mathrm{kHz}$ ) attached to the other end of the specimen with the adhesive. The mass of the crystal and the accelerometer used for the tests were $3.5 \mathrm{~g}$ and $1.5 \mathrm{~g}$, respectively. The measured signals were displayed, analyzed, and stored using a spectrum analyzer (ONO SOKKI CF6400).

\subsubsection{Axially confined vibration test setup}

Axially confined vibration tests were conducted using a similar experimental setup as the free vibration tests, but with the specimens under axial load. The load was applied to the specimens through a pair of PVC rings (Figure 3.1b). The dimensions of the rings were $7.02 \mathrm{~cm}$ in length and $2.66 \mathrm{~cm}$ and $3.28 \mathrm{~cm}$ in inner and outer diameters, respectively. The density of the rings was $1.07 \mathrm{~g} / \mathrm{cm}^{3}$. The accelerometer and the piezoelectric source were housed inside the rings during the tests.

\subsubsection{Measurement of frequency responses}

The force applied to a specimen by a piezoelectric crystal decreases with decreasing frequency of the applied electric signal. Therefore measured acceleration has to be corrected for this effect. An experimental frequency response function (FRF) is obtained as a spectral ratio between measured accelerations on a specimen and accelerations on the source piezoelectric crystal suspended in the air. To measure the acceleration on the crystal, an accelerometer was directly attached to the surface of the crystal. As the measured acceleration on the crystal is roughly proportional to the force applied to a specimen, the computed FRF is essentially an accelerance (acceleration response to unit force excitation). A typical acceleration response measured for a source piezoelectric crystal is shown in Figure 3.2. 


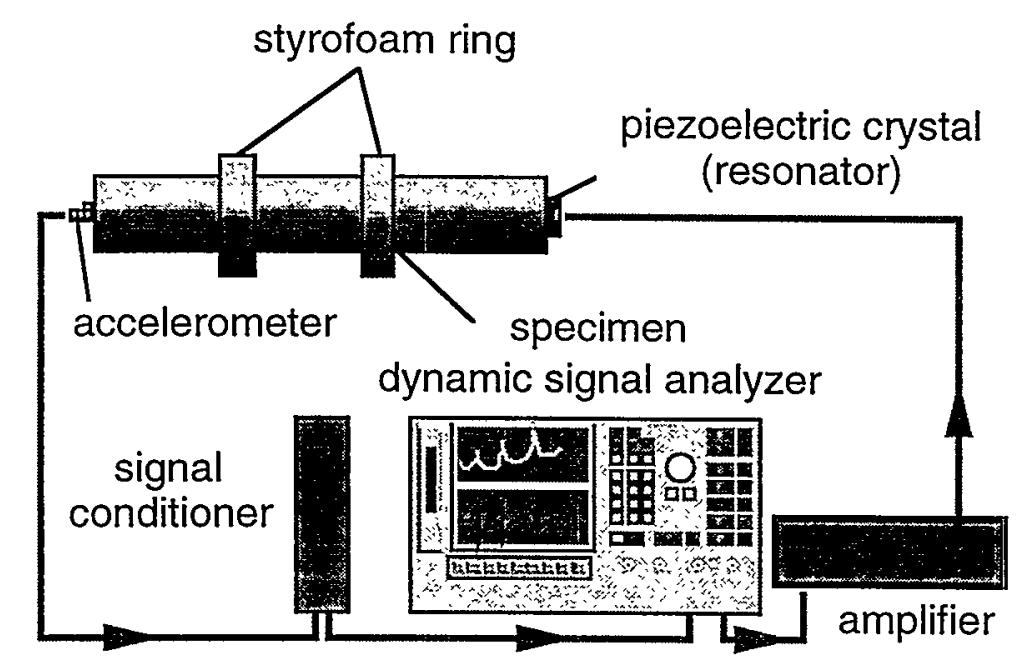

(a)free vibration test setup

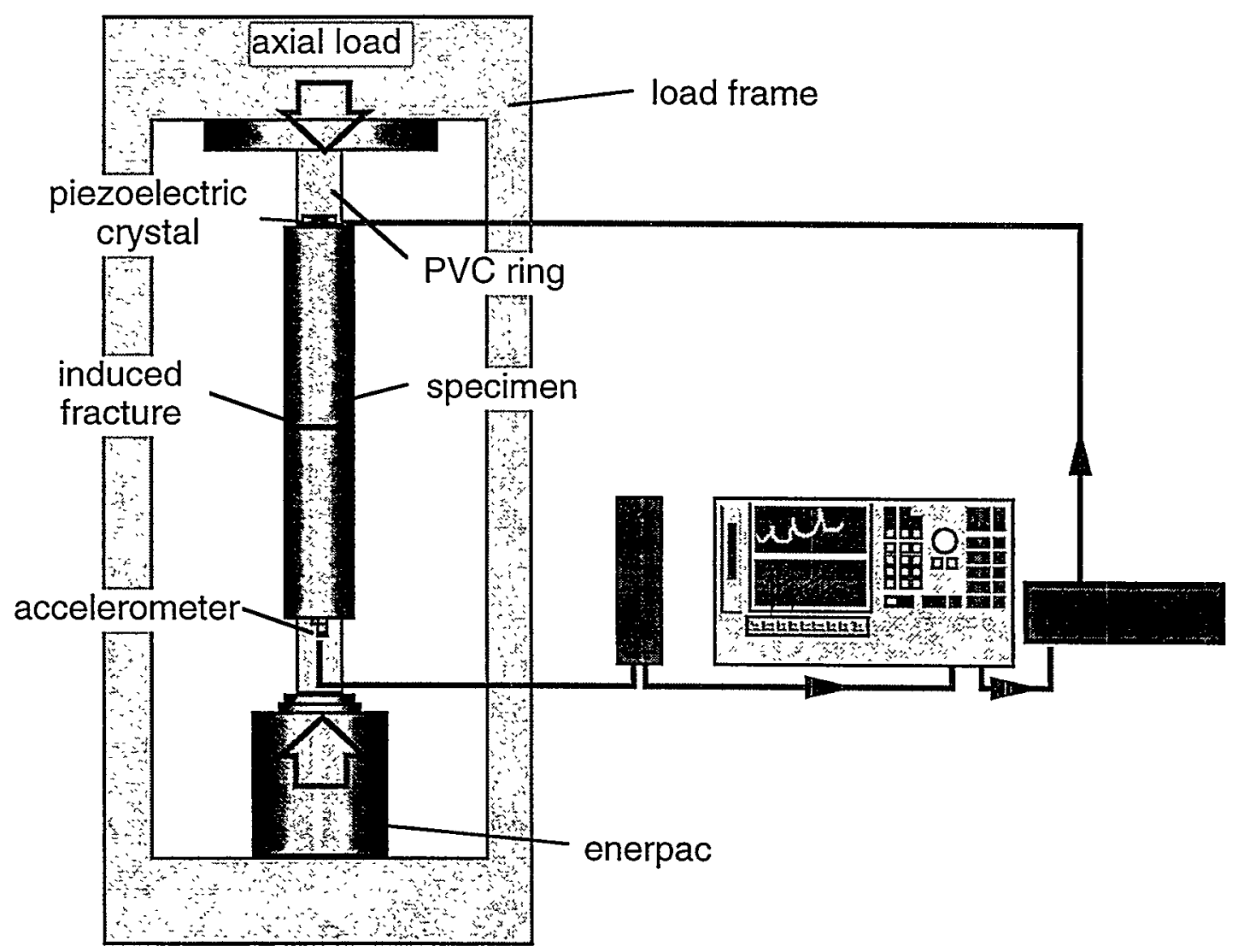

(b) axially confined vibration test setup

Figure 3.1 Experimental setup used for vibration tests on rod specimens. Free vibration tests were conducted with specimens suspended on styrofoam rings to acoustically isolate the vibration. For axially confined vibration tests, the specimens were loaded through plastic rings. 

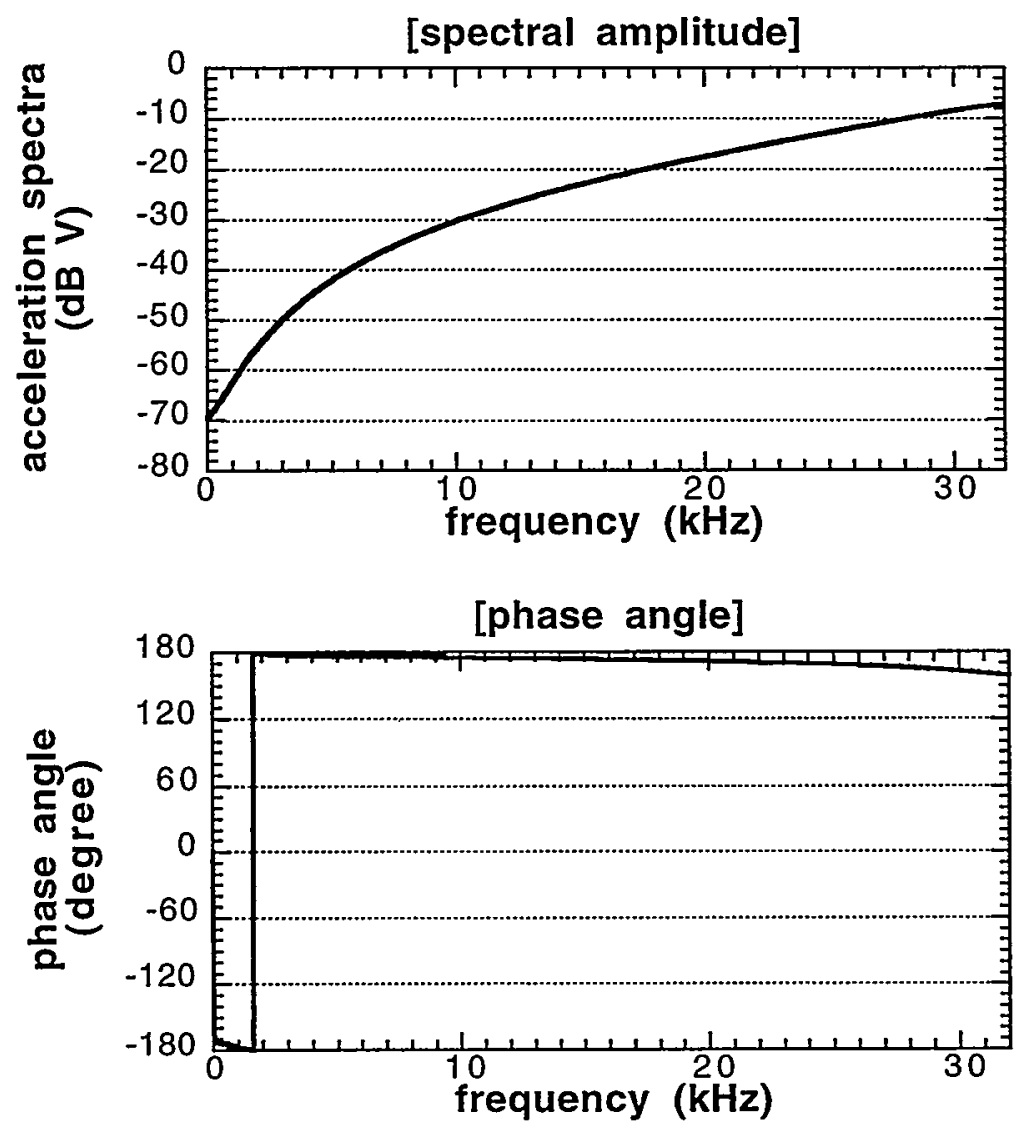

Figure 3.2 Acceleration spectra measured on a source piezoelectric crystal suspended in the air. An accelerometer was directly attached to the crystal with wax. The crystal was driven by single frequency sine waves that swept from 0 up to $32 \mathrm{kHz}$. Amplitude of the acceleration increases with increasing frequency. The phase spectra show approximately constant phase for the frequency range shown above.

\subsection{Free Vibration of Rod Specimens}

The dynamic Young's modulus along the axis of a thin finite rod can be measured from resonance frequencies of the longitudinal vibrations. If the vibration is truly onedimensional, the resulting resonance frequencies consist of harmonics of the primary mode that are equally spaced in frequency. From any of the harmonics, Young's modulus is determined by

$$
E=\rho c_{E}^{2}, c_{E}=f^{(n)} \cdot \frac{2 L}{n}
$$


where $\rho$ is the density, $c_{E}$ is the velocity of the wave propagating along the rod, $\mathrm{L}$ is the length of the rod, $\mathrm{n}$ is the mode number, and $f^{(n)}$ is the resonance frequency of the $\mathrm{n}^{\text {th }}$ mode (overtone) in $[\mathrm{Hz}]$. For a circular rod with a finite diameter, waves propagating along the rod become dispersive, decreasing the phase velocity from a "bar velocity" computed by $\sqrt{E / \rho}$ to a Rayleigh wave velocity with increasing frequency of the wave (Pochhammer, 1876; Graff, 1975). Corrections of Eq.(3.1) for a finite rod diameter and length are made by many researchers including Bancroft (1941), Love (1944), Rayleigh (1945), and Spinner and Tefft (1960). The corrected equation has a generic form

$$
E=\rho c_{E}^{2} \times \frac{1}{U}
$$

where $U$ is the correction factor that accounts for the effect of Poisson's ratio and the diameter to wavelength ratio (D/ $\lambda$ ) of a specimen. Rayleigh (1945) derived an approximate expression known as Rayleigh's correction for small $D / \lambda$. Love (1941) derived the complete governing equation of the resonance problem based on the variational principle. Bancroft (1941) derived the correction factor for an infinitely long rod by numerically solving the involved differential equations. Spinner et al. (1960) experimentally tested these results and found good agreement between predicted and measured resonance frequencies. A summary of the methods is provided by Spinner and Tefft (1961).

As the specimens used for the tests are relatively short (length to diameter ratio $\mathrm{L} / \mathrm{D}=6.46$ ), to avoid the buckling of the bar during the axially loaded tests, waves propagating along the rod can be dispersive even for the lowest order mode. To examine the effect of dispersion, wave velocity in the bar is calculated by Eq.(3.1) for the first several longitudinal mode resonances. The resulting velocities are normalized by the Young's modulus velocity $\sqrt{E / \rho}$ determined by a statically measured Young's modulus for a very small axial stress. Wave velocities for the aluminum and plexiglass specimens shown in Figure 3.3a (except for the static result) exhibit strong negative dispersion (velocity decreases with increasing order of mode). The large difference between the static and dynamic results for the plexiglass may be due to the viscoelastic behavior of the material. In contrast, the sandstone and granite specimens show much smaller dispersion. The different dispersion behaviors among the specimens are due to small Poisson's ratios of the rocks. Static stress-strain measurements reveal that the Poisson's ratios of the granite and sandstone specimens determined from their lateral expansion during uniaxial 


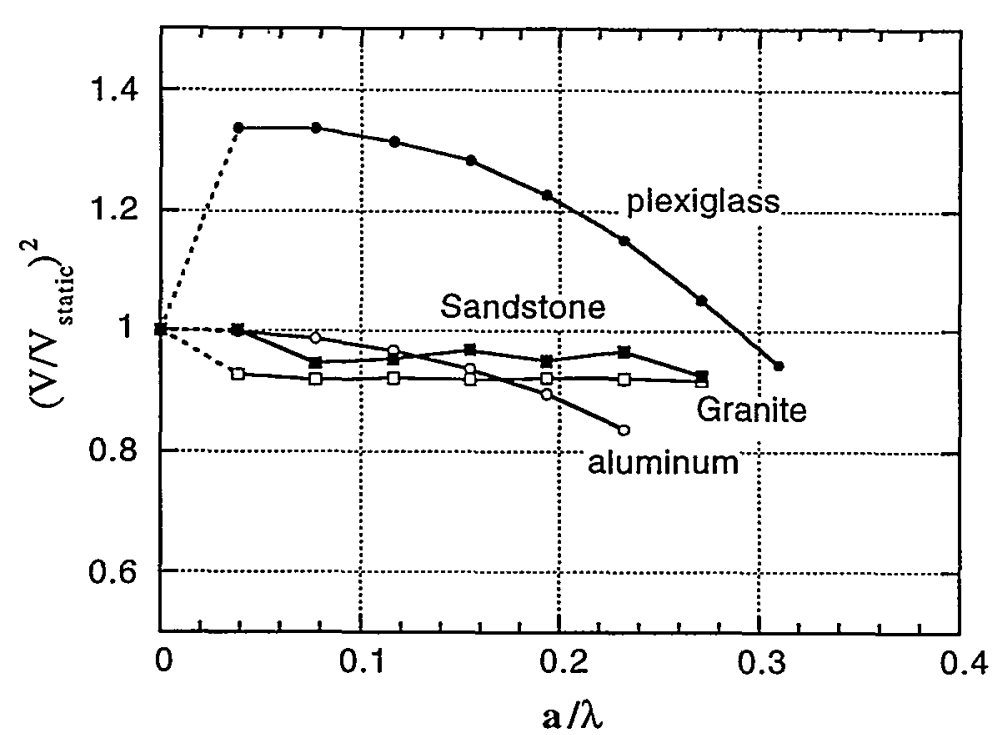

(a) Phase velocity measured by resonant bar test.

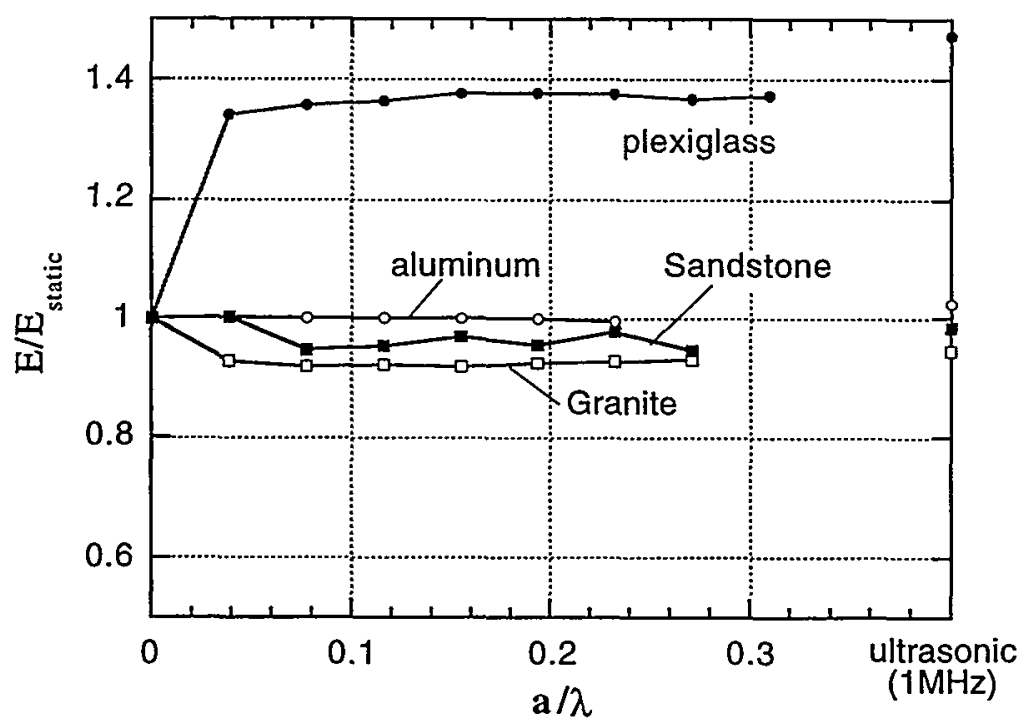

(b) Young's modulus determined from resonant bar test

Figure 3.3 Young's modulus determined from resonant bar tests. Top figure shows phase velocities computed from measured resonance frequencies and normalized by the phase velocities for the static properties of the specimen. Bottom figure shows the determined Young's modulus normalized by the static Young's modulus of the specimens after applying Bancroft's (1941) correction for Poisson's ratio and rod radius to wavelength ratio $(a / \lambda)$. The moduli obtained from ultrasonic transmission tests are shown on the far right ( $a / \lambda$ is not equal to 0.4 for these results). 
loading are negligibly small (less than 0.06). The marked low Poisson's ratios are attributed to the closure of thin flat microcracks in granite and loose grain contact in sandstone. Due to these small Poisson's ratios, waves propagating along the rod do not involve large surface motion in lateral directions. Therefore, dispersion due to the inertia effect of lateral motion does not occur. Aluminum and plexiglass specimens exhibit strong velocity dispersion due to their large Poisson's ratios $(0.35$ and 0.36 , respectively, from static loading tests).

The results were corrected for the geometry and Poisson's ratio (determined from static tests) of the specimens using Bancroft's. correction (Figure 3.3b). The same figure also includes the results obtained from ultrasonic transmission tests with $1 \mathrm{MHz} \mathrm{P}$ - and $\mathrm{S}$-wave sources for comparison. Although Young's modulus along the axis of the rod cannot be determined from the two velocities for transversely isotropic material, approximate values are obtained by assuming a zero Poisson's ratio for the granite (Young's modulus equals P-wave modulus) and elastic isotropy for the sandstone. The corrected results should give Young's modulus of the specimens that includes only the effect of velocity dispersion due to the dissipation of wave energy. Young's modulus of the aluminum specimen is constant for all the resonances. The modulus for the plexiglass specimen shows a large increase from 0 th mode (static) to the frequency for the first mode of resonance. Although the increase in velocity (or modulus) is small, a positive dispersion can be seen for most of the observed resonances. Young's modulus determined from the ultrasonic tests was the largest, which is consistent with the positive dispersion trend. The correction hardly changes the results for the rock specimens as the small Poisson's ratios make the correction factor $U$ very close to unity.

Attenuation of the specimens was measured by the half-power method for each resonance peak (Figure 3.4). Attenuation in aluminum appears to be rather large especially for higher order modes ( $Q$ becomes as low as 160). The other specimens exhibit roughly constant attenuation for all the observed resonances except for the highest order mode. This relatively high attenuation in aluminum is possibly due to the energy loss at the contacts with the external environment (coupling with source piezoelectric crystal, foam rings, air). As the attenuation in rock and plexiglass rods is much larger than the aluminum rod, the attenuation caused by the external coupling for rock and plexiglass rods is negligible. Therefore the measured attenuation for the rock and plexiglass specimens is approximately the intrinsic attenuation of these materials. 


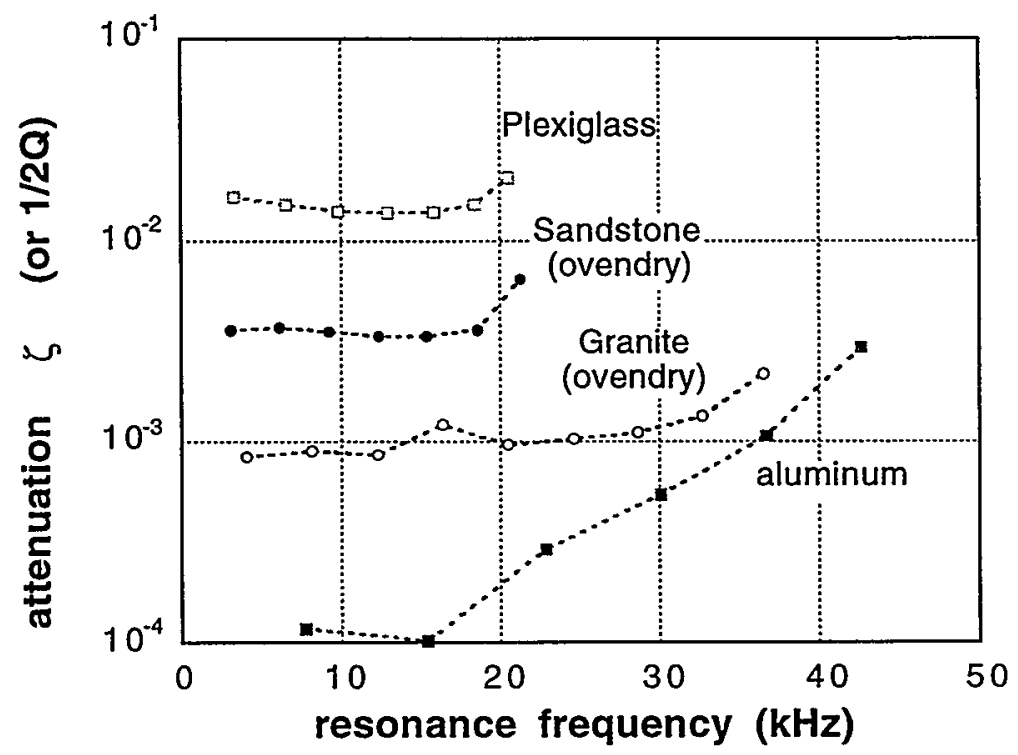

Figure 3.4 Attenuation of the specimens determined by the half-power method. Aluminum specimen shows rather large attenuation for metal possibly due to the coupling loss to the external environment. Rock and plexiglass specimens exhibit approximately constant attenuation for most of the observed resonances except for the last modes.

\subsection{Resonance of Axially Confined Rod Specimens}

\subsubsection{Effect of end coupling on resonance}

Typical crystalline rocks with microcracks and granular rocks with compliant grain contact exhibit an increase in elastic moduli when a compressional load is applied. The increase in moduli results in higher velocities of the propagating elastic waves that can be determined from the resonance frequencies of the rocks. Resonance measured using the experimental setup described in Section 3.2.3, however, may be affected by the mechanical coupling between the specimen and the surrounding load frame through the plastic rings.

To examine this effect, resonance frequencies and attenuation of the plexiglass and aluminum rods were first measured with increasing axial stress. Figure 3.5(a) and (b) shows the FRF's of the rods measured for a range of axial stresses. Shifts in the resonance frequencies due to the end coupling is shown in Figure 3.6(a) and(b). Measured resonance frequencies are normalized by those obtained for free vibration. The aluminum rod shows very small change in resonance frequencies for increasing axial stress. Although most of the resonances for the plexiglass specimen show only a small change in 

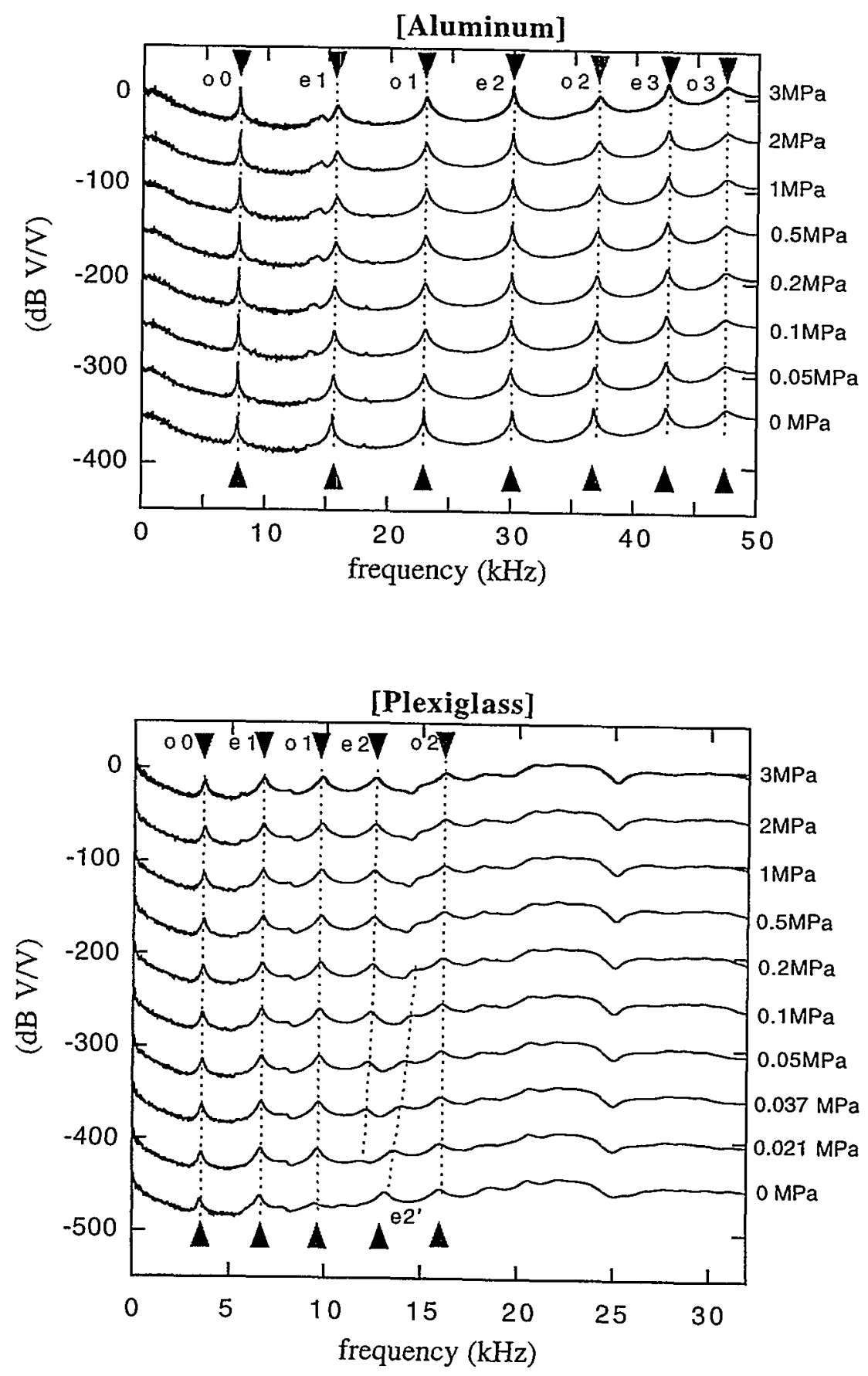

Figure 3.5 Frequency response spectra of uniaxially confined aluminum and plexiglass rods. (a) Resonance frequencies of the aluminum rod show very small changes while (b) some of the modes for the plexiglass rod show a large resonance frequency shift and changes in the structure of the FRF (e.g., e2 mode disappears as the load was decreased and is replaced by an emerging e2' mode). For both specimens, attenuation of the resonances changes significantly with applied load. 
[Aluminum]

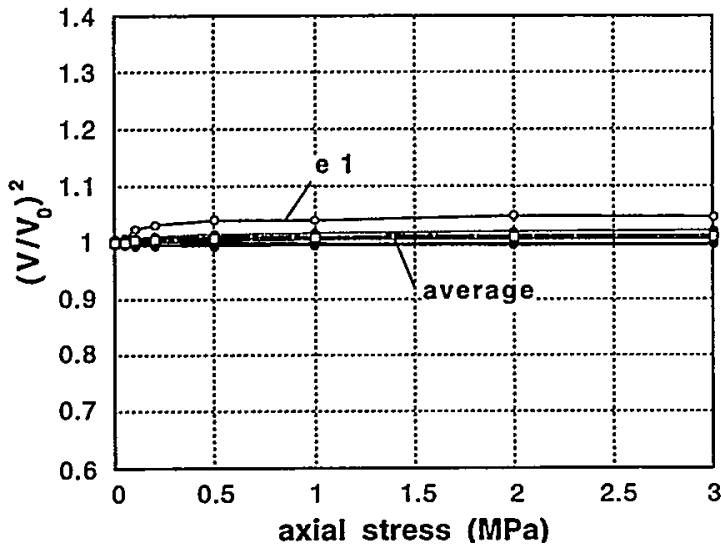

(a) phase velocity change in aluminum

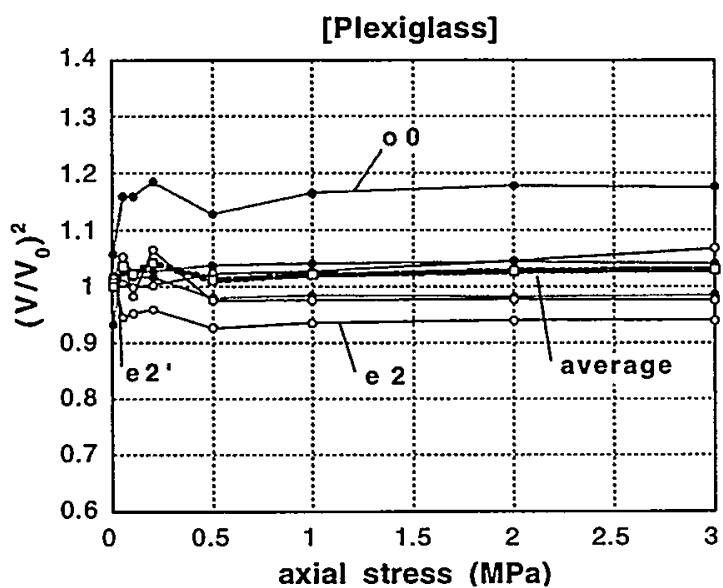

(b) phase velocity change in plexiglass

Figure 3.6 Effect of axial confinement on the resonance frequency (or phase velocity) of aluminum and plexiglass specimens. Resonance frequency of each mode is normalized by the mode's free vibration frequency. The aluminum specimen shows only a small change in the resonance frequency while the plexiglass resonance frequencies scatter around the free vibration frequencies. For both specimens, a large change in the resonance frequency occurs up to about $0.05 \mathrm{MPa}$ of the axial load. For higher axial loads, the effect becomes constant and the resonance frequencies do not show a large change.

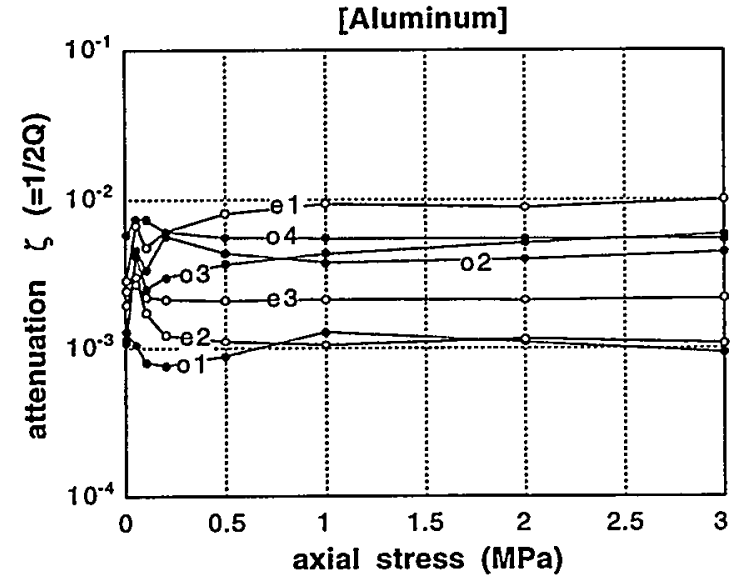

(a) attenuation change in aluminum

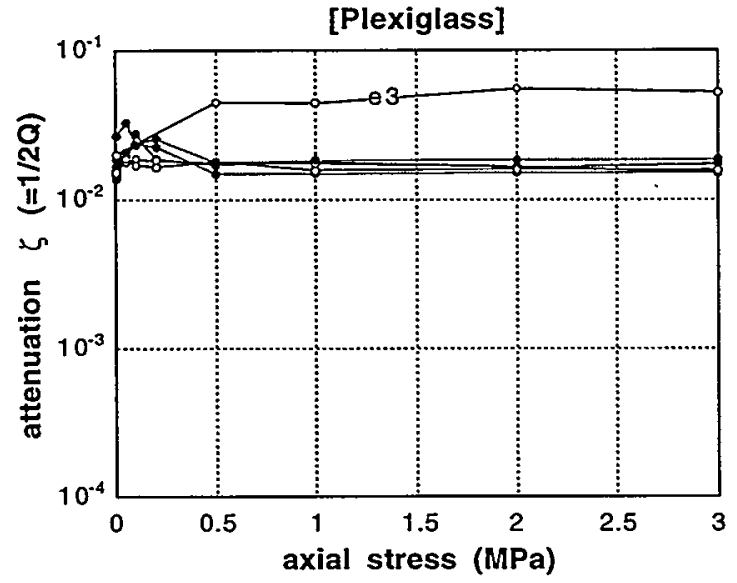

(b) attenuation change in plexiglass

Figure 3.7 Effect of axial confinement on the attenuation of aluminum and plexiglass specimens. The aluminum specimen shows a large increase in attenuation possibly due to the radiation of the vibration energy through the plastic rings. The plexiglass specimen shows relatively small change in attenuation except for the highest order mode (e3) shown here. Attenuation for the e2' mode is shown only up to $0.2 \mathrm{MPa}$ of axial stress after which it switches to the e 2 mode. The seemingly small increase in attenuation in the plexiglass specimen is due to a large intrinsic attenuation of the specimen that overwhelms the additional radiation damping. For both specimens, a large change in the attenuation occurs for small axial loads. 
the resonance frequencies, a few modes exhibit erratic behaviors such as a large frequency shift, and vanishing and emerging peaks (Figure 3.5). The erratic resonance behavior of the plexiglass rod is caused by a relatively weak impedance contrast between the specimen and the plastic rings. In contrast, the aluminum specimen has a much larger impedance contrast (impedance ratio between aluminum and plexiglass is 38 for the static moduli). Change in the attenuation is also shown in Figure 3.7 (a) and (b). As can be seen from the plot, attenuation of the aluminum rod increases significantly compared with the attenuation measured for free vibrations (Figure 3.4), and is not equal for all the modes. The plexiglass specimen, on the other hand, shows relatively small changes in attenuation except for the highest order mode ( $\mathrm{e} 3$ mode) in the plot. The seemingly small increase in attenuation in plexiglass is due to the large intrinsic attenuation of the material.

The results indicate that the determined stiffness of a rock from the resonance frequencies may not be accurate if the acoustic impedance of the rock is relatively small compared with the impedance of the load-bearing plastic rings. Attenuation of a specimen is also affected by the coupling with the surrounding media especially when the intrinsic attenuation of the specimen is small compared with the energy loss through the plastic rings.

\subsubsection{Resonance of intact rock specimens}

Frequency response functions (FRF's) of rock specimens are shown in Figure 3.8 (a) and (b) for increasing axial stress. From the plots, it can be clearly seen that the resonance frequencies of the rocks increase significantly with increasing axial stress. For relatively low axial stresses $(<0.1 \mathrm{MPa})$, the shifting behavior of the resonance peaks is severely disturbed by the improvement of the specimen-ring-frame coupling. The improved coupling both increases and decreases resonance frequencies. The behavior possibly depends on the relative contribution of the coupled mass (frequency decrease) and stiffness (frequency increase).

The normalized resonance frequencies are shown in Figure 3.9(a) and (b). Neglecting the effect of the dispersion due to the geometry and Poisson's ratio of the specimens, these results show an increase in Young's modulus of the rocks during uniaxial compression. For the granite specimen, the correction for the dispersion due to the inertia effect discussed in Section 3.3 is negligible because Poisson's ratio is as small as 0.06 at the maximum (measured statically). The modulus for the sandstone specimen, however, may include 


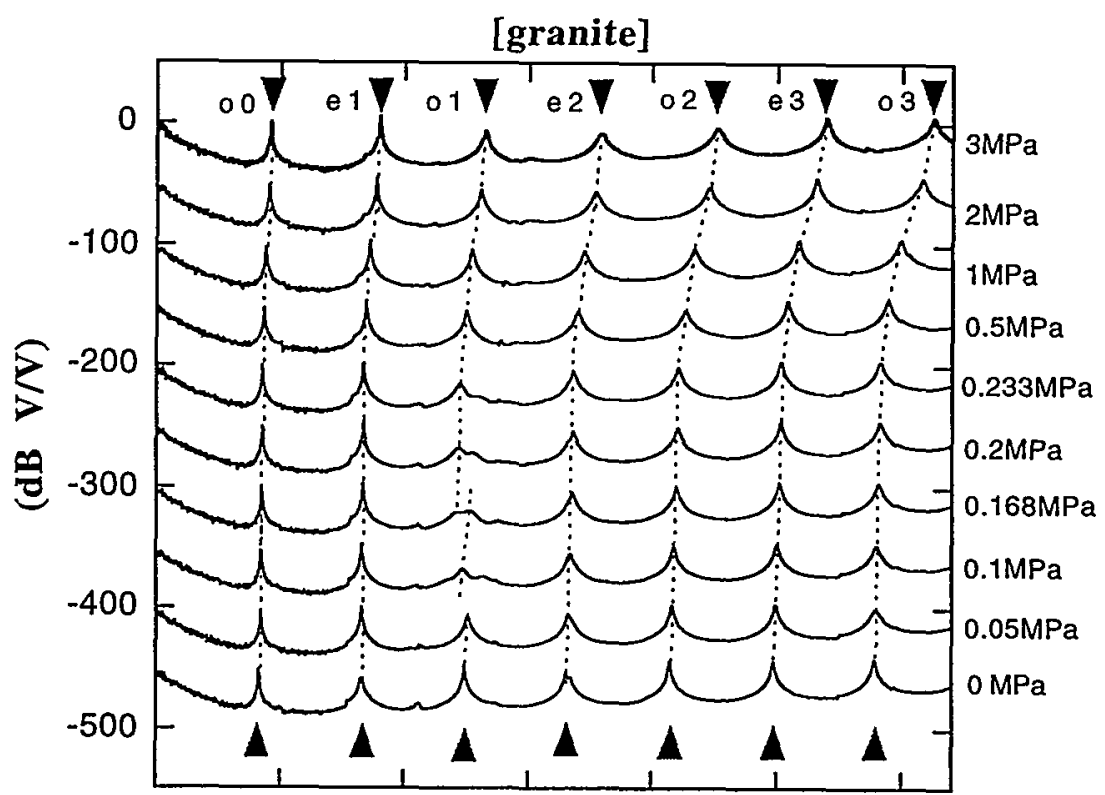

(a) FRF's of granite specimen

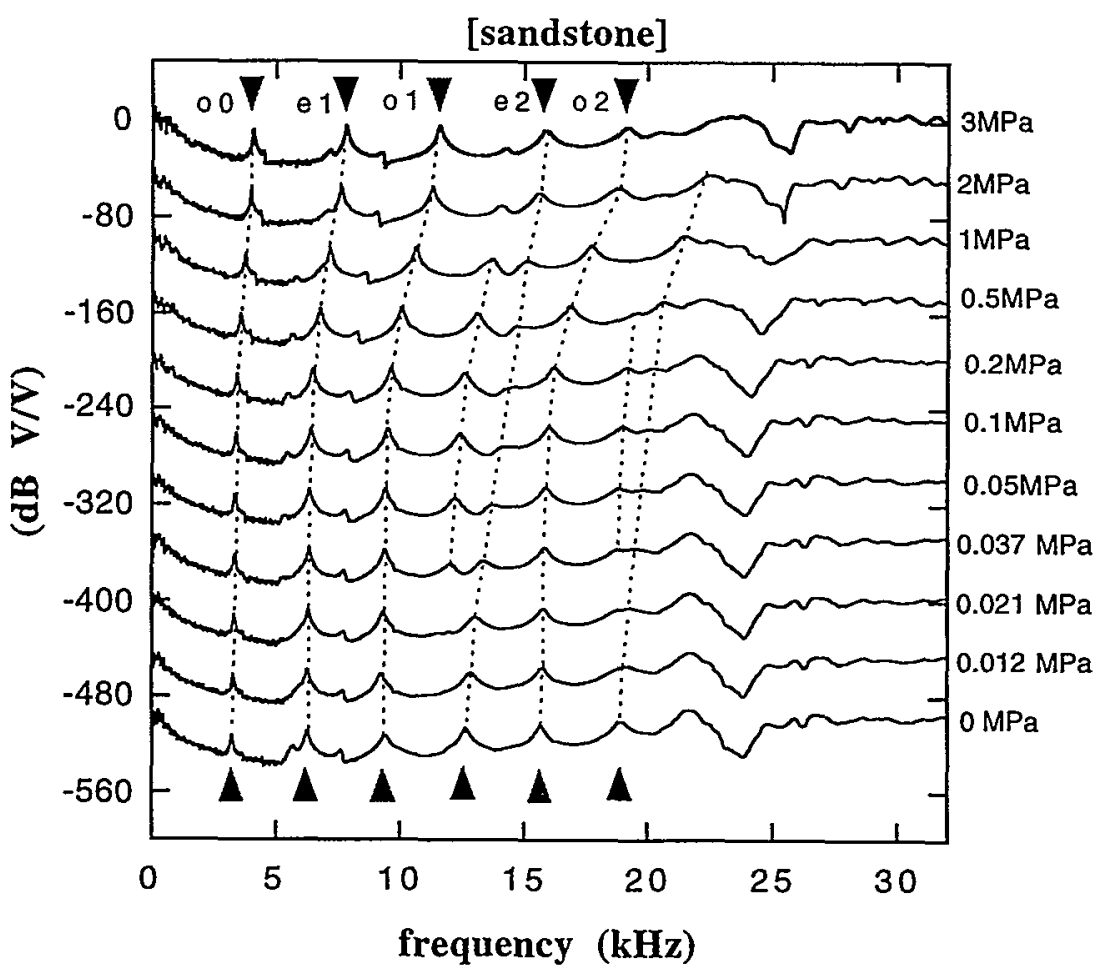

(b) FRF's of sandstone specimen

Figure 3.8 Measured FRF's of intact rock bars. Resonance frequencies of the specimens increase significantly with increasing axial stress. (a) 01 mode of the granite specimen shows slightly irregular behavior. (b) Resonances of the sandstone specimen show quite erratic behavior possibly due to the small acoustic impedance. 


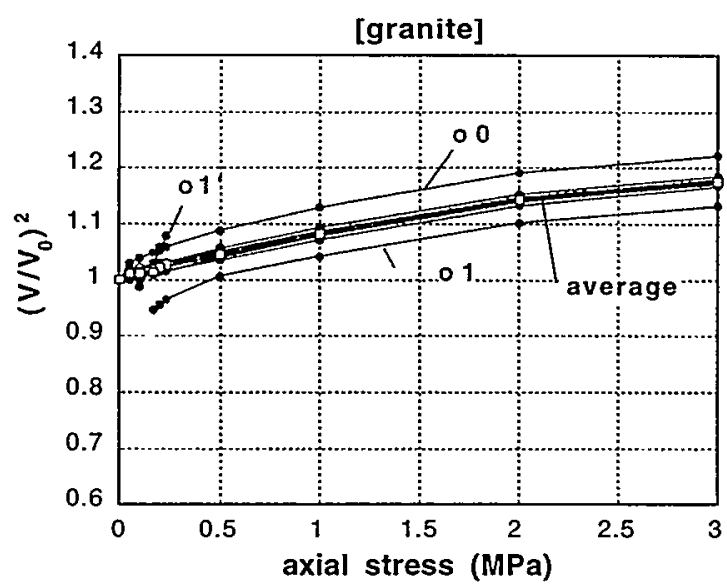

(a) phase velocity increase in granite

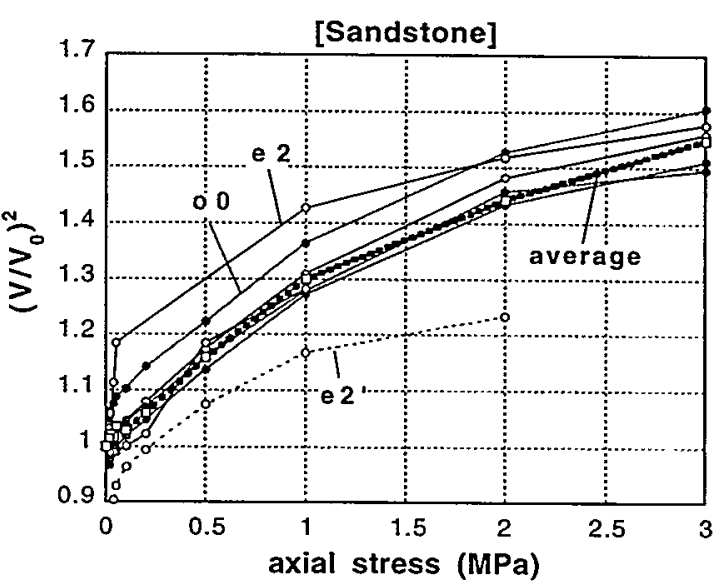

(b) phase velocity increase in sandstone

Figure 3.9 Increase in phase velocity of rock specimens with increasing axial stress determined from resonance frequencies. Velocities are normalized by the phase velocities computed from resonance frequencies of free vibrations. Increased axial stress increases the resonance frequencies, resulting in the increase in the phase velocity. Due to the small acoustic impedance, the velocities for the sandstone scatter around the mean.

significant error as Poisson's ratio increases up to 0.1 at $3 \mathrm{MPa}$ of axial stress. According to Bancroft's correction (Bancroft, 1941) with a Poisson's ratio of 0.1 , the 5 th and 6 th modes include $1.2 \%$ and $2.4 \%$ of dispersion in modulus, respectively. Although there is significant scattering among the velocities obtained from different modes, increase in the velocities with increasing axial stress is clearly seen for both rock specimens. Young's moduli measured from static stress-strain measurements and ultrasonic velocity measurements are also shown in Figure 3.10(a) and(b) for comparison. To compute Young's modulus from the ultrasonic velocity measurements, Poisson's ratio of the granite specimen was assumed to be zero, i.e., the P-wave modulus was assumed to be identical to the Young's modulus, and the sandstone was assumed to be isotropic (Young's modulus can be determined from $\mathrm{P}$ - and $\mathrm{S}$-wave velocities). The results obtained from the resonant bar tests show a basic agreement with the static tests. Results from the ultrasonic transmission tests, however, deviate from the static and resonant bar tests as the load is increased. This may be due to an increase in Poisson's ratio and the stress-induced anisotropy in the specimens. 


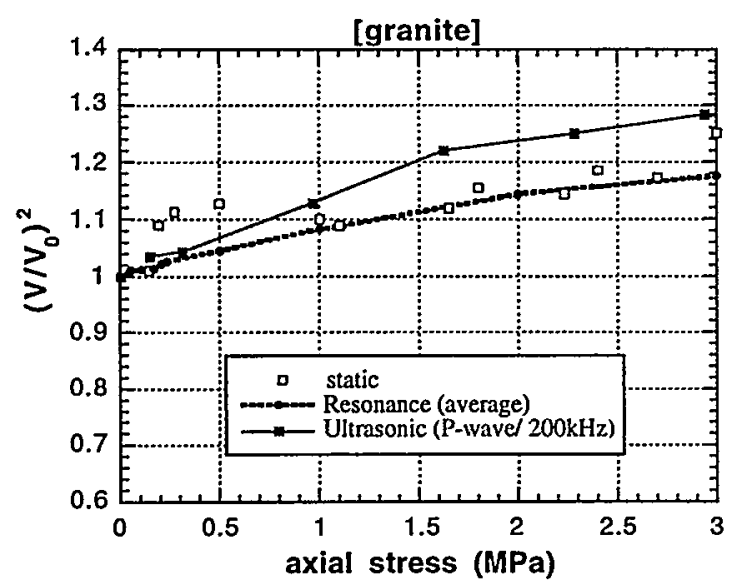

(a) comparison of moduli for granite

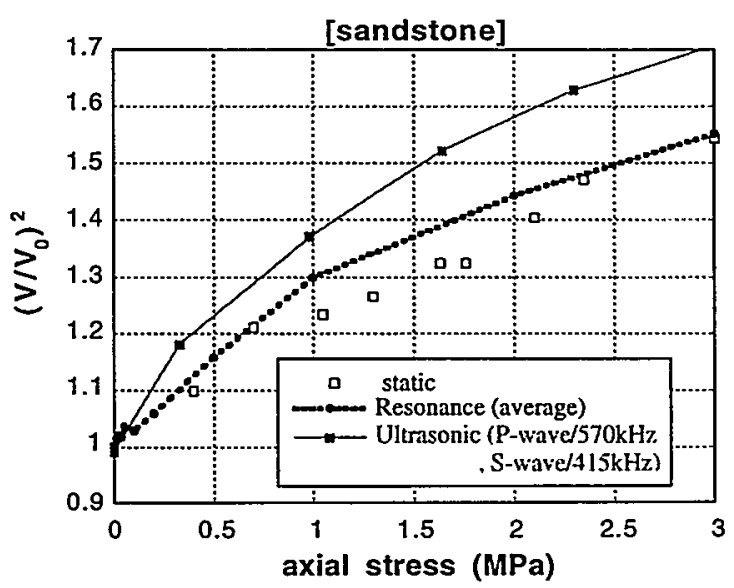

(b) comparison of moduli for sandstone

Figure 3.10 Comparison of phase velocities determined by static tests, ultrasonic transmission tests, and resonant bar tests. Results are normalized to the phase velocities at $0 \mathrm{MPa}$. Note that the normalized vertical scale is equivalent to the Young's modulus of the specimens. The averaged results for the resonant bar test show basic agreement with increase in statically determined Young's modulus. Phase velocities determined from the ultrasonic velocity measurements are consistently higher than the other results, possibly an effect of the increasing Poisson's ratio and stress-induced anisotropy in the specimens that are not taken into account in computing the phase velocity.

\subsubsection{Resonance of fractured rock specimen}

A single tensile fracture was introduced at the center of a granite rod specimen by Brazilian point loading. The orientation of the fracture was perpendicular to the rod axis and parallel to the average microcrack plane. The surfaces of the fracture were mated during the tests. The frequency response spectrum of the specimen is shown in Figure 3.11 for a range of axial stress. By comparing the resonance frequencies in Figure 3.11 and Figure 3.8(a), it can be seen that resonance frequencies of even modes were not affected by the introduced fracture while odd modes showed a decrease in frequencies. The difference in behaviors of resonance frequencies yields pairs of even and odd modes that were predicted in the analyses in the previous chapter (Section 2.2.1).

The dynamic stiffness of the fracture can be determined from the difference in the resonance frequencies of the odd modes between fractured and intact specimens. As the elastic moduli of the rock change with stress, the Young's modulus for the intact part of the fractured specimen was determined from the longitudinal resonance frequencies of the intact specimen at each stress. The density of the specimen was assumed to be constant during the test. For measured resonance frequencies, rod length, diameter, density, and 


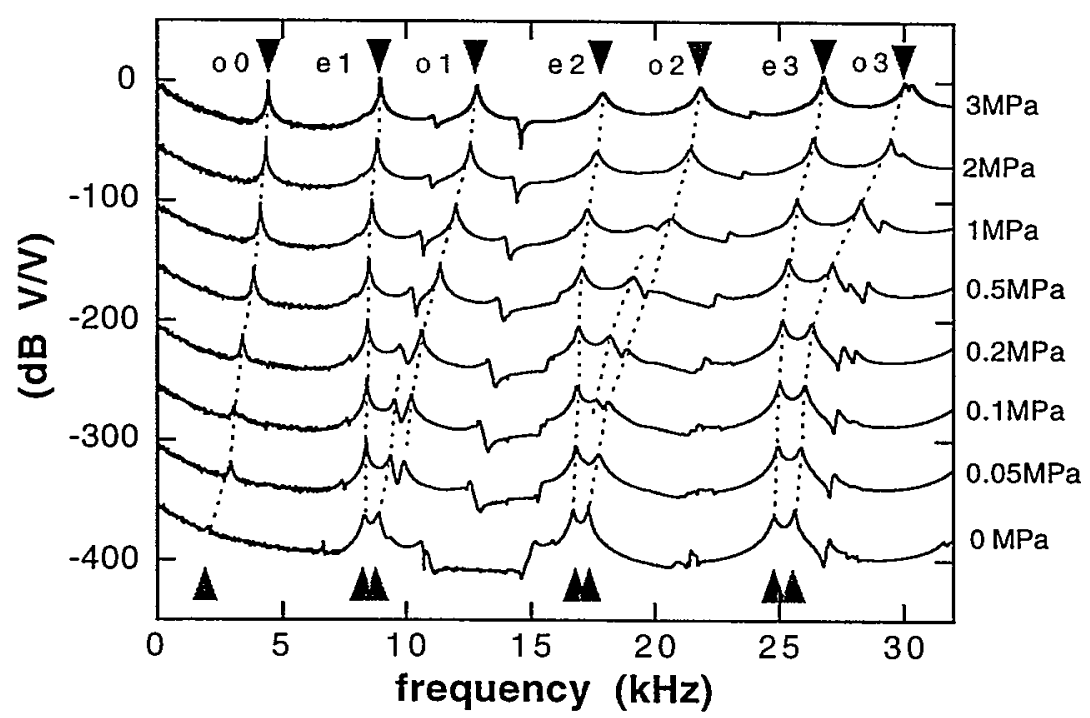

Figure 3.11 FRF's of granite bar specimen containing a single through-going fracture at the middle of the bar. As the axial stress decreases (as does the fracture stiffness), even and odd modes merge together. Comparison of this plot with Figure 3.8(a) reveals that the effect of the fracture is only on the odd modes and the even modes are not affected. Mode $o 1$ and 02 seem to be affected rather strongly by the improved coupling with the surrounding media through the plastic rings.

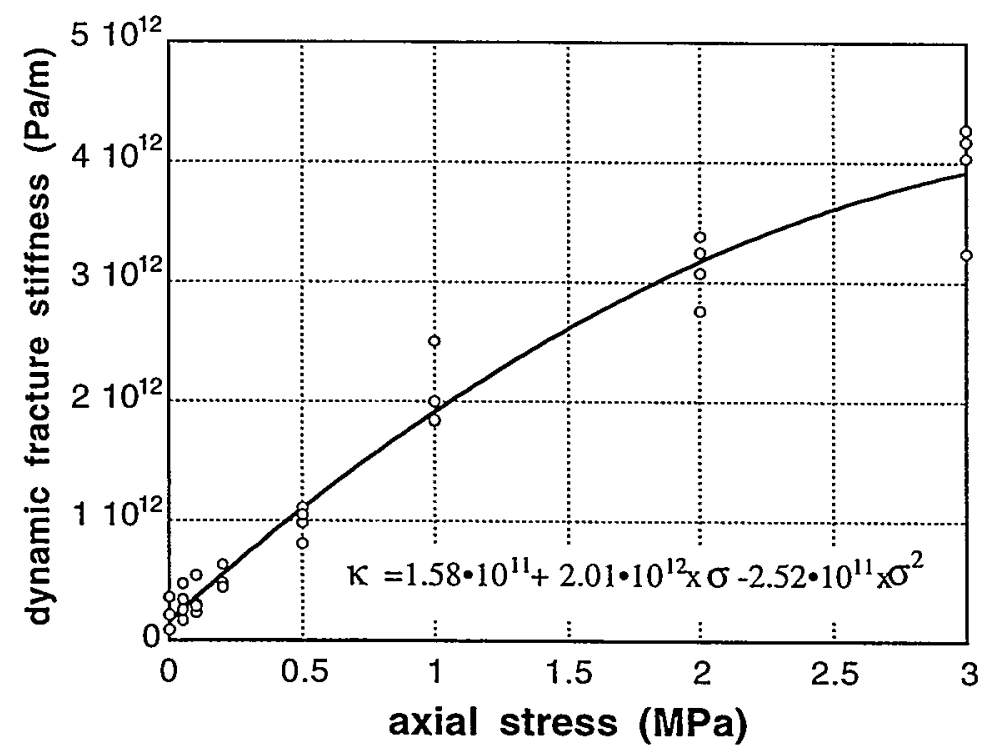

Figure 3.12 Dynamic fracture stiffness computed from the shift in the odd mode resonance frequencies. Each result represents the computed stiffness for each mode of resonance. The phase velocity in the intact part of the bar is obtained from the resonance frequencies of the bar without the fracture for each axial stress. The fracture stiffness increases as the specimen is loaded, resulting in the nonlinear load-displacement behavior of the fracture. A fitted polynomial is shown in the above plot. 
dynamic Young's modulus of the specimen, the dynamic fracture stiffness was calculated using Eq.(2.9) at each stress (Figure 3.12). For modes that showed erratic behaviors (splitting of resonance peaks), the most dominant resonance peak was chosen for computing the fracture stiffness. The plot shows the stiffness of the fracture increasing with applied axial stress. The increase in stiffness results in a nonlinear load-displacement (closing displacement) behavior of the fracture. An experimentally determined fracture stiffness-axial stress relation is:

$$
\kappa=\left(1.58+20.1 \cdot \sigma-2.52 \cdot \sigma^{2}\right) \times 10^{11}[\mathrm{~Pa} / \mathrm{m}]
$$

where $\sigma$ is the applied axial stress given in [MPa].

\subsubsection{Effect of fluid inside the fracture}

The fractured granite specimen in Section 3.4.4 was resonated first without axial load. After measuring resonance frequencies and attenuation of the longitudinal mode resonances, the fracture was filled with distilled water injected by a syringe and changes in the resonance frequencies and attenuation were measured. The same test was repeated with $0.5 \mathrm{MPa}$ of axial stress. Figure 3.13(a),(b) show the change in the experimental FRF's before and after the water was injected into the fracture. For each pair of the even and odd modes, clear increases in attenuation for the odd modes are present. Figure 3.14 and Figure 3.15 show the measured change in resonance frequencies and attenuation. For the higher fracture stiffness (axial stress $=0.5 \mathrm{MPa}$ ), odd mode resonance frequencies increased due to increase in viscosity of the fracture. On the other hand, even modes exhibited much smaller resonance frequency shifts. The resonance showed large increase in attenuation for odd modes and small increase for even modes. For the low-stiffness fracture, the resonance behaviors followed an opposite trend to the high-stiffness fracture: resonance frequencies of odd modes decreased while even modes exhibited increases. Attenuation behavior of the resonance was similar to the high fracture stiffness case, showing a large increase in attenuation for the odd modes.

The observed behaviors of the resonance are consistent with the theoretical prediction in the previous chapter (Section 2.2.2). As odd modes generate maximum stress amplitude at the center of the rod where the fracture is located, large relative motion results between the two partially contacting surfaces of the fracture. This motion leads to a squirting flow of fluid around the contacting asperities. The viscous drag between the fluid and the fracture 


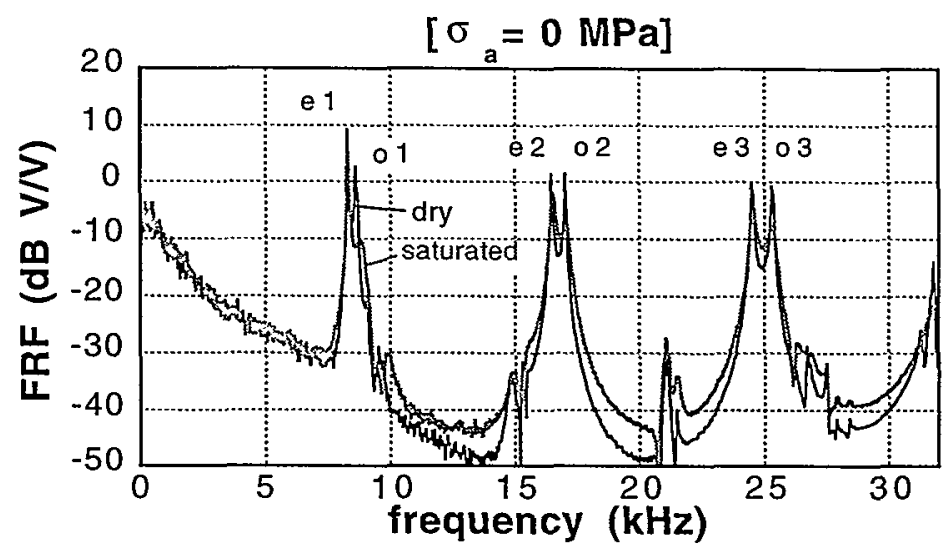

(a) OMPa axial stress

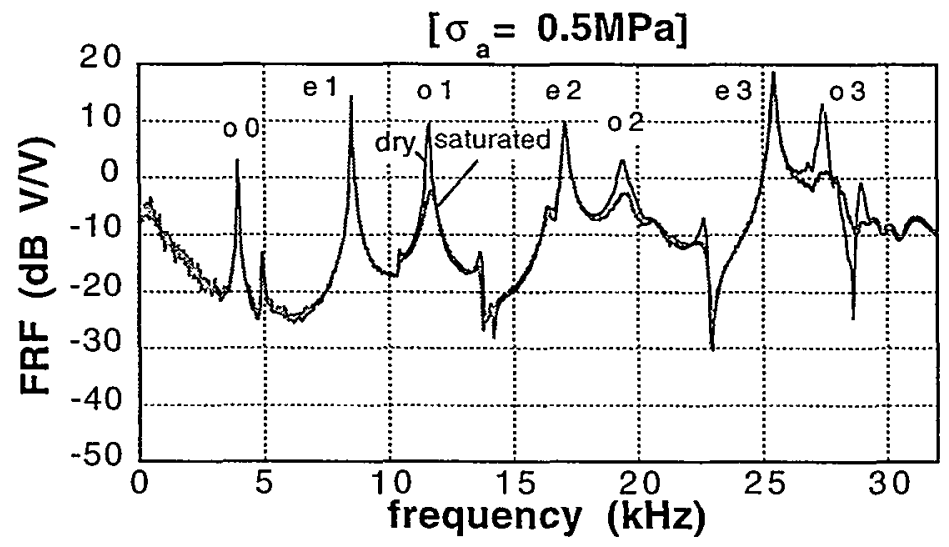

(b) $0.5 \mathrm{MPa}$ axial stress

Figure 3.13 FRF's of fractured granite bar showing the effect of fluid in the fracture. The fracture is saturated with distilled water. Increase in viscosity of the fracture attenuates odd mode resonances (labeled as ol 03). The tests are conducted on a low-stiffness fracture (axial stress=0MPa) and a high-stiffness fracture (axial stress $=0.5 \mathrm{MPa}$ ). The surfaces of the fracture are mated.

surface dissipates vibration energy. In contrast, vibrations for even modes do not generate stress at the center of the rod and attenuation remains small.

As was shown in Section 2.2.2, for high fracture stiffness, an increase of viscosity in the complex fracture stiffness leads to an increase in resonance frequency with increasing attenuation and a decrease in resonance frequency for a low-stiffness fracture. Theoretical curves that describe the behavior of resonance frequency versus relaxation time are plotted for fracture stiffnesses at 0 and $0.5 \mathrm{MPa}$ of axial load (Figure 3.16). The fracture stiffnesses are computed using the experimentally determined fracture stiffness-stress relation in Eq.(3.3). Although the mode o1 for low-stiffness fracture (axial stress $0 \mathrm{MPa}$ ) 


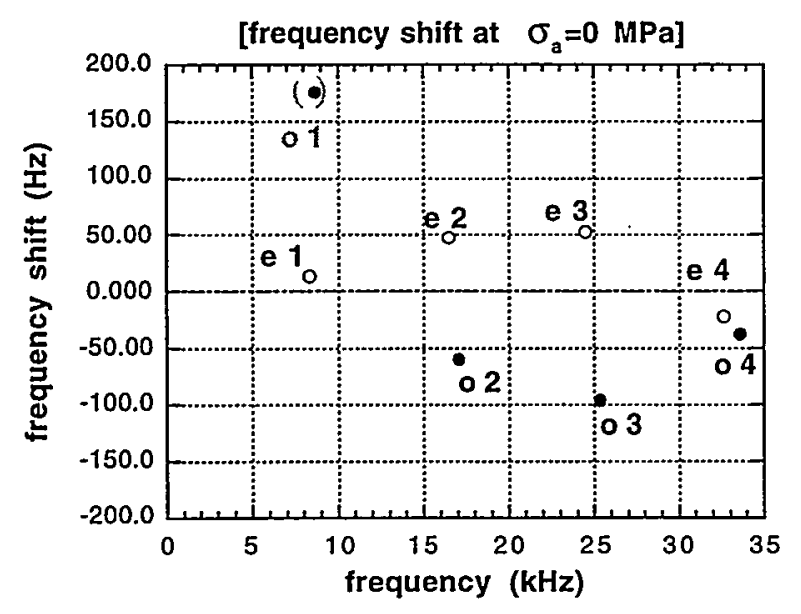

(a) OMPa axial stress

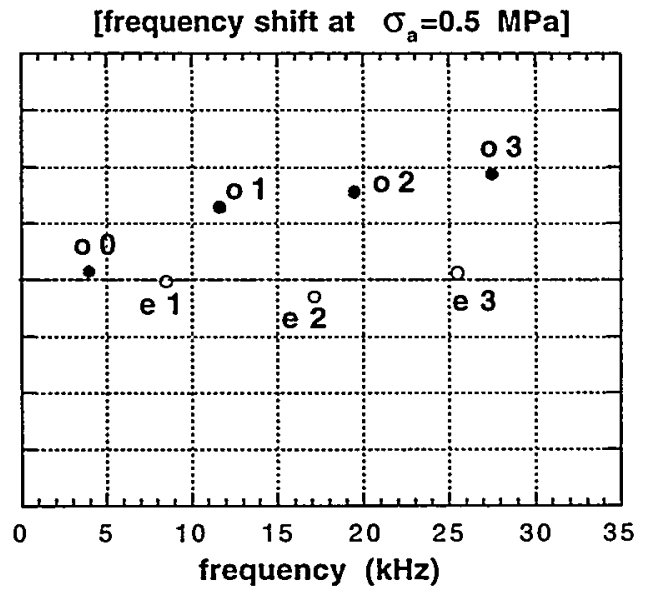

(b) $0.5 \mathrm{MPa}$ axial stress

Figure 3.14 Shift of resonance frequencies for a fracture injected with water. (a) For the low stiffness fracture, odd mode resonance frequencies decrease. Increase in the even mode frequencies cannot be explained by the model but may be due to the stiffening effect of water penetrated into the intact part of the specimen. o1 mode shows a different behavior than the other odd modes, showing an increasing resonance frequency. (b) For the high stiffness fracture, odd mode resonance shows opposite behavior to the low stiffness fracture, and shows an increase in resonance frequency by injected water.

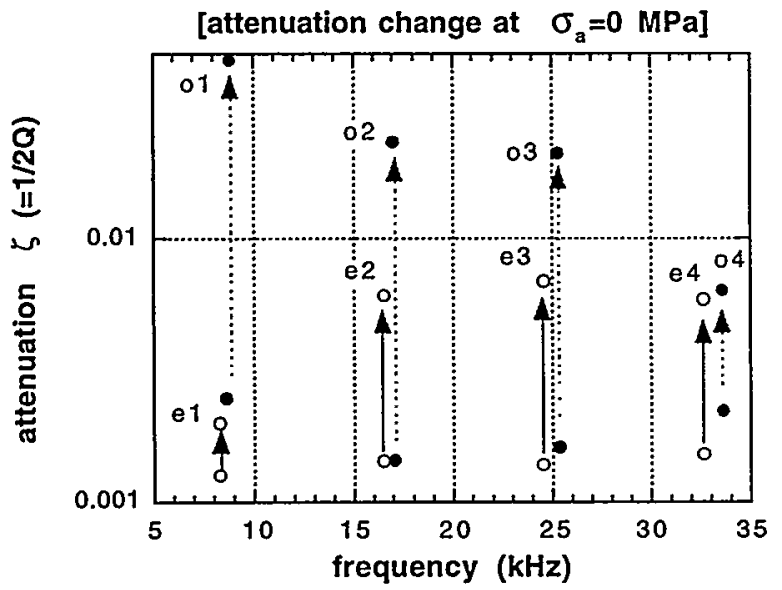

(a) OMPa axial stress

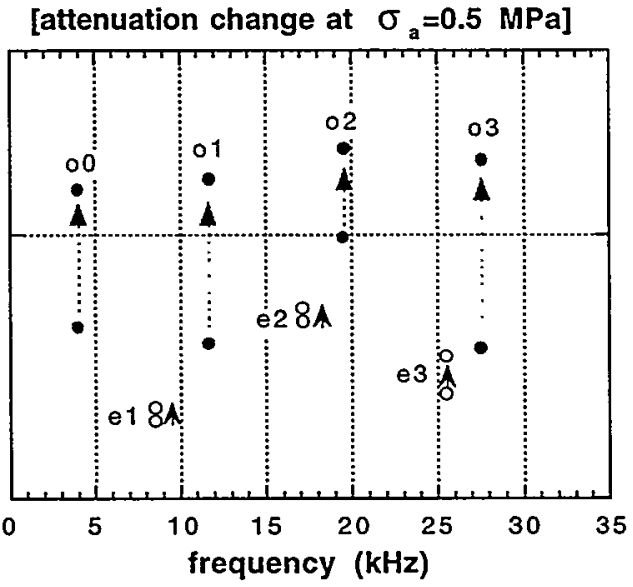

(b) $0.5 \mathrm{MPa}$ axial stress

Figure 3.15 Change in attenuation for a fracture injected with water. For both high and low fracture stiffnesses, odd mode attenuation increases much more than the even mode. Increase in attenuation is related to the amount of resonance frequency shift. (a) For the low stiffness fracture, even modes show an increase in attenuation corresponding to the resonance frequency shift while (b) the even modes of the high stiffness fracture show very small increase in attenuation. 
shows a different behavior than predicted, the experimental results show qualitative agreement with the theoretical predictions; odd mode resonance frequencies tend to increase for a high-stiffness fracture and decrease for a low-stiffness fracture due to a fluid induced increase in fracture viscosity.

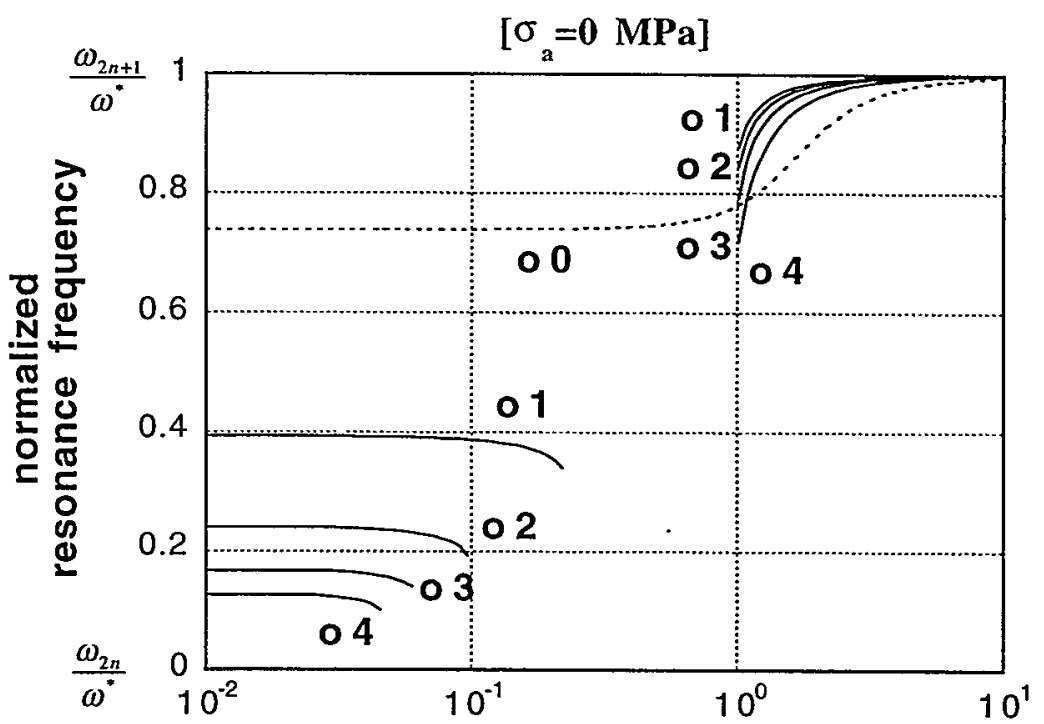

(a) Low stiffness fracture (OMPa axial stress)

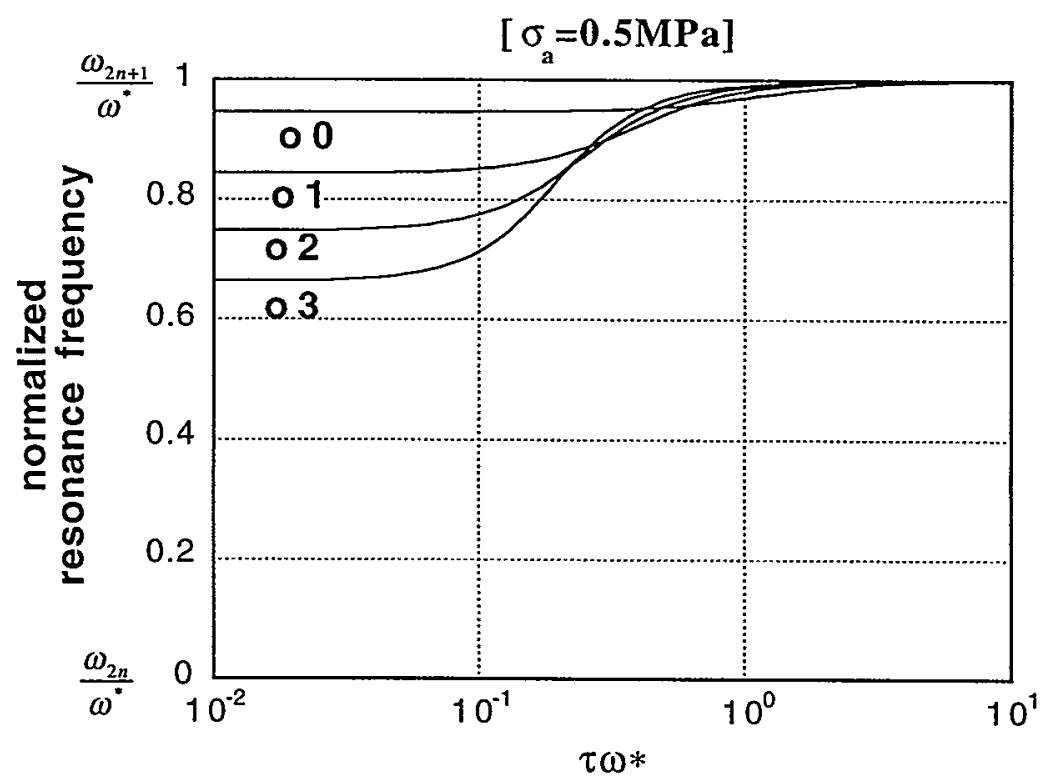

(b) High stiffness fracture (0.5MPa axial stress)

Figure 3.16 Resonance frequency shifts due to an increase in fracture viscosity. Experimentally determined phase velocity and fracture stiffness are used to compute the curves. The resonance frequency of each odd mode (on) is shifted by the paired even mode (en)'s resonance frequency $\omega_{2 n}$ and normalized by the fundamental mode's resonance frequency $\omega^{*}$ for.an intact bar . (a) For the low stiffness fracture, an increase in viscosity (shown by the normalized relaxation time in horizontal axis) results in a decrease in the resonance frequencies. On the other hand, (b) for the high stiffness fracture, an increase in viscosity increases the resonance frequencies. 


\subsubsection{Effect of attenuative filling}

A latex membrane was placed between two surfaces of a fracture in the specimen and axially loaded up to 3.0MPa. As the load was decreased, resonance frequencies and attenuation were measured. FRF's measured during unloading are shown in Figure 3.17. Change in the attenuation of the observed modes is shown quantitatively in Figure 3.18. As can be seen from the plots, attenuation of odd modes is maximum at an intermediate stress. Differences in the attenuation behavior between even and odd modes can be explained by the same mechanism described in Section 2.2.2. The non-monotonic increase and decrease in attenuation of the odd modes are due to the frictional and viscous dissipation in the latex membrane. A possible mechanism is shown schematically in Figure 3.19. When a large axial stress is applied, the stiffness of the fracture is high and only small relative motion between the surfaces of the fracture occurs during vibration. For this reason, attenuation of the odd modes for high axial stresses is small. When the stress is decreased, large shearing motion is introduced in the membrane as the closing motion of the fracture extrudes the latex out of the contact between asperities. For a very small axial stress, the stress introduced by passage of a wave becomes even within the membrane. As the shearing motion in the membrane is small, attenuation becomes small again. 


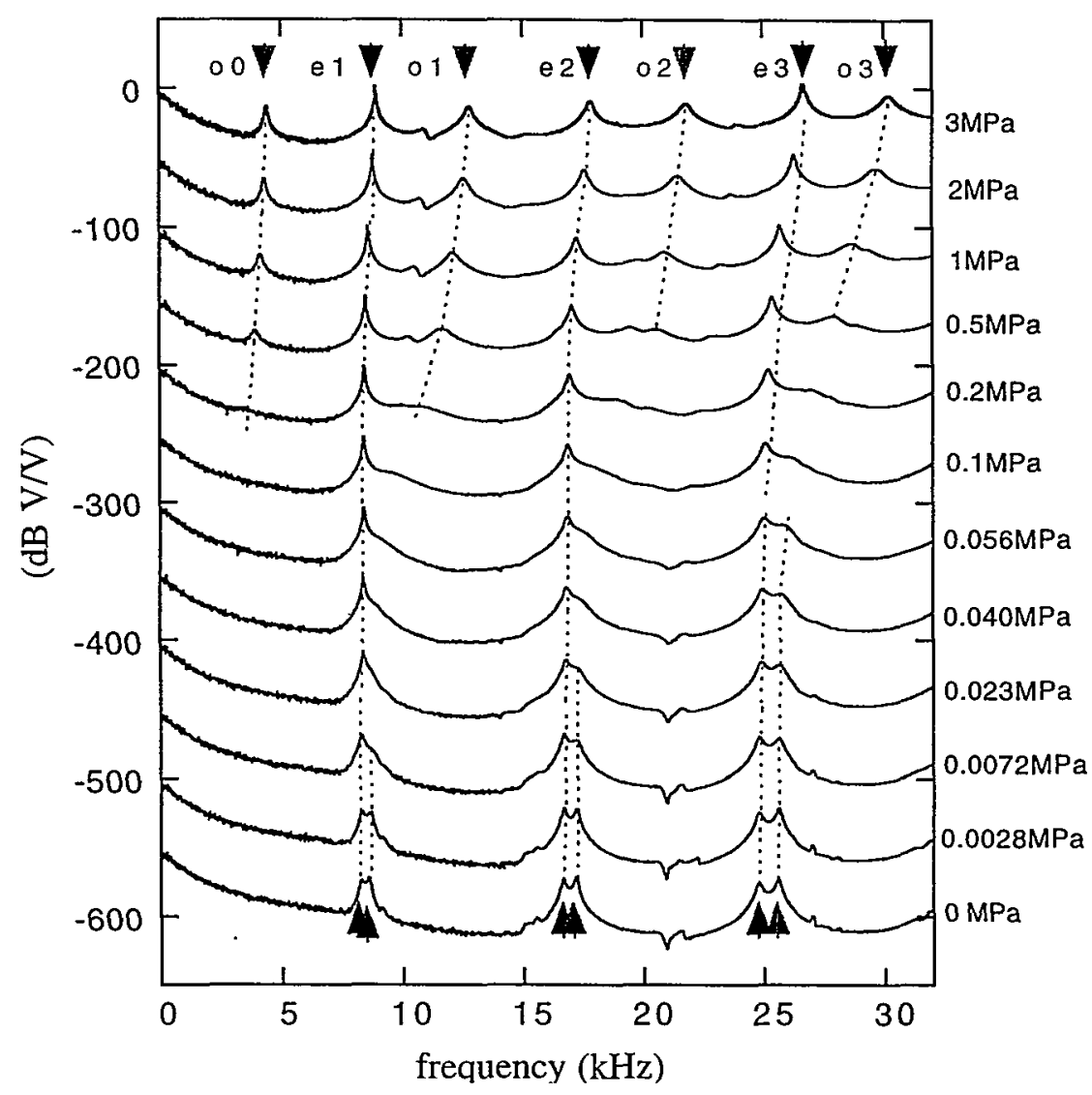

Figure 3.17 Attenuation of odd mode resonances due to a latex membrane in the fracture. Attenuation is relatively small for high and low axial loads (or fracture stiffnesses) while it is maximum for intermediate loads.

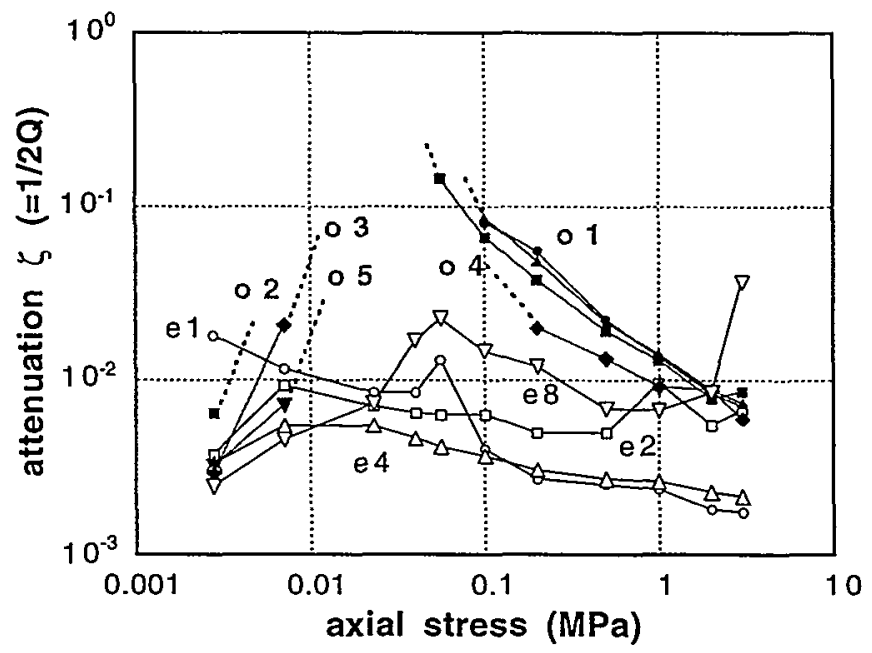

Figure 3.18 Attenuation in a fractured granite specimen with an inserted latex membrane. The attenuation coefficient was determined by the half-power method. For an intermediate stress range $(0.01 \sim 0.1 \mathrm{MPa})$, attenuation is maximum. Note that some of the resonance peaks for the odd modes vanish for maximum attenuation. 


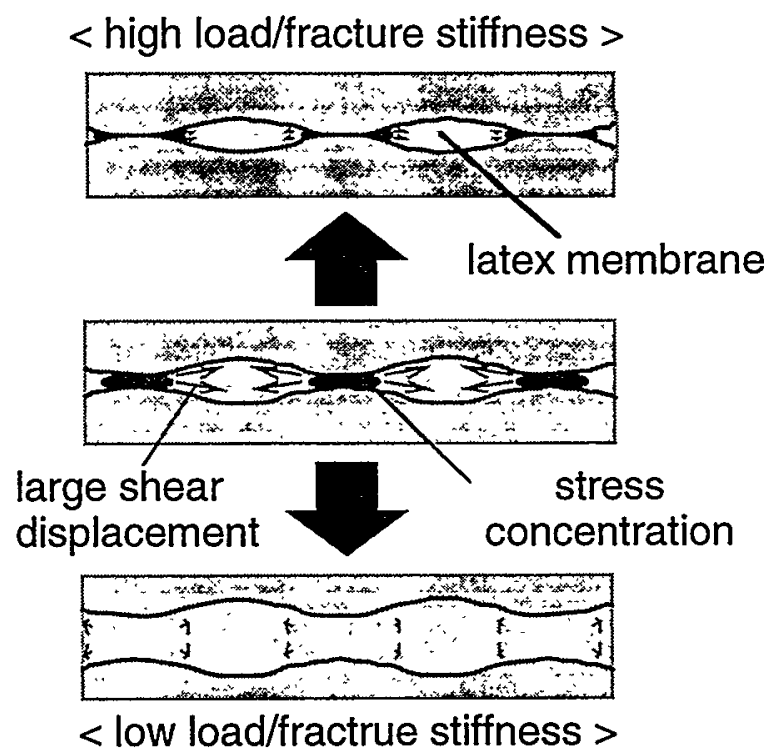

Figure 3.19 Possible mechanism for non-monotonic attenuation behavior of odd modes. For high stiffness fracture, asperities on two surfaces of a fracture are in contact and only a small stress change is caused in the latex membrane. At intermediate stresses, a large stress concentration is caused in the membrane between asperities, resulting in flow of the latex with a large shear motion. This shear motion causes large attenuation of resonance. For a small stress, stress concentration in the membrane becomes small and a passing wave causes a uniform fluctuation of stress. As the shear motion in the membrane is small, resulting attenuation decreases.

\subsection{Summary}

This chapter examined the resonance characteristics of rock specimens including a single fracture using the resonant bar test. To study the effect of fracture stiffness on resonance behavior, a bar specimen including a fracture was axially loaded through compliant plastic rings. The use of plastic rings made the boundary condition of the specimens approximately free-end although measured resonance frequencies and attenuation were affected by the coupling with the surrounding media to varying degrees depending on the acoustic impedance contrast between the specimen and the rings.

Axially loaded intact rock specimens exhibited a significant increase in velocity (or Young's modulus) with increasing axial load. This is due to the changes in rock microstructure by stress such as closing of microcracks and stiffening of grain contact. Although the measured resonances were affected by the improved end-coupling with 
increasing stress, the observed changes were significantly greater than the changes in resonance frequencies measured for aluminum and plexiglass specimens. For the range of frequencies used for the resonant bar tests, the results showed good agreement with the statically measured changes in Young's modulus. Dynamic Young's modulus values determined from high-frequency ultrasonic transmission tests were consistently greater than the moduli determined from other measurements especially for large axial stresses. Although the cause of such behavior is not known, changes in elastic behavior of the specimens such as increased Poission's ratio and stress-induced anisotropy may be responsible. The resonant bar test using longitudinal vibrations is essentially limited to measuring the dynamic Young's modulus along the bar axis, and Poisson's ratio, if the specimen is isotropic. Determination of elastic moduli for anisotropic rocks will be discussed in a later chapter (Chapter 5).

The effects of a compliant fracture on the resonance of a bar specimen were examined using a granite bar including a through-going center fracture. The stiffness of the fracture was changed by applying axial load to the specimen. Resonance frequencies of the odd modes changed significantly in contrast to the even modes that showed only a small shift, similar to the intact specimen. Although the resonance behavior was affected by end coupling to some degree, the behavior of experimentally measured resonances show qualitative agreement with the predictions of the analytic model described in the previous chapter. From the frequency shifts of the odd modes, dynamic fracture stiffness was determined for each axial load.

The fracture in the granite specimen was also filled with water and a latex membrane to see the effect on the attenuation of resonance. For both cases, attenuation of the odd modes increased as predicted in the previous chapter. The resonance frequency shift due to the water in the fracture varied depending on the stiffness of the fracture: for low fracture stiffness, an increase in viscosity of fracture lead to a decrease in the resonance frequencies while the resonance frequencies for the high-stiffness fracture showed the opposite behavior. These changes in behavior can be qualitatively predicted by using a rheological model (Kelvin-Voigt model) for fracture stiffness. A fracture with a latex membrane showed attenuation of resonance that changes as a function of stress applied to the fracture. The attenuation is maximum for intermediate stresses for which it is postulated that a large shear motion within the membrane occurs. 
The effects of the fracture stiffness on the resonance of the bar specimens predicted by the one-dimensional analyses in the pervious chapter were demonstrated successfully. Although some of the results presented in this chapter were affected by the boundary condition of the specimens, measured and predicted resonance behaviors for varying fracture stiffness and viscosity showed good qualitative agreement. This in turn indicates that the model (displacement-discontinuity boundary conditions) can be used for describing the dynamic behavior of a fracture during resonance. For a more quantitative measurement for relationship between fracture stiffness and characteristics of resonance such as resonance frequency shift and attenuation, an experimental setup that provides better acoustic isolation to the specimen while allowing application of axial stress is necessary.

Some of the problems with the resonant bar test that arise from the one-dimensionality of the vibration field can be solved by examining the resonances of arbitrarily shaped specimens. By examining resonance frequencies and mode shapes, anisotropic elastic moduli and fracture stiffness can be determined with the help of numerical models. In the following chapter, a numerical code that analyzes the normal mode vibration of arbitrarily shaped bodies with elastic anisotropy in their bulk material properties and with fractures is introduced. 


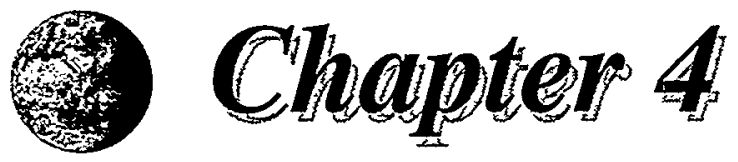

\section{Numerical Model for Resonance of 3-D Anisotropic Body Containing Fractures}

\subsection{Introduction}

Fractures in materials often cause serious structural hazards. As the introduction of a fracture in a solid body changes its vibration characteristics, attempts have been made to assess the presence and the degree of damage caused by fractures using the acoustic resonance of structures. Acoustic resonance has been used for characterizing bulk elastic properties of solids for many years. For solids of simple geometries such as slender bars (Birch \& Bancroft, 1938; Powers, 1938) or spheres (Soga \& Anderson, 1967; Birch, 1975), determination of elastic properties from measured resonance frequencies is based on the application of known analytic solutions. However, for many solid structures that have complex three-dimensional geometry, their resonance can hardly be analyzed by analytic methods. For this reason, numerical techniques such as finite element (FE) modeling are performed to simulate the effect of damage on resonance of structures. FE modeling is especially powerful for analyzing the vibration of beam and shell structures as it can make use of computationally efficient structural elements (e.g., Petyt, 1990). However, for massive solid structures such as dams, concrete bridge piers, and laboratory scale test 
specimens, the FE modeling loses its advantages as true three-dimensional modeling using three-dimensional elements is required.

In this chapter, a numerical technique is introduced for simulating the effect of compliant interface(s) and elastic anisotropy on the resonances of a body with a general three-dimensional geometry. Using this technique, anisotropic elastic constants and the stiffness of a fracture are determined from experimentally measured resonance frequencies.

For intact solids, many researchers including Ekstein \& Schiffman (1956), Holland (1967), and Demarest (1969) have investigated computational methods for determining elastic properties from resonance measurements. They showed that numerical techniques based on a variational method with the Rayleigh-Ritz discretization of the displacement field very accurately predicts resonance frequencies of small anisotropic elastic crystals with rectangular geometry. Ohno (1976) applied the method to crystals with rectangular geometry and determined their anisotropic elastic moduli by numerical inversion. Visscher et al.(1991) demonstrated that use of a truncated polynomial series instead of Legendre polynomials made it possible to analyze the normal mode vibrations of anisotropic elastic bodies with more complex geometries. These methods are important because they constitute fast and accurate forward analysis techniques that can be used in the back analysis of resonance measurements for general elastic moduli and mechanical properties of materials containing fractures.

To formulate the numerical technique presented in the following sections, a fracture is introduced as an interface between intact solid bodies with imperfect contact. The load displacement behavior of the fracture is described by the displacement-discontinuity boundary conditions (Schoenberg, 1980; Pyrak-Nolte et al., 1990a). A matrix equation that is to be solved for the normal modes of vibration is derived by applying the variational technique used by the researchers mentioned previously. The formulation of the problem is essentially an extension of Vissher et al.(1991)'s work. A numerical code developed for this model computes resonance frequencies, mode shapes, and frequency response functions for specified geometry and mechanical properties of a fractured elastic body (Nakagawa et al., 1996). Using the results of the forward computation for resonance frequencies and mode shapes, the numerical code can also be used for iterative inversions for anisotropic elastic moduli and fracture stiffness from observed resonance frequencies. 


\subsection{Theory}

\subsubsection{Hamilton's principle.}

For an elastic body undergoing a steady-state vibration, a Lagrangian is defined by

$$
L=\int_{V}\left(K_{E}-P_{E}\right) d V
$$

where $\mathrm{K}_{\mathrm{E}}$ and $\mathrm{P}_{\mathrm{E}}$ are the kinetic and potential energies, respectively (Vissher et al., 1991). When a fracture is introduced into the volume $\mathrm{V}$, the potential energy term now consists of two parts: elastic strain energy stored in the bulk part of the body $\left(P_{E}^{b}\right)$ and energy stored in the fracture $\left(P_{E}^{f}\right)$. If the body is separated by $\mathrm{N}_{\mathrm{frc}}$ fractures into $\mathrm{N}_{\mathrm{blk}}\left(=\mathrm{N}_{\mathrm{frc}}+1\right)$ blocks, noting that the $P_{E}^{f}$ is distributed over the surface of the fractures, the Lagrangian becomes

$$
L=\sum_{b}^{N_{b l k}} \int_{V_{b}}\left(K_{E}^{b}-P_{E}^{b}\right) d V+\sum_{f}^{N_{f r c}} \int_{S_{f}}\left(-P_{E}^{f}\right) d S .
$$

As subsequent discussion is made in the frequency domain, $e^{i \omega t}$ dependence of displacement is assumed. Behavior of the fracture is described using the displacementdiscontinuity boundary conditions. With a mass density function $\rho\left(x \in V_{b}\right)$, a general elasticity tensor $C_{i j k l}\left(x \in V_{b}\right)$, and a fracture stiffness matrix $\kappa_{i j}\left(x \in S_{f}\right)$, energy density terms are expressed as

$$
\begin{aligned}
& K_{E}^{b}=\frac{1}{2} \rho \omega^{2} u_{i}^{T} u_{i} \\
& P_{E}^{b}=\frac{1}{2} u_{i, j} C_{i j k l} u_{k, l} \\
& P_{E}^{f}=\frac{1}{2}\left[u_{i}\right] \kappa_{i j}\left[u_{j}\right],
\end{aligned}
$$

where $\left[u_{i}\right]:=u_{i}^{+}-u_{i}^{-}$represents a displacement-discontinuity across the fracture. A conceptual model for a fractured body is shown in Figure 4.1. For a fracture with a normal vector $\left\{n_{i}\right\}^{T}=(0,0,1)$, the diagonal form of the fracture stiffness matrix is

$$
\left[\kappa_{\mathrm{ij}}\right]:=\left[\begin{array}{ccc}
\kappa_{t x} & 0 & 0 \\
0 & \kappa_{t y} & 0 \\
0 & 0 & \kappa_{n}
\end{array}\right],
$$




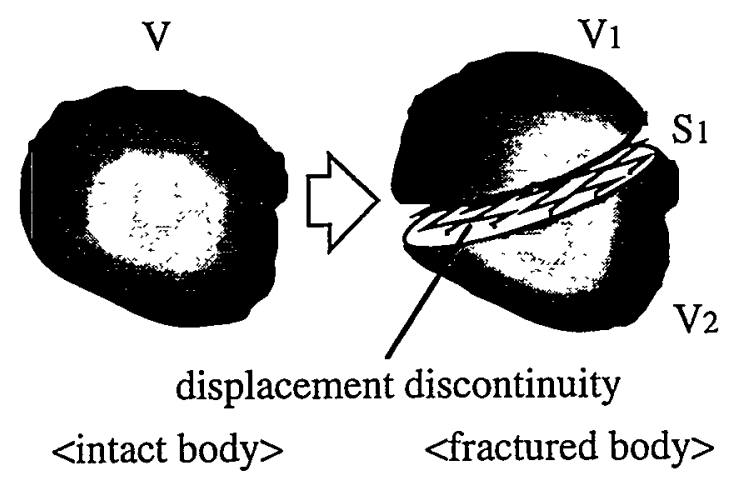

Figure 4.1 Modeling fractured elastic material with displacement-discontinuity boundary conditions.

where $\kappa_{t x}$ and $\kappa_{t y}$ are the tangential stiffnesses, and $\kappa_{n}$ is the normal stiffness of fracture. In general, fractures may also have non-zero off-diagonal stiffnesses. The physical meaning of the off-diagonal fracture stiffnesses for the above coordinate system is discussed in Chapter 8. It is noted that a rotation of the coordinate system can also make the fracture stiffness matrix fully populated.

Taking a variation of the Lagrangian with respect to displacement vector yields

$$
\delta L=\sum_{b}^{N_{b u}}\left\{\int_{V b}(E q . \mathrm{I}) \delta u_{i} d V+\int_{S b}(E q . \mathrm{II}) \delta u_{i} d S\right\}+\sum_{f}^{N_{\text {Ire }}} \int_{S f}(E q . \mathrm{II}) \delta\left[u_{i}\right] d S
$$

where

$$
\begin{aligned}
& E q . \mathrm{I} \equiv \rho \omega^{2} u_{i}+C_{i j k l} u_{k, l j} \\
& E q . \mathrm{II} \equiv C_{i j k l} u_{k, l} n_{j}=\sigma_{i j} n_{j}=t_{i} \\
& E q . \mathrm{II} \equiv \kappa_{i j}\left[u_{j}\right] .
\end{aligned}
$$

The surface of each block $S_{b}$ can be separated into an external surface $S_{b, e x t}$ and an internal surface (fracture surface) $S_{b \text {,int }}$. By combining a pair of displacement variations on the internal surfaces of adjacent blocks, a displacement-discontinuity variation $\delta\left[u_{i}\right]$ is formed. Consequently, the variation of the Lagrangian is modified as

$$
\delta L=\sum_{b}^{N_{b k}} \int_{V b}(E q \cdot \mathrm{I}) \delta u_{i} d V+\sum_{b}^{N_{b k}} \int_{S b, e x t}\left(E q \cdot \Pi^{\prime}\right) \delta u_{i} d S+\sum_{f}^{N_{f r r}} \int_{S f}\left(E q \cdot \mathrm{II}{ }^{\prime}\right) \delta\left[u_{i}\right] d S
$$

where

$$
\begin{aligned}
& E q . \Pi^{\prime} \equiv t_{i} \\
& E q . \mathrm{II}^{\prime} \equiv \kappa_{i j}\left[u_{i}\right]+t_{i} .
\end{aligned}
$$


In Eq.(4.7), variations of displacement in each term are independent. Therefore, requiring a stationarity of the Lagrangian is equivalent to solving the following boundary value problem

$E q . \mathrm{I} \equiv \rho \omega^{2} u_{i}+C_{i j k l} u_{k, l j}=0 \quad \ldots$. Wave equation

$E q . \Pi^{\prime} \equiv t_{i}=0$ or $\delta u_{i}=0 \quad$....Homogeneous B.C.

$E q . \mathrm{III}^{\prime} \equiv \kappa_{i j}\left[u_{j}\right]+t_{i}=0 . \quad$....Constitutive relationship for a fracture

This is a wave propagation problem in multiple intact blocks whose resulting surface displacements on the fracture are related by displacement-discontinuity boundary conditions. The above result demonstrates applicability of the variational technique to vibration problems of a solid elastic body with fractures.

\subsubsection{Rayleigh-Ritz method}

Using notations similar to those used by Visscher et al. (1991), a displacement field inside each block is expressed by a truncated polynomial series with unknown coefficients

$$
\begin{aligned}
& u_{i}\left(x \in V_{b}\right)=u_{i}^{(b)}=\sum_{\lambda=0}^{\lambda_{b}} a_{\lambda i}^{(b)} \Phi_{\lambda}^{(b)} \\
& \Phi_{\lambda}^{(b)}=x^{l} y^{m} z^{n}
\end{aligned}
$$

where $\lambda$ is defined for a combination of the order of polynomials

$$
\lambda \rightarrow\{l, m, n\} \quad \lambda=1,2, \ldots, R \quad l+m+n \leq N .
$$

If the maximum order of the polynomials $N$ is identical for the three directions $(x, y$, and $z), R$ is calculated from the $N$ by

$$
R=3(N+1)(N+2)(N+3) / 6 \text {. }
$$

This type of descretization is known as the Rayleigh-Ritz method. As pointed out by Visscher et al., this simple polynomial series is better suited than Bessel functions or Legendre polynomials used by other researchers for modeling mode shapes of a body with an arbitrary geometry. The set of basis functions $\left\{\Phi_{\lambda}^{(b)}\right\}$ can be chosen independently for each block. As $\left\{\Phi_{\lambda}^{(b)}\right.$ \}is a complete set, $i$. e., any polynomial of order less than $N$ can be expressed as a linear combination of $\Phi_{\lambda}^{(b)}$ 's, it can express any continuous displacement field if $\mathrm{N}$ is sufficiently large. 
By utilizing the equations (4.3a) (4.3c), a discrete version of the Lagrangian with a global coefficient vector $\boldsymbol{a}=\left\{a_{11}^{(1)}, a_{12}^{(1)}, \ldots, a_{R 3}^{\left(N_{b k}\right)}\right\}^{T}$ is obtained as

$$
\begin{aligned}
& L=\frac{1}{2} \omega^{2} a^{T} M a-\frac{1}{2} a^{T}\left(G+G^{F}\right) a \\
& M=\sum_{b}^{N_{b l k}} M^{(b)} \\
& G=\sum_{b}^{N_{b l k}} G^{(b)} \\
& G^{F}=\sum_{f}^{N_{f r c}} G^{F(f)} .
\end{aligned}
$$

where $\mathbf{M}$ is the global mass matrix, $\mathbf{G}$ is the global bulk stiffness matrix, and $\mathbf{G}^{\mathrm{F}}$ is the global fracture stiffness matrix. These are sums of submatrices $\mathbf{M}^{(\mathrm{b})}, \mathbf{G}^{(\mathrm{b})}$, and $\mathbf{G}^{\mathrm{F}(\mathrm{f}) \text {, }}$ respectively, that are defined for each block or fracture as

$$
\begin{aligned}
& M_{\lambda i \lambda^{\prime} i^{\prime}}^{(b)}=\delta_{i i^{\prime}} \int_{V_{b}} \Phi_{\lambda}^{(b)} \rho^{(b)} \Phi_{\lambda^{\prime}}^{(b)} d V \\
& G_{\lambda i \lambda^{\prime} i^{\prime}}^{(b)}=\int_{V_{b}} \Phi_{\lambda, j}^{(b)} C_{i j i^{\prime} j^{\prime}}^{(b)} \Phi_{\lambda, j^{\prime}}^{(b)} d V
\end{aligned}
$$

and

$$
\begin{aligned}
G_{\lambda i \lambda^{\prime} i^{\prime}}^{F(f)} & =\int_{S_{f}} \Phi_{\lambda}^{(f+)} \kappa_{i i^{\prime}}{ }^{(f)} \Phi_{\lambda^{\prime}}^{(f+)} d S \\
G_{\lambda i \lambda i^{\prime}}^{F(f)} & =\int_{S_{f}} \Phi_{\lambda}^{(f-)} \kappa_{i i i^{\prime}}^{(f)} \Phi_{\lambda^{\prime}}^{(f-)} d S,
\end{aligned}
$$

for the base functions for single blocks, and

$$
\begin{aligned}
G_{\lambda i \lambda^{\prime} i^{\prime}}^{F(f)} & =-\int_{S_{f}} \Phi_{\lambda}^{(f+)} \kappa_{i i^{\prime}}{ }^{(f)} \Phi_{\lambda^{\prime}}^{(f-)} d S \\
G_{\lambda i \lambda^{\prime} i^{\prime}}^{F(f)} & =-\int_{S_{f}} \Phi_{\lambda}^{(f-)} \kappa_{i i i^{\prime}}{ }^{(f)} \Phi_{\lambda^{\prime}}^{(f+)} d S,
\end{aligned}
$$

for the base functions in separate adjacent blocks. Through Eq.(4.16a) to (4.16d), $f+$ and $f$ - represents blocks on the positive and negative sides of a fracture, respectively. For numerical implementation, pairs of indices $(\lambda, i)$ and $\left(\lambda^{\prime}, i^{\prime}\right)$ in the above expressions are expressed by single indices so that the equation can be written in a matrix form.

Variation of the discrete Lagrangian with respect to the coefficient vector $a$ yields 


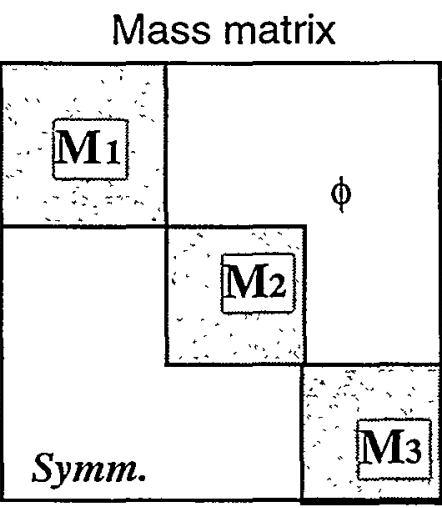

Block stiffness

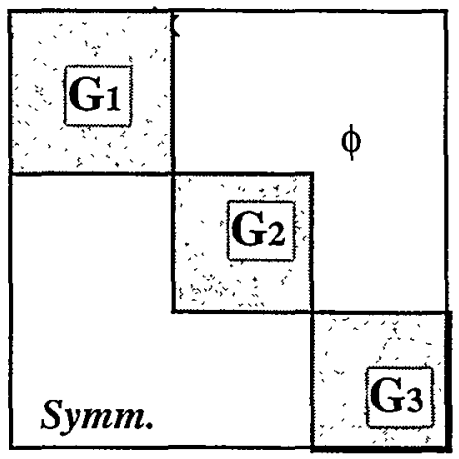

Fracture stiffness

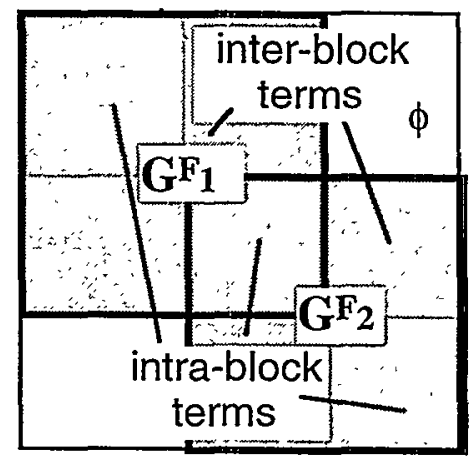

Figure 4.2 Structure of mass (M) and stiffness (G) matrices for a 3-block, 2-fracture system. Mass and block matrices are block-diagonal. The fracture stiffness matrix consists of block-diagonal intra-block terms and off-block-diagonal inter-block terms.

$$
\begin{aligned}
& \delta L=\delta a^{T}\left\{\omega^{2} M-\left(G+G^{F}\right)\right\} a=0 \text { for } \forall a \\
& \therefore \omega^{2} M a=\left(G+G^{F}\right) a .
\end{aligned}
$$

This is a matrix equation for a generalized eigenvalue problem. The mass matrix $M$ is symmetric positive definite and the stiffness matrices $G+G^{F}$ are symmetric. Therefore, the matrix equation can still be solved by standard eigenvalue solvers such as the Householder tri-diagonalization and the $\mathrm{QR}$ algorithm (Press et al., 1988). An example of the structure of the matrices for a 3-block, 2-fracture system is shown in Figure 4.2.

The numerical solution of Eq.(4.18) can be used to produce a frequency response function for a specified combination of source and receiver locations and directions. By employing a standard modal superposition technique (e.g., Newland, 1989), the displacement response (FRF) of a fractured body for a unit sine wave force excitation is given by

$$
w\left(\mathbf{x}^{(r)}, \mathbf{x}^{(s)}\right)=\sum_{n=1}^{N} \frac{\phi_{n}\left(\mathbf{x}^{(r)}\right) \phi_{n}\left(\mathbf{x}^{(s)}\right)}{\left(\omega^{2}-\omega_{n}^{2}\right) a^{(n) T} \mathbf{M} a^{(n)}},
$$

where $x^{(r)}$ and $x^{(s)}$ are location vectors for a receiver and a source, respectively, $\omega_{n}$ is the angular resonance frequency for mode $\mathrm{n}$, and $a^{(n)}$ is the $\mathrm{n}^{\text {th }}$ eigenvector. Weight functions $\phi_{n}$ are defined as 


$$
\begin{aligned}
\phi_{n}\left(\mathbf{x}^{(r)}\right) & =\sum_{i=1}^{3} u_{i} \cdot n_{i}^{(r)} \\
& =\sum_{i=1}^{3}\left(\sum_{\lambda=1}^{\lambda_{b}} a_{\lambda i}^{(b)} \Phi_{\lambda}^{(b)}\left(\mathbf{x}^{(r)}\right)\right) \cdot n_{i}^{(r)} \\
\phi_{n}\left(\mathbf{x}^{(s)}\right) & =\sum_{i=1}^{3} u_{i} \cdot n_{i}^{(s)} \\
& =\sum_{i=1}^{3}\left(\sum_{\lambda=1}^{\lambda_{b^{\prime}}} a_{\lambda_{i}}^{\left(b^{\prime}\right)} \Phi_{\lambda}^{\left(b^{\prime}\right)}\left(\mathbf{x}^{(s)}\right)\right) \cdot n_{i}^{(s)},
\end{aligned}
$$

where $n_{i}^{(r)}$ and $n_{i}^{(s)}$ are unit orientation vectors for receiver and source, respectively.

\subsubsection{Rigid and finite stiffness boundary conditions}

Resonance of a body on a rigid foundation with a compliant interface can be simulated by the same technique as the previous subsection: an elastic body is attached to a fixed (immobile) rigid body with displacement-discontinuity boundary conditions. As there is no displacement in the rigid body, assuming the rigid body is on the negative side of the interface, Eq.(4.16b d) vanish. The resulting mass and stiffness matrices are identical to those of the elastic body with traction-free boundaries except for additional intra-block terms in the stiffness matrix computed by Eq.(4.16a) (Figure 4.3). The stiffness of the interface between the elastic body and the rigid foundation can be specified by the fracture stiffness. A rigid boundary condition is realized by making the fracture stiffness of the boundary very large.

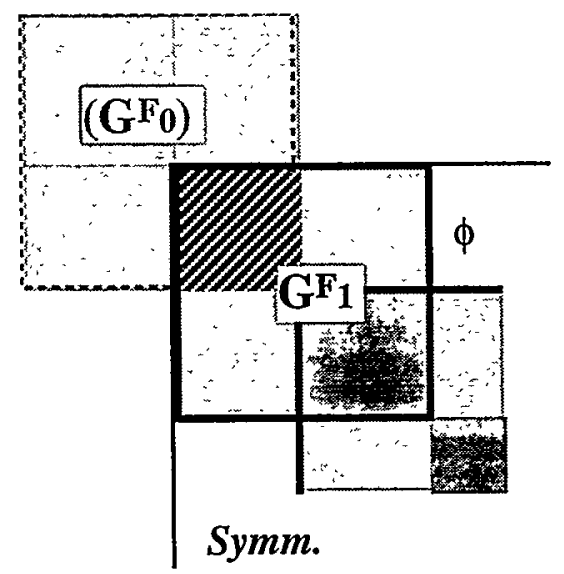

Figure 4.3 Structure of stiffness matrix for stiffness boundary (connected to rigid foundation). Modification of the matrix is required only for the block-diagonal intra-block terms of the $G^{F 1}$ submatrix. $\mathrm{G}^{\mathrm{F} 0}$ submatrix for the rigid foundation is not required as the displacement is always zero. 


\subsubsection{Symmetry of the problem}

When a problem has certain degrees of symmetry either in material properties or geometry, time and memory required for the computation can be greatly reduced by decomposing the eigenmodes into mutually independent groups. Demarest (1971) showed that normal modes of a cube specimen that exhibit elastic behavior symmetric with respect to the three mirror planes (planes that are parallel to the surfaces of a cube, cut through the center of the cube, and intersect each other at right angles) can be decomposed into eight mutually independent mode groups by selecting a certain combination of even and odd orthonormal basis functions for displacements. A detailed discussion is given by Ohno (1976). Although the current method does not use orthonormal basis functions employed by researchers such as Ohno, identical modal decomposition techniques can be used to rewrite the mass and the stiffness matrices into independent submatrices as long as the problem has a sufficient degree of symmetry.

When a fracture is introduced symmetrically into a symmetric elastic body, the symmetries of the problem can still be exploited with some modifications in the way the base functions are defined. These modifications are necessary because the displacementdiscontinuity boundary conditions between adjacent blocks break the symmetry of each block. For example, if a free vibration problem of a fractured body is to be solved, a block at a free end has a stress-free boundary at one end, but has a displacement-discontinuity boundary on the other end. To solve this problem, base functions for all the blocks are collected together to redefine global base functions. Odd and even global base functions are defined not by the order of power for the polynomial within each block but by the way the base functions are combined. For an even number of blocks (odd number of fracture(s)), an even global base function is constructed such that identical local base functions are chosen in the corresponding blocks across the plane of symmetry (Figure 4.4). Odd global base functions are constructed in the same way but with base functions with an opposite sign across the symmetry plane. If the number of the blocks is odd, base functions for the center block have to be either even or odd depending on the types of the global base function. 


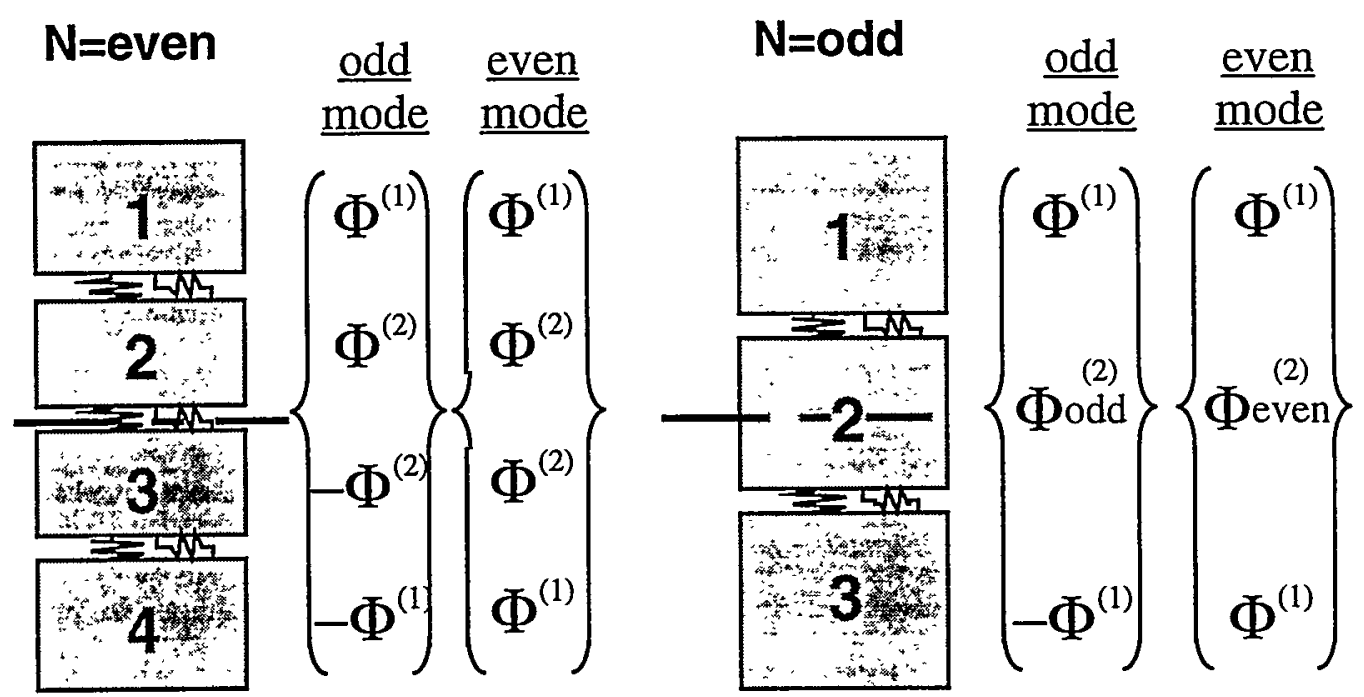

Figure 4.4 Examples of structure for global base functions for fracture(s) introduced symmetrically in a symmetric body. For even numbers of blocks, global odd and even base functions are defined using local base functions that can be either even or odd. For odd numbers of blocks, an even global base function requires an even local base function for the center block and odd global base functions require odd local base functions.

\subsection{Resonance Inversion}

\subsubsection{Perturbation of stiffness - Rayleigh's Quotient}

When the stiffness of an elastic system such as the elastic moduli of the intact part and the fracture stiffness changes, both resonance frequencies and related mode shapes are affected. The effects of a small change in the stiffness can be examined by applying small perturbations to the eigenvalue and eigenvector of the Eq.(4.17).

For an arbitrary mode $k$, the matrix equation for the elastic system is given by

$$
\omega_{k}^{2} \mathbf{M} a_{k}=\Gamma a_{k}=\left(\mathbf{G}+\mathbf{G}^{F}\right) a_{k} .
$$

When a small perturbation $\delta \Gamma$ is added to the stiffness matrix $\Gamma$, for the perturbed resonance frequency and mode vector, the following matrix equation has to be satisfied.

$$
\left(\omega_{k}^{2}+\delta \omega_{k}^{2}\right) \mathbf{M}\left(a_{k}+\delta a_{k}\right)=(\Gamma+\delta \Gamma)\left(a_{k}+\delta a_{k}\right)
$$

Subtracting Eq.(4.21) from (4.22) and ignoring small higher order terms yields 


$$
\left(\omega_{k}^{2} \mathbf{M}-\Gamma\right) \delta a_{k}+\left(\delta \omega_{k}^{2} \mathbf{M}-\delta \Gamma\right) a_{k}=0
$$

By multiplying the mode vector to Eq.(4.23) from left hand side of the equation and using the Eq.(4.21) to eliminate the first term, we get

$$
\begin{aligned}
& \delta \omega_{k}^{2} a_{k}^{T} \mathbf{M} a_{k}=a_{k}^{T} \delta \Gamma a_{k} \\
& \therefore \delta \omega_{k}^{2}=\frac{a_{k}^{T} \delta \Gamma a_{k}}{a_{k}^{T} \mathbf{M} a_{k}} .
\end{aligned}
$$

Eq.(4.25) is called Rayleigh's quotient and explicitly shows a change in resonance frequency due to a small perturbation in the stiffness of the system. This relation is particularly useful as the perturbation in the resonance frequency can be calculated without the knowledge of the perturbation in mode vector.

\subsubsection{Determination of elastic moduli for anisotropic material}

Suppose that the bulk stiffness matrix $\mathbf{G}$ contains $n$ elastic constants of the generalized Hooke's law $\left(\sigma_{i j}=C_{i j k l} \varepsilon_{k l}\right.$ ). First of all, an initial guess for the elastic constants is made and resulting resonance frequencies and mode vectors are computed. Assuming the initial guess is close to the elastic moduli of real material, Eq.(4.25) can be used to update the elastic constants of the system from differences between measured and computed resonance frequencies given by

$$
\delta \omega_{k}^{2}=\omega_{k}^{2^{(o b s)}}-\omega_{k}^{2^{(i)}}
$$

The superscript (obs) means a quantity from observation and (i) is an iteration counter. For the initial stage, $i=0$. By introducing Eq.(4.26) into Eq.(4.25)

$$
\frac{a_{k}^{(i) T} \delta \mathbf{G} a_{k}^{(i)}}{a_{k}^{(i) T} \mathbf{M} a_{k}^{(i)}}=\delta \omega_{k}^{2} \approx \omega_{k}^{2^{(i)}}-\omega_{k}^{2^{(o b s)}}
$$

As the left hand side of the equation is linear in terms of the perturbation of elastic constants $C_{i}$, the equation can be rewritten as

$$
A_{k}^{(i) T} \delta C=b_{k}^{(i)}
$$

where 


$$
\begin{aligned}
& C^{T}=\left\{C_{1}, C_{2}, \ldots, C_{n}\right\} \\
& b_{k}^{(i)}=\omega_{k}^{2^{(i)}}-\omega_{k}^{2(o b s)} .
\end{aligned}
$$

When $m$ resonance frequencies are observed, the following matrix equation is obtained

$$
\mathbf{A}^{(i)}(m \times n) \delta \mathbf{C}=\mathbf{b}^{(i)} .
$$

This is a generalized inverse problem for an increment in the elastic constants $\mathrm{C}_{\mathrm{i}}$. When the number of observations are more than unknowns $(m \geq n)$, assuming the matrix product has its inverse, the increment $\delta C$ is computed by

$$
\delta C=\left(A^{(i)^{T}} A^{(i)}\right)^{-1} b^{(i)}
$$

This is a least square solution of Eq.(4.30). The meaning of the least square minimization is graphically shown in Figure 4.5. The assumed elastic constants of the system are improved by

$$
\mathbf{C}^{(i+1)}=\delta \mathbf{C}+\mathbf{C}^{(i)}
$$

As the above discussion assumes infinitesimal perturbation of stiffness, elastic moduli computed from Eq.(4.32) do not yield resonance frequencies that agree with the observed resonance frequencies. The solution is obtained by iteratively applying the above procedure and minimizing the difference between observed and computed resonance frequencies. At each iteration, resonance frequencies and mode vectors are computed using updated elastic constants.

The iterative inversion method presented above is essentially identical to the method used by Ohno (1976) and Migliori et al. (1993). Migliori et al. derived the matrix equation Eq.(4.30) by minimizing the following subjective function

$$
I^{2} \equiv \sum_{k=1}^{m} w_{k}\left(\delta \omega_{k}^{2}\right)^{2} \approx \sum_{k=1}^{m} w_{k}\left(\omega_{k}^{2(i)}-\omega_{k}^{2^{(o b s)}}\right)^{2},
$$

where $w_{\mathrm{k}}$ is a weight reflecting the confidence and accuracy of each mode. The iteration procedure used here is known as Newton's method and the initial estimate for the elastic constants should be close to the final solutions for convergence. To improve the convergence of solution when an initial guess is relatively far from the final solutions, 
Migliori et al. employed the Levenberg-Marquardt method (e.g., Press, 1988) that combines the above with the steepest-descent method, which seeks the fastest-decreasing direction of the subjective function in the solution space. Increment of moduli by the Levenberg-Marquardt method is given by

$$
\delta \mathbf{C}=\left[\mathbf{A}^{(i)^{T}} \mathbf{A}^{(i)}+\Omega \cdot \operatorname{Diag}\left(\mathbf{A}^{(i)^{T}} \mathbf{A}^{(i)}\right)\right]^{-1} \mathbf{b}^{(i)}
$$

where $\Omega$ is a dimensionless positive parameter and Diag( ) is an operator that extracts a diagonal part of the matrix. When a solution is far from the final solution, a large $\Omega$ is taken to assure that the subjective function is decreased. When the solution becomes close to the final solution and the subjective function becomes small, $\Omega$ is brought to zero and Newton's method is used to achieve the converged solution.

Although implementing the Levenberg-Marquardt method to a computer code is not difficult, current research employed only the Newton's method to update solutions during iterations.

\subsubsection{Determination of anisotropic fracture stiffness}

The procedure shown in the previous subsection can be directly applied to determine anisotropic fracture stiffness. Noting that a perturbation in the stiffness is introduced only in the fracture component of the stiffness matrix, Eq.(4.27) becomes

$$
\frac{a_{k}^{(i) T} \delta \mathbf{G}^{F} a_{k}^{(i)}}{a_{k}^{(i) T} \mathbf{M} a_{k}^{(i)}}=\delta \omega_{k}^{2} \approx \omega_{k}^{2^{(i)}}-\omega_{k}^{2(o b s)} .
$$

Again, as the left hand side of the equation is linear in terms of the fracture stiffness, the above equation becomes

$$
D_{k}^{(i)^{T}} \delta \kappa=b_{k}^{(i)}
$$

where

$$
\kappa^{T}=\left\{\kappa_{1}, \kappa_{2}, \ldots, \kappa_{n}\right\}
$$

Note that the components of the fracture stiffness vector do not have to be for a single fracture. For $m(m \geq n)$ observations for resonance frequencies, the increment for the fracture stiffness vector is given as 


$$
\delta \kappa=\left(\boldsymbol{D}^{(i)^{T}} \boldsymbol{D}^{(i)}\right)^{-1} \boldsymbol{b}^{(i)}
$$

Using Eq.(4.38), a similar iterative procedure as in the previous section is taken.

\subsection{Accuracy of Computed Resonance Frequency}

\subsubsection{Lamé's mode}

Accuracy of the introduced method for computing resonance frequencies of intact elastic bodies have been demonstrated by many researchers. Holland(1968) compared his results and those of Ekstein and Schiffman (1956) for computed resonance frequencies of isotropic cubes with analytically available Lamé's mode resonance frequencies. Demarest (1969) showed that use of Legendre polynomials for base functions produces better results than the previous works and demonstrated the accuracy of his method by using the experimentally measured resonance of a steel cube.

For rectangular parallelepipeds, there exists a family of modes called Lamé's mode (Lamé, 1866) for which an analytic expression for the resonance frequency and mode shape are available. For free vibration of isotropic rectangular parallelepipeds, Lamé showed that the mode has resonance frequencies

$$
\omega=\frac{m \pi}{2 a} \sqrt{\frac{2 c_{66}}{\rho}},
$$

which satisfies $a / m=b / n$, where $2 \mathrm{a}$ and $2 \mathrm{~b}$ are any two lengths of the block, $\mathrm{m}$ and $\mathrm{n}$ are integers, $c_{66}$ is a shear modulus, and $\rho$ is density of material. A Lamé mode does not have any motion in the direction of the third length and tractions are zero on any plane normal to it. Lamé's mode for a more general type of elasticity is discussed by Mindlin (1956). For an isotropic cube, Eq.(4.39) becomes

$$
\omega=\frac{m \pi}{L} \sqrt{\frac{2 c_{66}}{\rho}},
$$

where $\mathrm{L}$ is a length of the cube. Due to the symmetry, each Lamé's mode is triply degenerate. 


\subsubsection{Accuracy of solution for an intact cube}

As general analytic solutions for the resonance frequency of an arbitrarily shaped elastic body do not exist, the accuracy of numerical solutions cannot be checked by reference to the analytic solution. An alternative method to check the accuracy is to examine the convergence behavior of the numerical solution as the order of the approximation for the employed base functions is increased. As any continuous displacement field can be expressed by a complete set of base functions with sufficiently high order of polynomials, numerical solutions can be considered to be close to the exact solutions if any change in the resonance frequencies with increasing order of base functions is sufficiently small.

Changes in computed resonance frequencies of an isotropic cube with traction free boundaries are shown in Figure 4.5. Each curve represents the difference between resonance frequencies for a single mode with increasing maximum order of base function polynomials, $\mathrm{n}$. The $\mathrm{n}$ is identical for three directions ( $\mathrm{x}, \mathrm{y}$, and $\mathrm{z}$ directions). The

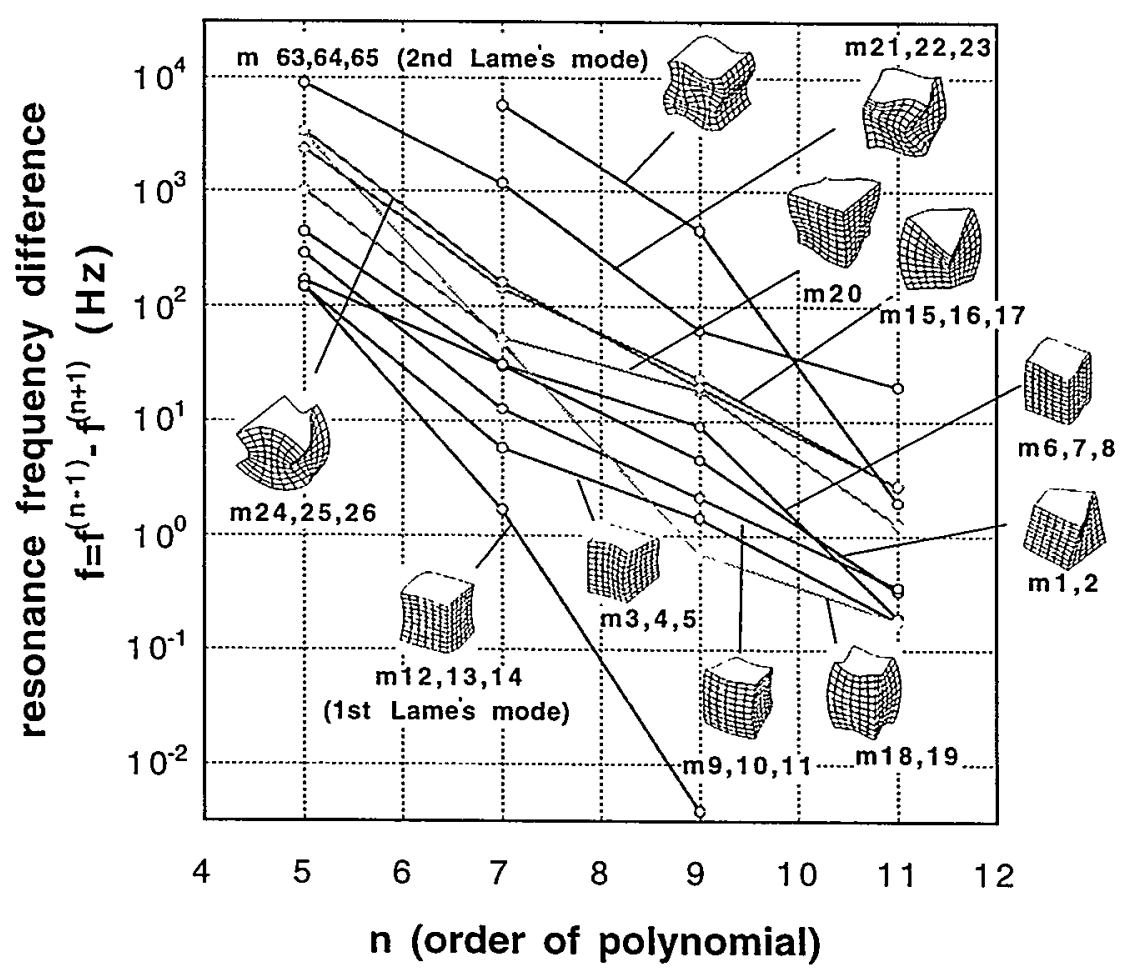

Figure 4.5 Convergence of computed resonance frequencies for an isotropic cube $(E=50 G P a, v=0.2$, $\left.\mathrm{L}=0.1 \mathrm{~m}, \rho=2600 \mathrm{~kg} / \mathrm{m}^{3}\right)$. Resonance frequency changes between $2 \mathrm{k}^{\text {th }}$ and $2(\mathrm{k}+1)^{\text {th }}$ order approximation $(\mathrm{n}=2 \mathrm{k}+1)$ are shown. The theoretical resonance frequency for the $1 \mathrm{st}$ Lamé's mode is $20.0160 \mathrm{kHz}$. Higher order modes require higher order base functions to compute the resonance frequency accurately. It is noted that the Lamé mode converges faster than other type of modes. 
accompanying mode shape is shown for each mode. It can be seen that higher order modes require higher order base functions for accuracy. The $12^{\text {th }}, 13^{\text {th }}, 14^{\text {th }}$ modes form the first Lamé's mode and exhibit faster convergence than other modes. The second Lamé's mode $\left(63^{\text {th }}, 64^{\text {th }}, 65^{\text {th }}\right.$ modes) are also shown for comparison. The computed resonance frequencies of the Lamé's modes are compared with the analytic solutions with increasing order of base function polynomials (Figure 4.6). It can be seen that the modes exhibit rapid convergence to the exact solutions as the order of approximation is increased.
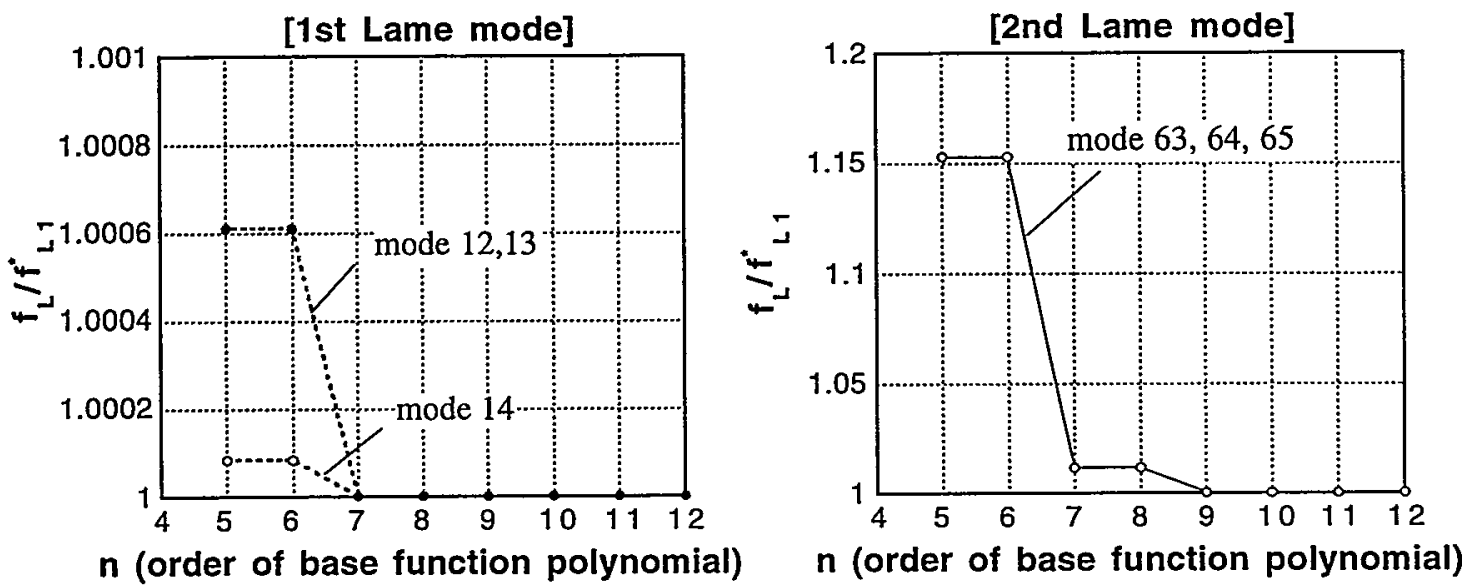

Figure 4.6 Convergence of the 1 st and 2 nd Lamé mode resonance frequencies for an isotropic cube. The vertical axis is normalized by theoretical resonance frequency. It is noted that $2 \mathrm{k}+1$ th and $2 \mathrm{k}$ th order base functions result in identical resonance frequencies of the Lamé's mode. The 1st mode (mode 12 14) resonance frequency is accurately computed even with relatively low order polynomials.

\subsubsection{Accuracy of solution for a fractured cube}

When a fracture is introduced into the cube discussed in Section 4.4.2, resonance frequency and mode shape are altered, depending on the stiffness of the fracture. As vibrations of the cube with very high and low fracture stiffness are close to free vibrations of intact block(s), an intermediate fracture stiffness $\left(\kappa=10^{11} \mathrm{~Pa} / \mathrm{m}\right)$ is chosen to understand the conversion behavior of numerical solutions. The fracture stiffness is assumed to be isotropic $\left(\kappa_{x}=\kappa_{y}=\kappa_{z}=\kappa\right)$.

Conversions of computed resonance frequencies for an isotropic cube containing a centered through-crack is shown in Figure 4.7. The maximum order of base function polynomials in each block separated by the fracture is $2 m(=n)$ for the $x$ and $y$ directions and $\mathrm{m}$ for the $\mathrm{z}$ direction (direction normal to the fracture). All material properties are identical 
to the intact cube in the previous subsection. From the plot it can be seen that introduction of a fracture does not slow down the convergence of solutions. It is also noted that convergence and mode shape of the $21^{\text {st }}$ mode are identical to the first Lamé's mode of an intact block. This is because a fracture introduced in parallel to the $x-y$ plane does not have any effect on the resonance of the mode as tractions on the plane are zero everywhere in the intact cube.

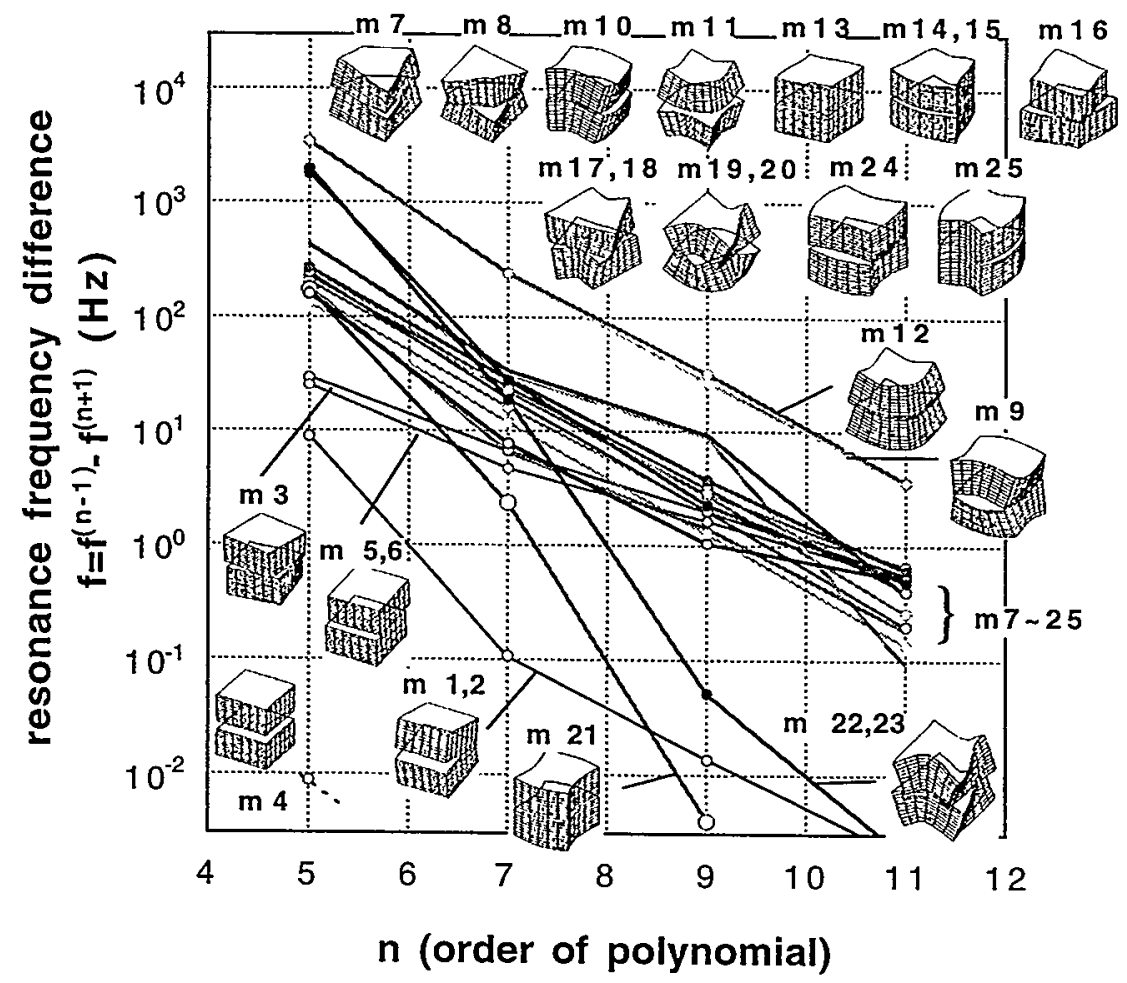

Figure 4.7 Convergence of computed resonance frequencies for a fractured block ( $E=50 \mathrm{GPa}, v=0.2$, $\mathrm{L}=0.1 \mathrm{~m}, \rho=2600 \mathrm{~kg} / \mathrm{m}^{3}$, fracture stiffness $k=10^{11} \mathrm{~Pa} / \mathrm{m}$ ). Resonance frequency changes between $2 \mathrm{k}$ th and $2(\mathrm{k}+1)^{\text {th }}$ order approximation $(\mathrm{n}=2 \mathrm{k}+1)$ are shown. $21^{\text {th }}$ mode is identical to the 1 st Lamé's mode $(20.0160$ $\mathrm{kHz}$ ). Higher order modes require higher order base functions to compute the resonance frequency accurately.

\subsection{Accuracy of Resonance Inversion}

\subsubsection{Inversion for isotropic elastic moduli}

The resonance inversion technique introduced in Section 4.3.2 was checked for its performance to determine isotropic elastic moduli of a cube from its resonance frequencies. Convergence and accuracy of determined solutions were examined. Resonance frequency and mode shape of the "observed" modes were computed using $12^{\text {th }}$ order base functions. 


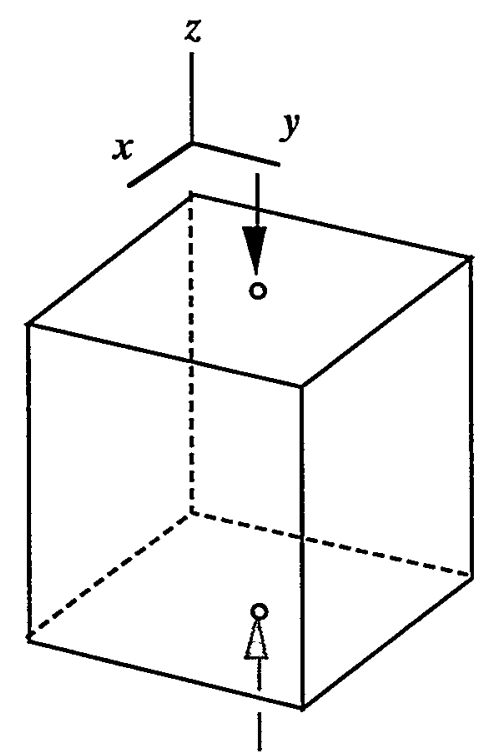

Figure 4.8 Source and receiver locations for observed resonance frequencies of an isotropic cube. Direction of the source and receiver are aligned.

Young's modulus and Poisson's ratio of the cube $(0.1 \mathrm{mx} 0.1 \mathrm{mx} 0.1 \mathrm{~m})$ were assumed to be $50 \mathrm{GPa}$ and 0.2 , respectively. Density of the cube was $2600 \mathrm{~kg} / \mathrm{m}^{3}$. For source and receiver locations shown in Figure 4.8, a FRF(compliance) was computed (Figure 4.9). Resonance frequencies of the first five modes were used for the inversion.

As the inversion process requires many iterations, lower $10^{\text {th }}$ order base functions were used to approximate the displacement field in the cube. As initial guesses, Young's modulus of $25 \mathrm{GPa}$ and Poisson's ratio of 0.2 were chosen. Figure 4.10 (a) shows changes in the standard deviation for the power of computed resonance frequency given by

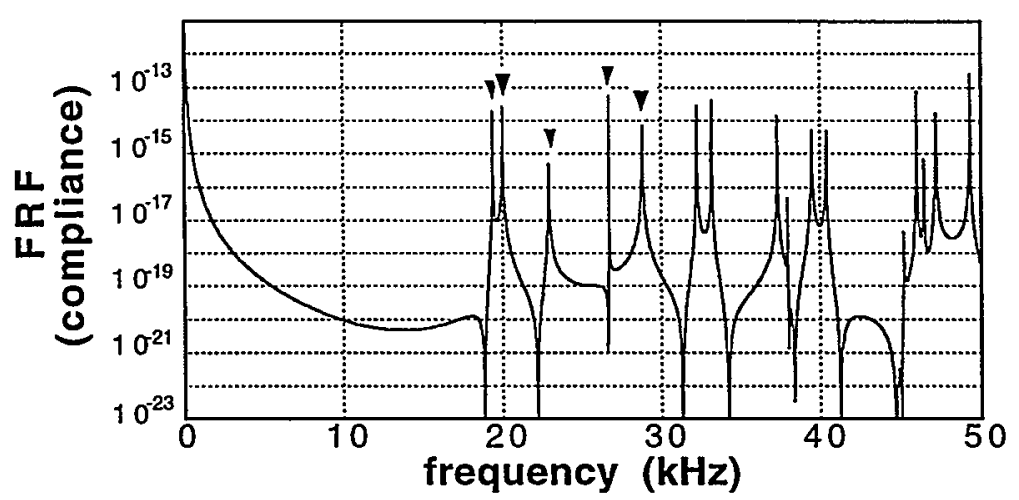

Figure 4.9 Computed FRF(compliance) for forced excitation of an isotropic cube. Modes marked by solid triangles were used for checking the accuracy of numerical inversion for elastic moduli. 


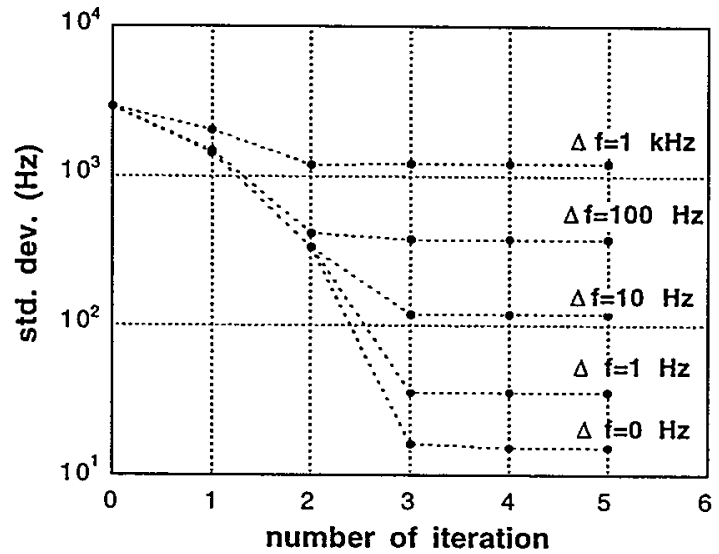

(a) difference between computed and observed resonance frequencies

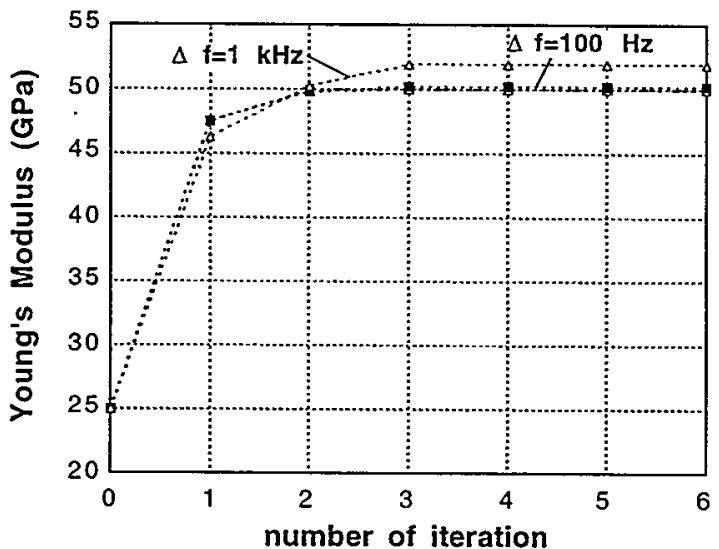

(b) convergence of Young's modulus

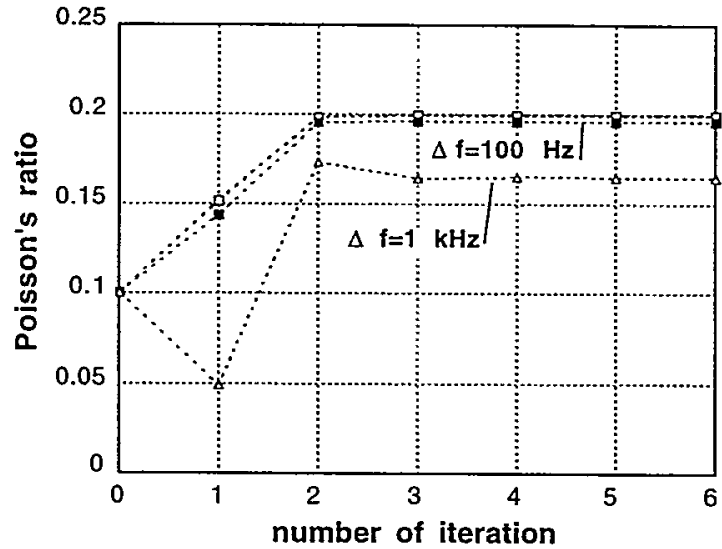

(c) convergence of Poisson's ratio

Figure 4.10 Convergence of the solution. The original model's Young's modulus and Poisson's ratio are $E=50 \mathrm{GPa}$ and $v=0.2$ (exact solutions), respectively. The initial guess is $E=25 \mathrm{GPa}$ and $v=0.1$. "Observed" resonance frequencies are computed using $12^{\text {th }}$ order base functions. Inversions are conducted using $10^{\text {th }}$ order base functions. The observed resonance frequencies are perturbed by the amount specified in the plot to see the effect of error in measured data on the convergence. 


$$
\sigma_{f^{2}}=\sqrt{\frac{1}{N} \sum_{k=1}^{m}\left(f_{k}^{2^{(i)}}-f_{k}^{2(a b s)}\right)^{2}}
$$

where $f_{k}^{2(o b s)}$ and $f_{k}^{2(i)}$ are the power of observed and computed resonance frequencies for the $\mathrm{i}$ th iteration, respectively, and $N$ is the number of observed modes (here, $N=5$ ). In the plot, the square root of $\sigma_{f 2}$ 's are shown to indicate an average difference between resonance frequencies of an inverted model and observed resonance frequencies. During the inversion, the weighting factors in Eq.(4.33) are assumed to be constant $(=1)$. To examine the effect of errors in the observed resonance frequencies, the frequencies were perturbed by the amount specified in the plot.

From the plot it can be seen that an increase in the perturbation leads to an increase in the difference between the inverted and observed resonance frequencies. A case with no perturbation ( $\Delta f=0 \mathrm{~Hz}$ ) also shows a small difference at convergence due to the difference in the order of base functions used for the inversion. Even though the initial guess is very different from the model's true elastic moduli, the convergence behavior is quite stable. Changes in the Young's modulus and Poisson's ratio at each iteration are also shown in Figure $4.10(\mathrm{~b})$ and (c). It is noticed that in spite of relatively large perturbations ( $\Delta f=100 \mathrm{~Hz}, 1 \mathrm{kHz}$ ), the inverted Young's moduli are reasonably accurate. Poisson's ratio, on the other hand, is more sensitive to the error in the observations.

\subsubsection{Inversion for isotropic fracture stiffness}

Performance of the resonance inversion technique for determining isotropic fracture stiffness was examined using the same method in Section 4.4.1. A model used for the tests was the same cube including a fracture as in Section 4.4 .3 (fracture stiffness $\left.\kappa=10^{11} \mathrm{~Pa} / \mathrm{m}\right)$. Locations of a source and a receiver are shown in Figure 4.11. Computed FRF and modes used for inversion are shown in Figure 4.12. An initial guess for the fracture stiffness was chosen as $10^{12} \mathrm{~Pa} / \mathrm{m}$. For a single case with no perturbation, an initial guess of $\kappa=10^{12} \mathrm{~Pa} / \mathrm{m}$ was also tested.

During the inversion, it was found that a direct application of Newton's method does not converge the solutions as the initial guesses were too far from the exact solutions. To ensure the convergence of the solutions, a correction for fracture stiffness at each iteration (Eq.(4.38)) was introduced as 


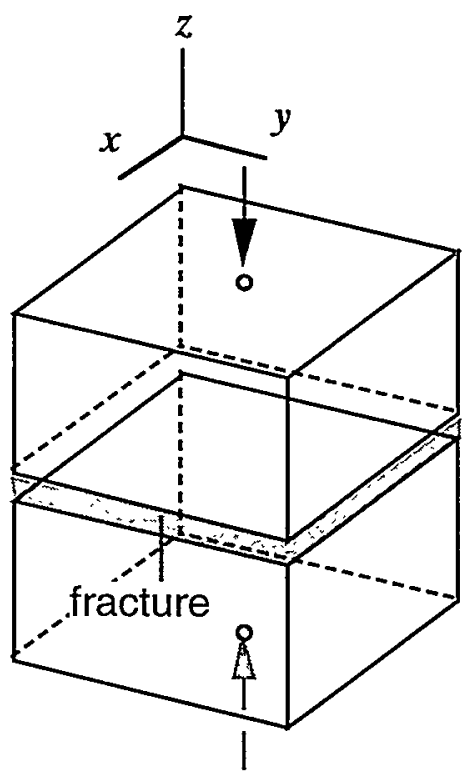

Figure 4.11 Source and receiver locations for observed resonance frequencies of an isotropic cube including a single through-crack. Direction of the source and receiver are aligned.

$$
\delta \kappa \rightarrow 0.5 \times \delta \kappa
$$

This modification decreases the speed of convergence but helps to stabilize the convergence process. Figure 4.13(a) and (b) shows changes in the averaged resonance frequency error and determined fracture stiffness, respectively. The speed of convergence is much slower than the inversion for elastic moduli of the intact cube discussed in Section 4.5.1. It is noticed that the determined fracture stiffnesses are relatively accurate except for a case with a large error in the observation ( $\Delta f=1 \mathrm{kHz}$ ).

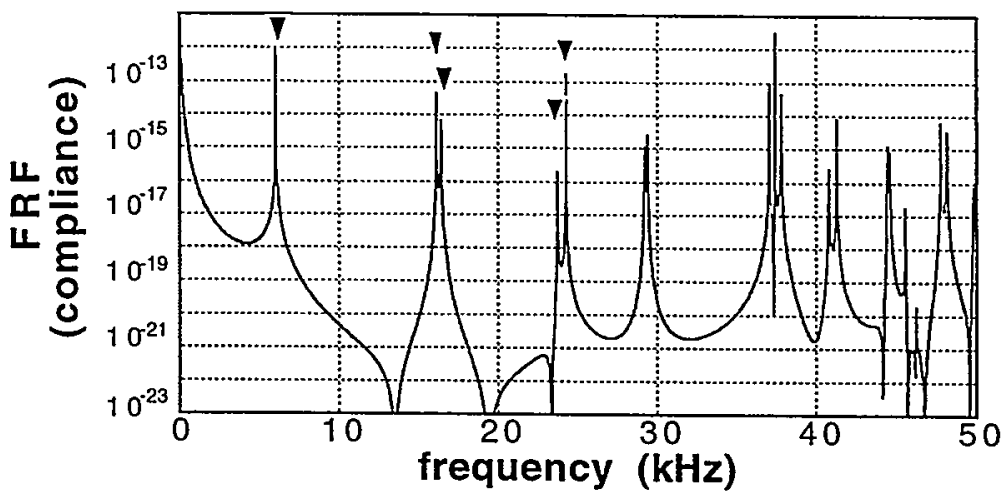

Figure 4.12 Computed FRF(compliance) for forced excitation of an isotropic cube containing a fracture. Modes marked by solid triangles were used for checking the accuracy of numerical inversion for elastic moduli. 


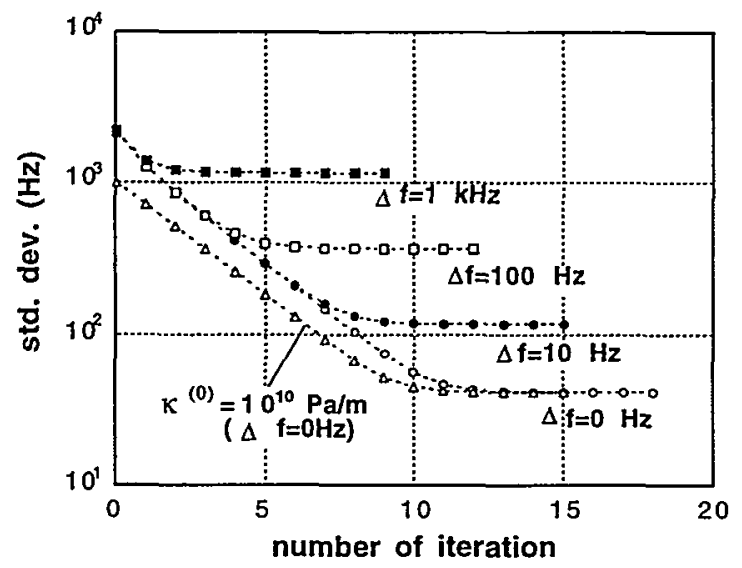

(a) difference between computed and observed resonance frequencies

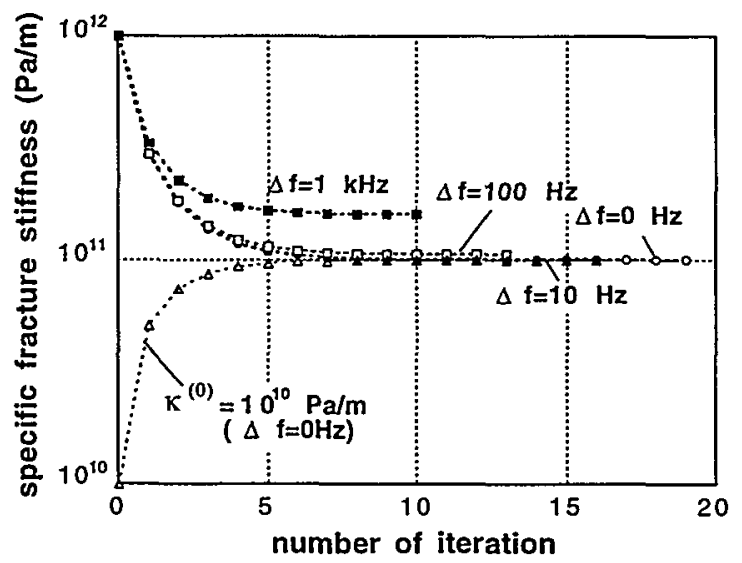

(b) Convergence of fracture stiffness

Figure 4.13 Convergence of solution. The original model's Young's modulus and Poisson's ratio are $\mathrm{E}=50 \mathrm{GPa}$ and $\mathrm{v}=0.2$, respectively, and fracture stiffness is $10^{11} \mathrm{~Pa} / \mathrm{m}$. The initial guess was made as $10^{12}$ $\mathrm{Pa} / \mathrm{m}$ for four cases with different perturbation in the observed resonance frequencies and $10^{10} \mathrm{~Pa} / \mathrm{m}$ for no perturbation. "Observed" resonance frequencies were computed using $12 \times 12 \times 6$ (x,y, and z-directions) ${ }^{\text {th }}$ order base functions in each block. Inversions were conducted using $8 \times 8 \times 4^{\text {th }}$ order base functions. 


\subsection{Summary}

In this chapter, a simple numerical method for computing normal mode vibrations of elastic body with fractures was introduced. Using this fast forward modeling technique, a numerical inversion technique for determining elastic moduli and fracture stiffness from observed resonance frequencies was developed.

As analytic solutions for an arbitrarily shaped three-dimensional body with fractures are not available, the accuracy of the numerical model is difficult to evaluate. However, from the convergence of computed resonance frequencies with increasing order of polynomial approximations, the accuracy of the numerical model is examined indirectly.

For a special case where analytic solutions were available (Lamé's mode), computed solutions were compared with analytic solutions showing good agreement. Accuracy and conversion of the inversion technique were checked for isotropic elastic moduli and fracture stiffness of cubes. The results indicated that even when relatively large error was present in the observed resonance frequencies, the method could determine the elastic parameters with reasonable accuracy. However, numerical results obtained for a specific problem have to be viewed with caution. This is because a resonance is affected by many geometrical and mechanical parameters of a specimen and the accuracy of the solution is expected to vary for different problems.

The numerical model developed in this chapter can be a very powerful tool for examining resonance of anisotropic and fractured bodies with general geometry. Using the forward-modeling part of the code, resonance frequencies, mode shapes, and expected FRF for specific source and receiver locations can be computed. Using the code, optimal source and receiver locations can be determined for exciting or avoiding large vibration motions to perform acoustic resonance tests. The inversion part of the code can determine unknown elastic parameters from measured resonance frequencies. As the moduli and fracture stiffness of the material can be related to the degree of damaged introduced, the code can be used to make the acoustic resonance technique a quantitative tool for characterizing material properties and diagnosing damage in a structure.

In the following chapters, the numerical models presented in this chapter are used for modeling experimentally observed resonances and determining stiffness parameters (elastic constants and fracture stiffness) of rock and concrete specimens. 


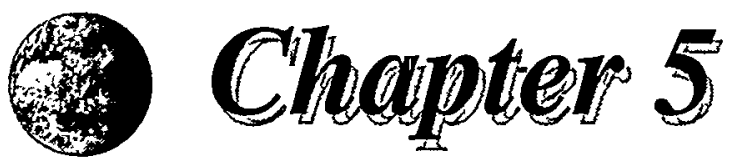

\section{Resonance of Anisotropic Rock}

\subsection{Introduction}

At the millimeter to submillimeter scale, rocks appear as a heterogeneous aggregate of crystals and mineral grains. Rocks may also contain numerous microcracks that are distributed uniformly within the matrix. When heterogeneities in rock occur in the form of systematically oriented mineral grains, bedding planes, and aligned microcracks, they result in anisotropic bulk stress-strain behavior. The bulk elastic behavior of the rock is described by elastic constants of an equivalent homogeneous anisotropic medium. These elastic constants can be determined either statically from load-displacement tests or dynamically from the velocities of $\mathrm{P}$ - and S-waves. Both the static and dynamic tests require measurements conducted in multiple directions with respect to the axes of elastic symmetry. For example, to determine the dynamic elastic moduli of a transversely isotropic rock, at least five independent $\mathrm{P}$ - and $\mathrm{S}$-wave velocities in three directions (typically $0^{\circ}, 45^{\circ}$, and $90^{\circ}$ to the axis of symmetry) have to be measured (e.g., King, 1969; Lo et al., 1984). Similarly, static load-displacement tests require axial compression and lateral expansion measurements on specimens cored in three directions (King, 1969). The 
latter approach for determination of the five anisotropic elastic constants is not common because it requires a cumbersome testing procedure that is susceptible to inaccuracies in measurements of the elastic deformation of a specimen resulting from nonelastic deformation (frictional slip).

Acoustic resonance spectroscopy is a technique for determining the dynamic elastic constants of a specimen using steady-state vibration of a specimen of known geometry. The technique consists of resonating the specimen over a broad range of frequencies, measuring the resonance frequencies, and computing the elastic constants by nonlinear inversion of the measured resonance frequencies. This technique is particularly interesting because it can determine anisotropic elastic constants of a specimen with general geometry from a single measurement of the frequency response.

The standard resonant bar technique in the Chapter 2 is restricted to longitudinal, flexural, and torsional resonances of a slender bar specimen with isotropic elastic constants. For this geometry, simple relations between the Young's modulus and the resonance frequencies of longitudinal and flexural modes, and the shear modulus and the resonance frequencies of torsional modes make the determination of the elastic constants almost trivial. No such relations exist, however, for use in determining the anisotropic elastic constants for a specimen of more general geometry. This more complicated problem requires inversion for the elastic constants using numerical techniques. Although such techniques have been used for determining the elastic constants of single crystals and minerals (Ohno, 1976; Maynard, 1992; Migliori et al., 1993), few attempts have made to characterize the anisotropic elastic properties of rocks whose anisotropy arises from the heterogeneous microstructures.

In this chapter, acoustic resonance spectroscopy is applied to determine the elastic constants of transversely isotropic rock specimens. For comparison purposes, static and ultrasonic measurements are first performed to determine the zero-frequency and highfrequency elastic moduli. These measurements are followed by acoustic resonance measurements on cube-shaped specimens. Measured resonance frequencies are used to invert for the five elastic constants using the numerical algorithm introduced in the previous chapter. Mode shapes of the anisotropic specimens are also measured using a laser Doppler vibrometer and compared with mode shapes computed from the elastic constants obtained from the numerical inversion of the measured resonance frequencies. 


\subsection{Static Elastic Properties}

\subsubsection{Description of specimens}

Rock specimens used in this chapter are Sierra White Granite, Berea Sandstone, and Tennessee Dolomite. These rocks are anisotropic due to different microstructures. The granite specimen contains a number of sub-millimeter size microcracks that are oriented perpendicular to a single direction. This granite is from the same quarry as the granite specimen used in the Chapter 2. The sandstone specimen has bedding structures that are visible as red oxidized minerals deposited along submillimeter-spaced bedding planes. The sandstone also contains minor cross-bedding that cross-cuts the dominant near parallel bedding structures (this Berea sandstone specimen is different from the specimen used in Chapter 2). The dolomite specimen contains a number of fractures that extend as long as a centimeter and are mostly filled with precipitated silica (Opal). The precipitated silica is also present in the form of millimeter size inclusions. These microstructures give rise to transversely isotropic static and dynamic properties. By visual inspection, the granite specimen appears to be the most homogeneous and the dolomite the most heterogeneous due to the presence of large cracks and inclusions. The densities of the ovendried granite, sandstone, and dolomite specimens are $2.62 \mathrm{~g} / \mathrm{cm}^{3}, 2.15 \mathrm{~g} / \mathrm{cm}^{3}$, and $2.70 \mathrm{~g} / \mathrm{cm}^{3}$, respectively.

The specimens were prepared for acoustic resonance spectroscopy by first determining the axis of symmetry of the rock using ultrasonic measurements and then cutting out a cube-shaped specimen from the block such that two of its surfaces are perpendicular to the axis of symmetry. The size of the cubes were $9.40 \mathrm{~cm}, 6.04 \mathrm{~cm}$, and $6.45 \mathrm{~cm}$ for the granite, sandstone, and dolomite, respectively. The specimens are shown in Figure 5.1. For the granite, cylindrical specimens $(9.81 \mathrm{~cm}$ in length, $3.73 \mathrm{~cm}$ in diameter) and short octagonal blocks $(5.08 \mathrm{~cm}$ across parallel surfaces) with their axis perpendicular to the surfaces of the cube specimen were also made. These specimens were used for measuring static and high frequency (ultrasonic) properties of the rock.

\subsubsection{Optical microscope observation for granite}

Sub-millimeter microstructures in the granite specimen such as aligned minerals, microcracks, and grain boundaries are not directly visible. Thin sections for optical microscopy were prepared from three cylindrical specimens of the granite cored 


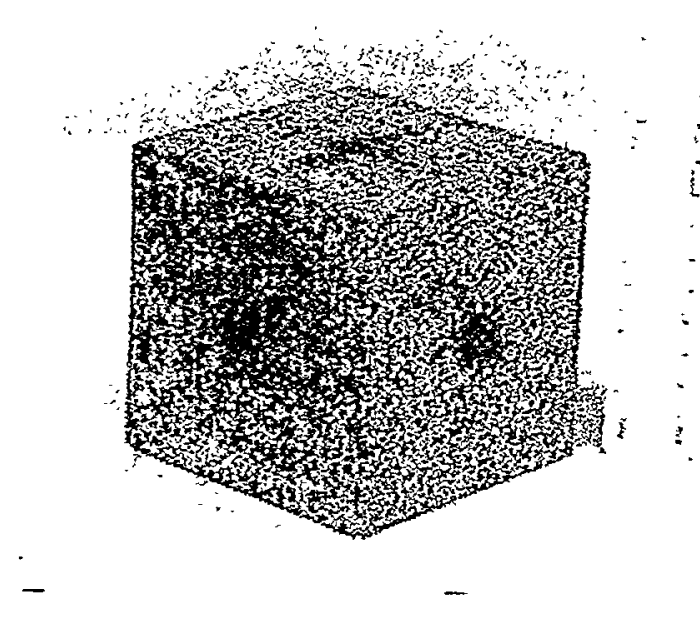

(a) Granite cube (Sierra White). No obvious microstructure can be detected by visual inspection. The rock appears to be homogeneous.

(b) Sandstone cube (Berea). Distinct bedding structures can be seen. Layers of red stain are spaced rather irregularly. Cross bedding structures that cut the dominant parallel bedding structures obliquely can be seen on the sides of the cube.

\section{bedding planes}
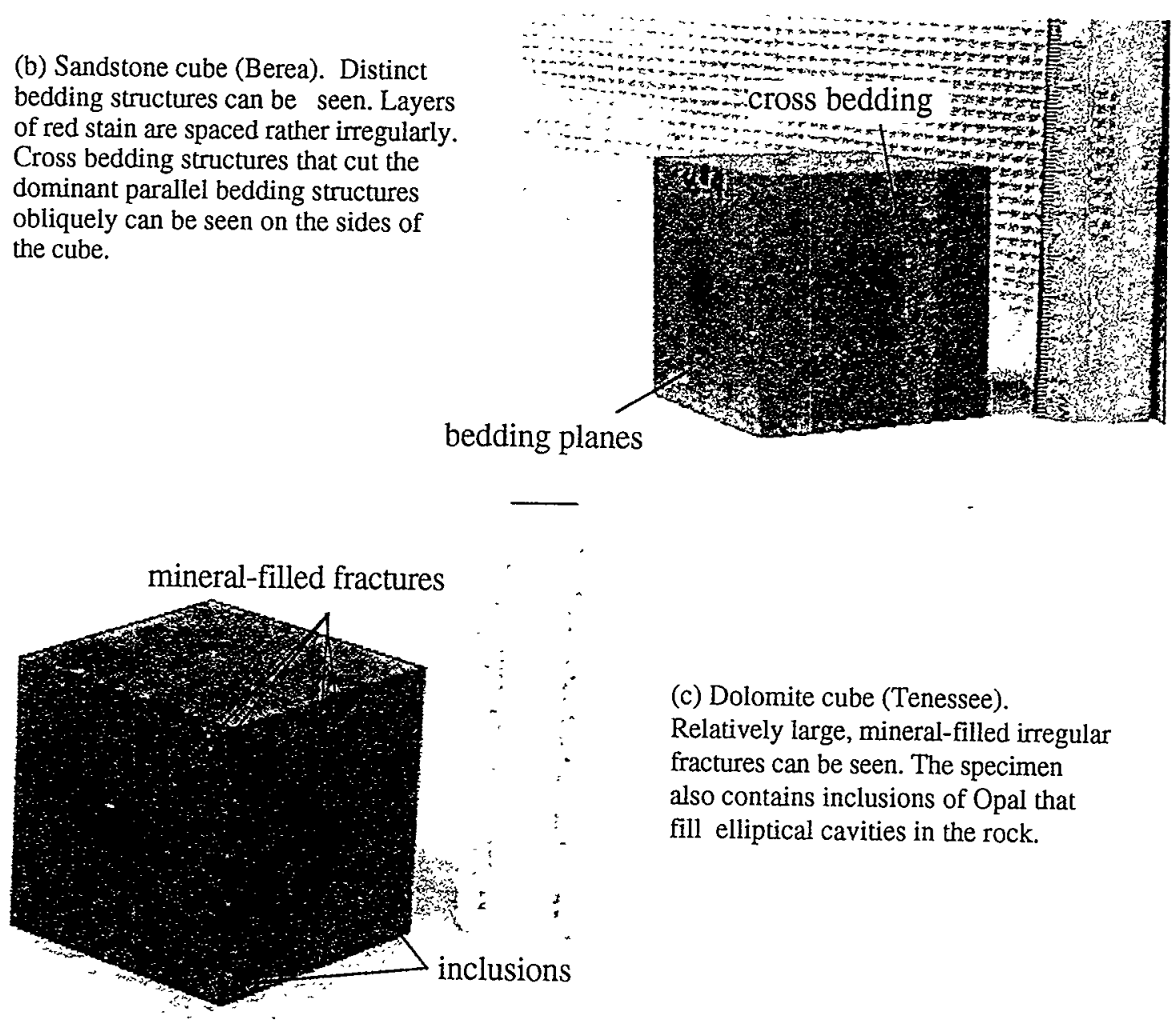

(c) Dolomite cube (Tenessee). Relatively large, mineral-filled irregular fractures can be seen. The specimen also contains inclusions of Opal that fill elliptical cavities in the rock.

Figure 5.1 Rock cube specimens used for resonance spectroscopy. The three rocks have different microstructures that lead to transversely isotropic elastic behavior: (a) granite, (b) sandstone, (c) dolomite 


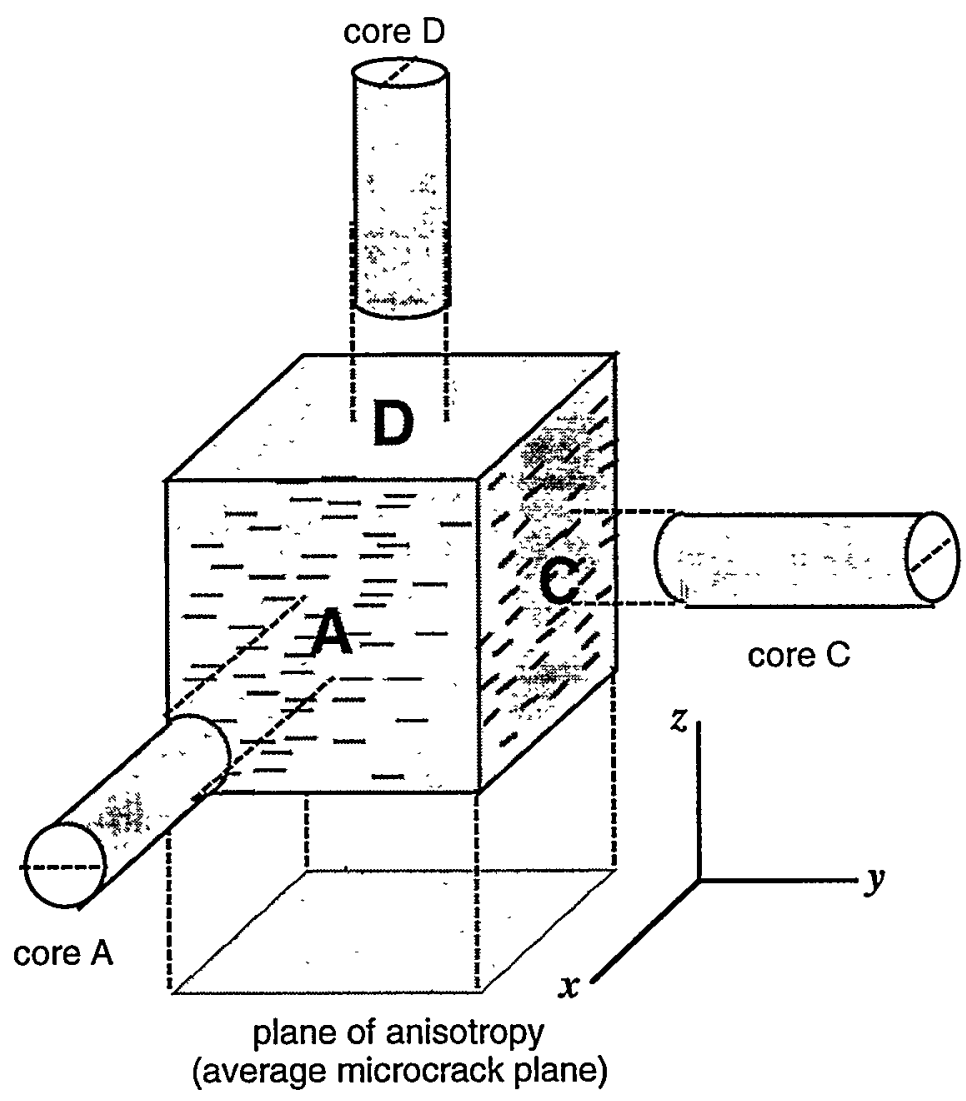

Figure 5.2 Core orientation of specimens used for optical microscope observation, imbibition tests, and static uniaxial load-displacement tests. Cores $\mathrm{A}$ and $\mathrm{C}$ are taken in the direction perpendicular to the isotropy axis (parallel to average microcrack planes). Core $\mathrm{D}$ is parallel to the isotropy axis. The reference direction of each core is shown by a dotted line along its diameter.

perpendicular to each other (Figure 5.2, National Petrographic Service Inc., Houston, Texas). The specimens were vacuum-impregnated with blue-dyed epoxy. Figure 5.3(a) (c) are the thin section optical-micrographs of microcracks observed in the three perpendicular directions. The observations are made using normal transmitted light. Microcracks impregnated by blue epoxy are best seen in the $\mathrm{C}$ core and are aligned roughly parallel in a single direction. In the A core, alignment of the microcracks are not as clear as in the $\mathrm{C}$ direction. In the $\mathrm{D}$ core, no preferential directions of the microcracks are observed. Figure 5.4(a) and (b) are the close-up photographs of the microcracks. Open cracks filled with epoxy are marked by solid triangles. Figure 5.5(a) is a thin section micrograph using polarized light. A micrograph of the same area using normal light is also shown (Figure 5.5(b)) for comparison. From the photographs, no obvious elongation or alignment of mineral grains is present. These observations indicate that the anisotropy of the granite is primarily due to open microcracks that are aligned in a single direction. 


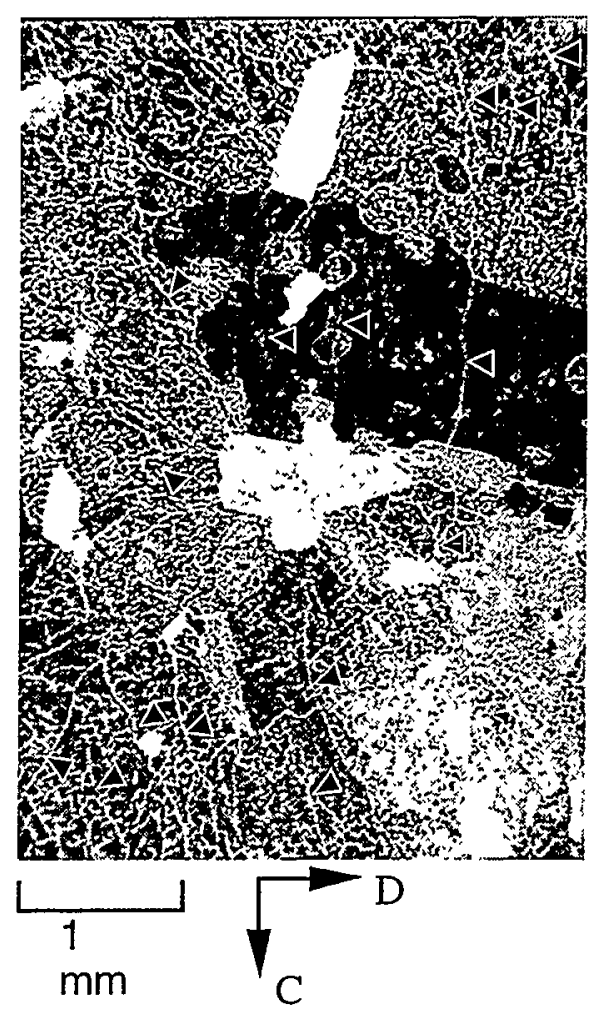

(a) core $\mathrm{A}$

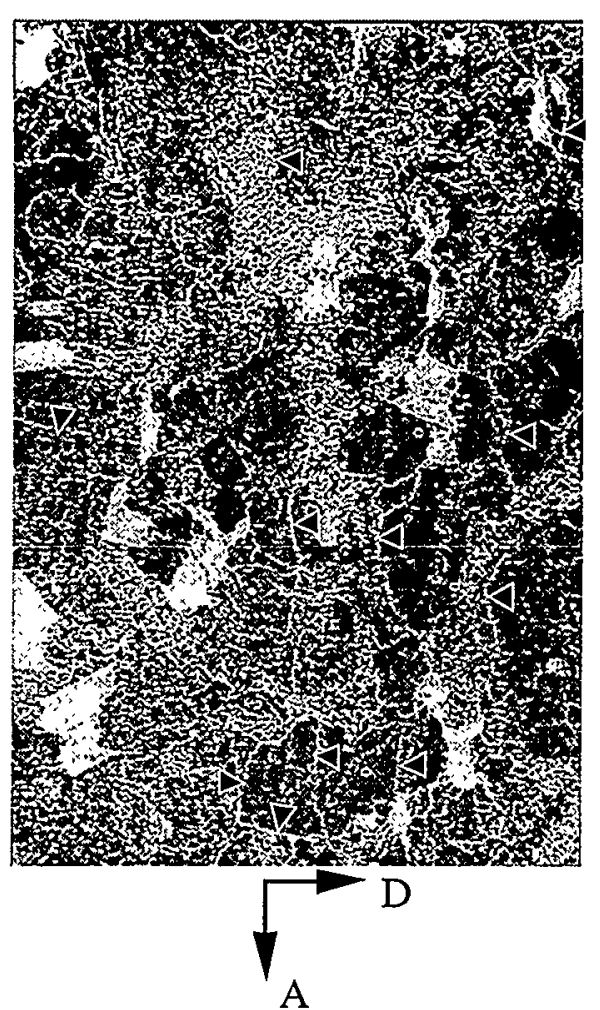

(b) core C

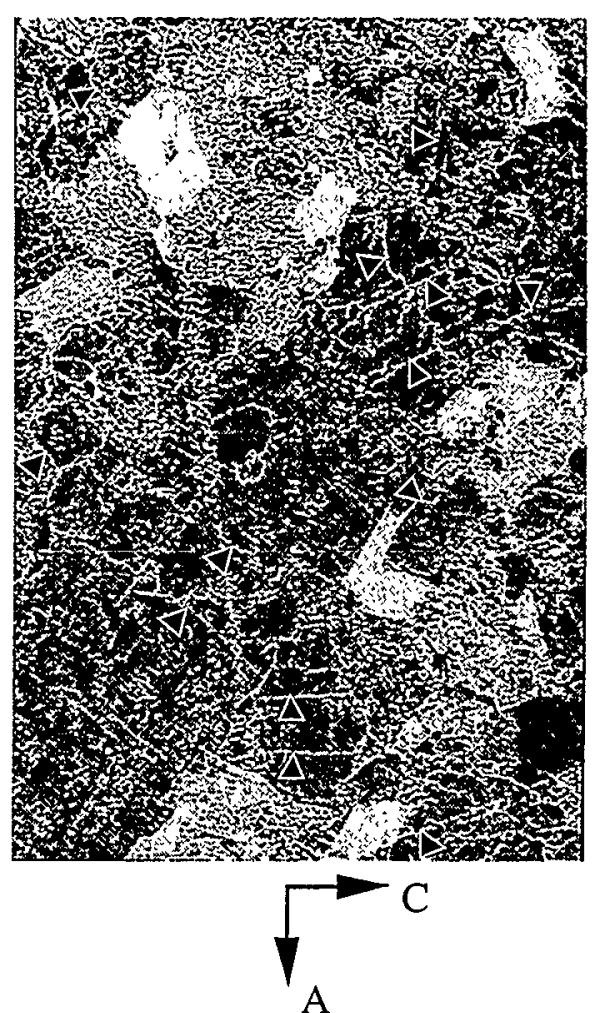

(c) core D

Figure 5.3 Thin section optical-micrographs of granite in three perpendicular directions. The color of the images is inverted to enhance the fractures. The bright white areas are the biotite grains. (a) Core $\mathrm{A}$ and (b) core $\mathrm{C}$ show microcracks approximately aligned in the direction perpendicular to the $\mathrm{D}$ direction (z-axis). (c) Microcracks in core $\mathrm{D}$ are more randomly oriented than the other directions. Core $\mathrm{C}$ exhibits epoxy-filled microcracks that are aligned parallel. 


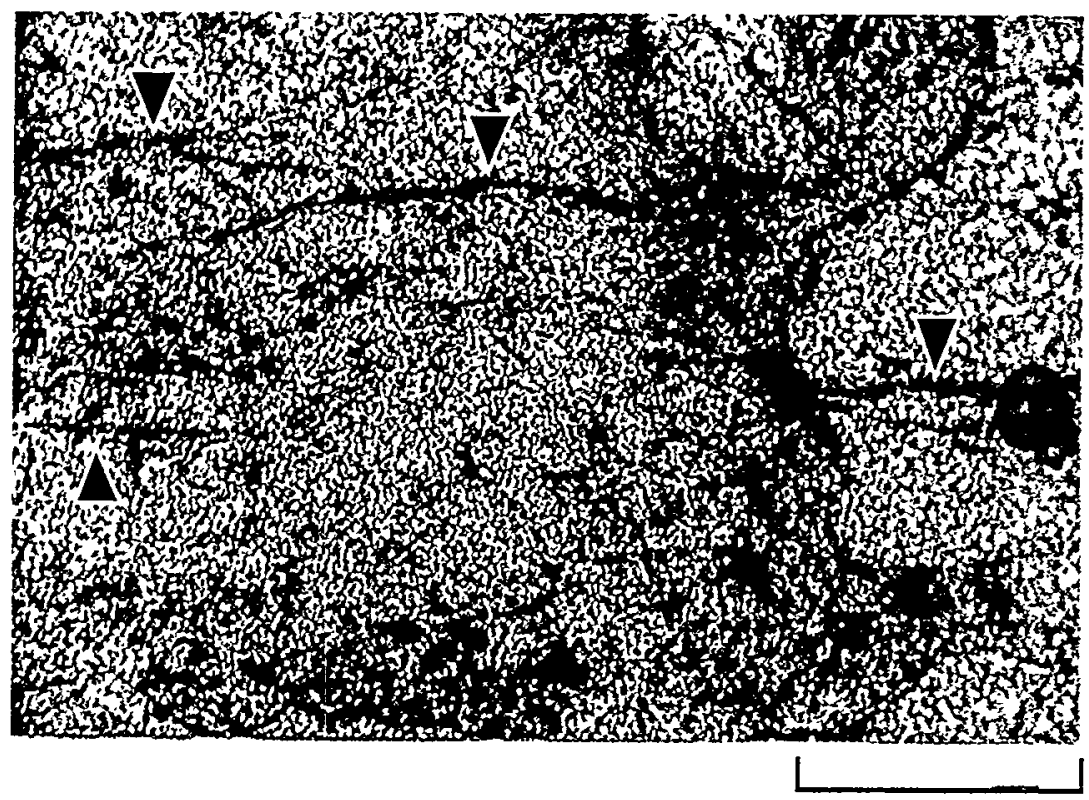

$0.25 \mathrm{~mm}$
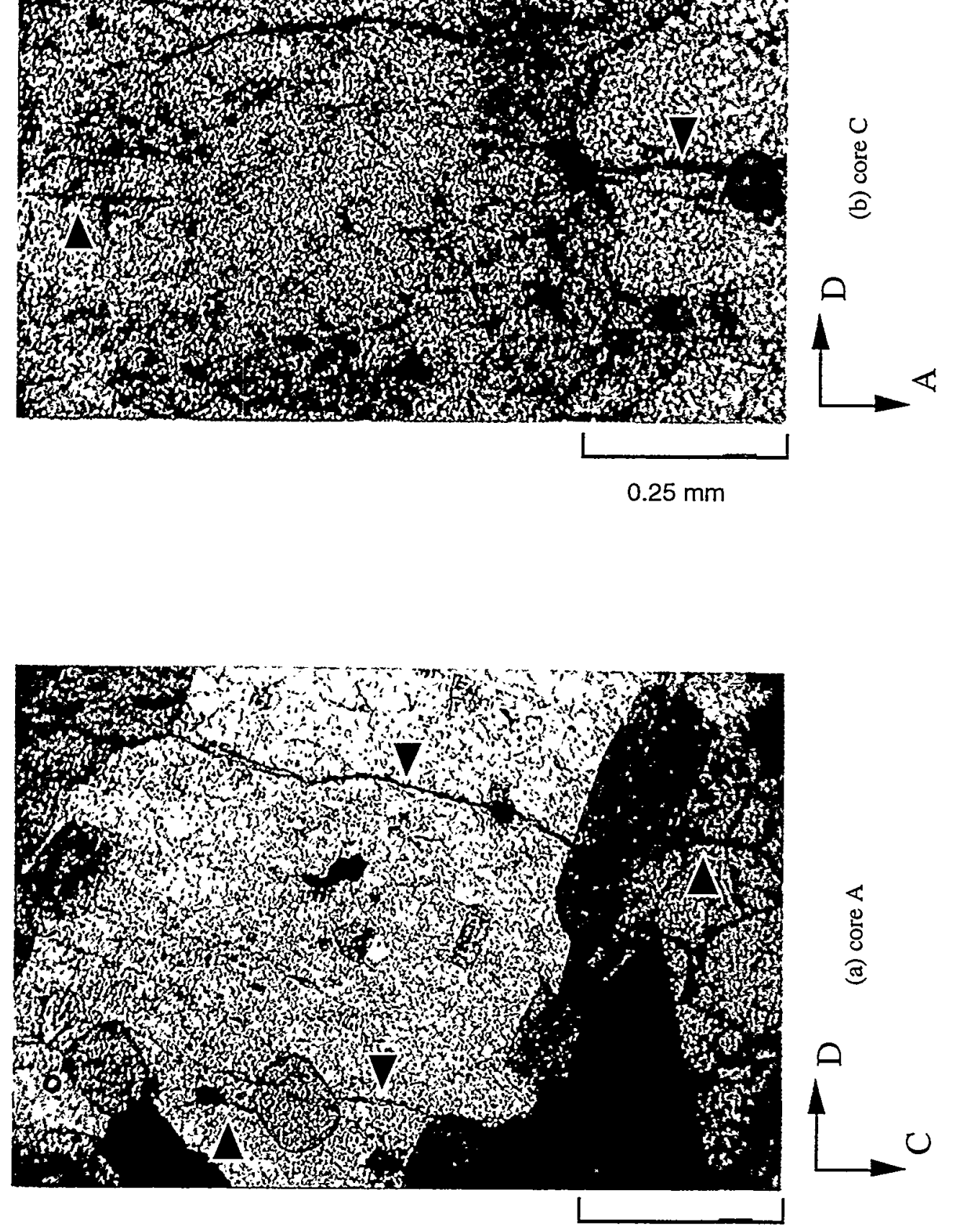

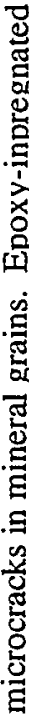

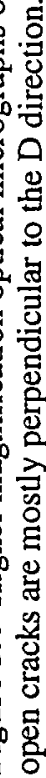

$0.5 \mathrm{~mm}$ 


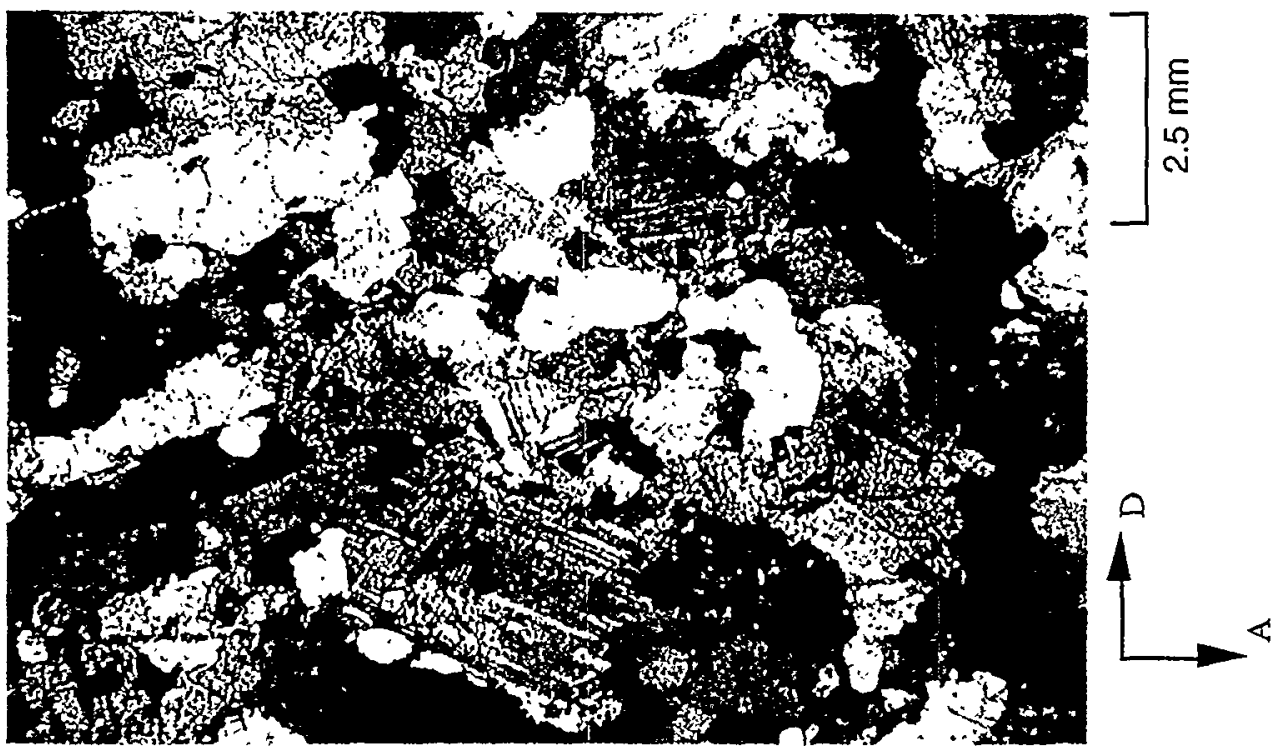

(a) Thin section micrograph of core $\mathrm{C}$ using polarized light.

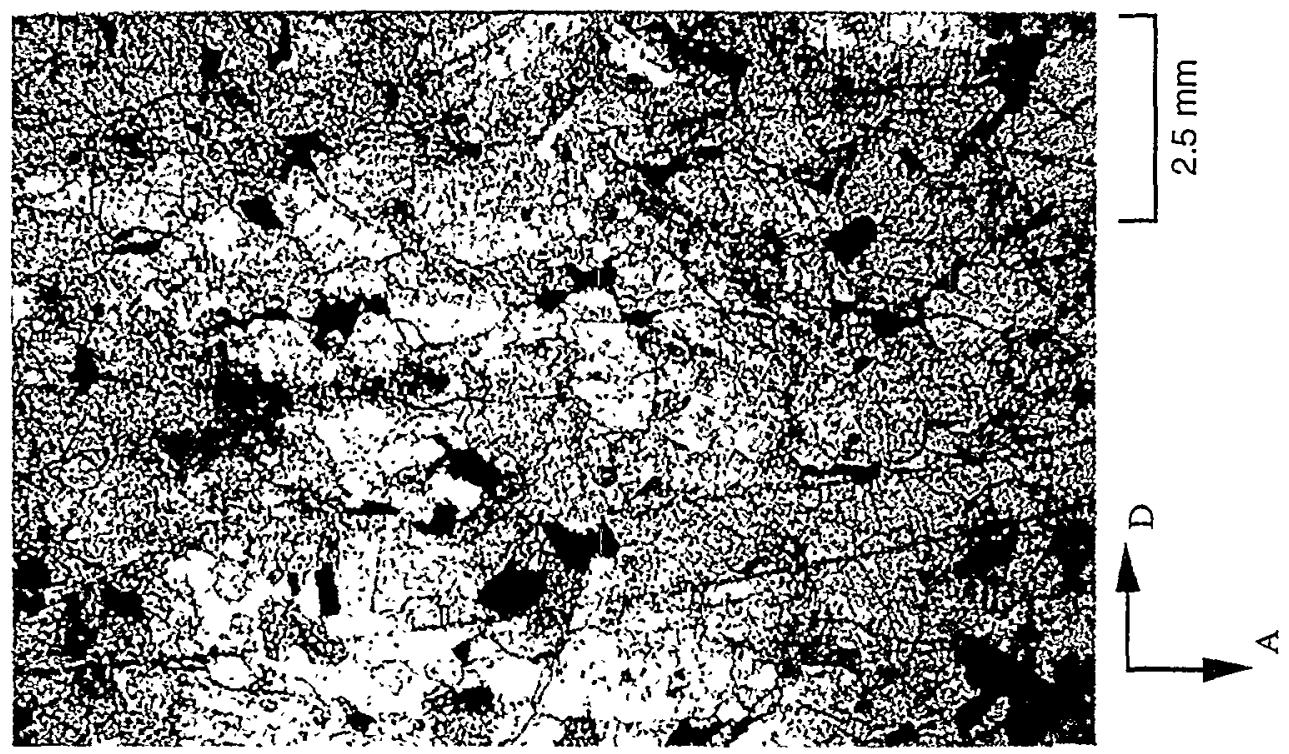

(b) Micrograph of similar area using non-polarized light (core C)

Figure 5.5 Shape and alignment of mineral grains in granite specimen. (a) Using polarized light, different types of minerals and their orientations can be distinguished. From the micrograph, no obvious elongation or alignment of crystals in any particular direction can be seen. (b) Úsing normal light, shapes and alignment of biotite grains (black regions) can be seen, which also show no systematic pattern that would lead to anisotropic behavior of the rock. 


\subsubsection{Capillary imbibition for granite}

In order to confirm that the microcracks in the granite were open, one-directional imbibition of water into the granite was performed using cylindrical specimens cored in mutually perpendicular directions. The specimens were $9.81 \mathrm{~cm}$ in height and $3.73 \mathrm{~cm}$ in diameter. The bottom of the specimens were cleaned prior to the experiment to expose fresh surfaces. After being ovendried for at least 48 hours at $103{ }^{\circ} \mathrm{C}$, the bottom of the specimens were immersed in distilled water up to $5 \mathrm{~mm}$ deep to allow the water to imbibe by the capillary force of the microcracks. The volume of water imbibed into the specimen was monitored by periodically measuring the total weight. During the test, the specimens were covered by glass jars to maintain the relative humidity of the air around the specimen at $100 \%$ (Figure 5.6). From the weight of oven-dried and vacuum saturated specimens, the effective porosity of the specimens was measured as $1.02 \%$.

Figure 5.7 shows the time history of the water-saturation resulting from imbibition into the specimens. From the figure, it can be seen that core $D$ initially shows a significantly slower rate of imbibition than the other specimens. Although the difference is subtle, core A exhibits a faster initial imbibition than core $\mathrm{C}$. For much later times, saturation ratios of all three cores asymptote to $80 \%$. The remaining $20 \%$ is due to the air trapped within the specimen.

The anisotropic rate of imbibition can be explained by the geometry and the alignment of microcracks in the granite (Figure 5.8) . Core D exhibits the slowest imbibition along the axis of the cylinder as the water has to flow through the most tortuous paths of connected microcracks. Directions along the axis of core $\mathrm{A}$ and $\mathrm{C}$ are the fastest imbibition directions as they have the least tortuousity. The small difference in imbibition rate for core $\mathrm{A}$ and $\mathrm{C}$ may be due to the larger misalignment of cracks in the $\mathrm{C}$ specimen. These results are consistent with the geometry and alignment of microcracks observed in the thin sections.

\subsubsection{Static behavior for granite}

Static uniaxial compression tests were conducted to examine the anisotropic elastic behavior of the granite core specimens. The specimens used for the static tests were identical to the ones used for the capillary imbibition tests (cores A, C, and D). The cores were tested under room-dry conditions (density of $2.62 \mathrm{~g} / \mathrm{cm}^{3}$ ). The experimental setup is 


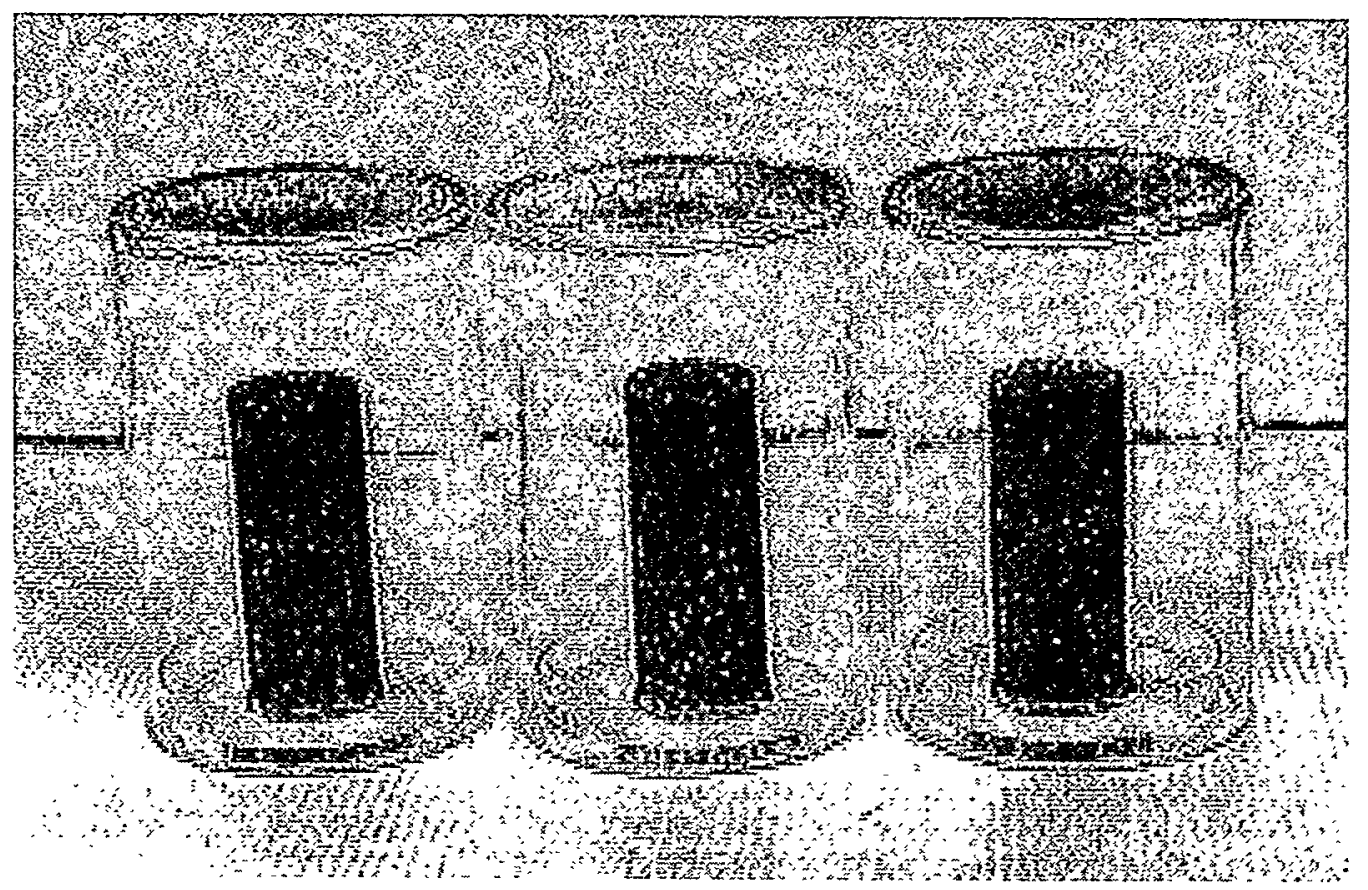

(a) Capillary imbibition test setup

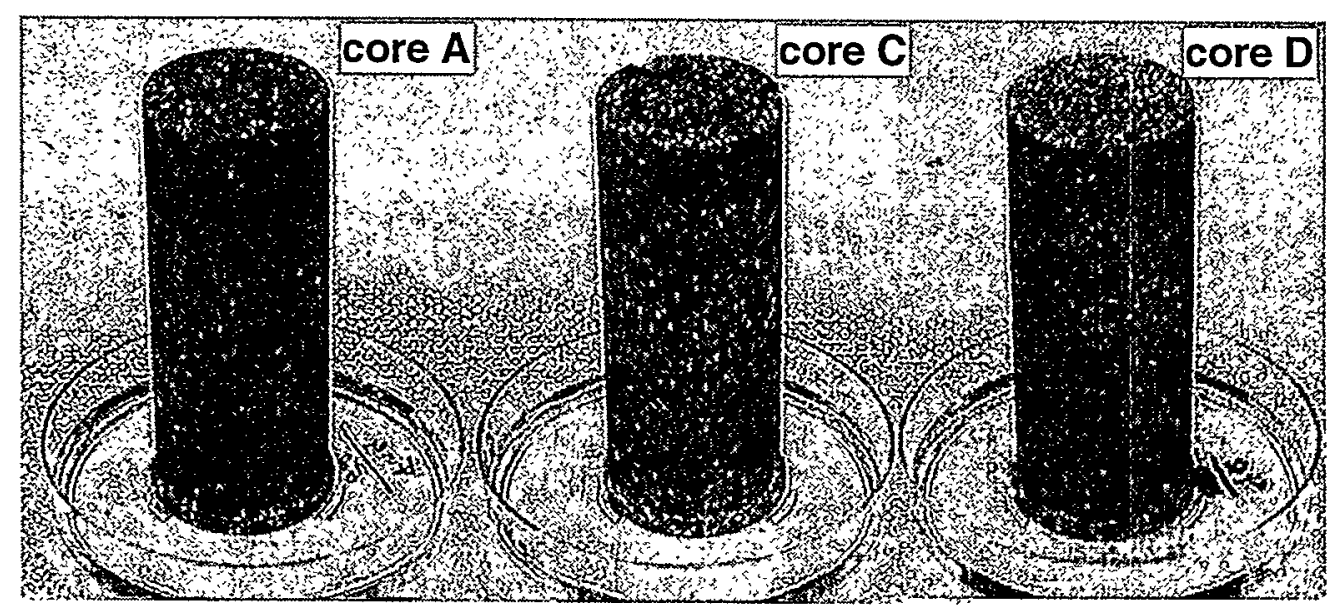

(b) Condition of specimens 50 hours after starting imbibition

Figure 5.6 Oven-dried core A, B, and C were placed in glass jars with their bases immersed in distilled water. Changes in their weight were measured as the water imbibed into the specimens. The bottom picture (Figure 5.6(b)) shows the specimens after 50 hours of imbibition. Water has reached the top of the specimens $A$ and $C$, but not core $D$. 


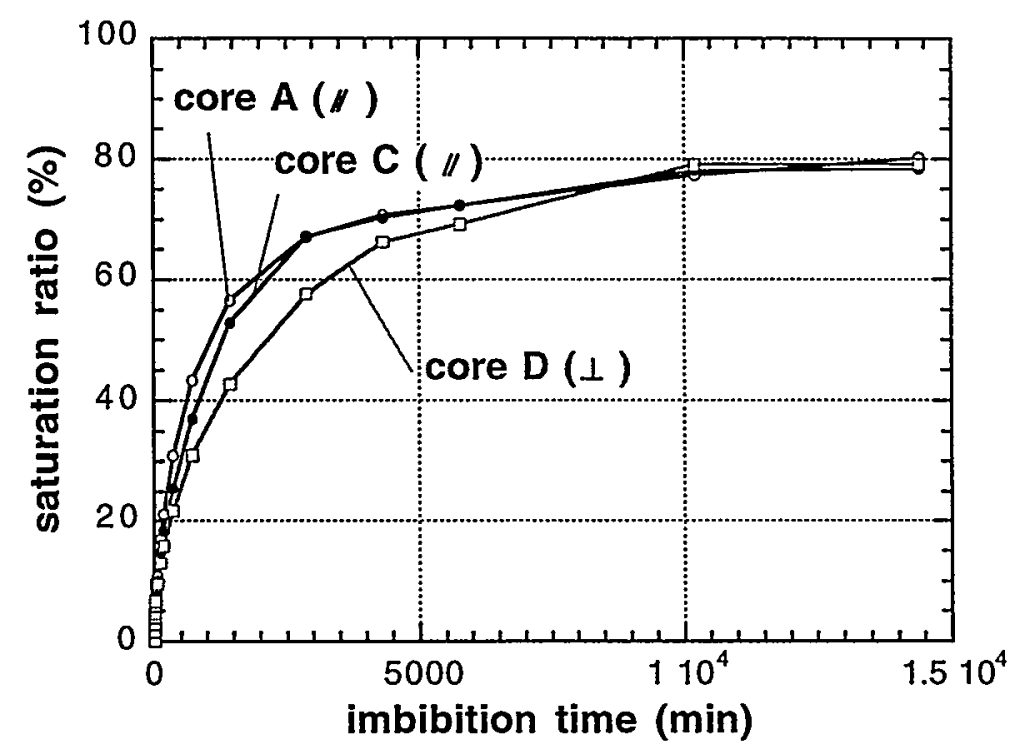

Figure 5.7 Time history of capillary imbibition into granite cores. All the cores are of equal size and shape. Core D exhibits much slower increase in the saturation ratio compared with the other two cores, indicating that the primary orientation of microcracks is not aligned with the core axis. Core A shows slightly faster imbibition than core C. Saturation ratios for the cores asymptote to approximately $80 \%$ after 10 days.

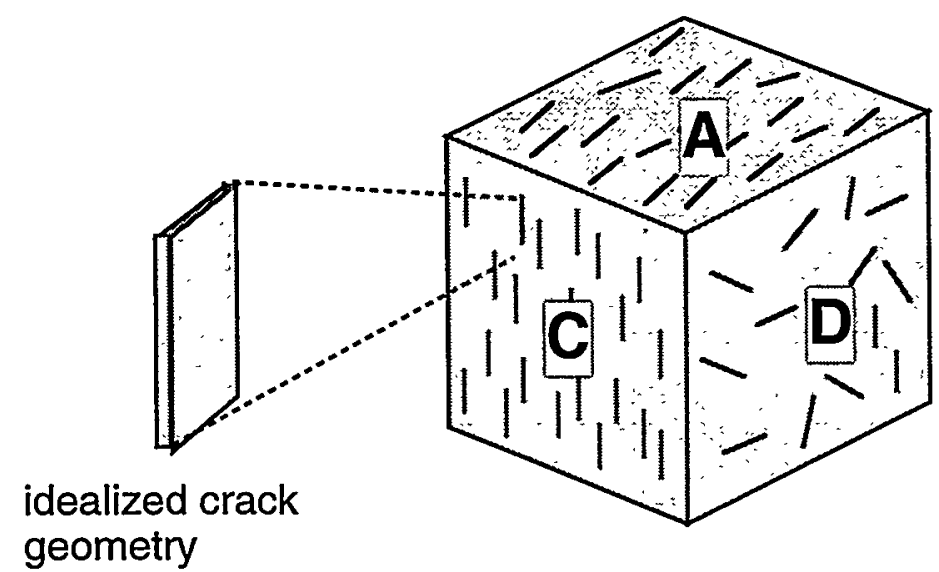

Figure 5.8 Idealized microcrack geometry and orientations from microscope observation and capillary imbibition tests. Open microcracks in the granite specimen are preferentially aligned perpendicular to the $D$ direction. Larger variations in the spatial distribution of the microcracks in the A surface of the block may be the reason for the slightly faster imbibition in the A direction than in the $\mathrm{C}$ direction. 
shown in Figure 5.9. A core specimen was uniaxially loaded in a load frame using a handoperated hydraulic jack. Both vertical and horizontal LVDT's were mounted on the specimen to measure vertical compression and lateral expansion of the specimen. The applied load was measured using a loadcell. Readouts from LVDT's and the loadcell were displayed and stored on a computer.

The static stress-strain relation of the rock also displays strong anisotropy. Figure 5.10(a) shows the axial stress-strain relations for three mutual perpendicular directions. Core D (z-axis direction) exhibits a significantly larger compliance than the other two directions. Small excursion loops from the main stress-strain loops are measured to obtain the elastic moduli of the rock free of frictional slip (e.g., Walsh, 1965; Cook and Hodgson, 1965). Figure 5.11(b) shows the relations between axial strain and radial strain. The direction of the measured expansion is $90^{\circ}$ from a reference direction (for core $\mathrm{A}$ and $\mathrm{C}$, in the direction parallel to the average microcrack planes; for core $\mathrm{D}$, in the direction parallel to core A). All specimens show very small Poisson's expansion for small axial load. This is due to the closing of microcracks oriented in the direction perpendicular to the loading direction. Core $\mathrm{D}$ shows the smallest radial strain versus axial strain ratio due to a large number of microcracks preferentially oriented perpendicular to the specimen axis. The difference between the Poisson's ratios for the specimens becomes small for larger axial stresses. As the hysteresis of the major loops is small, the slope of the curves is assumed to be identical to the slope of the small excursion loops that provides the elastic Poisson's ratio of the specimens.

Variations in Poisson's ratio around the axis of each specimen are shown in Figure 5.11(a) (c). For core A (Figure 5.11(a)), basic agreement between the Poisson's expansions for the $0^{\circ}$ and $90^{\circ}$ can be seen on the loading part of the curves. The unloading curve for $0^{\circ}$ shows a large amount of slip between the specimen and radial LVDT mount. In the Figure 5.11(b), core $\mathrm{C}$ exhibits similar behavior of Poisson's ratio for all directions around the axis. Radial strains in different directions are shifted for comparison. Unlike the other directions, core $\mathrm{D}$ exhibits small variations in the behavior of Poisson's expansion around its axis. This indicate that the behavior of the granite is not truly transversely anisotropic. Poisson's ratio is the smallest in the directions parallel to core $\mathrm{C}\left(90^{\circ}\right.$ and $270^{\circ}$, along y-axis). Although the difference in Poisson's ratio is large for large axial strains, all curves seem to have similar slopes for very small axial strain. Therefore, the granite is assumed to be transversely isotropic in the analysis of elastic moduli at zero confining stress presented in Sections 5.3 and 5.4. 


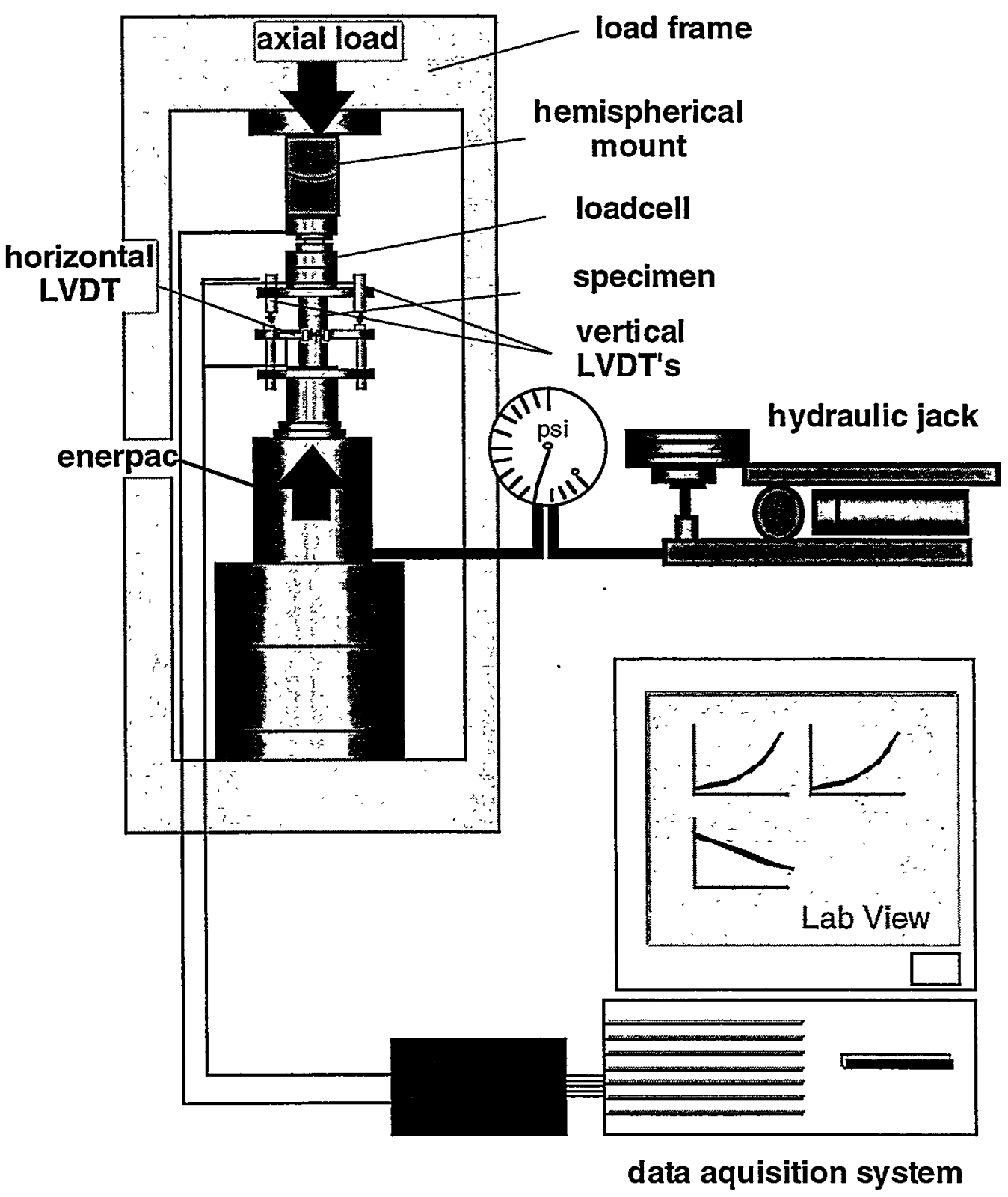

Figure 5.9 Experimental setup for static load-displacement test. Axial load is applied by a hydraulic jack. Both vertical displacement and lateral expansion are measured using LVDT's. Measured displacements are displayed and recorded on a data acquisition system on a computer running LabView software (National Instruments, Inc.). 


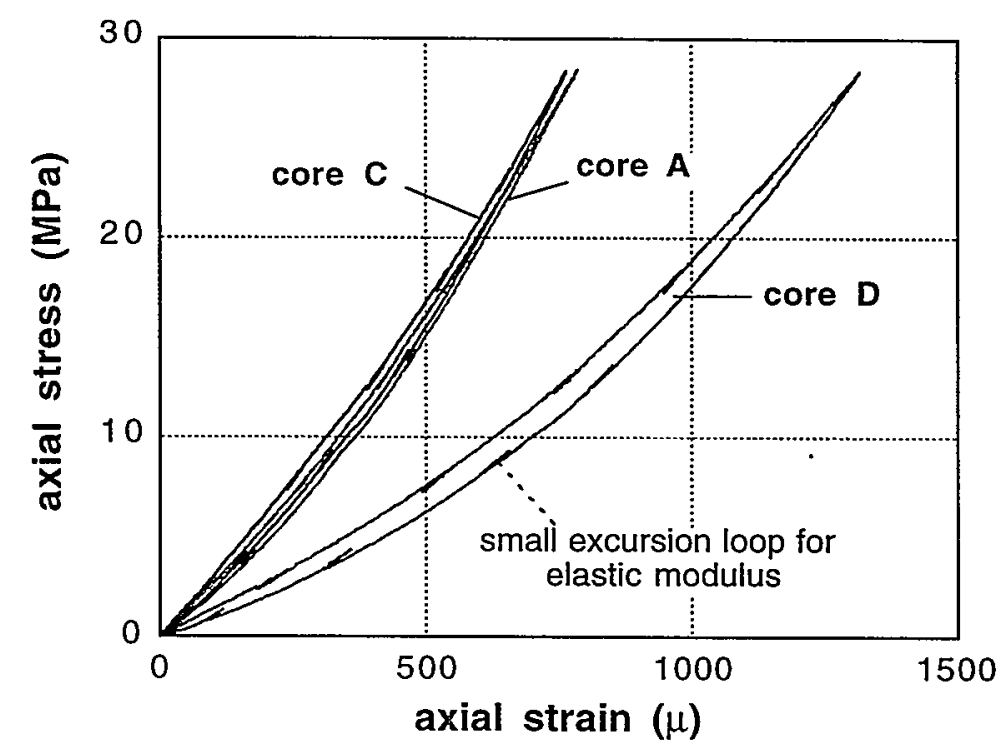

(a) Axial stress vs axial strain

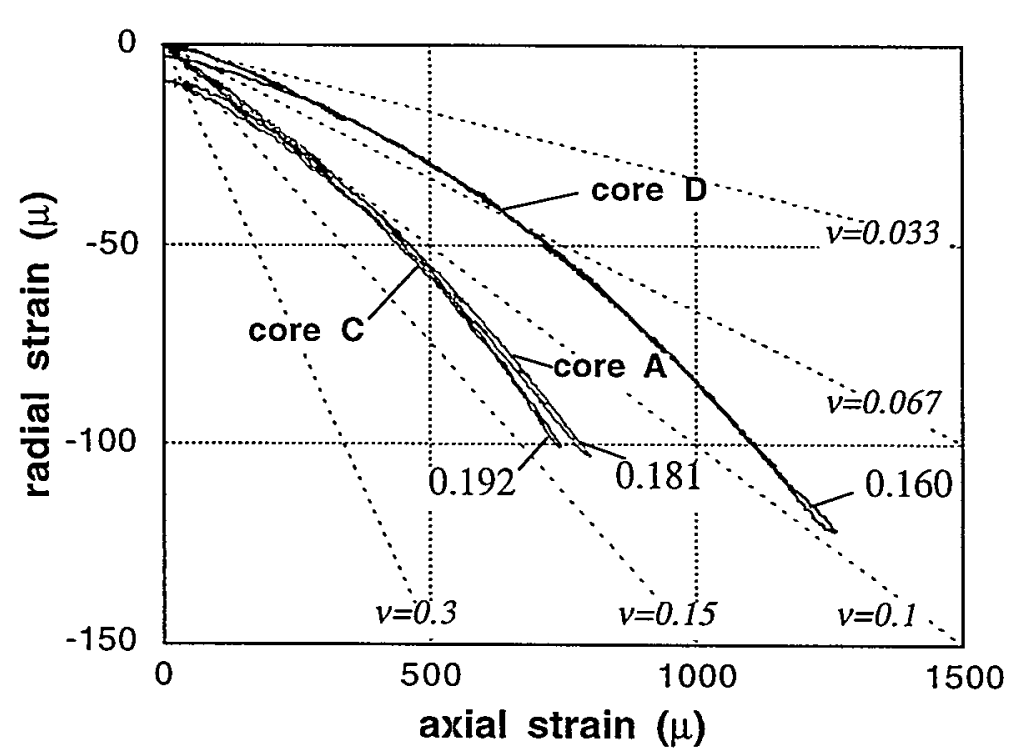

(b) Radial strain vs axial strain

Figure 5.10 Uniaxial compression tests on granite specimens cored in three mutually perpendicular directions. (a) core $\mathrm{D}$ shows much larger compliance than the other two cores due to closure of aligned microcracks. (b) Poisson's expansion was measured in the direction $90^{\circ}$ from the reference direction (direction parallel to the plane of anisotropy for core A and C, direction parallel to core A for core $\mathrm{D}$, see Figure 5.2). Permanent offset at zero axial strain is due to the slip between specimen and LVDT mount. Initial Poisson's ratio for the specimens are quite small $(V=0.033 \sim 0.066)$ due to the closing of microcracks. Young's moduli and Poisson's ratios for different specimens increase and become similar for high axial stresses. 


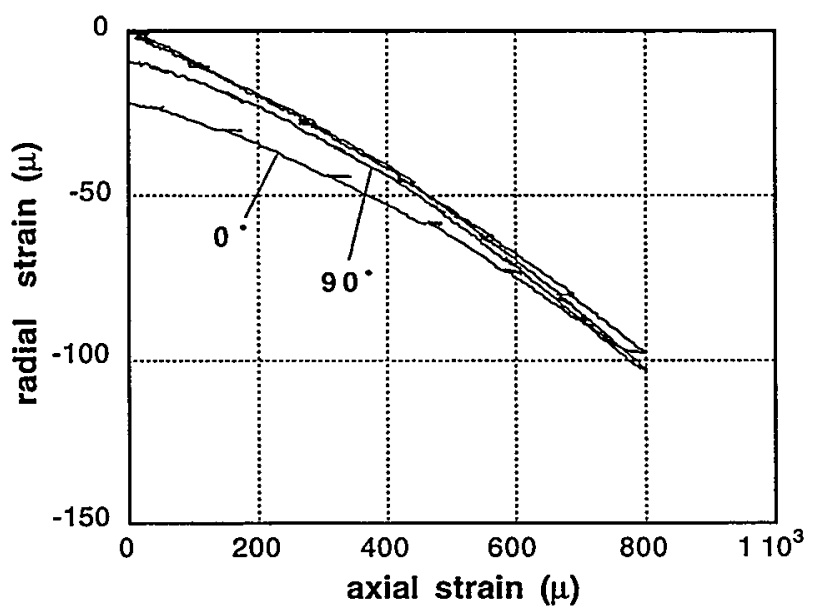

(a) Poisson's expansion of A core.

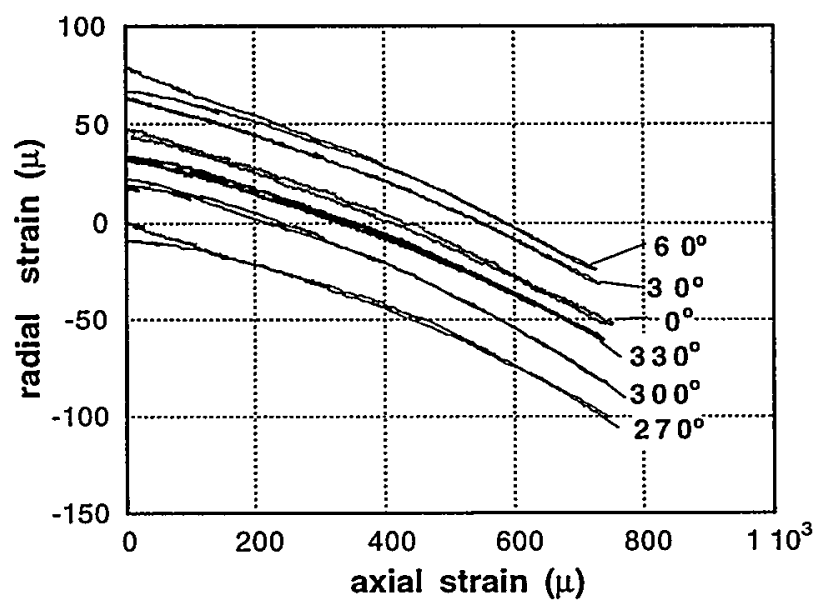

(b) Poisson's expansion of $\mathrm{C}$ core.

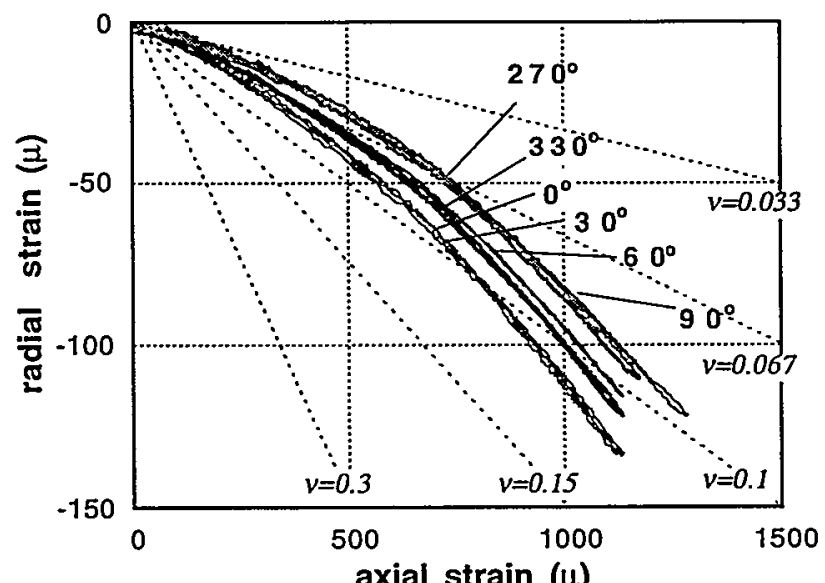

(c) Poisson's expansion of $\mathrm{D}$ core.

Figure 5.11 Poisson's expansion of specimens around the core axis. For core A, unloading curve for $0^{\circ}$ orientation deviates from the $90^{\circ}$ orientation due to large slip between specimen and the LVDT holder. Core $\mathrm{A}$ and $\mathrm{C}$ do not show significant difference in Poisson's expansions for different orientations. Core $\mathrm{D}$, however, exhibits small variations in Poisson's expansion, showing maximum expansion in the $0^{\circ}$ direction. 
Young's moduli of the specimens determined from small excursion loops in Figure 5.10(a) are shown in Figure 5.12. Due to the frictional slip in the rock and the limited resolution of the data, the accuracy of the determined Young's modulus and Poisson's ratio at low stresses is rather poor. By extrapolating the obtained modulus-stress relations, the Young's modulus at zero axial stress is estimated to be $19 \mathrm{GPa}$ for core $\mathrm{D}\left(\mathrm{E}_{3}\right)$ and $30 \mathrm{GPa}$ for core $A$ and $C\left(E_{1}=E_{2}\right)$. From the plot, Poisson's ratios are approximately 0.099 for core $A$ and $C\left(v_{12}=v_{13}=v_{21}=v_{23}\right)$ and 0.033 for $D$ core $\left(v_{31}=v_{32}\right)$. The upper left of the elastic compliance matrix for the measured elastic parameters are

$$
\left\{\begin{array}{l}
\varepsilon_{11} \\
\varepsilon_{22} \\
\varepsilon_{33}
\end{array}\right\}=\left[\begin{array}{ccc}
\frac{1}{E_{1}} & -\frac{v_{21}}{E_{2}} & -\frac{v_{31}}{E_{3}} \\
-\frac{v_{12}}{E_{1}} & \frac{1}{E_{2}} & -\frac{v_{32}}{E_{3}} \\
-\frac{v_{13}}{E_{1}} & -\frac{v_{23}}{E_{2}} & \frac{1}{E_{3}}
\end{array}\right]\left\{\begin{array}{l}
\sigma_{11} \\
\sigma_{22} \\
\sigma_{33}
\end{array}\right\}=\left[\begin{array}{ccc}
0.0333 & -0.00333 & -0.00174 \\
-0.00333 & 0.0333 & -0.00174 \\
-0.00333 & -0.00333 & 0.0526
\end{array}\right]\left\{\begin{array}{l}
\sigma_{11} \\
\sigma_{22} \\
\sigma_{33}
\end{array}\right\} \times 10^{-9}
$$

The above compliance matrix $S$ is not symmetric, possibly due to the experimental error and data resolution. Assuming the error is caused by the measurements for the Poissons ratios, the compliance matrix is redefined by

$$
\bar{S}=\frac{S+S^{T}}{2},
$$

where the superscript ${ }^{T}$ is a matrix transpose operator. The stiffness matrix is obtained as

$$
\begin{aligned}
C & =\bar{S}^{-1}=\left[\begin{array}{ccc}
0.0333 & -0.00333 & -0.00252 \\
-0.00333 & 0.0333 & -0.00252 \\
-0.00252 & -0.00252 & 0.0526
\end{array}\right]^{-1} \times 10^{9} \\
& =\left[\begin{array}{ccc}
30.4 & 3.16 & 1.61 \\
3.16 & 30.4 & 1.61 \\
1.61 & 1.61 & 19.2
\end{array}\right] \times 10^{9}[\mathrm{~Pa}]
\end{aligned}
$$




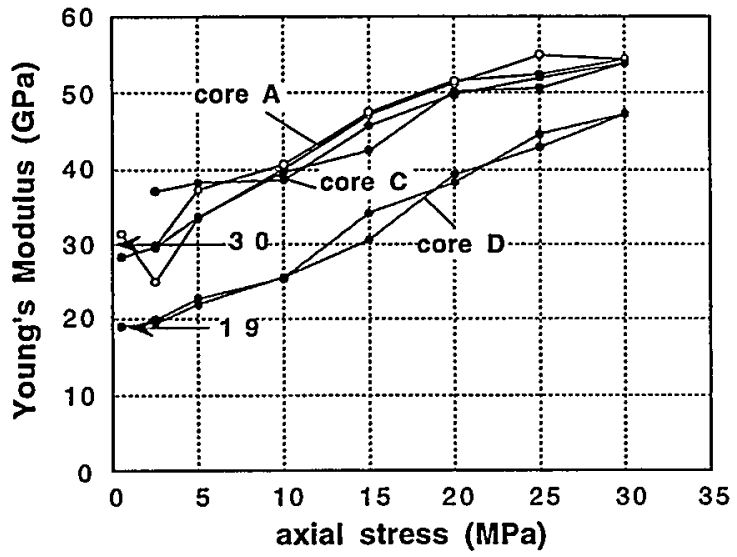

(a) Young's Modulus

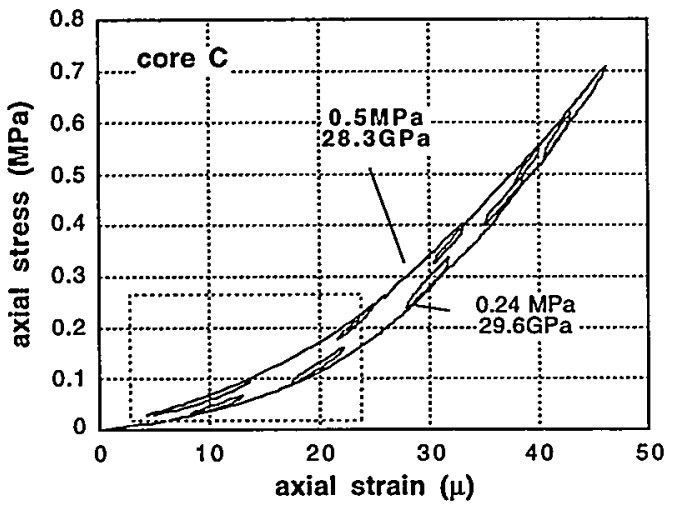

(c) core $\mathrm{C}$ (small stress)

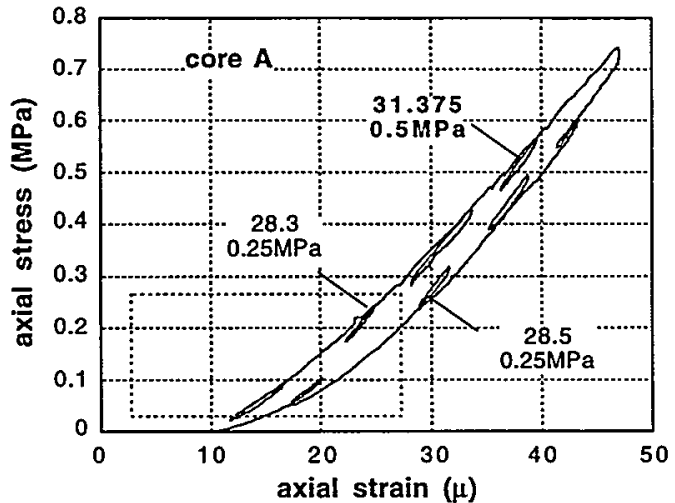

(b) core A (small stress)

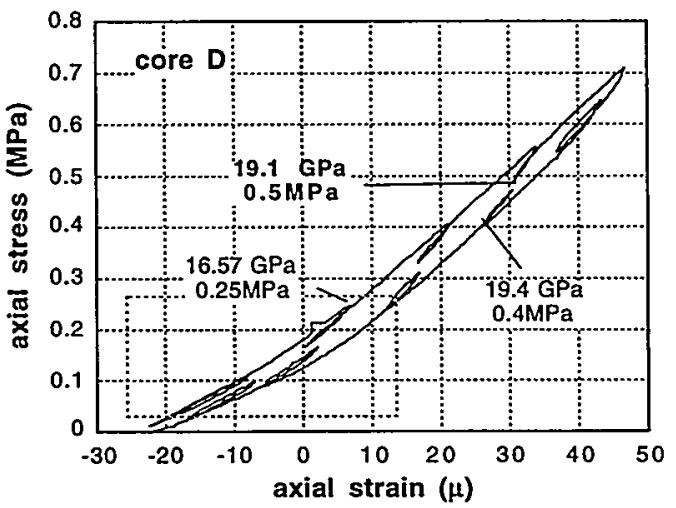

(d) core D (small stress)

Figure 5.12 (a) Young's modulus determined from small excursion loops in Figure 5.10(a) and ((b)-(d)) low-axial stress load-displacement tests. Young's moduli at zero axial stress are estimated as $30 \mathrm{GPa}$ and 19 $\mathrm{GPa}$ for directions parallel and perpendicular to the microcracks, respectively. The square regions in the above plots show the initial nonlinear behaviors due to compliant contact between specimen and loading plates. Young's modulus for all three directions increases with increasing axial stress. Difference between directions parallel to the microcracks (core A and C) and perpendicular to the cracks (core D) gradually decreases as the stress increases. 


\subsection{Ultrasonic Transmission Test}

\subsubsection{P-wave anisotropy for granite}

To check for the anisotropy of transmitted P-waves, the granite cores were vacuum saturated and placed in a water bath (Figure 5.13(a)). The water bath has a turn table at the bottom and a pair of immersion ultrasonic transducers (central frequency of $1 \mathrm{MHz}$ ) attached to the side walls. The P-wave anisotropy of the specimen can be examined by rotating the table and measuring transmitted $\mathrm{P}$-waves as a function of angle (Figure 5.14). Both cores $\mathrm{A}$ and $\mathrm{C}$ show peaks in velocity and amplitude in the direction parallel to the microcracks while core D shows consistently low velocity and amplitude for all directions.

\subsubsection{S-wave anisotropy}

S-wave anisotropy was measured on the granite cores and the cube-shaped sandstone and dolomite specimens using the experimental setup shown in Figure 5.13(b). The specimens were tested under room dry conditions. To establish repeatable acoustic coupling between the specimen and the ultrasonic transducers, the specimen was loaded between the transducers through thin lead foil disks in a load frame (5MPa for granite, 6.9MPa for sandstone, and 5.9MPa for dolomite). The measured S-wave waveforms are shown in Figure 5.15. The granite cores show two distinct quasi-shear waves that result from shear-wave splitting. For the direction of wave propagation parallel to the plane of anisotropy (labeled as ' $/ /$ '), components of an S-wave polarized parallel to the microcracks (labeled " $\mathrm{H}^{\prime}$ ) propagate faster than the components polarized perpendicular to the microcracks (labeled "V"). In the direction perpendicular to the plane of anisotropy (labeled " $\perp$ "), the velocity and amplitude of the S-wave are constant. The sandstone specimen shows similar splitting of $\mathrm{S}$-waves but the splitting is rather small indicating a smaller degree of anisotropy. Although the amplitudes of the S-waves for the dolomite cube show similar patterns as other specimens, the shear wave splitting was not observed.

\subsubsection{Velocity anisotropy and axial load}

Changes in wave velocities with increasing axial load are shown in Figure 5.16 for Pwaves and in Figure 5.17 for S-waves. For granite and sandstone, both P and S-waves initially show a rapid increase in velocity with increasing load. This is due to closing 


\section{vertical load}

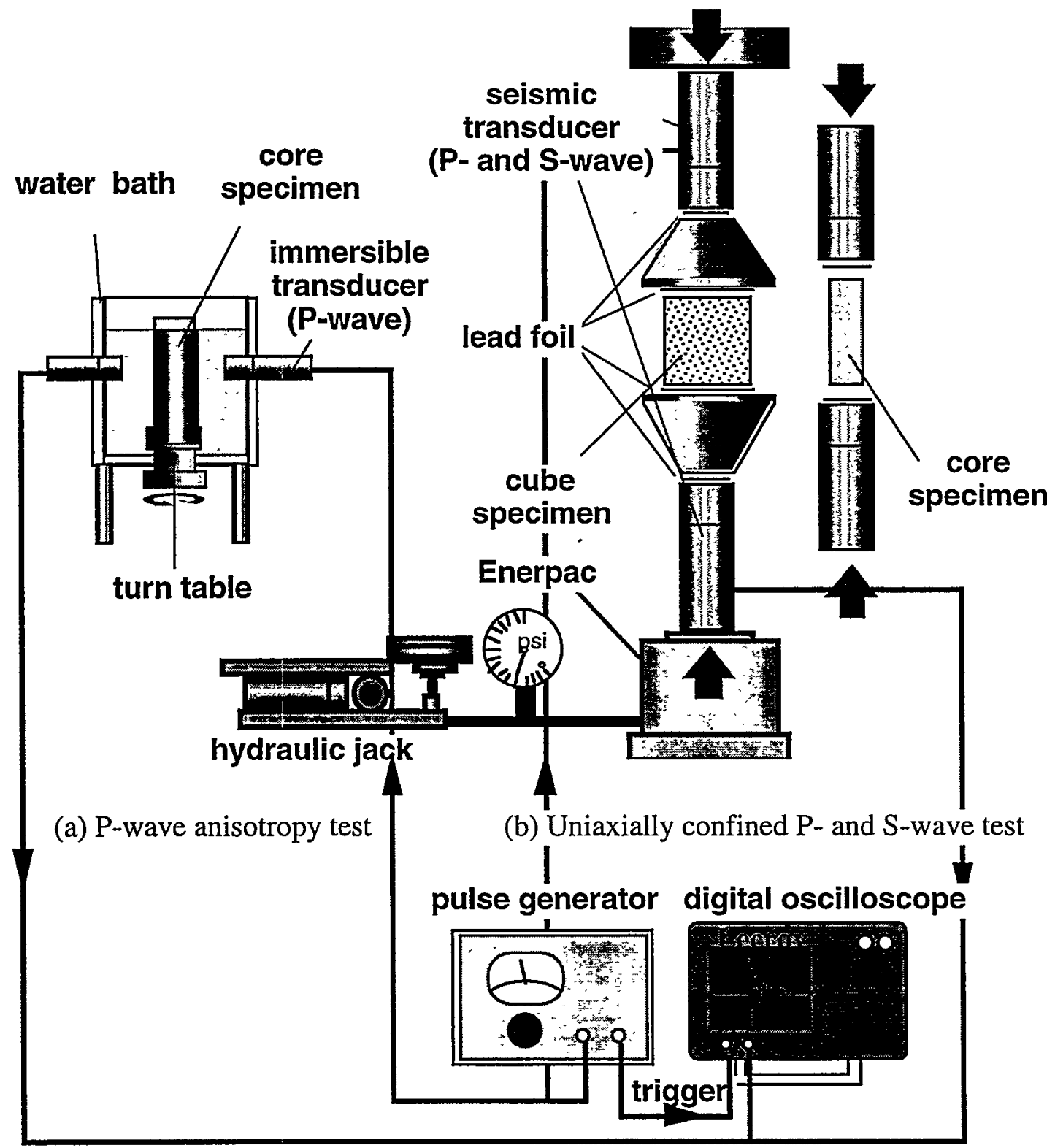

Figure 5.13 Experimental setup for ultrasonic wave transmission tests. (a) For granite core specimens, anisotropy of $\mathrm{P}$-wave transmission was measured using immersible transducers (central frequency $=1 \mathrm{MHz}$ ). The specimen was rotated around its axis with a turn table attached to a water bath. (b) The granite core specimens and sandstone and dolomite cubes were uniaxially loaded to measure S-wave anisotropy and stress dependency of $\mathrm{P}$ and $\mathrm{S}$-waves. For cube specimens, aluminum cones were used to apply a uniformly distributed load to the specimens. Piezoelectric crystals in the transducers were excited by a high-voltage pulse generator and measured waves were displayed and stored on an oscilloscope. 

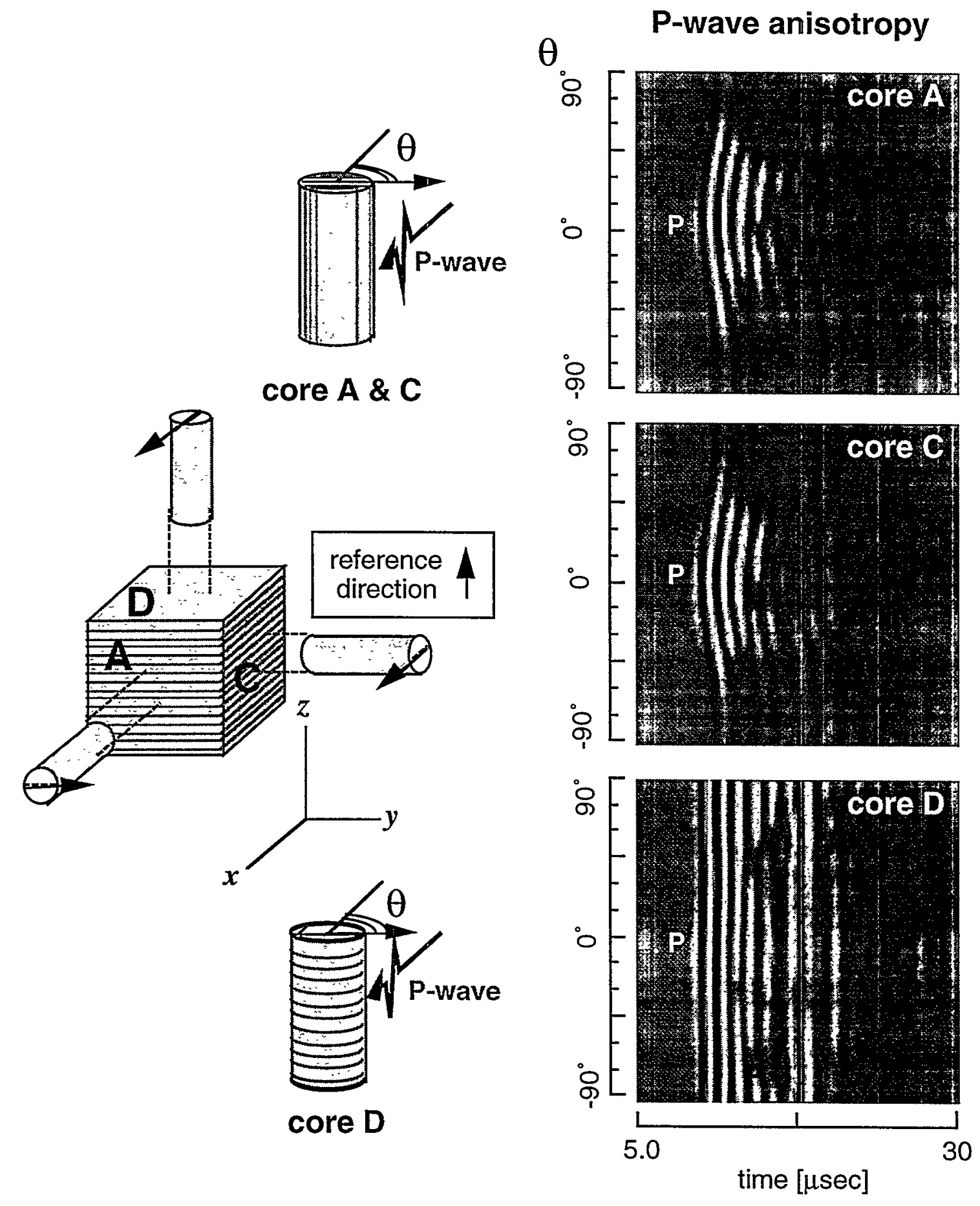

Figure 5.14 P-wave anisotropy of granite core specimens (vacuum saturated with water). A waveform was measured at every 15 degrees. Angles in the plots are measured from a reference direction of each specimen. Large positive and negative amplitude is shown in light and dark grays, respectively. P-wave shows maximum amplitude and velocity in the direction perpendicular to the isotropy axis (perpendicular to microcrack planes). 


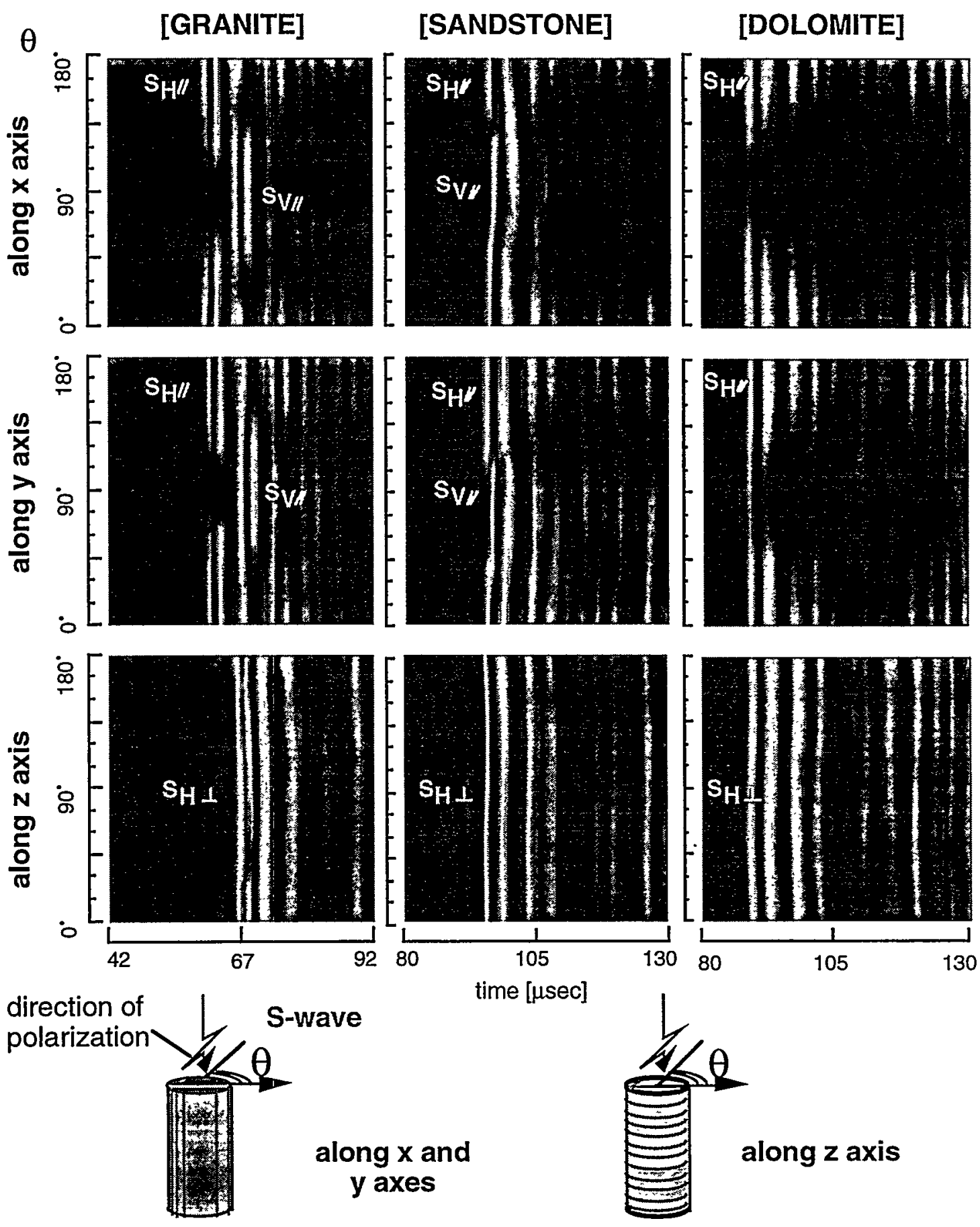

Figure 5.15 S-wave anisotropy of granite core specimens and sandstone and dolomite cubes (room-dry). The polarization direction of source and receiver were rotated around each coordinate axis and transmitted Swave was measured for every 15 degrees of rotation. For granite and sandstone, shear wave splitting due to transverse isotropy of the rock is observed (SH and SV-waves). Although it shows clear S-wave amplitude anisotropy, the dolomite specimen does not exhibit splitting of shear waves. 


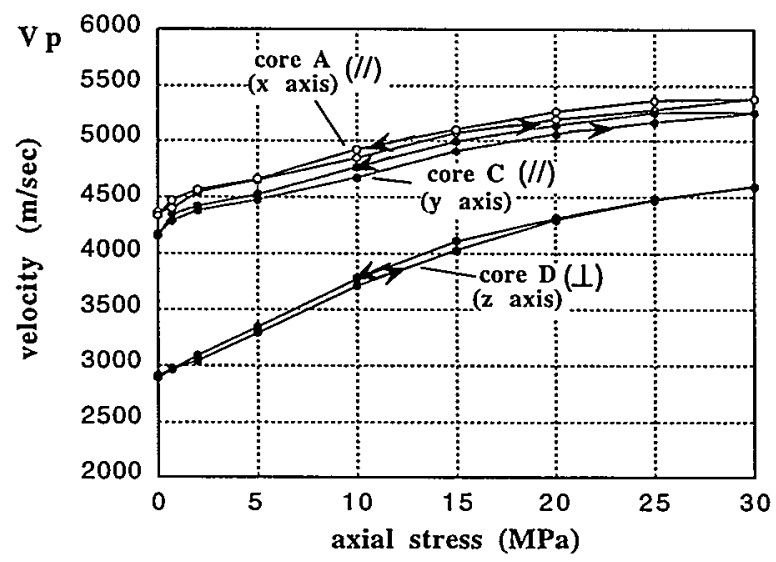

(a) P-wave velocities for granite core specimen

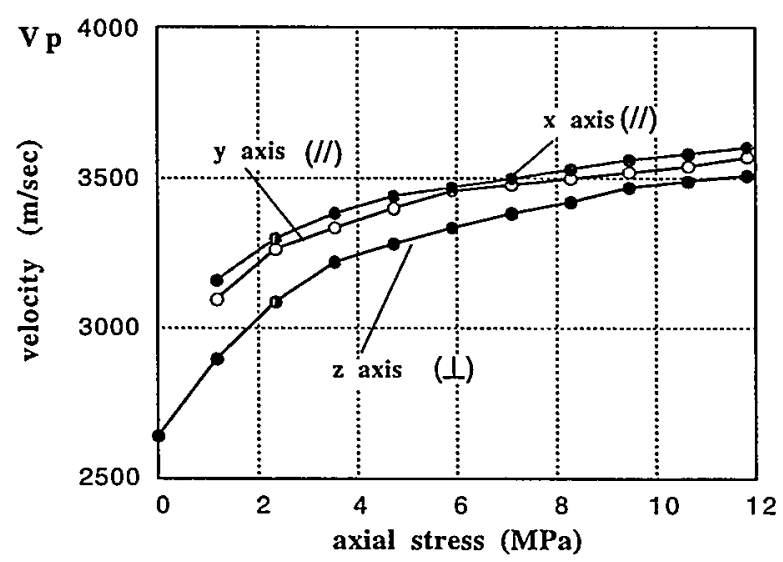

(b) P-wave velocities for sandstone cube specimen

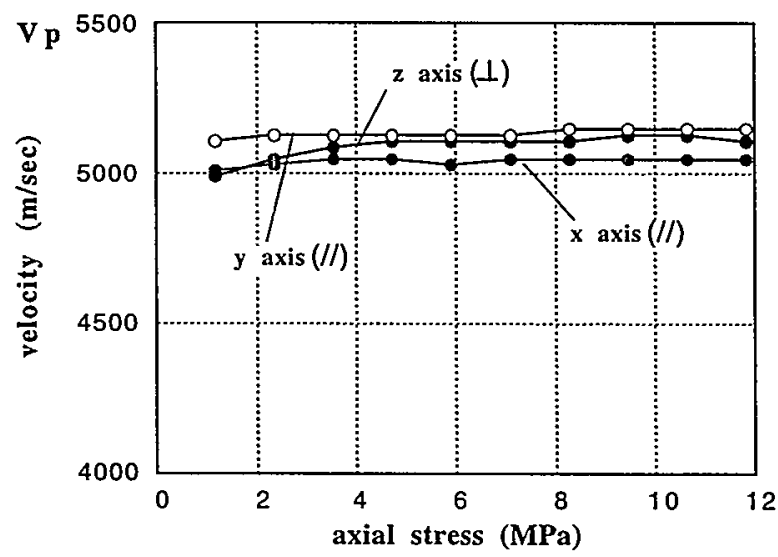

(c) P-wave velocities for dolomite cube specimen

Figure 5.16 Stress dependence and anisotropy of P-wave velocity. (a) Granite shows strong stress dependence due to microcracks that close under uniaxial stress. (b) Sandstone also shows strong stress dependence due to improved grain contact by the axial load. For both specimens, a P-wave parallel to the isotropy axis shows the smallest velocity. (c) For the axial loads shown above, the dolomite specimen exhibits very small stress dependence possibly because the open cracks are filled with precipitated mineral. The velocity anisotropy is also very small. 

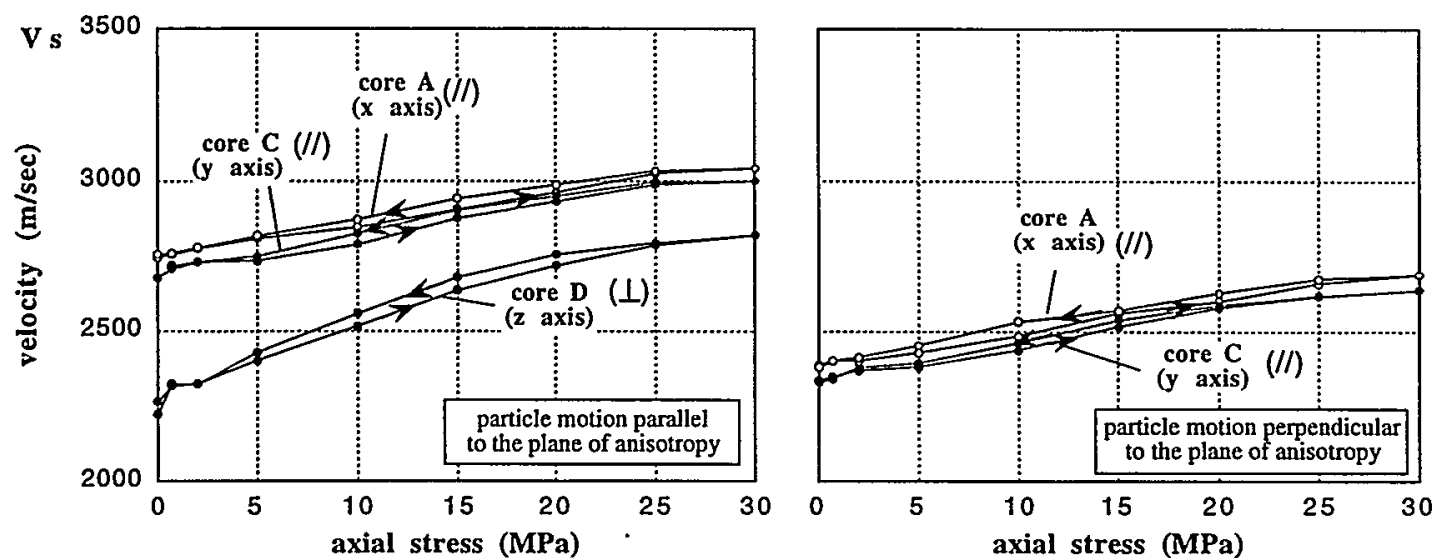

(a) S-wave velocities for granite core specimen
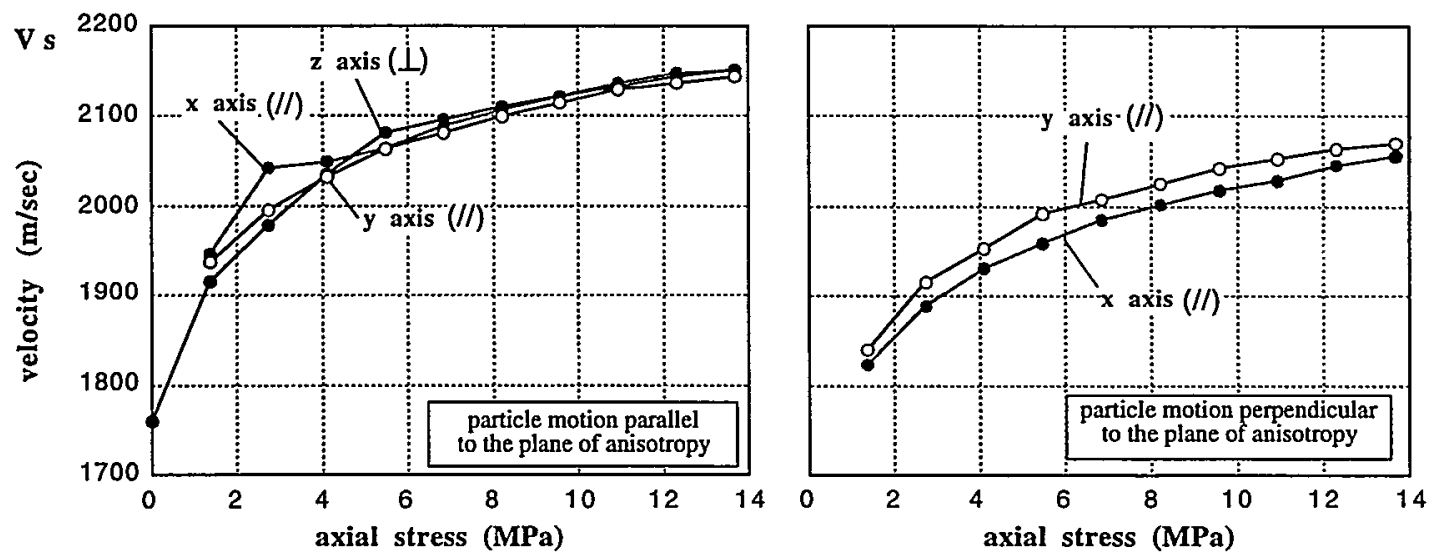

(b) S-wave velocities for sandstone cube specimen
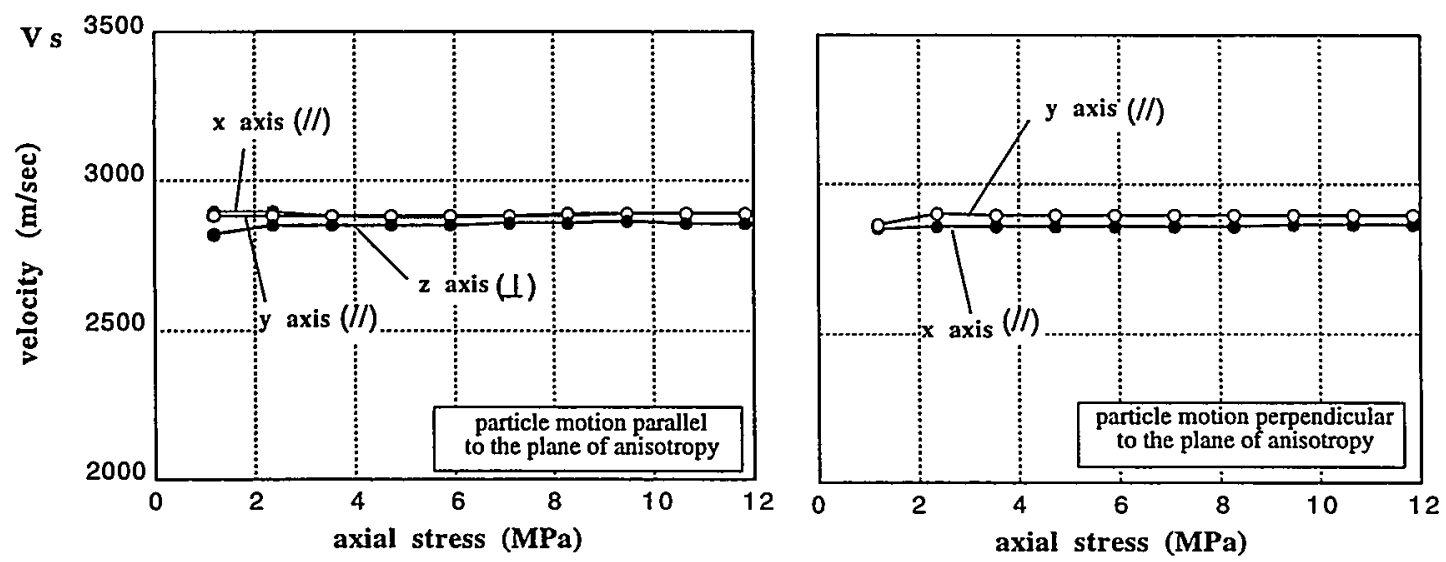

(c) S-wave velocities for dolomite cube specimen

Figure 5.17 Stress dependence and anisotropy of S-wave velocity. Granite (a) and sandstone (b) specimens show similar stress dependence of S-waves as P-waves. Similar to P-wave velocity, dolomite (c) specimen shows very small stress dependence and velocity anisotropy. 
microcracks for the granite and the stiffening of grain contacts for the sandstone. In contrast, the dolomite shows a very small stress dependence and a small velocity anisotropy. The behavior of the waves in the dolomite specimen may be caused by scattering of waves off the thin mineral inclusions that fill the open flat fractures. Here, the acoustic impedance contrast between the matrix and the inclusions causes amplitude anisotropy of the waves by scattering, but because they are thin relative to the wavelength, they have little effect on the wave velocities.

\subsubsection{High-frequency dynamic elastic moduli}

High-frequency dynamic elastic moduli of the rocks were measured using P and Swave contact transducers (central frequency $1 \mathrm{MHz}$ ). The stress applied to the specimen was approximately zero. Lower frequency transducers $(250 \mathrm{kHz})$ were used when the attenuation was too severe to assess accurate first arrivals. Velocities of the granite were measured using both the cube specimen and the octagonal blocks (Figure 5.18). Velocities for other types of rock were measured using only the cube specimens. For this series of tests, oven dried specimens were used. Table 5.1 shows measured velocities of $\mathrm{P}$ and Swaves. Propagation and polarization directions are shown in Figure 5.18. For a transversely isotropic elastic material, the five independent elastic constants can be determined from $\mathrm{P}$ and S-wave velocities using the following equations (Lo et al., 1985)

Table 5.1 $\mathrm{P}$ and $\mathrm{S}$-wave velocities for cube specimens

\begin{tabular}{|c|c|c|c|c|c|c|c|c|c|}
\hline & \multicolumn{3}{|c|}{ granite } & \multicolumn{3}{|c|}{ sandstone } & \multicolumn{3}{|c|}{ dolomite } \\
\hline density & \multicolumn{3}{|c|}{$2622\left(\mathrm{~kg} / \mathrm{m}^{3}\right)$} & \multicolumn{3}{|c|}{$2148\left(\mathrm{~kg} / \mathrm{m}^{3}\right)$} & \multicolumn{3}{|c|}{$2701\left(\mathrm{~kg} / \mathrm{m}^{3}\right)$} \\
\hline axis & $\begin{array}{c}\mathrm{VP} \\
(\mathrm{m} / \mathrm{sec})\end{array}$ & $\begin{array}{l}\mathrm{VSH} \\
(\mathrm{m} / \mathrm{sec})\end{array}$ & $\begin{array}{l}\text { Vsv } \\
(\mathrm{m} / \mathrm{sec})\end{array}$ & $\begin{array}{c}\mathrm{VP} \\
(\mathrm{m} / \mathrm{sec})\end{array}$ & \begin{tabular}{|c}
$\mathrm{VSH}$ \\
$(\mathrm{m} / \mathrm{sec})$
\end{tabular} & $\begin{array}{l}\text { VSV } \\
(\mathrm{m} / \mathrm{sec})\end{array}$ & $\underset{(\mathrm{m} / \mathrm{sec})}{\mathrm{VP}}$ & $\begin{array}{c}\mathrm{VSH} \\
(\mathrm{m} / \mathrm{sec})\end{array}$ & $\begin{array}{l}\text { VsV } \\
(\mathrm{m} / \mathrm{sec})\end{array}$ \\
\hline$x$ & 4285 & 2700 & 2320 & 2828 & 1914 & 1846 & 4952 & 2839 & 2637 \\
\hline$y$ & 4296 & 2668 & 2290 & 2808 & 1902 & 1841 & 5070 & 2814 & 2692 \\
\hline$z$ & 3093 & 2302 & 2326 & 2602 & 1810 & -.--- & 4474 & $2569^{\mathrm{a}}$ & ---- \\
\hline $\begin{array}{c}\text { source } \\
\text { frequency }\end{array}$ & $\begin{array}{l}500 \\
\mathrm{kHz}\end{array}$ & $\begin{array}{l}25 \\
\mathrm{kF}\end{array}$ & & & $1 \mathrm{MHz}$ & & $\begin{array}{l}500 \\
\mathrm{kHz}\end{array}$ & & \\
\hline
\end{tabular}

a. Due to strong scattering, the first arrival of this wave was not clearly observed. Velocities shown in bold are compared with the velocities for the octagonal specimens 

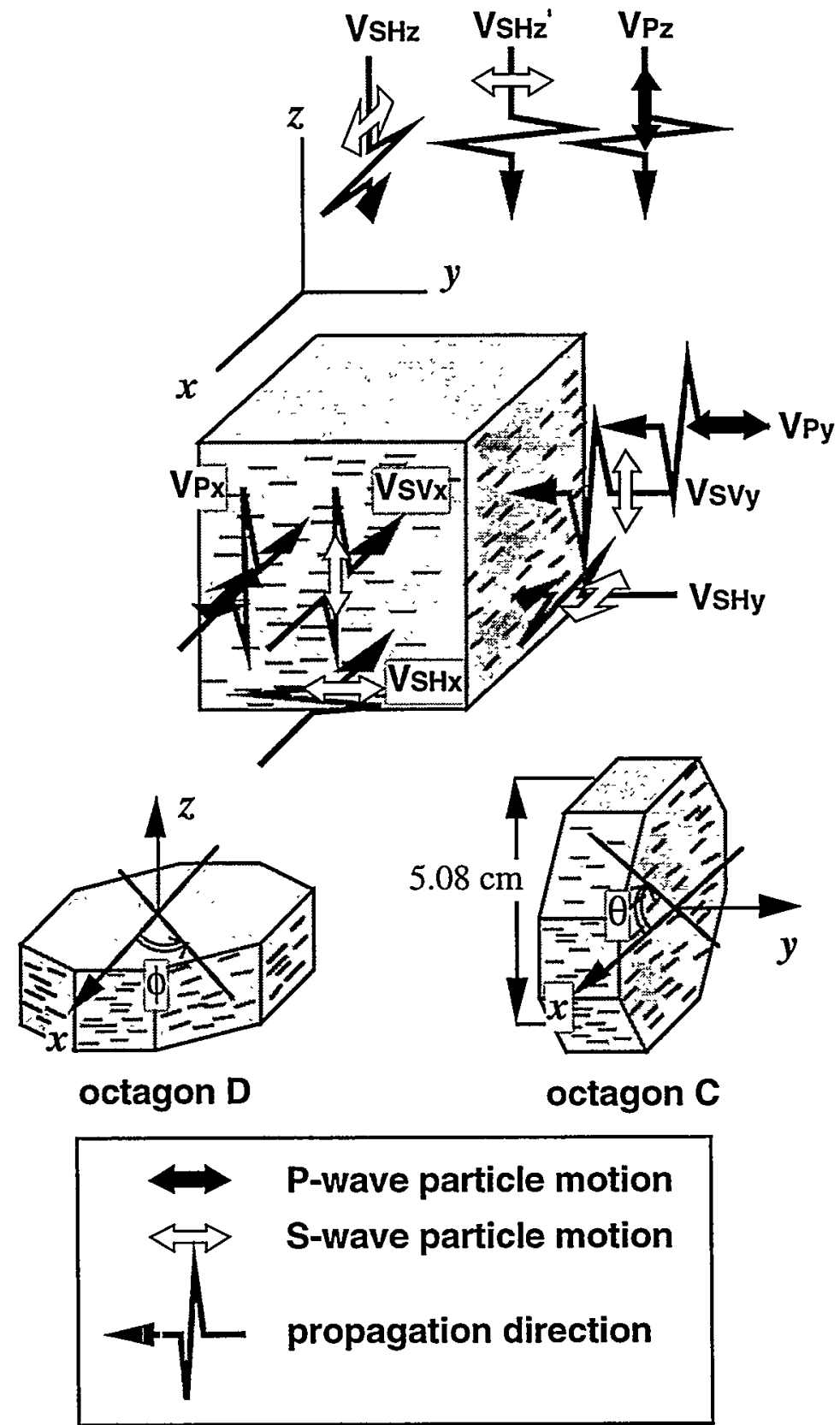

Figure 5.18 Directions of propagation and polarization (particle motion) of $\mathrm{P}$ and $\mathrm{S}$-waves measured in the cube specimens. The name for the velocity of each wave is shown in the plot. S-wave velocities with particle motion parallel and normal to the plane of isotropy (x-y plane) are shown with a subscript "SH" and "SV", respectively. For the granite specimen, octagonal blocks were also prepared for measuring Pwaves propagating obliquely to the isotropy axis (perpendicular to $x-y$ plane). 


$$
\begin{aligned}
& C_{11}=\rho V_{P x}^{2}=\rho V_{P y}^{2}, \\
& C_{12}=C_{11}-2 \rho V_{S H x}^{2}=C_{11}-2 \rho V_{S H y}^{2}, \\
& C_{33}=\rho V_{P z}^{2}, \\
& C_{44}=\rho V_{S H z}^{2}, \\
& C_{13}=-C_{44}+\sqrt{4 \rho^{2} V_{P 45^{\circ}}^{4}-2 \rho V_{P 45^{\circ}}^{2}\left(C_{11}+C_{33}+2 C_{44}\right)+\left(C_{11}+C_{44}\right)\left(C_{33}+C_{44}\right)}
\end{aligned}
$$

where $\rho$ is density of material, $V_{P x}$ and $V_{P y}$ are P-wave velocities measured perpendicular to the isotropy axis, $V_{P_{z}}$ is the P-wave velocity parallel to the isotropy axis. $V_{S H x}$ and $V_{S H y}$ are velocities of the $S$-waves propagating perpendicular to the isotropy axis with particle motion perpendicular to the axis. $V_{S H z}$ is the S-wave velocity parallel to the isotropy axis. $V_{P 45^{\circ}}$ is the P-wave velocity in the direction oblique $\left(45^{\circ}\right)$ to the isotropy axis. Other commonly measured velocities $V_{S V x}$ and $V_{S V y}$ (S-wave velocities perpendicular to the isotropy axis, particle motion parallel to the axis) are not used in the Eq.(5.4a) (5.4e). This is because these velocities are identical to $V_{S H z}$. Notice that some velocities for the dolomite specimen are significantly smaller than the velocities measured for finite axial stresses in Figure 5.16 and 5.17, indicating large velocity anisotropy. This may be caused by thin, compliant debonding cracks between the dolomite matrix and the mineral inclusions that close when subjected to small axial loads.

From the velocities in Table 5.1, four out of five elastic constants for transversely isotropic rocks can be determined using Eq.(5.4a) (5.4d). Results are shown in Table 5.2. Average velocities were used to compute the moduli for velocities that should be identical (for example, $V_{P_{x}}$ and $V_{P y}$ ). In Table 5.1, $V_{S H_{z}}$ for the dolomite is much smaller than $V_{S V x}$ and $V_{S V y}$. This is probably due to a large error in reading the arrival of the strongly scattered and distorted wave. Therefore, this velocity was not used for computing the moduli.

Table 5.2 Dynamic Elastic Moduli (250kHz 1MHz)

\begin{tabular}{|l|c|c|c|}
\hline & granite & sandstone & dolomite \\
\hline $\mathrm{C}_{11}$ & 48.27 & 17.06 & 67.82 \\
$\mathrm{C}_{12}$ & 10.49 & 1.42 & 24.67 \\
$\mathrm{C}_{33}$ & 25.08 & 14.54 & 53.99 \\
$\mathrm{C}_{44}$ & 13.99 & 7.21 & 19.18 \\
\hline
\end{tabular}

The moduli are shown in [GPa] 
From the velocities in Table 5.1, modulus $\mathrm{C}_{13}$ cannot be computed. This is because it requires a $\mathrm{P}$ or $\mathrm{S}$-wave measured at an oblique angle to the axis of isotropy. For granite, octagonal specimens were prepared for measuring waves at this orientation. Table 5.3 shows measured velocities in the direction and polarization shown in Figure 5.18. Velocities for the octagon $\mathrm{D}$ measured using $1 \mathrm{MHz} \mathrm{P}$ and $\mathrm{S}$ - waves show good agreement with the velocities for cube specimens measured using $500 \mathrm{kHz} \mathrm{P}$ and $250 \mathrm{kHz}$ S-sources. From the table, octagon $C$ shows smaller velocities than octagon $D$. This is possibly due to local heterogeneity of the rock. Due to the anomalously low velocities, the $45^{\circ} \mathrm{P}$-wave velocity of the octagon $C$ cannot be directly used for determining modulus $C_{13}$. The $45^{\circ} \mathrm{P}$ wave velocity $\left(V_{P 45^{\circ}}\right)$ was inferred from $V_{P x}$ and $V_{P z}$, using the relative magnitude of $V_{P 45^{\circ}}$ compared with $V_{P x}$ and $V_{P z}$ for the octagon C. The estimated $V_{P 45^{\circ}}$ was $3727 \mathrm{~m} / \mathrm{sec}$. From Eq.(5.4e) and the moduli in Table 5.2, $\mathrm{C}_{13}$ for the granite was determined to be 4.93 GPa.

Table 5.3 Velocities Determined for Granite Octagons at $1 \mathrm{MHz}$ Source Frequency

\begin{tabular}{|l|c|c|c|c|c|c|c|}
\hline & \multicolumn{3}{|c|}{ octagon C } & & \multicolumn{3}{c|}{ octagon D } \\
\hline axis $/ \theta$ & $\begin{array}{c}\text { VP } \\
(\mathrm{m} / \mathrm{sec})\end{array}$ & $\begin{array}{c}\text { VsH } \\
(\mathrm{m} / \mathrm{sec})\end{array}$ & $\begin{array}{c}\text { VSV } \\
(\mathrm{m} / \mathrm{sec})\end{array}$ & axis $/ \phi$ & $\begin{array}{c}\text { VP } \\
(\mathrm{m} / \mathrm{sec})\end{array}$ & $\begin{array}{c}\text { VSH } \\
(\mathrm{m} / \mathrm{sec})\end{array}$ & $\begin{array}{c}\text { VSV } \\
(\mathrm{m} / \mathrm{sec})\end{array}$ \\
\cline { 2 - 6 } \cline { 6 - 9 }$x / 0^{\circ}$ & $\mathbf{4 0 4 2}$ & $\mathbf{2 5 8 2}$ & 2189 & $x / 0^{\circ}$ & $\mathbf{4 2 7 1}$ & 2677 & 2297 \\
$x / 45^{\circ}$ & 3564 & 2420 & 2191 & $x / 45^{\circ}$ & 4271 & 2680 & 2306 \\
$z / 90^{\circ}$ & 3008 & 2341 & 2293 & $y / 90^{\circ}$ & 4271 & 2680 & 2326 \\
$z / 135^{\circ}$ & 3493 & 2526 & 2176 & $y / 135^{\circ}$ & 4164 & 2749 & 2281 \\
\hline
\end{tabular}

Velocities shown in bold are $\mathrm{P}, \mathrm{SH}$, and $\mathrm{SV}$ waves having the same directions of wave propagation and polarization (particle motion) as in Table 5.1. 


\subsection{Resonance of Anisotropic Rocks}

\subsubsection{Experimental setup}

Resonance of the anisotropic rocks tested in both Section 5.2 for the static properties and Section 5.3 for the high-frequency dynamic properties was examined in the cube specimens. A similar setup to the resonant bar tests in the Chapter 3 was adopted (Figure 5.19). Specimens were suspended using thin steel wires and excited with a sine wave swept over a broad frequency range by the internal signal generator in an Ono Sokki CF6400 spectrum analyzer. This signal was amplified by a voltage amplifier (Krohn-Hite 7602) and then passed to a small piezoelectric crystal attached to the specimen with bonding wax. The resulting accelerations were measured using a miniature high frequency accelerometer (PCB Piezotronics, 309A, resonance frequency $>120 \mathrm{kHz}$ ) and then displayed and analyzed on the spectrum analyzer.

\subsubsection{Frequency response of an isotropic cube}

Before examining the resonance of anisotropic rocks, the resonance of an isotropic cube made of lead glass was conducted to determine the accuracy of the measurements and the inversion technique presented in Chapter 4. The length of the specimen was $10.16 \mathrm{~cm}$ and the density was $6275 \mathrm{~kg} / \mathrm{cm}^{3}$. As the specimen was too heavy to be supported by steel wires, it was placed on a foam pad to approximately simulate stress-free boundary conditions. High frequency ultrasonic transmission tests (central frequency of $1 \mathrm{MHz}$ ) gave $\mathrm{P}$ and S-wave velocities of $3187 \mathrm{~m} / \mathrm{sec}$ and $1792 \mathrm{~m} / \mathrm{sec}$, respectively. The dynamic Young's modulus and Poisson's ratio computed from these velocities were 51.14 GPa and 0.269 , respectively.

Measured experimental frequency response functions are shown in Figure 5.20(a) and (b) for source and receiver locations indicated in the plots. Measured accelerations were normalized by the values determined with an accelerometer attached to the back of the piezoelectric crystal suspended in air. Accelerations for the crystal were multiplied by the crystal's mass $(3.5 \mathrm{~g}$ ) to obtain the force applied to the specimen. In the plots, frequency response functions (accelerance) were computed with the numerical model introduced in Chapter 4 that used the measured dynamic elastic moduli. The highest order of polynomials used for approximating the displacement field was $12(12 \times 12 \times 12)$. The measured and computed FRF's show good agreement over the full frequency range. 


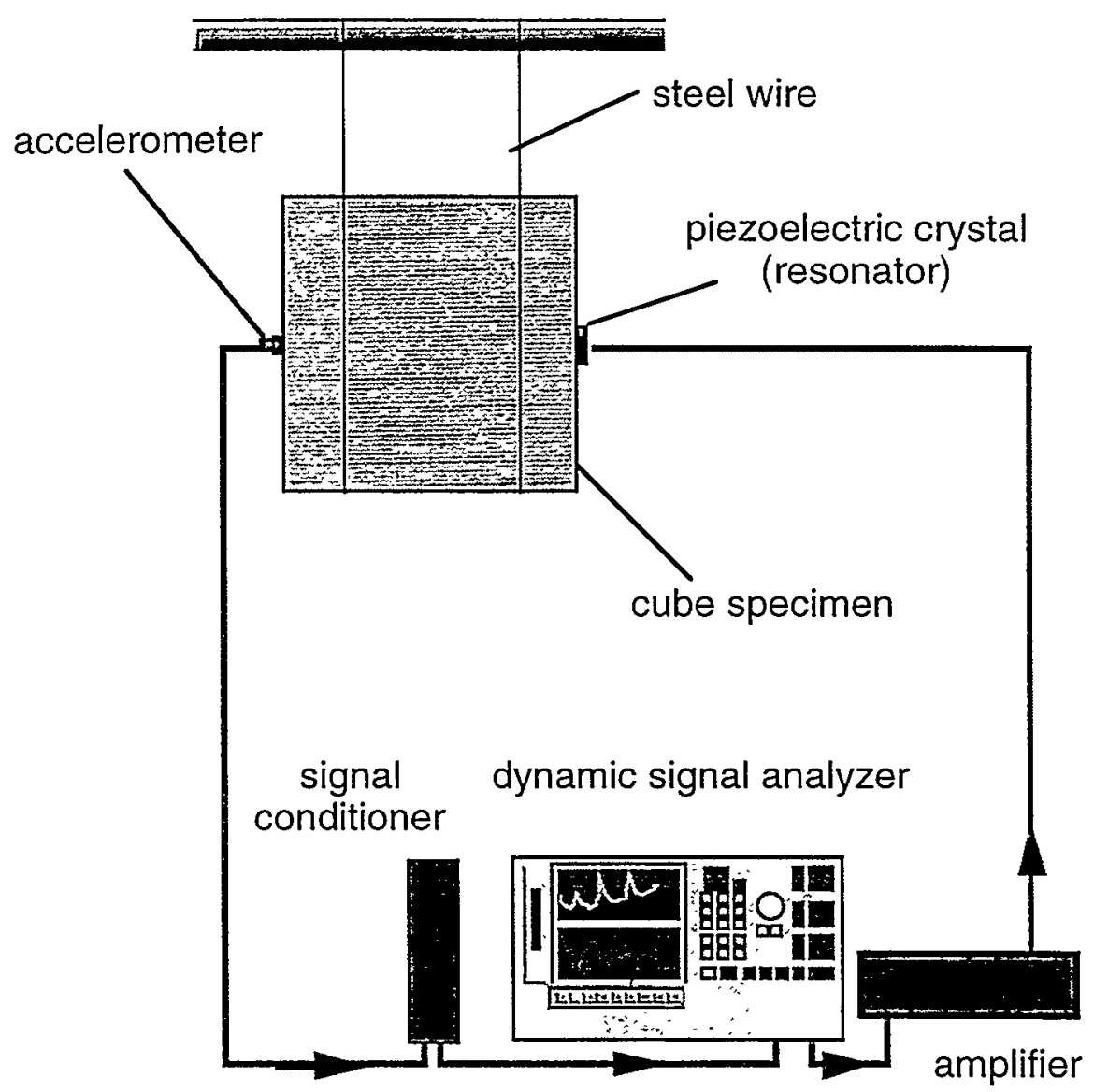

Figure 5.19 Experimental setup used for free vibration tests of rock cubes. Specimens were suspended in air using thin steel wires. A small piezoelectric crystal attached to the specimen was excited by a sine wave. Resulting accelerations were measured by an accelerometer attached to the opposite side of the cube. The measured accelerations were displayed, analyzed, and stored on a spectrum analyzer. 


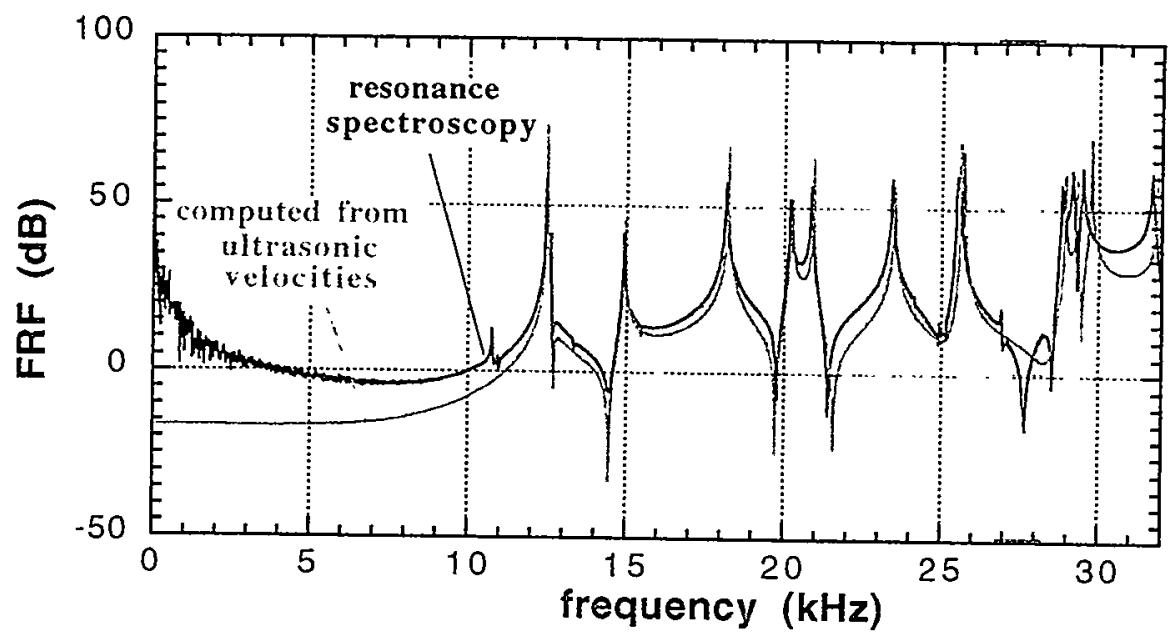

(a) "Face-to-face" resonance test

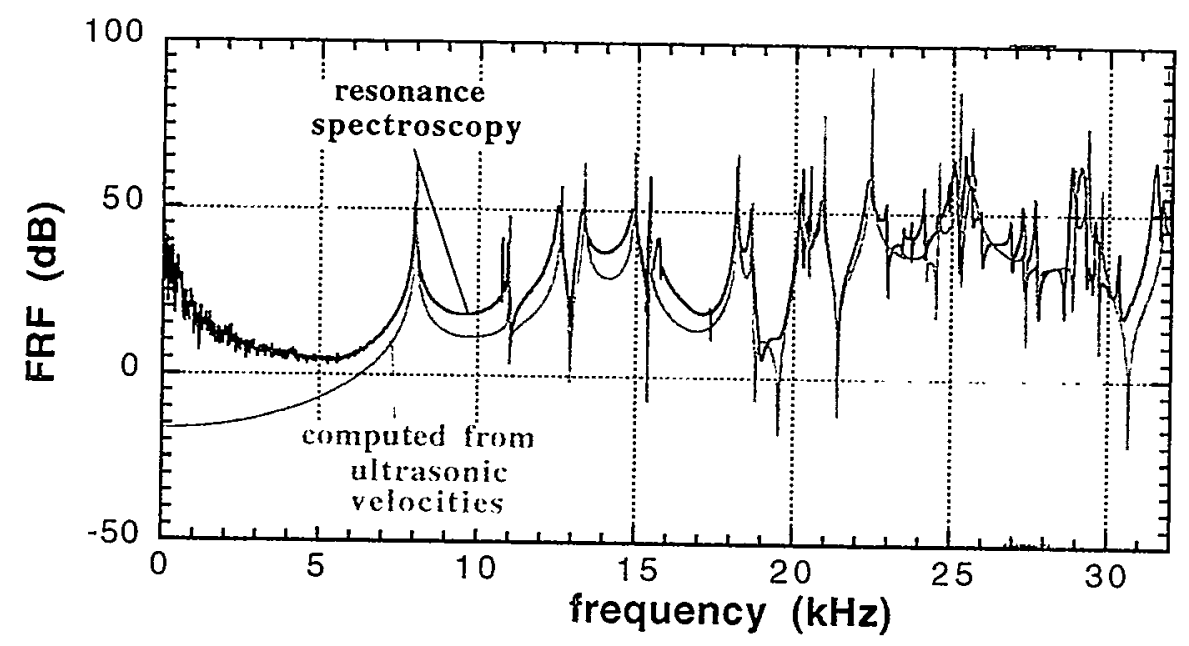

(b) "Twist" resonance test

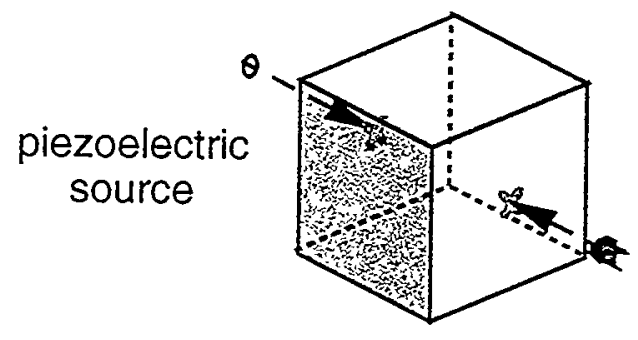

'Face-to-face' test

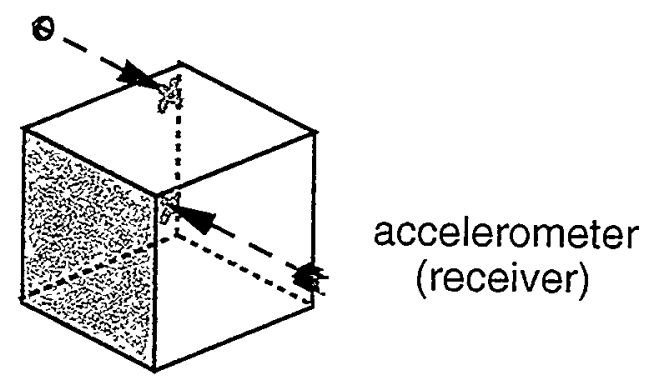

'Twist' test

Figure 5.20 Measured and computed (from measured ultrasonic velocities) frequency response functions. Both measured and computed FRF's show good agreement. 
Resonance inversion for elastic moduli was performed using the numerical algorithm introduced in the previous chapter. Several low frequency modes were selected for matching the observed and computed resonance frequencies. The types of the modes were identified by comparing the shape of experimental and computed (using an initial guess for elastic moduli) FRF's. Measured and computed resonance frequencies for converged numerical model are shown in Table 5.4 as well as the results of the ultrasonically determined resonance frequencies. Young's modulus and Poission's ratio determined by the resonance inversion were $50.76 \mathrm{GPa}$ and 0.270 , respectively. These moduli show very good agreement with the results from ultrasonic transmission tests. In Figure 5.21 (a) and (b), measured FRF's are compared with the computed FRF's for the elastic parameters determined from the resonance inversion.

Table 5.4 Resonance Frequencies for the Lead Glass Cube

\begin{tabular}{|l|c|c|c|c|}
\hline $\begin{array}{l}\text { mode } \\
\text { number }\end{array}$ & $\begin{array}{c}\text { (1) observed } \\
\text { resonance } \\
(\mathrm{kHz})\end{array}$ & $\begin{array}{c}\text { (2)resonance } \\
\text { inversion } \\
(\mathrm{kHz})\end{array}$ & $\begin{array}{c}\text { (3)ultrasonic } \\
(\mathrm{kHz})\end{array}$ & $\begin{array}{c}(4) \text { error } \\
{[(2)-(1)] /(1)} \\
(\%)\end{array}$ \\
\hline $1,2^{\mathrm{a})}$ & 8.0000 & 7.9892 & 8.0220 & -0.14 \\
$3,4,5$ & 10.740 & 10.736 & 10.779 & -0.039 \\
$6,7,8$ & 10.940 & 10.935 & 10.979 & -0.048 \\
$9,10,11^{\mathrm{a})}$ & 12.440 & 12.428 & 12.479 & -0.093 \\
$12,13,14^{\mathrm{a}}$ & 12.560 & 12.567 & 12.612 & 0.060 \\
$15,16,17$ & 13.300 & 13.295 & 13.346 & -0.041 \\
$18,19 \mathrm{a})$ & 14.900 & 14.892 & 14.945 & -0.052 \\
20 & 15.380 & 15.383 & 15.445 & 0.021 \\
$\left.30,31,32^{\mathrm{a}}\right)$ & 18.140 & 18.146 & 18.218 & 0.036 \\
$34,35,36$ & 20.180 & 20.170 & 20.251 & -0.051 \\
44,45 & 20.840 & 20.840 & 20.922 & -0.00072 \\
51,52 & 23.420 & 23.399 & 23.492 & -0.089 \\
$56,57,58$ & 24.120 & 24.084 & 24.176 & -0.15 \\
$61,62,63$ & 24.920 & 24.857 & 24.959 & -0.25 \\
$67,68,69$ & 25.480 & 25.499 & 25.597 & 0.073 \\
70.71 .72 & 25.580 & 25.511 & 25.611 & -0.27 \\
\hline
\end{tabular}

a) Modes used for resonance inversion 


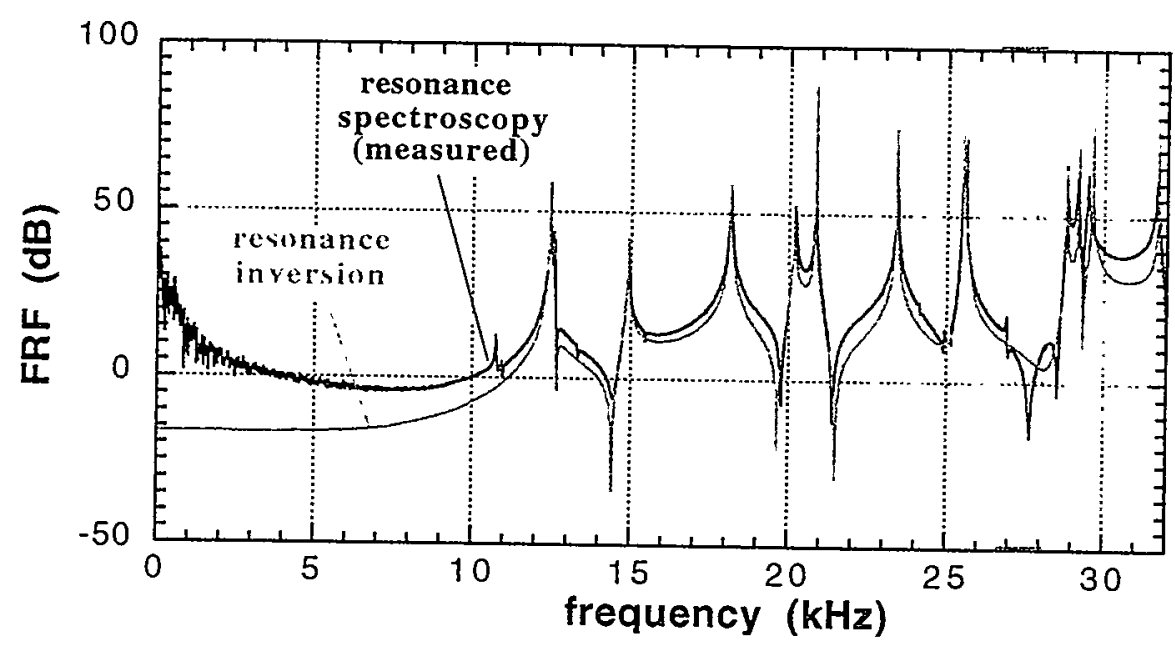

(a) "Face-to-face" resonance test

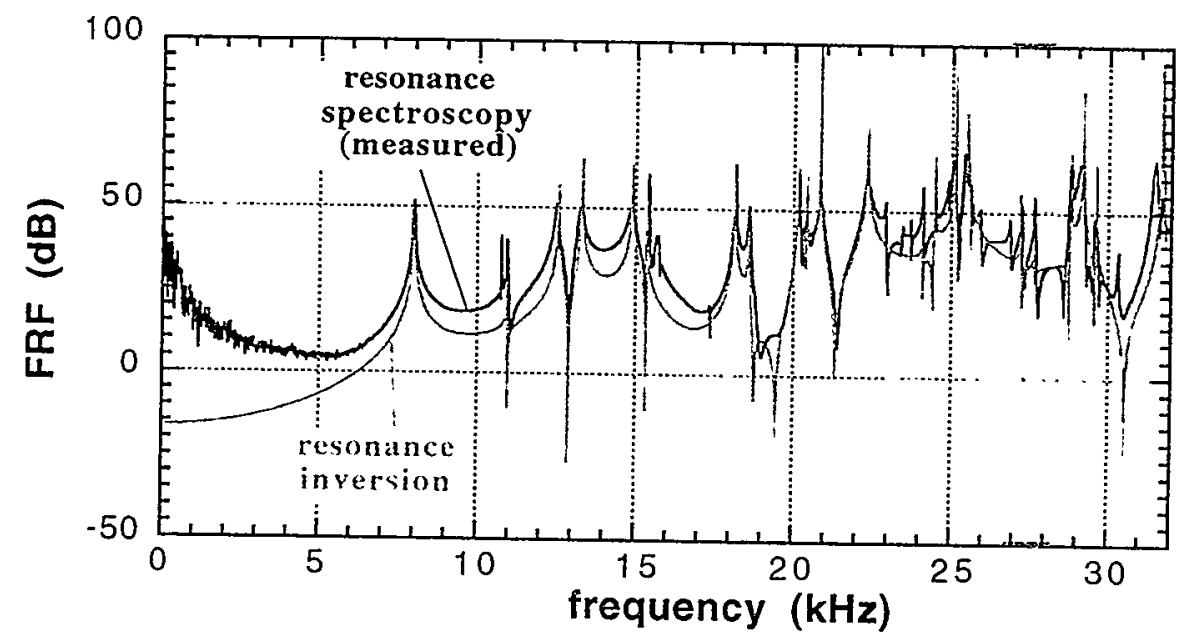

(b) "Twist" resonance test

Figure 5.21 Measured and computed FRF's. The computed FRF is shown for Young's modulus and Poisson's ratio determined by the inversion that matches the experimentally measured and computed resonance frequencies. The FRF's show excellent agreement. 


\subsubsection{Frequency response of anisotropic rocks}

For conducting the numerical inversion, one type of mode has to be specified for each experimentally measured resonance frequency. The proper mode type is identified by comparing the experimentally measured FRF and simulated FRF using an initial guess for the elastic moduli. From Figure 5.21, it can be seen that the "twist" type source-receiver configuration yields a much more complicated FRF than the "face-to-face" configuration. This is because the latter eliminates many of the modes by the symmetries in the configuration such as location and orientation of the source and receiver. As mode types can be identified more easily with fewer modes, measurements for anisotropic rocks are performed primarily using the "face-to-face" testing configuration.

Measured FRF's for each type of rock with the source and receiver located along the three orthogonal coordinate axes are shown in Figure 5.22(a) (c). The FRF's were measured by sweeping over 0 to $32 \mathrm{kHz}$ for granite and 0 to $50 \mathrm{kHz}$ for sandstone and dolomite using sine waves. Oven-dried specimens were suspended in air using thin steel wires. FRF's for the granite specimen (Figure 5.22(a)) show distinct differences perpendicular to the isotropy axis (along $\mathrm{x}$ - and $\mathrm{y}$-axes) and parallel to the isotropy axis (along z-axis). The sandstone specimen shows similar FRF's for all three directions. However, there are some noticeable differences between FRF's measured parallel and perpendicular to the isotropy axis (z-axis). The dolomite specimen shows quite different FRF's for all directions. This is possibly due to the strong heterogeneity of the specimen that disturbs the symmetry of the acoustic properties. Due to the distortion of the FRF's, mode types of the measured resonances could not be identified successfully. For this reason, resonance inversion was not conducted for the dolomite specimen.

Using the measured resonance frequencies in the first several modes, resonance inversion for anisotropic elastic moduli was performed for the granite and sandstone specimens. Comparisons between experimentally measured and computed FRF's for the inverted elastic moduli are shown in Figure 5.23 for granite and in Figure 5.24 for sandstone. The overall shapes of the FRF's show good agreement for both rocks. Although the magnitudes of the computed FRF's for the granite cube overestimate the experimentally measured FRF, it should be noted that the inversion technique used in this research requires only the resonance frequencies and the general shape of the FRF for determining the elastic moduli. 


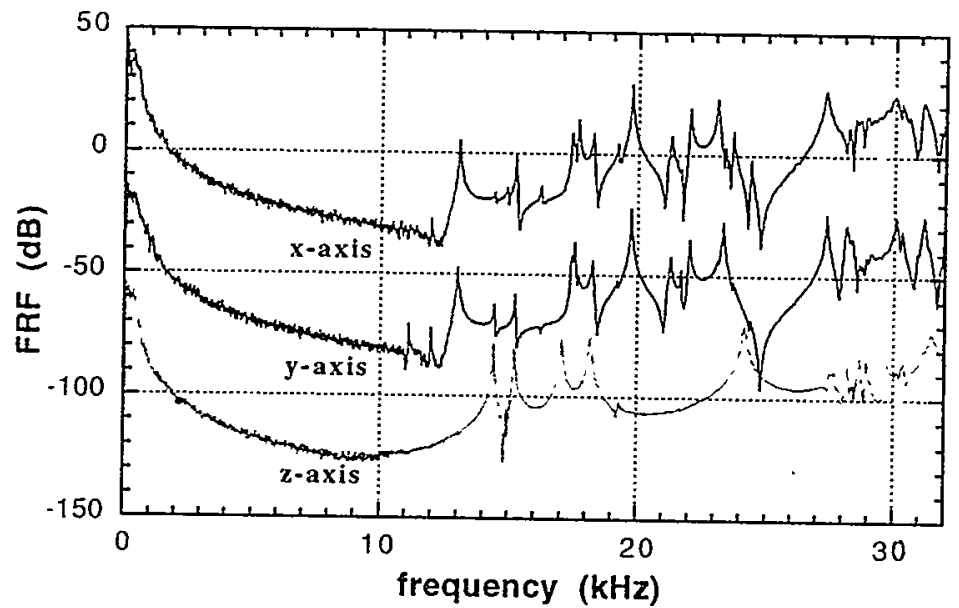

(a) FRF's for granite cube

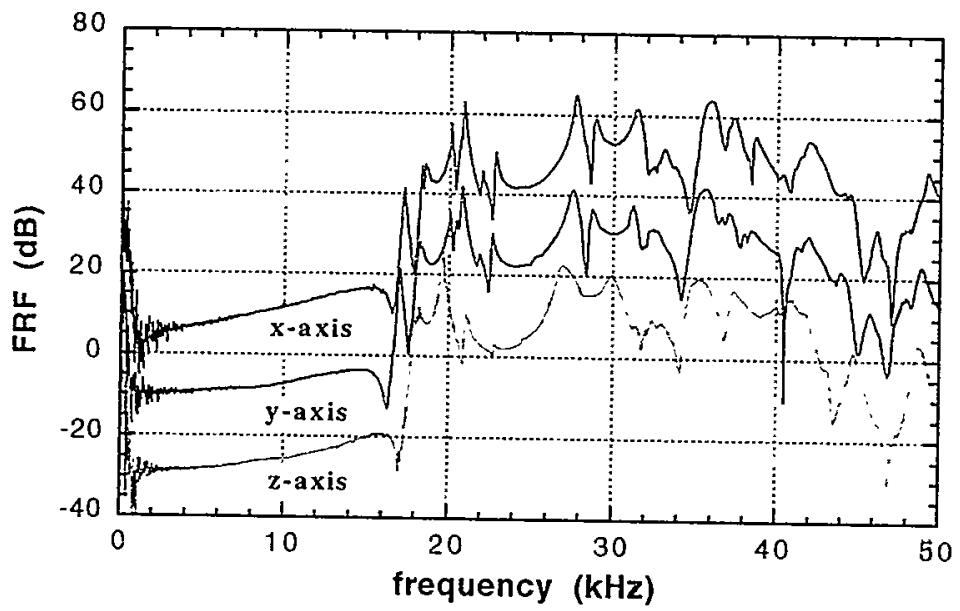

(b) FRF's for sandstone cube

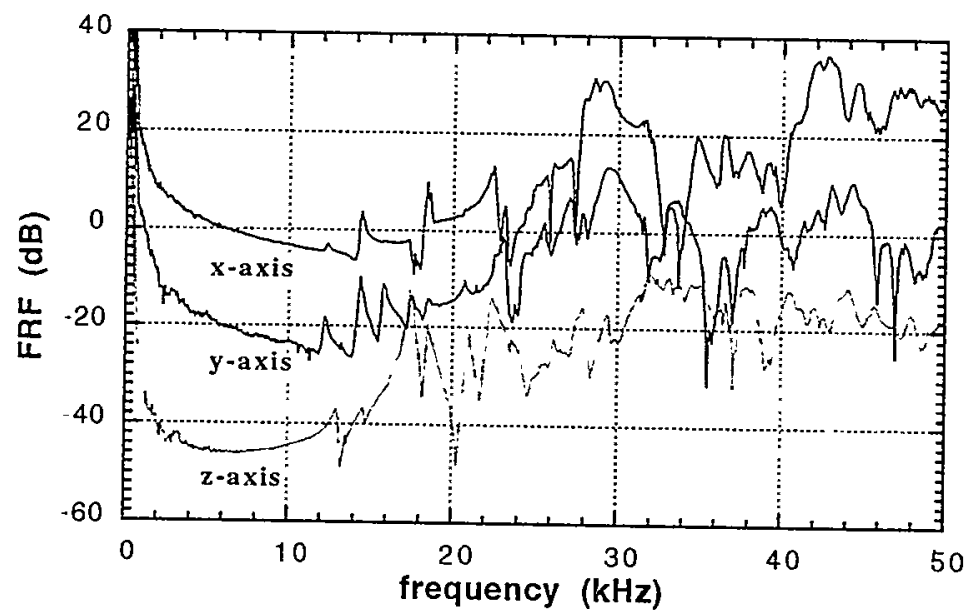

(c) FRF's for dolomite cube

Figure 5.22 FRF's for rock cubes. FRF's in each plot are shifted by $50 \mathrm{~dB}$ for (a) and $20 \mathrm{~dB}$ for (b) and (c). Although granite and sandstone cubes show clear similarities between FRF's along $x$ - and $y$-axes, FRF's for the dolomite cube are very different along all three directions. 


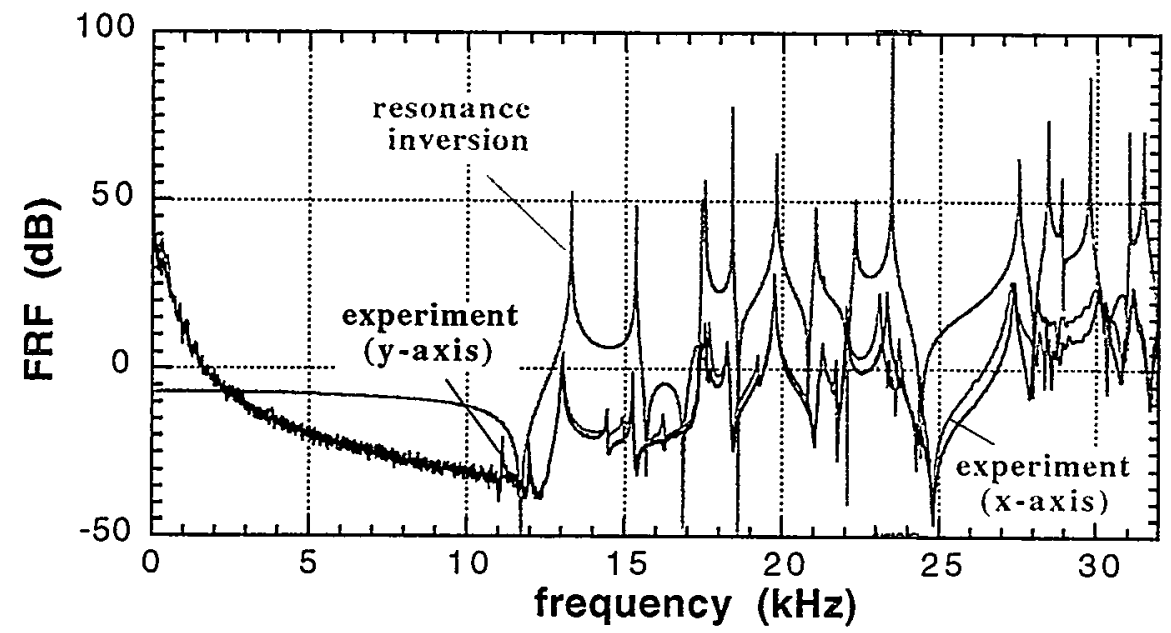

(a) Resonance along $x$ and $y$ - axes

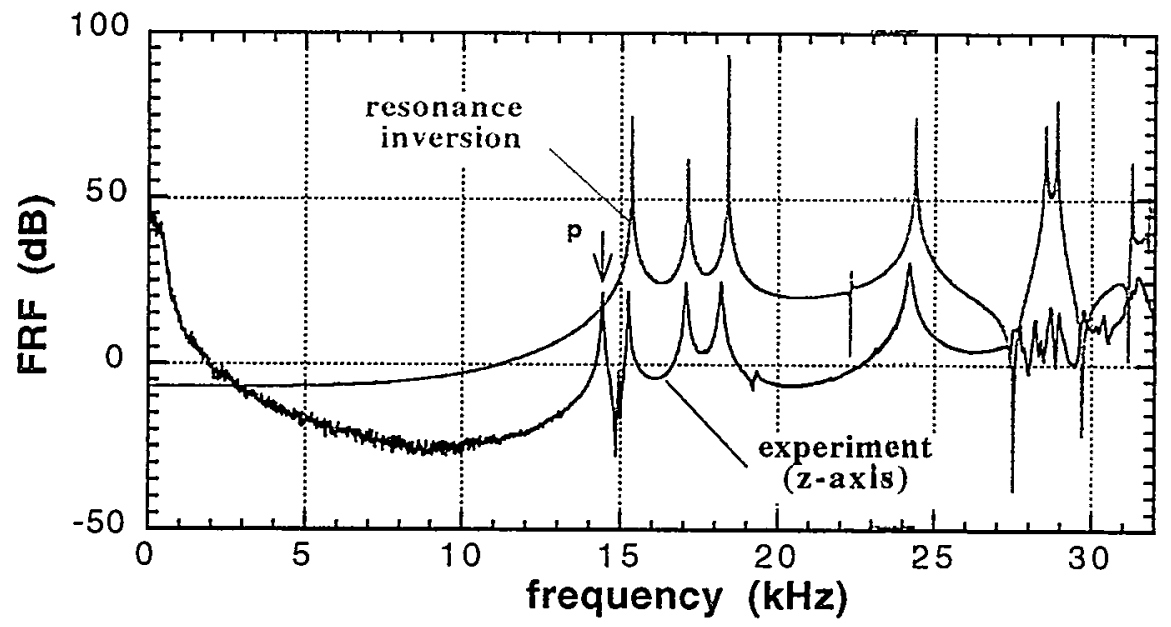

(b) Resonance along $\mathrm{z}$ - axis

Figure 5.23 Measured and computed FRF's for the granite cube. Computed FRF (resonance inversion) is for the five elastic constants inverted by matching experimentally measured and numerically computed resonance frequencies. Resonance frequencies of both FRF's show good agreement except for the first resonance (labeled as "P") in the bottom plot. This resonance is likely caused by the heterogeneity and higher order anisotropy of the specimen. 


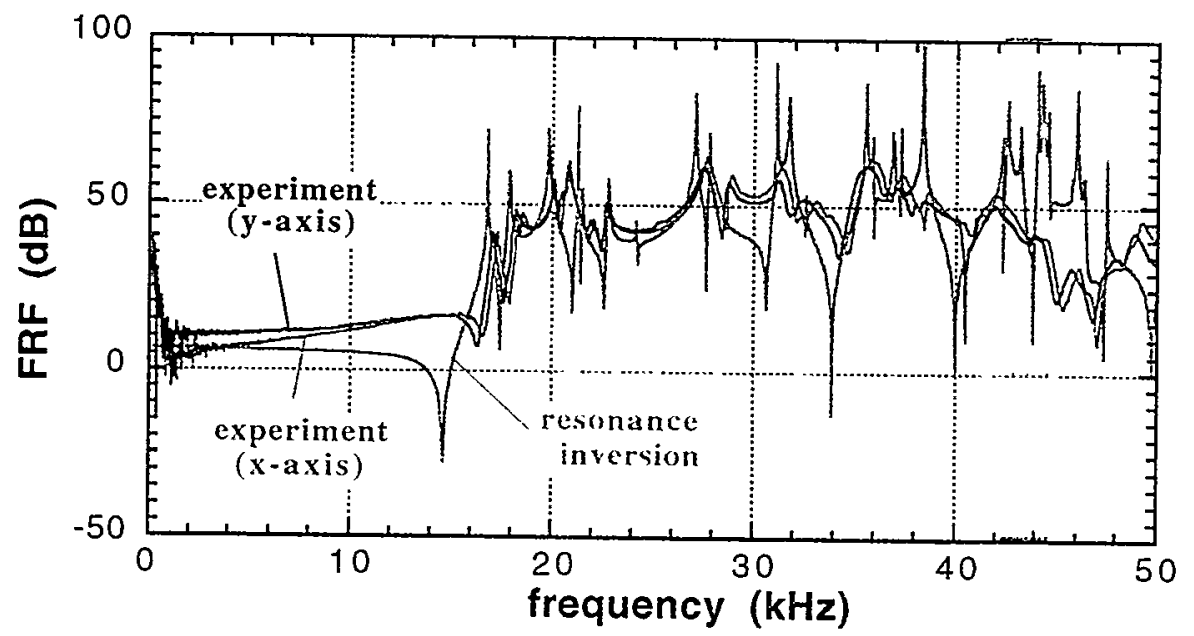

(a) Resonance along $x$ and $y$ - axes

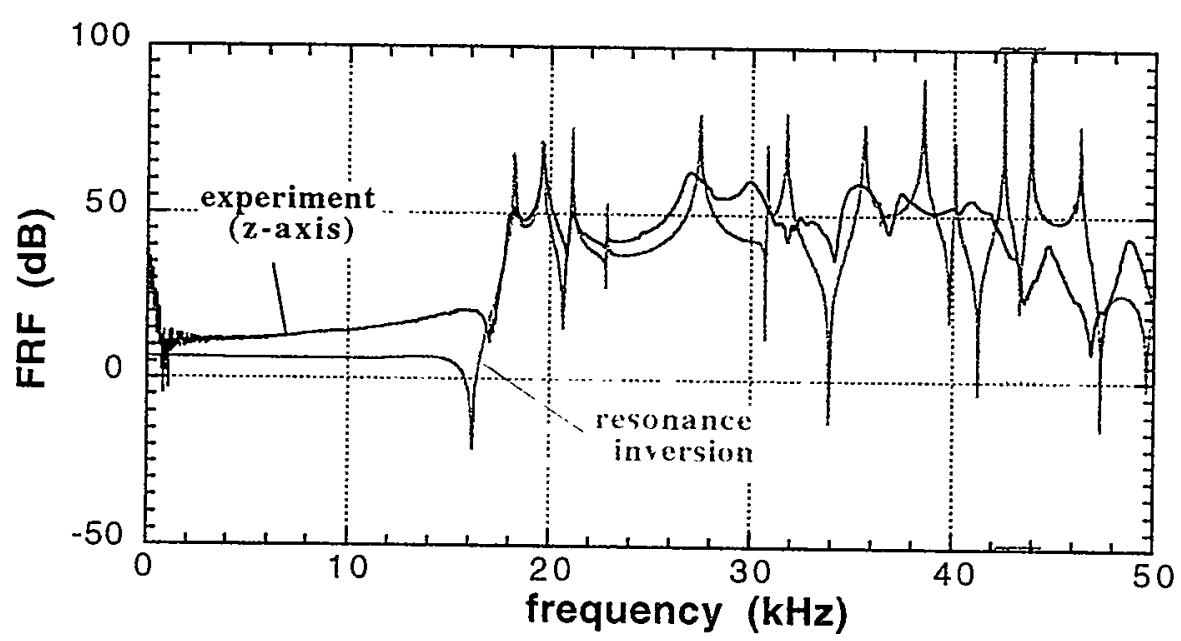

(b) Resonance along $\mathrm{z}$ - axis

Figure 5.24 Measured and computed FRF's for the sandstone cube. Computed FRF (resonance inversion) is for the five elastic constants inverted by matching experimentally measured and numerically computed resonance frequencies. As the mass for the source and receiver was not negligibly small, numerical simulations were performed modeling the source and receiver as point masses on the surface of the cube. Only the resonance frequencies measured along the $z$-axis were used for inversion. Although low frequency resonances in the bottom plot show excellent agreement between the experimental and computed resonance frequencies, the top plot shows rather large differences. 
Table 5.5 shows a comparison between the measured and computed resonance frequencies for the granite cube. Although some of the low frequency resonances show up to a few percent of error, most of the resonances show good agreement. In Figure 5.23(b), there is a mode that does not appear in the computed FRF (labeled as "p"). This resonance emerges in the computed FRF if the source or receiver is located off-center on the surface of the cube. The mode also exhibits a splitting and its counterpart is observed at $14.94 \mathrm{kHz}$. Such behavior of the measured resonance is possibly due to heterogeneities in density and stiffness that break the symmetry of the problem and to higher order anisotropy of the rock.

Moduli of the sandstone cube were also determined by resonance inversion. Due to the relatively small dimension and density of the cube specimen, the mass added by the source and receiver ( $3.5 \mathrm{~g}$ and $1.5 \mathrm{~g}$, respectively) was significant compared to the mass of the specimen ( $0.86 \%$ of the cube mass). The additional mass increases the inertia of the specimen and consequently decreases its resonance frequencies. To account for this effect,

Table 5.5 Resonance Frequencies for the Granite Cube

\begin{tabular}{|l|c|c|c|}
\hline $\begin{array}{l}\text { mode } \\
\text { number }\end{array}$ & $\begin{array}{c}\text { (1) observed } \\
\text { resonance } \\
(\mathrm{kHz})\end{array}$ & $\begin{array}{c}\text { (2)resonance } \\
\text { inversion } \\
(\mathrm{kHz})\end{array}$ & $\begin{array}{c}(3) \text { error } \\
{[(2)-(1)] /(1)} \\
(\%)\end{array}$ \\
\hline 1 & 11.120 & 10.981 & -1.25 \\
2 & 11.980 & 11.968 & -0.097 \\
3,4 & 13.000 & 13.278 & 2.14 \\
& 13.040 & & 1.83 \\
5,6 & 14.420 & 14.916 & 3.44 \\
& 14.940 & & -0.16 \\
7 & 15.260 & 15.342 & 0.54 \\
8 & 16.220 & 16.220 & -0.0026 \\
10 & 17.060 & 17.129 & 0.41 \\
12 & 17.420 & 17.424 & 0.025 \\
13,14 & 17.540 & 17.528 & -0.071 \\
& 17.720 & & -1.09 \\
15 & 18.240 & 18.402 & 0.89 \\
16,17 & 19.220 & 19.244 & 0.12 \\
& 19.320 & 19.244 & -0.40 \\
18 & 19.740 & 19.794 & 0.27 \\
31 & 24.180 & 24.379 & 0.82 \\
\hline
\end{tabular}

Note: All the measured modes were used for inversion 
the numerical code was modified to handle the source and receiver as point masses attached to the surface of the specimen. As individual modes were most easily identified in the direction of $\mathrm{z}$-axis, the inversion was conducted using five resonance frequencies measured in this direction. Table 5.6 compares resonance frequencies of the measured and computed modes. The first five resonance frequencies for the measured and computed modes in the z-direction fit exactly since the number of observations is identical to the number of unknowns (elastic constants). On the other hand, directions along the plane of anisotropy (along $\mathrm{x}$ and $\mathrm{y}$-axes) show rather large differences between the measured and computed resonance frequencies. The misfit may be caused by heterogeneities in the specimen, such as unevenly spaced bedding planes and cross bedding structures.

Table 5.6 Resonance Frequencies for the Sandstone Cube

\begin{tabular}{|l|c|c|c|}
\hline $\begin{array}{l}\text { mode } \\
\text { number }\end{array}$ & $\begin{array}{c}\text { (1) observed } \\
\text { resonance } \\
(\mathrm{kHz})\end{array}$ & $\begin{array}{c}\text { (2)resonance } \\
\text { inversion } \\
(\mathrm{kHz})\end{array}$ & $\begin{array}{c}\text { (3)error } \\
(2)-(1)] /(1) \\
(\%)\end{array}$ \\
\hline \multicolumn{4}{|c|}{ along z-axis } \\
\hline $11^{\mathrm{a})}$ & 18.219 & 18.219 & 0.00 \\
$13^{\mathrm{a})}$ & 19.625 & 19.625 & 0.00 \\
$18^{\mathrm{a})}$ & 21.094 & 21.094 & 0.00 \\
$20^{\mathrm{a})}$ & 22.844 & 22.844 & 0.00 \\
$26^{\mathrm{a})}$ & 24.500 & 24.500 & 0.00 \\
28 & 26.969 & 27.172 & 0.75 \\
\hline \multicolumn{4}{|c|}{ along $\mathrm{x}$ and y-axis (average) } \\
\hline 4 & 17.172 & 16.797 & -2.18 \\
9 & 18.266 & 17.871 & -2.16 \\
13 & 20.094 & 19.820 & -1.36 \\
18 & 20.797 & 21.252 & 2.19 \\
19 & 22.359 & 21.486 & -3.90 \\
20 & 22.734 & 22.779 & 0.20 \\
30 & 27.591 & 27.089 & -1.82 \\
31 & 28.812 & 27.788 & -3.55 \\
\hline
\end{tabular}

a) Modes used for resonance inversion 


\subsubsection{Anisotropic elastic constants}

Anisotropic elastic constants for granite and sandstone determined by the resonance inversion technique are shown in Table 5.7. These elastic constants are compared with the statically and ultrasonically measured elastic constants in Figure 5.25. Granite exhibits a consistent trend that shows higher elastic moduli for the higher-frequency test. Sandstone has a similar trend showing higher elastic moduli for the ultrasonic results. Frequencydependent behavior of the elastic moduli was not observed for the lead glass, which showed almost identical Young's modulus and Poisson's ratio for the resonance and ultrasonic measurements.

For the granite specimen, static moduli determined from the small excursion loops are significantly smaller than dynamic moduli, especially in the direction perpendicular to the isotropy axis $\left(C_{11}\right)$. Although the type of rocks is different, this result contradicts the measurements on Berea sandstone conducted by Hilbert et al.(1994) that showed good agreement between statically (uniaxial strain load-displacement tests) and ultrasonically measured elastic moduli. On the other hand, similar measurements conducted by Plona and Cook (1995) with uniaxial stress compression tests showed a rather large difference between the static and dynamic moduli. The difference is possibly due to the slip motion between sand grains that is more easily mobilized under the low confining stress state of the uniaxial stress tests. It is also noted that the difference was larger for lower axial stresses.

As the static moduli in this research were determined by uniaxial compression tests at very low confining stress, it is likely that slip along microcracks was present even for the small excursion loops. During the uniaxial compression tests on the core specimens

Table 5.7 Inverted Elastic Moduli $(10 \mathrm{kHz} \sim 30 \mathrm{kHz})$

\begin{tabular}{|l|l|r|}
\hline & granite & sandstone \\
\hline $\mathrm{C}_{11}$ & 42.16 & 14.71 \\
$\mathrm{C}_{12}$ & 5.62 & 1.93 \\
$\mathrm{C}_{13}$ & 4.47 & 0.68 \\
$\mathrm{C}_{3}$ & 22.59 & 12.74 \\
$\mathrm{C}_{44}$ & 12.87 & 6.08 \\
\hline
\end{tabular}

The moduli are shown in [GPa] 


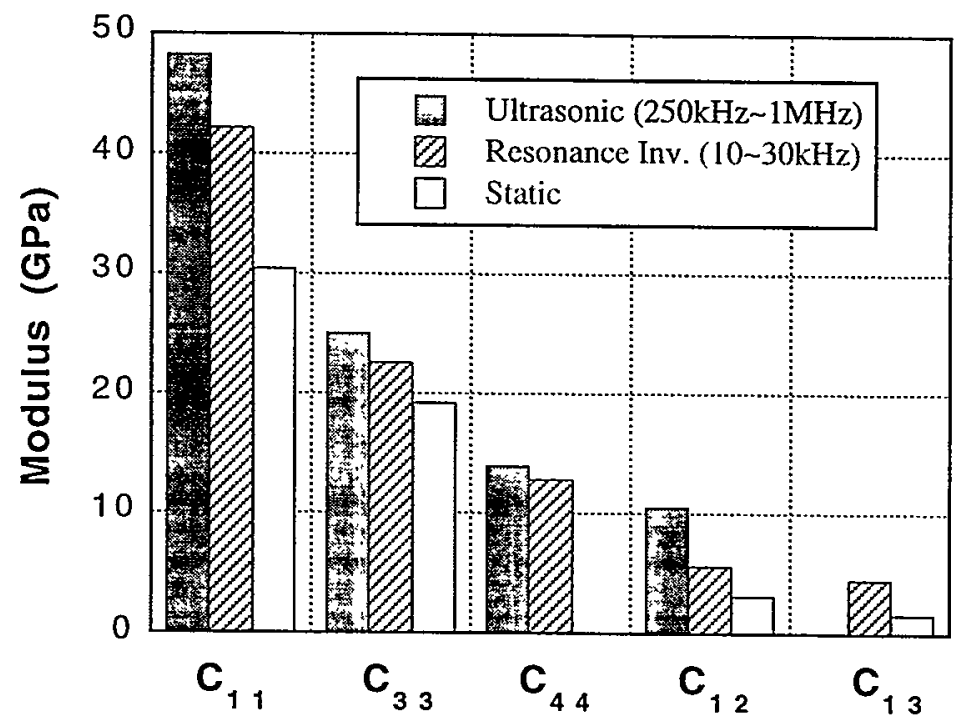

(a) Elastic moduli for granite

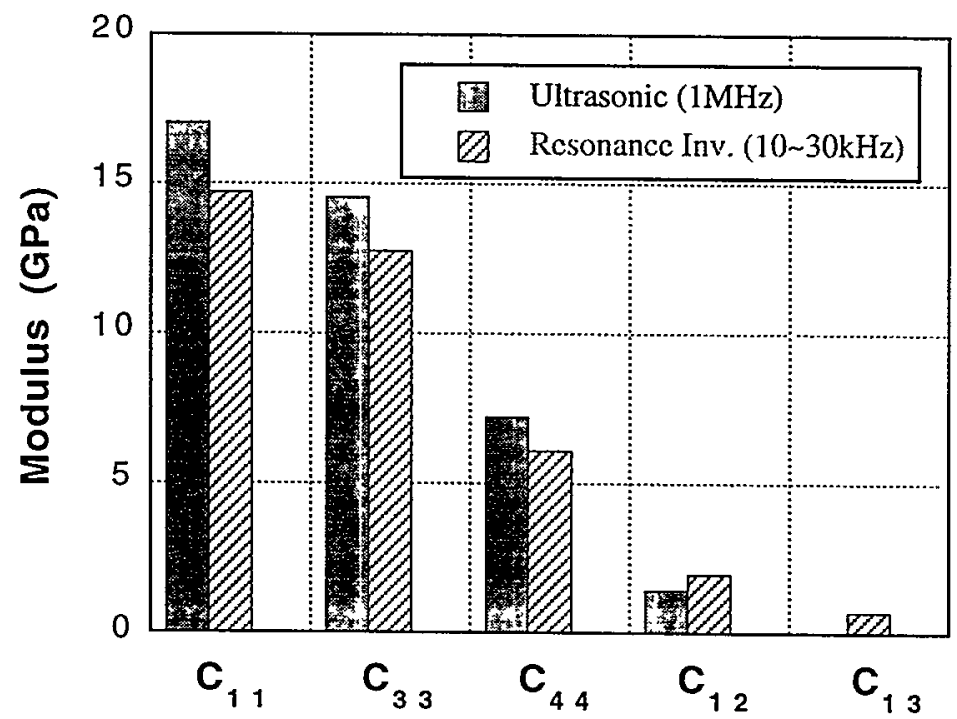

(b) Elastic moduli for sandstone

Figure 5.25 Experimentally determined elastic moduli for granite and sandstone cubes. Results for the resonance inversion are consistently smaller than the moduli determined from high-frequency ultrasonic transmission tests. Static (zero frequency) results for. granite show moduli much smaller than both ultrasonic and resonance tests, especially in the direction perpendicular to the isotropy axis. The small static moduli may be due to sliding along microcracks that is present even for the small excursion loops of the load-displacement curves. 
perpendicular to the isotropy axis, it was also found that the linear elastic part of the excursion loops was hard to identify, indicating that the loops were not free of slip motion. In the direction parallel to the isotropy axis, excursion loops showed very small hysteresis and the moduli were relatively easily identified. The different slip behavior of the granite may be due to the microcracks that are nearly perpendicular to the isotropy axis and slip easily by small axial load applied to the crack surface at a shallow angle.

Elastic moduli of granite and sandstone determined by ultrasonic and resonance measurements show similar values. The small difference may be due to the dispersion of waves in the rocks caused by scattering off microstructures such as compliant microcracks and grain contacts. Small amounts of clay present also leads to dispersion due to viscoelastic behavior (Nihei, 1992; Suárez-Rivera, 1992). The velocity of the wave propagating through such a system monotonically increases with increasing wave frequency.

\subsubsection{Mode shapes}

The determination of elastic moduli using resonance spectroscopy requires identification of each mode. In order to verify that a mode specified for the numerical inversion is indeed the mode that corresponds to the observed resonance peak, the mode shape of the resonance can be used. Determination of mode shape is a common practice for measuring the vibration of machinery, automobiles, and airplanes using a large number of accelerometers. However, for measuring the mode shapes of laboratory-scale specimens, the use of accelerometers is neither accurate nor practical. This is because using a large number of accelometers increases the inertia of a specimen. Moving a single accelerometer on the surface of the specimen is not only work intensive but introduces poor repeatability of the measurement. A slight change of coupling between an accelerometer and a specimen causes a large change in the amplitude of the measured acceleration.

A laser doppler vibrometer measures the velocity on the surface of a specimen by measuring the interference between coherent incident light (laser) and reflected light by the specimen. The non-contacting nature of the method leads to good repeatability of measurements. As the measurement is done from a distance, the surface of a specimen can be scanned in a relatively short period of time.

The lead glass cube examined in Section 5.4.2 was scanned with a laser doppler vibrometer for mode shapes at several selected resonances. The experimental setup is 
shown in Figure 5.26. On one side of the cube, $1 \mathrm{~cm} \mathrm{x} 1 \mathrm{~cm}$ grids were drawn for a $10 \mathrm{~cm} \mathrm{x}$ $10 \mathrm{~cm}$ square area. At each grid point, a small reflector was glued so that the vibration of the surface could be measured. Velocity spectra were measured at each grid point using 0 to $32 \mathrm{kHz}$ swept sine waves (Chirp signal). The spectra were averaged over 20 measurements and normalized by the velocity spectra measured on the surface of the piezoelectric source suspended in the air. Figure 5.27 shows a comparison between several of the measured and computed mode shapes. Magnitudes of the normalized spectra at each resonance frequency are shown. Modes that did not have significant amplitude were not plotted as they were affected by neighboring major resonances. For degenerate modes (modes with identical resonance frequencies), only the mode shape for a single mode is shown. Most of the modes show very good agreement between measured and computed results, demonstrating that the modes are correctly identified. Some differences in the experimental mode shapes may be caused by interference between degenerate modes and distortion due to neighboring dominant modes.

Mode shapes were measured also for the granite cube examined in Section 5.4.3. Comparison between the selected mode shapes that had significant velocity spectral amplitudes are shown in Figure 5.28. A marked characteristic is that the mode shape for the surface perpendicular to the isotropy axis ( $z$-plane) is quite different from the surfaces parallel to the isotropy axis ( $x$ and $y$-planes). Most of the modes show good agreement between measured and computed mode shapes. Rather large differences are seen for the mode shapes with low-frequency resonances $\left(5,6\right.$, and $7^{\text {th }}$ modes). Although there is a chance that these modes were not correctly identified, the good fit between computed and experimental FRF's and mode shapes for many high-frequency resonances indicate that there may be some other reasons that causes the distortion of these mode shapes such as heteregeneity of the specimen and higher order anisotropy. 


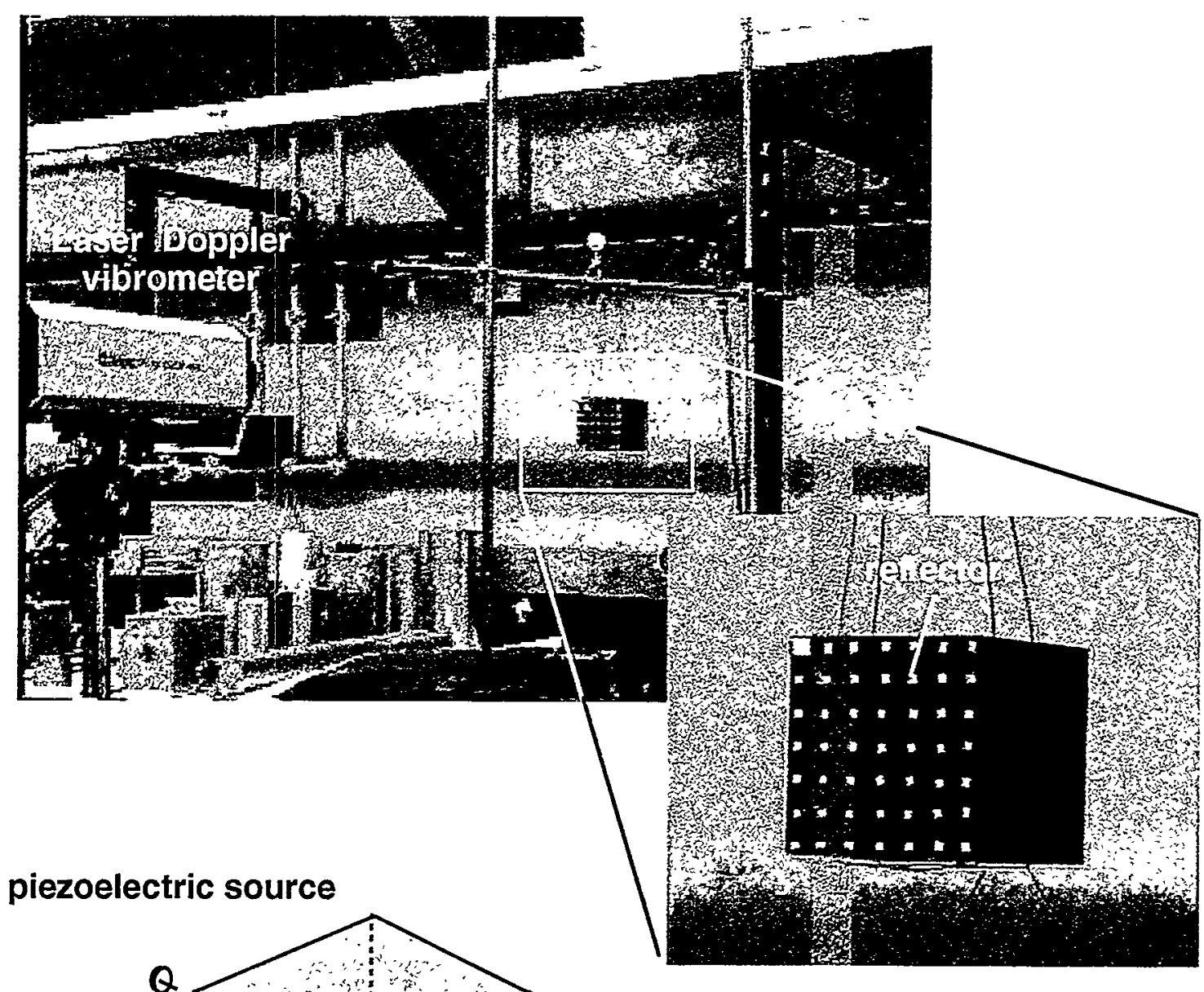

Q

Laser doppler vibrometer

\section{$1.0 \mathrm{~cm} \times 1.0 \mathrm{~cm}$ grids}

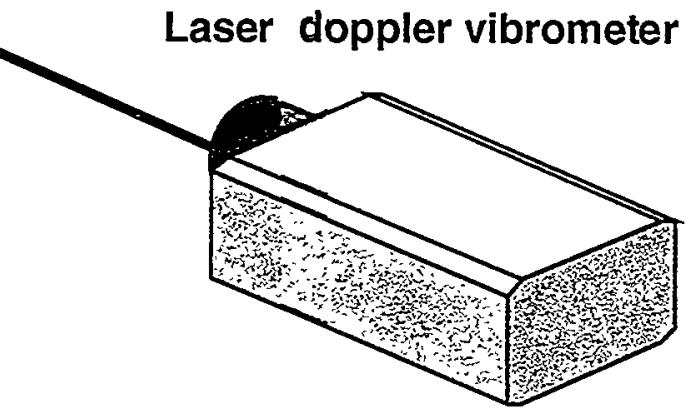

Figure 5.26 Experimental setup for measuring mode shapes of rock cubes. The cubes were resonated using a piezoelectric source swept by sine waves for frequencies ranging from 0 to $32 \mathrm{kHz}$. Velocities of the resulting surface vibration were measured by a laser Doppler vibrometer and then displayed, analyzed, and recorded on a spectrum analyzer. 

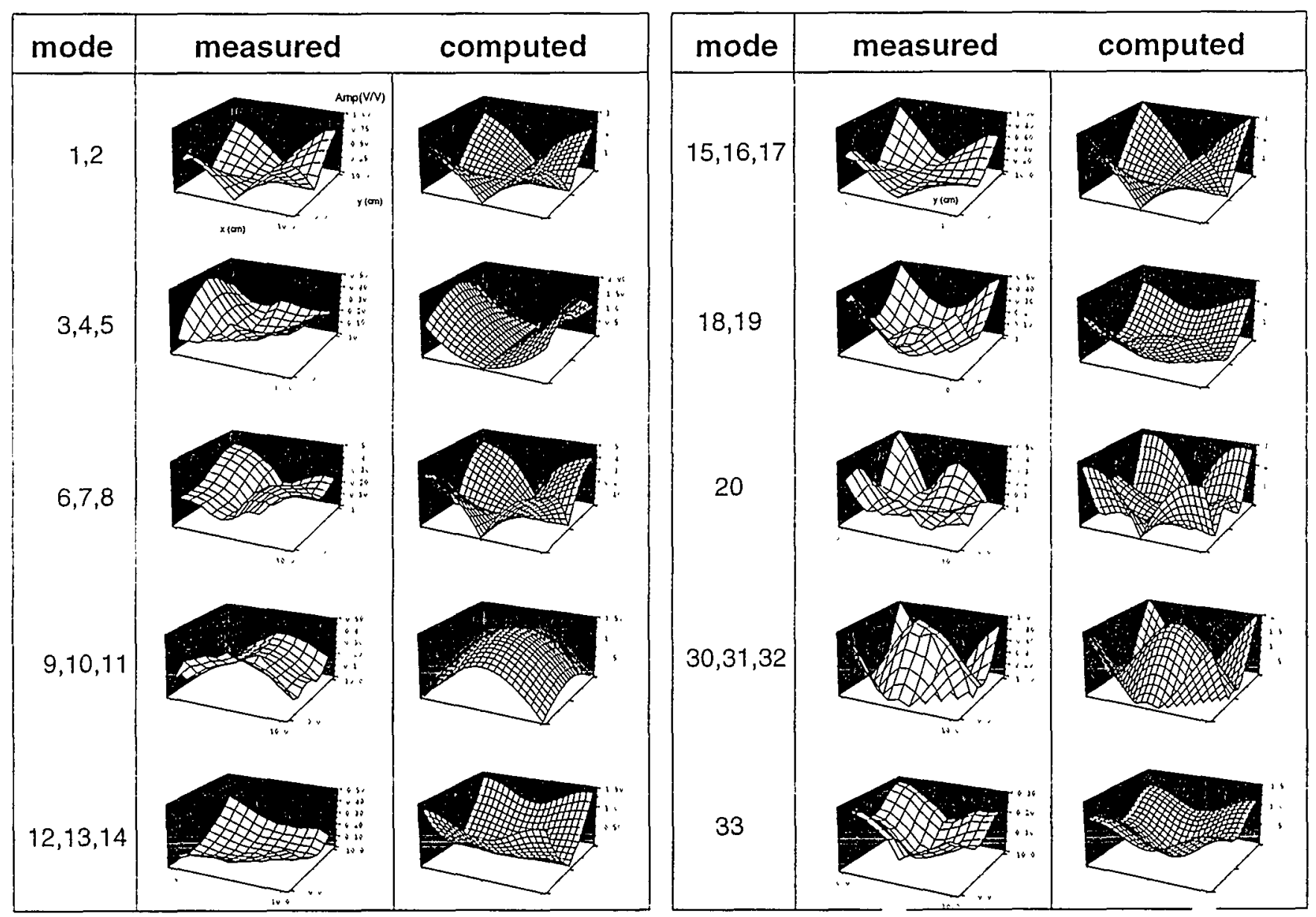

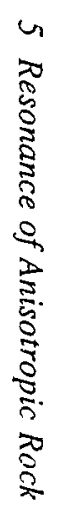

Figure 5.27 Several of the observed and computed mode shapes for the lead glass cube. Mode shapes (absolute amplitude is shown) were determined by scanning a surface of the cube with a laser-doppler vibrometer. Computed mode shapes used the Young's modulus and Poisson's ratio determined from the resonance inversion. 


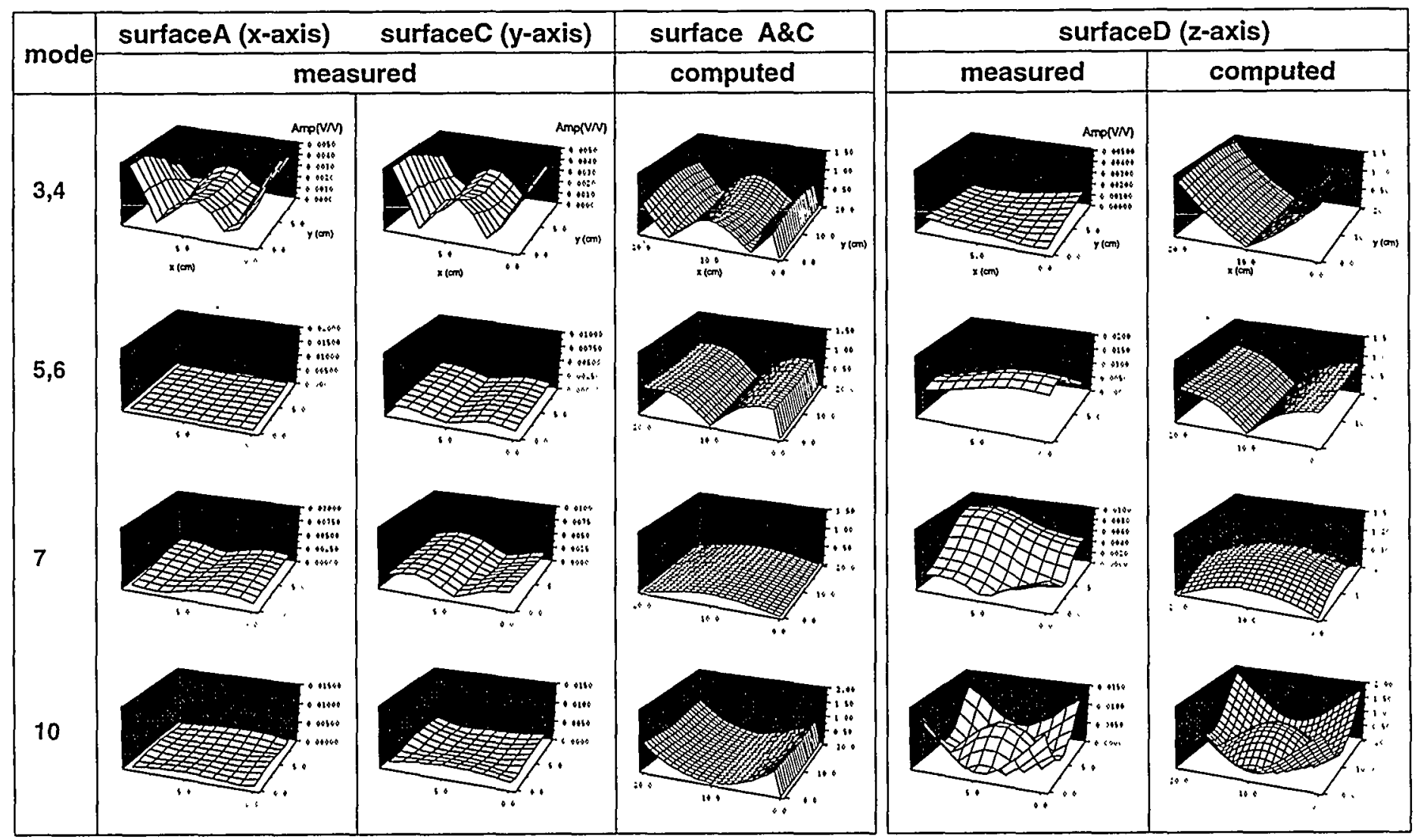

Figure 5.28 (a) Experimental and computed mode shapes for the granite cube. Experimental mode shapes are measured using a laser doppler vibrometer. Due to the strong anisotropy, mode shapes observed for surface $D$ are quite different from those for surface A and C. Some of the low-frequency modes show rather different shapes for the experimental and computed results. 


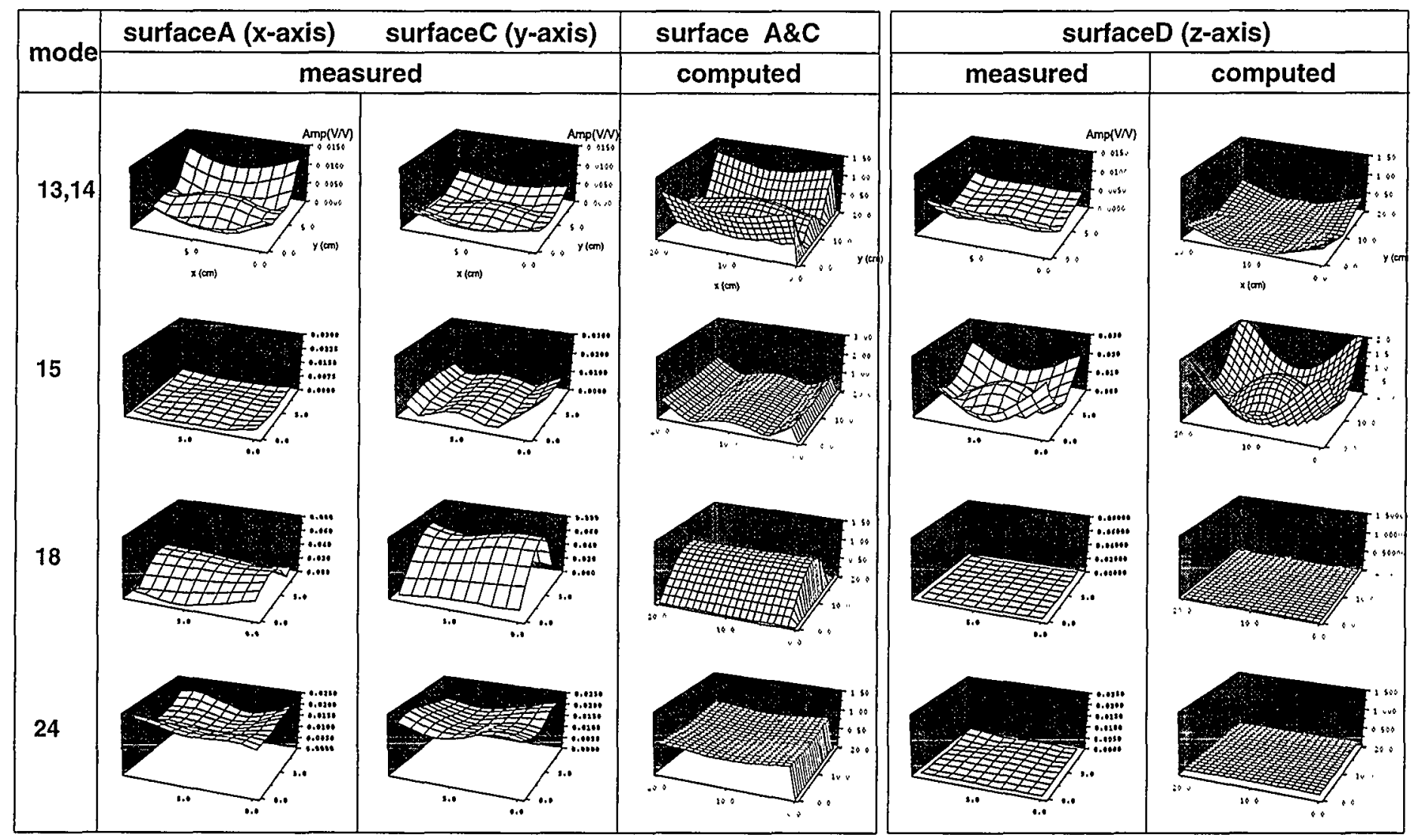

Figure 5.28 (b) Experimental and computed mode shapes for the granite cube. Experimental mode shapes are measured using a laser doppler vibrometer. The higher-frequency modes in the above plots show a very good agreement between the experimental and computed results. 


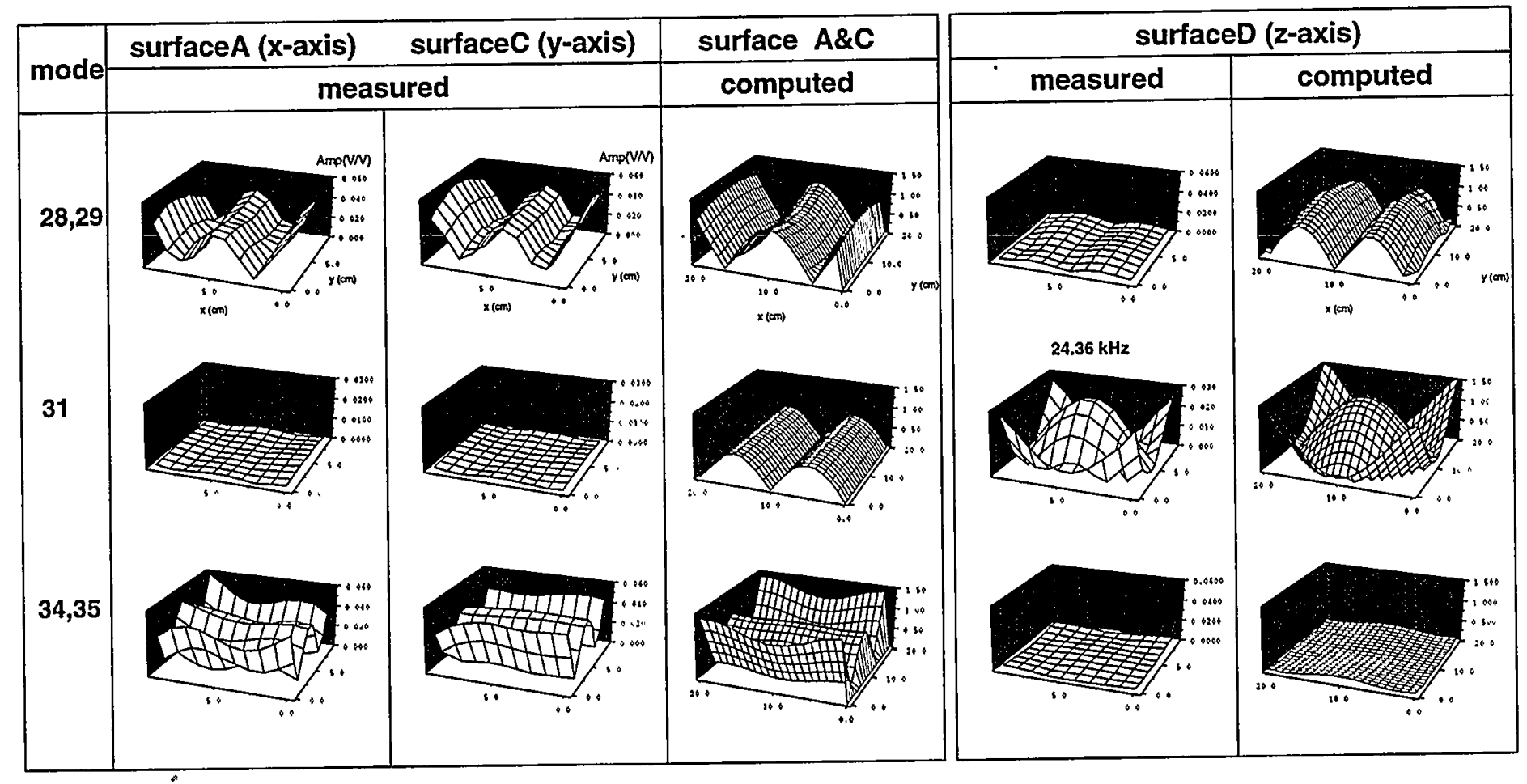

Figure 5.28 (c) Experimental and computed mode shapes for the granite cube. Experimental mode shapes are measured using a laser doppler vibrometer. The higher-frequency modes in the above plots show a good agreement between the experimental and computed results. 


\subsection{Summary}

In this chapter, an acoustic resonance spectroscopy technique is used for quantitative characterization of the bulk elastic properties of rocks. Using this technique, elastic moduli of an anisotropic rock are determined from a single measurement of the frequency response. Although such techniques have been applied to homogeneous materials such as single crystals and mineral grains, application to rocks whose elastic anisotropy arises from a variety of inherent microstructures has not been previously reported.

Microstructural features in rocks such as systematically oriented microcracks, bedding planes, and mineral inclusions result in anisotropic elastic behaviors. Using cube specimens of rocks, the medium to low frequency $(10 \mathrm{kHz}-30 \mathrm{kHz})$ elastic moduli of rocks are determined by the resonance inversion technique introduced in the previous chapter. As the acousic resonance spectroscopy method uses wave frequencies that are lower than those of the ultrasonic transmission tests $(100 \mathrm{kHz} \sim$ several $\mathrm{MHz})$ in the laboratory, measured dynamic properties of rock are closer to the properties measured in the field, especially for crosshole seismic measurements. Elastic moduli of a granite specimen show intermediate values between static moduli and moduli from high-frequency ultrasonic transmission tests. A sandstone specimen also shows moduli slightly lower than from the high-frequency wave transmission tests. Since the frequency-dependent behavior of the wave can be related to the micromechanical structure of rocks, such as compliant open microcracks and grain contacts, acoustic resonance spectroscopy can be a valuable tool for characterizing micromechanical rock properties if combined with other tests such as static loading tests and ultrasonic transmission tests. For example, oriented open microcracks that are indicated by frequency-dependent anisotropic dynamic properties of granite may be related to the rock's anisotropic hydraulic properties as is demonstrated in the experiment for granite specimens in Section 5.2.3.

Although a dolomite cube appeared to be approximately transversely isotropic from its ultrasonic transmission tests, the frequency responses of the specimen did not show any transverse isotropy. This result is possibly due to millimeter to centimeter-scale cracks and mineral inclusions. It is interesting that resonance spectroscopy, which uses longer wavelengths than the ultrasonic transmission test, is more sensitive to the heterogeneity of this specimen. Although the current research does not examine the effect of heterogeneities 
on acoustic resonance, the results indicates that it can be a diagnostic tool for detecting and locating heterogeneites in a rock.

Besides the measurements for frequency response, a laser doppler vibrometer was used to measure mode shapes of vibrations. For a homogeneous isotropic cube of lead glass, experimentally measured and numerically computed mode shapes showed good agreement. Results for a granite cube also showed good agreement confirming that the elastic moduli of the specimen used to compute the mode shapes were accurately determined from resonance inversion of the FRF's. Although the mode shapes for the dolomite specimen were not presented in this work, heterogeneity of this specimen may be characterized by measuring the mode shapes and inverting for distribution of elastic moduli or density. Development of such a technique is a topic for future research.

This chapter has demonstrated that acoustic resonance microscopy has many aspects suited for characterizing unique properties of rocks, such as frequency-dependent elastic moduli due to microstructure and heterogeneity. Although there are still many issues to be resolved to make acoustic resonance spectroscopy an accurate and robust rock characterization tool, its potential capabilities in the future will make the development of this technique an important research field. 


\section{Resonance of a Fractured Three-Dimensional Body}

\subsection{Introduction}

Semi-brittle materials such as rock and concrete often include partially or fully open microcracks and macroscale fractures that can serve as fast paths for fluid and gas transport, pore space for their storage, and structural weaknesses that increase the compliance of a structure and decrease its strength. It is important, therefore, to develop robust geophysical methods for locating and characterizing fractures. This chapter examines the effect of a fracture on the acoustic resonance of a three-dimensional body. From changes in the acoustic resonance characteristics such as resonance frequencies and related mode shapes, the characteristics of the fracture such as its location and stiffness can be assessed.

If fractures occur as microcracks uniformly distributed in a homogeneous medium, the bulk elastic moduli of the material decrease due to the additional compliance of individual cracks (Walsh, 1965a,b; Budiansky and O'Connell, 1976; Walsh and Grosenbaugh, 
1979). As was discussed in the previous chapter (Chapter 5), such an effect can be anisotropic if the microcracks are oriented in any preferred directions. Due to a decrease in elastic moduli, materials containing microcracks exhibit a decrease in wave velocities and resonance frequencies of vibration. Therefore, by measuring changes in the dynamic properties of materials, damage introduced into materials can be evaluated. For example, Powers (1938) measured resonance frequencies of concrete specimens to determine their dynamic Young's modulus and Poisson's ratio. Based on his study, Powers suggested to use the changes in the resonance frequencies to evaluate degradation of specimens. Such an approach is valid if the dynamic behavior of damaged material can be described by an equivalent homogeneous material with decreased elastic moduli.

If a medium contains a distinct fracture in the form of partially contacting interface or a zone of locally concentrated microcracks (damage), the dynamic behavior of the medium cannot be explained by the behavior of an equivalent homogenous medium. Waves propagating in such materials exhibit attenuation and multiple arrivals due to scattering by the fractures as well as a decrease in wave velocity. The resonance characteristics of such materials are also different from a homogenous medium, showing changes in spectral response and mode shapes. Therefore, explicit interaction between waves and fractures has to be taken into account in examining the dynamic behavior of a medium with distinct fractures.

Carino et al. (1984) and Lin and Sansalone (1994) experimetally and numerically studied the effects of fractures on the spectral characteristics of the echo for waves generated by a surface impact (Impact Echo method). An interval between echoes can be seen as a period for one-dimensional resonance of the medium between a fracture and a free surface. Davis and Hertlein (1987) examined the transient dynamic response of structures including fractures subjected to a hammer impact (Impact Response method). Unlike the method using wave echoes, Davis and Hertlein's method excites part of the resonance modes for the entire structure. Both methods examine the spectral characteristics of measured waves and relate them to the location and properties of fractures. In applying the Impact Echo method and the Impact Response method, fractures are usually considered as open gaps. Sakata and Ohtsu (1995) applied ultrasonic spectroscopy to concrete members with a notch by resonating the specimens using sine waves swept over a range of frequencies. The effect of the fracture on the frequency responses of the concrete members was studied by varying the depth of the notch from $0 \%$ to $90 \%$ of the cross section. Bamnios and Trochidis (1995) performed similar measurements on a cantilever beam with 
a notched cross section.

If a fracture is modeled as an open notch or thin void, no mechanical coupling is allowed across the fracture. However, in a structure that is subjected to compressional load, the surfaces of a fracture can be partially in contact allowing some degree of mechanical coupling. In such cases, the stiffness of a fracture plays an important role in determining the resonance characteristics of a damaged structure. Pyrak-Nolte et al. (1990a) showed that the velocities and spectral amplitudes of waves transmitted through single fractures in rock strongly depend on the fracture stiffness. Hesler (1995) examined resonance of a material containing a fracture with finite stiffness. In Hesler's work, the dynamic behavior of a fracture was modeled using the displacement-discontinuity boundary conditions (Schoenberg, 1980; Pyrak-Nolte et al., 1990a). Results of the analysis showed that the stiffness of a fracture in a slender bar alters the resonance frequency and mode shape of the vibrations significantly. Hesler also conducted laboratory resonance measurements on a plexiglass rod with a saw-cut interface. The interface was glued with wax, providing a thin layer of increased compliance analogous to a fracture with an imperfect contact. Measured resonance frequencies showed good agreement with analytical results, which demonstrated that the model provides a good approximation for describing the dynamic behavior of a fracture during resonance.

The objective of this chapter is to extend Hesler (1995)'s work to include resonance of three-dimensional bodies containing single fractures. The primary focus of the research is on the effect of fracture stiffness on resonance. Free vibrations of a cylindrical concrete specimen with a through-going fracture are examined experimentally. During the experiment, the stiffness of the fracture is changed by applying axial load through a pair of compliant plastic rings. Due to the strong impedance contrast between the rings and the specimen, the rings have little effect on the resonance frequencies of the specimen. The observed resonance is simulated using the numerical model developed in Chapter 4. From measured resonance frequencies, the dynamic stiffnesses of the fracture are determined and agree favorably with the statically measured stiffnesses. 


\subsection{Experiment}

\subsubsection{Properties of the intact specimen}

A cylindrical specimen of concrete was fabricated for a series of static and dynamic tests. The specimen was $10.16 \mathrm{~cm}$ (4 inches) in diameter and $19.2 \mathrm{~cm}$ ( 7.55 inches) in length. The water to cement ratio of the specimen was 0.5 and the volumetric ratio of the aggregate was $73 \%$ which included a coarse aggregate (limestone, $30 \%$ ) and sand (43\%). The specimen had been immersed in lime saturated water for several months before the tests. During the tests, the specimen was wrapped with a plastic film to avoid evaporation of the pore water. The density of the specimen was $2.47 \mathrm{~g} / \mathrm{cm}^{3}$.

Before a fracture was introduced, the intact concrete cylinder was loaded axially to measure its Young's modulus. The compression of the specimen was measured using a pair of LVDT's directly mounted to the sides of the cylinder. The applied axial stress was measured using a loadcell. Figure 6.1 shows the measured stress-strain relation for the intact specimen. For the measured range of axial stresses, the specimen exhibited an approximately linear elastic stress-strain relation with very small hysteresis. Such behavior

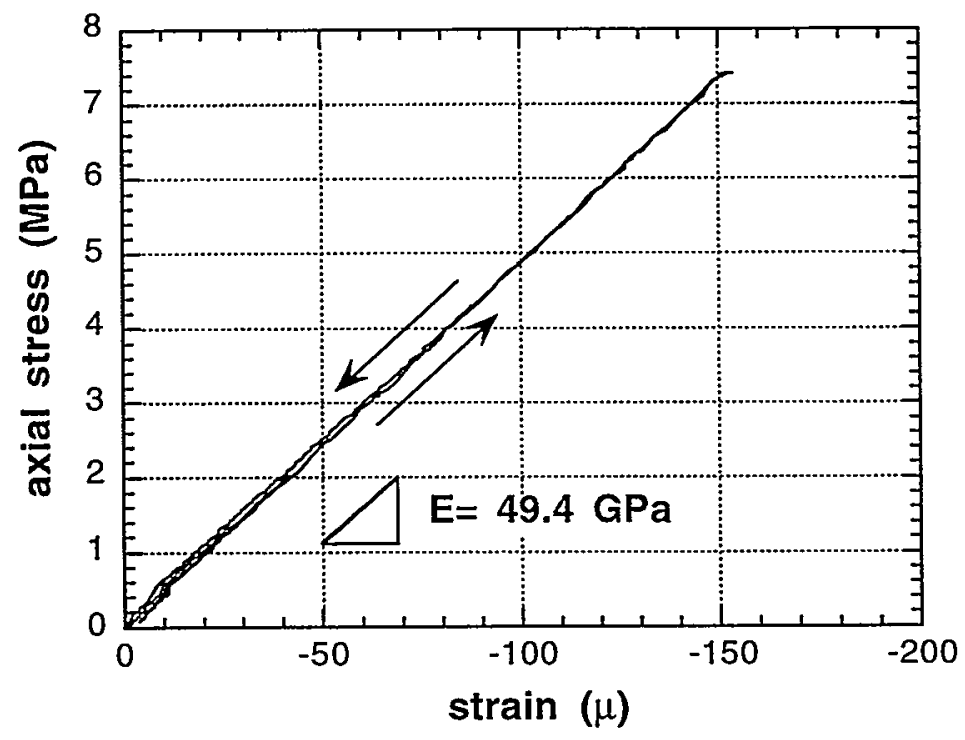

Figure 6.1 Uniaxial compression of an intact concrete cylinder. The measured stress-strain relation shows very small nonlinearlity and hysteresis. 
indicates that the specimen does not contain any significant number of compliant microcracks that would result in non-linear and hysteretic behavior. This is possibly because the specimen was tested in its virgin condition (no load had been applied). From the slope of the stress-strain curve, the Young's modulus of the specimen was determined to be $49.4 \mathrm{GPa}$. This value is large for concrete and is possibly due to the large volumetric content of the aggregate. However, actual moduli of the aggregate are unknown.

Dynamic elastic properties of the specimen were also measured using ultrasonic waves. Measured velocities of $P$ and S-wave components were $5233 \mathrm{~m} / \mathrm{sec}$ and $2961 \mathrm{~m} / \mathrm{sec}$, respectively, yielding a dynamic Young's modulus of $54.8 \mathrm{GPa}$ and a Poisson's ratio of 0.26 . Central frequencies of the source $P$ and $S$ - waves were $850 \mathrm{kHz}$ and $500 \mathrm{kHz}$, respectively. The difference between dynamically and statically measured Young's moduli may be due to errors in the lower resolution static measurement. For this reason, the numerical analysis performed later in this chapter uses the results of the dynamic measurements.

\subsubsection{Static behavior of the fracture}

A through-going tensile fracture was created by applying a point load along the diameter of the cylindrical specimen (Brazilian loading). The surface of the fracture was perpendicular to the axis of the specimen and located $5.08 \mathrm{~cm}$ ( 2 inches) from the top. To measure the closure of the fracture under axial load, LVDT's were attached to the sides of the specimen across the fracture (Figure 6.2).

An example of the measured stress-displacement behavior of the fracture during the tests is shown in Figure 6.3. Strong nonlinearity and hysteric behavior is seen as is the time-dependent closure of the fracture (the fracture "creeps" under constant axial load). Displacements measured by the two LVDT's are different for low axial stresses because of the uneven contact of the fracture surface. The closing behavior on both sides become similar for higher axial stresses as the distribution of contacting asperities becomes uniform.

The nonlinear deformation of the fracture is caused by imperfect contact between fracture surfaces. As stress applied on the fracture increases contact between the surface asperities, stiffness of the fracture increases with increasing stress (Brown and Sholz, 1985). The closure of a fracture is also accompanied by frictional slip and wear that are 


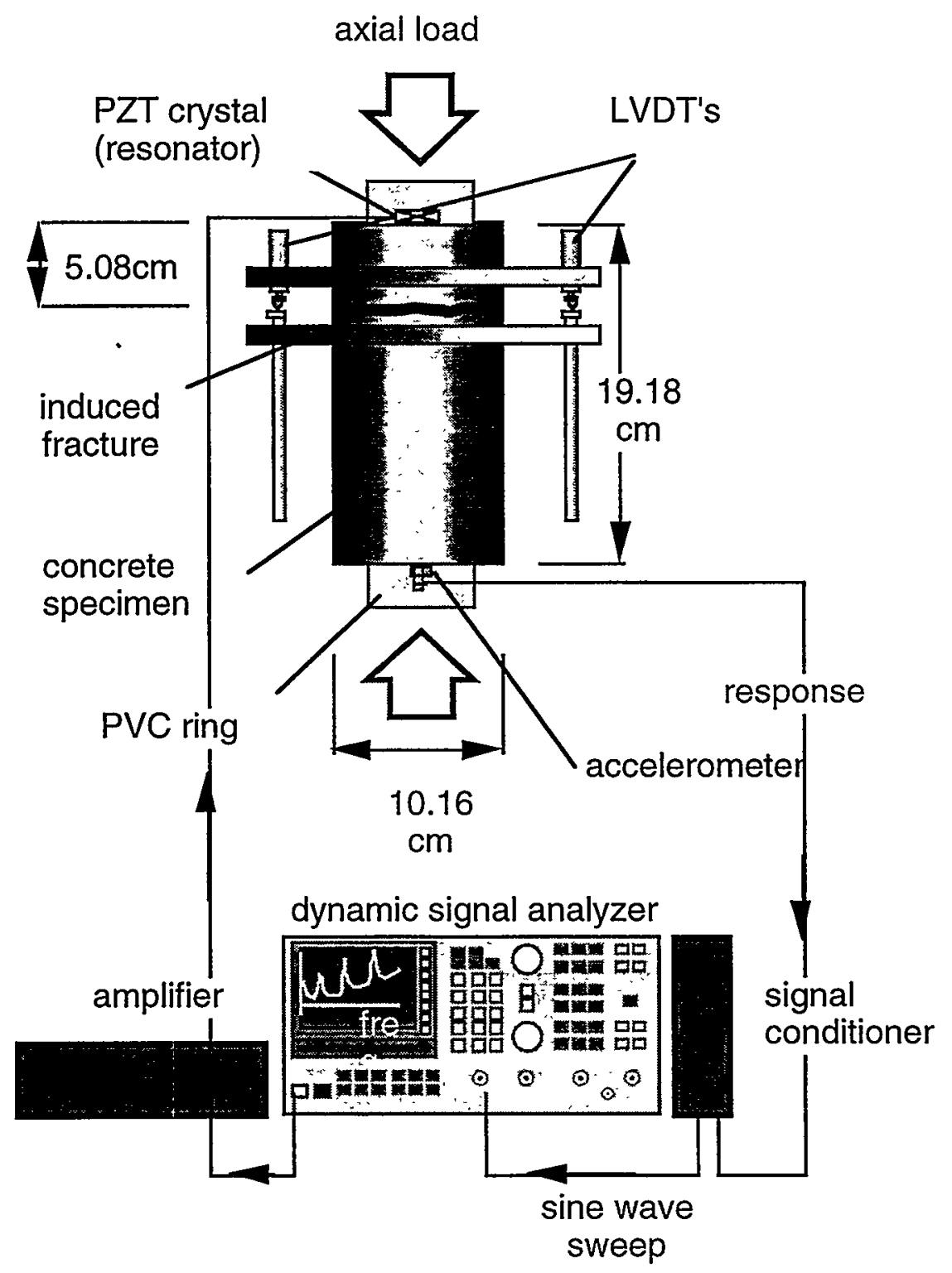

Figure 6.2 Experimental setup used for resonance test of a concrete cylinder containing a single throughgoing fracture. Axial stress was applied through PVC rings at the top and bottom of the specimen. Static closure of the fracture was measured by LVDT's attached to the sides of the specimen. 


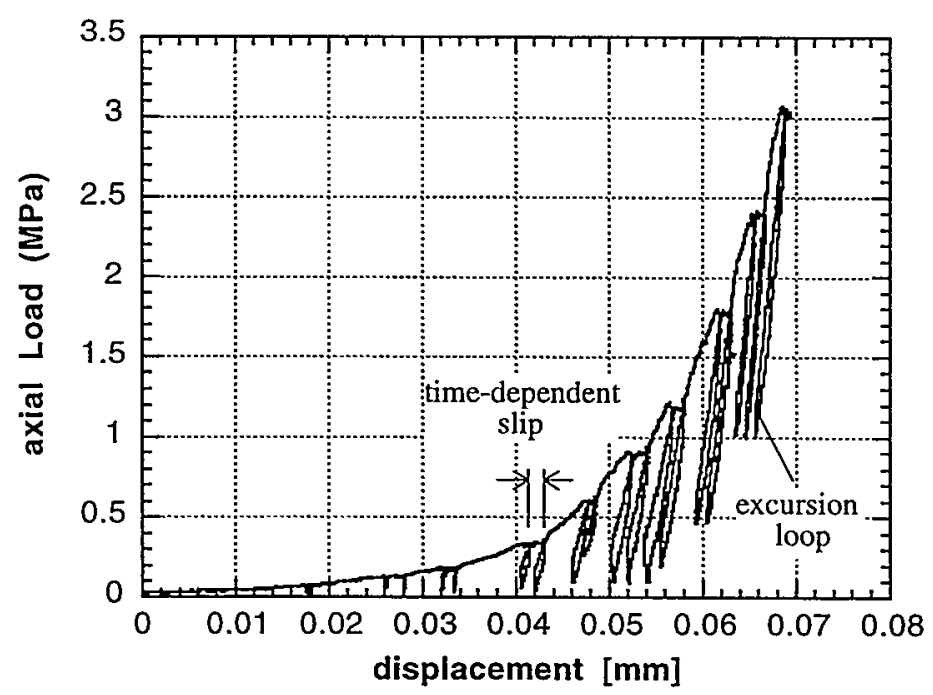

(a) LVDT 1 (left side of specimen)

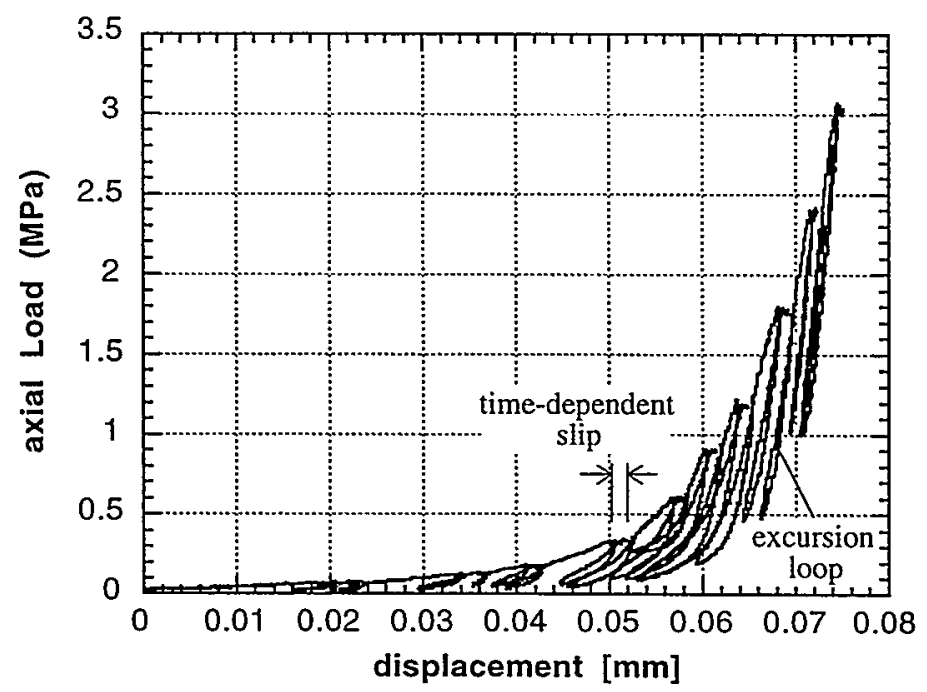

(b) LVDT 2 (right side of specimen)

Figure 6.3 Examples of closing displacement of fracture measured by LVDT's on both sides of specimen. Due to uneven contact distribution on the fracture, displacements measured by the two LVDT's show different displacements especially for low axial stresses. Small excursion loops are made before and after each resonance test at each loading stage and used to determine the elastic stiffness of the fracture. 
seen as stress-displacement hysteresis. It was anticipated that the time-dependent slip could cause a change in fracture stiffness during the measurements. To avoid errors resulting from this effect, resonance measurements were postponed for at least fifteen minutes after each load increment and decrement.

Closing displacement of the fracture can be obtained by subtracting the deformation of intact part of the specimen from the measured displacement across the fracture (Goodman, 1976). As the deformation of the intact part was not measured concurrently, the Young's modulus of the intact specimen $(49.5 \mathrm{GPa})$ was used to estimate the deformation. As resonance of the specimen measured in this study involves only a small displacement, the dynamic behavior of the specimen depends only on the elastic stress-strain relation (Walsh, 1965b; Cook and Hodgson, 1965). For this reason, slopes of the small excursion loaddisplacement loops were used to estimate the elastic moduli of the specimen. The excursion loops were made at each loading stage before and after the resonance of the specimen was measured. The slope of the loops was corrected for the deformation of the intact part of the specimen to obtain the stiffness of the fracture. Measured static fracture stiffnesses are shown later in this chapter. However, it should be noted that the fracture stiffnesses measured for low axial stresses are not accurate due to the uneven contact of the fracture surface.

\subsubsection{Experimental setup for the resonance tests}

Resonance behavior was examined by vibrating the specimen with continuous sine waves, accomplished by the experimental setup shown in Figure 6.2. A small piezoelectric crystal was attached to the top of the specimen and was driven electronically over a frequency range of $50 \mathrm{~Hz}$ to $50 \mathrm{kHz}$. A miniature accelerometer (PCB Piezotronics, 309A, resonance frequency $>120 \mathrm{kHz}$ ) was attached to the specimen to measure the resulting acceleration of the surface. The measured acceleration was analyzed and stored on a dynamic signal analyzer that was also used for generating the source signals. A range of axial stresses were applied from 0 to $3.0 \mathrm{MPa}$ and acceleration spectra were measured at each stress. Two locations on the specimen were selected to measure its surface vibrations: For "T-configuration" tests, an accelerometer was attached to the bottom of the specimen. For "R-configuration" tests, it was attatched to the specimen next to the source (piezoelectric crystal). The T-configuration was used for measurements conducted during the loading cycle up to axial stress of 3.0MPa while R-configuration was used for the unloading cycle. 


\subsubsection{Effect of axial stress on intact specimen}

In order to change the stiffness of the fracture during the test, an axial load was applied to the specimen through a pair of PVC rings designed to isolate the vibrations of the specimen from the load frame. As the stiffness of the PVC rings is much smaller than the stiffness of the concrete cylinder or the fracture, the resonance behavior of the specimen approximates free vibrations. To examine the effect of the axial load applied through the PVC rings, an intact specimen was tested for its resonances with increasing axial stress. The result shown in Figure 6.4 compares measured frequency response functions (FRF's) for the specimen with axial stresses of OMPa, 1.5MPa, and 3MPa. To compute the FRF's, measured acceleration spectra were normalized by mass and a spectrum measured for the source suspended in air. As a product between mass and acceleration provides force, approximate frequency response functions (accelerance) are obtained by such normalization. Resonance frequencies of the first three modes are shown in the plot. The result demonstrates that the axial loading through the plastic rings did not cause significant effects on the resonance frequencies of an intact concrete. The insensitivity of resonance frequencies to stress also confirms that the intact specimen did not include compliant microcracks that would cause change in the overall stiffness of the material.

\subsubsection{Resonance of the fractured specimen}

Resonances of the fractured concrete specimen were measured at each load level for both $\mathrm{T}$ and R-configurations. Figure 6.5(a) and (b) show FRF's measured at the bottom ( $\mathrm{T}$ - configuration) and top (R-configuration) of the specimen, respectively. Each FRF is plotted with $20 \mathrm{~dB}$ of vertical offset. Both plots show a monotonic increase in resonance frequencies with increasing axial stress. For the maximum applied stress (3MPa), the FRF's for both configurations become close to those for an intact specimen.

Although most of the resonance peaks persist throughout the test, the FRF's measured at the bottom of the specimen show peaks (such as the mode " $F$ ") that can be seen only for low to medium axial stresses. By comparing the Figure 6.5(a) and (b), it is also noted that some of the resonance peaks observed for FRF's measured at the bottom of the specimen disappear from the FRF's measured at the top of the specimen for small axial stresses (such as the mode "E2").

The resonance peaks observed for low to intermediate stresses with a T-configuration are possibly the modes associated with flexural motions. Although the accelerometer 


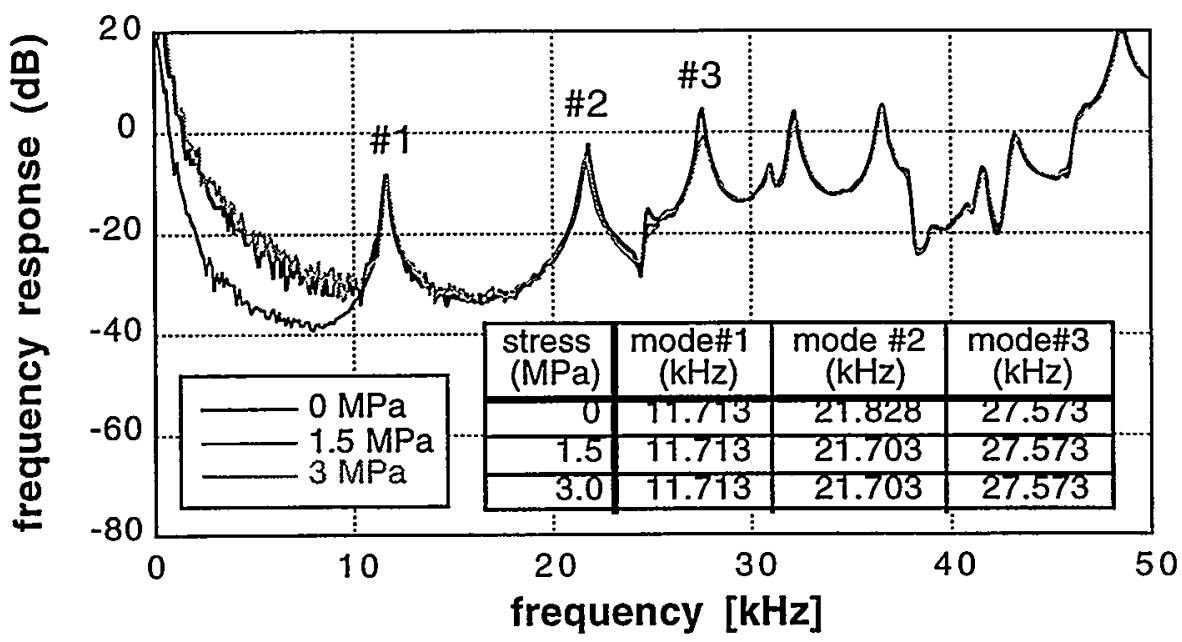

Figure 6.4 Measured FRF for an intact specimen. An accelerometer was attached to the bottom of the specimen ( $\mathrm{T}$-config). Axial stress was increased from 0 to $3 \mathrm{MPa}$. Resonance frequencies of the low-order modes do not show a significant change. A small frequency shift for the second mode is comparable to the resolution of the spectra $(125 \mathrm{~Hz})$.

located on and along the axis of the specimen should not detect the flexural motions, uneven distribution of the contact stiffness along the fracture breaks the symmetry of the specimen, introducing small axial motion to the flexural modes. The resonances that were seen only at the bottom block for small axial load are the localized resonances in the bottom block. Such behavior of a fractured system has also been observed in the one-dimensional analysis performed in an earlier chapter (Chapter 2, Section 2.2.1. Also Figure 2.1). This phenomenon is particularly interesting because large vibration is occurring in the bottom block even though the source is located on the top block. As the stiffness of the fracture increases, resonance in the bottom block progressively extends its motion to the top block, making the vibration less localized.

The amplitudes of FRF's measured at the bottom of the specimen become significantly smaller for small axial loads. On the contrary, the amplitudes of the FRF's at the top of the specimen either do not change or they become even greater. Some of the modes shown in Figure 6.5(b) become especially pronounced for low axial stresses (shown as "M1" and "M2"). Such effects are due to the isolation of vibration energy in the top block. Due to the large compliance of the fracture, most of the energy in waves incident on the fracture is reflected and causes localized vibration motions in the section of the specimen near the source. 
[Measured FRF at the top of the specimen]
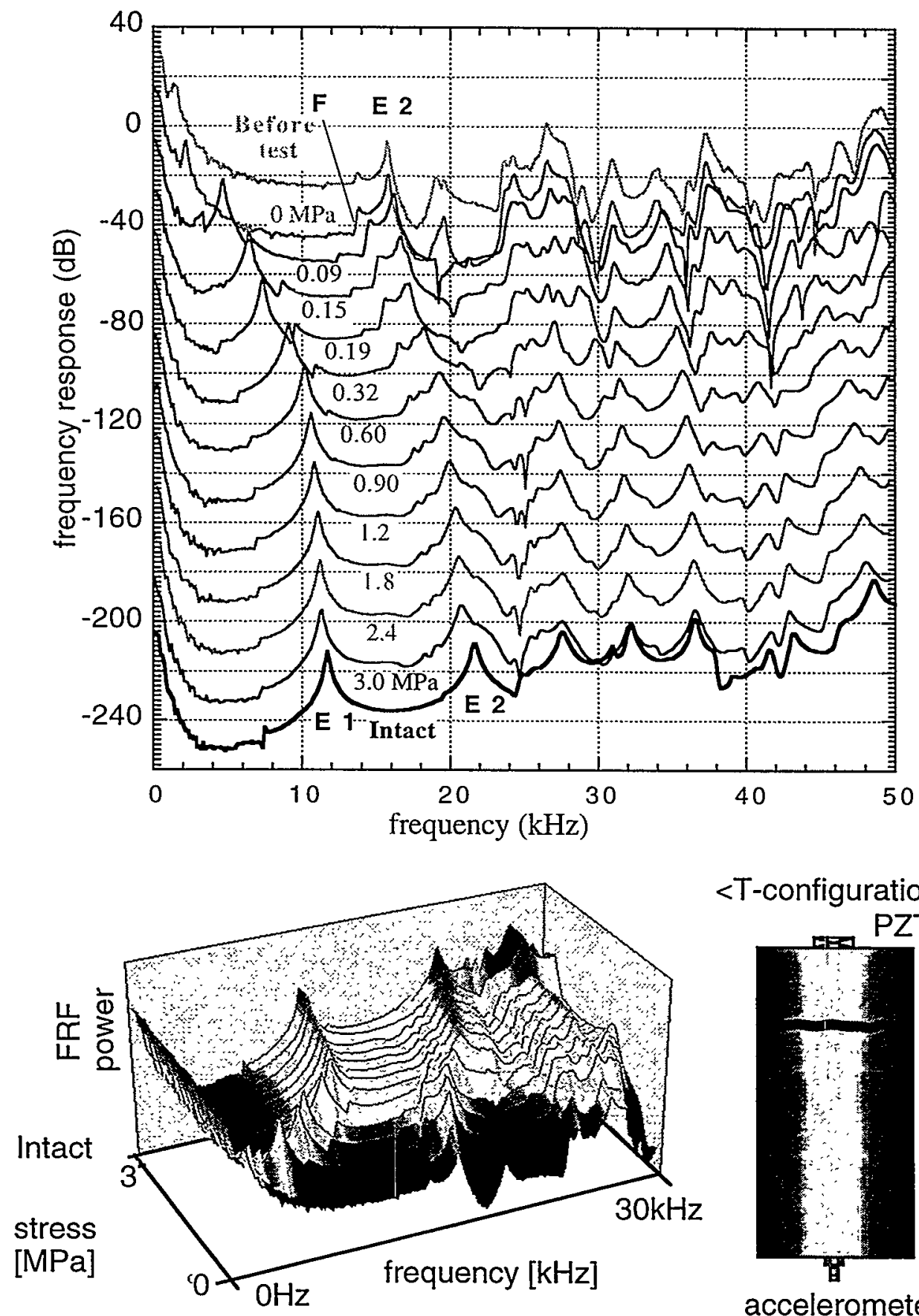

$<T$-configuration $>$

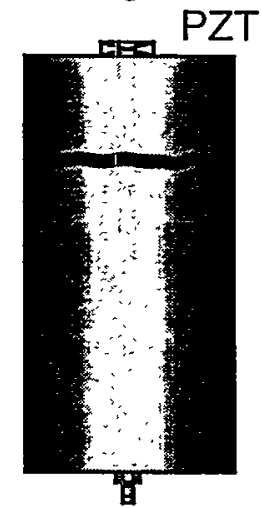

accelerometer

Figure 6.5 (a) Measured FRF's of fractured concrete cylinder. FRF's are offset by 20dB. An accelerometer was attached to the bottom of the specimen (T-config.). As axial stress is increased, resonance frequencies increase, approaching those for an intact specimen. It can be seen that the lower order modes show larger shifts than the higher order modes. 
[Measured FRF at the bottom of the specimen]
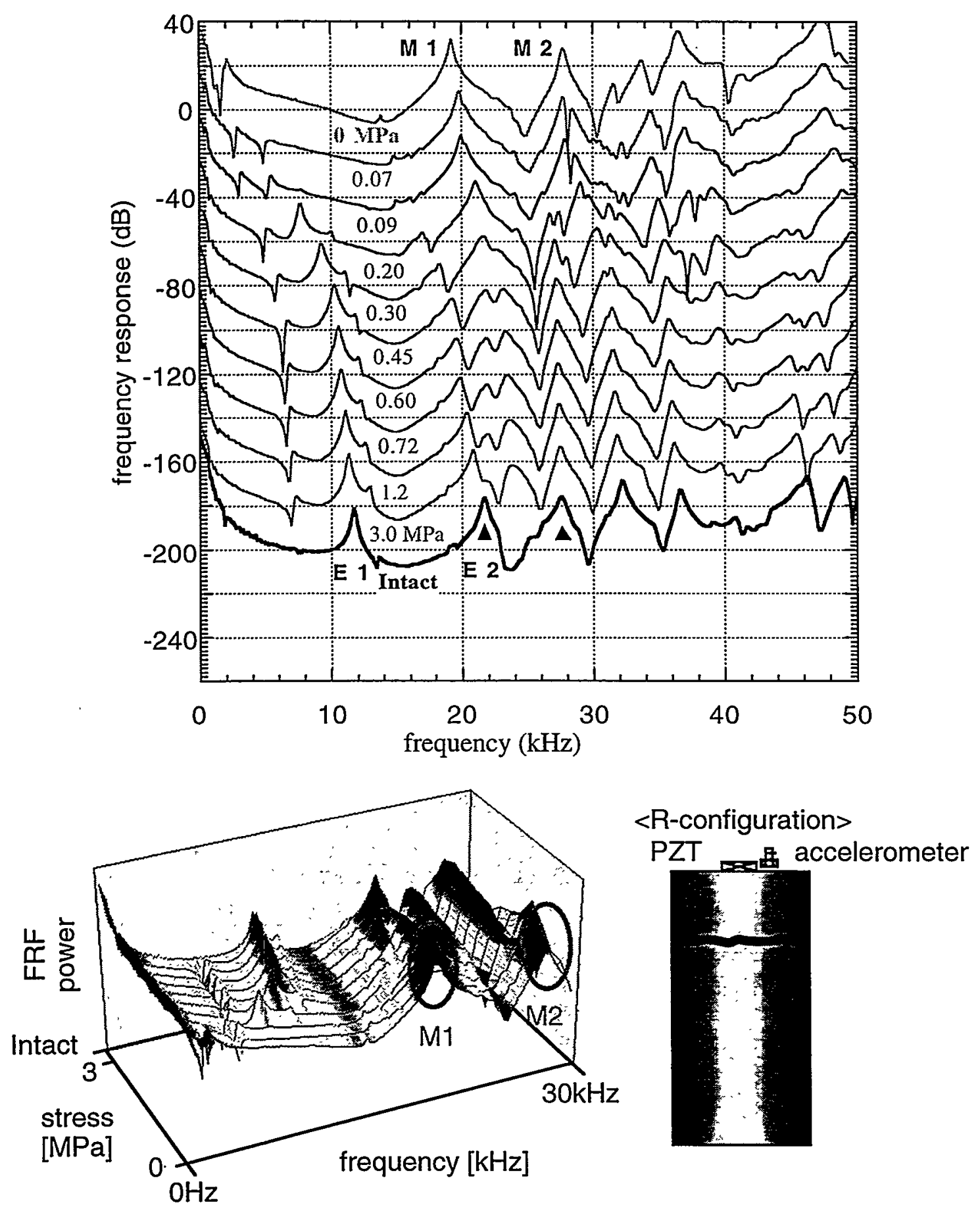

Figure 6.5 (b) Measured FRF's of a fractured concrete cylinder. An accelerometer is attached to the top of the specimen (R-config.). Unlike the T-configuration tests, some of the modes (shown by solid triangles) seem to disappear from the FRF's for low axial stresses. As a result, the FRF's for low axial stresses become much simpler than those for the T-configuration. The resonance amplitude in the FRF's for low axial stresses become very large due to the trapped vibration energy in the top block. 


\subsection{Numerical Simulation}

\subsubsection{Computed FRF's}

The numerical code developed in Chapter 4 was used to simulate the resonance of a fractured concrete cylinder. A numerical model was built assuming that the intact part of the specimen was isotropic and homogeneous, and that the fracture had a planar geometry. A vertical source was located at the center of the top surface as in the experiment. The dynamic Young's modulus and the Poisson's ratio from high-frequency seismic transmission tests were used for the elastic properties of the model. For simplicity, normal and tangential stiffnesses of the fracture were assumed to be identical. The highest order of polynomials used for approximating the displacement field in the block were $(l, m, n)=(5$, $5,4)$ for the smaller top block and $(5,5,6)$ for the larger bottom block, where the components of displacement are given by

$$
u_{i}=\sum_{\lambda=1}^{N} a_{i \lambda(l, m, n)} \cdot x^{l} y^{m} z^{n}, \quad(i=x, y, z)
$$

In the above expression, $a_{i \lambda}$ is a coefficient for each basis function. The $z$-axis was taken along the axis of the cylinder. Although the orders of the polynomials used for the displacement field approximation were relatively low, they were found to provide sufficient accuracy for low order modes. Table 6.1 shows the first three resonance frequencies of both torsional and longitudinal resonance (singlet modes) for models with different orders of the polynomial approximation. Figure 6.6 shows computed FRF's for the Tconfiguration. Cases $\mathrm{a}, \mathrm{b}$, and $\mathrm{c}$ were computed for a single block and $\mathrm{d}$ was computed for two blocks with a welded fracture (stiffness of fracture $=10^{20} \mathrm{~Pa} / \mathrm{m}$ ). The table shows that case $d$ (low order approximation with two blocks) yields results similar to higher order approximations for low-frequency resonances considering the resolution of the experimental data $(125 \mathrm{~Hz})$. Doublet modes also show good results (Table.6.2) with slightly greater differences than the singlet modes. As the numerical inversions performed later in this chapter require many computational iterations for resonance frequencies and vectors, the use of an unnecessarily high order approximation was not desirable. Therefore, justified by the above results, a lower order approximation was used for simulating the resonance of the fractured specimen. 


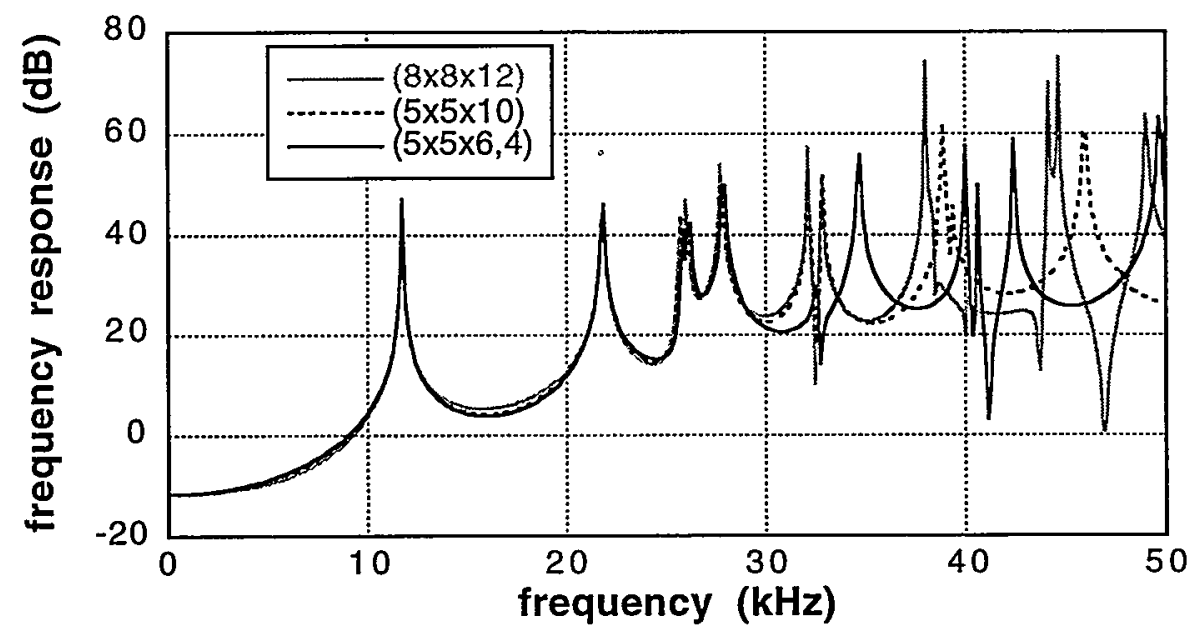

Figure 6.6 Comparison of computed FRF's with different degree of approximations. Source and receiver are located on top and bottom in the numerical model of the concrete cylinder. The plot shows that the low-order approximation used for the subsequent analyses compares well with the higher-order approximations up to about $30 \mathrm{kHz}$.

Frequency response functions for the fractured concrete cylinder were computed for a range of fracture stiffnesses. Figure 6.7(a) and (b) show computed FRF's for a receiver located at the bottom and top of the specimen, respectively. The computed FRF's have very sharp resonance peaks as no attenuation was assumed in the numerical model. From a comparison between numerical (Figure 6.7) and experimental results (Figure 6.5), it can be seen that changes in FRF's with increasing fracture stiffness of the numerical model are similar to those for the experimental FRF's with increasing axial stress.

Table 6.1 Comparison of computed resonance frequencies (in $\mathrm{Hz}$ ) for singlet modes.

\begin{tabular}{|c|c|c|c|c|c|c|}
\hline mode & $\mathrm{T} 1$ & $\mathrm{~T} 2$ & T3 & E1 & E2 & E3 \\
\hline $\begin{array}{l}\text { a. }(8 \times 8 \times 12) \\
\text { b. }(8 \times 8 \times 10) \\
\text { c. }(5 \times 5 \times 10) \\
\text { d. }(5 \times 5 \times 4,6)\end{array}$ & $\begin{array}{l}7473.7 \\
7473.7 \\
7473.7 \\
7482.7\end{array}$ & $\begin{array}{l}14947 \\
14947 \\
14947 \\
14967\end{array}$ & $\begin{array}{l}22421 \\
22421 \\
22421 \\
22564\end{array}$ & $\begin{array}{l}11722 \\
11722 \\
11722 \\
11735\end{array}$ & $\begin{array}{l}21106 \\
21106 \\
21106 \\
21106\end{array}$ & $\begin{array}{l}27787 \\
27791 \\
27795 \\
27938\end{array}$ \\
\hline $\mathrm{d}-\mathrm{a}(\mathrm{Hz})$ & 9.0 & 20 & 143 & 13 & 0 & 151 \\
\hline
\end{tabular}

Table 6.2 Comparison of computed resonance frequencies (in $\mathrm{Hz}$ ) for doublet modes.

\begin{tabular}{|c|c|c|c|c|c|c|}
\hline mode & $\mathrm{F} 1$ & $\mathrm{~F} 2$ & F3 & $\mathrm{F} 4$ & F5 & F6 \\
\hline $\begin{array}{l}\text { a. }(8 \times 8 \times 12) \\
\text { b. }(8 \times 8 \times 10) \\
\text { c. }(5 \times 5 \times 10) \\
\text { d. }(5 \times 5 \times 4,6)\end{array}$ & $\begin{array}{l}7485.0 \\
7485.1 \\
7485.1 \\
7499.7\end{array}$ & $\begin{array}{l}13474 \\
13474 \\
13474 \\
13496\end{array}$ & $\begin{array}{l}19034 \\
19034 \\
19034 \\
19079\end{array}$ & $\begin{array}{l}19248 \\
19249 \\
19249 \\
19286\end{array}$ & $\begin{array}{l}19583 \\
19583 \\
19584 \\
19704\end{array}$ & $\begin{array}{l}19685 \\
19693 \\
19694 \\
19753\end{array}$ \\
\hline $\mathrm{d}-\mathrm{a}(\mathrm{Hz})$ & 14.7 & 22 & 45 & 38 & 121 & 68 \\
\hline
\end{tabular}


[Computed FRF at the top of the cylinder]
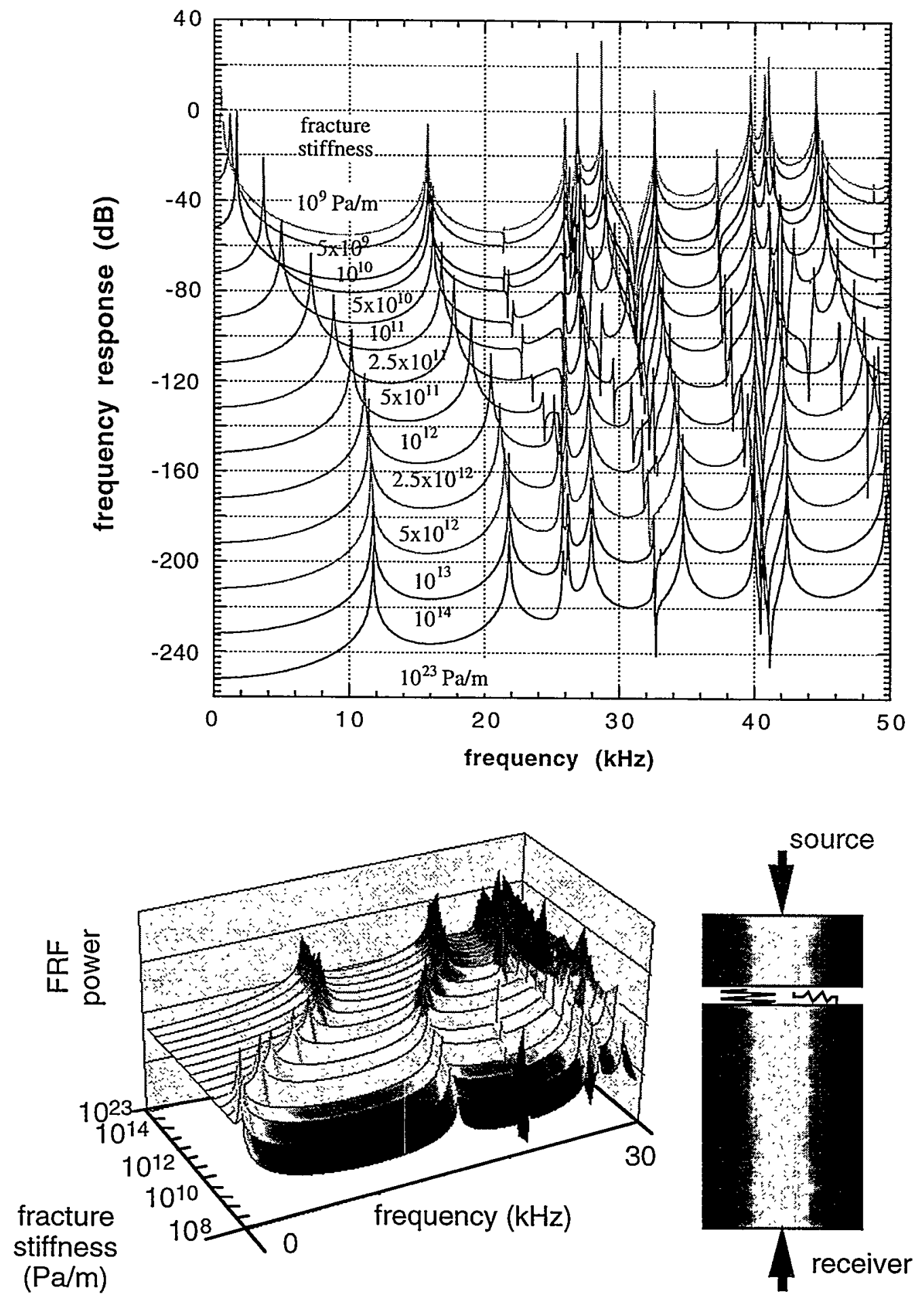

Figure 6.7 (a) Computed FRF's of a fractured concrete cylinder. FRF's are offset by 20dB. The source and receiver are located on the top and bottom of the model. Increasing fracture stiffness results in changes in the FRF's similar to the changes in experimental FRF's with increasing axial stress. 
[Computed FRF at the top of the cylinder]
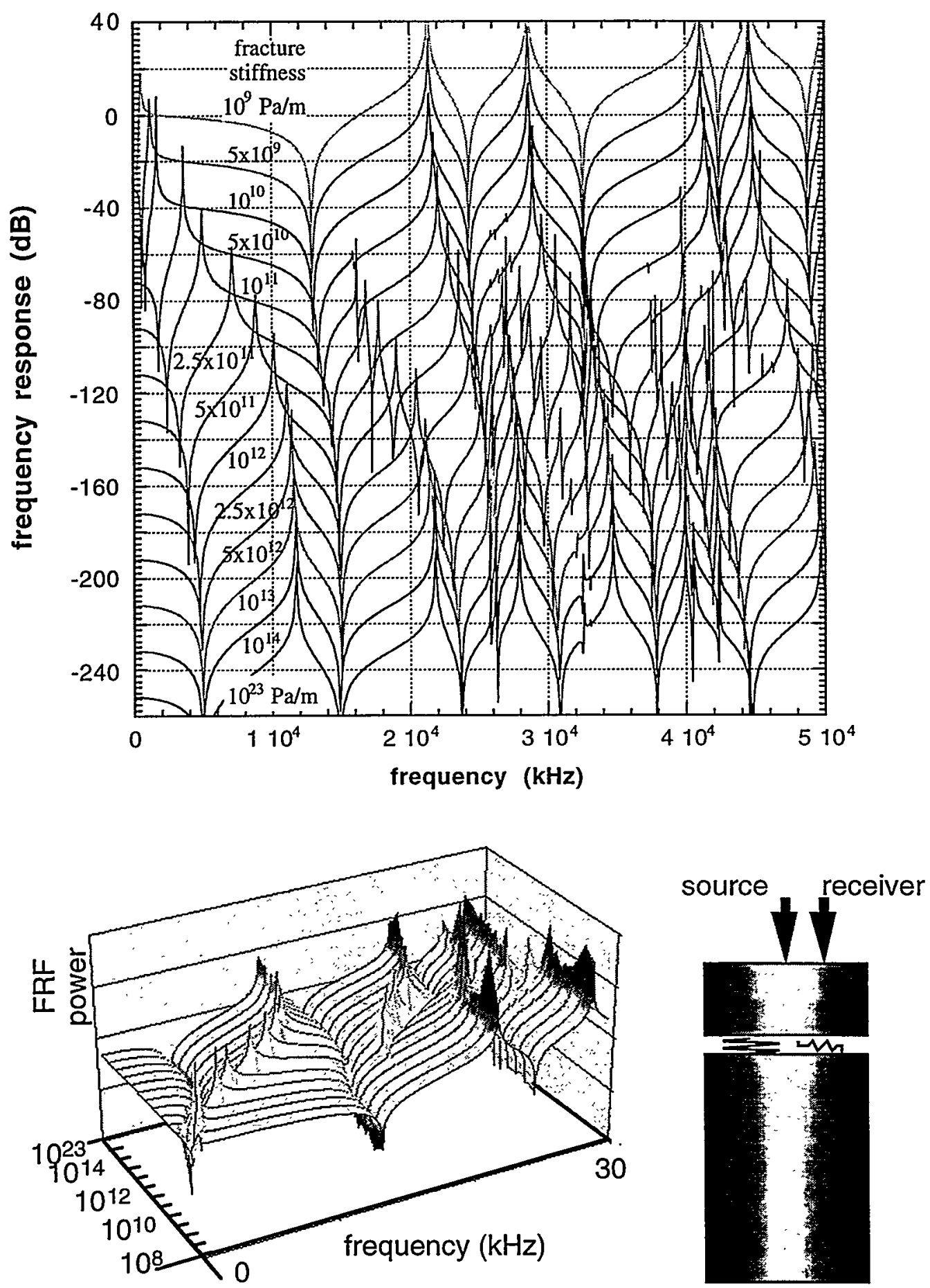

Figure 6.7 (b) Computed FRF's of fractured concrete cylinder. FRF's are offset by 20dB. Both source and receiver are located on top of the model. Increase in the fracture stiffness results in changes in the FRF's similar to the changes in experimental FRF's with increasing axial stress. 
Computed resonance frequencies for a range of fracture stiffnesses are shown in Figure 6.7 (a). Each curve represents the behavior of a mode with varying fracture stiffness. Unlike the resonance of the one-dimensional system discussed in Chapter 2 and 3, the resonance-frequency curves for a three-dimensional body containing a fracture can intersect each other. In Figure 6.8(a), open circles are singlet modes (unique eigenvalues of the matrix Eq.(4.18)) and closed circles are doublet modes (degenerate eigenvalues). A singlet mode is either an axial extension (labeled "E1", "E2") or torsion ("T1", "T2") as is seen from their mode shapes shown later. Motion of the other doublet modes (for example, "F1", "F2", and "F3") are primarily flexural and shear. For comparison, experimental resonance frequencies for the T-configuration are shown in Figure 6.8(b).

The resonance frequencies of the first six modes (two singlet modes $\mathrm{E} 1$ and $\mathrm{T} 1$, and two doublet modes) degenerate to zero as the stiffness of the fracture approaches zero. This is because these modes become the rigid body translation and rotation modes for zero fracture stiffness. For two bodies with free surfaces, there should be twelve possible rigid body motions. The remainder of the twelve rigid body motions are those arising from the two blocks being coupled.

\subsubsection{Computed mode shapes}

The shape of the individual modes can be computed from the eigenvectors (mode vectors) provided by the numerical code. Figure 6.9 shows mode shapes for several of the modes shown in Figure 6.8(a). Three representative fracture stiffnesses were chosen: $\kappa=10^{14}$ (high stiffness), $10^{12}$ (medium stiffness), and $10^{10}$ (low stiffness) $[\mathrm{Pa} / \mathrm{m}]$. The corresponding nondimensional fracture stiffness defined by $b=\kappa L / E$ (L and $\mathrm{E}$ are the length and Young's modulus of the specimen, respectively) is 350 (high stiffness), 3.5 (medium stiffness), or 0.035 (low stiffness). $b$ can be interpreted as a ratio between static deformation in the intact part and the fracture.

From the plot, torsion modes (T) and longitudinal modes (E) are singlets. The other modes $(\mathrm{F})$ are doublets, having mode pairs with identical resonance frequency and mode shapes that are a mirror image of each other. When the stiffness of the fracture is high, the mode shapes are close to those of an intact cylinder. As the stiffness of the fracture decreases, deformations in the top block and bottom block become discontinuous.

At low fracture stiffness, mode shapes in the two blocks are nearly decoupled. For the first six modes, individual intact blocks show very small deformation. Different mode 


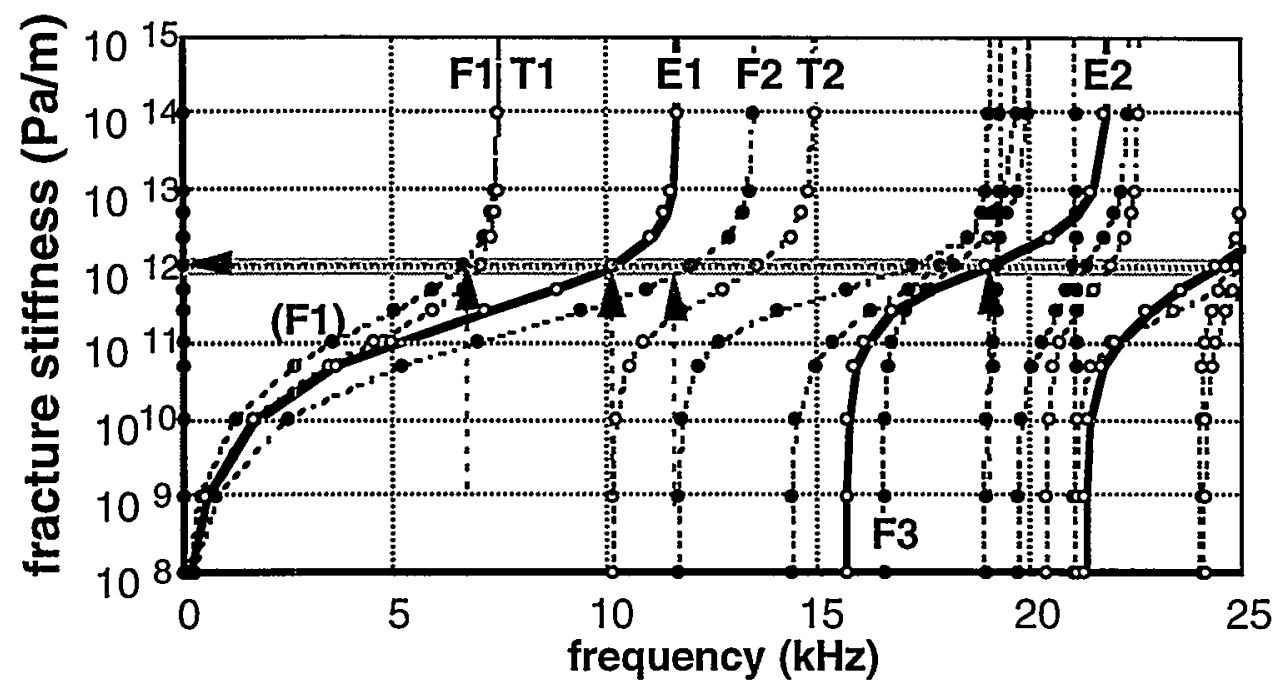

(a)computed resonance frequencies

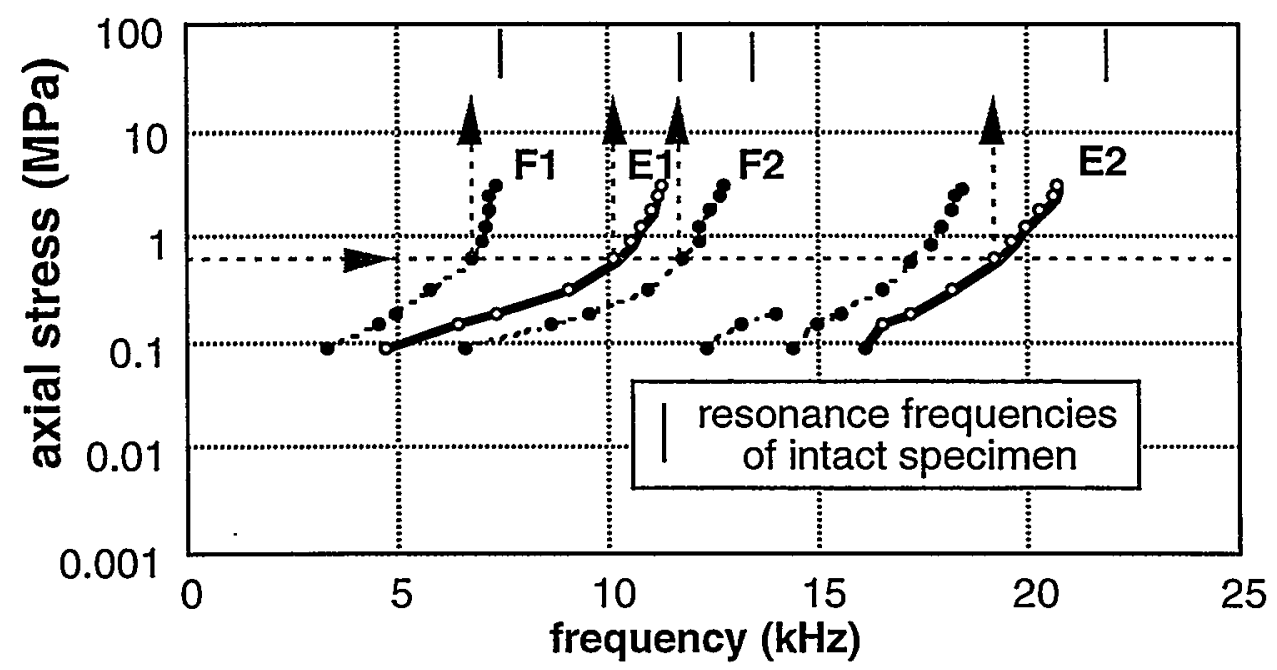

(b) experimental resonance frequencies

Figure 6.8 Comparison between experimentally measured and computed resonance frequencies for a range of axial stresses and fracture stiffnesses, respectively. Each curve corresponds to a normal mode and is labeled according to the mode shown in Figure 6.9. Fracture stiffness for an axial stress of $0.9 \mathrm{MPa}$ is determined to be about $10^{12} \mathrm{~Pa} / \mathrm{m}$ from the above plot.

shapes are due to different relative motions of the blocks across the fracture. This suggests that these modes become rigid body motions for zero fracture stiffness. The higher order modes have different behaviors, showing deformation of either one of the blocks. This is a three-dimensional analogue of the vibration localization discussed in Chapter 2 (Section 2.3). For example, as the $\mathrm{E} 2$ mode shows, resonance localizes in the bottom block even though the source is located at the top of the numerical model. This behavior of the E2 mode was also observed in the laboratory measurements (Figure 6.5). 


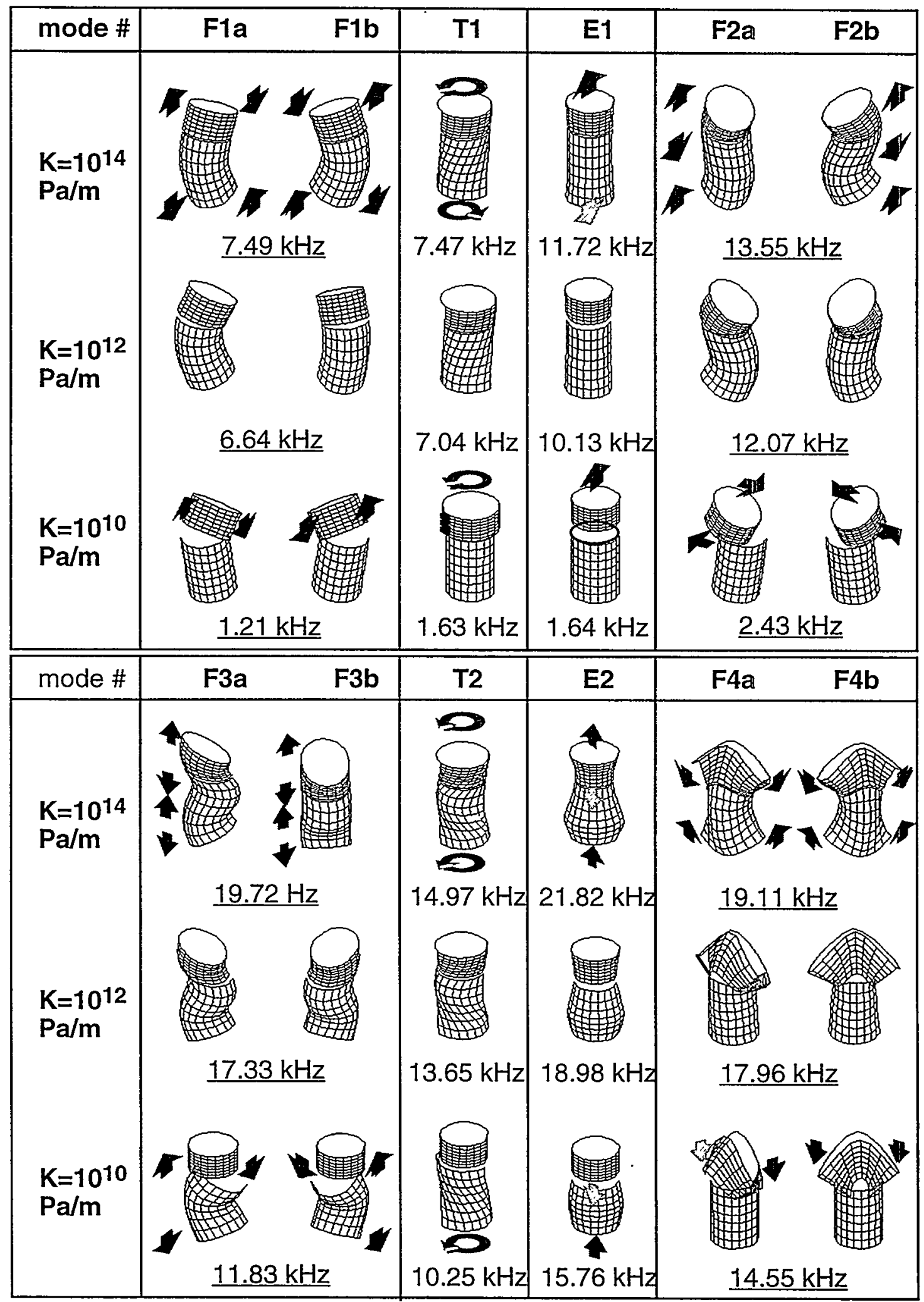

Figure 6.9 Effects of fracture stiffness on the mode shapes of a fractured concrete cylinder. With decreasing fracture stiffness, sharp changes in the mode shapes across the fracture are observed as is the localization of vibration in both top and bottom blocks. 


\subsection{Numerical Inversions}

\subsubsection{Inversion for elastic moduli}

Dynamic elastic moduli of an intact specimen can be determined by performing the numerical inversion described in Chapter 4. The assumed elastic moduli for a numerical model were changed iteratively until the differences between the experimentally measured and numerically computed resonance frequencies are minimized. Assuming the material was isotropic and homogeneous, Young's modulus and Poisson's ratio of the specimen were inverted from the first two longitudinal modes (E1 and E2) as $51.3 \mathrm{GPa}$ and 0.26 , respectively. These values were close to the result from the high frequency ultrasonic measurements (Young's modulus of $54.8 \mathrm{GPa}$ and Poisson's ratio of 0.26). Measured FRF and computed FRF using the inverted elastic moduli are compared in Figure 6.10. Polynomials of the order of $(l, m, n)=(8 \times 8 \times 10)$ were used. Although the FRF's start to show differences for higher frequencies, both FRF's are in good agreement for low frequencies. The initial misfit up to about $10 \mathrm{kHz}$ is due to noise in the experimental data. The plot also shows an FRF computed for moduli determined from the high frequency measurements. Within the resolution of the plot, no significant difference between the two computed FRF's can be seen.

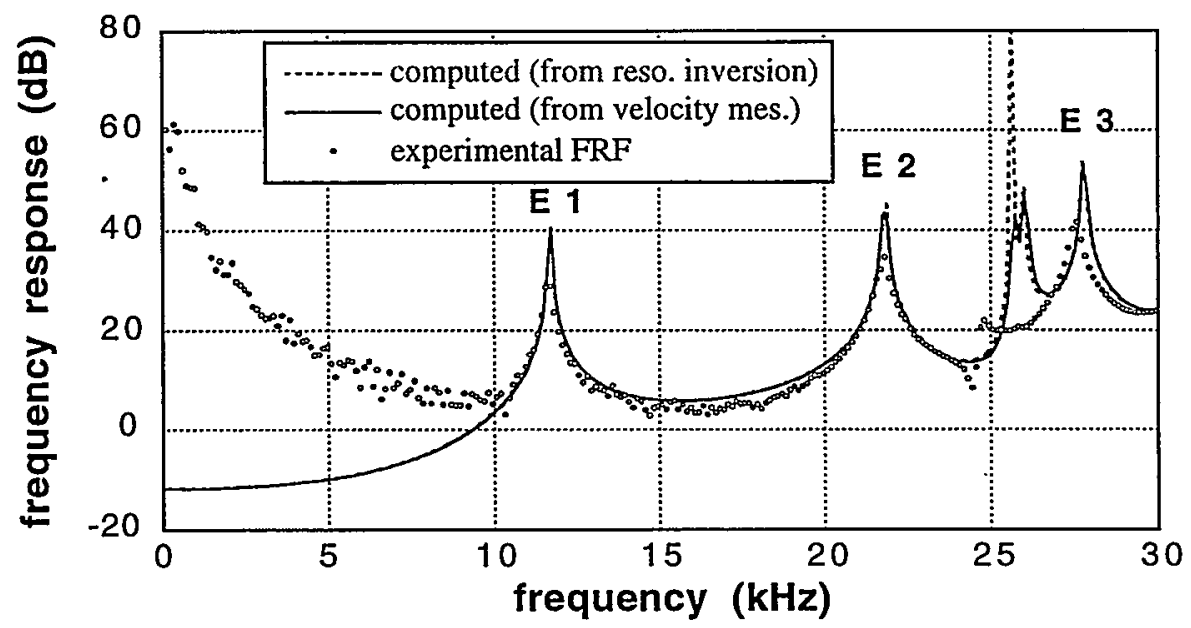

Figure 6.10 Comparison between experimentally measured and computed FRF's. The computed FRF's were obtained for the Young's modulus and Poisson's ratio that were determined from the resonance inversion (shown in dotted line) and high-frequency ultrasonic velocity measurements (shown in solid line). The experimental FRF was measured by an acclerometer attached to the bottom of the specimen (T-config). Both computed FRF's show good agreement with the experimental FRF. Due to the noise and limited sensitivity of the accelerometer, the experimental FRF deviates from the numerical FRF for low frequencies up to $10 \mathrm{kHz}$. 


\subsubsection{Inversion for fracture stiffness}

Dynamic stiffnesses of the fracture were determined from measured resonance frequencies of the fractured specimen. Elastic moduli determined from the high frequency ultrasonic measurements were assumed for the intact part of the specimen. This method involves finding a fracture stiffness for the numerical model that yields resonance frequencies similar to the ones measured at each load. For example, in Figure 6.8, the stiffness of the fracture at an axial stress of $0.9 \mathrm{MPa}$ is found to be approximately $10^{12}$ $\mathrm{Pa} / \mathrm{m}$ by matching the resonance frequencies for the $\mathrm{F} 1, \mathrm{~F} 2, \mathrm{E} 1$, and $\mathrm{E} 2$ modes. The numerical code performed this task automatically by changing the stiffness of the fracture instead of the elastic moduli.

By assuming identical normal and tangential stiffnesses, dynamic stiffness of the fracture during loading and unloading stages was determined from measured resonance frequencies of the first longitudinal mode (E1). Figure 6.11 compares inverted dynamic stiffnesses from resonance tests and (elastic) static stiffnesses from uniaxial loading tests. Erratic behavior of the static stiffness for the loading eycle is possibly due to uneven closure of the fracture. Although both results show stiffnesses of the same order of magnitude, inverted dynamic stiffnesses are significantly higher than the static stiffnesses.

Resonance inversion for fracture stiffness was also performed assuming dissimilar normal and tangential stiffnesses. To invert for the two stiffnesses, measured resonance frequencies for the four or five lowest order modes were used. Inversion was performed only for resonances measured during the loading cycle ( $\mathrm{T}$-configuration). If modes used for inversion are not sensitive to changes in the fracture stiffness, inversion instability of can occur. In this research, such a problem was avoided by using multiple (more than two) modes. For all of the cases presented here, this inversion process was found to be stable. Inverted fracture stiffnesses are shown with static stiffnesses in Figure 6.12. Better agreement is noticed between static and dynamic normal fracture stiffnesses than the previous model with assumed identical normal and tangential fracture stiffnesses. Experimentally measured and numerically computed FRF's using the inverted fracture stiffnesses are compared in Figure 6.13. Both FRF's show good agreement particularly for large axial stresses. This may be because the stiffness of the fracture has become more or less uniform at high axial stresses. 


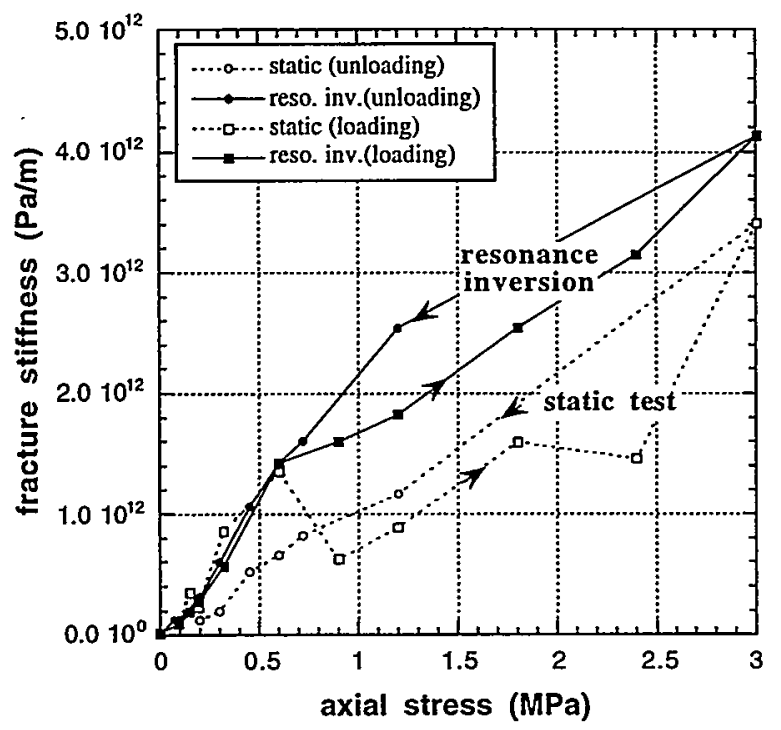

Figure 6.11 Comparison between static normal fracture stiffnesses from loading tests and dynamic fracture stiffnesses determined using the resonance inversion technique. Normal and tangential fracture stiffnesses were assumed to be identical for the inversions, and the resonance frequencies of the first longitudinal mode were used.

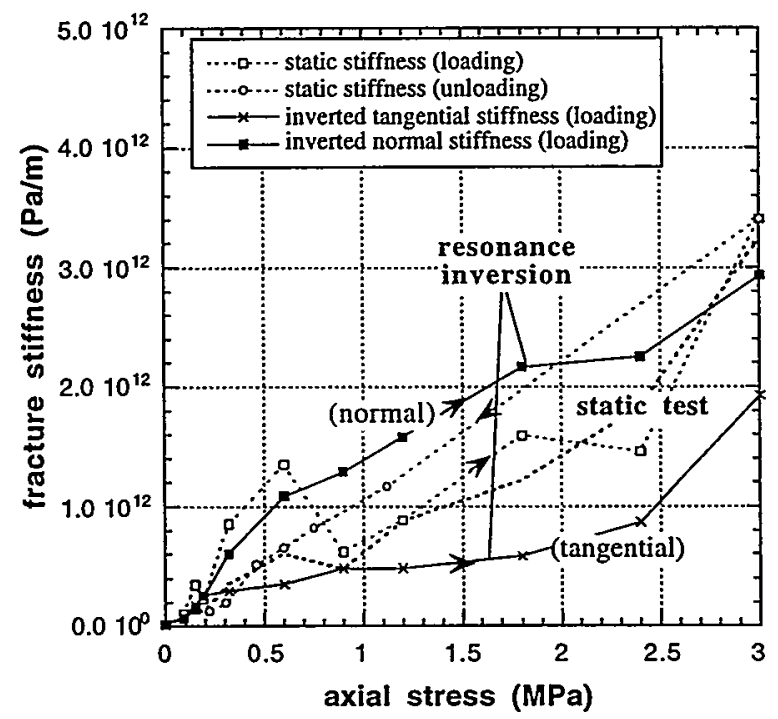

Figure 6.12 Comparison between static normal fracture stiffnesses from loading tests and dynamic fracture stiffnesses from the resonance inversion technique. For the resonance inversion, an anisotropic fracture stiffness was assumed. 


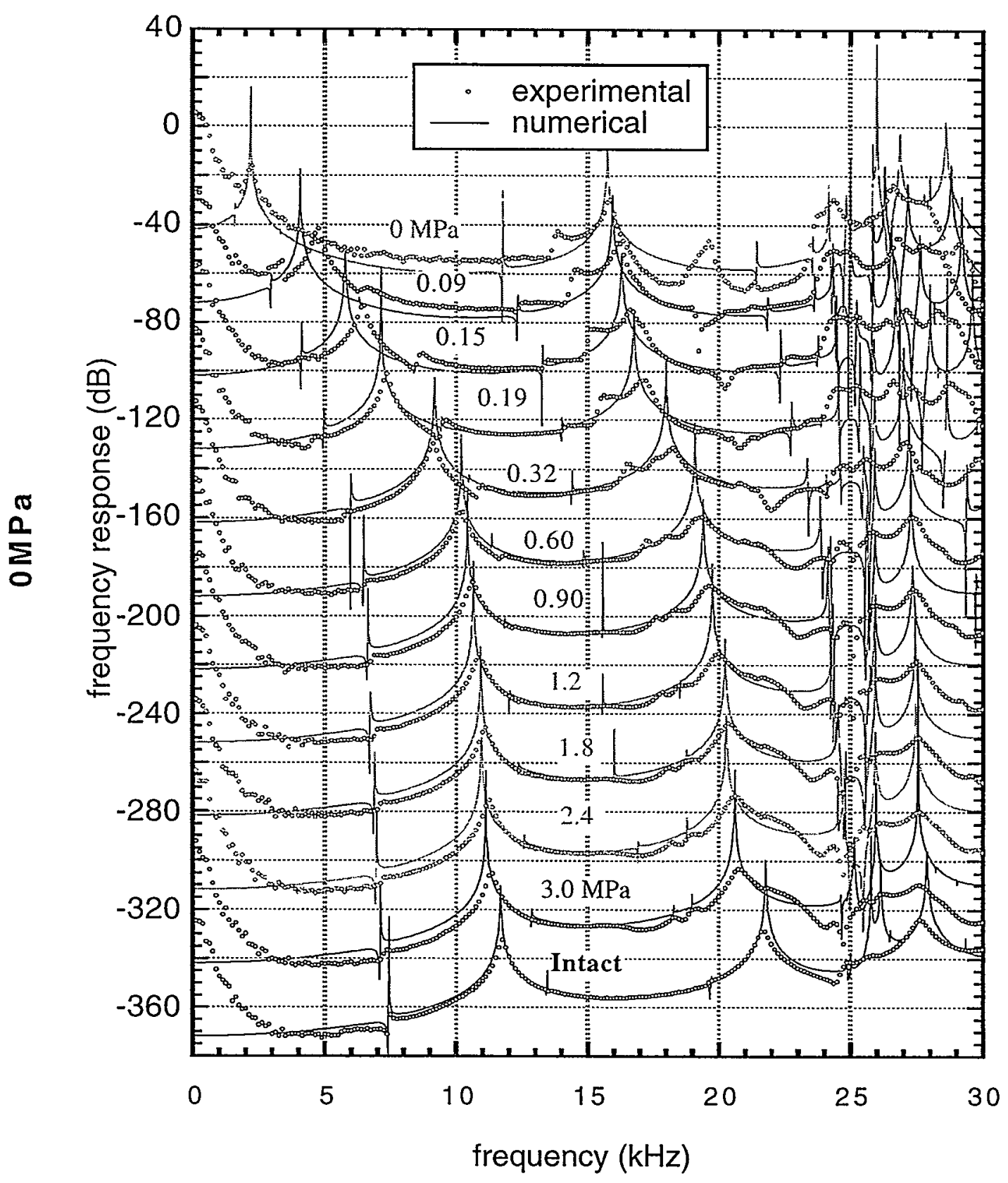

Figure 6.13 Comparison between experimentally measured and numerically computed FRF's using the inverted fracture stiffnesses. The FRF's show good agreement particularly for large axial stresses. For small axial stresses, misfit between the FRF's is relatively large possibly due to the uneven contact between the surfaces of the fracture. 


\subsection{Summary}

This chapter examined the resonance of a concrete cylinder containing a single throughgoing fracture both experimentally and numerically. The specimen was forced to resonate using a piezoelectric source. By applying an axial load, the stiffness of the fracture in the specimen was changed. Although the specimen was loaded on a load frame, vibrations in the specimen were approximated to be free vibrations since the strong impedance contrast between the specimen and compliant plastic rings isolates the vibrations.

With increasing axial stress, the resonance frequencies of the fractured specimen increased systematically, approaching those of the intact specimen. For small axial stresses, FRF's measured by two accelerometers attached to the top and bottom of the specimen revealed different types of localized resonance in the top and bottom sections of the specimen. The top block on which the source was attatched exhibited strong resonance peaks as the fracture prohibited the transmission of vibration to the bottom section. In contrast, the bottom section also showed strong localized resonances even though it was located on the opposite side of the fracture as the source. This is experimental evidence for the localized resonance in a irregular system discussed in Chapter 2 (top and bottom blocks have different geometries, resulting in different resonance characteristics). These results are significant because they indicate that both types of localized resonances can be used for detecting a section of damaged rock and concrete structures isolated by fractures.

The numerical code developed in Chapter 4 was used to examine the effect of fracture stiffness on the mode shape and resonance frequencies. With decreasing stiffness of the fracture, both a decrease in resonance frequencies and changes in mode shapes were observed. The mode shape exhibited sharp changes across the fracture, which is a good indicator for locating fractures. The code was also used for determining the dynamic stiffnesses of the fracture from measured resonance frequencies. Both statically measured and numerically inverted fracture stiffnesses showed good agreement. The results demonstrate the applicability of the resonance inversion technique for quantitative diagnosis of damage in rock and concrete structures.

In the following chapter, an application of the resonance inversion technique to a semisite scale concrete structure is presented. Changes in the dynamic stiffness of the structures due to introduced damage and repair are determined from the resonance frequencies and compared with the measured static stiffnesses. 


\section{Resonance of a Fractured Concrete Infrastructure}

\subsection{Introduction}

Many elements of the civil infrastructure such as tunnels, dams, port piers, buildings, and highway ramps are built of rock and concrete. In these structures, severe loading from excessive traffic, earthquakes, and aging of building materials can cause cracking of the matrix. The resultant cracks and fractures can affect the integrity of a structure by decreasing its stiffness and strength, thereby promoting further degradation. Earthquakerelated damage in particular has been a major problem in heavily populated urban and suburban areas susceptible to frequent seismic activity because the resultant damage is often severe, widespread, and requires prompt restoration. Consequently, delays in the restoration of highway and public transportation systems lead to serious traffic problems that could impair the function of major cities. How soon a damaged structure is restored depends upon both economic and technical factors. Demolishing and replacing the whole structure can be both costly and time consuming. As long as the original performance of 
the structure (load capacity, stiffness etc.) can be restored by repair, it is desirable that a damaged structure be repaired on site.

For effective and successful post-earthquake repair of the civil infrastructure, quantitative, accurate, and practical techniques for assessing the degree of damage and the effect of repair are essential. For assessing the structural damage due to microcracking and fracturing of building materials, non-destructive tests based on acoustic methods are among the most promising as they are sensitive to changes in the stiffness of a structure that are caused by damage. In this chapter, the acoustic characteristics of semi-site scale bridge columns are examined by resonance and seismic transmission tests. The tests were conducted before and after damage is introduced to the columns, and after the columns are repaired using two different repair techniques. The results presented in this chapter are obtained as a part of a research program conducted at the Earthquake Engineering Research Center at University of California, Berkeley for development of post-earthquake repair techniques for reinforced bridge columns (contract No. RTA-59X517).

\subsection{Experimental setup}

\subsubsection{Description of specimens}

The tested specimens are one-third scale models of actual bridge columns (Lehman, et al., 1996). The geometry and dimensions of the column are shown in Figure 7.1. The columns have 0.25 inch $(6.4 \mathrm{~mm})$ diameter spiral reinforcement with 1.25 inch $(3.2 \mathrm{~cm})$ spacings and vertical steel reinforcement that occupies $1.5 \%$ of the total cross section.

Specimens \#415M and \#415S are identical in structure and concrete mix design. Before the columns were loaded, \#415M was tested for its dynamic properties. Subsequently, the specimens were loaded by both cyclic lateral load and constant axial load (Figure 7.2). The applied axial load was $0.653 \mathrm{MN}(2.24 \mathrm{MPa}$ in the column cross section: $10 \%$ of gross cross section load capacity). During the loading tests, the specimens were cemented and braced to the concrete floor and the load-displacement behavior of the column was recorded. For specimen $\# 415 S, 7$ inches $(17.8 \mathrm{~cm})$ of lateral cyclic displacement was applied at 8 feet $(2.44 \mathrm{~m})$ above the surface of the concrete footing to introduce severe damage at the bottom of the column. After the last load cycle, the specimen was accidentally displaced up to 10 inches $(25.4 \mathrm{~cm})$, causing further damage. 


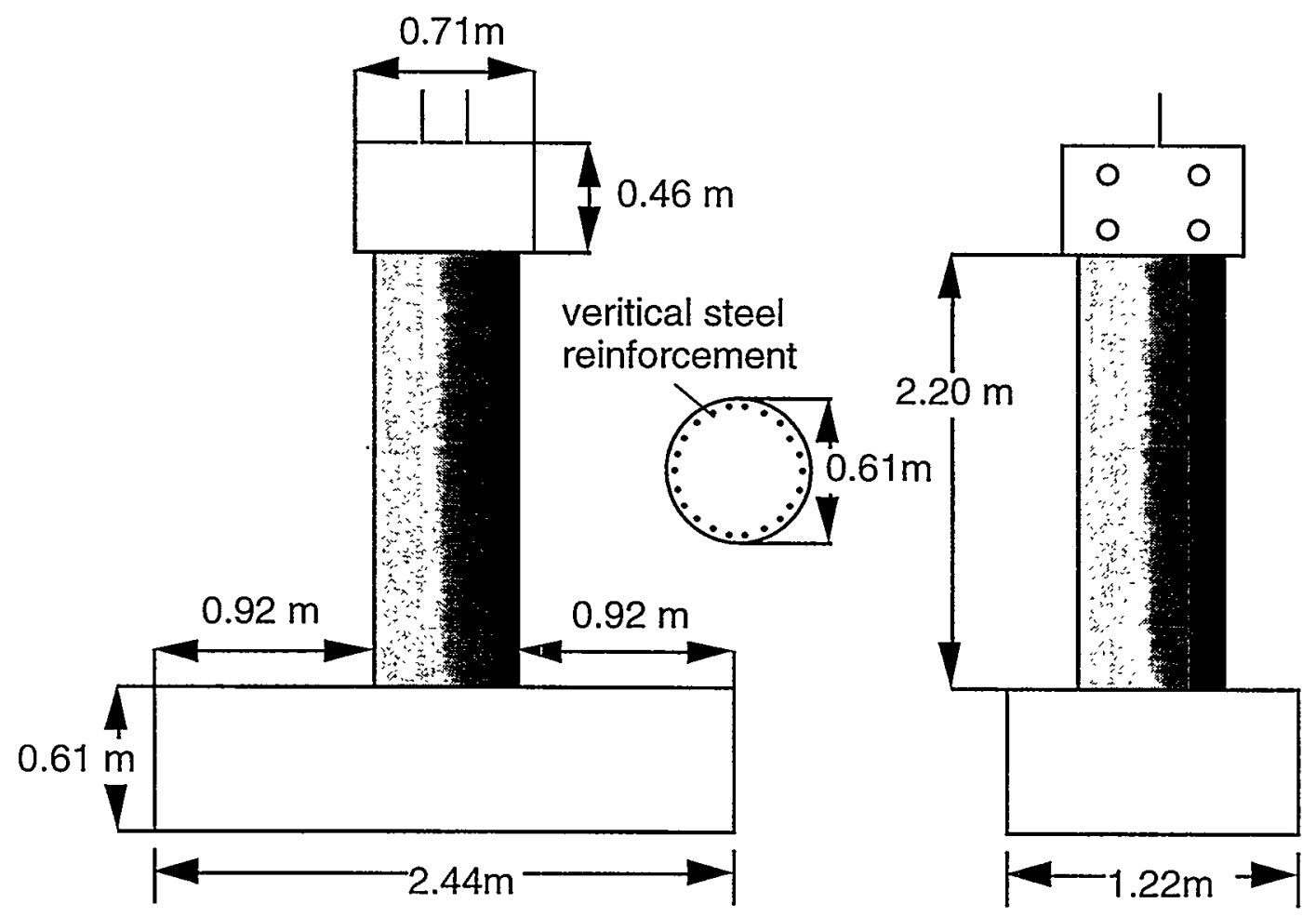

Figure 7.1 Geometry of column specimen. The column part has both vertical reinforcement (22 No.5 steel reinforcement bars) and spiral reinforcement (No.2 with 1.25 " (3.18 cm) spacing) (Lehman, et al., 1996). The column is on a rectangular block that is longer in the loading direction.

Specimen \#415M was loaded by 3 inches $(7.6 \mathrm{~cm})$, which lead to less severe damage than specimen \#415S. Estimated yield displacement of the column was approximately 0.75 inches $(1.9 \mathrm{~cm})$. Both specimens experienced severe cracking over the middle to bottom sections of the column wall, spalling of the concrete cover, crushing of the concrete core, and yielding and rupturing of steel reinforcement bars at the bottom of the column (Figure 7.3).

After the changes in the dynamic properties were measured, the columns were repaired by two different techniques (Figure 7.4). For specimen \#415M, the fractures were injected with epoxy (low-viscosity epoxy resin, Concresive TM 1360) and cementitious patching material (Thorite ${ }^{\mathrm{TM}}$ ) was applied to the spalled concrete cover at the bottom. For specimen \#415S, the yielded and ruptured reinforcement was replaced by new reinforcement that was connected to the remaining reinforcement in the column by couplers. The crushed core at the bottom section was removed and replaced by fresh concrete. The diameter of the repaired cross section was larger than the original by 5.8 inches $(15 \mathrm{~cm})$. When the epoxy 

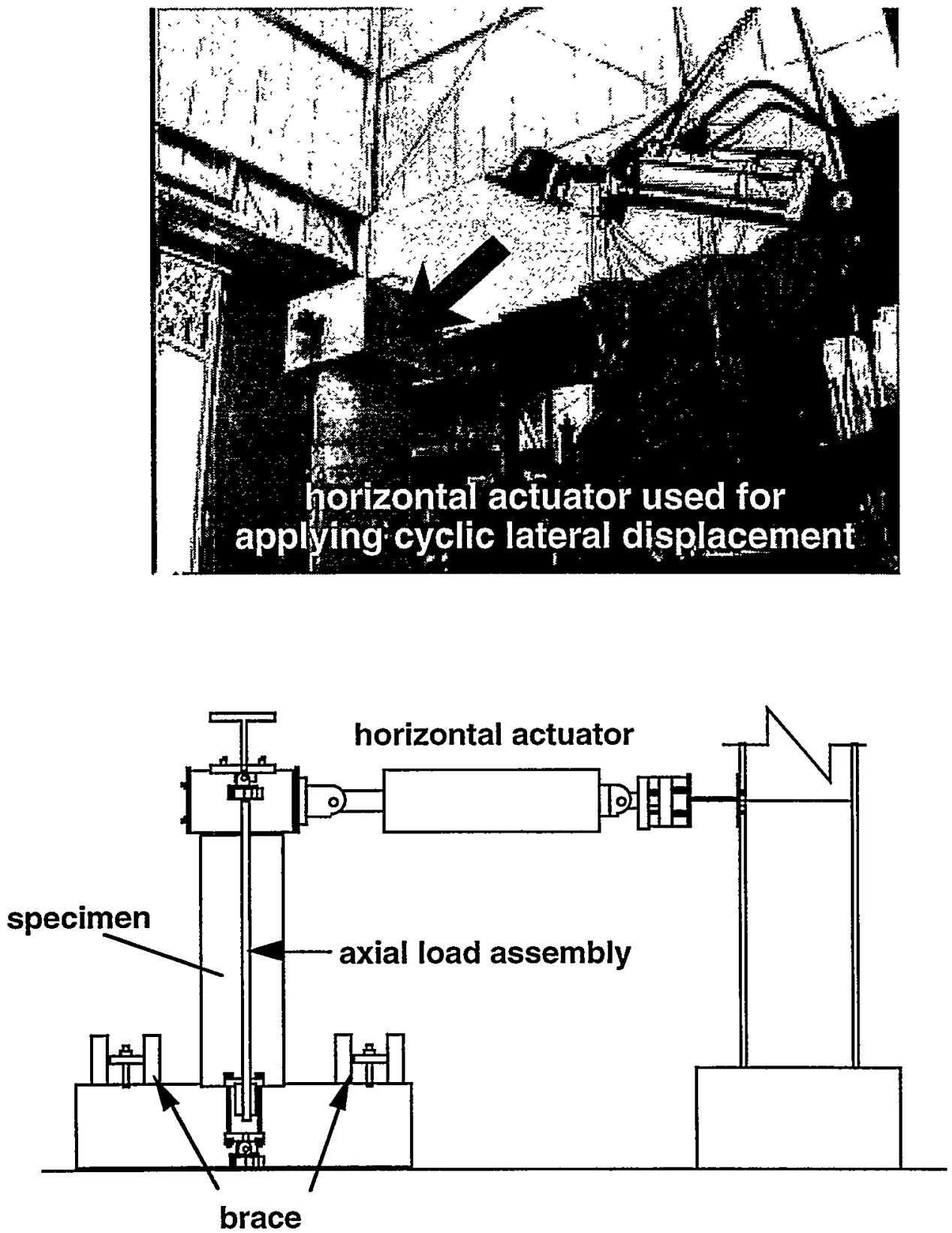

Figure 7.2 Experimental setup for cyclic loading tests for model columns (after Elkin et al., 1998). Lateral displacement was applied using a horizontal actuator attached to the center of the top block section. The footing was cemented and braced to the floor. 


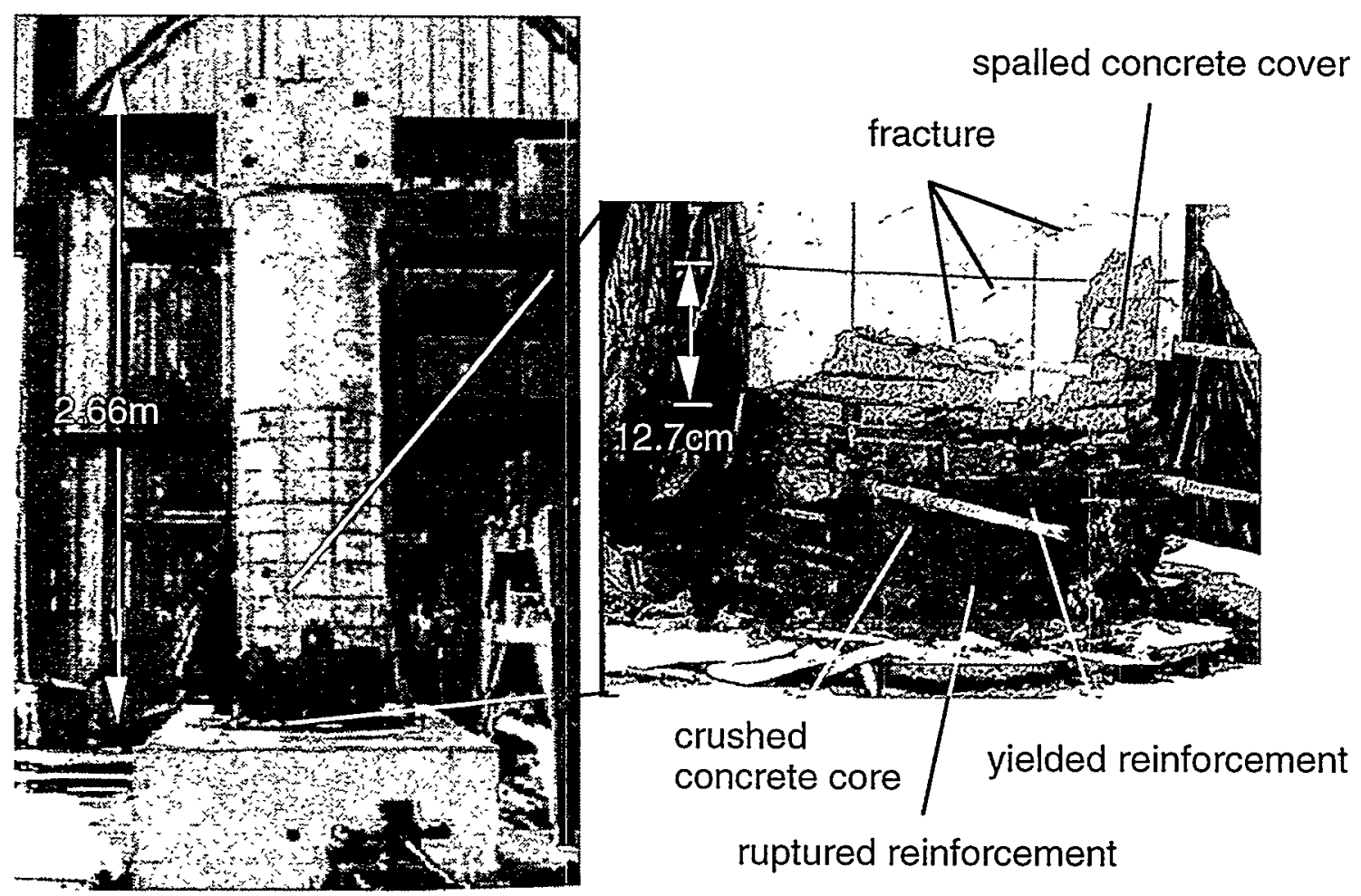

Figure 7.3 Damage introduced by cyclic loading of the column. Above photos are for the severely damaged column (415S). The damage localized near the bottom of the column exhibiting yielding and rupturing of reinforcement, crushed concrete core, and spalling of concrete cover. The middle section did not suffer major damage but many horizontal tensile fractures were observed.

and concrete cured, resonance and wave transmission tests were repeated for the dynamic properties of columns.

The repaired specimens were loaded once again for their ultimate strength and stiffness. The load-displacement behavior of the columns for the second loading test was recorded for comparison with the results of the first loading test.

\subsubsection{Seismic wave transmission test}

Wave transmission tests were performed along the diameters of the columns. A source and receiver pair was moved along the axis of the column to construct a vertical profile of $\mathrm{P}$-wave travel times and waveforms. The propagation path between a source and a receiver was either parallel to or normal to the direction of the applied lateral displacement (Figure 7.5). Waves were generated using a small calibrated hammer (Impulse Force Hammer, PCB Piezotronics, 086C80). The resulting accelerations were measured by an 


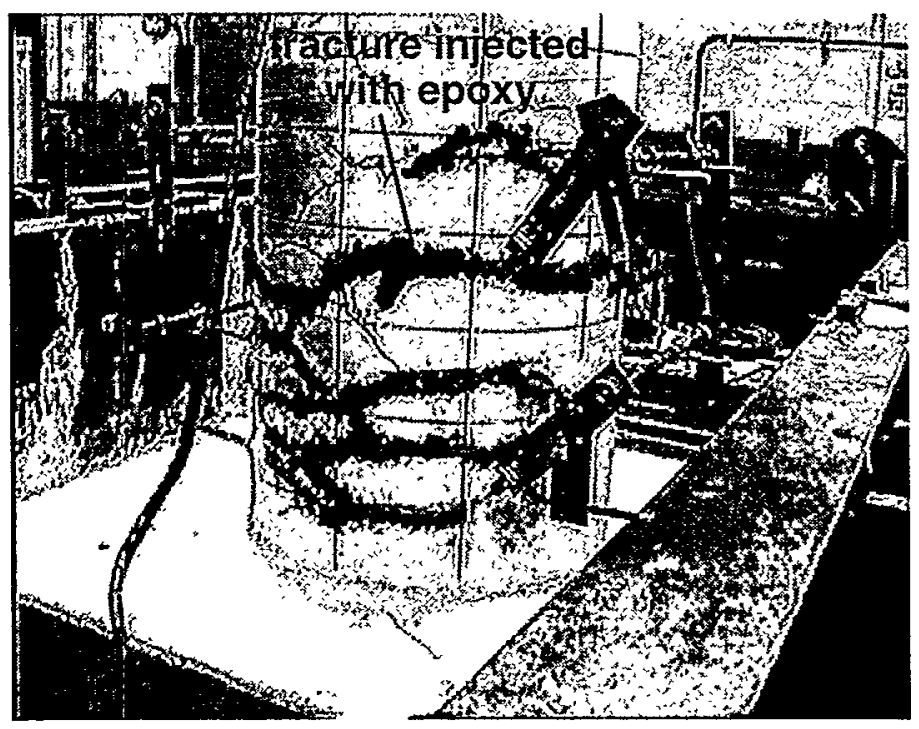

(a) Repair of a moderately damaged column (415M)

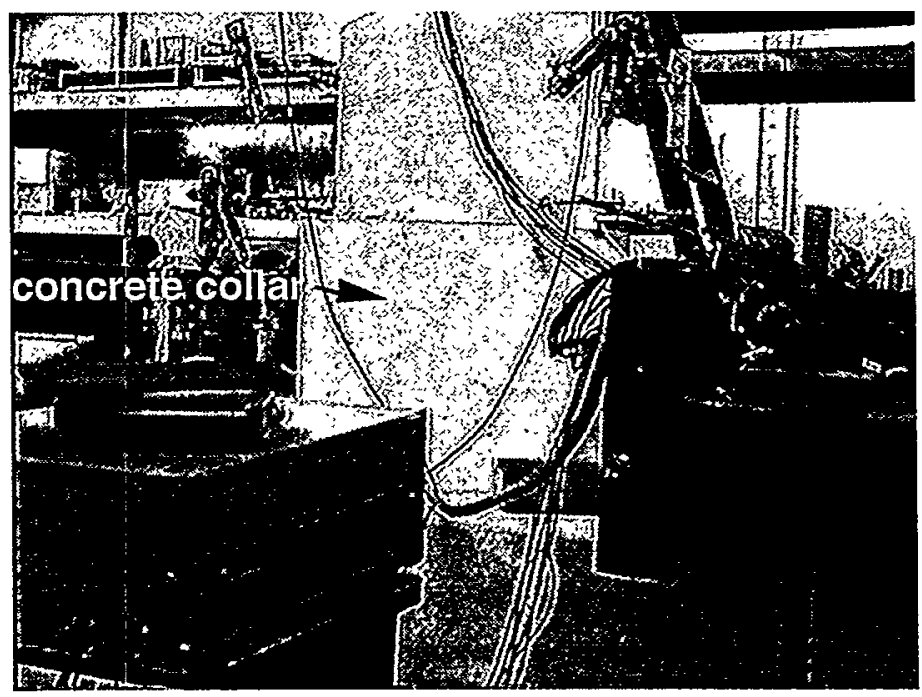

(b) Repair of a severely damaged column (415S)

Figure 7.4 Repair of the damaged columns. (a) A moderately damaged column (415M) was repaired by injecting epoxy resin into the fractures at the bottom to middle sections. Spalled concrete was patched with cementitous material. (b) A severely damaged column (415S)was repaired by replacing damaged reinforcement and concrete core and increasing the diameter of the bottom cross section. 


\section{Measurement parallel to loading direction}

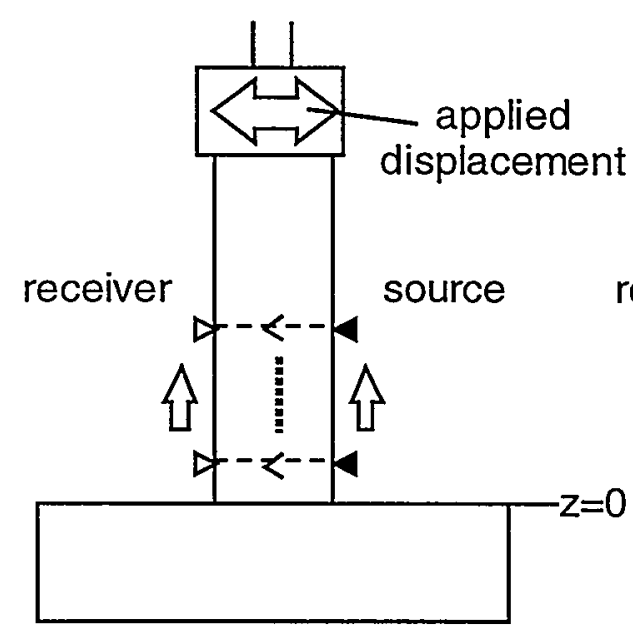

\section{Measurement perpendicular to loading direction}

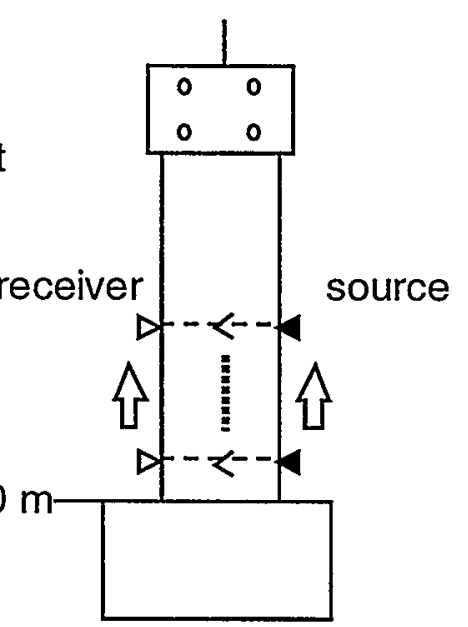

Figure 7.5 Source and receiver configuration for seismic transmission tests. Waves propagating along the diameter of the columns were measured. The source and receiver pair was moved along the length of the column to construct travel time and waveform profiles.

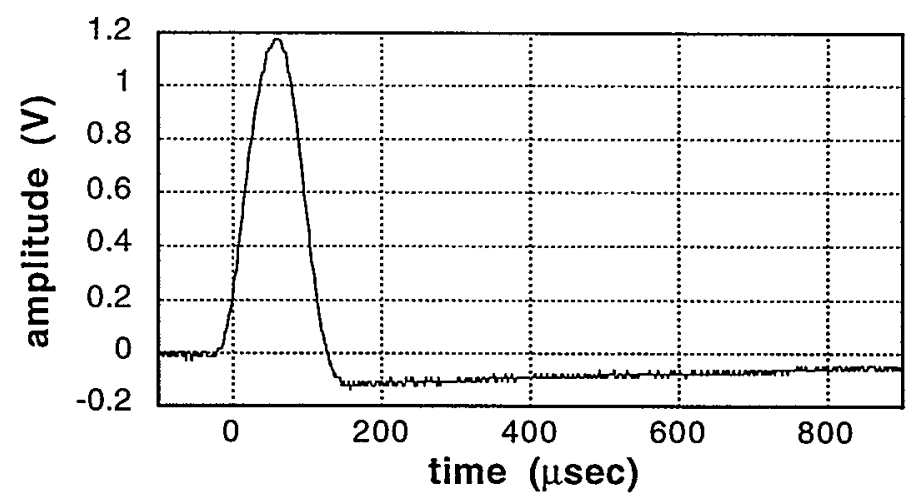

(a) time-domain response of the impact hammer

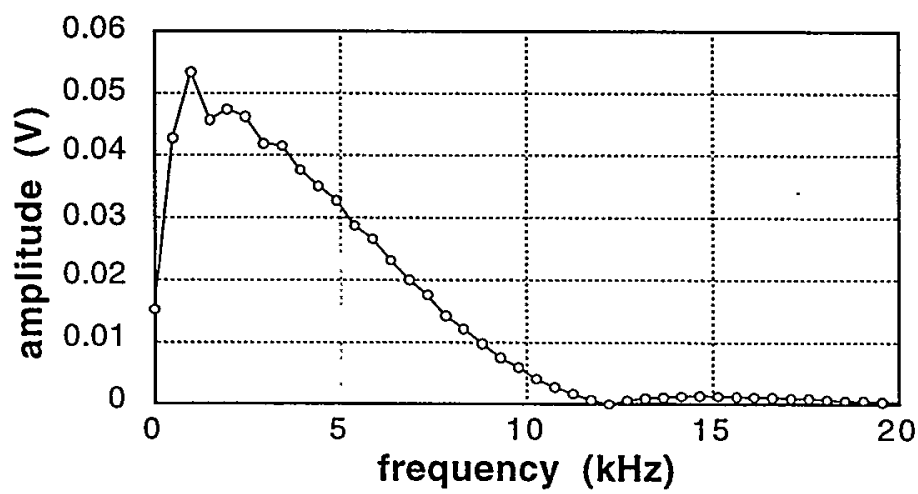

(b) frequency-domain response of the impact hammer

Figure 7.6 Response of calibrated impact hammer hit on the surface of an intact column. The spectrum shows that the source generates waves with frequencies of 0 to approximately $12 \mathrm{kHz}$. 
accelerometer (PCB Piezotronics, 353B68) and analyzed and recorded on a digital oscilloscope (LeCroy 9424E). The stress history of the applied impact was also measured and used to normalize the amplitude of the measured waves. The impact hammer generated waves containing spectra ranging from 0 to approximately $10 \mathrm{kHz}$. The hammer's impact response and its spectrum are shown in Figure 7.6.

\subsubsection{Resonance test}

The specimens were vibrated using either an impact from a $5 \mathrm{lb}$. plastic sledge hammer, or a mechanical shaker (LDS vibrator, V102) that was driven by continuous single frequency sine waves sweeping over a desired frequency range. Force from the shaker was transmitted to the column through a thin piano wire that was connected to the top of the column (Figure 7.7). Resulting accelerations were measured by an accelerometer attached to the concrete block at the top. Measured accelerations were displayed, analyzed, and recorded by a spectrum analyzer (ONO SOKKI CF6400). It should be noted that damaged column \#415S was tested on wooden blocks and the intact column \#415M was tested on plywood laid over a concrete floor. These differences in the boundary conditions may have affected the results of the resonance tests. 


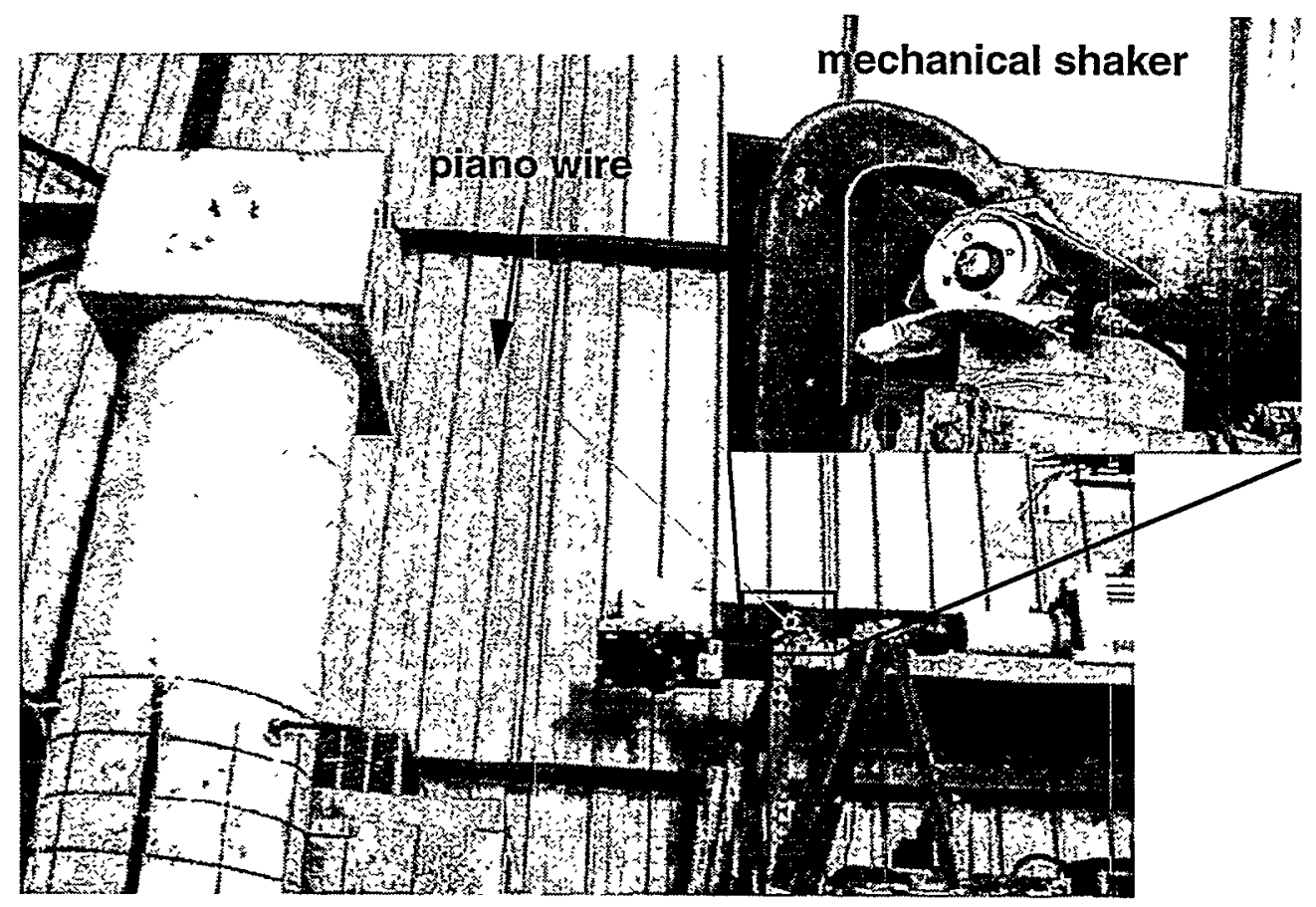

loaded direction

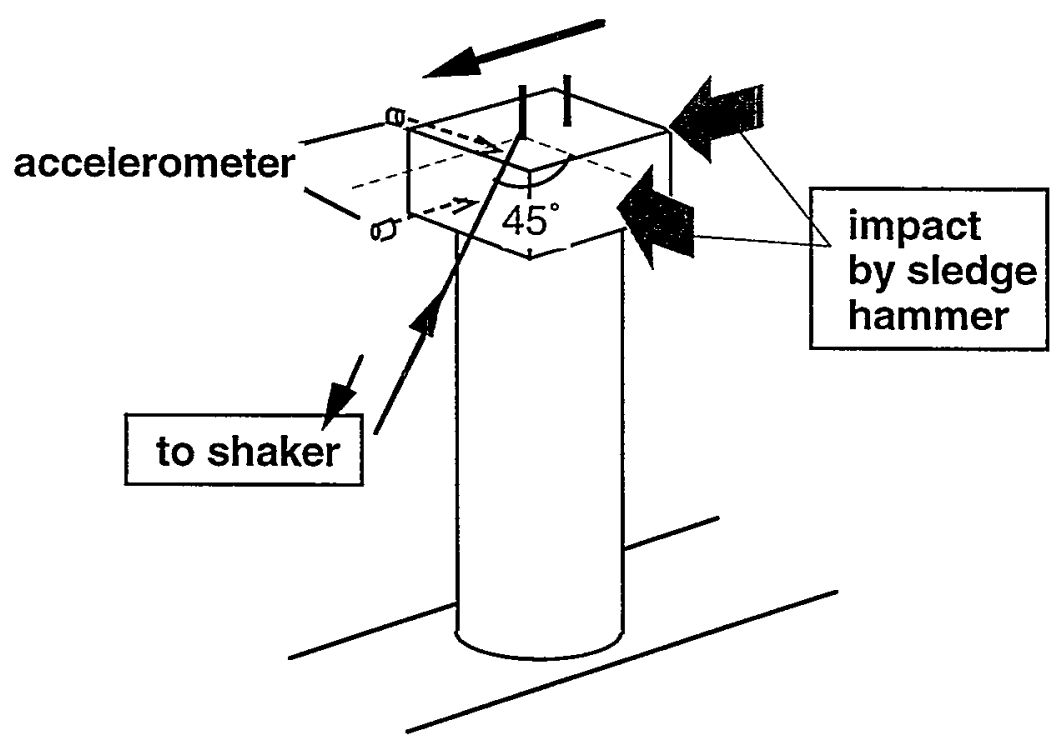

Figure 7.7 Experimental setup for vibration tests. Resonance of the columns was introduced by either continuous sine wave excitation using a mechanical shaker or by the impact from a sledge hammer. Force from the shaker was transmitted to the column at approximately $45^{\circ}$ to the loading direction. Impact from the sledge hammer was applied in both directions parallel and perpendicular to the loading direction. Resulting accelerations at the top of the column were measured by accelerometers. 


\subsection{Experimental Results}

\subsubsection{Static behavior}

The static load-displacement response of the specimens during two successive loading tests before and after the repair is shown in Figure 7.8. For both specimens, secant stiffness of the columns decreases significantly at the onset of yield. The measured loaddisplacement relations are strongly hysteretic, indicating large frictional sliding along the introduced fractures and debonded steel reinforcement. In order to relate the static behavior to the dynamic response that results only from small deformation of the specimen, elastic properties that do not include frictional sliding in the specimen have to be measured (Walsh, 1965; Cook and Hudgson, 1965). For this purpose, tangent stiffnesses of the load-displacement curves were measured for the initial slope of small unloading loops (Figure 7.9). The slope provides the approximate elastic stiffness of the specimen as the frictional sliding "locks up" when the load is reversed.

The initial load-displacement loops of columns \#415M and \#415S showed clear linear behavior and small hysteresis. After damage was introduced, the load-displacement curve became strongly hysteretic and the elastic stiffness decreased significantly. The initial stiffness of the repaired specimen \#415M was found to be significantly lower than the intact column. This may be due to the crushed concrete core at the bottom section and cracks in the middle section of the column that were not injected with epoxy. However, small hysteresis in the first loading loop for the repaired column indicates the effect of the remaining open fractures is rather small. The low stiffness of the epoxy injected into fractures might also be responsible for the observed decreased stiffness. Repaired heavily damaged column \#415S showed almost identical stiffness as the intact initial state. Although the cracks in the middle section of the column may have some effect on decreasing the stiffness of the column, increase in stiffness due to the thick concrete cover at the bottom section seemed to compensate for the effect. Load capacities of the repaired columns were similar to or even larger than those of the original intact columns, indicating successful repairs. 


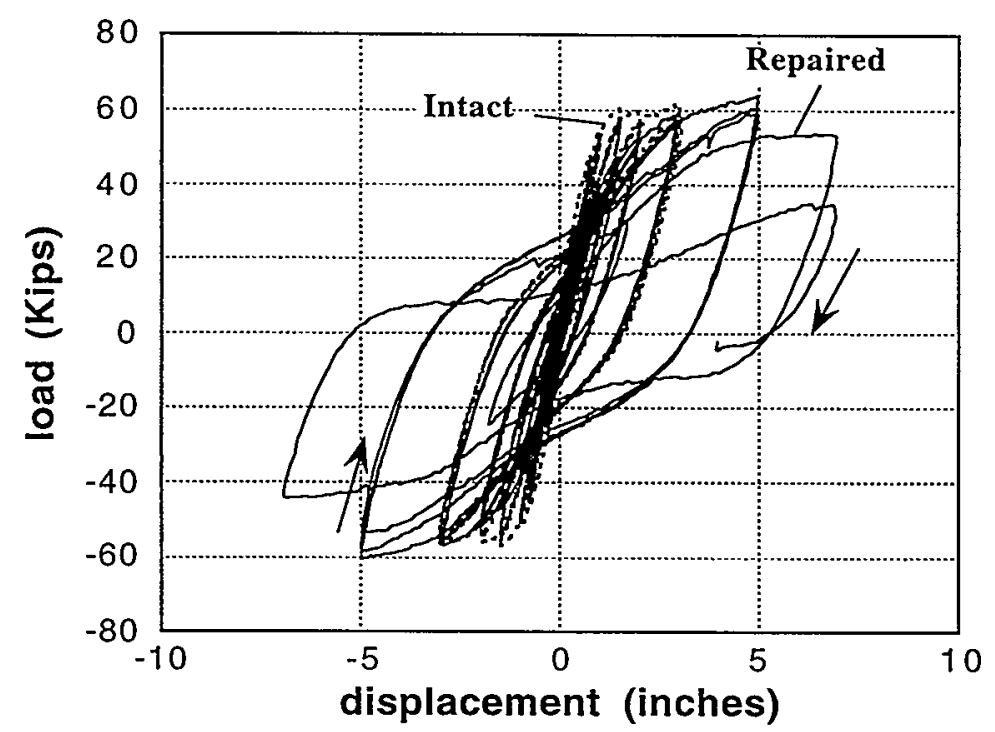

(a) Intact and repaired column $415 \mathrm{M}$ (moderately damaged)

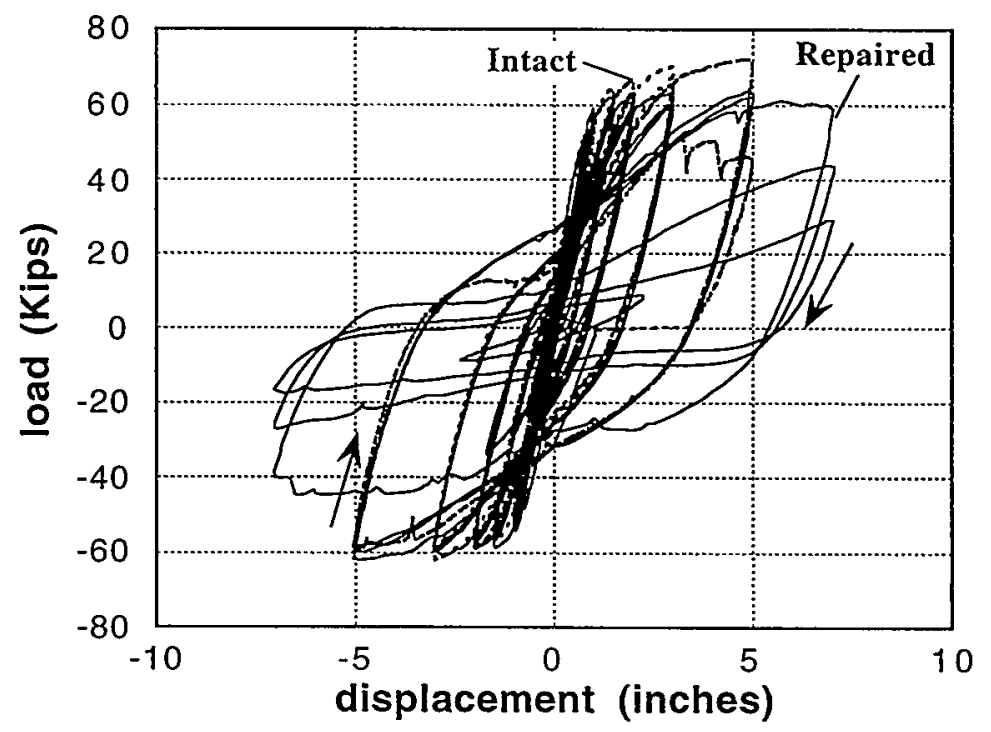

(b) Intact and repaired column $415 S$ (severely damaged)

Figure 7.8 Load-displacement behavior of intact and repaired columns. The stiffness (secant stiffness) of the columns decreased significantly after the columns underwent yield. Although the repaired column 415 $M$ showed reduced initial stiffness, both columns showed equal or even larger maximum load capacities (after Elkin et al., 1998) 


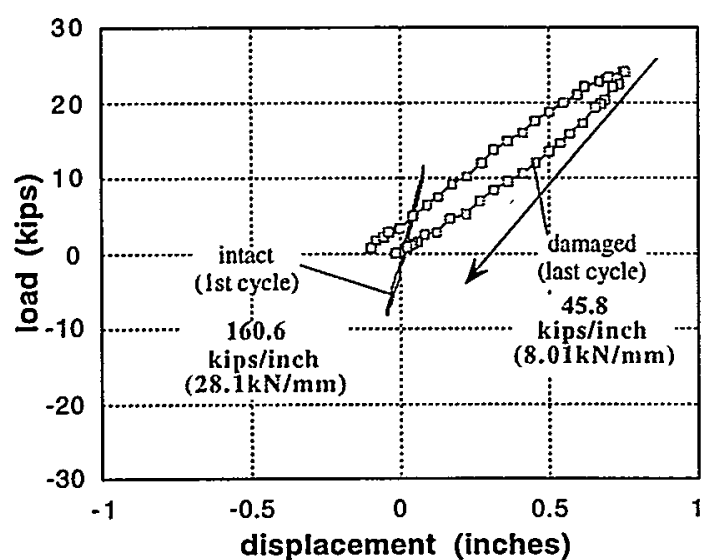

(a) $415 \mathrm{M}$ intact and damaged columns

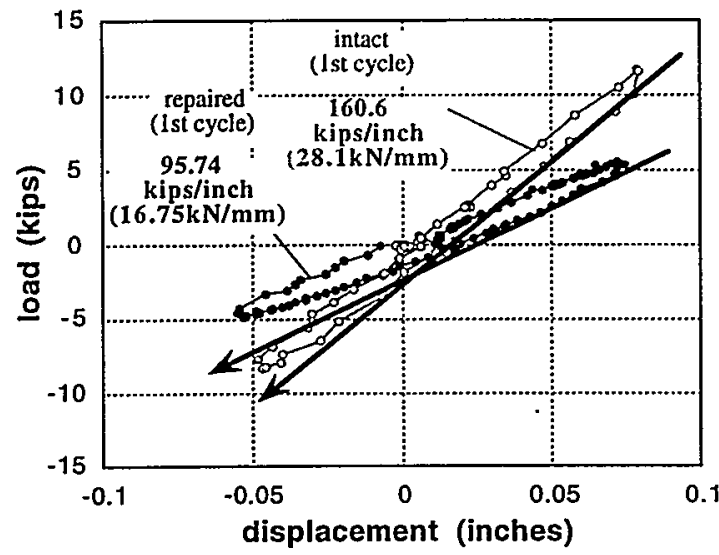

(c) $415 \mathrm{M}$ intact and repaired columns

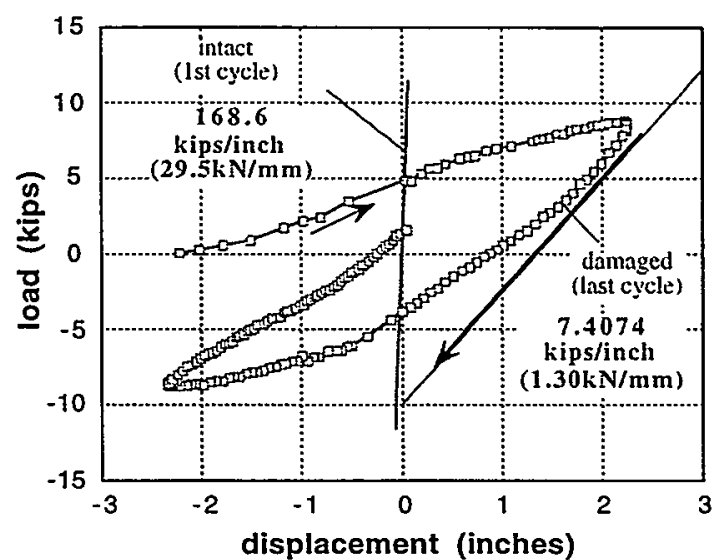

(b) $415 \mathrm{~S}$ intact and damaged columns

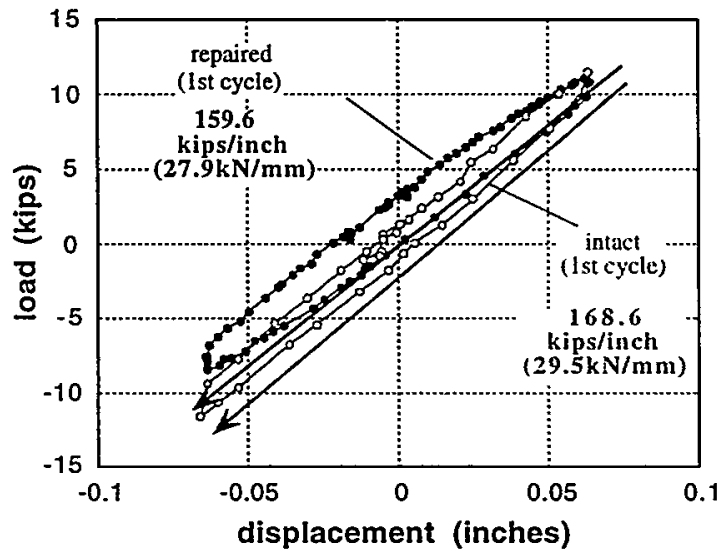

(d) $415 \mathrm{~S}$ intact and repaired columns

Figure 7.9 Stiffness of intact, damaged, and repaired columns. Damaged columns show significantly lower stiffness than intact columns. Repaired column $415 \mathrm{~S}$ shows almost full recovery of the stiffness while the column $415 \mathrm{M}$ still shows significantly reduced stiffness.

\subsubsection{Wave transmission tests}

The intact specimen \#415M was tested for wave velocities before the first loading test. Waves were generated by an impact from a small calibrated hammer. As the wavelength of the wave generated by the hammer was relatively long (wavelength to column diameter ratio $\lambda / D \sim 0.64$ at $10 \mathrm{kHz}$ ), scattering and fast channeling of the waves due to the steel reinforcement were negligible. 
For the specimen \#415M, both $\mathrm{P}$ and $\mathrm{S}$ - waves were measured along diameters while moving the source and receiver pair along the length of the column. An S-wave was generated by applying a lateral impact on a small metal piece glued on the side of the column. Figure 7.10 shows examples of the resultant waveforms. Besides the $\mathrm{P}$ and $\mathrm{S}-$ waves propagating directly from the source to the receiver, strong Rayleigh surface waves propagating along the perimeter of the column were also present. The averaged velocities of the $\mathrm{P}$ and S-waves along the column length were $3929 \mathrm{~m} / \mathrm{sec}$ for the $\mathrm{P}$-wave and 1885 $\mathrm{m} / \mathrm{sec}$ for the S-wave. At these velocities, the theoretical velocity of Rayleigh wave is 1761 $\mathrm{m} / \mathrm{sec}$, and the expected arrivals of the Rayleigh waves are shown in the plots. From the averaged measured velocities of the intact column, Young's modulus and Poisson's ratio of the column were determined as $23.7 \mathrm{GPa}$ and 0.35 , respectively, assuming that the column was homogeneous. The average density of the column was computed as $2473 \mathrm{~kg} / \mathrm{m}^{3}$ using known densities of steel reinforcement $\left(7832 \mathrm{~kg} / \mathrm{m}^{3}\right)$ and concrete $\left(2398 \mathrm{~kg} / \mathrm{m}^{3}\right)$.

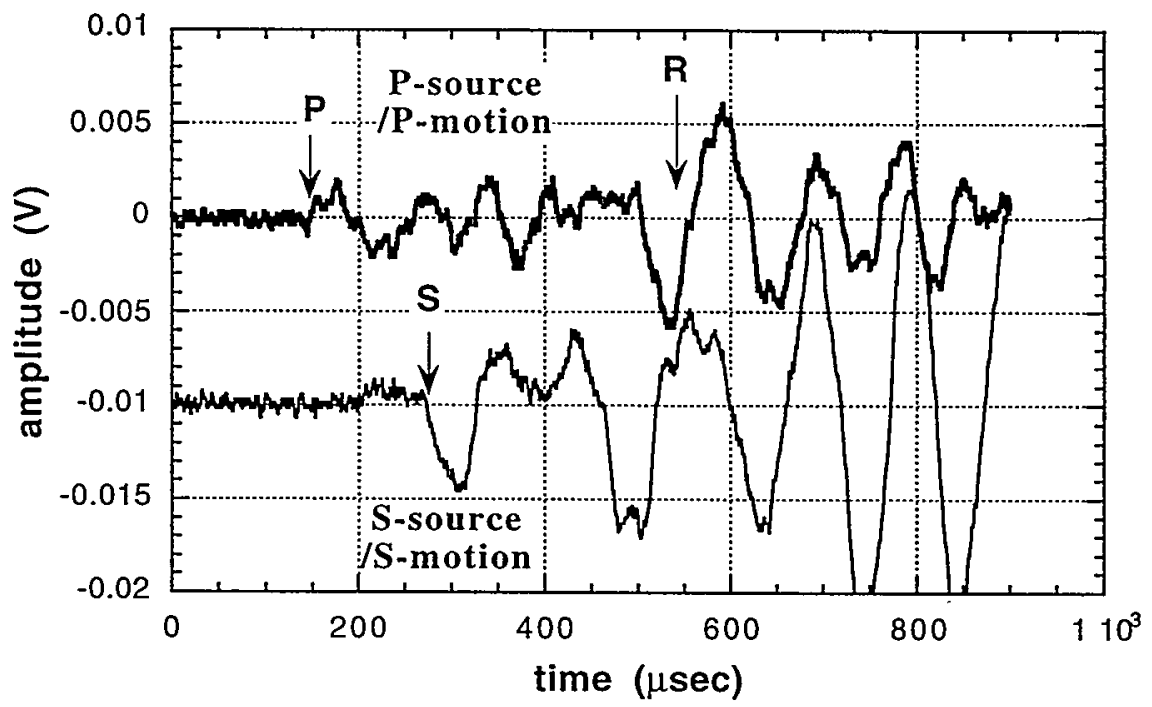

Figure 7.10 Examples of waveforms measured at the middle section of an intact column. The top waveform was obtained by applying an impact normal to the surface and measuring the normal particle motion on the other side of the column. After the arrival of the P-wave (labeled as "P"), a strong Rayleigh surface wave (labeled as "R") that propagates along the perimeter of the cross section of the column can be seen. The bottom waveform was obtained by applying a lateral impact to a small metal piece glued to the surface. The resulting particle motion on the other side of the column was measured by an accelerometer attached to the surface horizontally. The waveform shows a clear arrival of the S-wave (labeled as "S"). 
Once damage was induced in the columns, the characteristics of the waves propagating in the specimens were significantly altered. Figure 7.11 shows profiles of the travel time for the first-arriving wave (propagating along the diameter of the columns). From the plots, changes in the travel time due to the presence of fractures and microcracks can be seen clearly. For both columns, travel times increased significantly in the direction parallel to the direction of column loading. In some cases, the travel time became as large as twice the travel time for the intact specimen. In the direction normal to the applied load, the changes in the travel time and distortion of the waveform were small for both columns. Figure 7.12(a) and (b) show waveforms measured along the axis of the column specimens. In the direction parallel to the loading direction, the amplitude of the first-arriving P-wave diminishes and the travel time of the wave increases significantly. In the direction normal to the loading direction, the P-wave shows a clear first arrival even after the columns were damaged. However, in the section where cracking of the concrete was observed, a large amplitude wave that propagates slower than the P-wave but faster than both S- and Rayleigh waves can be seen (Figure 7.13).

The different characteristics of the waves propagating in the two directions (parallel and perpendicular to the loading direction) reflects the geometry of the fractures introduced in the columns. In both directions in the columns, an increase in the travel time is caused by microcracks and fractures that cause frequency-dependent time delays in the propagating waves (e.g., Pyrak-Nolte et al., 1990a). The extreme time delays observed in the direction parallel to the loading direction are possibly due to the tortuous paths through which those waves have to propagate. As the waves cannot directly propagate across the fractures, they instead are multiply reflected by the fractures and free surface of the column before reaching the receiver. Conversely, the large amplitude of the late-arriving wave in the normal direction is possibly due to the waves trapped and multiply reflected between approximately parallel fractures. Such a wave is known as the fracture channel wave (Nihei et al., 1998) and can propagate for a long distance without attenuating. The geometry of fractures that cause these differences in the characteristics of wave propagation in each direction is schematically shown in Figure 7.14. 

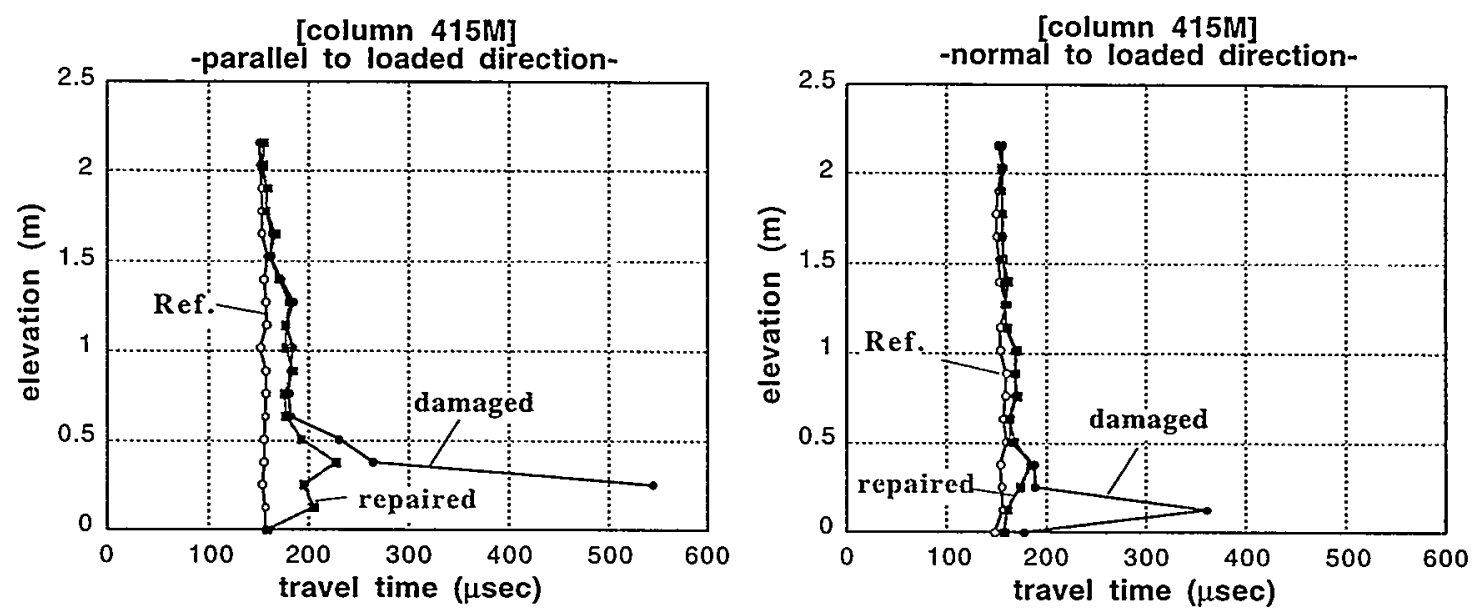

(a) Moderately damaged column (415M)
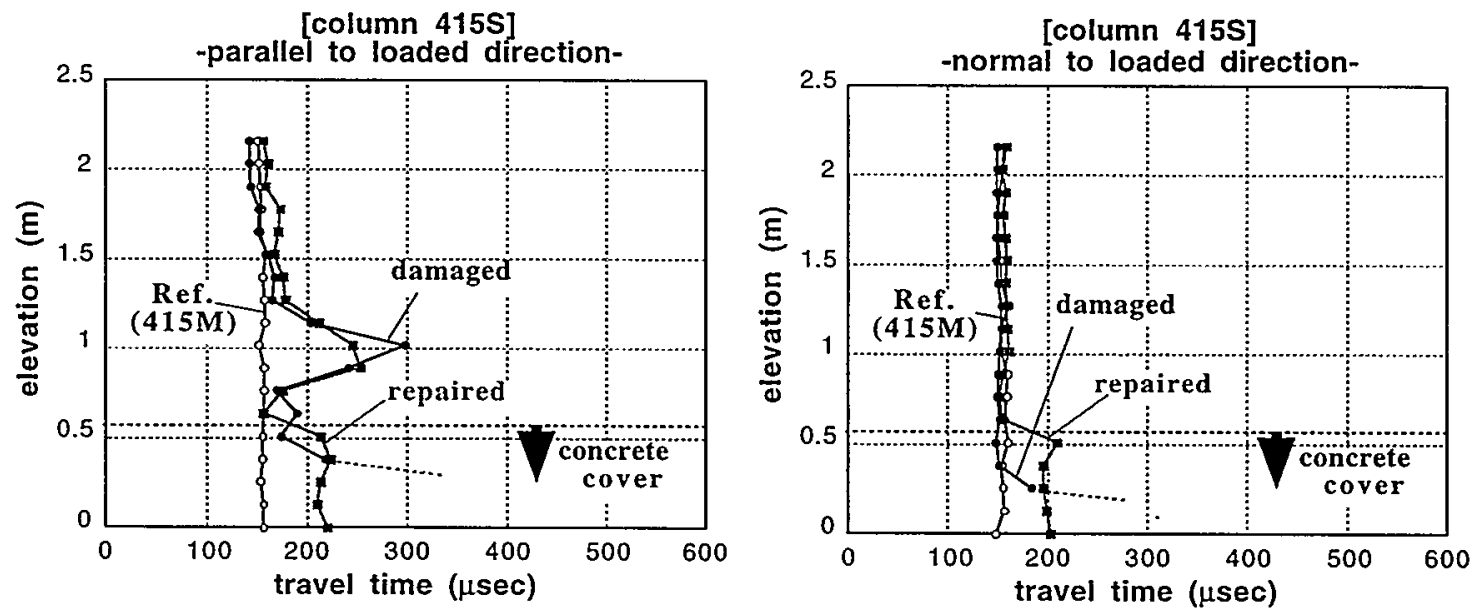

(b) Severely damaged column (415S)

Figure 7.11 Vertical profiles of P-wave travel time along the diameter of column specimens. The profiles for the intact specimen (labeled "Ref.") were measured only for specimen 415M. Damaged specimens show large increases in travel times. For specimen $415 \mathrm{M}$, travel time increases monotonically as it gets close to the bottom of the column. Specimen $415 \mathrm{~S}$ exhibits a local peak at elevation $H=1.0 \mathrm{~m}$, which indicates localized damage in the middle section. It is noted that the direction perpendicular to the loading direction exhibits a much smaller change in the travel time for the middle section than in the loading direction. Although the bottom section of the repaired specimen $415 \mathrm{M}$ showed a significant decrease in the wave travel time ( $415 \mathrm{M})$, this was not true over the entire epoxy-injected section. The apparent increase in travel time for the repaired specimen $415 \mathrm{~S}$ is due to the increased diameter of the column cross section. 


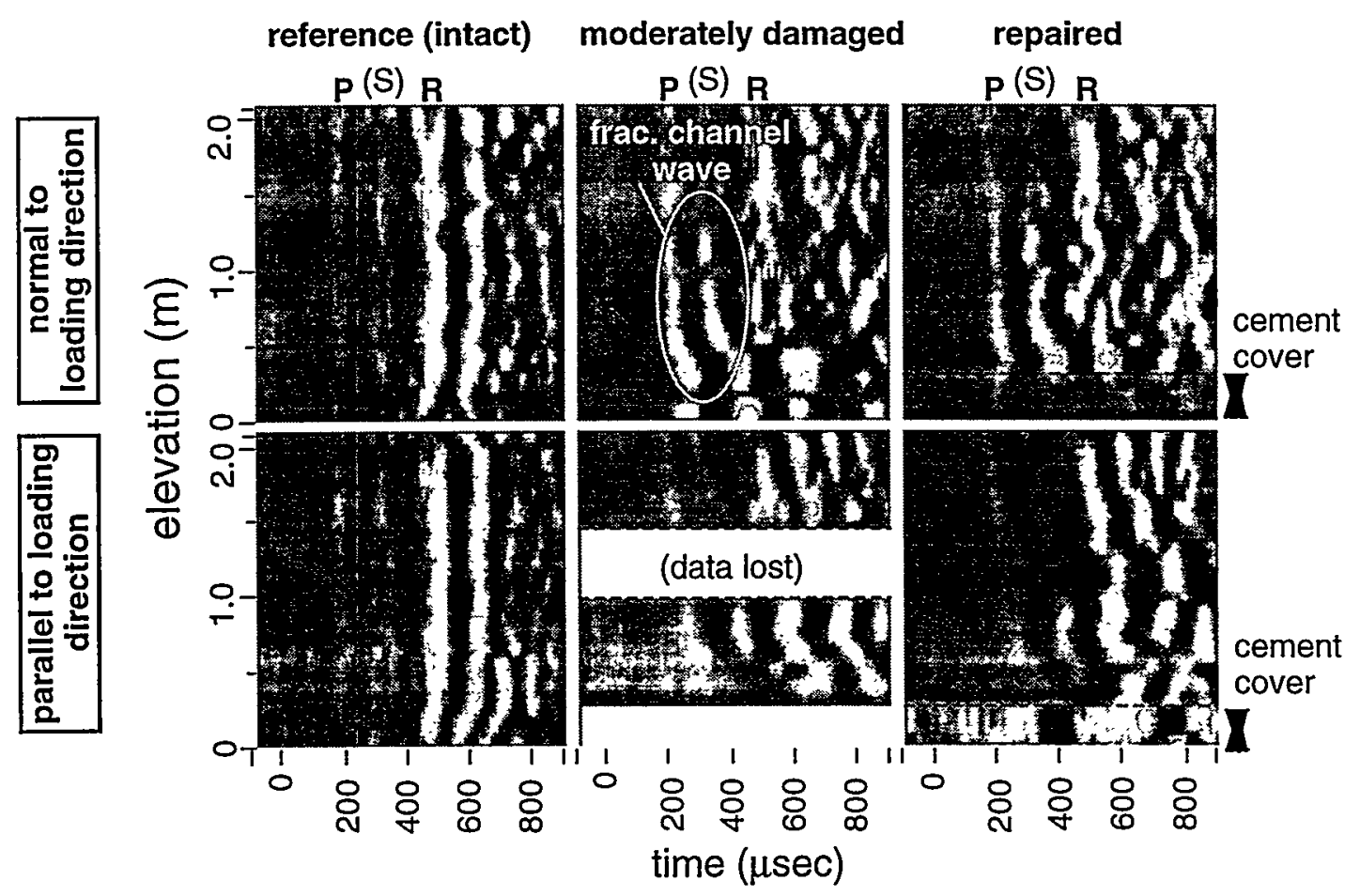

(a) Seismograms for column $415 \mathrm{M}$ (moderately damaged)

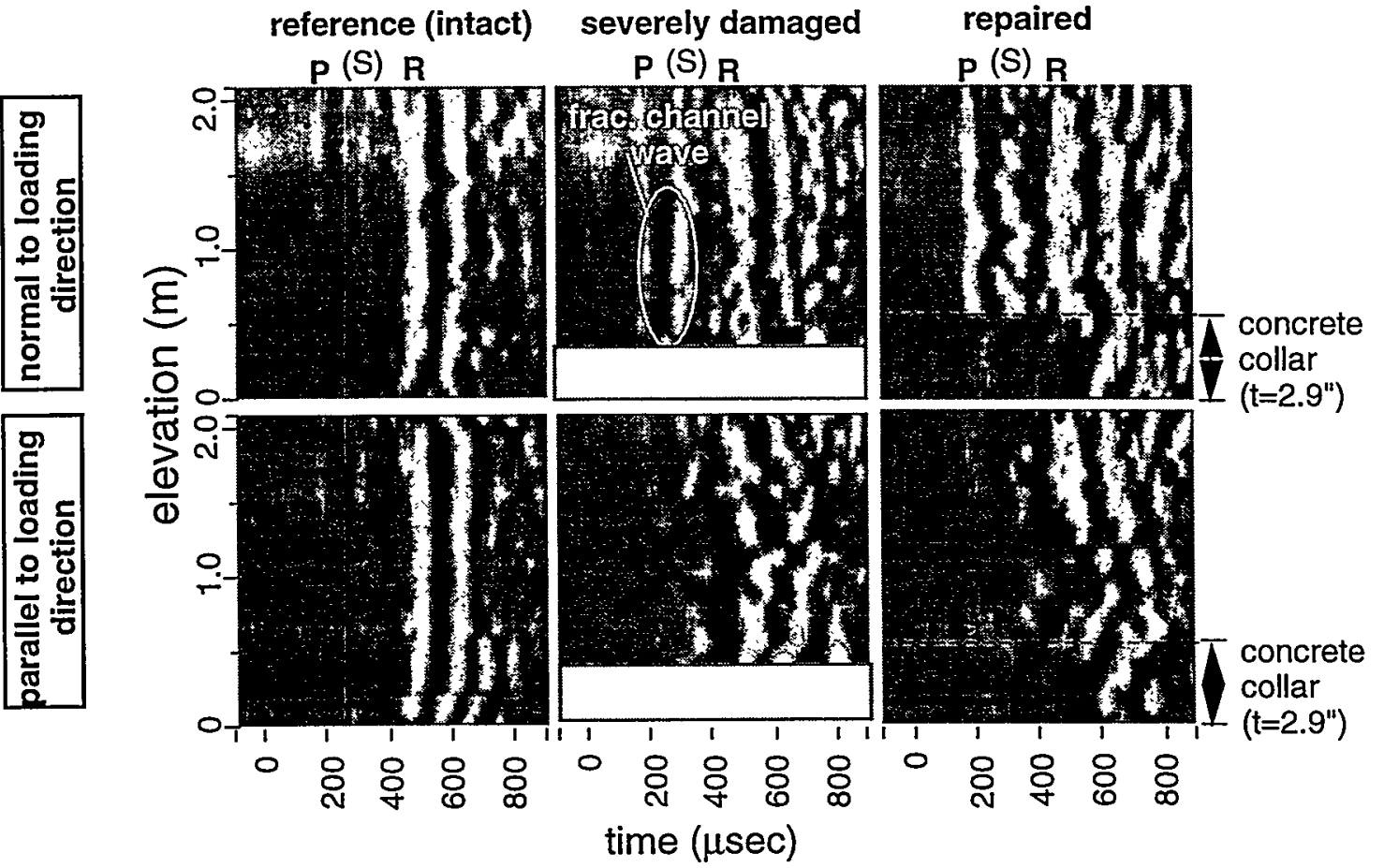

(b) Seismograms for column $415 \mathrm{~S}$ (severely damaged)

Figure 7.12 Seismograms for specimens before and after damage introduction and repair. Large amplitude is shown by white (positive) and black (negative). Damaged specimens show significant change in the velocity and amplitude of the first-arriving part of the wave in the direction parallel to the loading direction while the change normal to the loading direction is small. 


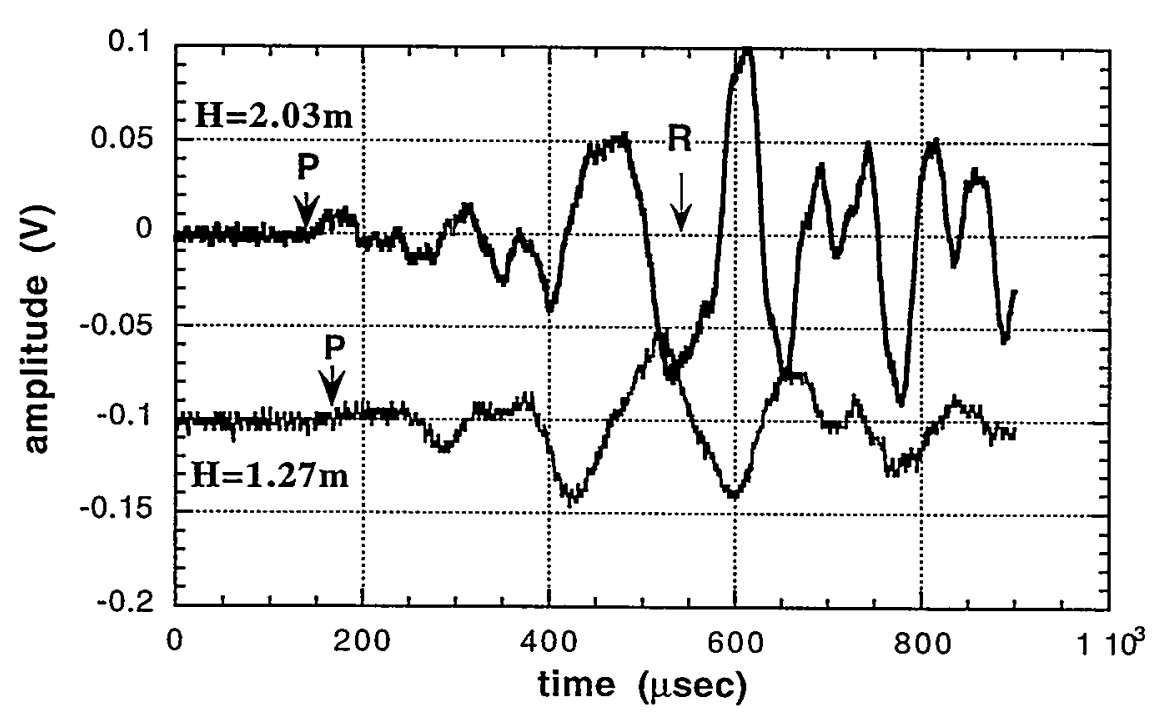

(a) parallel to loading direction

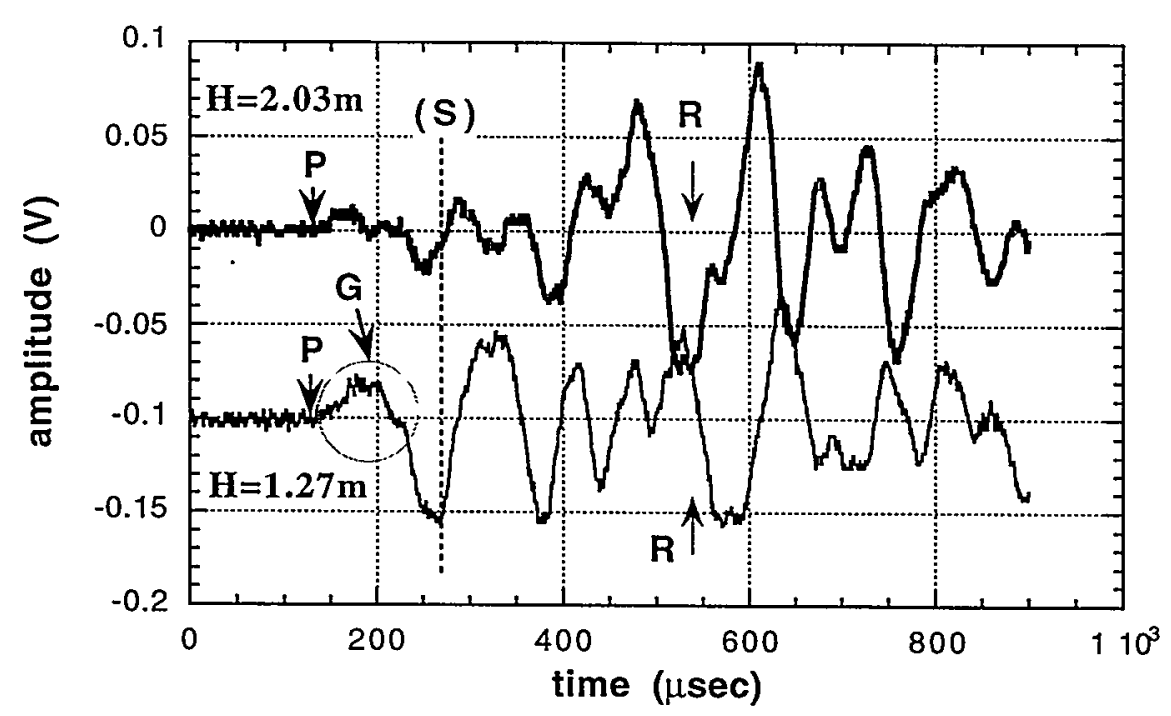

(b) perpendicular to loading direction

Figure 7.13 Example of measured waveforms for column 415S. At level $\mathrm{H}=2.03 \mathrm{~m}$, no surface fractures were observed. Whereas level $\mathrm{H}=1.27 \mathrm{~m}$ is in a section where distinct horizontal surface fractures were observed. (a) In the direction parallel to the loading direction, the first-arriving P-wave velocity and amplitude is smaller for the damaged section. (b) In the direction perpendicular to the loading direction, the velocity of the first-arriving $\mathrm{P}$-wave does not show significant change. However, there is a distinct arrival of wave energy between the first-arriving P-wave and expected S-wave (circled and labeled as " $G$ "). 


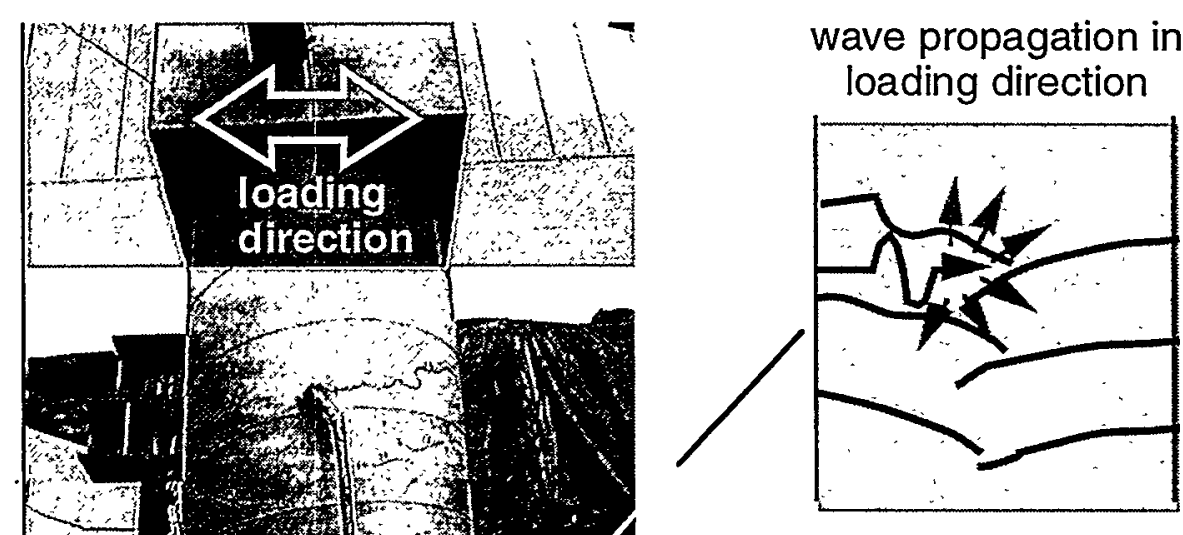

- Large decrease in velocity - Wave is greatly attenuated due to reflection by fractures

\section{wave propagation normal to loading direction}

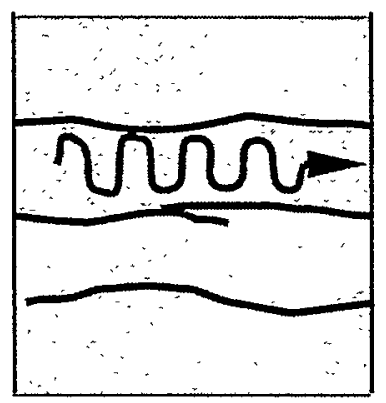

- Slight decrease in velocity - Strong secondary arrival of fracture-guided wave

Figure 7.14 Explanation for different wave behaviors in directions normal and perpendicular to the lateral displacement applied to the columns. Direction normal to the loading direction shows small change in its velocity and generation of a fracture channel wave. In the direction parallel to the loading direction, both velocity and amplitude of the wave decreases significantly as the wave is scattered by fractures curving down in the column. 


\subsubsection{Resonance tests}

\subsubsection{Resonance frequency}

Resonance frequencies of the columns were measured either by continuous excitation from a mechanical shaker or by an impact from a plastic sledge hammer. For most of the tests, however, the sledge hammer source was preferred because a sweep with the shaker requires an impractically long time (typically several hours to sweep over a desired frequency range with sufficient resolution). The hammer source, on the other hand, requires only a few minutes to complete all of the measurements needed for averaging. Figure 7.15 shows a comparison between spectra measured by the two methods on the damaged column \#415S. Resonance frequencies of the first mode show good agreement indicating that measurements made from the hammer source are sufficient for determining resonance frequencies of the specimens.

Figure 7.16(a) and (b) show measured spectra using the hammer source for the specimens. Amplitudes of the individual spectra are normalized by their maxima. In the plots, measurements conducted in the loading direction are labeled as " $\mathrm{A}$ " and measurements in the direction perpendicular to the loading direction are labeled as "B". From Figure 7.16(a), it can be clearly seen that resonance frequencies decrease as more severe damage is introduced to the specimen. Figure 7.16(b) shows recovery of the resonance frequencies after repair. For the severely damaged and repaired specimens, the resonance frequencies are even higher than the intact specimen, possibly due to the increased stiffness of the column at the bottom section.

\subsubsection{Splitting of resonance}

Although the frequencies of the modes "A" and "B" are different, these are essentially the same type of mode. Figure 7.17 shows mode shapes of the observed resonances for the intact \#415M and damaged \#415S specimens. The mode shapes were measured by resonating the specimen at a single frequency using the mechanical shaker and measuring the resulting amplitude of acceleration along the columns. The amplitudes were normalized to the maximum for comparison. The measured mode shapes indicate that these are the first-order flexural mode. If the bending stiffness of a column is isotropic around its axis, the first two flexural modes are degenerate, having identical resonance frequencies. Nondegenerate flexural modes with different resonance frequencies (splitting of resonance) 


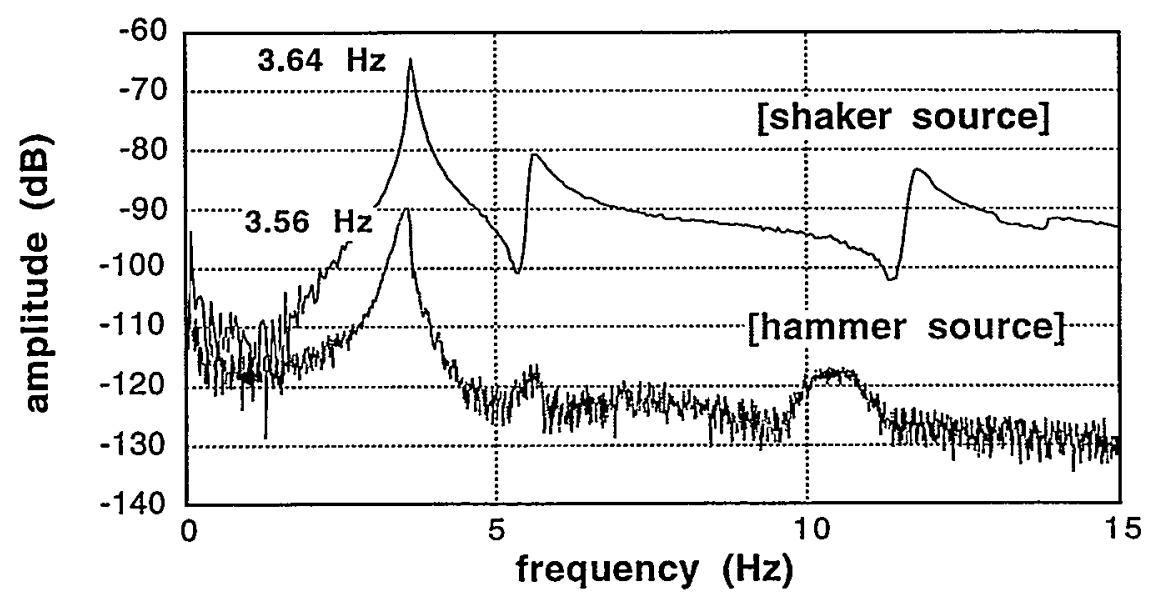

(a) resonance parallel to the loading direction

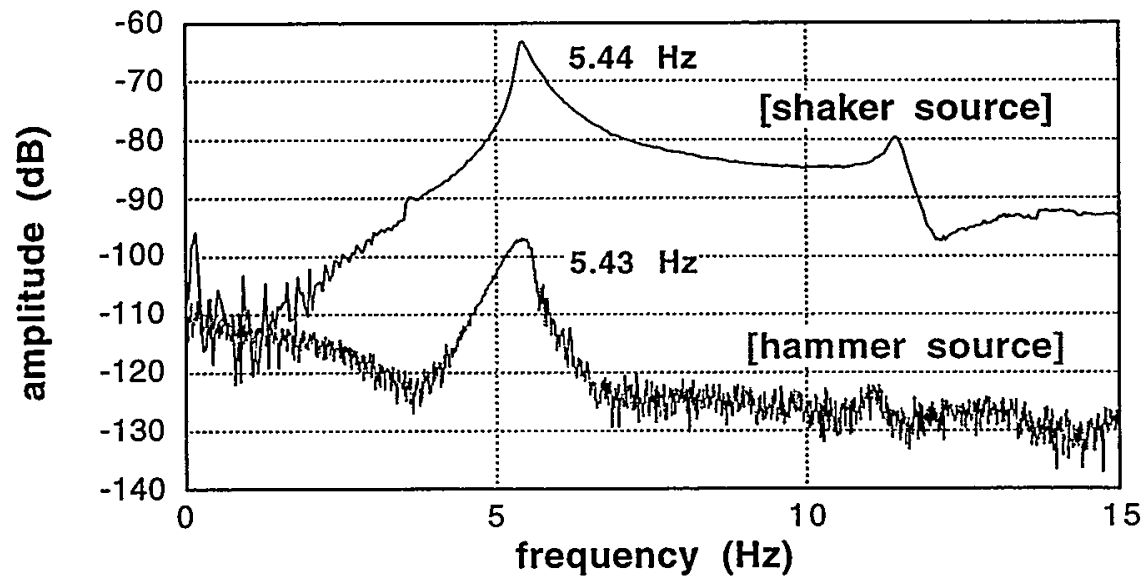

(b) resonance perpendicular to the loading direction

Figure 7.15 Comparison between resonances introduced by different types of sources. The tested specimen was damaged 415S. Although the shapes of the spectra are quite different, both sources yield resonances with similar resonance frequencies. 


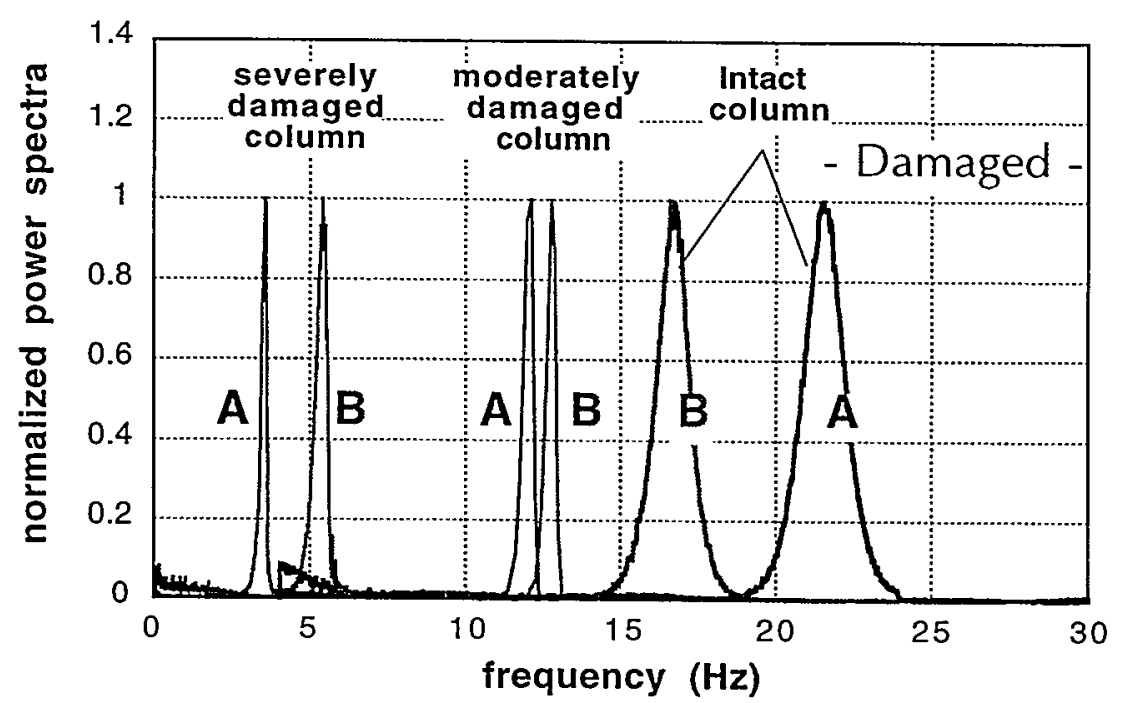

(a) before and after the first loading test

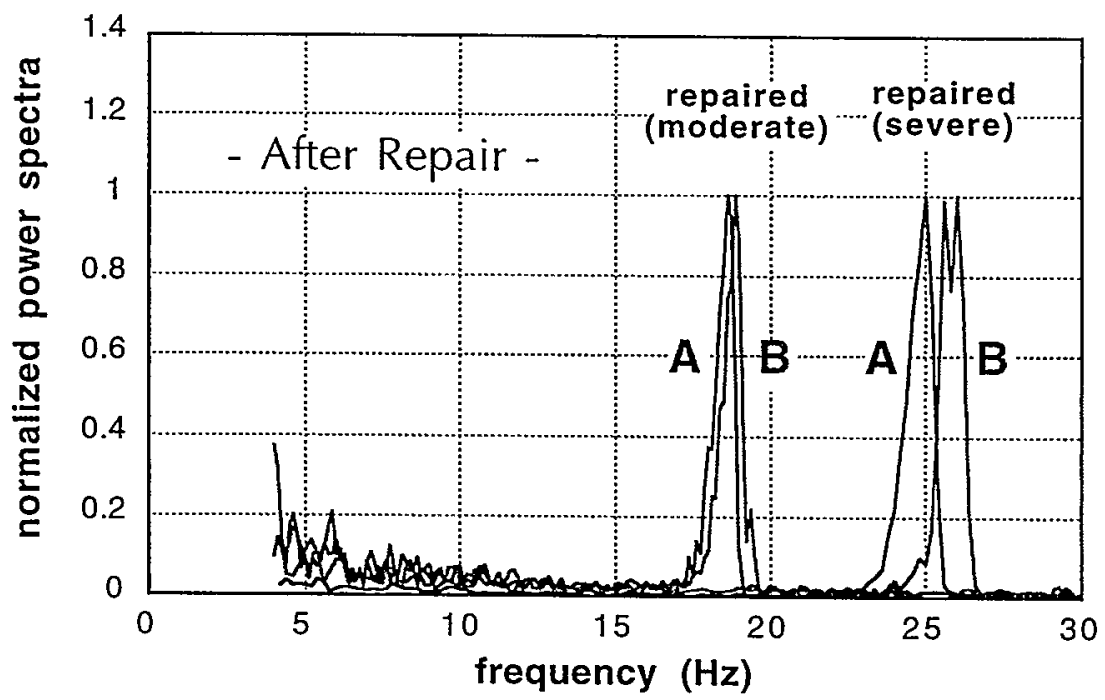

(b) after repair

Figure 7.16 Normalized spectra measured using a sledge-hammer source. Each spectrum has a clear single resonance peak. Resonance frequencies are different for directions of vibration parallel $(A)$ and perpendicular (B) to the direction of the applied displacement during the static loading test. 


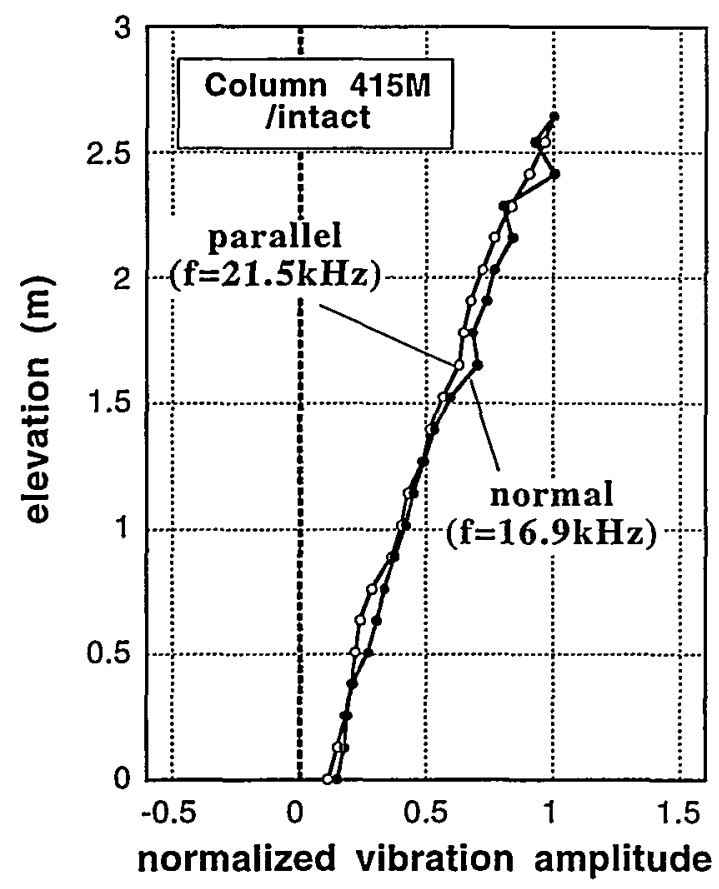

(a) column $415 \mathrm{M} /$ intact

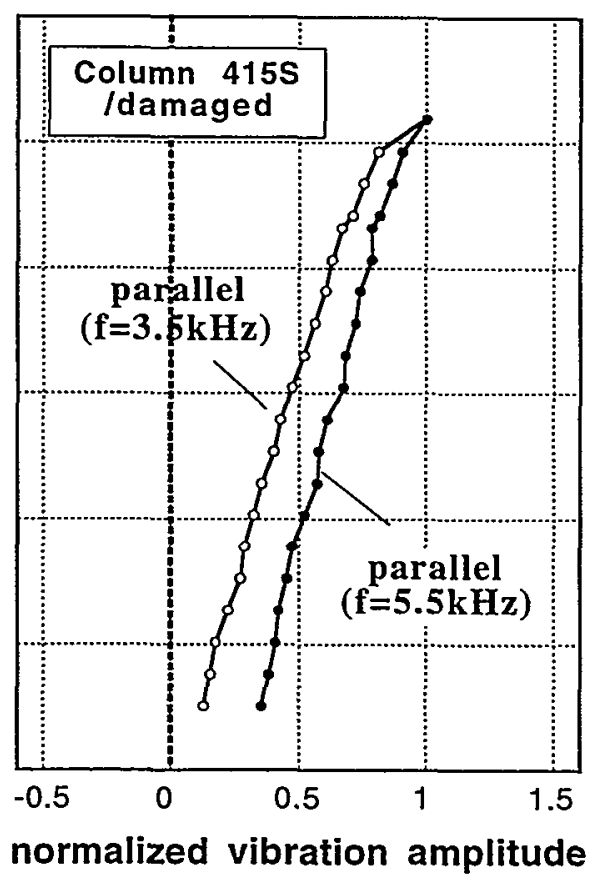

(b) column $415 S /$ damaged

Figure 7.17 Normalized amplitude of mode shapes measured for the observed resonances. Shapes of the modes indicate these are of the same mode type (first flexural mode). For column 415S, mode shape in the direction normal to the loading direction shows a relatively large amplitude at the bottom of the column. This may indicate a large motion in the footing supported by wooden blocks.

indicate that the stiffness of the columns are not isotropic around their axes. For the damaged columns, this anisotropy is primarily due to the damage introduced by directional loading. As a result, stiffness of the column became lower in the loading direction, yielding a lower resonance frequency. The splitting of the resonance for the intact column is possibly due to the geometry of the footing that is longer in the loading direction. As the deformation of the footing on the plywood is larger in the direction perpendicular to the loading direction, the column exhibits a lower resonance frequency in this direction. Although even larger effects from the footing may be expected for the damaged column \#415S tested on wooden blocks, resonance of the column is largely decoupled from the footing due to the formation of a compliant "hinge" at the bottom of the column. The mode shapes in Figure 7.17(b), however, indicate relatively large footing motion in the direction perpendicular to the loading. 


\subsubsection{Attenuation (Damping)}

Assuming a linear visco-elastic response for the resonating specimens, the resonance frequency and attenuation were determined by fitting the following equation to the measured response

$$
u_{x}(t)=A \cdot \exp (-\zeta \cdot t) \cdot \sin \left\{2 \pi\left(f_{n} \sqrt{1-\zeta^{2}}-f_{0}\right) \cdot t\right\}
$$

where $f_{n}$ is the undamped natural frequency (resonance frequency) of the structure and $\zeta$ is the damping coefficient. As $\zeta$ is usually small, $f_{n} \sqrt{1-\zeta^{2}}$ in the equation can be replaced by $f_{n}$. Although the attenuation could also be determined by the half-power method, it was not employed as the method resulted in unreasonably large or small attenuation. This inaccuracy might be due to the contamination of measured signals by the large transient waves that arrive before the development of resonance. Figure 7.18 shows

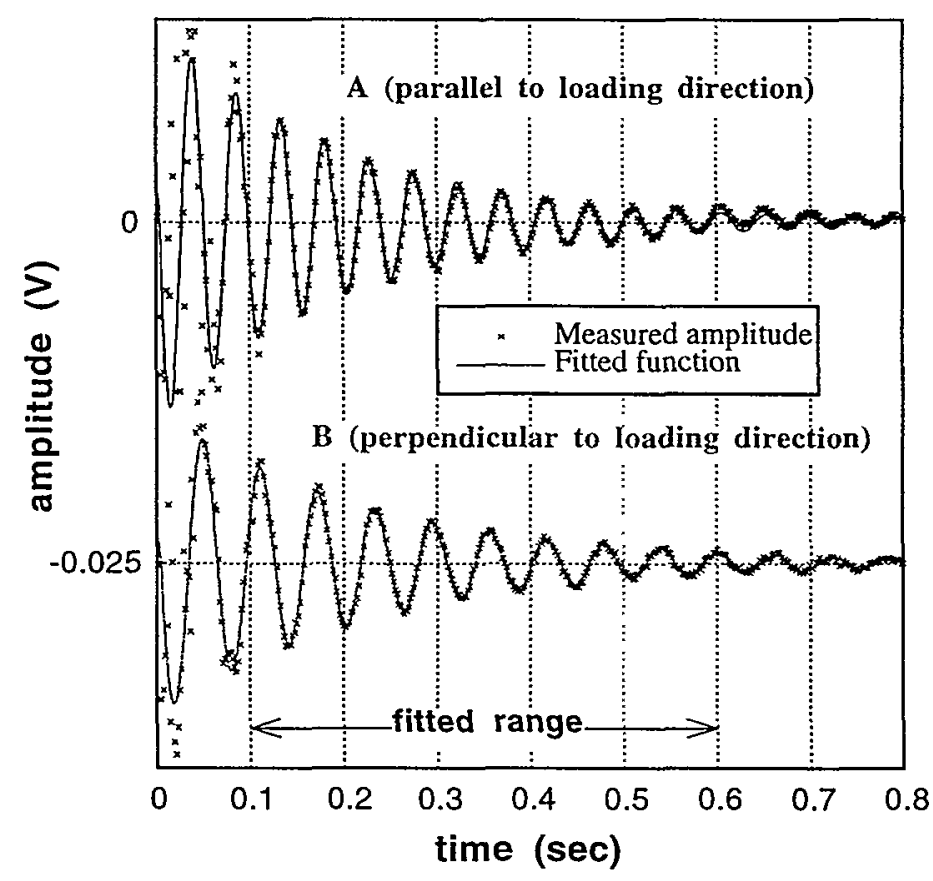

Figure 7.18 The measured transient vibration introduced by an impact from a sledge hammer was fitted with a function of unknown frequency and attenuation parameters. The above examples are for the intact column $415 \mathrm{M}$. 
Table 7.1 Resonance frequency and attenuation of column specimens

\begin{tabular}{|c|l|l|l|l|}
\hline Specimen & direction & \multicolumn{1}{|c|}{ Intact } & \multicolumn{1}{|c|}{ Damaged } & \multicolumn{1}{c|}{ Repaired } \\
\hline 415M & parallel & $\mathrm{fn}=21.15 \mathrm{~Hz}$ & $\mathrm{fn}=11.50 \mathrm{~Hz}$ & $\mathrm{fn}=17.64 \mathrm{~Hz}$ \\
(moderate & & $\zeta=0.038$ & $\zeta=0.018$ & $\zeta=0.025$ \\
damage) & \multirow{2}{*}{ normal } & $\mathrm{fn}=16.31 \mathrm{~Hz}$ & $\mathrm{fn}=12.45 \mathrm{~Hz}$ & $\mathrm{fn}=18.08 \mathrm{~Hz}$ \\
& & $\zeta=0.043$ & $\zeta=0.016$ & $\zeta=0.024$ \\
& & $\mathrm{fn}=3.53 \mathrm{~Hz}$ & $\mathrm{fn}=23.77 \mathrm{~Hz}$ \\
$415 \mathrm{~S}$ & parallel & & $\zeta=0.019$ & $\zeta=0.026$ \\
(severe & & & & \\
damage) & normal & & & \\
& & & $\zeta=0.02 \mathrm{~Hz}$ & $\mathrm{fn}=25.14 \mathrm{~Hz}$ \\
& & & $\zeta=0.029$ \\
\hline
\end{tabular}

Note: The "direction" shows the direction of vibration motions with respect to the loading direction.

an example of measured acceleration response and the fitted equation. As the resonance was not sufficiently developed during early times and noise prevailed during later times, the fitting was performed for intermediate times. The choice of the selected period was somewhat arbitrary. The determined resonance frequencies and attenuation are shown in Table 7.1 and Figure 7.19.

In general, attenuation decreases with damage and increases with repair. The smallest attenuation, however, was observed for the moderately damaged specimen $\# 415 \mathrm{M}$. The general trend observed for the column specimens can be explained by invoking the onedimensional resonance of a delaminated halfspace, as discussed in Chapter 2. Because localized damage at the bottom of the columns serves as a compliant interface between the column part and the footing part of the specimen, resonance of a damaged specimen exhibits smaller attenuation than an intact specimen due to the trapping of vibration energy in the column part. The observed attenuation behavior indicates that attenuation due to the dissipation of vibration energy in the column from damage is overcome by attenuation due to radiative loss of energy into the footing and the foundation. The cause of the relatively large attenuation in the severely damaged specimen may be due to an increase in dissipation of energy within the column. 


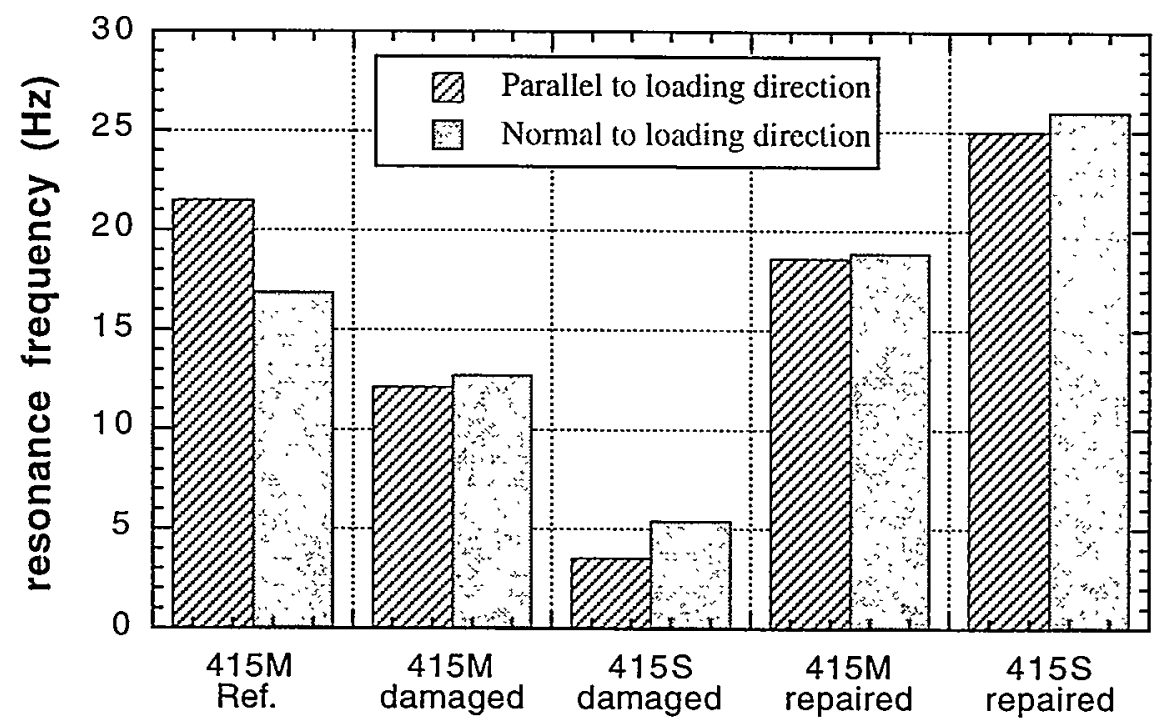

(a) resonance frequencies of time-domain vibration signal

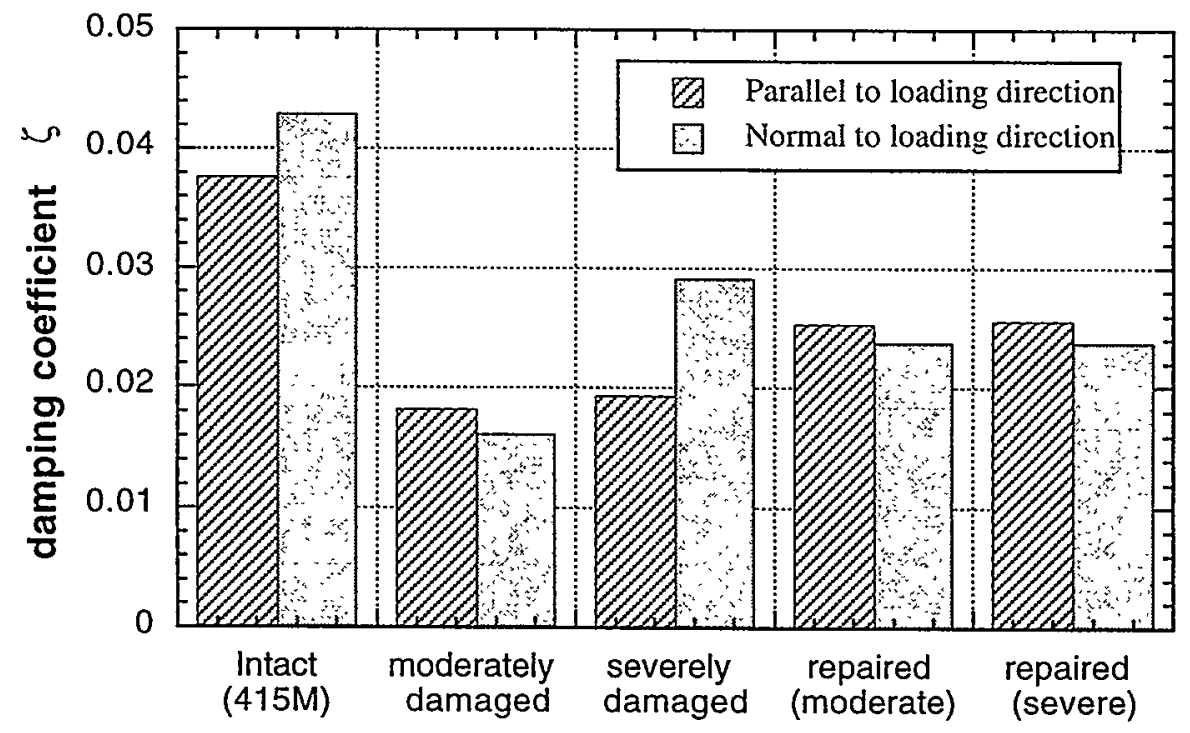

(b) attenuation of time-domain vibration signal

Figure 7.19 Resonance frequencies and attenuation (damping coefficients) for measured vibration of columns hit by a sledge hammer. Resonance frequency decreases as damage is introduced to the specimen and recovers after the repair. Although attenuation shows a similar trend as the resonance frequency, the severely damaged specimen exhibits rather large attenuation especially in the direction normal to the loading direction. 


\subsection{Static stiffnesses and resonance frequencies}

\subsubsection{Displacement-discontinuity B.C. for column containing a compliant interface}

The degree of damage introduced into a structure is reflected by an increase in its elastic compliance that can be measured statically by load-displacement tests. The change in the compliance (or stiffness) can also be measured from the changes in resonance frequencies of the structure. The statically (load-displacement) and dynamically (resonance) determined elastic compliances of a structure should ideally be identical if the soundness of a structure is to be accurately determined from resonance tests.

From measured static load-displacement behaviors, the elastic stiffness of the columns can be determined. Table 7.2 shows the experimentally determined flexural stiffness of the columns before and after the damage was introduced and repaired (see Figure 7.9). As the laterally loaded columns show strongly localized damage at the bottom, a damaged specimen can be, to a first-order approximation, modeled by a circular column attached to a rigid foundation via a compliant interface. The load-displacement behavior of the interface is described by displacement-discontinuity boundary conditions (Schoenberg, 1980; PyrakNolte et al., 1990a) using normal and tangential specific interface stiffnesses (fracture stiffnesses). For an interface located on the $x-y$ plane with lateral load applied along the $x-$ axis, the boundary conditions are

$$
\begin{aligned}
& t_{x}^{-}=\kappa_{f}\left(u_{x}^{+}-u_{x}^{-}\right), \\
& t_{z}^{-}=\kappa_{n}\left(u_{z}^{+}-u_{z}^{-}\right), \\
& t_{x}^{-}=-t_{x}^{-} \equiv 1 \cdot \tau, \\
& t_{z}^{-}=-t_{z}^{-} \equiv 1 \cdot \sigma,
\end{aligned}
$$

Table 7.2 Measured static bending stiffness of columns

\begin{tabular}{|c|c|c|c|}
\hline Specimen & Intact & Damaged & Repaired \\
\hline $415 \mathrm{M}$ & $161 \mathrm{kips} / \mathrm{inch}$ & $45.8 \mathrm{kips} / \mathrm{inch}$ & $95.7 \mathrm{kips} / \mathrm{inch}$ \\
(moderate damage) & $(28.1 \mathrm{kN} / \mathrm{mm})$ & $(8.01 \mathrm{kN} / \mathrm{mm})$ & $(16.8 \mathrm{kN} / \mathrm{mm})$ \\
$415 \mathrm{~S}$ & $169 \mathrm{kips} / \mathrm{inch}$ & $7.41 \mathrm{kips} / \mathrm{inch}$ & $160 \mathrm{kips} / \mathrm{inch}$ \\
$\begin{array}{c}\text { (severe damage) } \\
(29.5 \mathrm{kN} / \mathrm{mm})\end{array}$ & $(1.30 \mathrm{kN} / \mathrm{mm})$ & $(27.9 \mathrm{kN} / \mathrm{mm})$ \\
\hline
\end{tabular}


where $\kappa_{n}$ and $\kappa_{t}$ are normal and tangential specific fracture stiffnesses, respectively, $u_{x}$ and $u_{z}$ are displacements, $t_{x}$ and $t_{z}$ are tractions, and $\sigma$ and $\tau$ are normal and tangential stress on the interface. Superscripts " + " and "-" denote the column side and the foundation side of the interface, respectively. If the bending moment $M$ and lateral load $Q$ are applied to the interface, assuming that the interface remains plane after deformation, displacement in $\mathrm{z}$-direction is given by

$$
u_{z}=\theta \cdot x
$$

Where $\theta$ is the inclination angle for the cross section plane of the column originally parallel to the $\mathrm{x}-\mathrm{y}$ plane. From the above equation,

$$
u_{z}^{+}-u_{z}^{-}=\left(\theta^{+}-\theta^{-}\right) x \text {. }
$$

The bending moment on the interface is given by

$$
M=\int_{A} \sigma \cdot x d A
$$

From Eq.(7.3), (7.4), and (7.5),

$$
M=\int_{A} \kappa_{n}\left(u_{z}^{+}-u_{z}^{-}\right) x d A=\kappa_{n}\left(\theta^{+}-\theta^{-}\right) \int_{A} x^{2} d A \equiv \kappa_{n} I\left(\theta^{+}-\theta^{-}\right),
$$

where I is the moment of inertia for the column's cross-section. Therefore, the displacement-discontinuity boundary conditions in the z-direction for the interface become

$$
\begin{aligned}
& M=\kappa_{n} I \cdot\left(\theta^{+}-\theta^{-}\right), \\
& M^{+}=M^{-} \equiv M .
\end{aligned}
$$

In the x-direction,

$$
Q=\int_{A} \tau d A
$$

Therefore,

$$
\begin{aligned}
& Q=\kappa_{t} A \cdot\left(u_{x}^{+}-u_{x}^{-}\right), \\
& Q^{+}=Q^{-} \equiv Q .
\end{aligned}
$$

Using Eq.(7.7a) (7.9b), elementary beam (Euler-Bernoulli beam) theory yields the flexural compliance of the column measured at the top for a lateral load applied at the center of the top concrete block in the form 


$$
\frac{1}{K_{M}} \equiv \frac{\Delta u_{x}}{F}=\frac{L_{c}^{3}}{E I}\left\{\frac{1}{3}+\frac{1}{2} \frac{L-L_{c}}{L_{c}}+\frac{1}{\kappa L_{c} / E}\left(1+\alpha^{2}+\frac{L-L_{c}}{L_{c}}\right)\right\},
$$

where $\mathrm{E}$ is the Young's modulus of the specimen, $\mathrm{I}$ is the cross-sectional inertia, $\mathrm{L}$ is the length of the column and $\mathrm{Lc}_{\mathrm{c}}$ is the elevation of the loading point. In the above expression, the specific stiffness of the interface was assumed to be isotropic $\left(\kappa_{n}=\kappa_{t}=\kappa\right)$. The parameter $\alpha$ is a ratio between the diameter of the column $\mathrm{R}$ and $\mathrm{Lc}$.

From the measured static stiffnesses of the intact columns and Eq.(7.10) with $\kappa=\infty$ (welded interface), the Young's modulus of the columns were determined to be $21.65 \mathrm{GPa}$ for specimen \#415M and $22.72 \mathrm{GPa}$ for specimen \#415S. Considering the error in measuring the static stiffness of the column, these values are sufficiently close to the dynamically determined Young's modulus of $23.7 \mathrm{GPa}$ from velocities of propagating waves. For a finite $\kappa$, Eq.(7.10) is used to compute resulting bending stiffness of the columns.

\subsubsection{Dynamic response of the structure}

Using the determined dynamic Young's modulus of the intact column and the assumed stiffness of the interface, the resonance frequency of the first flexural vibration can be computed using the numerical code described in Chapter 5 and Chapter 6 . As the geometry of the column specimen is too complex to be modeled by the code, and the code cannot model the dynamic interaction between the specimen and the foundation, some idealizations of the geometrical and mechanical properties of the column were made.

Two idealized models were examined for their resonance frequencies using the numerical code, assuming a simple cylindrical geometry of the column and a rigid footing and foundation (Figure 7.20). The first model has an identical height and column density as the actual specimens. The effect of the mass added by the top concrete block was accounted for by placing a point mass at its center of gravity in the column. The first flexural mode resonance frequency for this model was computed to be $31.2 \mathrm{~Hz}$. The computed resonance frequency seemed to be much higher than the measured resonance frequency of $21.15 \mathrm{~Hz}$ (loading direction) or $16.31 \mathrm{~Hz}$ (perpendicular to the loading direction). The primary cause for the difference in the computed and measured resonance frequency is possibly the vibration within the footing and the foundation of the actual specimen that is modeled as a rigid half-space in the numerical model. This speculation is 
MODEL I

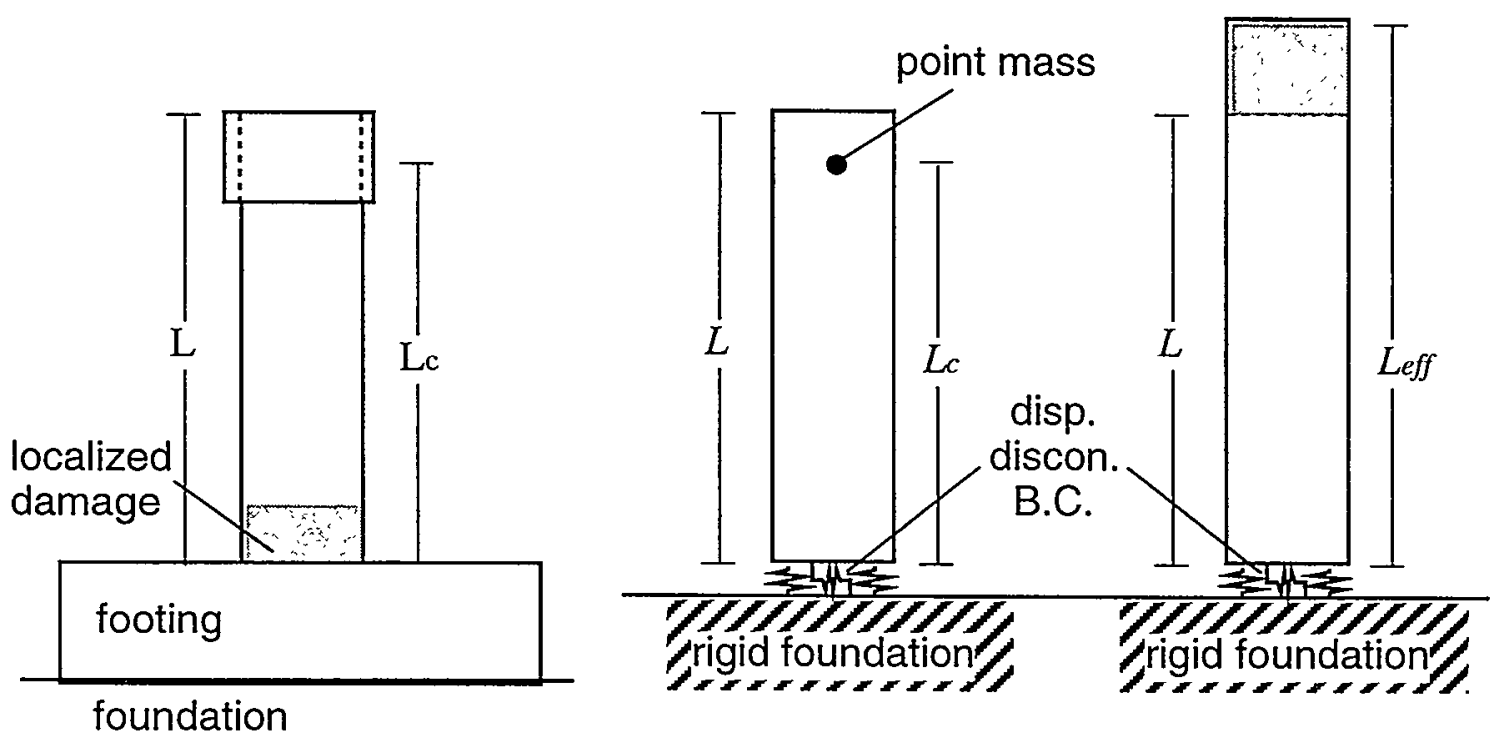

Figure 7.20 Idealization of the damaged column. Both model I and II consist of a circular column attached to a rigid foundation via an interface with displacement-discontinuity boundary conditions. Model I includes a point mass that accounts for the effect of the additional mass due to the rectangular block at the top. Model II is a simple circular column but the length of the column is determined by matching the theoretical resonance frequency of the intact model and the experimentally measured resonance frequency.

also supported by the measured mode shape of the flexural resonance (Figure 7.17(a)). The amplitude of the measured vibration does not become zero at the bottom of the column, indicating that there are finite vibrations in the footing part of the specimen.

To account for the vibration within the footing and the foundation, the effective length of the column $L_{e f f}$ was defined by matching the resulting resonance frequency of the intact column. For this model, the effect of the mass added by the top concrete block was also accounted for by introducing the effective length of the column. Using a solution for the resonance frequency of a cantilever beam (Graff, 1975), $L_{e f f}$ is computed from

$$
f_{n}=\frac{\left(\beta_{1} L_{e f f}\right)^{2}}{2 \pi L_{e f f}^{2}} \sqrt{\frac{E I}{\rho A}}, \quad \beta_{1} L_{e f f}=1.875 .
$$

For the measured resonance frequency of the intact column in the loading direction $(21.15 \mathrm{~Hz})$, the effective length of the column was determined to be $3.52 \mathrm{~m}$. 
Computed resonance frequencies of the first flexural mode for a range of interface stiffnesses are shown in Figure 7.21. For resonance frequencies measured in the loading direction, the corresponding stiffnesses of the interface are determined. It should be noted that for a resonance frequency higher than the resonance frequency of an intact column, no solution for the interface stiffness exists. Interface stiffness determined in this way is not necessarily the same as the stiffness used in Eq.(7.10), since deformation of the footing and foundation are accounted for in defining the length of the column. However, it should still approximate the stiffness of the damaged section that causes a decrease in the bending stiffness of the column. By introducing this value of stiffness into the Eq.(7.10), the resulting bending stiffness of the columns is determined (Table.2.3). Figure 7.22 compares the measured static bending stiffnesses and dynamic stiffnesses determined from measured resonance frequencies. The stiffnesses for damaged specimens and repaired, moderately damaged specimens show very good agreement. The stiffness of the repaired, severely damaged column cannot be determined as the resonance frequency is higher than the intact column.

The above results demonstrate that the elastic stiffness of a column can be determined from its resonance frequency. As the level of damage and the effect of repair can be directly related to the bending stiffness of the column, the above analysis provides a technique to assess the soundness of a structure quantitatively from its resonance frequency.

Table 7.3 Bending stiffness of columns determined from resonance frequency

\begin{tabular}{|c|c|c|c|}
\hline Specimen & Intact & Damaged & Repaired \\
\hline $\begin{array}{c}415 \mathrm{M} \\
\text { (moderate damage) }\end{array}$ & $29.5 \mathrm{kN} / \mathrm{mm}$ & $8.61 \mathrm{kN} / \mathrm{mm}$ & $20.7 \mathrm{kN} / \mathrm{mm}$ \\
$\begin{array}{c}415 \mathrm{~S} \\
\text { (severe damage) }\end{array}$ & $-0.81 \mathrm{kN} / \mathrm{mm}$ & $>29.5 \mathrm{kN} / \mathrm{mm}^{\mathrm{a}}$ \\
\hline
\end{tabular}

a Bending stiffness could not be determined as the resonance frequency of the repaired column was higher than the intact column 


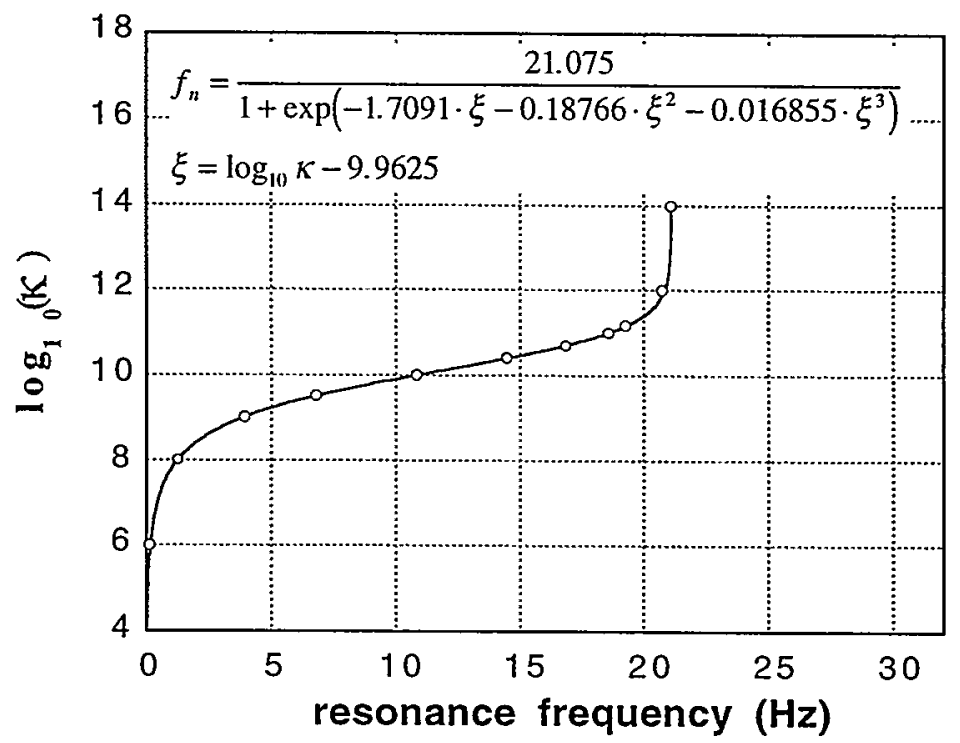

Figure 7.21 Computed resonance frequencies of the first flexural mode as a function of interface stiffness $\kappa$. A fitted function is shown in the plot.

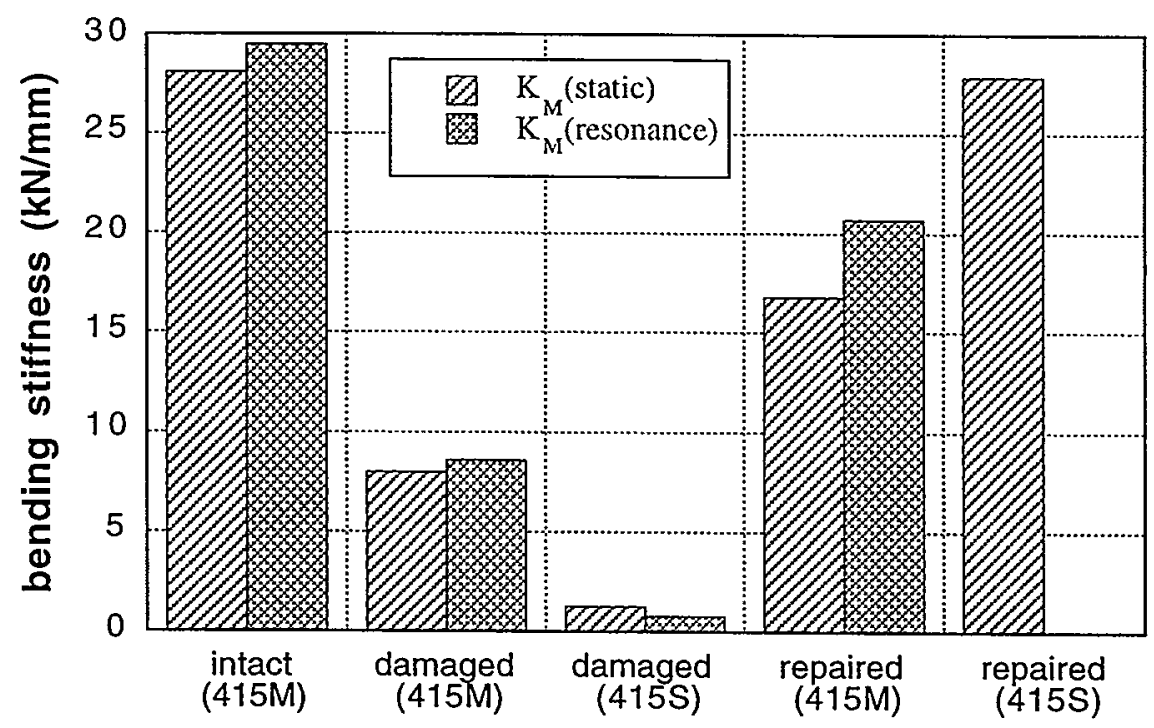

Figure 7.22 Comparison between measured static bending stiffnesses and determined bending stiffnesses from measured resonance frequencies. Intact $(415 \mathrm{M})$ and damaged columns show very good agreement between the stiffnesses obtained by the two different methods. A repaired column (415M) shows a rather large difference but the relative magnitude of the stiffness compared with other columns is consistent. Dynamically determined stiffness of the repaired $415 \mathrm{~S}$ column is not shown as the current method cannot determine the stiffness if the measured frequency is larger than the resonance frequency of an intact specimen. 


\subsection{Summary}

It is found that the velocities and waveforms of a propagating wave $(\sim 10 \mathrm{kHz})$ are sensitive to the fractures and microcracks introduced into a damaged column. Amplitudes and velocities both decrease. Waves measured for different directions along the diameters of the columns show different velocity, amplitude, and time of arrival. Such characteristics can be used for assessing the geometry of fractures in a structure that in turn may be used to examine the load applied to the structure when the fractures are created. Although propagating waves show good sensitivity to the introduced damage, the effect of repair is not clearly seen from changes in wave velocity and wave amplitude.

Low frequency vibration tests were conducted to measure the change in elastic bending stiffness of the column specimens. Resonance frequencies and attenuation decreased significantly as the damage was introduced. Attenuation increased because the localized damage at the bottom of a column isolated vibration within the column, reducing the radiation damping. Resonance frequencies decreased corresponding to the degree of damage (moderate and severe damage). Repaired columns showed significant increase in resonance frequencies and attenuation. Localized damage at the bottom of the columns was modeled as an interface with displacement-discontinuity conditions and used to determine the bending stiffness of the columns from measured resonance frequencies. Attenuation of the vibrations was not examined quantitatively because the dynamic interaction between the columns and the foundation that resulted in radiative loss of vibration energy were too complicated to be analyzed by the current model.

Although high-frequency measurements using transmitted waves are good for detecting small cracks and mapping the location of damage in a structure, they did not reflect the overall integrity of a structure. In contrast, results from low-frequency acoustic resonance measurements correspond remarkably well to the degree of introduced damage, repair, and measured static stiffness of the structure. Moreover, the dynamic stiffnesses determined from resonance frequencies show very good agreement with statically measured stiffnesses except for a column that had a resonance frequency higher than the original intact column. These results demonstrate that the acoustic resonance technique can be used as a quantitative diagnostic tool for assessing the degree of damage and the effect of repair in concrete infrastructure. 


\section{4}

In conclusion, damage in the concrete structure discussed in this chapter can be thoroughly diagnosed using either local seismic transmission tests or global vibration tests. However, the degree of damage introduced and the effect of repair can be measured by the acoustic resonance tests more accurately. Therefore, if the primary objective of any test of a structure is an assessment of its global performance, the acoustic resonance test is highly recommended. 


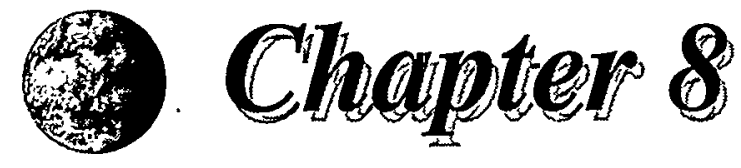

\section{Dynamic Behavior of a sheared Fracture and the Extension of Displacement-Discontinuity Theory}

\subsection{Introduction}

The strength and elastic moduli of materials such as rocks, ceramics, and concrete are significantly influenced by inherent fractures. As the load required to cause slip along a fracture is usually much less than the strength of an intact material, the shear strength of the fracture often determines the material's overall strength. Many researchers including Jaeger (1959), Byerlee (1967a, b), and Jaeger and Rosengren (1969) examined the frictional behavior of fractures to understand the behavior of rocks under shear. For a rock mass containing distinct fractures (joints), the stability of structures such as rock slopes and mine pillars depends greatly on the shear strength and stress of the inherent fractures (Hoek, 1970; Jaeger, 1971). At a much larger scale, stick-slip behavior of faults that involves the accumulation of shear strain and resultant slip failure is claimed to be a major cause of earthquakes (Byerlee and Brace, 1968; Dieterich, 1978).

For predicting and, if possible, preventing the slip failure along a fracture, it is important to know the stress acting on that fracture. Methods using seismic waves are 
among the most promising for measuring the stress remotely. This is because a seismic wave is sensitive to the stiffness of a fracture, which is stress dependent. Stress applied normal to an interface between two rough surfaces, such as a fracture, alters the characteristics of waves interacting with this interface. Changes in the wave's travel time, amplitude, and spectral characteristics are used to determine the normal and shear stiffness of a fracture. Kendall and Tabor (1971) used the transmission coefficient of waves to determine the stiffness of an interface between a variety of materials including steel and glass. Pyrak-Nolte et al. (1990a) also measured waves transmitted across single natural fractures in rocks and attempted to determine the normal and shear stiffness of the fractures by matching experimental and theoretical wave spectra.

If the geometry of the contacting surfaces is known, the stress applied to a fracture can be determined from the fracture stiffness assessed by seismic measurements. For nominally flat and smooth metal surfaces, Haines (1980) proposed a model that predicts the average contact stress of an interface from its specific stiffness. Using this model, Buck et al. (1982) determined the normal stress on a fracture from the spectra of transmitted and diffracted waves. Rehbein et al. (1984) applied a similar technique to determine the normal stress on the interface of a metal coupler from waves reflected off the interface. For the contact between rough surfaces in rock-like materials, however, the full geometry of the contact is not known or it is too complicated to find the relation between the applied stress and resulting stiffness. For such cases, the stress-stiffness relation has to be measured experimentally.

Shear stress applied to a fracture also changes the normal and tangential stiffness of a fracture. However, in many cases, shear stress on a fracture is also accompanied by a normal stress component that prevents slip along the interface. As the effect of the normal stress is usually larger than the shear stress, shear stress on a fracture is difficult to determine by the seismic measurements described above. Furthermore, changes in the waves do not carry any information about the direction of the shear stress applied to the fracture.

To determine the presence, direction, and magnitude of shear stress on a fracture, the conventional measurements for transmitted and reflected $\mathrm{P}$ and $\mathrm{S}$-waves do not provide sufficient information. Therefore, an alternative measurement that is sensitive to the change in the shear stress on a fracture is desirable. In this chapter, a new understanding about the behavior of seismic waves interacting with a sheared fracture is presented. Laboratory 
experiments show that the shear stress applied to a fracture in a rock specimen partially converts normal incident compressional (P)-wave and shear ( $\mathrm{S}$ )-wave to $\mathrm{S}$ and $\mathrm{P}$-waves, respectively. As the waves are normally incident on a fracture, this phenomenon cannot be explained by the Snell's law or the conventional displacement-discontinuity theory (Schoenberg, 1980; Pyrak-Nolte et al., 1990a). This behavior of seismic waves may provide a tool for detecting and measuring the shear stress on a fracture. The results of laboratory ultrasonic transmission tests performed on a granite specimen containing a through-going fracture and a fractured steel specimen with a regular surface geometry are presented. Theoretical transmission and reflection coefficients for a sheared fracture were obtained by applying an extended form of the displacement-discontinuity boundary conditions that included cross-coupling terms in the fracture stiffness matrix. The model predicts increasing conversion of waves as the cross-coupling stiffness increases, which is directly related to an increase in the magnitude of the shear stress on a fracture. Using this extended form of the displacement-discontinuity boundary conditions for modeling dynamic interactions between a fracture and seismic waves, numerical simulations were conducted using a boundary element method to demonstrate the effect of the shear-induced cross-coupling fracture stiffness on propagating seismic waves.

\subsection{Mechanism of Shear-induced Wave Conversion}

The mechanical properties of a fracture can be examined by modeling a fracture as a non-welded interface between two solid media with imperfect contacts (Greenwood and Williamson, 1966). The surface of a fracture consists of irregularities at many scales ranging from atomic scale roughness to macroscopic undulations with a scale comparable to the dimension of the fracture itself. Brown et al. (1986) showed that the spectral components of the two surface profiles across a fracture are more "correlated" (in phase) at longer spatial wavelength than at shorter wavelength especially for a mated fracture. Compliance of a fracture decreases as the two surfaces are pressed together primarily due to increase in contact between irregularities with relatively short spatial wavelength (Brown and Scholz, 1985, 1986). Velocity and amplitude of the transmitting P and S-waves increase due to an increase in mechanical coupling between two surfaces.

One of the simplest models for a fracture surface consists of elastic hemispheres attached to rigid halfspaces (Greenwood and Williamson, 1966; Brown and Scholz, 1985, 1986; Yoshioka and Sholtz, 1989a,b). The nonlinear closing behavior is explained by 
deformation of individual asperities predicted by the Hertz-Mindlin model (Mindlin, 1949). However, such a model ignores the effect of the deformation in the half-space behind the asperities. Kendall and Tabor (1971) showed that the stiffness of an interface and therefore the velocity and amplitude of transmitted and reflected waves depend not only on the nonlinear increase in contact area between single asperities but also on the interaction between neighboring contacts. Using numerical simulations for a variety of contact patterns and spacings, Hopkins et al. $(1987,1990)$ demonstrated the significant effect of the interacting contacts on the stiffness of an interface. Their results indicate that the stiffness of a rough interface cannot be understood by simply examining the deformation of single asperities. Instead, the contact distribution has a large effect in determining the overall stiffness of an interface.

When a natural fracture is subjected to shear stress, the local stiffness of the fracture due to contacting asperities becomes either stiffened or relaxed, depending on the relative approaching motion of the fracture surfaces and angle of contact. Such behavior is governed by the long spatial wavelength component of the fracture surface profiles that are more or less correlated across the fracture. The multi-scale surface irregularity of a fracture would be idealized by a geometry of the surface that consists of small random (uncorrelated) asperities superposed on regular saw-tooth profiles that are identical across the fracture (Figure 8.1). In Figure 8.1, the regular long-wavelength profile determines whether the short-wavelength asperities across the fracture approach or separate when the two surfaces are sheared. On the approaching side of the slopes, the local stiffness of the fracture increases due to a non-linear increase in contact stiffness between the micro-scale asperities while the local stiffness decreases on the other side of the slopes. If the applied shear stress is large, the stiffness of the relaxed contacts approaches zero and the stiffness tends to infinity for the stiffened contacts. In this limit, the fracture effectively behaves as an array of inclined open flat microcracks. Statically, (i.e. at the zero-frequency limit) such cracks exhibit dilation (coupled) behavior: application of a normal stress on the fracture is accompanied by a relative lateral displacement across the fracture, and shear stress results in relative normal displacement. It should be noted that this behavior is elastic and no slip along the fracture is involved. Dynamic interactions between such cracks and propagating elastic waves were studied by Mikata and Achenbach (1988) using a boundary integral equation method. Their results showed that, for $\mathrm{P}$ and $\mathrm{S}$-waves normally incident on a mean-crack plane of the microcracks, transmission and reflection coefficients of the crosscoupled waves are finite, i.e., the converted waves are generated. 


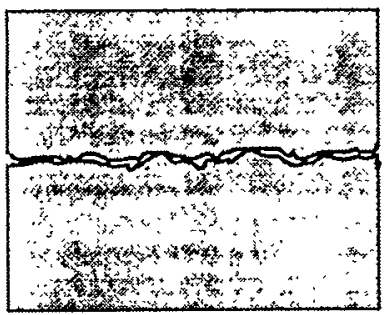

(a) imperfect interface (fracture)

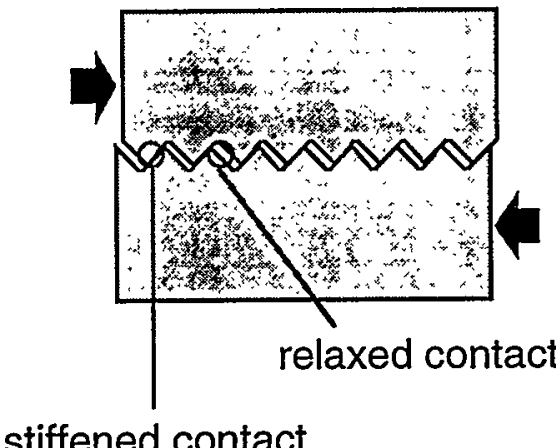

(c) idealized interface (sheared)

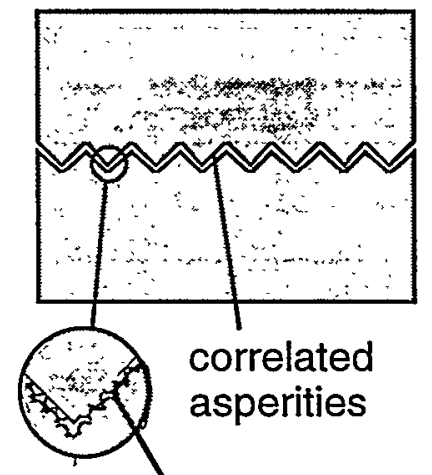

uncorrelated asperities

(b) idealized interface (without shear)

transmitting

P-wave

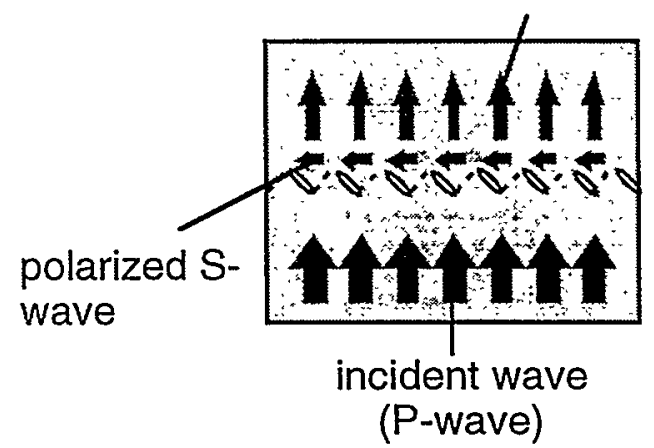

(d) generation of converted waves

Figure 8.1 Mechanism generating cross-coupled waves for normal-incident plane waves. Surface profile of a natural fracture (a) is modeled by correlated long-wavelength undulations and uncorrelated (random) short-wavelength asperities (b). (c) Application of shear stress changes the local contact stiffness of the fracture that becomes (d) inclined flat microcracks that exhibit the elastic dilation for applied normal and shear load. In the above case, generation of a converted S-wave by a normal-incident P-wave is shown.

In the following section, experimentally observed conversion of elastic waves normally incident on a sheared fracture in a rock specimen is presented. The effect of an idealized surface geometry for generating cross-coupled waves is demonstrated using a steel block specimen. 


\subsection{Experiment}

\subsubsection{Wave transmission tests across a fracture in rock}

\subsubsection{Experimental setup}

Ultrasonic transmission tests were conducted using a pair of identical transducers that contained both $\mathrm{P}$ and $\mathrm{S}$ - type piezoelectric crystals. By selectively generating and measuring different types of waves, both directly transmitted and converted $\mathrm{P}$ and $\mathrm{S}$-waves propagating through a specimen were observed. The central frequencies of the generated $P$ and S-waves were $850 \mathrm{kHz}$ and $500 \mathrm{kHz}$, respectively. Particle motion of the S-wave generated by the transducer was polarized in a single direction. Both waves were generated by $500 \mathrm{~V}$ square pulses sent from a high-voltage pulse generator (Cober 605P). Received waves were displayed and recorded by a digital oscilloscope (LeCroy 9424E). The experimental setup is shown in Figure 8.2

A series of laboratory ultrasonic measurements was performed on a cylindrical specimen of granite $(5.08 \mathrm{~cm}$ in diameter and $10.16 \mathrm{~cm}$ in length). The granite used for this test exhibited approximately transversely isotropic behavior due to preferentially oriented microcracks similar to the granite specimens used in the previous chapters (Chapter 3 and 5). The density of the specimen was $2.66 \mathrm{~g} / \mathrm{cm}^{3}$ (room dry). The specimen was cored along the average microcrack plane. Velocities of $\mathrm{P}$ and $\mathrm{S}$-waves (polarized parallel to the microcrack planes) measured along the axis of the intact specimen were $4606 \mathrm{~m} / \mathrm{sec}$ and $2851 \mathrm{~m} / \mathrm{sec}$, respectively. The applied axial stress was $2.46 \mathrm{MPa}$. Thin pieces of lead foil were used to establish good acoustic coupling between the transducers and the specimen.

Figure 8.3 shows $\mathrm{P}$ and $\mathrm{S}$ - waves transmitted through the specimen before and after the fracture was introduced. From the plot, it can be seen that both directly transmitted $\mathrm{P}$ and $\mathrm{S}$-waves (P-source to $\mathrm{P}$-receiver and S-source to S-receiver) and converted waves (Psource to S-receiver and S-source to P-receiver) show a large decrease in amplitude and an increase in travel time. The small converted waves are observed even for the intact specimen as the transducer does not generate pure P or S-waves. It was found that the changes in the velocity of the waves are too large to be caused by any increase in compliance from the fracture. Although most of the changes were possibly due to the effect of the fracture, a slight decrease in the water content of the specimen between tests 


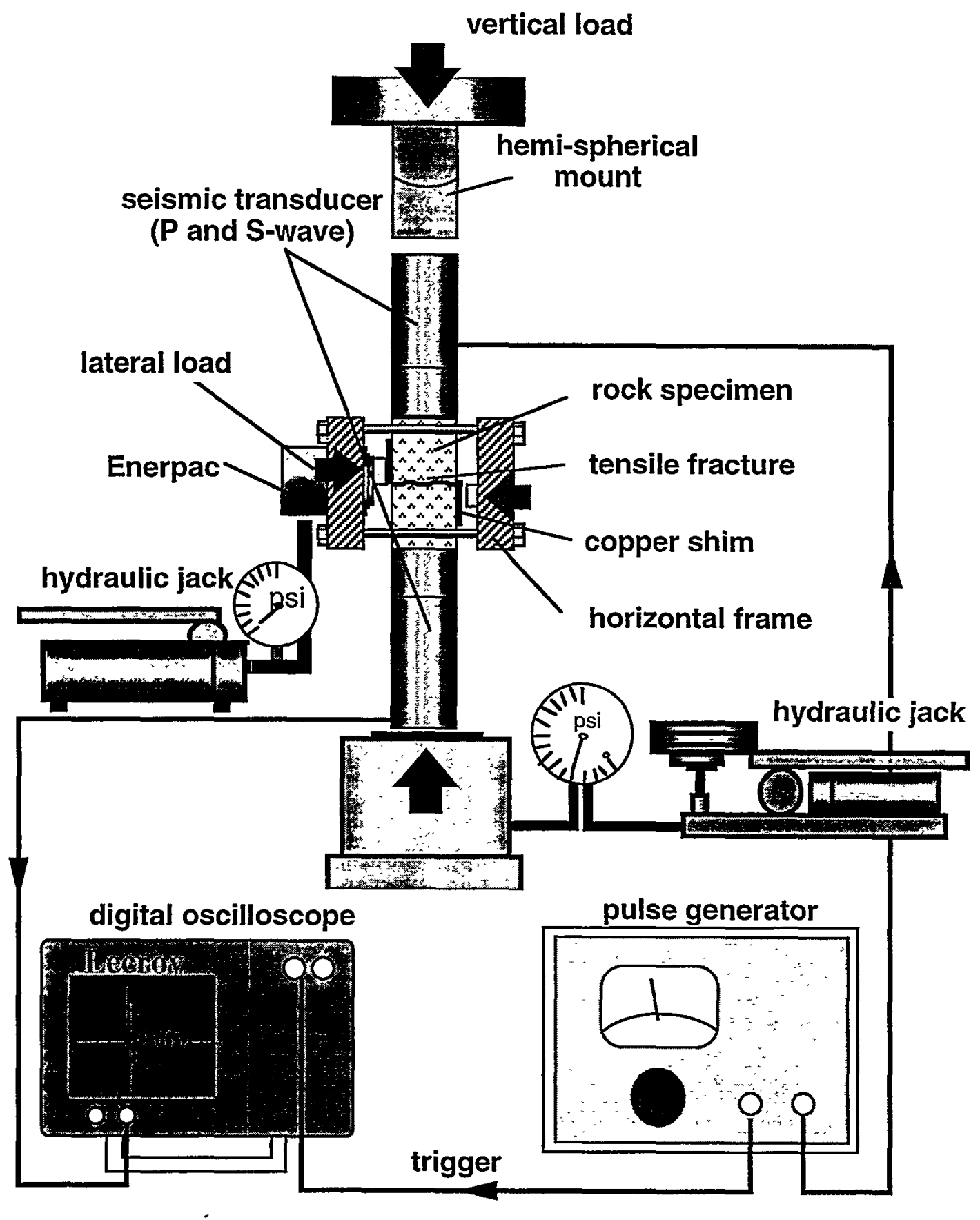

Figure 8.2 Experimental setup for seismic wave conversion tests across a fracture in a rock specimen. During the tests, the specimen was axially loaded. Waves were generated by piezoelectric crystals inside the transducers. The effect of shear stress on the conversion behavior of the waves was examined by applying a shear force across the fracture using a lateral load frame. 


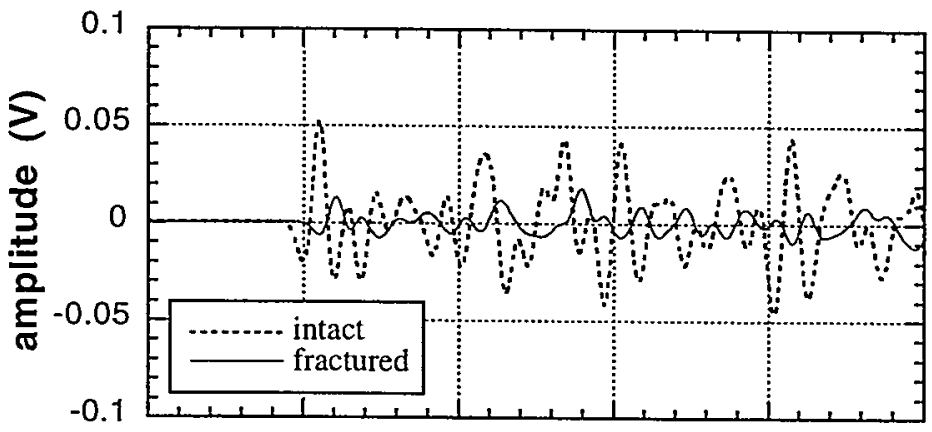

(a) $\mathrm{P}$ to $\mathrm{P}$ transmitted waves

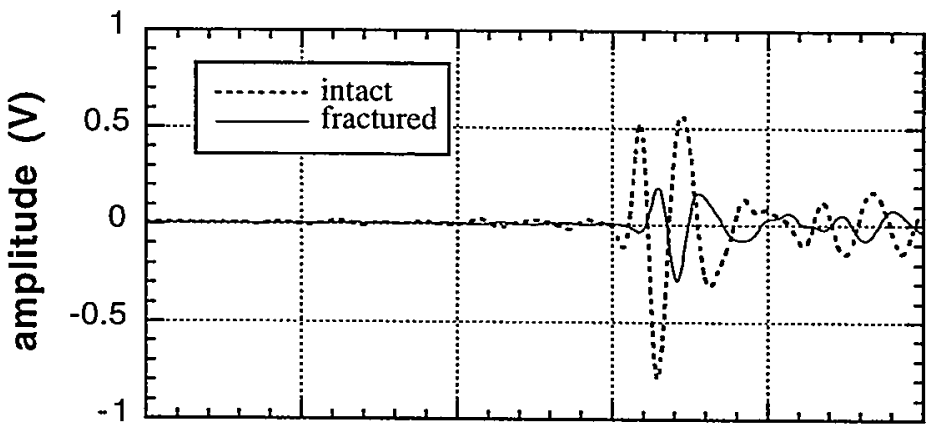

(b) $\mathrm{S}$ to $\mathrm{S}$ transmitted waves

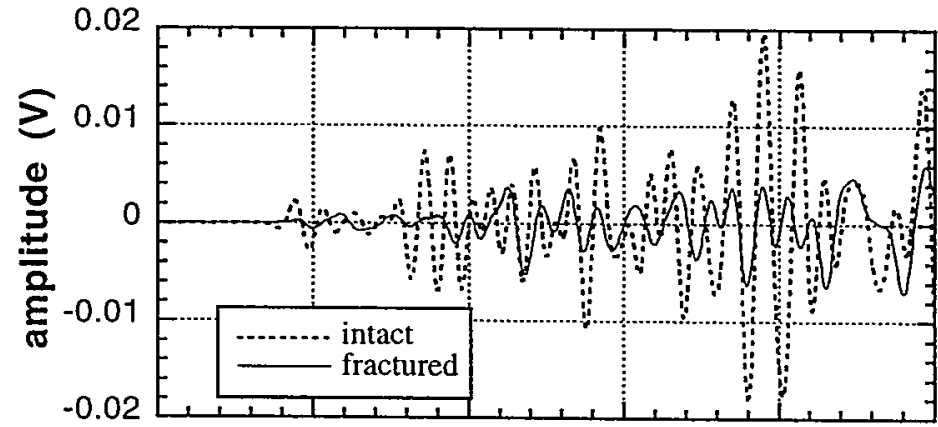

(c) $\mathrm{P}$ to $\mathrm{S}$ transmitted waves

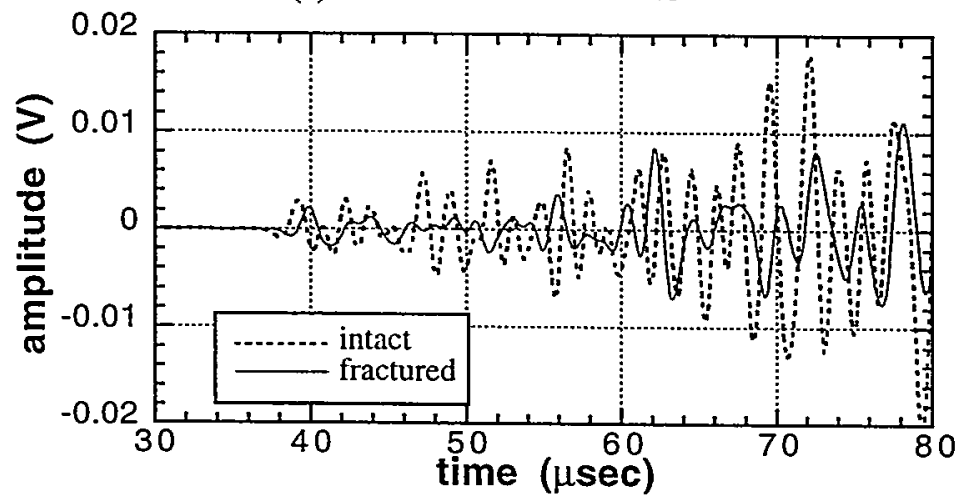

(d) $S$ to $P$ transmitted waves

Figure 8.3 Changes in transmitted and converted waves through a granite specimen before and after a fracture is introduced. All the waves show decreases in velocities and amplitudes due to the frequencydependent scattering by the fracture. It is noted that the changes in the waveforms may also be caused by a slight change in the water content of the specimen between the times the two tests were conducted. 
was also suspected to be the cause. For this reason, no attempts were made to determine the specific stiffness of the fracture from the dynamic measurements.

After measuring the ultrasonic properties of the intact specimen, a through-going tensile fracture was created perpendicular to the core axis by applying a point load along a diameter of the core (Brazilian loading). The specimen was held in a horizontal load frame that applied a lateral load, shearing the specimen across the fracture. The two surfaces of the fracture were mated. Before the measurements were conducted, the fracture was uniaxially cycled for several times up to $3 \mathrm{MPa}$. This was done to minimize the amount of permanent deformation on the fracture surface such as crushing of asperities that may occur during the tests. During the tests, a constant axial stress $(2.46 \mathrm{MPa})$ was applied to the specimen placed between the transducers.

\subsubsection{Effect of shear stress}

In the first series of tests, the lateral load was varied to see the effect of shear stress on seismic waves propagating across the fracture. The shearing direction was aligned with the polarization direction of the S-wave. These directions were also aligned parallel to the average microcrack plane in the granite. A range of shear stress from 0 to $3 \mathrm{MPa}$ (called "right-lateral shear") were applied followed by shear with reversed direction from 0 to -3 MPa (called "left-lateral shear").

The measured values of the directly-transmitted waves across the fracture are shown in Figure 8.4(a) for P-waves and in Figure 8.4(b) for S-waves. Waves measured before and after application of the right-lateral shear are also shown for comparison. During shearing, directly transmitted waves showed small changes with increasing shear stress. P-wave amplitudes decreased approximately $10 \%$ for both right-lateral and left-lateral shear. S-wave amplitude showed a small increase for left-lateral shear $(540 \mathrm{mV}$ increased up to $562 \mathrm{mV}$ ) but release in shear stress resulted in higher amplitude $(571 \mathrm{mV})$ and, consequently, the left-lateral shear amplitude decreased by approximately $15 \%$. It appears that application of shear stress tends to decrease the non-converted transmitted waves. The small increase in S-wave amplitude for right-lateral shear may be due to slip of the fracture that will greatly increase the tangential stiffness of the fracture by changing the contact geometry. As can be seen from the above results, although the shear stress does cause some change in the directly transmitted (non-converted) $\mathrm{P}$ and $\mathrm{S}$-waves, its effect is relatively small and the direction of the shear cannot be known from these measurements. 


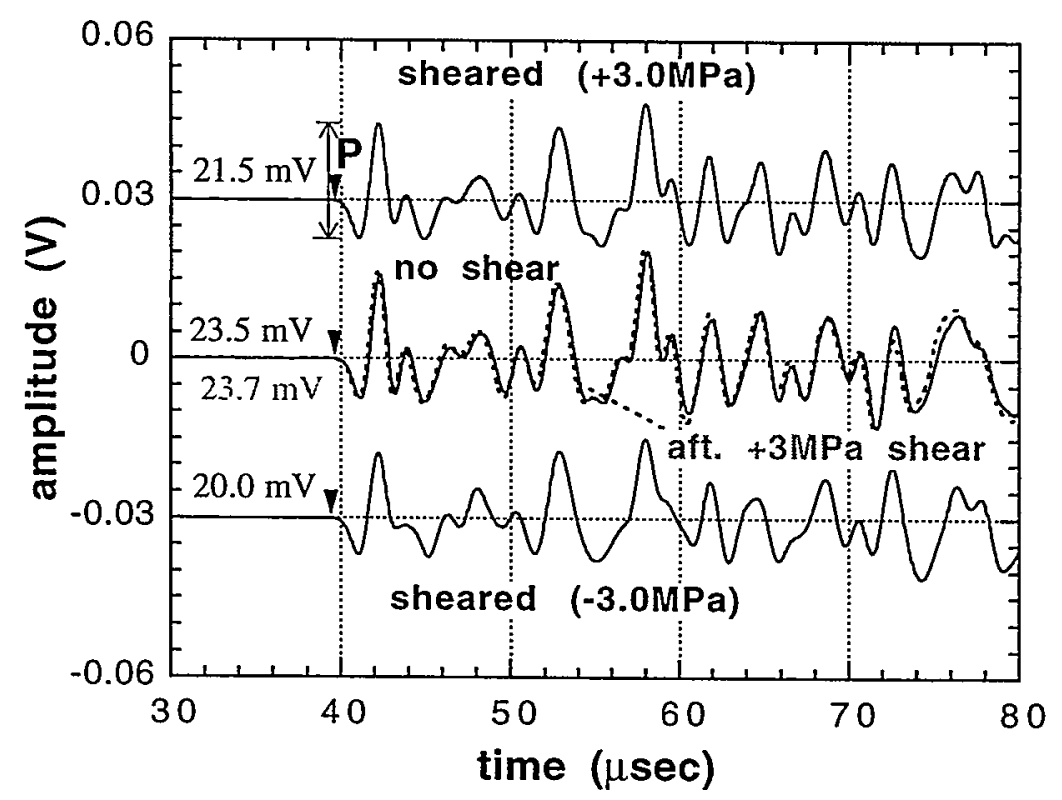

(a) $\mathrm{P}$ to $\mathrm{P}$ transmitted waves

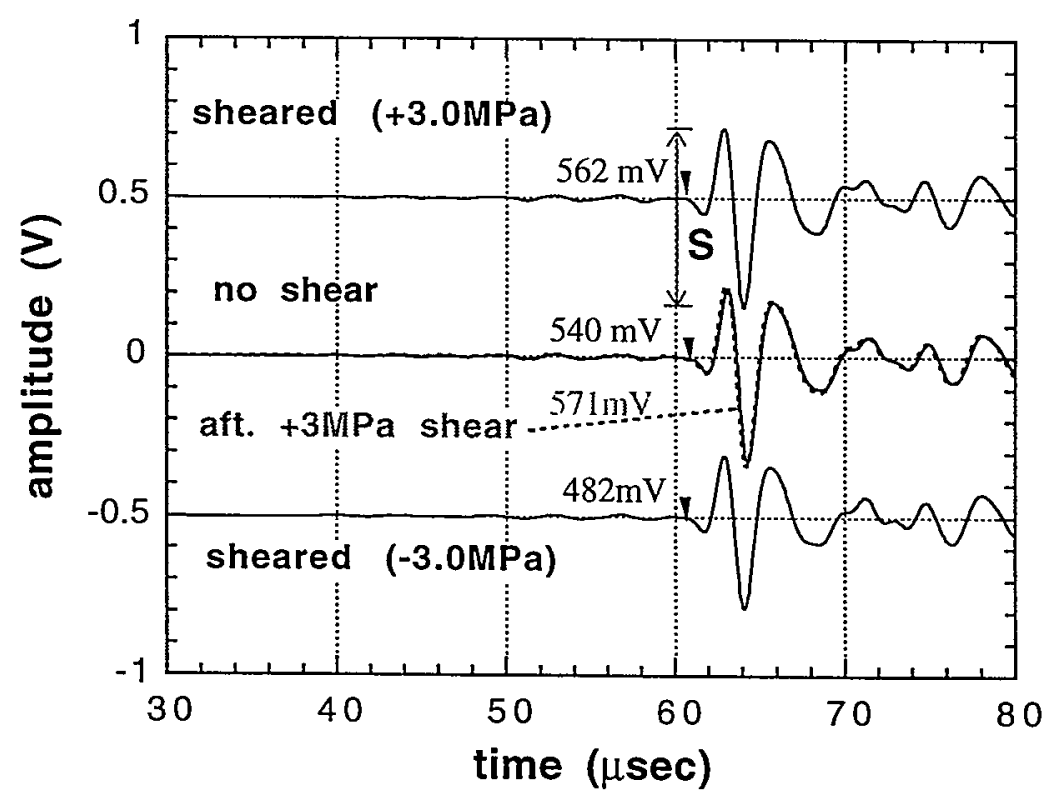

(b) $S$ to $S$ transmitted waves

Figure 8.4 Waves transmitted and converted on a single fracture in a granite specimen. Both directly transmitted (a) P and (b) S-waves show little change. Shear stress was increased from 0 to $3 \mathrm{MPa}$ in both directions (left-lateral shear and right-lateral shear). 


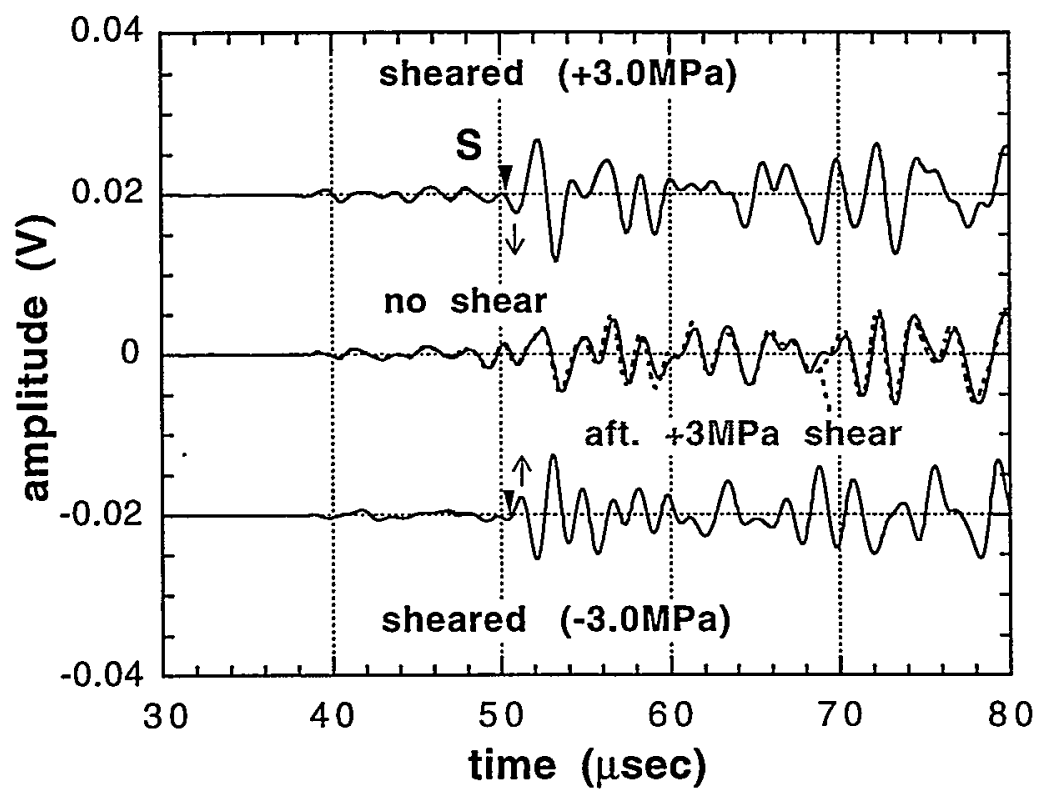

(c) $\mathrm{P}$ to $\mathrm{S}$ transmitted waves

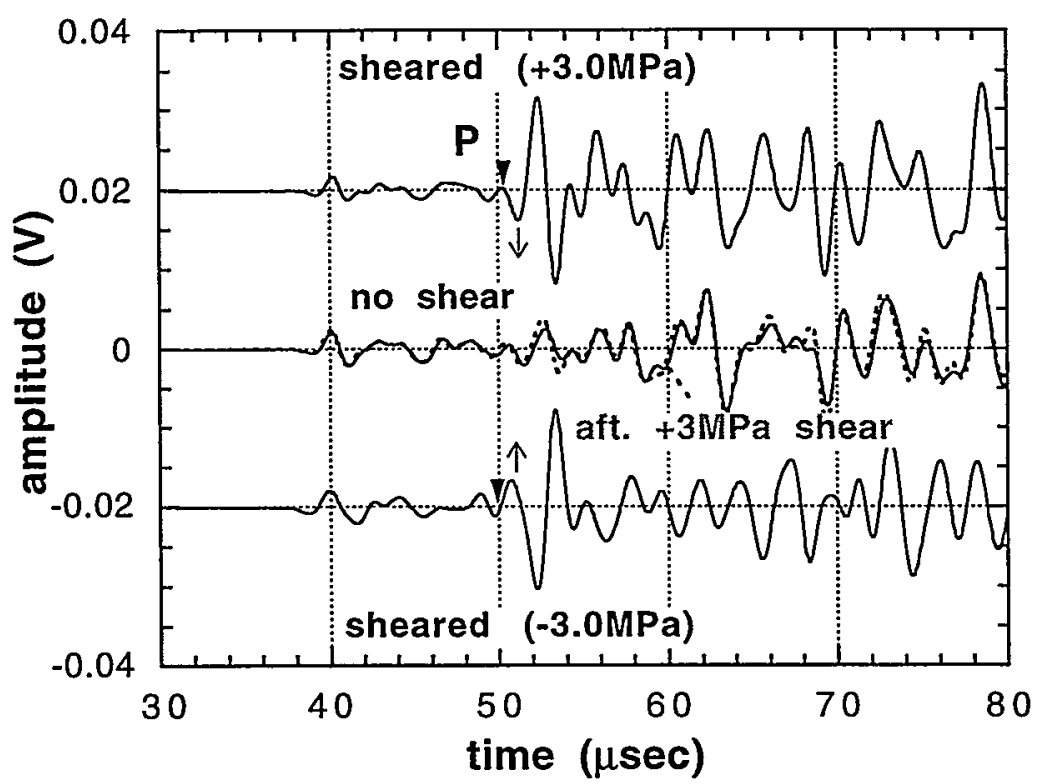

(d) $\mathrm{S}$ to $\mathrm{P}$ transmitted waves

Figure 8.4 Waves transmitted and converted on a single fracture in a granite specimen. Amplitudes of both converted (c) S and (d) P-waves increase with applied shear stress. Particle motions of the waves change their phase by $180^{\circ}$ when the direction of the shear is reversed. 


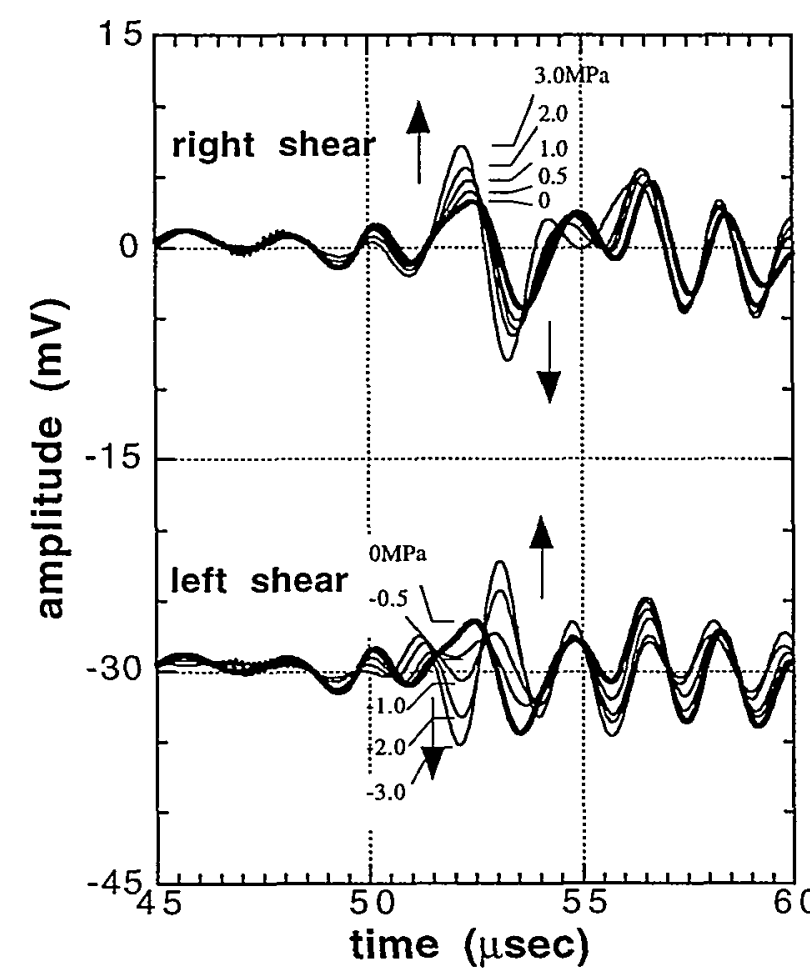

(a) $\mathrm{P}$ to $\mathrm{S}$ transmitted waves

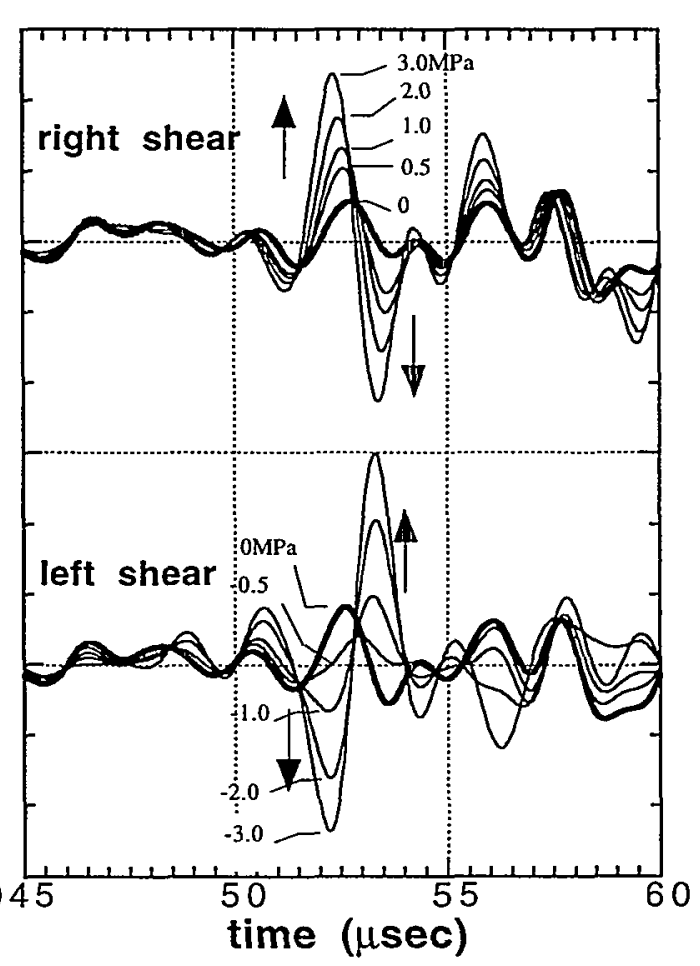

(b) $\mathrm{S}$ to $\mathrm{P}$ transmitted waves

Figure 8.5 Changes in converted (a) S and (b) P-waves for different shear stresses. Normal stress on the fracture is maintained at $2.46 \mathrm{MPa}$. The amplitude of the converted waves increases in accordance with increases in shear stress.

The shear stress applied to the fracture resulted in converted $\mathrm{S}$ and $\mathrm{P}$ - waves generated from incident $\mathrm{P}$ and S- waves, respectively (Figure 8.4 (c) and (d)). Both waves showed a small amplitude without any shear stress. This may be due to the waves converted by the fracture that was not perfectly perpendicular to the axis of the specimen. The converted waves showed a monotonic increase in amplitude with increasing shear stress (Figure 8.5). Phase for the particle motion of the converted waves changed by $180^{\circ}$ when the direction of the shear was reversed. A larger increase in the amplitude of converted S-waves than converted $\mathrm{P}$-waves is due to the difference in the characteristics of the sending and receiving transducers. This is confirmed by the observation of identical waveforms when the receiving transducer is used to generate waves and the sending transducer to receive the converted waves. This demonstrates the reciprocity in wave propagation. 


\subsubsection{Effect of shear angle}

The second series of tests was conducted by changing the direction of the lateral shear around the axis of the sample. Both axial and lateral loads were maintained at $2.46 \mathrm{MPa}$. Figure 8.6 shows waveforms for varying shear directions. From the plots, clear arrivals of the converted waves can be seen when the fracture is sheared in the polarization direction of the S-wave. Directly transmitted waves do not show significant changes in their arrival times and waveforms. The converted waves vanish when the polarization direction is normal to the shear direction $\left(90^{\circ}\right.$ and $\left.270^{\circ}\right)$. As was seen for the first series of tests, particle motion of the wave changes its phase when the direction of the shear is reversed by $180^{\circ}$.

\subsubsection{Transmission tests across a regular saw-tooth fracture}

\subsubsection{Experimental setup}

As was explained in Section 8.2, a shear force applied to the surface of a fracture changes the distribution of contacts on the fracture. The altered distribution of the contacts can be idealized by an interface between two solid halfspaces with a sawtooth geometry. A physical model of such a fracture was fabricated using cylindrical steel blocks (Figure 8.7). The spacing between the teeth was $3 \mathrm{~mm}$ and the angle of the peaks and valleys was $90^{\circ}$. The density of the specimen was $7.82 \mathrm{~g} / \mathrm{cm}^{3}$ and the velocities of ultrasonic waves measured using an identical steel block without sawteeth were $5908 \mathrm{~m} / \mathrm{sec}(\mathrm{P}$-wave, $850 \mathrm{kHz}$ ) and $3237 \mathrm{~m} / \mathrm{sec}$ (S-wave, 500kHz), respectively. The wavelengths of $P$ and Swaves in the block were $7 \mathrm{~mm}$ and $6.5 \mathrm{~mm}$, respectively.

For the first series of tests, an array of inclined flat slits was introduced between the two blocks (Figure 8.8a). Two brass shims were placed on the edge of the fracture to introduce mismatch between the two surfaces. Strips of lead foil were placed on the contacting slopes of the saw teeth, and the specimen was axially loaded by $12.3 \mathrm{MPa}$ to establish good acoustic coupling. During the tests, no shear load was applied to the interface.

The second series of tests was conducted with a thin layer of clean glass beads (diameter 50 to $100 \mu \mathrm{m}$ ) on the interface between the sawtooth surfaces (Figure 8.8b). During these tests, the axial load was maintained at $2.46 \mathrm{MPa}$. The thickness of the glass 

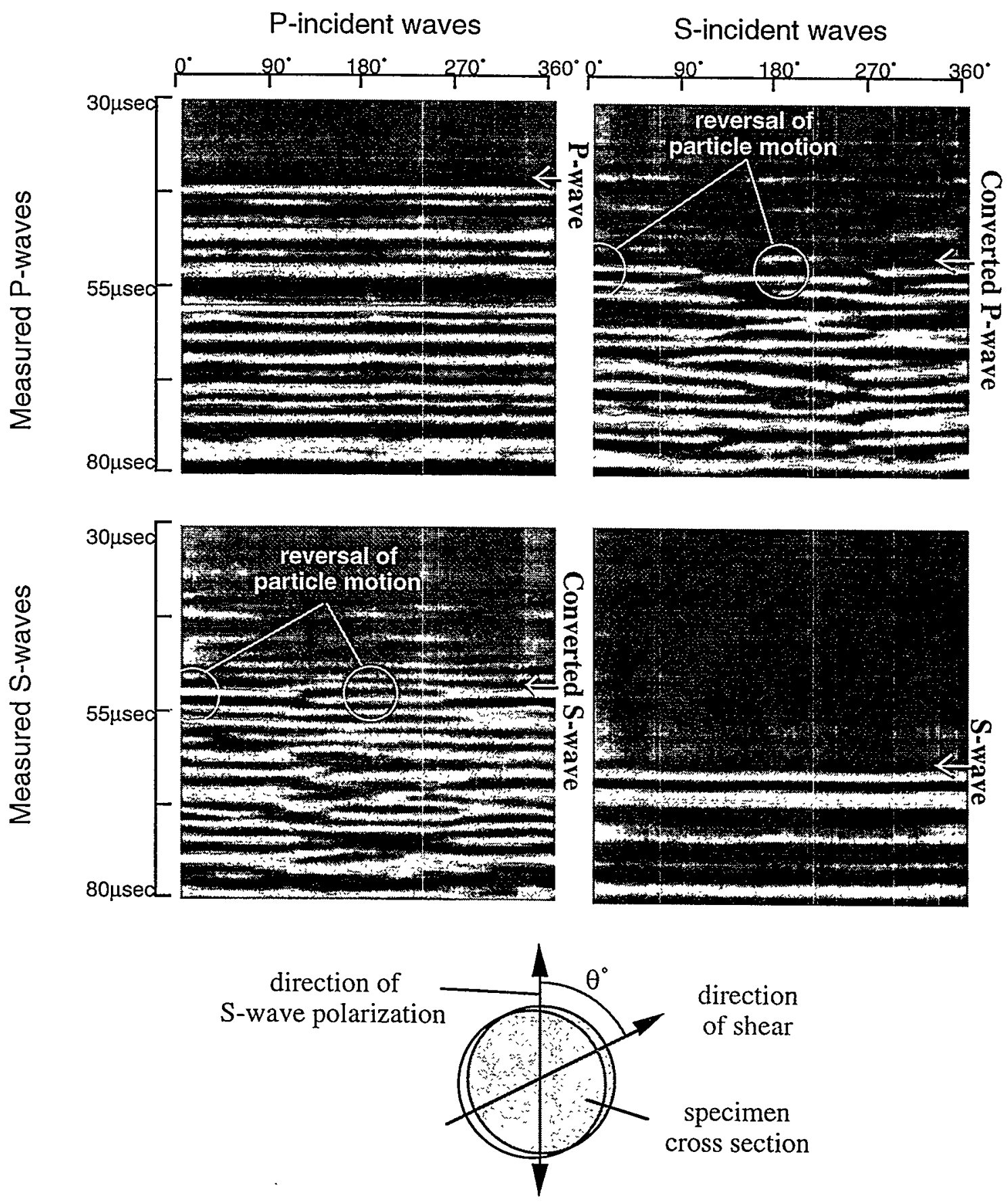

Figure 8.6 Measured waves along the axis of a fractured rock specimen for a range of shear angles. The amplitude of each plot is normalized against the maximum amplitude of the plot. Measurements were made every $15^{\circ}$. The applied axial stress was $2.46 \mathrm{MPa}$ and the shear stress was approximately $2.5 \mathrm{MPa}$. When the shear direction is aligned with the polarization direction of the shear piezoelectric crystal, large conversions between $\mathrm{P}$ and S-waves on the fracture are observed. Non-converted P and S- waves show little change for different shear angles. 

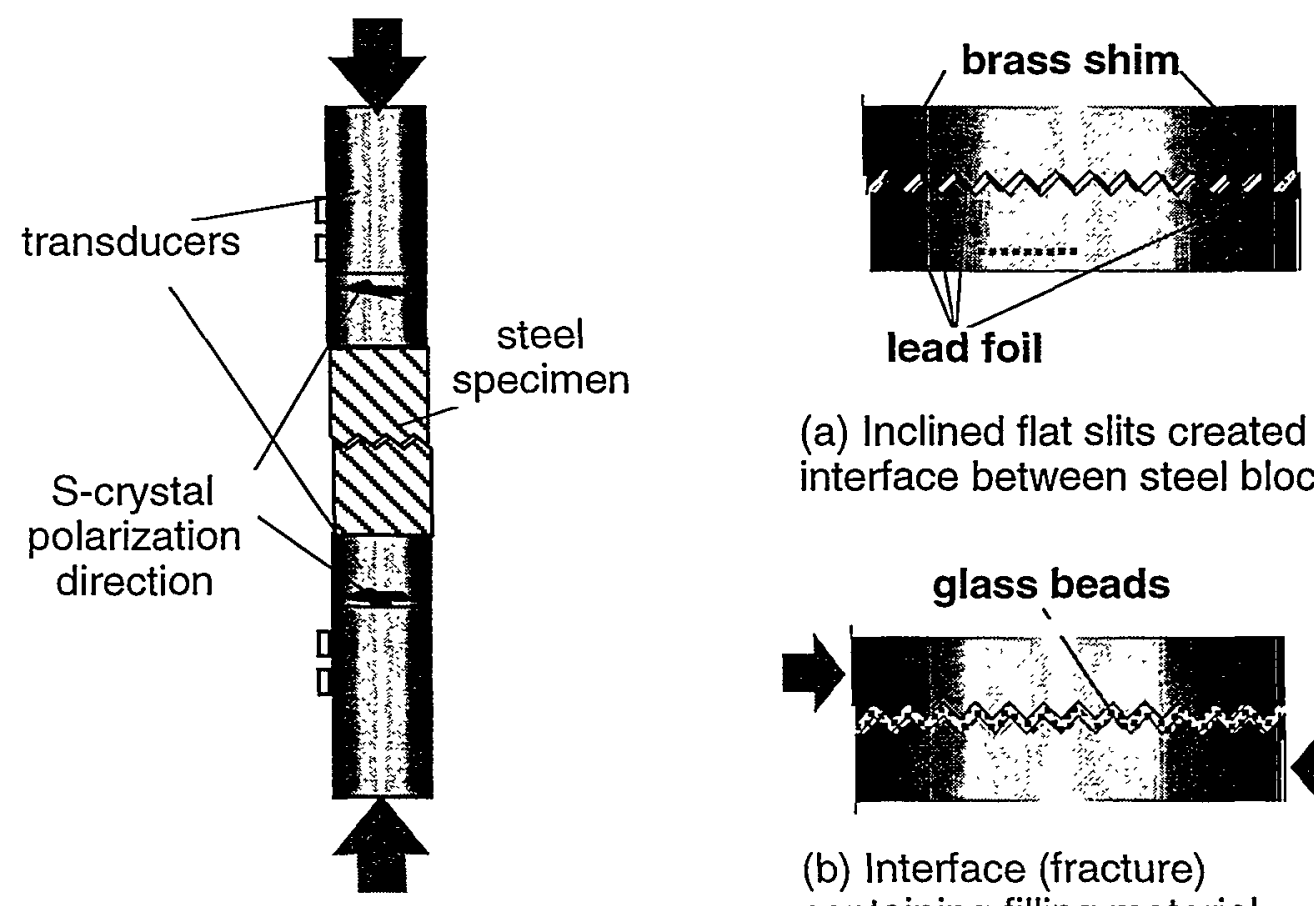

(a) Inclined flat slits created at interface between steel blocks

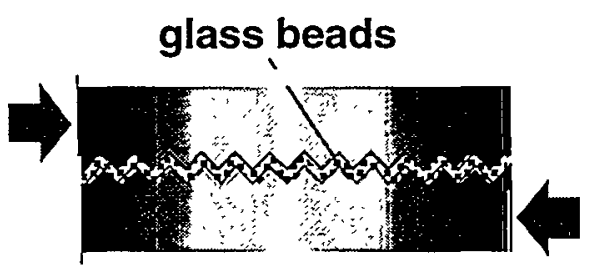

(b) Interface (fracture)

containing filling material

Figure 8.8 (a) A series of inclined flat slits was created by introducing slight lateral offset between two steel blocks with a regular sawtooth surface. No shear stress was applied to this specimen during the tests. (b) To see the effect of sheared filling material on the converted seismic waves, fine glass beads were deposited on the steel block interface.

\subsubsection{Effect of filling material}

The glass bead mediated fracture surface can be considered a fracture containing debris, such as fault gouge. Before the tests, the specimen was axially loaded up to $2.46 \mathrm{MPa}$ and all transmitted waves were measured. Subsequently, shear stress was applied to the fracture using a horizontal load frame. Figure 8.10 compares waves with and without the applied shear stress. The figure also includes waves measured when the shear stress is decreased to zero. It is noted that both non-converted $\mathrm{P}$ and $\mathrm{S}$-waves show a decrease in amplitude when the shear is applied, analogous to the behavior of the waves observed for the fracture in a rock specimen. The results clearly show a large increase in the amplitude of converted waves. A small initial conversion may be due to the random scattering of waves off the glass bead layer that has a strong acoustic impedance contrast against the steel blocks. 
The mechanism of wave conversion for a fracture containing debris is similar to that of a clean fracture with imperfect contacts. As a fracture is sheared, the glass beads between approaching surfaces are compacted, increasing their contact stiffness, while the beads between the parting surfaces are not, decreasing stiffness. As a result, the stiffness distribution of the interface becomes similar to inclined open cracks that exhibit an elastic dilation behavior.

The above tests were repeated for a glass bead layer saturated with distilled water. Without shear, the directly transmitted $\mathrm{P}$ and $\mathrm{S}$-waves show much larger amplitude than for fracture with dry glass beads (Figure 8.11). This is due to the stiffening effect of the water that fills the pore space among glass beads. On the other hand, converted waves for a sheared wet fracture show smaller amplitude than for the dry fracture. This may be because the overall increase in the stiffness of a fracture makes its behavior close to a welded fracture.

The experimental results shown in this section demonstrate that the changes in the surface geometry of the surface of a fracture by shear leads to a property of the fracture that introduces conversion of normally incident waves. Results for the steel block specimen show that the geometry of a sheared fracture can be modeled by an array of inclined cracks along the interface. 
[Steel block with open dry slits]

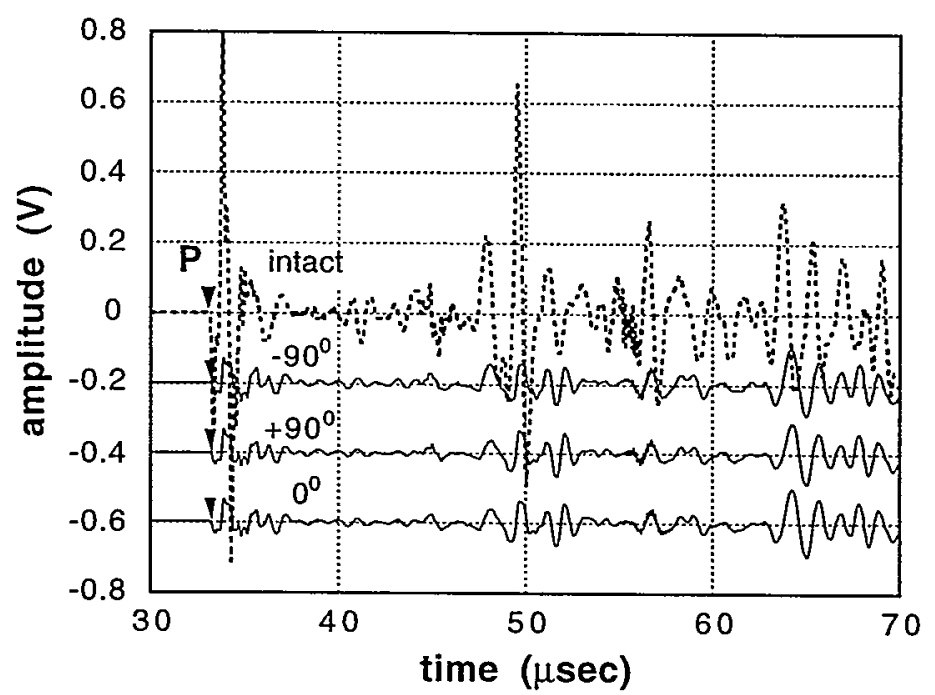

(a) P-source, P-receiver

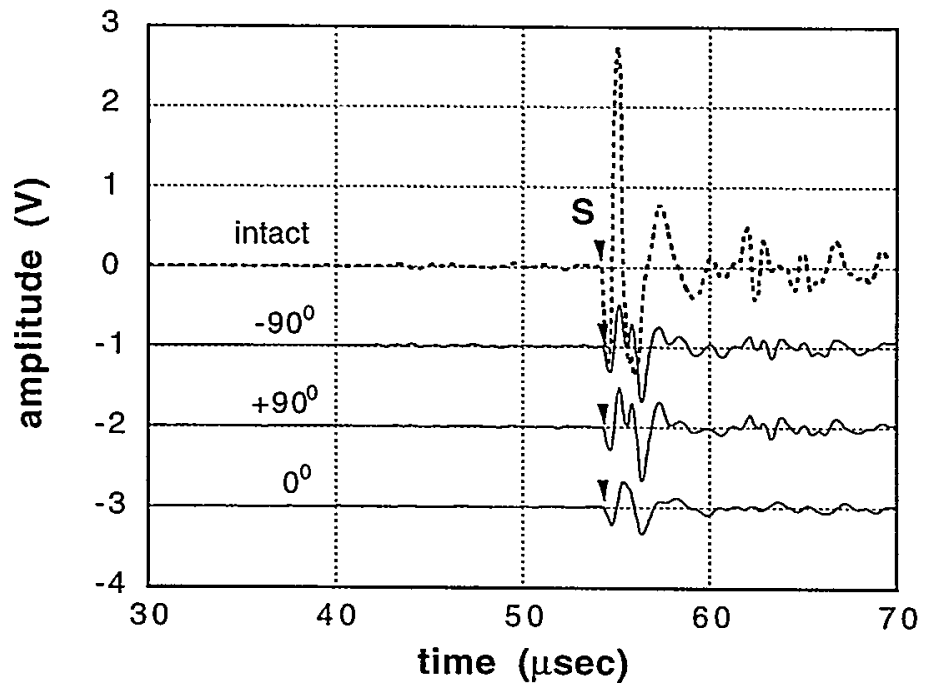

(b) S-source, S-receiver

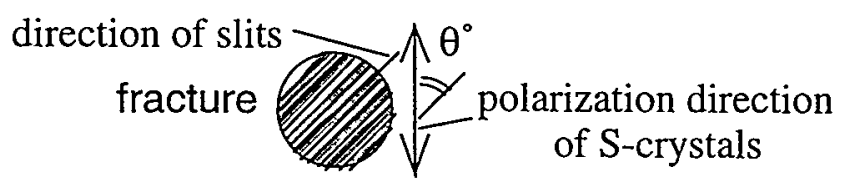

Figure 8.9 Comparison of directly transmitted waves through intact and fractured steel specimens. In the plots, the angle between the open slits and the polarization direction of the S-wave crystals is shown. Arrival of the P and S-waves is indicated by solid triangles. Directly transmitted (a) P and (b) S-waves show large decreases in wave amplitudes. It is noted that when the angle between the slits and $\mathrm{S}$-wave particle motion is $0^{\circ}$, the $\mathrm{S}$-wave shows even smaller amplitude. 
[Steel block with open dry slits]

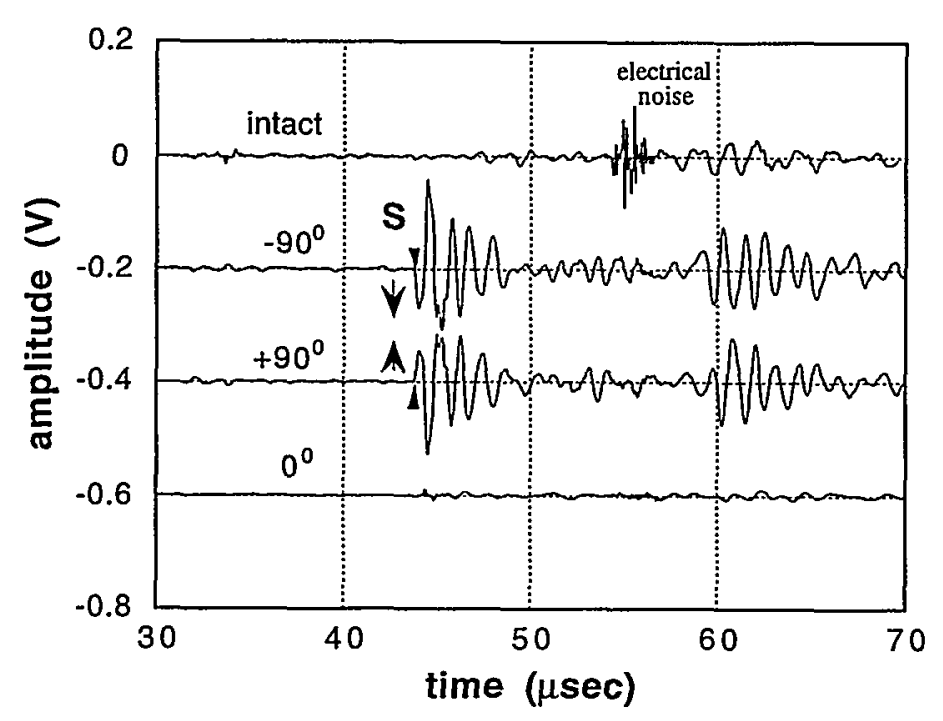

(c) P-source, S-receiver

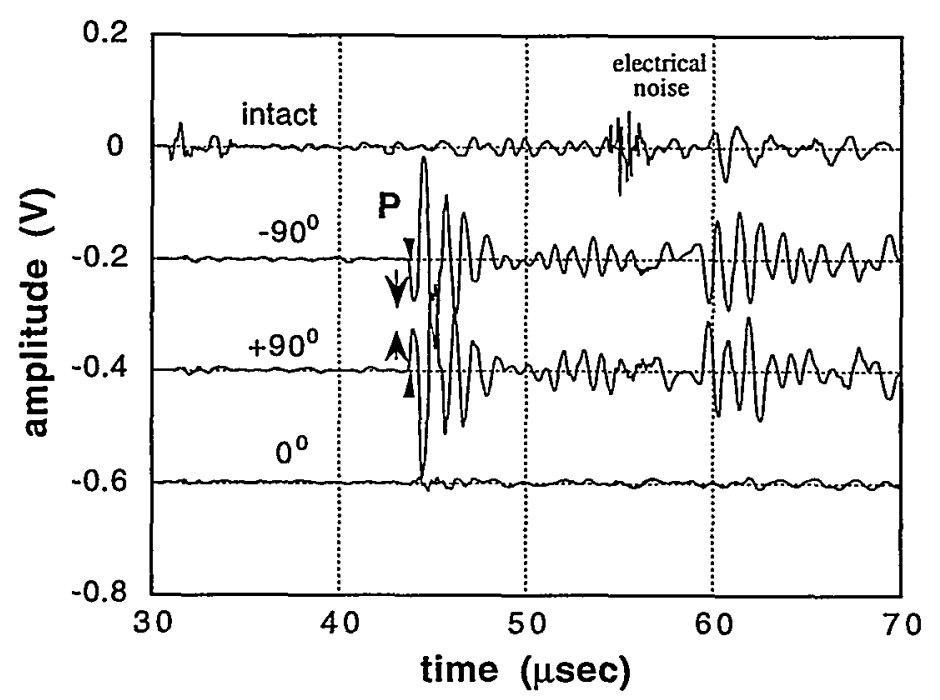

(d) S-source, P-receiver

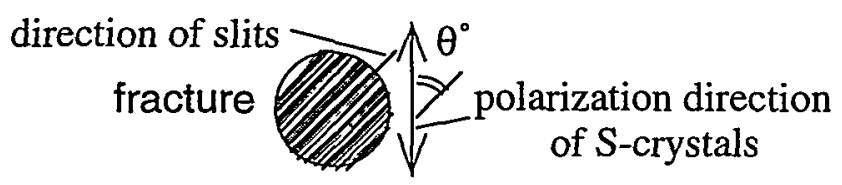

Figure 8.9 (c) (d) The fractured specimen shows very strong and clear converted (c) $S$ - and (d) $P$ - waves. The particle motion of the converted waves reverses when the direction of slits is rotated by $180^{\circ}$. 


\section{[Steel block with fracture containing dry glass beads]}

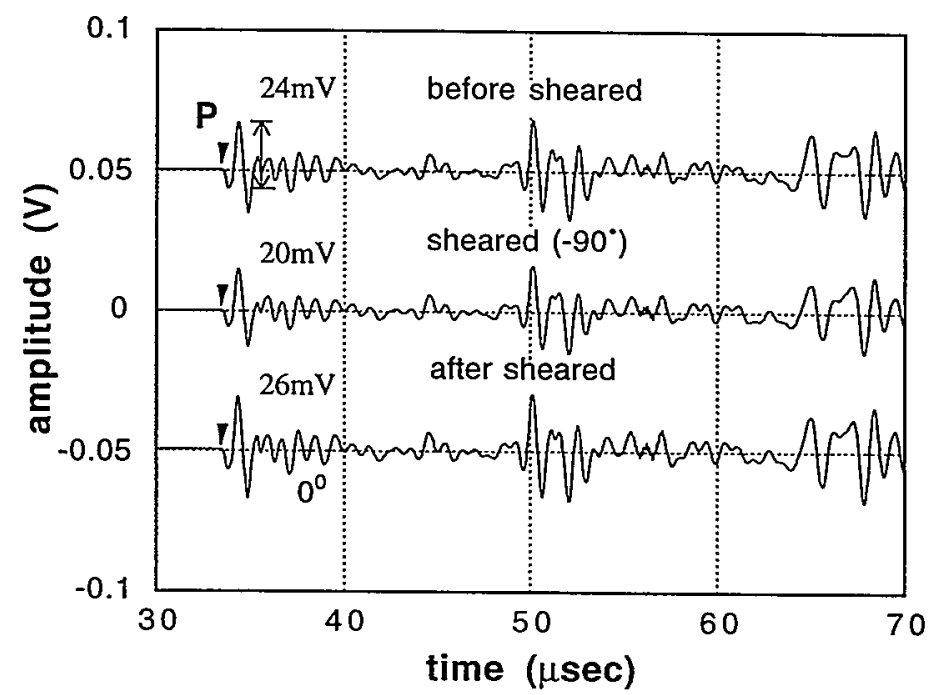

(a) P-source, P-receiver

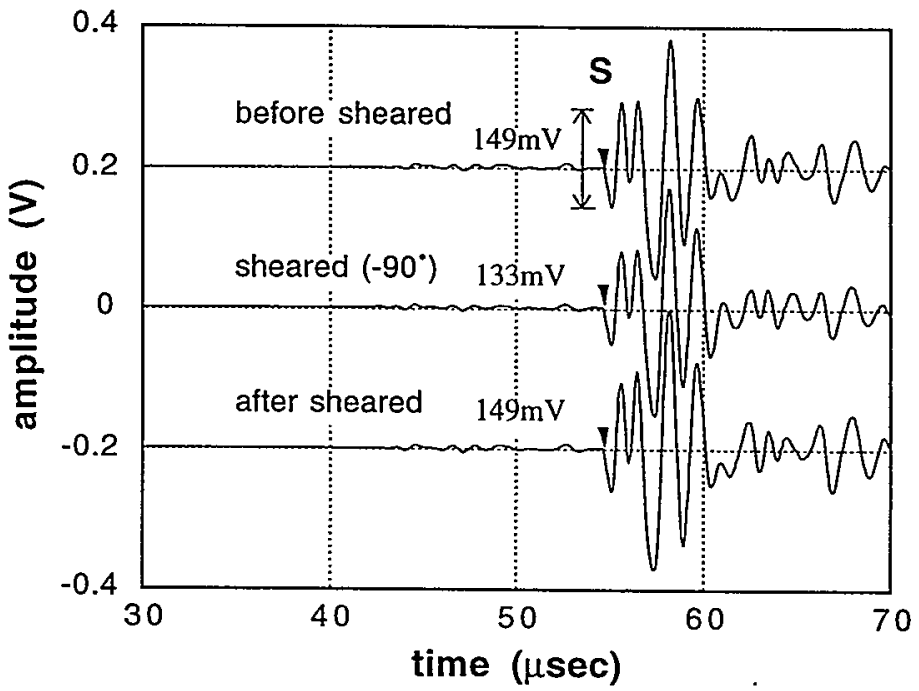

(b) S-source, S-receiver

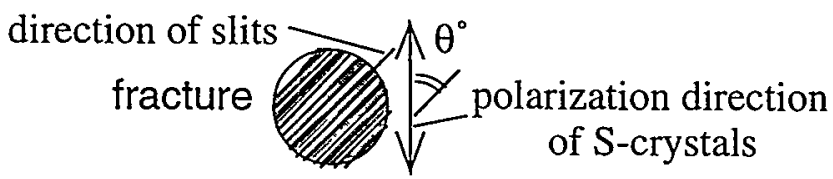

Figure 8.10 Directly transmitted waves through a fracture containing dry glass beads. Axial stress is maintained at 2.46 MPa and shear stress was increased up to approximately $2.5 \mathrm{MPa}$. Waves measured before and after the shear are compared with waves during the shear. Shear stress slightly decreases the amplitude of directly transmitted (a) P and (b) S-waves 


\section{[Steel block with fracture containing dry glass beads]}

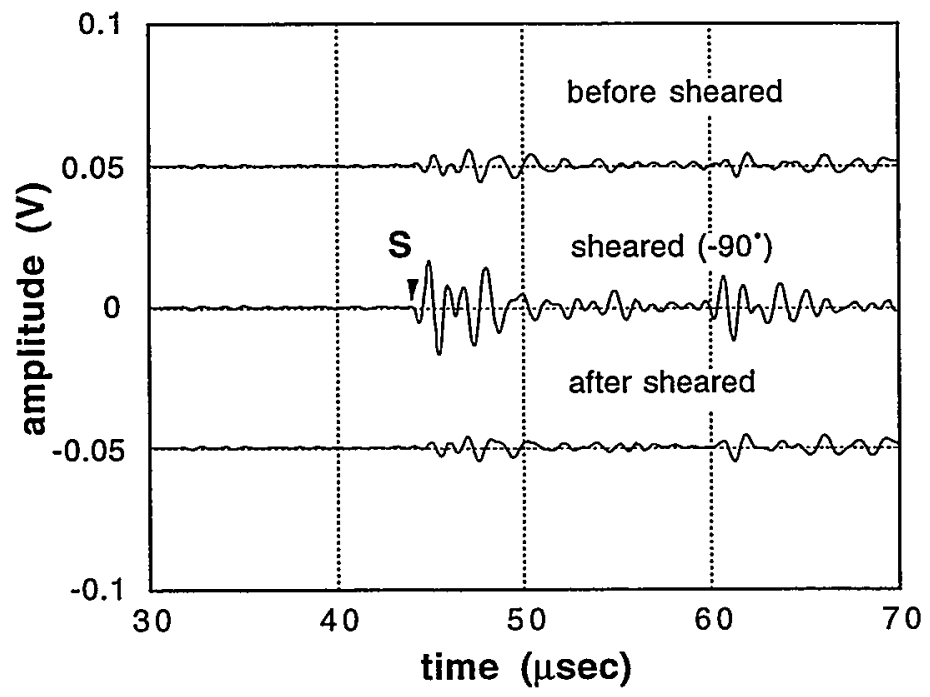

(a) P-source, S-receiver

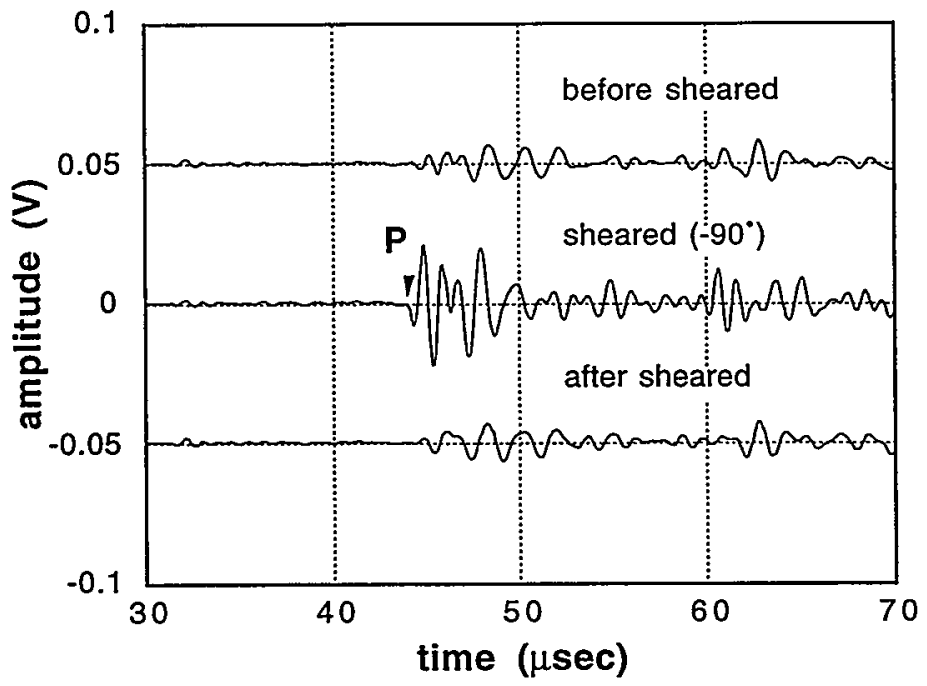

(b) S-source, P-receiver

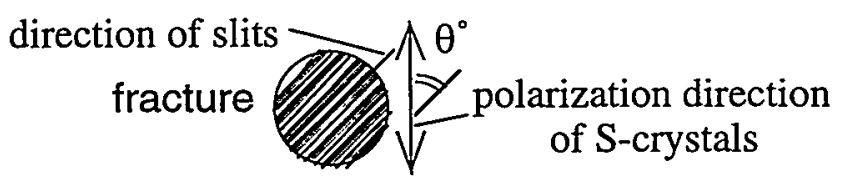

Figure 8.10 (c) (d) Converted waves through a fracture containing dry glass beads. Clear arrival and increase in amplitude of converted waves are observed. Small converted waves observed before and after the shear are possibly due to random scattering from the glass bead layer between the two steel blocks. 
[Steel block with fracture containing water-saturated glass beads]

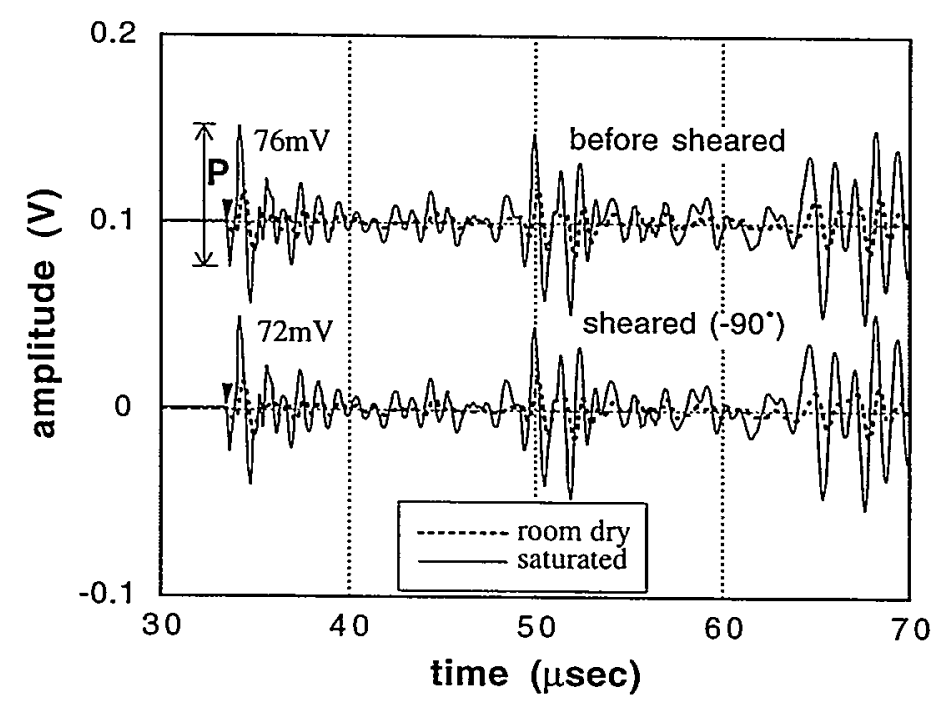

(a) P-source, P-receiver

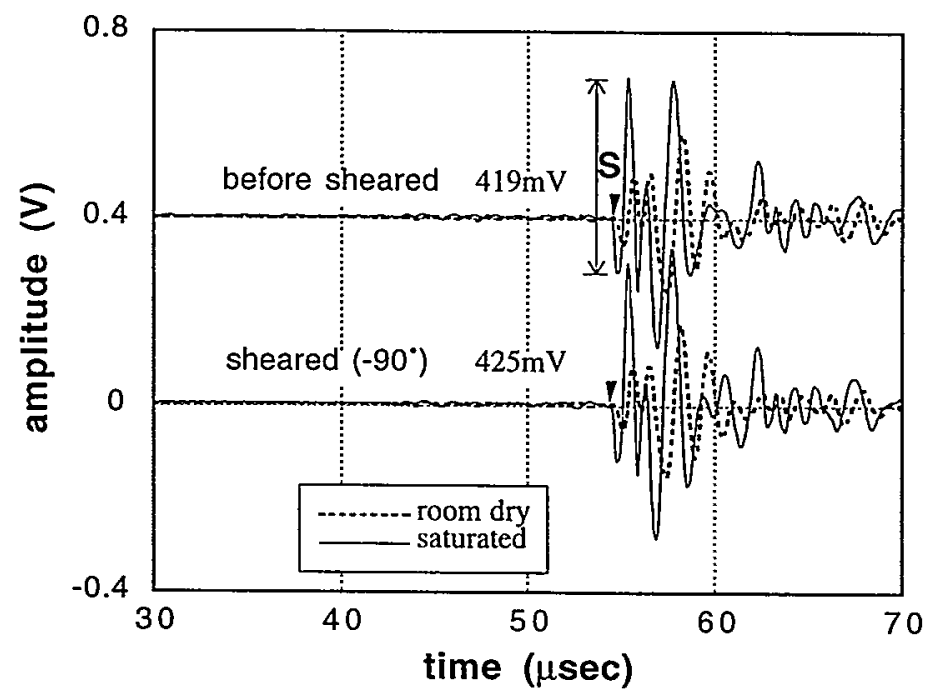

(b) S-source, S-receiver

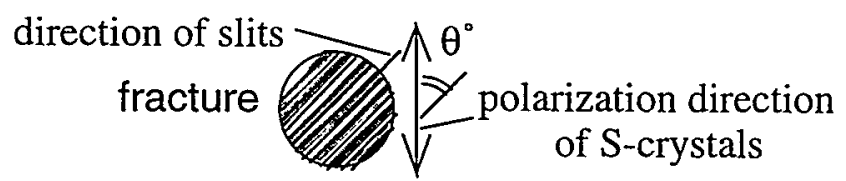

Figure 8.11 Directly transmitted waves through a fracture containing water saturated glass beads. Axial stress is maintained at $2.46 \mathrm{MPa}$ and shear stress is increased up to approximately $2.5 \mathrm{MPa}$. Compared with the waves through dry glass beads, velocity and amplitude of the directly transmitted wave increase for both (a) P and (b) S-waves. 


\section{[Steel block with fracture containing water-saturated glass beads]}

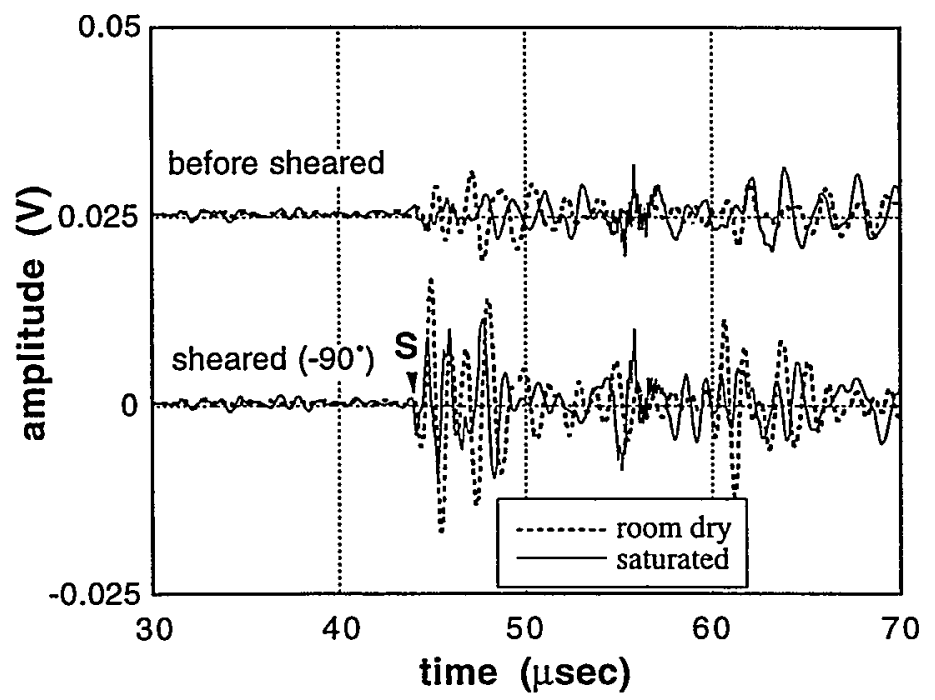

(c) P-source, S-receiver

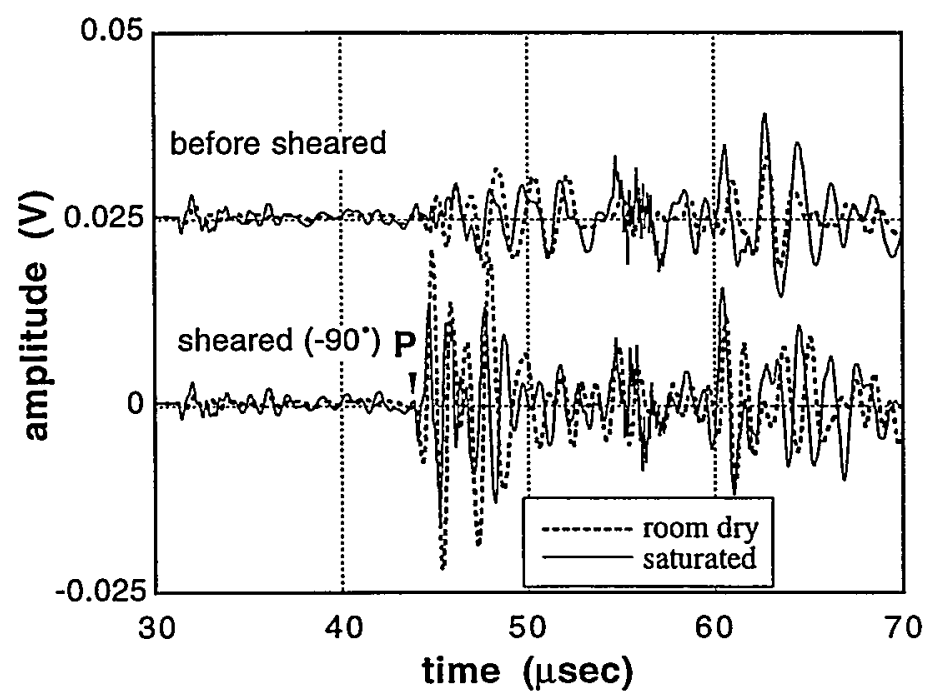

(b) S-source, P-receiver

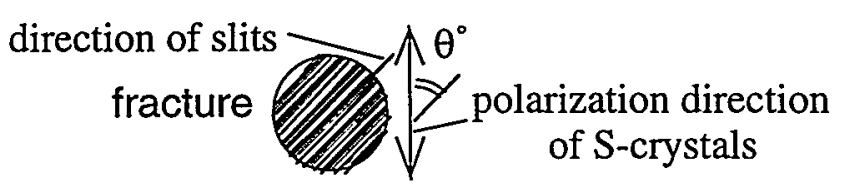

Figure 8.11 (c) (d) Converted waves transmitted through a fracture containing water saturated glass beads. Axial stress is maintained at $2.46 \mathrm{MPa}$ and shear stress is increased up to approximately $2.5 \mathrm{MPa}$. Unlike the directly transmitted waves, the converted waves show smaller amplitudes than the dry specimen. 


\subsection{Extension of the Displacement-Discontinuity Theory}

\subsubsection{Applicability of displacement-discontinuity model for dynamic problems}

When a static load is applied to a medium that contains a compliant fracture, large displacements localize around the fracture due to deformation of contacting asperities and their supporting halfspace. Many researchers including Angel and Achenbach (1985) and Baik and Thompson (1984) idealized a fracture as an interface consisting of a periodic array of flat microcracks (Figure 8.12). The contact between the two halfspaces is represented by bridges between cracks. The stress and displacement fields around the fracture are obtained analytically using a Westergarrd stress function (Westergaard, 1939).

The displacement-discontinuity model (Schoenberg, 1980; Pyrak-Nolte et al., 1990a) assumes that the additional displacement introduced by a fracture is localized within an interface with infinitesimal thickness. Therefore, the model replaces the complicated distribution of stress and strain around the fracture by uniform stress and strain in halfspaces and displacement discontinuity across the interface. The effect of the fracture on

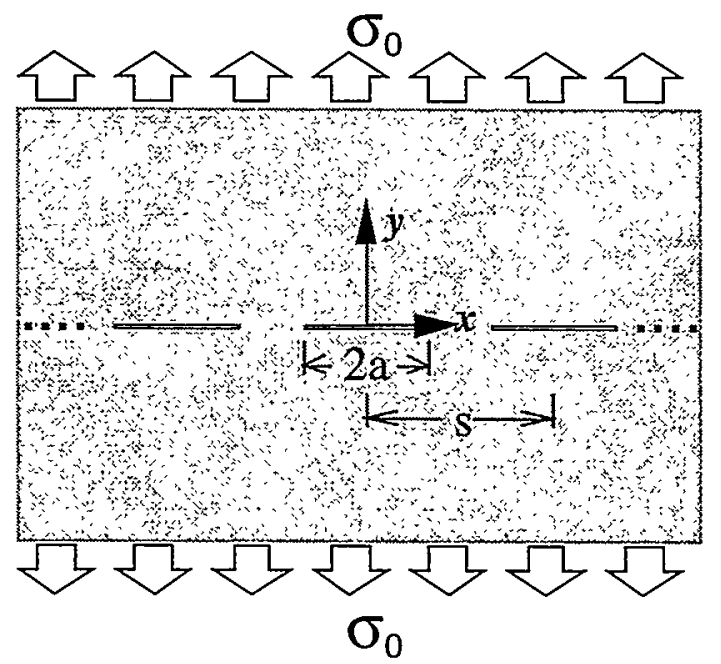

Figure 8.12 Interface consists of a periodic array of flat open microcracks. Intact bridges between cracks represent contacts and bonds between the two surfaces of a fracture. 
the far-field displacement is described by a specific compliance, defined as an additional displacement introduced into the medium by a fracture for applied unit far-field stress. Specific stiffness of a fracture is defined as the inverse of the specific compliance.

Applicability of the displacement-discontinuity model for dynamic problems was examined by Angel and Achenbach (1985) for transmission and reflection of plane waves impinging on a periodic array of flat cracks. Transmission and reflection coefficients of the waves were derived numerically using a boundary element method computing the dynamic interaction between waves and individual cracks. The computed coefficients were compared with analytic solutions obtained using the displacement-discontinuity model. The stiffness of a fracture used in the model was obtained analytically using the Westergaard stress function. For a periodic array of cracks with center spacing of $s$ and width $2 a$, a normal fracture stiffness that yields equivalent additional far-field displacement is given by (Angel and Achenbach, 1985; Baik and Thompson, 1984)

$$
\kappa=E^{\prime} \kappa^{*}=\frac{E}{1-v^{2}} \frac{1}{s} \kappa^{*}\left(\frac{2 a}{s}\right),
$$

where $\kappa^{*}$ is a non-dimensional fracture stiffness given by

$$
\kappa^{*}=\frac{\pi}{4} / \ln \left[\sec \frac{\pi(1-2 a / s)}{2}\right] .
$$

The above solution is for plane strain problems. Results obtained by Angel and Achenbach showed that the computed coefficients agree well with the analytic results given by the displacement-discontinuity theory for wavelengths longer than the size and spacing of the cracks. Therefore, by using the displacement-discontinuity model, the quasi-static behavior of a fracture can be used to examine the dynamic interaction between the waves and the fracture.

\subsubsection{Static behavior of an idealized sheared fracture}

To understand the interaction between a sheared fracture and waves, the static behavior of the fracture was examined for the idealized surface geometry (a periodic array of inclined flat cracks) discussed in the previous section. As analytic solution for displacement around such cracks is not known, the static plane-strain boundary element method was used to compute the displacement for an applied far-field stress. Using static Green's functions, 
displacements and tractions on a boundary of a computational domain are related by (for example, Brebbia and Dominguez, 1989)

$$
c_{i j} u_{j}(x)=\int_{\Gamma} u_{i j}^{*}\left(x, x^{\prime}\right) p_{j}\left(x^{\prime}\right) d \Gamma\left(x^{\prime}\right)-\int_{\Gamma} p_{i j}^{*}\left(x, x^{\prime}\right) u_{j}\left(x^{\prime}\right) d \Gamma\left(x^{\prime}\right),
$$

where $u_{i j}^{*}$ and $p_{i j}^{*}$ are the static displacement and traction Green's functions, and $u_{j}$ and $p_{j}$ are displacement and traction on the boundary, respectively. No body force is assumed. Integrations are performed in the Cauchy's sense and the $c_{i j}$ is a coefficient matrix that includes the jump-term resulting from the singularity of the Green's functions. By performing the numerical integration, the above expression leads to a linear system of equations that relate displacements and tractions on the boundary

$$
H_{I J} u_{J}=G_{I J} p_{J}
$$

where $u_{I}$ and $p_{I}$ are known and unknown displacements and tractions on the boundary. The above matrix equation can be decomposed to known and unknown parts as

$$
[\mathbf{H} \overline{\mathbf{H}}]\left\{\begin{array}{l}
\mathbf{u} \\
\overline{\mathbf{u}}
\end{array}\right\}=[\mathbf{G} \overline{\mathbf{G}}]\left\{\begin{array}{l}
\mathbf{p} \\
\overline{\mathbf{p}}
\end{array}\right\} \Rightarrow[\mathbf{H}-\mathbf{G}]\left\{\begin{array}{l}
\mathbf{u} \\
\mathbf{p}
\end{array}\right\}=[-\overline{\mathbf{H}} \overline{\mathbf{G}}]\left\{\begin{array}{l}
\overline{\mathbf{u}} \\
\overline{\mathbf{p}}
\end{array}\right\} \equiv \mathbf{b}
$$

where $\mathbf{u}$ and $\mathbf{p}$ are unknown displacement and traction, and $\overline{\mathbf{u}}$ and $\overline{\mathbf{p}}$ are known displacement and traction specified by boundary conditions, respectively. By solving Eq.(8.5), all unknown displacements and tractions are determined. In this research, a crack is modeled as a part of the boundary between two domains. Such a method is known as the multiple-domain technique in solving crack problems using the boundary element method (Blandford et al., 1981). Quadratic-continuous elements are used for the current simulations except for the elements on the interface between two domains and elements adjacent to the interface (Figure 8.13). For these, quadratic-discontinuous elements (all or part of the nodes are located within an element) are used. The quadratic-discontinuous element provides accurate results for computing displacement and stress concentration around cracks (Portela et al., 1992). For simulating the behavior of an infinite series of cracks, periodic boundary conditions are applied to the sides of the interconnected domains.

To check the accuracy of the numerical code, computed results are compared with known analytic solutions for a periodic array of flat cracks. The cracks lie on a single plane 


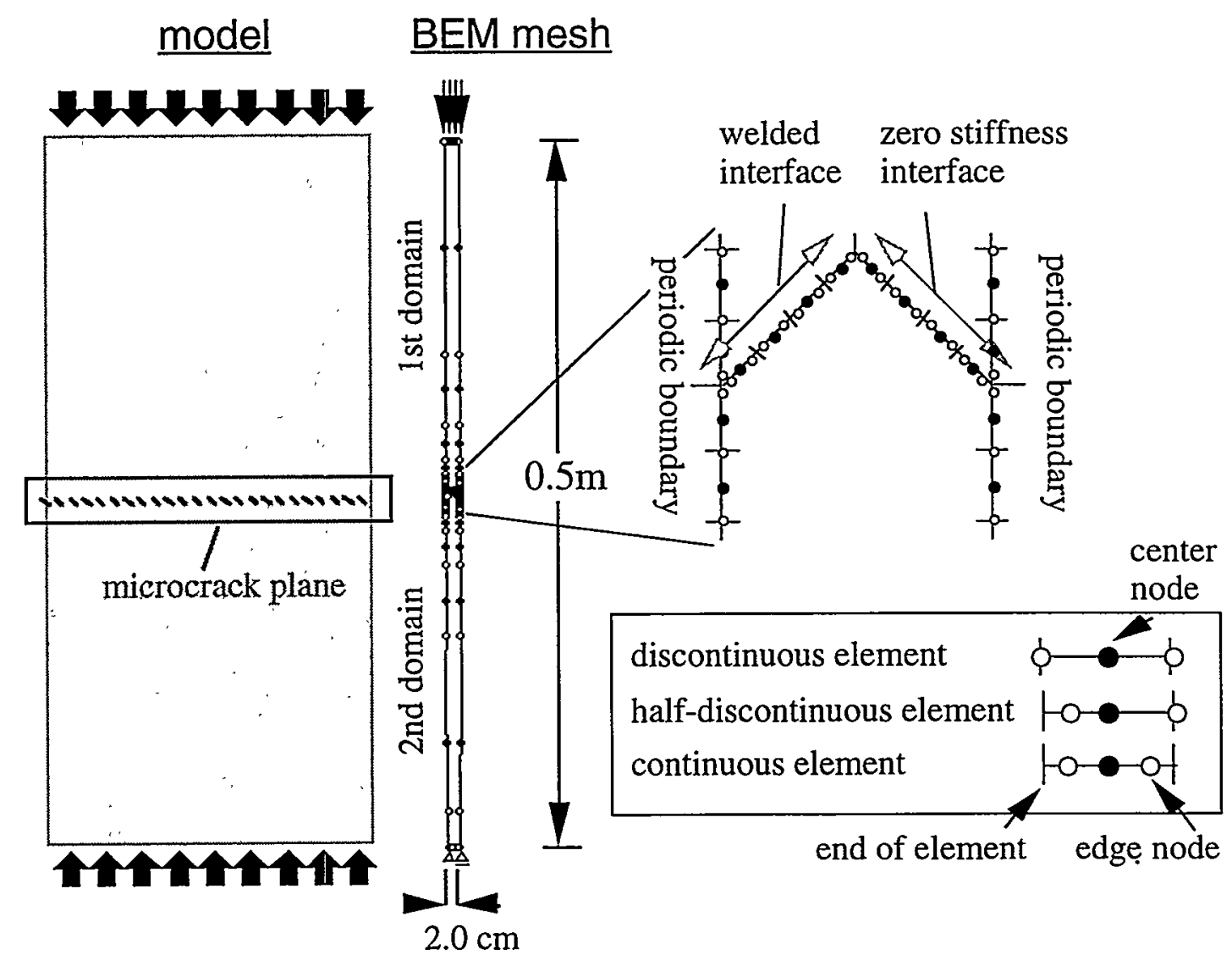

Figure 8.13 Boundary element mesh used for computing displacement around a periodic array of inclined flat cracks subjected to far-field stress. Due to periodicity of the problem, a single strip with a crack was used for the simulations.

and are subjected to a given far-field stress. An analytic solution for opening displacement for a crack is derived from a stress function given by (Westergaard, 1939)

$$
Z_{I}(z)=\frac{\sigma_{o} \sin (\pi z / s)}{\sqrt{\sin ^{2}(\pi z / s)-\sin ^{2}(\pi a / s)}},
$$

where $\sigma_{o}$ is the stress applied normal to the interface at infinite distance. In the above expression, $z$ is a complex variable defined by $z=x+i y$ where $x$ and $y$ are real numbers. Noting that $z=x,|x| \leq a$ within a crack, crack surface displacement is computed by

$$
u_{z}(x)=\frac{1-v}{G} \operatorname{Im}\left\{\int_{x}^{a} Z_{l}(x) d x\right\} .
$$




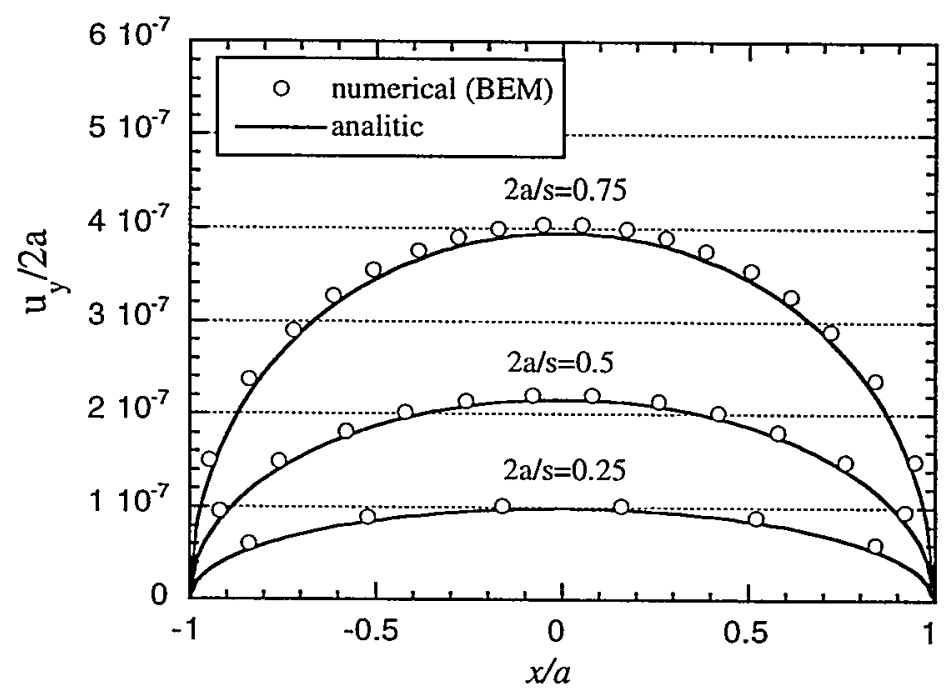

Figure 8.14 Comparison between numerical and analytical solutions for crack-surface displacement for the model in Figure 8.11. The numerical results were obtained using the boundary element model described in Figure 8.13. Both results show good agreement for a range of crack sizes with respect to the crack interval.

The analytic solution for the lateral crack-surface displacement due to a far-field shear stress is obtained by replacing the $\sigma_{o}$ in the Eq.(8.6) by the shear stress $\tau_{o}$ and $u_{y}$ in the Eq.(8.7) by $u_{x}$. Figure 8.14 shows a comparison between computed and analytic solutions for the crack-opening displacement. The result demonstrates the accuracy of the numerical model for solving problems with an infinite periodic array of cracks.

The numerical code was used to compute the displacement around a periodic array of open flat cracks. An example of a computed displacement field is shown in Figure 8.15. From the plot, it can be seen that the perturbation of the displacement field due to the cracks is strongly localized in the vicinity of the cracks, which indicates that a fracture can be modeled by a displacement-discontinuity boundary with zero-thickness. The relation between the displacement discontinuity and the applied stress is described by the specific fracture compliance. This compliance can be measured as an additional displacement in the far-field due to the fracture for an applied unit far-field traction. Normal and tangential fracture compliances are computed by

$$
\begin{aligned}
& \chi_{y y}=\left(u_{y}^{f a r / f r a c ' d}-u_{y}^{f a r / \text { intacl }}\right) / \sigma_{y y}^{f a r}, \\
& \chi_{x x}=\left(u_{x}^{f a r / \text { frac'd }}-u_{x}^{f a r / \text { intact }}\right) / \sigma_{y x}^{f a r},
\end{aligned}
$$



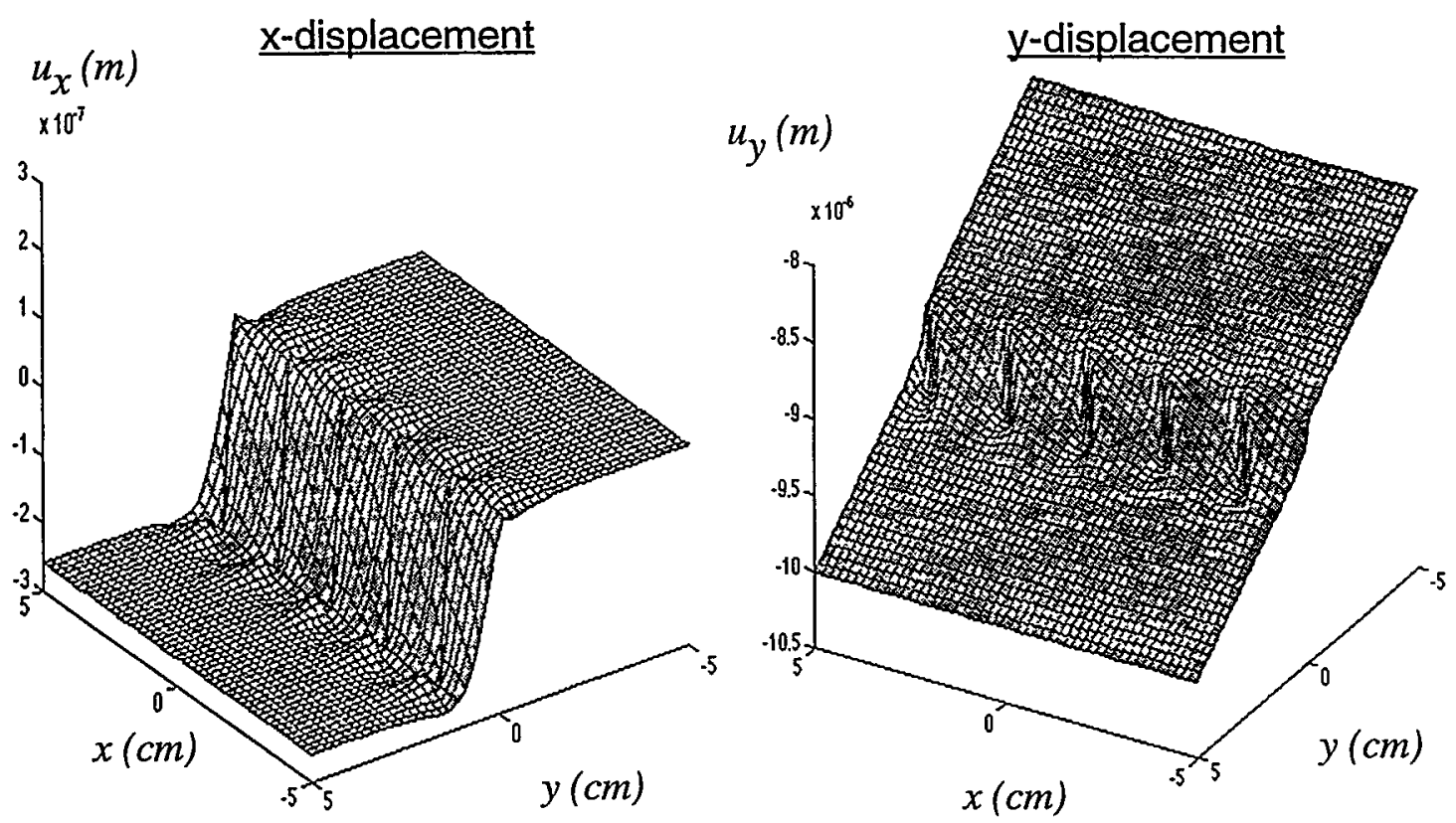

Figure 8.15 Computed displacement field for cracks with crack size to interval ratio of $2 \mathrm{a} / \mathrm{s}=0.5$. Inclination of the cracks is $45^{\circ}$. A compressional stress of $1 \mathrm{MPa}$ was applied at far-field. Young's modulus of the half-space was $50 \mathrm{GPa}$ and Poisson's ratio was 0.2 . Resulting displacement localizes strongly nearby the array of inclined cracks. Finite lateral displacement (x-displacement) in the upper domain $(y>0)$ indicates dilation of the interface.

where superscript " far" stands for far-field, " frac' $d$ " for fractured medium, and "intact" for intact medium. Coupling compliances are computed from tangential and normal displacements for normal and tangential tractions, respectively, by

$$
\begin{aligned}
& \chi_{y x}=\left(u_{x}^{\text {far/frac'd }}-u_{x}^{\text {far/intact }}\right) / \sigma_{y y}^{f a r}, \\
& \chi_{x y}=\left(u_{y}^{f a r / \text { frac d }^{\prime}}-u_{y}^{\text {far/intact }}\right) / \sigma_{y x}^{\text {far }} .
\end{aligned}
$$

Figure 8.16(a) shows computed compliances for an array of infinite number of cracks. The compliances are computed for a range of crack inclination angles. The compliances are normalized by the normal compliance of an array of $0^{\circ}$-inclination cracks whose fracture stiffness is given by Eq.(8.1). As can be seen from the plot, the coupling compliances increase monotonically with increasing inclination of the cracks. It is also noted that both coupling compliances show similar values in accordance with reciprocity of elasticity. The small difference observed is due to numerical errors. Fracture stiffness is computed by inverting the compliance matrix whose off-diagonal terms are given by the coupling compliances (Figure 8.16b). It is found that the stiffnesses of the fracture becomes 
infinitely large for a certain inclination of the cracks. This is because the determinant of the compliance matrix approaches zero at this angle (Figure 8.16c). The relative magnitude of the coupling compliance or stiffness of a fracture is defined by

$$
R=\sqrt{\frac{\kappa_{x y} \cdot \kappa_{y x}}{\kappa_{x x} \cdot \kappa_{y y}}}=\sqrt{\frac{\chi_{x y} \cdot \chi_{y x}}{\chi_{x x} \cdot \chi_{y y}}} .
$$

The computed ratios are plotted in Figure 8.16d. It can be seen that the ratio approaches unity as the determinant of the compliance matrix approaches zero.

\subsubsection{Some basic properties of fracture stiffness and compliance matrices}

For a general three-dimensional coupling between displacement-discontinuities and tractions across a fracture, the fracture compliance and stiffness matrices are $3 \times 3$ square matrices. As the additional strain energy stored by the fracture has to be non-negative, the matrices must be positive definite. Therefore, the matrices have real non-negative eigenvalues and are symmetric. For the plain strain problem discussed in Section 8.4.2, the matrices can be treated as $2 \times 2$, since the third components are decoupled from the rest. Eigenvalues of the $2 \times 2$ fracture compliance matrix are given by

$$
\chi^{ \pm}=\frac{\chi_{x x}+\chi_{y y} \pm \sqrt{\left(\chi_{x x}+\chi_{y y}\right)^{2}-4\left(\chi_{x x} \cdot \chi_{y y}-\chi_{x y} \cdot \chi_{y x}\right)}}{2} .
$$

Therefore, for non-negative normal and tangential compliances $\chi_{y y}$ and $\chi_{x x}$, a necessary and sufficient condition to have non-negative eigenvalues is

$$
\operatorname{det}\left[\chi_{i j}\right] \equiv \chi_{x x} \cdot \chi_{y y}-\chi_{x y} \cdot \chi_{y x} \geq 0
$$

This condition is satisfied in all previous simulations (Figure 8.16c). An identical condition is required for the stiffness matrix to have non-negative normal and tangential stiffnesses. These conditions provide a constraint on the coupling ratio $\mathrm{R}$ defined by Eq.(8.9) as

$$
R \equiv \sqrt{\chi_{x y} \cdot \chi_{y x} / \chi_{x x} \cdot \chi_{y y}}=\sqrt{\kappa_{x y} \cdot \kappa_{y x} / \kappa_{x x} \cdot \kappa_{y y}} \leq 1 .
$$

This constraint is also satisfied for the numerical simulation (Figure 8.16d). Note that Eq.(8.12) is an equality only when the determinant of the compliance matrix becomes zero. 


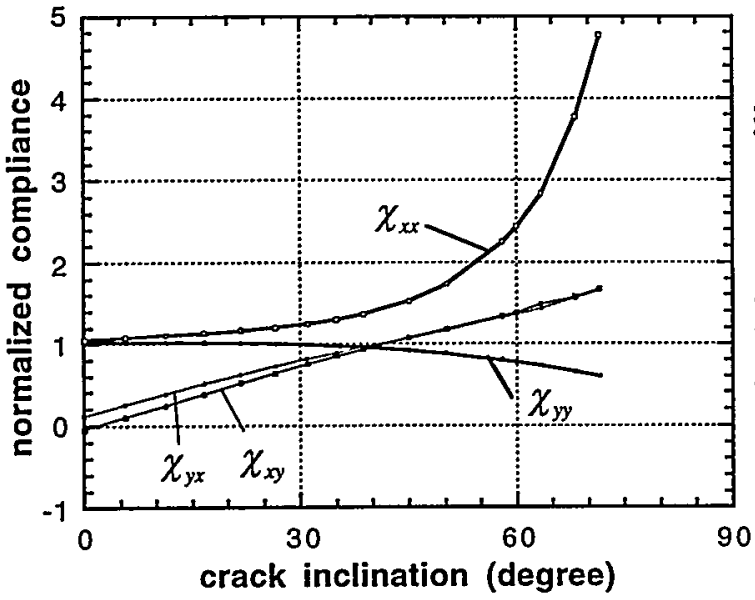

(a) fracture compliance

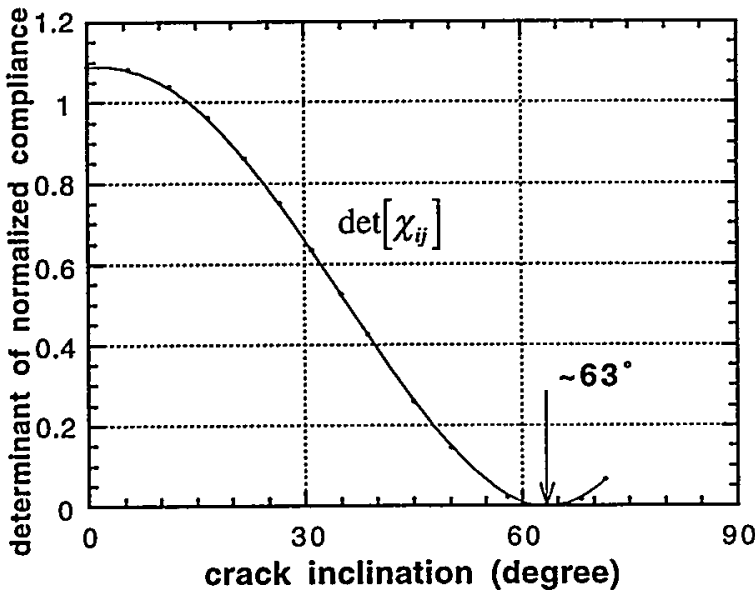

(c) determinant of compliance matrix

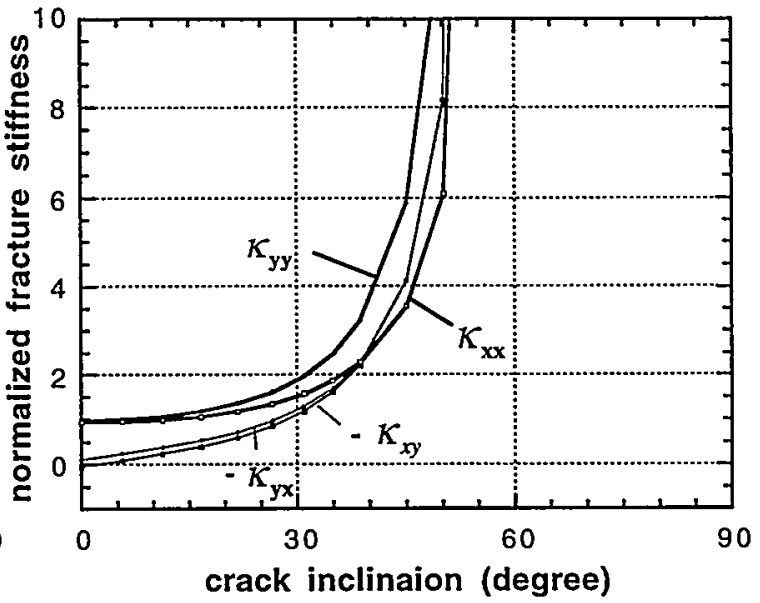

(b) fracture stiffness

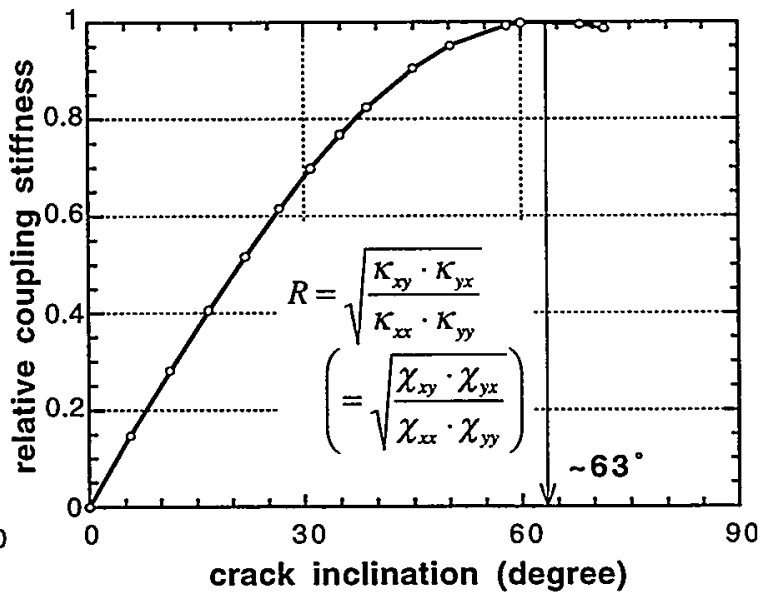

(d) coupling stiffness (compliance) ratio

Figure 8.16 Computed compliances and stiffnesses of a fracture consisting of a periodic array of inclined cracks. Coupling compliance (a) and stiffness (b) that show dilation behavior of the fracture increase monotonically as the inclination of the cracks increases. The fracture stiffnesses become infinitely large at an angle (approximately $63^{\circ}$ ) where the determinant of the compliance matrix becomes zero (c). The coupling stiffness ratio $R$ increases monotonically with increasing inclination of the cracks until it reaches unity (d). The angle at which the ratio $\mathrm{R}$ is maximum is identical to the angle where the determinant of the compliance matrix becomes zero. 


\subsubsection{General scattering matrix for plane waves incident on fracture}

The results in Section 8.4.2 demonstrated that a periodic array of inclined cracks (an idealized geometry for a sheared fracture) exhibits a significant dilation. Such behavior is described quantitatively by the coupling (off-diagonal) components of the fracture stiffness and compliance matrices. In the following analysis, the effect of the dilation behavior on those waves incident upon a fracture is examined using the displacement-discontinuity model.

The displacement-discontinuity model provides a set of boundary conditions that require continuous stress and discontinuity in displacement that is proportional to the traction on the fracture. For a fracture located on an $x-y$ plane, these boundary conditions are

$$
\begin{aligned}
& t_{1 i}=-t_{2 i}=\sigma_{z i}, \\
& \sigma_{z i}=\kappa_{i j}\left[u_{j}\right](i, j=x, y, z),
\end{aligned}
$$

where $\sigma_{z i}(i=x, y, z)$ are components of the stress tensor on the fracture ( $\sigma_{z x}$ and $\sigma_{z y}$ are tangential stresses, $\sigma_{z z}$ is normal stress), $t_{i}$ is the traction force on the surface, and $\left[u_{j}\right]$ is the displacement-discontinuity across the fracture defined by $\left[u_{j}\right] \equiv u_{2 j}-u_{1 j}(j=x, y, z)$. Subscripts "1" and "2" denote halfspaces on either side of a fracture.

For a fracture without shear, the cross-coupling stiffness becomes zero and the fracture stiffness matrix $\kappa_{i j}$ becomes diagonal. Therefore the above matrix equation yields three sets of independent scalar equations

$$
\begin{array}{ll}
\sigma_{z x}=\kappa_{x x}\left[u_{x}\right], & t_{1 x}=-t_{2 x} \equiv \sigma_{z x}, \\
\sigma_{z y}=\kappa_{y y}\left[u_{y}\right], & t_{1 y}=-t_{2 y} \equiv \sigma_{z y}, \\
\sigma_{z z}=\kappa_{z z}\left[u_{z}\right], & t_{1 z}=-t_{2 z} \equiv \sigma_{z z} .
\end{array}
$$

The displacement-discontinuity boundary conditions without coupling fracture stiffness have been successfully used to investigate the interaction between elastic waves and fractures in rock when the fractures were subjected to normal loading only (Pyrak-Nolte $e t$ al., 1990a). If the $\mathrm{x}$-coordinate axis is defined such that the incident wave propagates parallel to the $\mathrm{x}-\mathrm{z}$ plane, Eq.(8.15a) and (8.15c) are used to derive transmission and reflection coefficients for $\mathrm{P}$ and SV- waves. Eq.(8.15b) is used to derive the transmission 
and reflection coefficients for an SH-wave that is decoupled from the other waves. The following analysis will show that if coupling fracture stiffnesses are non-zero, $\mathrm{P}, \mathrm{SV}$, and SH-waves will become coupled.

For plane waves incident on a fracture (Figure 8.17), the displacement of the waves is given by

$$
\begin{aligned}
& \mathbf{u}_{I S V}=A_{I S V} \cdot\left(\begin{array}{c}
\cos \phi_{1} \\
0 \\
-\sin \phi_{1}
\end{array}\right) \cdot \exp \left\{i \omega\left(\frac{\sin \phi_{1}}{c_{S 1}} x+\frac{\cos \phi_{1}}{c_{S 1}} z\right)\right\}, \quad \text { (incident SV-wave) } \\
& \mathbf{u}_{I S H}=A_{I S H} \cdot\left(\begin{array}{l}
0 \\
1 \\
0
\end{array}\right) \cdot \exp \left\{i \omega\left(\frac{\sin \phi_{1}}{c_{S 1}} x+\frac{\cos \phi_{1}}{c_{S 1}} z\right)\right\}, \quad \text { (incident SH-wave) } \\
& u_{I P}=A_{I P} \cdot\left(\begin{array}{c}
\sin \theta_{1} \\
0 \\
\cos \theta_{1}
\end{array}\right) \cdot \exp \left\{i \omega\left(\frac{\sin \theta_{1}}{c_{P 1}} x+\frac{\cos \theta_{1}}{c_{P 1}} z\right)\right\}, \quad \text { (incident P-wave) } \\
& \mathbf{u}_{R S V}=A_{T P} \cdot\left(\begin{array}{c}
\cos \phi_{1} \\
0 \\
\sin \phi_{1}
\end{array}\right) \cdot \exp \left\{i \omega\left(\frac{\sin \phi_{1}}{c_{S 1}} x-\frac{\cos \phi_{1}}{c_{S 1}} z\right)\right\}, \quad \text { (reflected SV-wave) (8.16d) } \\
& \mathbf{u}_{R S H}=A_{r P} \cdot\left(\begin{array}{l}
0 \\
1 \\
0
\end{array}\right) \cdot \exp \left\{i \omega\left(\frac{\sin \phi_{1}}{c_{S 1}} x-\frac{\cos \phi_{1}}{c_{S 1}} z\right)\right\}, \quad \text { (reflected SH-wave) (8.16e) } \\
& \mathbf{u}_{R P}=A_{R P} \cdot\left(\begin{array}{c}
-\sin \theta_{1} \\
0 \\
\cos \theta_{1}
\end{array}\right) \cdot \exp \left\{i \omega\left(\frac{\sin \theta_{1}}{c_{P 1}} x-\frac{\cos \theta_{1}}{c_{P 1}} z\right)\right\}, \quad \text { (reflected P-wave) } \\
& \mathbf{u}_{T S V}=A_{T S V} \cdot\left(\begin{array}{c}
\cos \phi_{2} \\
0 \\
-\sin \phi_{2}
\end{array}\right) \cdot \exp \left\{i \omega\left(\frac{\sin \phi_{2}}{c_{S 2}} x+\frac{\cos \phi_{2}}{c_{S 2}} z\right)\right\},(\text { transmitted SV-wave })(8.16 \mathrm{~g}) \\
& \mathbf{u}_{T S H}=A_{T S H} \cdot\left(\begin{array}{l}
0 \\
1 \\
0
\end{array}\right) \cdot \exp \left\{i \omega\left(\frac{\sin \phi_{2}}{c_{S 2}} x+\frac{\cos \phi_{2}}{c_{S 2}} z\right)\right\}, \quad(\text { transmitted SH-wave) }(8.16 \mathrm{~h}) \\
& \mathbf{u}_{T P}=A_{T P} \cdot\left(\begin{array}{c}
\sin \theta_{2} \\
0 \\
\cos \theta_{2}
\end{array}\right) \cdot \exp \left\{i \omega\left(\frac{\sin \theta_{2}}{c_{P 2}} x+\frac{\cos \theta_{2}}{c_{P 2}} z\right)\right\}, \text { (transmitted P-wave) }
\end{aligned}
$$



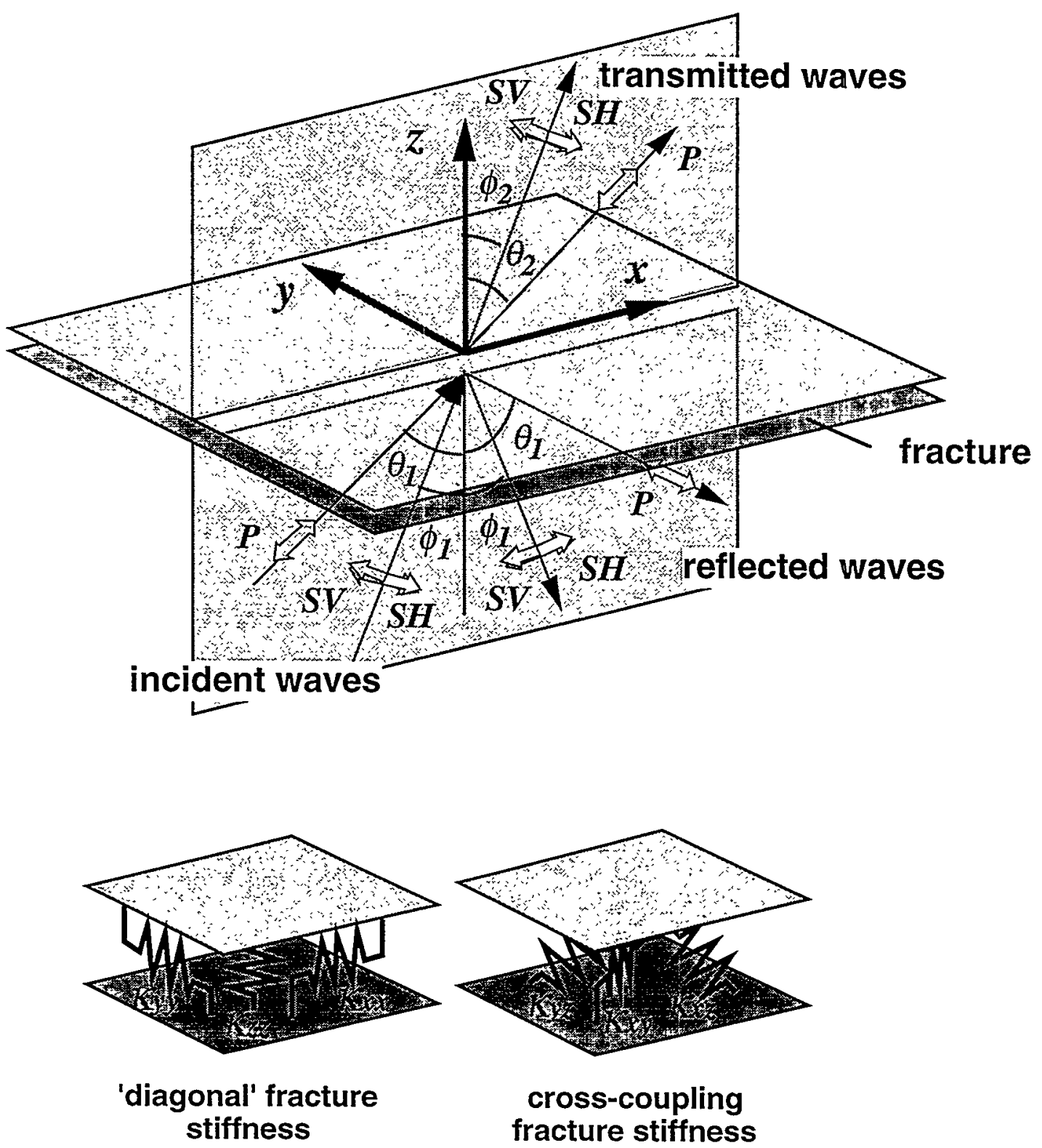

cross-coupling fracture stiffness

Figure 8.17 Scattering of waves incident upon a compliant interface (fracture). The coordinate system is defined such that the incident waves propagate parallel to the $\mathrm{x}-\mathrm{z}$ plane. $\mathrm{P}, \mathrm{SH}$, and SV-waves incident on the interface result in transmitted and reflected $\mathrm{P}, \mathrm{SH}$, and SV-waves. The components of the fracture stiffness matrix consist of diagonal stiffnesses and off-diagonal cross-coupling stiffnesses that lead to dilation behavior of the fracture. 
where $c_{P}$ and $c_{S}$ are the velocity of $\mathrm{P}$ and $\mathrm{S}$-waves in the halfspace, respectively. The terms $\theta$ and $\phi$ are incidence angles of $\mathrm{P}$ and S- waves, respectively, that satisfy Snell's law, and $\omega$ is the angular frequency of wave. Displacement in the half-spaces is

$$
\begin{aligned}
& \mathbf{u}_{1}=\mathbf{u}_{I P}+\mathbf{u}_{I S V}+\mathbf{u}_{I S H}+\mathbf{u}_{R P}+\mathbf{u}_{R S V}+\mathbf{u}_{R S H}, \\
& \mathbf{u}_{2}=\mathbf{u}_{T P}+\mathbf{u}_{T S V}+\mathbf{u}_{T S H} .
\end{aligned}
$$

The displacement-discontinuity vector is

$$
[u]=u_{2}-u_{1} \text {. }
$$

The constitutive relations in the halfspaces are

$$
\begin{aligned}
& \sigma_{1 z x}=\mu_{1}\left(u_{1 x, z}+u_{1 z, x}\right), \\
& \sigma_{2 z x}=\mu_{2}\left(u_{2 x, z}+u_{2 z, x}\right), \\
& \sigma_{1 z y}=\mu_{1}\left(u_{1 y, z}+u_{1 z, y}\right), \\
& \sigma_{2 z y}=\mu_{2}\left(u_{2 y, z}+u_{2 z, y}\right), \\
& \sigma_{1 z z}=\left(2 \mu_{1}+\lambda_{1}\right) u_{1 z, z}+\lambda_{1} u_{1 x, x}, \\
& \sigma_{2 z z}=\left(2 \mu_{2}+\lambda_{2}\right) u_{2 z, z}+\lambda_{2} u_{2 x, x} .
\end{aligned}
$$

The relation between the amplitude of incident and scattered waves is obtained by introducing the displacement of the wave given by Eq.(8.16a) (8.16i) into the six displacement-discontinuity boundary conditions in Eq.(8.13) and (8.14). Stresses and displacement discontinuities are then computed from Eq.(8.18) and (8.19a f). The result is expressed in the form of a matrix equation that is solved for the transmission and reflection coefficients. The transmission coefficient matrix [T] and a reflection coefficient matrix $[R]$ are defined as

$$
[T] \equiv\left[\begin{array}{lll}
T_{S V}^{(S V)} & T_{S V}^{(S H)} & T_{S V}^{(P)} \\
T_{S H}^{(S)} & T_{S H}^{(S H)} & T_{S H}^{(P)} \\
T_{P}^{(S)} & T_{P}^{(S H)} & T_{P}^{(P)}
\end{array}\right],[R] \equiv\left[\begin{array}{lll}
R_{S V}^{(S V)} & R_{S V}^{(S H)} & R_{S V}^{(P)} \\
R_{S H}^{(S V)} & R_{S H}^{(S H)} & R_{S H}^{(P)} \\
R_{P}^{(S V)} & R_{P}^{(S H)} & R_{P}^{(P)}
\end{array}\right] .
$$

The superscript on the coefficients identifies the type of incident waves (SV-, SH-, and Pwaves) and the subscript identifies the type of scattered waves. The matrix equation for the transmission and reflection coefficients is shown in Figure 8.18. For the sake of simplicity, sine and cosine functions are abbreviated as $\mathrm{s} 0$ and $\mathrm{c}()$. 


\subsubsection{Normal incident waves with cross-coupling fracture stiffnesses}

For the special case when the incident wave propagates normal to the fracture between two identical media, the scattering matrix becomes

$$
\begin{aligned}
& {\left[\begin{array}{cccccc}
Z_{s} & 0 & 0 & Z_{S} & 0 & 0 \\
0 & Z_{S} & 0 & 0 & Z_{S} & 0 \\
0 & 0 & Z_{P} & 0 & 0 & Z_{P} \\
\kappa_{x x}-i \omega Z_{S} & \kappa_{z y} & \kappa_{z x} & -\kappa_{x x} & -\kappa_{x y} & -\kappa_{z z} \\
\kappa_{y x} & \kappa_{y y}-i \omega Z_{S} & \kappa_{y z} & -\kappa_{y x} & -\kappa_{y y} & -\kappa_{y z} \\
\kappa_{z x} & \kappa_{z y} & \kappa_{z z}-i \omega Z_{P} & -\kappa_{z x} & -\kappa_{z y} & -\kappa_{z z}
\end{array}\right] \mathrm{x}\left[\begin{array}{l}
{[T]} \\
{[R]}
\end{array}\right]} \\
& =\left[\begin{array}{ccc}
Z_{S} & 0 & 0 \\
0 & Z_{S} & 0 \\
0 & 0 & Z_{P} \\
\kappa_{x x} & \kappa_{x y} & \kappa_{x z} \\
\kappa_{y x} & \kappa_{y y} & \kappa_{y z} \\
\kappa_{z x} & \kappa_{z y} & \kappa_{z z}
\end{array}\right]
\end{aligned}
$$

where $Z_{P}$, and $Z_{S}$ are $\mathrm{P}$-wave and $\mathrm{S}$-wave acoustic impedance of the half-space, respectively. By introducing an impedance ratio matrix

$$
[\beta] \equiv\left[\begin{array}{ccc}
\beta_{x x}^{s} & \beta_{x y}^{s} & \beta_{x z}^{s} \\
\beta_{y x}^{s} & \beta_{y y}^{s} & \beta_{y z}^{s} \\
\beta_{z x}^{P} & \beta_{z y}^{P} & \beta_{z z}^{P}
\end{array}\right] \equiv\left[\begin{array}{lll}
2 \kappa_{x x} / \omega Z_{S} & 2 \kappa_{x y} / \omega Z_{S} & 2 \kappa_{x z} / \omega Z_{S} \\
2 \kappa_{y x} / \omega Z_{S} & 2 \kappa_{y y} / \omega Z_{S} & 2 \kappa_{y z} / \omega Z_{S} \\
2 \kappa_{z x} / \omega Z_{P} & 2 \kappa_{z y} / \omega Z_{P} & 2 \kappa_{z z} / \omega Z_{P}
\end{array}\right],
$$

the matrix equation becomes

$$
\left[\begin{array}{cccccc}
1 & 0 & 0 & 1 & 0 & 0 \\
0 & 1 & 0 & 0 & 1 & 0 \\
0 & 0 & 1 & 0 & 0 & 1 \\
2+i \beta_{x x}^{S} & i \beta_{x y}^{S} & i \beta_{x z}^{S} & -i \beta_{x x}^{S} & -i \beta_{x y}^{S} & -i \beta_{x z}^{S} \\
i \beta_{y x}^{S} & 2+i \beta_{y y}^{S} & i \beta_{y z}^{S} & -i \beta_{y x}^{S} & -i \beta_{y y}^{S} & -i \beta_{y z}^{S} \\
i \beta_{z x}^{P} & i \beta_{z y}^{P} & 2+i \beta_{z z}^{P} & -i \beta_{z x}^{P} & -i \beta_{z y}^{P} & -i \beta_{z z}^{P}
\end{array}\right] \times\left[\begin{array}{c}
{[T]} \\
{[R]}
\end{array}\right]=\left[\begin{array}{ccc}
1 & 0 & 0 \\
0 & 1 & 0 \\
0 & 0 & 1 \\
i \beta_{x x}^{S} & i \beta_{x y}^{S} & i \beta_{x z}^{S} \\
i \beta_{y x}^{S} & i \beta_{y y}^{S} & i \beta_{y z}^{S} \\
i \beta_{z x}^{P} & i \beta_{z y}^{P} & i \beta_{z z}^{P}
\end{array}\right]
$$

From the first three rows of the matrix equation, it follows that

$$
[T]+[R]=\mathbf{I}
$$




\begin{tabular}{|c|c|c|c|c|c|}
\hline$Z_{s 2} c 2 \phi_{2}$ & 0 & $2 Z_{s 2} c \theta_{2} s \phi_{2}$ & $Z_{S 1} c 2 \phi_{1}$ & 0 & $-2 Z_{S 1} c \theta_{1} s \phi_{1}$ \\
\hline 0 & $Z_{S 2} c \phi_{2}$ & 0 & 0 & $Z_{S 1} c \phi_{1}$ & 0 \\
\hline$-Z_{s 2} s 2 \phi_{2}$ & 0 & $Z_{P 2}-2 Z_{s 2} s \theta_{2} s \phi_{2}$ & $Z_{S 1} s 2 \phi_{1}$ & 0 & $Z_{P 1}-2 Z_{s 1} s \theta_{1} s \phi_{1}$ \\
\hline$\kappa_{x x} c \phi_{2}-\kappa_{z x} s \phi_{2}-i \omega Z_{s 2} c 2 \phi_{2}$ & $\kappa_{x y}$ & $\kappa_{x x} s \theta_{2}+\kappa_{z x} c \theta_{2}-i \omega 2 Z_{s 2} c \theta_{2} s \phi_{2}$ & $-\kappa_{x x} c \phi_{1}-\kappa_{x x} s \phi_{1}$ & $-\kappa_{x y}$ & $\kappa_{z x} s \theta_{1}-\kappa_{z z} c \theta_{1}$ \\
\hline$\kappa_{y x} c \phi_{2}-\kappa_{y z} s \phi_{2}$ & $\kappa_{y y}-i \omega Z_{S 2} c 2 \phi_{2}$ & $\kappa_{y x} s \theta_{2}+\kappa_{y z} c \theta_{2}$ & $-\kappa_{y x} c \phi_{1}-\kappa_{y z} s \phi_{1}$ & $-\kappa_{y y}$ & $\kappa_{y x} s \theta_{1}-\kappa_{y z} c \theta_{1}$ \\
\hline$\kappa_{z x} c \phi_{2}-\kappa_{z z} s \phi_{2}+i \omega Z_{S 2} s 2 \phi_{2}$ & $\kappa_{z y}$ & $\kappa_{z x} s \theta_{2}+\kappa_{z z} c \theta_{2}-i \omega\left(Z_{P 2}-2 Z_{s 2} s \theta_{2} s \phi_{2}\right)$ & $-\kappa_{z x} c \phi_{1}-\kappa_{z z} s \phi_{1}$ & $-\kappa_{z y}$ & $\kappa_{z x} s \theta_{1}-\kappa_{z z} c \theta_{1}$ \\
\hline
\end{tabular}

$$
\mathrm{X}\left[\begin{array}{c}
{[T]} \\
{[R]}
\end{array}\right]=\left[\begin{array}{ccc}
Z_{S 1} c 2 \phi_{1} & 0 & 2 Z_{S 1} c \theta_{1} s \phi_{1} \\
0 & Z_{S 1} c \phi_{1} & 0 \\
-Z_{s 1} s \phi_{1} & 0 & Z_{P 1}-2 Z_{s 1} s \theta_{1} s \phi_{1} \\
\kappa_{x x} c \phi_{1}-\kappa_{x z} s \phi_{1} & \kappa_{x y} & \kappa_{x x} s \theta_{1}+\kappa_{x z} c \theta_{1} \\
\kappa_{y x} c \phi_{1}-\kappa_{y z} s \phi_{1} & \kappa_{y y} & \kappa_{y x} s \theta_{1}+\kappa_{y z} c \theta_{1} \\
\kappa_{z x} c \phi_{1}-\kappa_{z z} s \phi_{1} & \kappa_{z y} & \kappa_{z x} s \theta_{1}+\kappa_{z z} c \theta_{1}
\end{array}\right]
$$

\section{Note}

$$
\begin{aligned}
& \sin () \equiv s() \\
& \cos () \equiv c()
\end{aligned}
$$

Figure 8.18 Matrix equation for the general scattering problem of a fracture including coupling between all displacement discontinuity and traction components. Subscripts " 1 " and " 2 " represent media on either sides of the fracture. By solving the matrix equation,

transmission and reflection coefficient matrices ([T] and [R]) are determined for any specified angle of incidence. 
where I is an identity matrix. Therefore the matrix equation is reduced to as

$$
\left[\begin{array}{ccc}
1+i \beta_{x x}^{S} & i \beta_{x y}^{S} & i \beta_{x z}^{S} \\
i \beta_{y x}^{s} & 1+i \beta_{y y}^{S} & i \beta_{y z}^{S} \\
i \beta_{z x}^{P} & i \beta_{z y}^{P} & 1+i \beta_{z z}^{P}
\end{array}\right][T]=\left[\begin{array}{ccc}
i \beta_{x x}^{S} & i \beta_{x y}^{S} & i \beta_{x z}^{s} \\
i \beta_{y x}^{S} & i \beta_{y y}^{S} & i \beta_{y z}^{S} \\
i \beta_{z x}^{P} & i \beta_{z y}^{P} & i \beta_{z z}^{P}
\end{array}\right] .
$$

For the special case when the coupling stiffnesses between $y$ and the other directions are zero, the above matrix equation is decomposed to a P-SV problem and a SH problem. Such fracture stiffness is expected for a fracture sheared along the $\mathrm{x}$-axis. By setting the coupling impedance parameters $\beta_{x y}^{s}, \beta_{y x}^{s}, \beta_{y z}^{s}$, and $\beta_{z y}^{s}$ equal to zero, the matrices become block-diagonal, yielding

$$
\begin{aligned}
& {\left[\begin{array}{cc}
1+i \beta_{x x}^{S} & i \beta_{x z}^{S} \\
i \beta_{z x}^{P} & 1+i \beta_{z z}^{P}
\end{array}\right]\left[\begin{array}{ll}
T_{S V}^{(S V)} & T_{s V}^{(P)} \\
T_{P}^{(S V)} & T_{P}^{(P)}
\end{array}\right]=\left[\begin{array}{cc}
i \beta_{x x}^{S} & i \beta_{x z}^{S} \\
i \beta_{z x}^{P} & i \beta_{z z}^{P}
\end{array}\right],} \\
& \left(1+i \beta_{y y}^{S}\right) T_{s H}^{(S H)}=i \beta_{y y}^{S}, \\
& T_{S H}^{(S V)}=T_{S H}^{(P)}=T_{S V}^{(S H)}=T_{P}^{(S H)}=0 .
\end{aligned}
$$

Therefore, the transmission coefficients for the P-SV problem are

$$
\begin{aligned}
& {\left[\begin{array}{ll}
T_{s V}^{(S V)} & T_{s V}^{(P)} \\
T_{P}^{(S V)} & T_{P}^{(P)}
\end{array}\right]=\frac{1}{\Delta}\left[\begin{array}{cc}
i \beta_{x x}^{S}\left(1+i \beta_{z z}^{P}\right)+\beta_{x z}^{S} \beta_{z x}^{P} & i \beta_{x z}^{S} \\
i \beta_{z x}^{P} & i \beta_{z z}^{P}\left(1+i \beta_{x x}^{S}\right)+\beta_{z x}^{P} \beta_{x z}^{S}
\end{array}\right],} \\
& \Delta=\left(1+i \beta_{x x}^{S}\right)\left(1+i \beta_{z z}^{P}\right)+\beta_{x z}^{S} \beta_{z x}^{P} .
\end{aligned}
$$

The transmission coefficient for the $\mathrm{SH}$ problem is

$$
T_{S H}^{(S H)}=\frac{i \beta_{y y}^{S}}{1+i \beta_{y y}^{S}} .
$$

Once the transmission coefficients are obtained, reflection coefficients are derived from Eq.(8.25). The matrix equation Eq.(8.28) shows that the transmission (and reflection) coefficients for converted waves (converted SV wave from P-wave and converted P-wave from SV-wave) do not vanish for finite coupling fracture stiffnesses. The scattering coefficients of the transmitted, reflected, and converted waves for a normal incident $\mathrm{P}$-wave are shown in Figure 8.19. Normal and tangential fracture stiffnesses are assumed to be identical ( $\kappa_{x x}=\kappa_{2 z} \equiv \kappa$ ) and the relative magnitude of the coupling stiffness is given by the coupling stiffness ratio $R \equiv \kappa_{z x} / \kappa$. The horizontal axis is the inverse of impedance ratio 

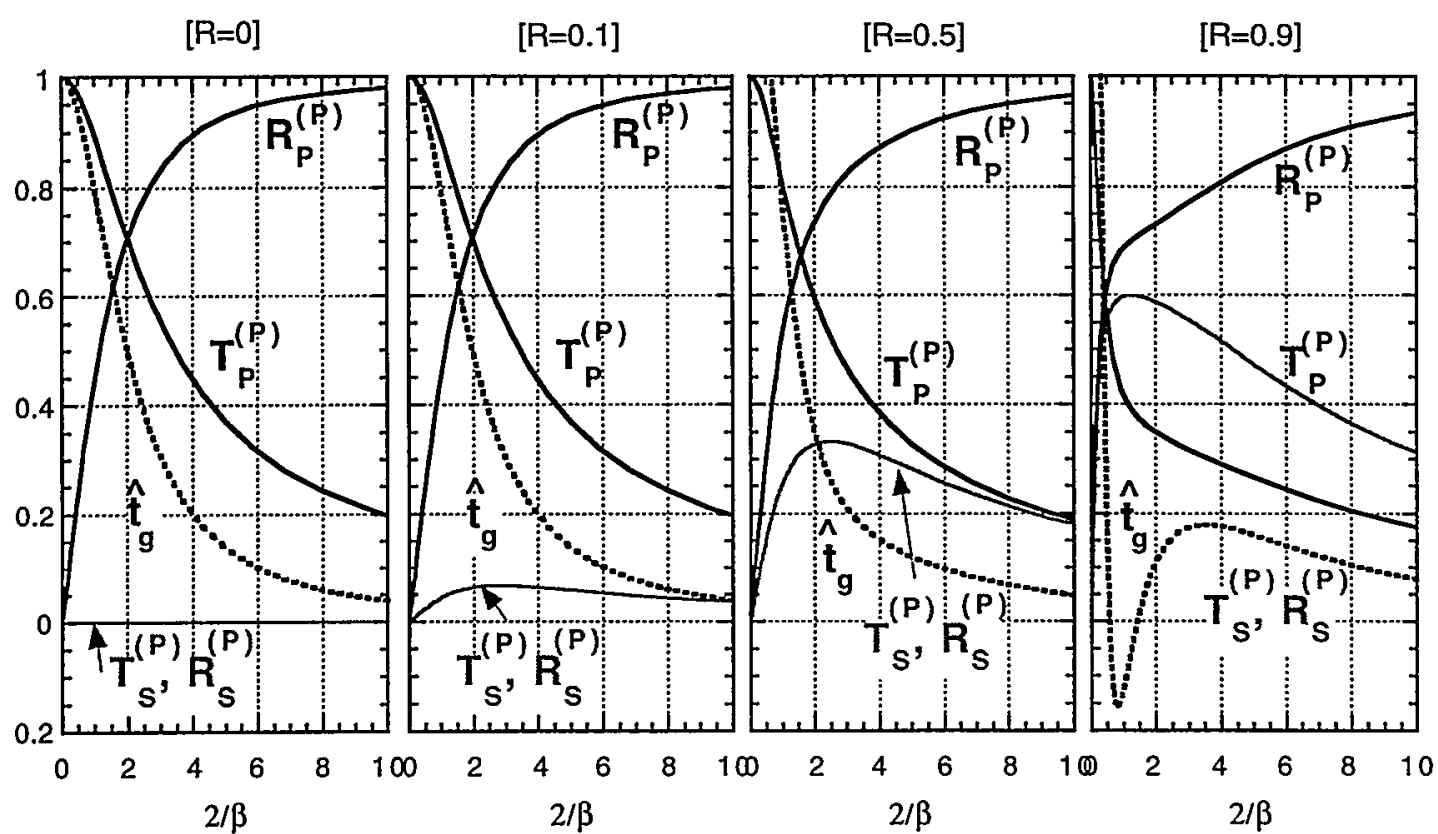

Figure 8.19 Transmission and reflection coefficients for a P-wave normally incident on a fracture. Poisson's ratio of the half-space is 0.2. Normal and tangential fracture stiffnesses are assumed to be identical $(=K)$. The second subscript of each coefficients shows the type of scattered wave. The horizontal axis shows an inverse of impedance ratio defined by $2 / \beta=\omega Z P / K$ where $Z P$ is the $P$-wave impedance of the half-space and $\omega$ is the angular frequency of wave. As the coupling fracture stiffness $R\left(=\kappa_{x z} / \kappa\right)$ increases, transmission and reflection coefficients of the converted waves $\left(T_{p s}\right.$ and $\left.R_{p s}\right)$ increase monotonically. The plots also include normalized group time delay for the directly transmitted P-wave $\left(\hat{i}_{g}=t_{g} \cdot 2 \mathrm{~K} / Z_{P}\right)$. For the case with very large coupling fracture stiffness $(\mathrm{R}=0.9)$, the group time delay becomes negative for a certain range of frequency and fracture stiffness.

$\beta$ between the fracture and its halfspace. The parameter $2 / \beta$ is proportional to the wave frequency and inversely proportional to the normal stiffness of the fracture. It can be seen that the transmission and reflection coefficients for the converted wave become significantly larger with increasing coupling stiffness. On the other hand, the transmission coefficient of the $\mathrm{P}$-wave for a constant $\beta$ decreases slightly. Such behaviors of the waves have been observed during the laboratory wave transmission tests presented earlier in this chapter. Unlike the monotonically decreasing P-to-P transmission coefficient or monotonically increasing P-to-P reflection coefficient, coefficients of converted waves have a peak for $\beta \approx 1$. This indicates that the converted waves are best generated for intermediate fracture stiffnesses and wave frequencies.

In the Figure 8.19 , changes in the group time delay for the transmitting P-wave are also shown. The group time delay increases for low frequency (or high stiffness) and decreases for high frequency (or low stiffness) as the coupling stiffness increases. For a very high 
coupling stiffness $(R=0.9)$, the group time delay becomes negative for a certain range of frequency and fracture stiffness. Such a result appears to be unphysical as it indicates that wave energy propagates across the fracture during negative travel time. The cause of the negative group time delay and its effects on an actually measured wave is left for further investigation.

If coupling stiffness between the $\mathrm{x}$ and $\mathrm{z}$ directions is not present, the $\mathrm{P}$ and $\mathrm{SV}$-waves are also decoupled, yielding

$$
\begin{aligned}
& T_{S V}^{(S V)}=\frac{i \beta_{x x}^{S}}{1+i \beta_{x x}^{S}}, \\
& T_{P}^{(P)}=\frac{i \beta_{z z}^{P}}{1+i \beta_{z z}^{P}}, \\
& T_{S V}^{(P)}=T_{P}^{(S V)}=0 .
\end{aligned}
$$

These results are identical to the transmission coefficients of a fracture without coupling stiffnesses (for example, Pyrak-Nolte et al., 1990a).

\subsubsection{P-SV cross coupling for obliquely incident waves}

When a wave is obliquely incident on a compliant interface (fracture), conversions between $\mathrm{P}$ and S-waves occur even without the cross-coupling fracture stiffnesses. Gu et al. (1996) conducted a detailed analysis of the characteristics of transmitted and reflected waves obliquely incident on a fracture using the displacement-discontinuity model. However, their analysis did not include the effect of cross-coupling fracture stiffnesses. The results for the transmission and reflection coefficients of normal incident waves suggest that the cross-coupling stiffness has a significant effect on the transmission and reflection coefficients of obliquely incident waves as well.

In the following analyses, a virtual shear on the fracture is applied along the $\mathrm{x}$-axis and the incident wave impinges on the fracture in the $\mathrm{x}-\mathrm{z}$ plane. In this case, coupling fracture stiffnesses $\kappa_{z y}, \kappa_{y z}, \kappa_{x y}$, and $\kappa_{y x}$ can be assumed to be zero due to the symmetry of the problem. Because the $\mathrm{SH}$-wave has a particle motion that is parallel to the fracture and is decoupled from the $\mathrm{P}$ and $\mathrm{SV}$-waves, its behavior is not affected by the remaining coupling fracture stiffnesses $\kappa_{x z}$ and $\kappa_{z x}$. Therefore its behavior is not discussed here. Identical material is assumed on both sides of the fracture and Poisson's ratio of the halfspaces is 
assumed to be 0.2. Normal and tangential fracture stiffnesses are also assumed to be equal $\left(\kappa_{x x}=\kappa_{z z} \equiv \kappa\right)$ for the sake of simplicity.

For a range of incidence angles, the matrix equation Eq.(8.17) was solved to determine the resulting transmission and reflection coefficients. Figure 8.20 shows the computed coefficients for varying incidence angles of the $\mathrm{P}$-wave. The vertical axis shows the angle of incidence and the horizontal axis shows the inverse of the fracture impedance ratio with the P-wave acoustic impedance of the halfspace. An increase in the parameter $2 / \beta$ corresponds to an increase in wave frequency or a decrease in normal and tangential fracture stiffnesses. When the relative coupling fracture stiffness $\left(R=\kappa_{x z} / \kappa\right)$ is zero, all the coefficients are symmetric about the normal incident direction $\left(\theta=0^{\circ}\right)$. For obliquely incident waves $(\theta \neq 0)$, transmission and reflection coefficients of the S-wave are not zero due to the conversion of the waves. As the relative coupling fracture stiffness increases, the symmetry of the coefficients about the normal incidence direction is distorted, resulting in finite coefficients in the normal incidence direction. The direction in which large transmission and reflection coefficients are observed changes with increasing relative coupling stiffness and varies from coefficient to coefficient. Unlike the other coefficients, the coefficient for the reflected P-wave maintains its symmetry about the normal incident direction with increasing coupling fracture stiffness.

For an S-incident wave, similar behavior of the scattered wave was observed (Figure 8.21). Since critical angle of incidence was $38^{\circ}$, the S-waves incident beyond $38^{\circ}$ result in invalid data as the scattered waves are inhomogeneous (e.g., Aki and Richards, 1980). As for the P-incident case, an increase in the relative coupling fracture stiffness distorts the symmetry in the transmission and reflection coefficients. However, the coefficient for the reflected S-wave maintains symmetry.

For the results shown above, it is noted that the increasing and decreasing trends in the amplitude of the transmission and reflection coefficients reverse when the sign of the relative coupling fracture stiffness is reversed. This can be easily achieved by reversing the sign of the $\mathrm{x}$-axis for the system. 


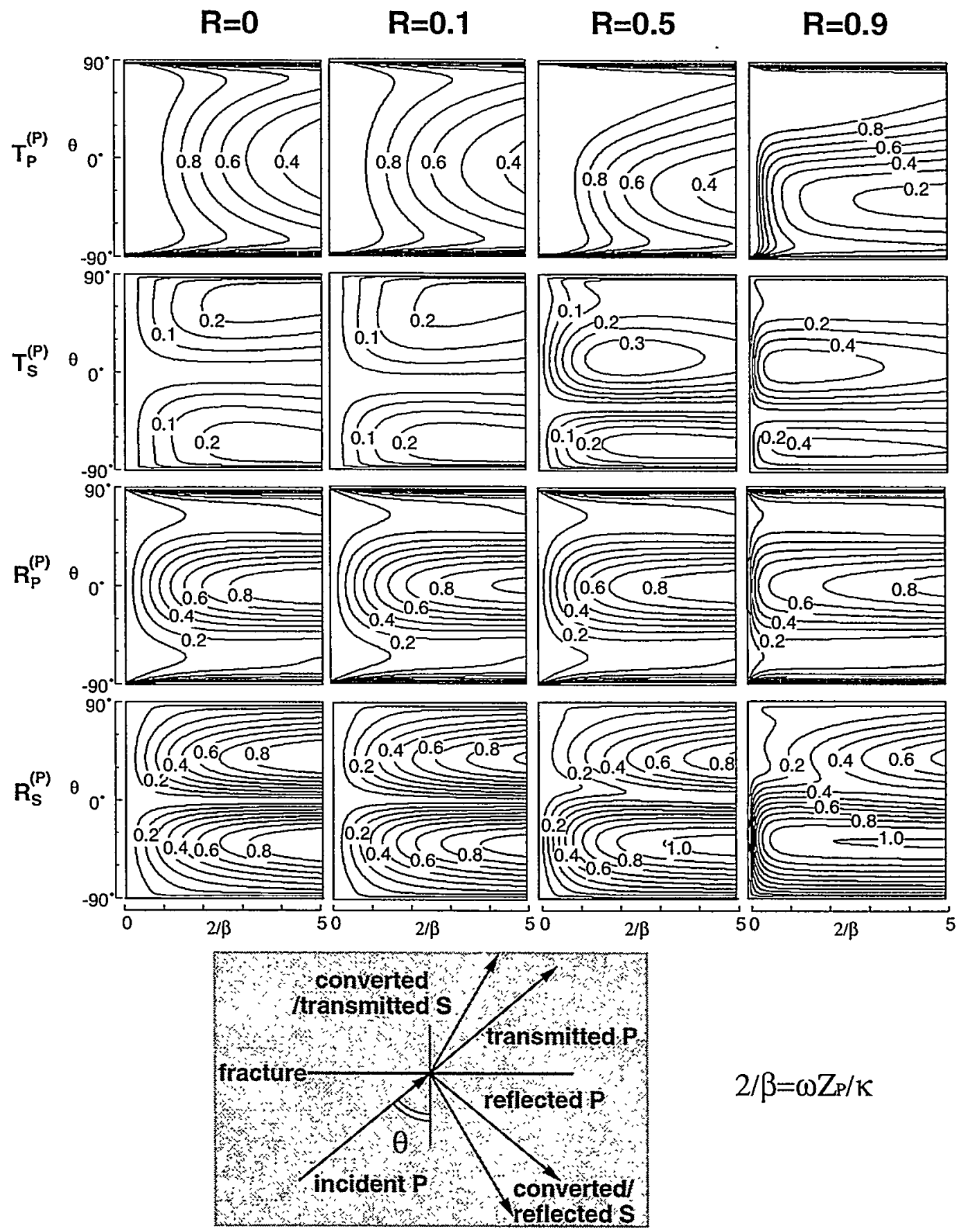

Figure 8.20 Transmission and reflection coefficients for an obliquely incident $\mathrm{P}$-wave. An increase in the coupling stiffness breaks the symmetry in the plots for directly transmitted $\mathrm{P}$ waves $\left(T^{(P)}\right)$, reflected $S$-waves $\left(R^{(P)}\right)$, and transmitted $S$-waves $\left(T^{(P)}\right)$. Reflected $P$-waves ( $\left.R^{(P)}\right)$ keep their symmetry around the normal incidence direction $\left(\theta=0^{\circ}\right)$. 


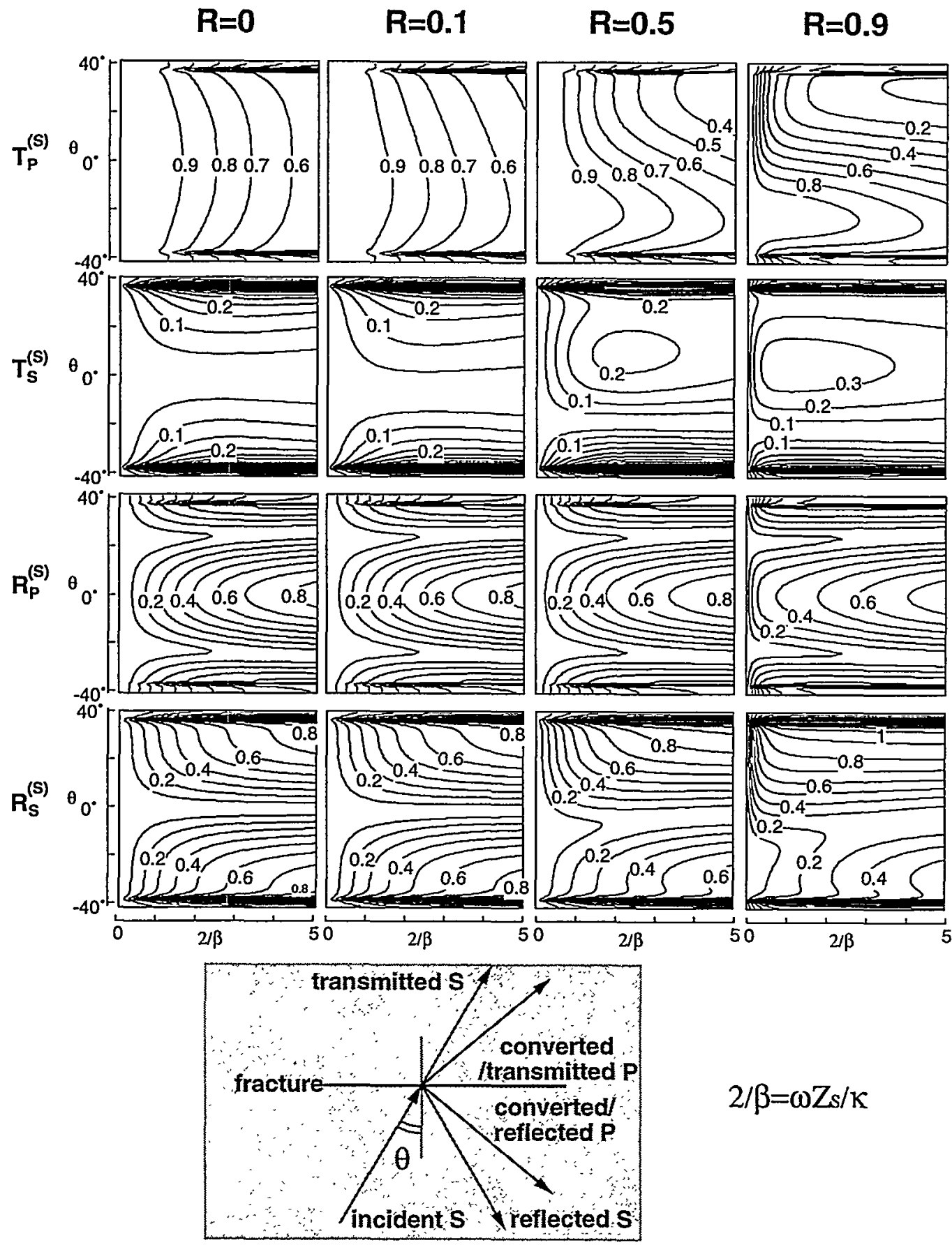

Figure 8.21 Transmission and reflection coefficients for an obliquely incident $S$-wave. The critical angle of incidence is $38^{\circ}$ (Poisson ratio is 0.2 ). An increase in the coupling stiffness breaks the symmetry in the plots for directly transmitted S-waves (TS), reflected P-waves $\left(R^{(S)}\right)$, and transmitted $P$-waves $\left(T^{(S)}\right)$. Reflected S-waves $\left(R S^{(S)}\right)$ keep their symmetry around the normal incidence direction $\left(\theta=0^{\circ}\right)$. 


\subsection{Numerical Simulation}

\subsubsection{Frequency-domain boundary element code (BIEMPS)}

The effect of coupling fracture stiffness on the transmission and reflection of waves incident on a fracture was examined using a frequency-domain plane strain boundary element method. The fracture was modeled by pairs of constant boundary elements (straight elements with a single node at the center) located along a single interface between two domains. Each element pair was mechanically coupled by displacement-discontinuity boundary conditions. The displacement-discontinuity boundary conditions were used for modeling the dynamic interaction between a compliant interface and wave. Such a method was used by Nihei (1992) for simulating acoustic wave propagation through a granular medium (a frequency-domain code), and by $\mathrm{Gu}$ et al. (1994) for simulating a fracture interface wave (a time-domain code). At each frequency $\omega$, displacement at a node was computed by a similar expression as the static expression in Eq.(8.3)

$$
\begin{aligned}
c_{i j} u_{j}(x: \omega)= & \int_{\Gamma} u_{i j}^{*}\left(x, x^{\prime}: \omega\right) p_{j}\left(x^{\prime}: \omega\right) d \Gamma\left(x^{\prime}\right)-\int_{\Gamma} p_{i j}^{*}\left(x, x^{\prime}: \omega\right) u_{j}\left(x^{\prime}: \omega\right) d \Gamma\left(x^{\prime}\right) \\
& +\int_{\Omega} u_{i j}^{*}\left(x, x^{\prime}: \omega\right) f_{j}\left(x^{\prime}: \omega\right) d \Omega\left(x^{\prime}\right)
\end{aligned}
$$

where $u_{i j}^{*}$ and $p_{i j}^{*}$ are dynamic displacement and traction Green's functions for angular frequency $\omega$ (e.g., Brebbia and Dominguez, 1989). The last term in the equation includes a body force $f_{j}$ that represents a point source for generating waves. Integrations were performed only along the boundary of the domain where the displacement is computed. A matrix equation equivalent to Eq.(8.5) was constructed and solved at each frequency for unknown boundary displacement and traction. Once the displacement and traction for the desired range of frequencies were computed, the time-domain response (waveform) was obtained by applying an inverse Fourier transform.

A model used for simulating the waves transmitted and reflected at a compliant interface (fracture) is shown in Figure 8.22. A total of 140 pairs of constant boundary elements (280 nodes) are located along a straight interface between two domains. The total length of the fracture is $12.0 \mathrm{~m}$ and a single point source is located $3.5 \mathrm{~m}$ below the center of the fracture. The source generates a three-lobe Ricker wavelet with central frequency of $2.65 \mathrm{kHz}$. Young's modulus of the half spaces is $50 \mathrm{GPa}$, Poisson's ratio is 0.2 , and the 


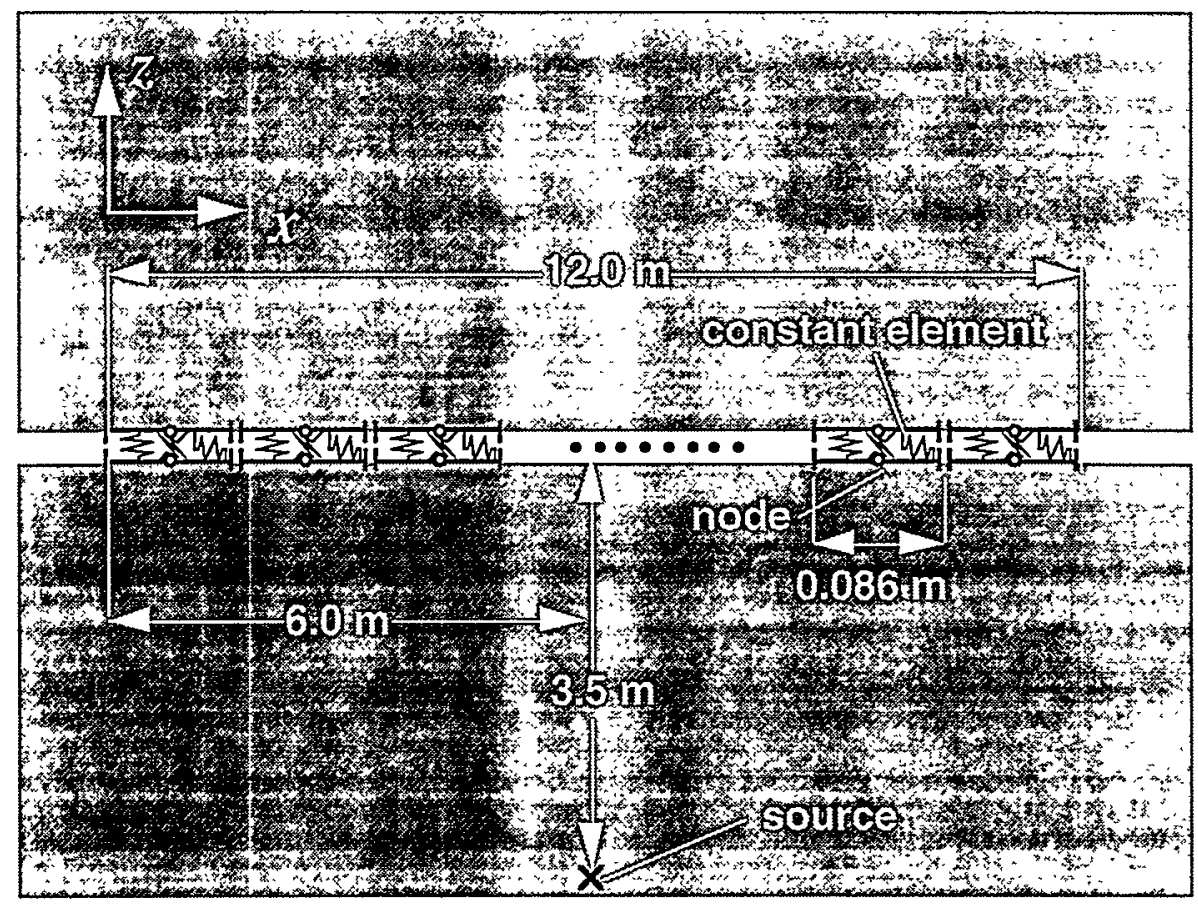

Figure 8.22 A numerical model for simulating scattering of waves incident on a compliant fracture. Constant boundary element pairs are placed along an interface between two half spaces. Each element pair is connected by normal, tangential, and coupling displacement-discontinuity fracture stiffnesses. A directional force is applied from below the center of the fracture.

density is $2600 \mathrm{~kg} / \mathrm{m}^{3}$. For the $S$-wave, the element size to wavelength ratio is 0.08 , yielding sufficient resolution for varying displacement and traction along the fracture. Throughout the following simulations, normal and tangential fracture stiffnesses are assumed to be equal to $\kappa\left(\equiv \kappa_{z z}=\kappa_{x x}\right)=10^{11} \mathrm{~Pa} / \mathrm{m}$. For $\mathrm{P}$ and $\mathrm{S}$-wave velocities of the half-space and the central frequency of the incident wave, the resulting fracture impedance ratios $(\beta=2 \kappa / \omega Z)$ are 1.0 for the $\mathrm{P}$-wave and 1.63 for the $S$-wave. Therefore, from the magnitude of the resulting transmission and reflection coefficients in Figure 8.20 and Figure 8.21 , the waves should have a sufficiently strong interaction with the fracture.

\subsubsection{Simulations}

The first test was conducted using an explosion source. The left column in Figure 8.23 shows snapshots and the particle displacement distribution for the case without coupling fracture stiffness. The right column shows the case for a coupling fracture stiffness of $R=\kappa_{x z} / \kappa=0.5$. Snapshots show the magnitude of the particle displacement given by $u=\sqrt{u_{x}^{2}+u_{z}^{2}}$ where $u_{x}$ and $u_{z}$ are $\mathrm{x}$ and $\mathrm{z}$-direction particle displacements. 


\section{Explosion Source}

\section{without coupling with coupling $(\mathrm{R}=0.5)$}

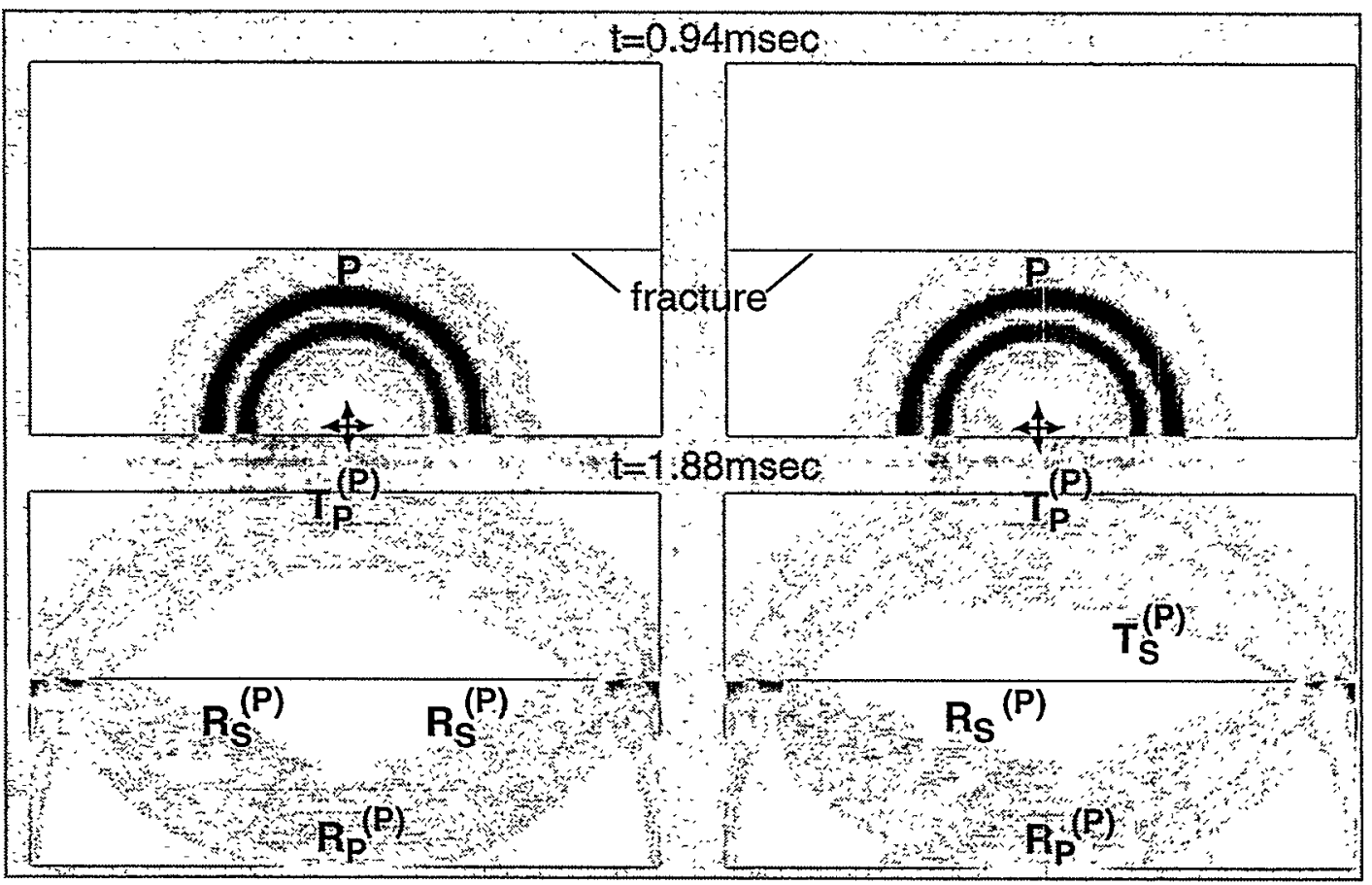

\section{particle displacement $(t=1.88 \mathrm{msec})$}
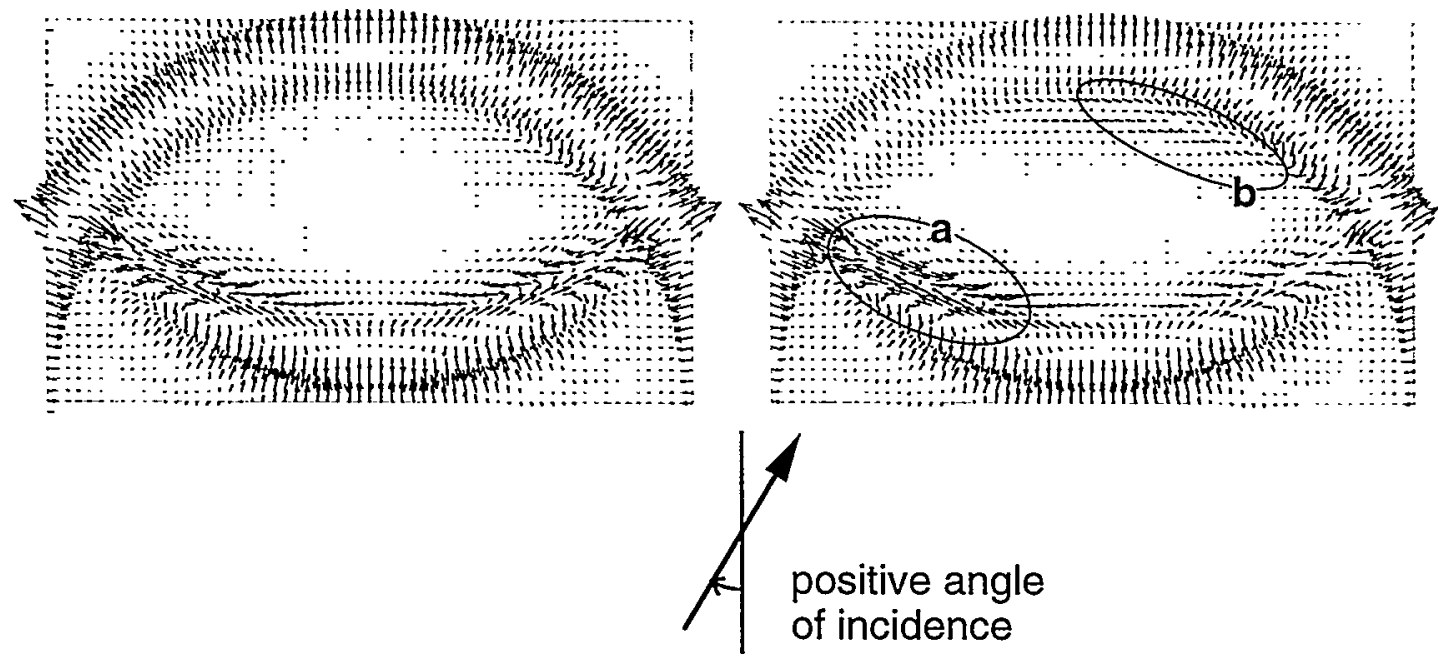

Figure 8.23 Snapshots of the wavefield interacting with a compliant fracture. Waves are generated by an explosive source. Absolute magnitude of particle displacement is shown. Normal and tangential fracture stiffnesses are $1011 \mathrm{~Pa} / \mathrm{m}$. Introduction of coupling fracture stiffness $(R=0.5)$ results in an increase in (a) reflected and (b) transmitted, converted S-waves at oblique directions. 
At $t=0.94 \mathrm{msec}$, there is only a $\mathrm{P}$-wave with a wavefront propagating radially from the source. As the wave propagates, its wavefront curvature decreases and the wave becomes approximately a plane wave. Therefore, the circular wavefront can be seen as a series of plane waves with continuously changing angle of incidence. At $t=1.88 \mathrm{msec}$, the plots show many wavefronts generated as a result of interaction between the P-incident wave and the fracture. For the case without coupling, the amplitudes of the transmitted and reflected waves are distributed symmetrically about the vertical axis. The reflection conversion from $\mathrm{P}$ - wave to S- wave can be seen at oblique angles. When the coupling fracture stiffness is introduced, the symmetry about the vertical axis is destroyed. For the reflected S-wave (converted from $\mathrm{P}$-wave), amplitude of the wave decreases in directions with positive incidence angle (labeled as " $a$ ") and increases for negative incidence angle. The transmitted $\mathrm{S}$-wave (converted from $\mathrm{P}$-wave) also shows increase in amplitude, primarily in the directions with a positive incidence angle (labeled as "b").

The second test was conducted with a vertical point source (Figure 8.24) that generated a strong $\mathrm{P}$-wave in the vertical direction and an S-wave in the horizontal direction. The resulting wavefield generated by the interaction between the P-incident wave and the fracture was similar to the wavefield generated by the explosion source.

In addition, a horizontal source was used to generate strong S-wave motion in the vertical direction (Figure 8.25 , snapshot at $t=1.25 \mathrm{msec}$ ). From the snapshots at $\mathrm{t}=2.19$ msec, a slight increase in the amplitude of reflected P-wave (converted from S-wave) can be seen in directions with a positive incidence angle (labeled as "a"). The transmitted Swave shows a slight increase for negative incidence angles (labeled as "b"). The plot also shows an increased amplitude of the reflected S-wave converted from the incident $\mathrm{P}$-wave (labeled as "c").

In the above results, the observed changes in the amplitude of the scattered wave are consistent with the results of the previous analysis using plane waves. The results show that sheared fractures re-direct wave energy for both converted and non-converted waves. 
Vertical Source

without coupling with coupling $(R=0.5)$

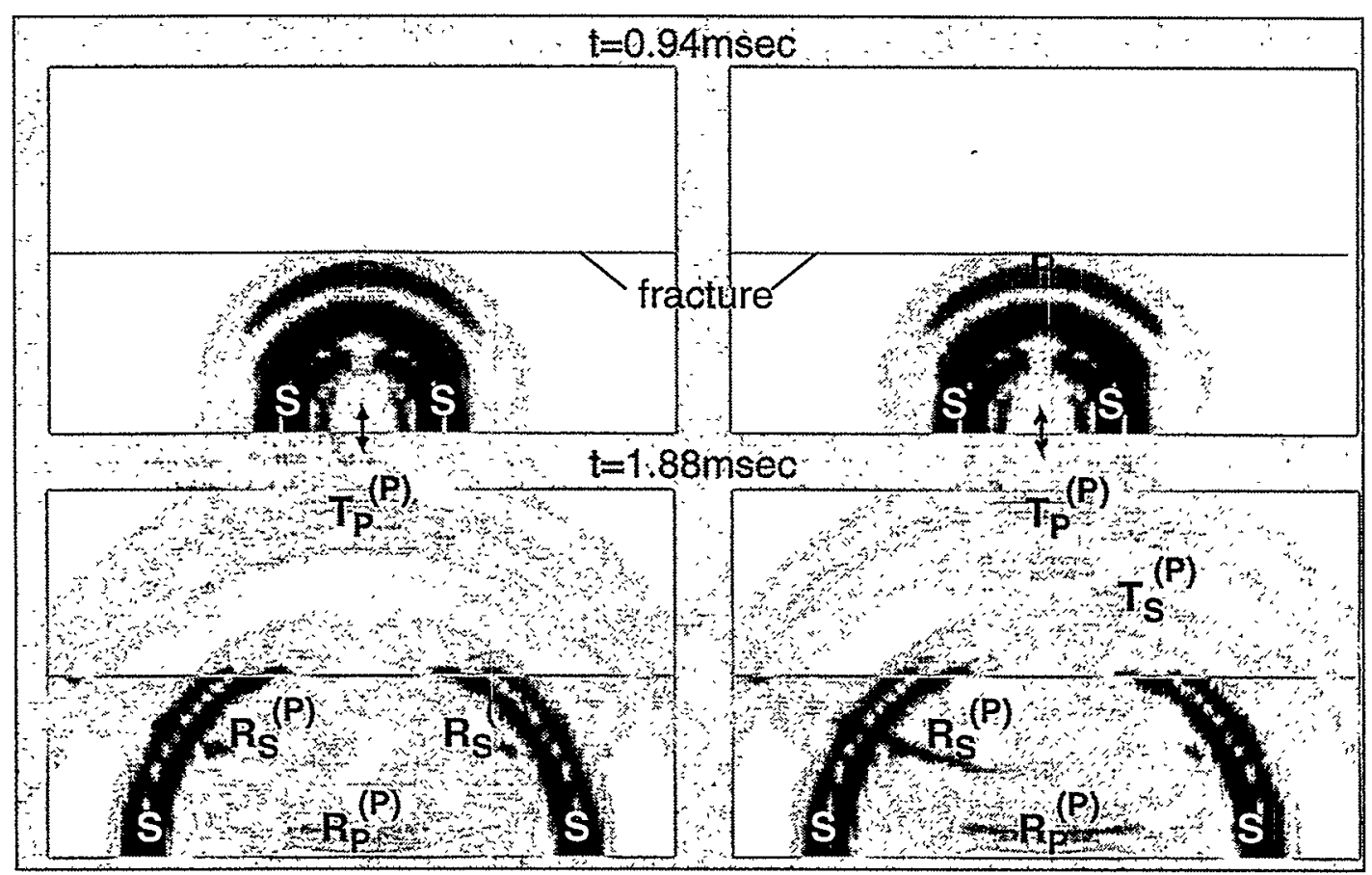

particle displacement $(\mathrm{t}=1.88 \mathrm{msec})$
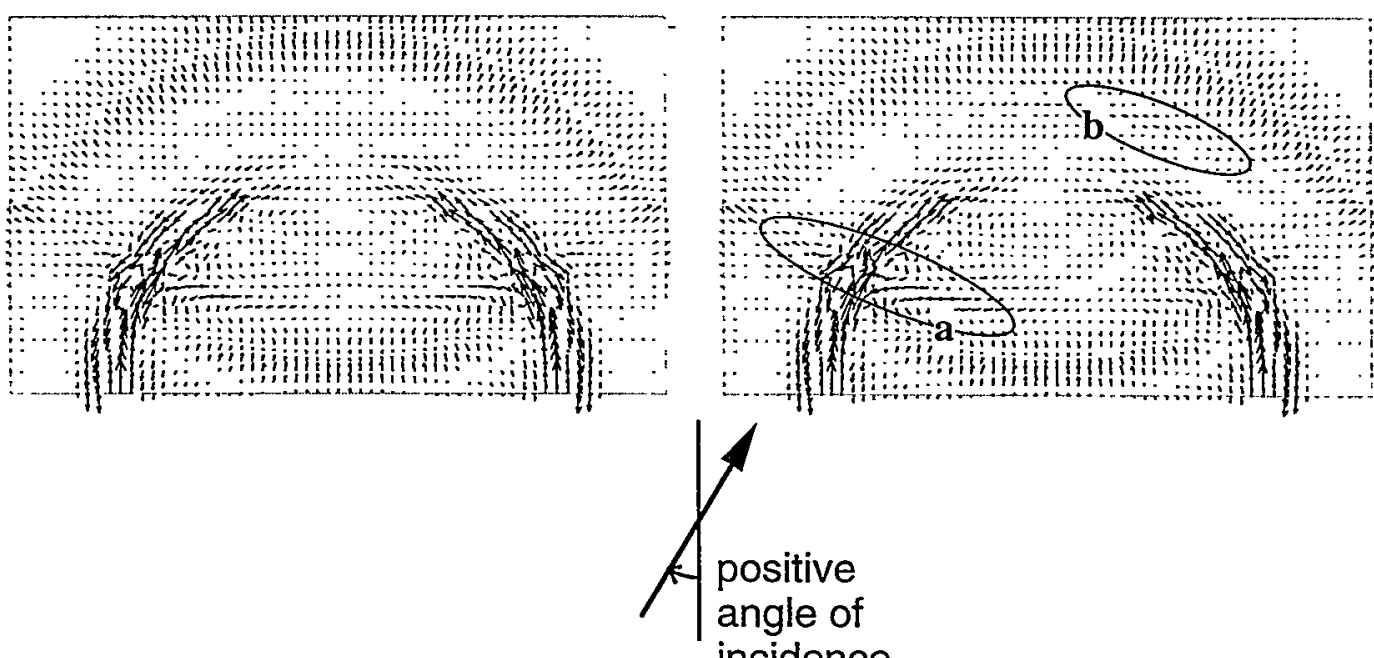

Figure 8.24 Snapshots of the wavefield interacting with a compliant fracture. Waves are generated by a vertical source. Similar to the waves generated by an explosion source, introduction of coupling fracture stiffness $(R=0.5)$ results in an increase in reflected and transmitted, converted S-waves ( $\mathbf{a}$ and $\mathbf{b}$ ) at oblique directions. 


\section{Horizontal Source}

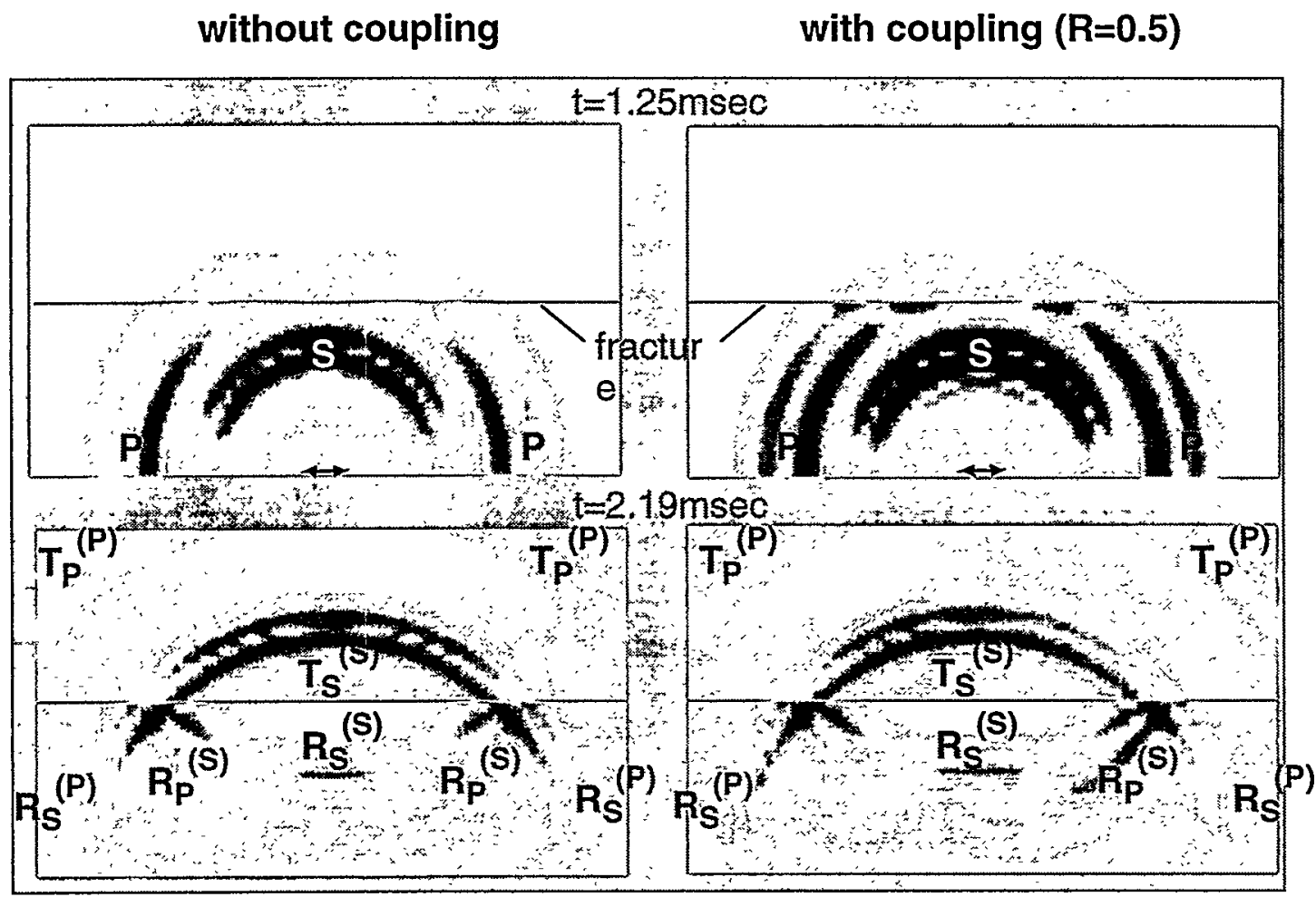

particle displacement $(t=2.19 \mathrm{msec})$
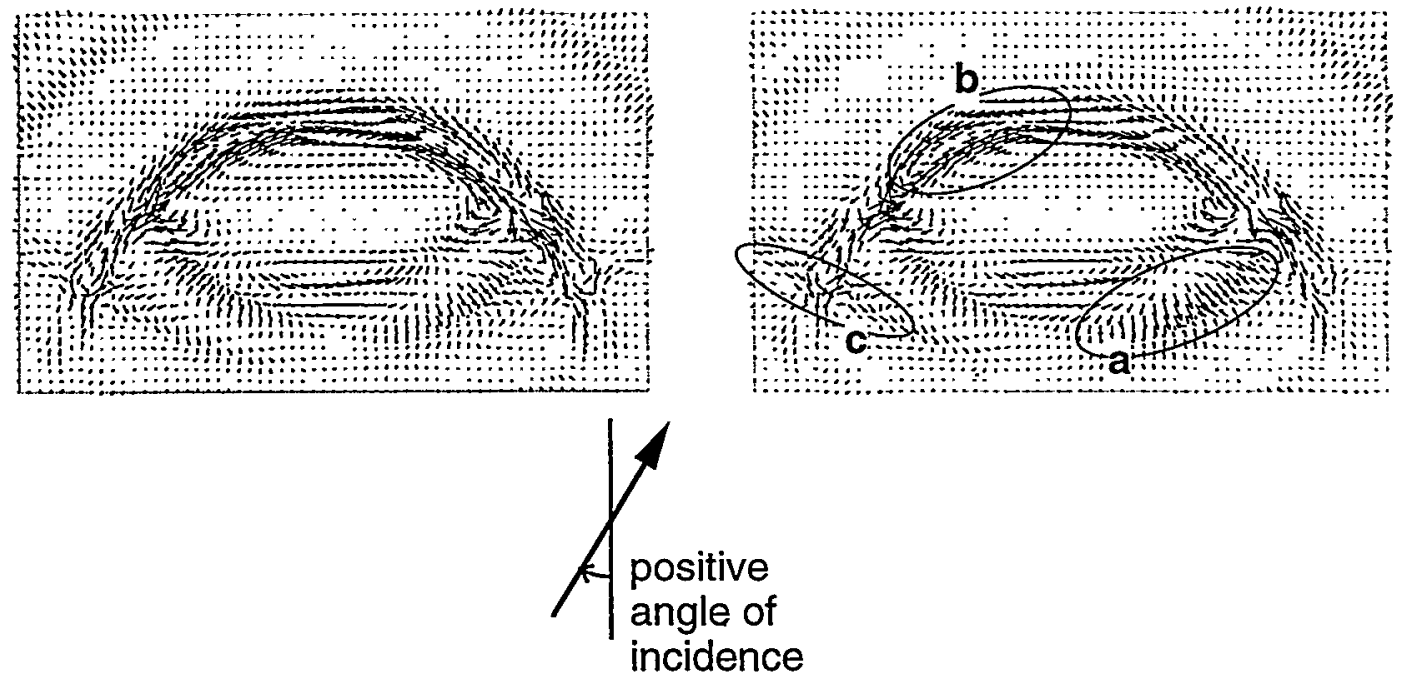

Figure 8.25 Snapshots of the wavefield interacting with a compliant fracture. Waves are generated by a horizontal source. Introduction of coupling fracture stiffness $(R=0.5)$ results in an increase in converted and reflected P-waves (a) and transmitted $S$-waves (b) at oblique directions. In the plot, a converted, reflected $S$-wave generated by an obliquely incident $P$-wave can also be seen (c). 


\subsection{Summary}

In this chapter, the interaction between propagating seismic waves and a sheared fracture is examined experimentally, numerically, and analytically. Because this chapter specifically discussed propagating waves rather than standing waves (resonances), the results provide a fundamental understanding of the dynamic properties of a sheared fracture. The extended form of the displacement-discontinuity boundary conditions can be easily incorporated into the model described in Chapter 4 to simulate the resonance of a structure containing a sheared fracture (e.g., by including the cross-coupling terms in the fracture stiffness matrix in Eq.(4.4)).

Laboratory experiments reveal that a sheared fracture in rock introduces conversions of normally incident seismic waves. The amplitude of the converted waves increases monotonically with an applied shear stress. When the direction of the shear is reversed, the particle motion of the converted wave changes its phase by $180^{\circ}$. The observed behaviors of the wave show that the conversions are not caused by random scattering of waves off the rough surface of a fracture. A mechanism that leads to the conversion of waves is postulated and confirmed by a physical analogue (steel block specimen with regular surface geometry) and numerical simulations. A static numerical simulation shows that changes in the geometry of a sheared fracture leads to dilation behavior of the fracture that manifests itself as conversions of seismic waves normally incident on the fracture. Analytic solutions for transmission and reflection coefficients of scattered waves off a fracture are derived using an extended form of the displacement-discontinuity boundary conditions that include cross-coupling terms in the fracture stiffness matrix.

Although the dilation behavior of a fracture subjected to static loading has been studied by researchers for many years, few have investigated the effects of dilation on wave propagation. The experimental results presented in this chapter are quite novel to the knowledge of the author. The shear-induced conversion of seismic waves may have significant applications as a diagnostic tool for detecting and measuring the shear stress on a rough interface between solids. As the underlying physics is very fundamental, the phenomenon has a broad scope of applications. For example, it may be applied to develop a sensor that measures shear stress in manufactured parts, and structures such as buildings, columns, piers, tunnels, and dams. In the latter area, such a sensor can be used to measure the stress state on a fracture that poses potential slip failure or to monitor the stress state on 
a geological fault that may cause an earthquake by accumulation of shear stress. It is also significant that the shear-induced conversion behavior of the waves can be modeled and examined using a simple extension of an existing model (displacement-discontinuity model). Many interesting new wave phenomena are expected for single and multiple fractures subjected to shear stress. An example is shown in the following chapter for fracture interface waves propagating along a sheared fracture.

For future research, it would be necessary to quantify the magnitude of the conversions that can happen under realistic conditions. For this purpose, the relation between geometry of contacting fracture surfaces and their elastic behavior when subjected to load has to be investigated. Unlike the simple idealized two-dimensional interface (inclined flat microcracks) discussed in this chapter, surfaces of a real fracture tend to be in contact at small patches and islands (Baik and Thompson, 1984). Although the dynamic dilation that leads to conversion of the wave still can be treated using the extended displacementdiscontinuity boundary conditions, the relation between the geometry and the stiffness of the fracture is difficult to determine. To examine the dilation behavior of the fracture, threedimensional analysis as performed by Hopkins et al. (1990), which considers the interaction between individual contacts, may be required. This analysis also leads to the determination of the relation between stress applied to the fracture (both normal and tangential) and its resulting fracture stiffnesses. 
2hapter 9

\section{Wave Propagation along Sheared Fractures}

\subsection{Introduction}

A compliant interface between two solids such as a fracture or a bond can support waves that propagate for a long distance without attenuating. Such waves are called the fracture interface waves and can be shown mathematically to be a generalized form of the classic Rayleigh surface wave. The existence of fracture interface waves was predicted by Murty (1975) for an interface with a finite shear stiffness, and by Pyrak-Nolte and Cook (1987) for a fracture with finite normal and tangential stiffnesses. Pyrak-Nolte et al. (1992) have confirmed the existence of these wave through laboratory measurements of waves propagating along a model fracture in an aluminum specimen. The properties of fracture interface waves have been examined both analytically (Pyrak-Nolte and Cook, 1987) and numerically (Gu et al., 1996) by modeling the behavior of a fracture using the displacement-discontinuity boundary conditions (Schoenberg, 1980; Pyrak-Nolte et al., 1990a). Analysis by Pyrak-Nolte and Cook (1987) predicts two types of fracture interface 
waves, a fast wave with symmetric particle motion across a fracture and a slow wave with anti-symmetric particle motion. The velocities and particle motions of the interface wave depend on both the wave frequency and the fracture stiffness. Gu et al. (1996) show that the behavior of the fast and slow waves are each governed by independent dispersion equations.

In the previous chapter (Chapter 8), a sheared fracture was shown to generate converted $\mathrm{P}$ and S- waves for normally incident $\mathrm{S}$ and $\mathrm{P}$-waves, respectively. This is attributed to the elastic dilation of a fracture that involves mechanical cross-coupling between normal stress and tangential displacement, and shear stress and normal displacement across the fracture (Nakagawa et al., 1997). The elastic dilation occurs as the shear stress applied to the surface of a fracture systematically changes the distribution of local contact stiffness. Relations between stress and relative displacement across a fracture can be described using an extended form of the displacement-discontinuity boundary conditions with coupling fracture stiffnesses.

The purpose of this chapter is to examine the effects of the shear-induced crosscoupling fracture stiffness on the properties of fracture interface waves. A dispersion equation for the interface wave is derived by applying the displacement-discontinuity boundary conditions to a plane wave solution. It is shown that the generalized fracture interface waves for a sheared fracture no longer have pure symmetric or anti-symmetric particle motion. Furthermore, introduction of coupling stiffnesses change the velocities of the interface wave, which may be misinterpreted as changes in normal and tangential fracture stiffnesses if the effect of the shear is not taken into account. Changes in wave velocity and accompanying particle motion are examined for a range of fracture stiffnesses and coupling fracture stiffnesses. Numerical simulations are performed using a dynamic boundary element method to demonstrate the predicted behaviors of fracture interface waves for a sheared fracture. Observed changes in the velocity and particle motion can potentially be used for characterizing the stress state on a fracture.

\subsection{Theory}

\subsubsection{Generalized interface wave}

A dispersion equation for the fracture interface wave propagating along a sheared fracture is derived following Gu et al.(1996)'s work for a fracture without shear. For a 


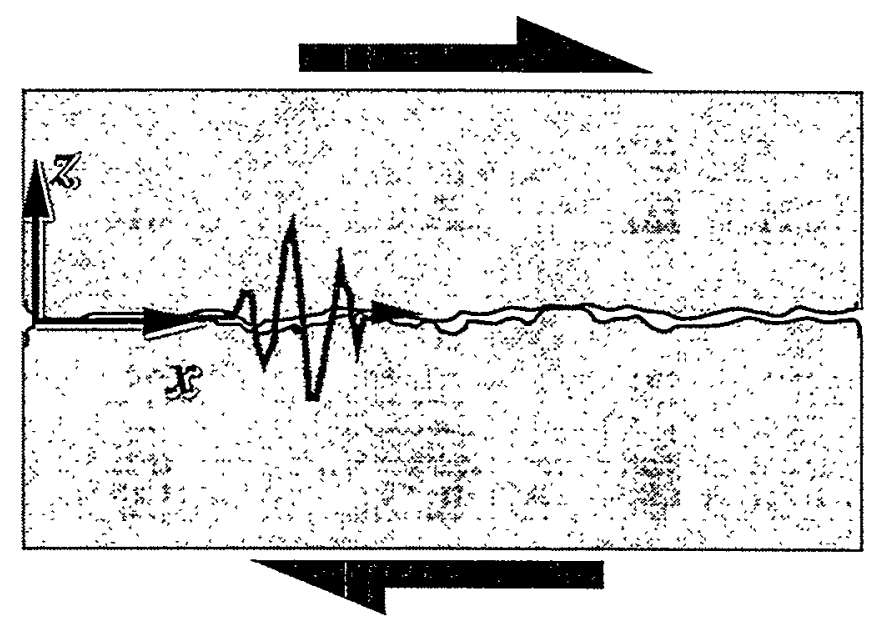

(a) Sheared compliant interface (fracture) with partial contacts and bonds
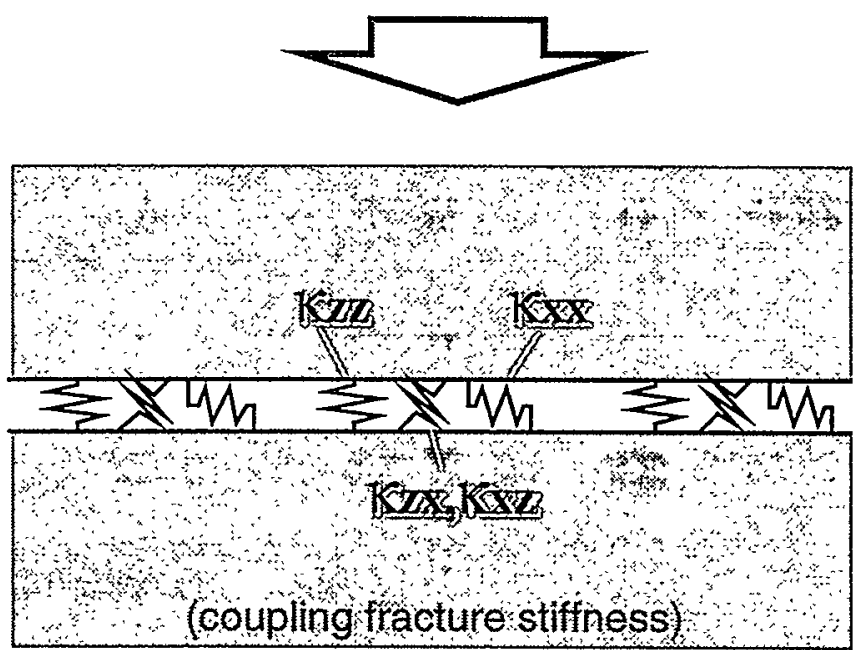

(b) Displacement-discontinuity model including coupling fracture stiffness

Figure 9.1 Fracture interface wave propagating along a sheared fracture. (a) The fracture is assumed to be on an $x-y$ plane at $z=0$ and the shear force is applied along the $x$-axis. (b) The quasi-static behavior of the fracture is described using the displacement-discontinuity boundary conditions with coupling (dilation) fracture stiffness. 
fracture located on an $x-y$ plane at $z=0$ (Figure 9.1), scalar Helmholtz potentials for a interface wave propagating along the $\mathrm{x}$-axis are given by

$$
\begin{array}{ll}
\phi_{2}=A_{2} e^{-p \omega z} e^{i \omega(x / c-t)} & , z \geq 0, \\
\phi_{1}=A_{1} e^{+p \omega z} e^{i \omega(x / c-t)} & , z \leq 0,
\end{array}
$$

for P-wave motion. The vector Helmholz potentials for the in-plane S-wave motion are given by

$$
\begin{array}{ll}
\psi_{2}=B_{2} e^{-q \omega z} e^{i \omega(x / c t)} & , z \geq 0, \\
\psi_{1}=B_{1} e^{+q \omega z} e^{i \omega(x / c t)} & , z \leq 0 .
\end{array}
$$

Subscripts " 1 " and "2" designate the half-space $z \leq 0$ and $z \geq 0$, respectively. In the above expressions, $p$ and $q$ are given by

$$
p=\sqrt{\frac{1}{c^{2}}-\frac{1}{c_{P}^{2}}}, q=\sqrt{\frac{1}{c^{2}}-\frac{1}{c_{S}^{2}}},
$$

where $c_{P}$ and $c_{S}$ are $\mathrm{P}$ and $\mathrm{S}$-wave velocities in the isotropic homogeneous halfspaces.

Using the potentials defined above, particle displacements in the $\mathrm{x}$ and $\mathrm{z}$ - directions are derived from the following relations

$$
u_{x}=\frac{\partial \phi}{\partial x}-\frac{\partial \psi}{\partial z}, u_{z}=\frac{\partial \phi}{\partial z}+\frac{\partial \psi}{\partial x} .
$$

The tractions on the surface of the isotropic homogeneous halfspace are obtained from Hooke's Law

$$
\begin{aligned}
& t_{1 z}=2 \mu \frac{\partial u_{1 z}}{\partial z}+\lambda\left(\frac{\partial u_{1 x}}{\partial x}+\frac{\partial u_{1 z}}{\partial z}\right) \\
& -t_{2 z}=2 \mu \frac{\partial u_{2 z}}{\partial z}+\lambda\left(\frac{\partial u_{2 x}}{\partial x}+\frac{\partial u_{2 z}}{\partial z}\right) \\
& t_{1 x}=\mu\left(\frac{\partial u_{1 z}}{\partial x}+\frac{\partial u_{1 x}}{\partial z}\right) \\
& -t_{2 x}=\mu\left(\frac{\partial u_{2 z}}{\partial x}+\frac{\partial u_{2 x}}{\partial z}\right)
\end{aligned}
$$

for each halfspace. The tractions and displacements of the surfaces are related by the displacement-discontinuity boundary conditions (Schoenberg, 1980; Pyrak-Nolte et al., 
1990). In the previous chapter (Chapter 8), it was shown that the coupling fracture stiffnesses that describe the elastic dilation behavior of the fracture can have a significant effect on $\mathrm{P}$ and $\mathrm{S}$-waves propagating across the fracture. A generalized form of the displacement-discontinuity boundary conditions for a fracture sheared along the $\mathrm{x}$-axis was

$$
\begin{aligned}
& \sigma_{z x}=\kappa_{x x}\left[u_{x}\right]+\kappa_{x z}\left[u_{z}\right] \\
& \sigma_{z z}=\kappa_{z x}\left[u_{x}\right]+\kappa_{z z}\left[u_{z}\right] \\
& \sigma_{z x} \equiv t_{1 x}=-t_{2 x} \\
& \sigma_{z z} \equiv t_{1 z}=-t_{2 z} .
\end{aligned}
$$

By introducing Eq.(9.1) through (9.5) into the above expressions, the following homogeneous matrix equation is obtained

$$
\begin{aligned}
& {\left[\begin{array}{cccc}
-\frac{2 i \omega p \mu}{c}-\frac{i \kappa_{x x}}{c}+p \kappa_{x z} & \frac{i \kappa_{x x}}{c}+p \kappa_{x z} & -\mu \omega\left(\frac{2}{c^{2}}-\frac{1}{c_{s}^{2}}\right)-q \kappa_{x x}-\frac{i \kappa_{x z}}{c} & -q \kappa_{x x}+\frac{i \kappa_{x z}}{c} \\
\mu \omega\left(\frac{2}{c^{2}}-\frac{1}{c_{s}^{2}}\right)-\frac{i \kappa_{z x}}{c}+p \kappa_{z z} & \frac{i \kappa_{z x}}{c}+p \kappa_{z z} & -\mu \omega \frac{2 i q}{c}-q \kappa_{z x}-\frac{i \kappa_{z z}}{c} & -q \kappa_{z x}+\frac{i \kappa_{z z}}{c} \\
\frac{2 i p}{c} & \frac{2 i p}{c} & \frac{2}{c^{2}}-\frac{1}{c_{s}^{2}} & -\left(\frac{2}{c^{2}}-\frac{1}{c_{s}^{2}}\right) \\
\frac{2}{c^{2}}-\frac{1}{c_{s}^{2}} & -\left(\frac{2}{c^{2}}-\frac{1}{c_{s}^{2}}\right) & -\frac{2 i q}{c} & -\frac{2 i q}{c}
\end{array}\right]} \\
& \times\left\{\begin{array}{l}
A_{2} \\
A_{1} \\
B_{2} \\
B_{1}
\end{array}\right\}=0,
\end{aligned}
$$

where that $\rho c_{P}^{2}=\lambda+2 \mu$ and $\rho c_{S}^{2}=\mu$.

To simplify the above expression, coefficients of the potentials are decomposed into their symmetric and antisymmetric components as follows

$$
A^{\text {sym }}=\frac{A_{2}+A_{1}}{2}, \quad A^{\text {asym }}=\frac{A_{2}-A_{1}}{2}, \quad B^{\text {sym }}=\frac{B_{2}-B_{1}}{2}, \quad B^{a s y m}=\frac{B_{2}+B_{1}}{2} .
$$

It is noted that the symmetry is defined with respect to the interface located at $z=0$. By substituting the variables, the matrix equation becomes 


$$
\begin{aligned}
& {\left[\begin{array}{cccc}
-\mu \omega \frac{2 i p}{c}+2 p \kappa_{x z} & -\mu \omega\left(\frac{2}{c^{2}}-\frac{1}{c_{s}^{2}}\right)-\frac{2 i \kappa_{x z}}{c} & -\mu \omega \frac{2 i p}{c}-\frac{2 i \kappa_{x x}}{c} & -\mu \omega\left(\frac{2}{c^{2}}-\frac{1}{c_{s}^{2}}\right) \\
\mu \omega\left(\frac{2}{c^{2}}-\frac{1}{c_{s}^{2}}\right) & -\mu \omega \frac{2 i q}{c}-\frac{2 i \kappa_{z z}}{c} & \mu \omega\left(\frac{2}{c^{2}}-\frac{1}{c_{s}^{2}}\right)-\frac{2 i \kappa_{z x}}{c} & -\mu \omega \frac{2 i q}{c}-2 q \kappa_{z x} \\
+2 p \kappa_{z z} & \frac{2}{c^{2}}-\frac{1}{c_{s}^{2}} & 0 & 0 \\
\frac{2 i p}{c} & 0 & \frac{2}{c^{2}}-\frac{1}{c_{s}^{2}} & -\frac{2 i q}{c} \\
0 & &
\end{array}\right]} \\
& \times\left\{\begin{array}{c}
A^{\text {sym }} \\
B^{\text {sym }} \\
A^{a s y m} \\
B^{\text {asym }}
\end{array}\right\}=0 .
\end{aligned}
$$

The equations in the third and fourth rows independently determine the relative magnitudes of the $\mathrm{P}$ and S- type motions for the symmetric and anti-symmetric particle motions independently. Using these relations, the coefficients are given by

$$
\left\{\begin{array}{l}
A^{s y m} \\
B^{\text {sym }}
\end{array}\right\}=\text { const. } \times\left\{\begin{array}{c}
\frac{2}{c^{2}}-\frac{1}{c_{s}^{2}} \\
-\frac{2 i p}{c}
\end{array}\right\},\left\{\begin{array}{l}
A^{u s y m} \\
B^{\text {assm }}
\end{array}\right\}=\text { const. } \times\left\{\begin{array}{c}
\frac{2 i q}{c} \\
\frac{2}{c^{2}}-\frac{1}{c_{S}^{2}}
\end{array}\right\} .
$$

By introducing non-dimensional slowness parameters $\xi=c_{S} / c, \zeta=c_{S} / c_{P}$, these relations become

$$
\begin{gathered}
\left\{\begin{array}{l}
A^{s y m} \\
B^{s y m}
\end{array}\right\}=U^{s y m} \cdot c_{s}^{2}\left\{\begin{array}{c}
\frac{2}{c^{2}}-\frac{1}{c_{s}^{2}} \\
-\frac{2 i p}{c}
\end{array}\right\}=U^{s y m}\left\{\begin{array}{c}
2 \xi^{2}-1 \\
-2 i \xi \sqrt{\xi^{2}-\zeta^{2}}
\end{array}\right\} \\
\left\{\begin{array}{l}
A^{a s y m} \\
B^{a s s m}
\end{array}\right\}=U^{a . s y m} \cdot c_{s}^{2}\left\{\begin{array}{c}
\frac{2 i q}{c} \\
\frac{2}{c^{2}}-\frac{1}{c_{S}^{2}}
\end{array}\right\}=U^{a . s m m}\left\{\begin{array}{c}
2 i \xi \sqrt{\xi^{2}-1} \\
2 \xi^{2}-1
\end{array}\right\} .
\end{gathered}
$$

Substitution of variables into the matrix equation reduces the size of the matrix from a $4 \times 4$ to a $2 \times 2$ as follows 


$$
\left[\begin{array}{cc}
f(\xi)-\beta_{z z}^{s} \sqrt{\xi^{2}-\zeta^{2}} & \beta_{z x}^{s} \sqrt{\xi^{2}-1} \\
\beta_{x z}^{s} \sqrt{\xi^{2}-\zeta^{2}} & f(\xi)-\beta_{x x}^{s} \sqrt{\xi^{2}-1}
\end{array}\right]\left\{\begin{array}{c}
U^{s y m} \\
U^{a s y m}
\end{array}\right\}=\mathbf{0},
$$

where the stiffness of the interface is expressed by non-dimensional impedance parameters

$$
\begin{aligned}
& \beta_{x x}^{s}=\frac{2 \kappa_{x x} / \omega}{\rho c_{s}}, \quad \beta_{z z}^{s}=\frac{2 \kappa_{z z} / \omega}{\rho c_{s}}, \\
& \beta_{x z}^{s}=\beta_{z x}^{s}=\frac{2 \kappa_{x z} / \omega}{\rho c_{s}}=\frac{2 \kappa_{z x} / \omega}{\rho c_{s}},
\end{aligned}
$$

where $\rho$ is the material density of the half-spaces and

$$
f\left(\xi^{2}\right)=\left(1-2 \xi^{2}\right)^{2}-4 \xi^{2} \sqrt{\xi^{2}-1} \sqrt{\xi^{2}-\zeta^{2}}
$$

Note that $f\left(\xi^{2}\right)=0$ is a Rayleigh-wave equation (for example, Aki and Richards, 1980). Non-trivial solutions of the matrix equation are obtained by equating the determinant of the matrix to zero

$$
\begin{aligned}
P\left(\xi^{2}\right) & \equiv \operatorname{det}|M| \\
& =\left\{f\left(\xi^{2}\right)-\beta_{z z}^{s} \sqrt{\xi^{2}-\zeta^{2}}\right\}\left\{f\left(\xi^{2}\right)-\beta_{x x}^{s} \sqrt{\xi^{2}-1}\right\}-\beta_{x z}^{s} \beta_{z x}^{s} \sqrt{\xi^{2}-\zeta^{2}} \sqrt{\xi^{2}-1}=0
\end{aligned} .
$$

Solutions of Eq.(9.15) provide the velocity of the interface wave for given frequencies. For a solution of the equation, the corresponding mode vector is derived as

$$
\left\{\begin{array}{c}
U^{s y m} \\
U^{a s y m}
\end{array}\right\}=\left\{\begin{array}{c}
\beta_{z x}^{s} \sqrt{\xi^{2}-1} \\
\beta_{z z}^{s} \sqrt{\xi^{2}-\zeta^{2}}-f\left(\xi^{2}\right)
\end{array}\right\} \text { or }\left\{\begin{array}{c}
\beta_{x x}^{s} \sqrt{\xi^{2}-1}-f\left(\xi^{2}\right) \\
\beta_{x z}^{s} \sqrt{\xi^{2}-\zeta^{2}}
\end{array}\right\} \text {. }
$$

For the special case when there is no coupling fracture stiffness, two independent equations arise from Eq.(9.15)

(a) $f(\xi)=\beta_{z z}^{S} \sqrt{\xi^{2}-\zeta^{2}}$,

(b) $f(\xi)=\beta_{x x}^{S} \sqrt{\xi^{2}-1}$.

These equations are identical to those derived by $\mathrm{Gu}$ et al.(1996). The accompanying mode vectors of the equations are

(a) $\left\{\begin{array}{c}U^{s y m} \\ U^{\text {asym }}\end{array}\right\}=\left\{\begin{array}{l}1 \\ 0\end{array}\right\}$, 
(b) $\left\{\begin{array}{c}U^{\text {sym }} \\ U^{\text {asym }}\end{array}\right\}=\left\{\begin{array}{l}0 \\ 1\end{array}\right\}$.

The horizontal particle motions of the fracture interface wave described by the mode vectors given by Eq.(9.18a) and Eq.(9.18b) are symmetric and anti-symmetric across the interface, respectively. The velocity of the symmetric interface wave is faster than the anti-symmetric wave for identical normal and tangential fracture stiffnesses (Gu et al., 1996). On the other hand, as is shown by the analyses performed later in this chapter, particle motions of the interface wave derived from Eq.(9.16) for a fracture with coupling fracture stiffness are no longer symmetric or anti-symmetric. As these particle motions approach symmetric and anti-symmetric motions for small coupling fracture stiffnesses, the fast wave is called the "quasi-symmetric Rayleigh interface wave (q-symmetric RIW)" and the slow wave is called the "quasi-antisymmetric Rayleigh interface wave (q-antisymmetric RIW)".

\subsubsection{Dispersion relations}

For a given wave frequency and fracture stiffness, the dispersion equation Eq.(9.15) can be solved to determine the velocity of the interface wave. The velocity can be either a real or a complex number. Because an interface wave with a complex velocity decays exponentially as it propagates along a fracture (leaky wave), the fracture can support only waves with real velocity. The real solution (velocity) of the dispersion equation is found only for velocities smaller than the S-wave velocity $(\xi \geq 1)$ or equal to the P-wave velocity $(\xi=\zeta)$. This can be proven as the $\sqrt{\xi^{2}-1}$ term in the equation can be explicitly expressed by all the other real-valued terms. For this reason, the q-symmetric RIW becomes attenuative (leaky) when its velocity increases beyond the S-wave velocity.

The behavior of the interface wave velocity can be predicted by examining the structure of the dispersion equation. For the sake of simplicity, the following discussion assumes identical normal and tangential fracture stiffnesses $\left(\kappa_{x x}=\kappa_{z z}=\kappa\right.$, and therefore $\beta_{z z}^{s}=\beta_{x x}^{s}=\beta$ ). The relative magnitude of the coupling fracture stiffness is expressed by a fracture stiffness ratio $R$ defined by

$$
R \equiv \frac{\kappa_{z x}}{\sqrt{\kappa_{x x} \kappa_{z z}}}=\frac{\kappa_{z x}}{\kappa}
$$

The dispersion equation $P\left(\xi^{2}\right)$ (Eq.(9.15)) is expressed by the following components 


$$
\begin{aligned}
& P\left(\xi^{2}\right)=F-G, \\
& F=(f-\beta \cdot \Theta)(f-\beta \cdot \Phi), \\
& G=(R \beta)^{2} \Theta \Phi \\
& \Theta=\sqrt{\xi^{2}-1} \\
& \Phi=\sqrt{\xi^{2}-\zeta^{2}}
\end{aligned}
$$

The behaviors of functions $f, \beta \cdot \Theta$, and $\beta \cdot \Phi$ for a range of interface wave velocities are shown in Figure 9.2(a). The Poisson's ratio of the halfspaces is assumed to be 0.2. A zero of the function $f$ provides the Rayleigh wave velocity $\left(c_{R}\right)$. As the stiffness of the fracture increases, $\beta \cdot \Theta$ and $\beta \cdot \Phi$ increase monotonically. For a fracture without coupling stiffness, intercepts between these functions and $f$, or zeros of the function $F$ (Figure 9.2(b)) provide velocities of the symmetric $\left(c_{R s y m}\right)$ and anti-symmetric $\left(c_{\text {Rasym }}\right)$ waves. When the coupling fracture stiffness is introduced $(R \neq 0)$, the function $G$ increases monotonically (Figure 9.2(b)). As can be seen from the plot, an increase in the coupling fracture stiffness results in a slight increase in the velocity of the (quasi-)symmetric wave and a relatively large decrease in the velocity of the (quasi-)anti-symmetric wave.

The computed velocity dispersion of the interface waves for a range of fracture stiffnesses (represented by the dimensionless impedance parameter $\beta$ ) is shown in Figure 9.3. The vertical axis is the velocity of the interface wave normalized by the $S$-wave velocity. Curves are computed for a range of coupling fracture stiffnesses specified by $R$. When the stiffness of the fracture is very low or the frequency of the wave is high ( $\beta \rightarrow 0$ ), the two solutions of the dispersion equation degenerate to the Rayleigh wave velocity of the halfspace. On the other hand, when the stiffness of the fracture is very high (fracture is welded) or the frequency of the wave is low $(\beta \rightarrow \infty)$, the two solutions become $\mathrm{P}$-wave and $\mathrm{S}$-wave velocities of the halfspaces ( $\mathrm{P}$-wave velocity is not shown in the plot). For an intermediate stiffness and wave frequencies, two kinds of waves with velocity between the Rayleigh wave velocity and S-wave velocity exist. For a fracture without the coupling fracture stiffness, these are the fast wave with symmetric particle motion and the slow wave with anti-symmetric particle motion (Pyrak-Nolte and Cook, 1987). For a sheared fracture with a finite coupling fracture stiffness $(R \neq 0)$, these waves are no longer purely symmetric or antisymmetric. 


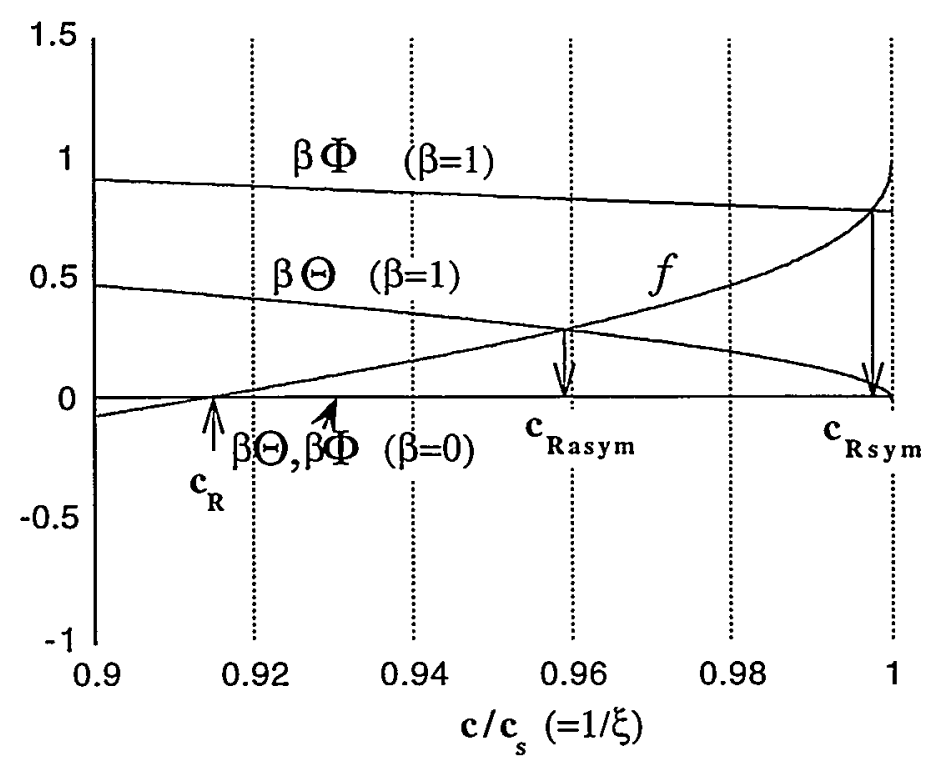

(a) Solutions of non-coupled dispersion equation

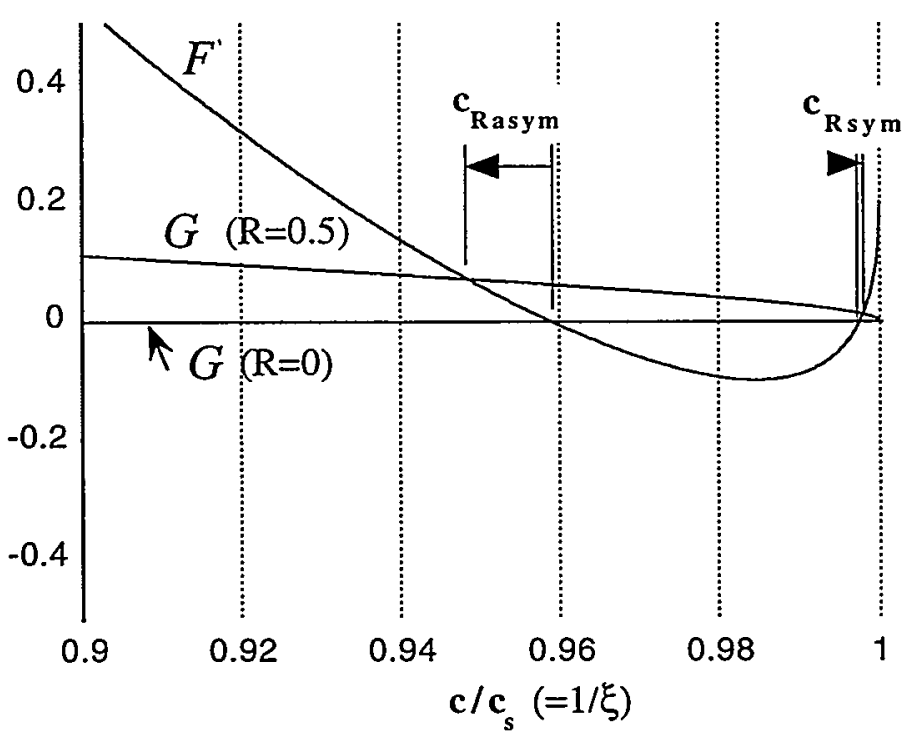

(b) Solutions of coupled dispersion equation

Figure 9.2 Graphic representation of individual terms in the dispersion equation for the fracture interface wave. The above plots are for a special case of $b=1$ and $R=0.5$. (a) A zero of the function $f$ yields the Rayleigh wave velocity. As the stiffness of the fracture increases, functions $\beta_{\Phi}$ and $\beta_{\Theta}$ increase monotonically, their intersections with $f$ yielding the velocities of the symmetric and antisymetric interface waves, respectively. (b) When the coupling fracture stiffness is introduced, function $G$ increases monononically, decreasing the velocity of the (quasi-) anti-symmetric wave and increasing the velocity of the (quasi-) symmetric wave.

Note: Dispersion equation $P=(f-\beta \cdot \Theta)(f-\beta \cdot \Phi)-G=F-G$ where, $\Theta=\sqrt{\xi^{2}-1}$, $\Phi=\sqrt{\xi^{2}-\zeta^{2}}, f=\left(2 \xi^{2}-1\right)^{2}-4 \xi^{2} \sqrt{\xi^{2}-1} \sqrt{\xi^{2}-\zeta^{2}}$ and $G=(R \beta)^{2} \Theta \Phi$ 


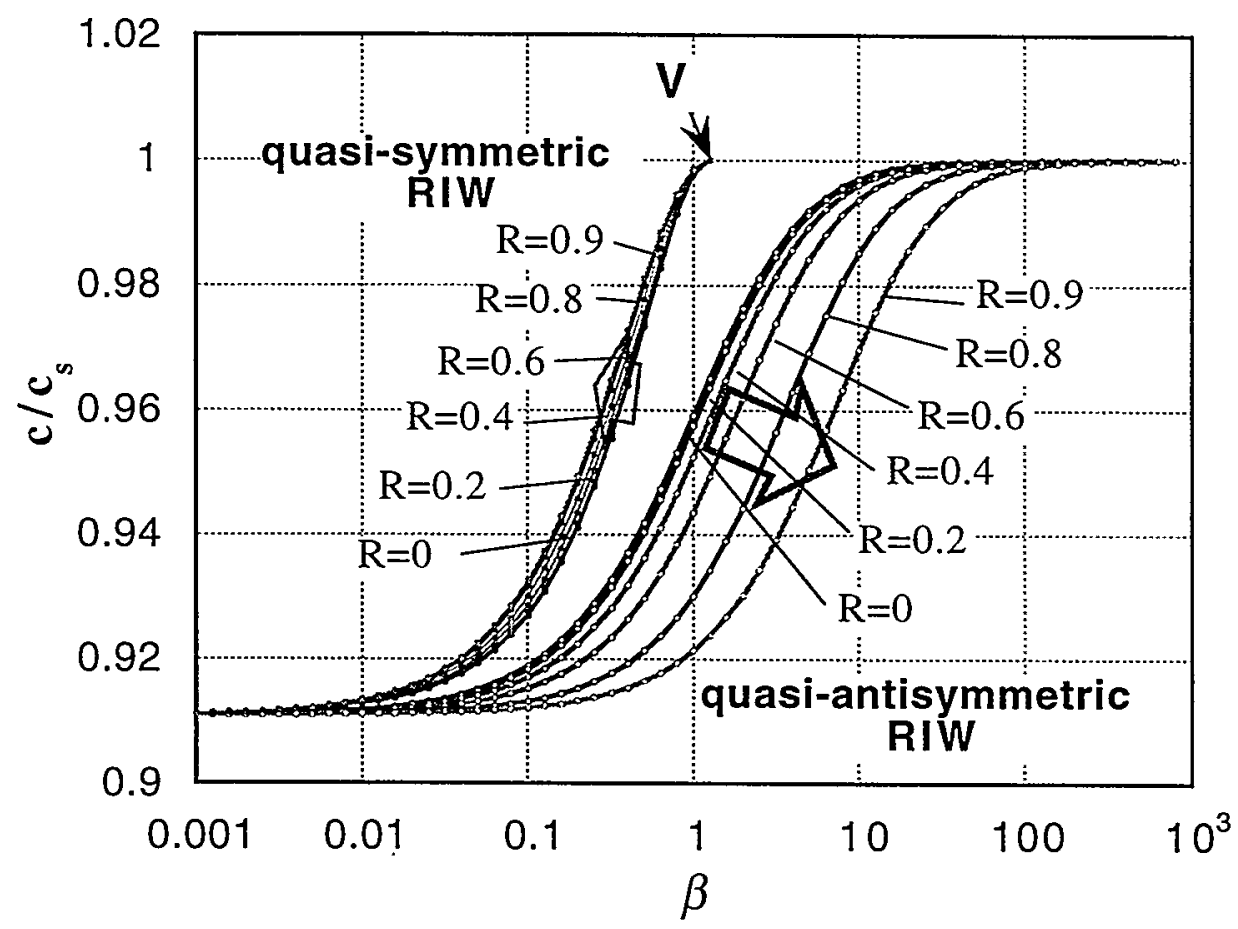

Figure 9.3 Computed dispersion of phase velocities for interface waves with a coupling fracture stiffness. Poisson's ratio of the material is 0.2 and normal and tangential fracture stiffnesses are assumed to be equal $\left(K_{z z}=\kappa_{x x}=\kappa\right)$ ). Increase in the coupling stiffness $\left(\mathrm{R}=\kappa_{z x} / K\right)$ increases the velocity of the quasi-symmetric wave while it decreases the velocity of the quasi-antisymmetric wave. For any possible magnitude of the coupling fracture stiffness, the curves for the quasi-symmetric wave share a common point at $c=c_{S}$ (S-wave velocity) where the wave becomes attenuative (corresponding $\beta$ gives the cut-off frequency).

From the plot, it can be seen that an increase in the coupling fracture stiffness decreases the velocity of the slow wave (q-asymmetric $\mathrm{RIW}$ ) and slightly increases the velocity of the fast wave ( $\mathrm{q}$-symmetric RIW) for a given frequency and fracture stiffness. As $\beta$ increases beyond $\beta=1$, the fast wave approaches the $\mathrm{S}$-wave velocity and then vanishes. By setting $\xi \equiv c_{S} / c=1$, the dispersion equation yields a corresponding non-dimensional impedance parameter (Gu et al., 1996)

$$
\beta=\frac{1}{\sqrt{1-\zeta^{2}}} .
$$

It is noted that the above expression does not include the coupling fracture stiffness parameter R. Therefore, all type curves for the fast wave ( $q$-symmetric RIW) share a common cut-off point (shown as "V" in the plot). 


\subsubsection{Particle motion}

Particle motions associated with the fracture interface waves are obtained by introducing the solution of the dispersion equation (Eq.(9.15)) into Eq.(9.16) and Eq.(9.11) to determine the coefficients of the Helmholtz potentials. The $\mathrm{x}$ and z-direction displacements on the surface of the fracture are computed from the Eq.(9.4) as

$$
\left\{\begin{array}{l}
u_{1 x} \\
u_{1 z} \\
u_{2 x} \\
u_{2 z}
\end{array}\right\}=\frac{\omega}{c_{S}}\left[\begin{array}{cc}
i \Gamma & \Theta \\
-\Phi & i \Gamma \\
i \Gamma & -\Theta \\
\Phi & i \Gamma
\end{array}\right]\left\{\begin{array}{l}
U^{s y m} \\
U^{a . s y m}
\end{array}\right\} e^{i \omega(x / c-t)},
$$

where

$$
\Gamma=\xi\left\{\left(2 \xi^{2}-1\right)-2 \sqrt{\xi^{2}-1} \sqrt{\xi^{2}-\zeta^{2}}\right\}
$$

Parameters $\Theta, \Phi$, and the above parameters can take only real non-negative values for an admissible range of $\xi(\zeta<1 \leq \xi)$ that is given as a real-valued solution of the dispersion equation. If the coupling fracture stiffness is zero, Eq.(9.18a) and (9.18b) hold, and Eq.(9.22) yields

$$
\begin{gathered}
\text { (a) }\left\{\begin{aligned}
u_{1 x} & =u_{2 x} \\
u_{1 z} & =-u_{2 z} \\
u_{1 z} / u_{1 x} & =-u_{2 z} / u_{2 x}=i \cdot(\Phi / \Gamma)
\end{aligned}\right. \\
\text { (b) }\left\{\begin{aligned}
-u_{1 x} & =u_{2 x} \\
u_{1 z} & =u_{2 z} \\
-u_{1 x} / u_{1 z} & =u_{2 x} / u_{2 z}=i \cdot(\Theta / \Gamma)
\end{aligned}\right.
\end{gathered}
$$

Eq.(9.24a) and (9.24b) represent symmetric and anti-symmetric particle motions, respectively. For both cases, the phase for the $\mathrm{z}$-direction motion is $90^{\circ}$ ahead of the $\mathrm{x}$ direction on the halfspace $z<0$, and $90^{\circ}$ behind on the halfspace $z>0$. This leads to an elliptic retrograding motion whose principal axes are normal and parallel to the interface. The corresponding displacement components across the interface are either in phase or $180^{\circ}$ off-phase. Note that $u_{1 x}=u_{2 x}=0$ for $c=c_{S}(\xi=1)$ and $u_{1 z}=u_{2 z}=0$ for $\mathrm{c}=c_{P}(\xi=\zeta)$.

When the coupling fracture stiffness is non-zero, the phase of the z-direction particle motion is faster than the $\mathrm{x}$-direction's particle motion by:

$$
\arg \left(\frac{u_{1 z}}{u_{1 x}}\right)=-\arg \left(\frac{u_{2 z}}{u_{2 x}}\right)=\tan ^{-1}\left\{\frac{\Gamma\left(\Theta \cdot U^{u s s m}+\Phi \cdot U^{s y m}{ }^{2}\right)}{\left(\Gamma^{2}-\Theta \Phi\right) U^{s y m} \cdot U^{a s y m}}\right\} .
$$


For $\xi \geq 1, \Gamma, \Theta, \Phi$, and $\Gamma^{2}-\Theta \Phi$ are non-negative. Therefore, the sign of the expression in the major bracket depends on the sign of $U^{\text {sym }} \cdot U^{\text {asym }}$. For a quasi-symmetric wave, the second expression in Eq.(9.16) yields

$$
U^{s y m} \cdot U^{a s m m}=R \Theta(\beta \cdot \Phi-f) .
$$

From Figure 9.2, $\beta \cdot \Phi-f<0$ due to the increase in the velocity of the quasi-symmetric wave caused by the increase in coupling fracture stiffness. Therefore,

$$
\arg \left(\frac{u_{1 z}}{u_{1 x}}\right)=-\arg \left(\frac{u_{2 z}}{u_{2 x}}\right)=\frac{\pi}{2}+\delta_{s},
$$

where the sign of the phase shift $\delta_{s}$ is given by the sign of R. For a quasi-antisymmetric wave, the first expression in the Eq.(9.16) yields

$$
U^{s y m} \cdot U^{a s g m}=R \Phi(\beta \cdot \Theta-f) .
$$

As $\beta \cdot \Theta-f>0$ for the decreased velocity of the quasi-antisymmetric wave,

$$
\arg \left(\frac{u_{1 z}}{u_{1 x}}\right)=-\arg \left(\frac{u_{2 z}}{u_{2 x}}\right)=\frac{\pi}{2}-\delta_{a}
$$

where $\delta_{a}$ is the phase shift whose sign is given by the sign of R. Using these phase angles, relations between $\mathrm{x}$ and $\mathrm{z}$ - direction particle motions on the surface of the halfspaces are obtained as

(a) $\left\{\begin{array}{l}u_{1 x}=\operatorname{Re}\left\{a \cdot e^{-i \omega t}\right\}=a \cos (\omega t) \\ u_{1 z}=\operatorname{Re}\left\{b \cdot e^{-i \omega t+i\left(\pi / 2+\delta_{s}\right)}\right\}=b \sin \left(\omega t-\delta_{s}\right) \\ u_{2 x}=\operatorname{Re}\left\{c \cdot e^{-i \omega t}\right\}=c \cos (-\omega t) \\ u_{2 z}=\operatorname{Re}\left\{d \cdot e^{-i \omega t-i\left(\pi / 2+\delta_{s}\right)}\right\}=d \sin \left(-\omega t-\delta_{s}\right)\end{array}\right.$
(b) $\left\{\begin{array}{l}u_{1 x}=\operatorname{Re}\left\{a \cdot e^{-i \omega t}\right\}=a \cos (\omega t) \\ u_{1 z}=\operatorname{Re}\left\{b \cdot e^{-i \omega t+i\left(\pi / 2-\delta_{a}\right)}\right\}=b \sin \left(\omega t+\delta_{a}\right) \\ u_{2 x}=\operatorname{Re}\left\{c \cdot e^{-i \omega t}\right\}=c \cos (-\omega t) \\ u_{2 z}=\operatorname{Re}\left\{d \cdot e^{-i \omega t-i\left(\pi / 2-\delta_{a}\right)}\right\}=d \sin \left(-\omega t+\delta_{a}\right)\end{array}\right.$. 
Coefficient $a \sim d$ are real and positive. Eq.(9.30a) and (9.30b) are for the quasi-symmetric wave and the quasi-antisymmetric wave, respectively. If the coupling fracture stiffness is positive, both $\delta_{s}$ and $\delta_{a}$ are positive. The above expressions also reveal that both particle motions are retrograde. From Eq.(9.30a), it can be seen that the ellipse of the particle motion for a quasi-symmetric wave tilts into the second and fourth quadrants around the origin $(\pi / 2<\theta<\pi$ and $3 \pi / 2<\theta<2 \pi$, where $\theta$ is an angle defined from the positive $\mathrm{x}-$ axis in the counter-clockwise direction) by $\delta_{s}$ as the coupling fracture stiffness is increased $(\mathrm{R}>0)$. On the other hand, from Eq.(9.30b), the ellipse tilts into the first and the third quadrants by $\delta_{a}$ for a quasi-antisymmetric wave.

Computed particle motions on the fracture surface for a range of impedance ratios and coupling fracture stiffnesses are shown in Figure 9.4. When the stiffness of the fracture is low (or the wave frequency is high), both q-symmetric and q-antisymmetric waves exhibit elliptic retrograde motions. For this stiffness $(\beta=0.1)$, an increase in the coupling stiffness (R) has little effect on the shape of the particle motion. For an intermediate fracture stiffness $(\beta=1)$, the q-symmetric wave exhibits elongation and tilting of the elliptic motion in the predicted direction, while the q-antisymmetric wave exhibits slight widening and tilting in the opposite direction. For a high fracture stiffness $(\beta=10)$, particle motion of the q-antisymmetric wave exhibits similar behaviors as the intermediate fracture stiffness case. A slight change is that the elliptic trajectory becomes narrower in the $\mathrm{z}$-direction, approaching the particle motion of the S-wave. The quasi-symmetric wave becomes a leaky $\mathrm{P}$-wave in this range, as can be seen by the nearly horizontal particle motion.

The distribution of particle motion in the vicinity of the fracture is shown in Figure 9.5 for the (a) q-symmetric and (b) q-antisymmetric interface waves. When there is no crosscoupling stiffness $(R=0)$, the q-symmetric wave's particle motion is symmetric and the qantisymmetric wave's particle motion is anti-symmetric across the interface located at $\mathrm{z}=0$. Therefore, for the symmetric wave, the $\mathrm{x}$-direction motions are in phase and the $\mathrm{z}$-direction motions are $180^{\circ}$ out of phase across the fracture. Conversely, the anti-symmetric wave has in-phase $\mathrm{z}$-direction motions and $180^{\circ}$ out-of-phase $\mathrm{x}$-direction motions. The most marked effect of the shear or cross coupling stiffness is the altering of the phase difference between the particle motions on both surfaces of the fracture. These shifts occur in the same direction for both q-symmetric and q-antisymmetric waves. 

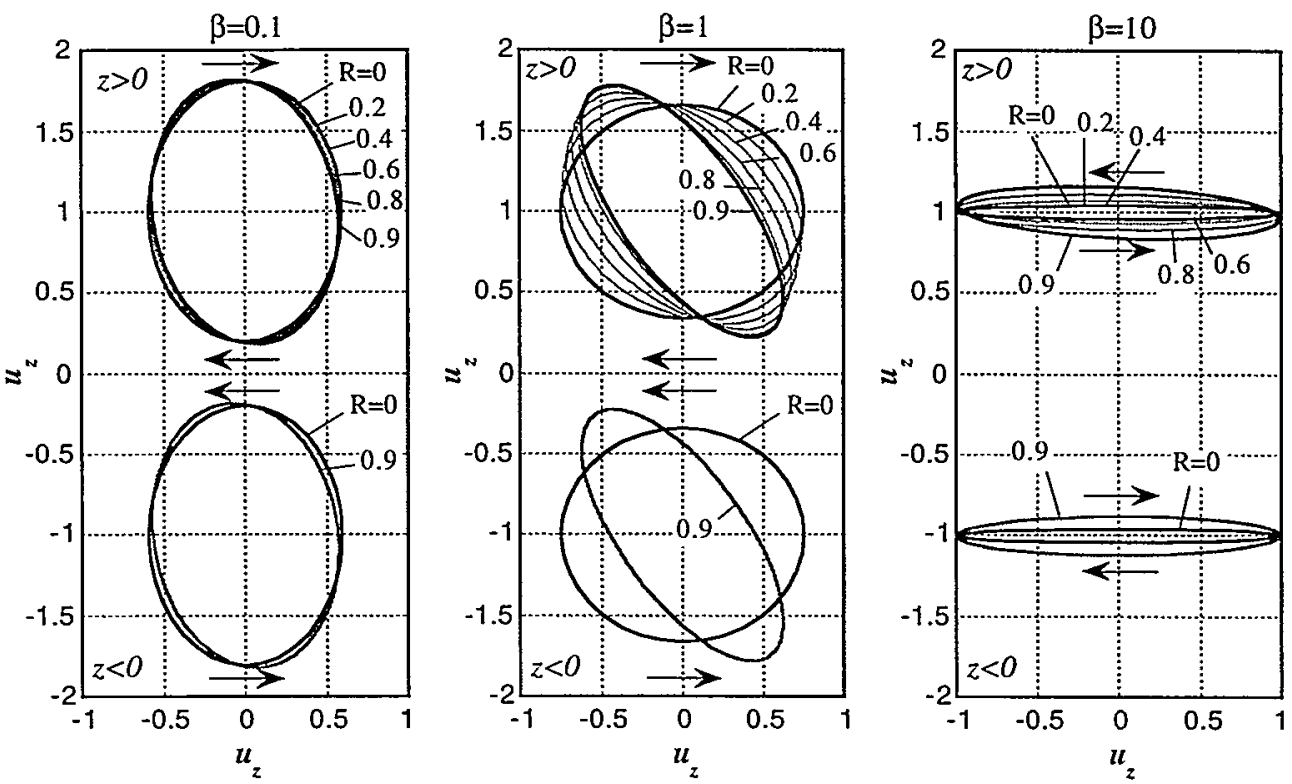

(a) Quasi-symmetric mode
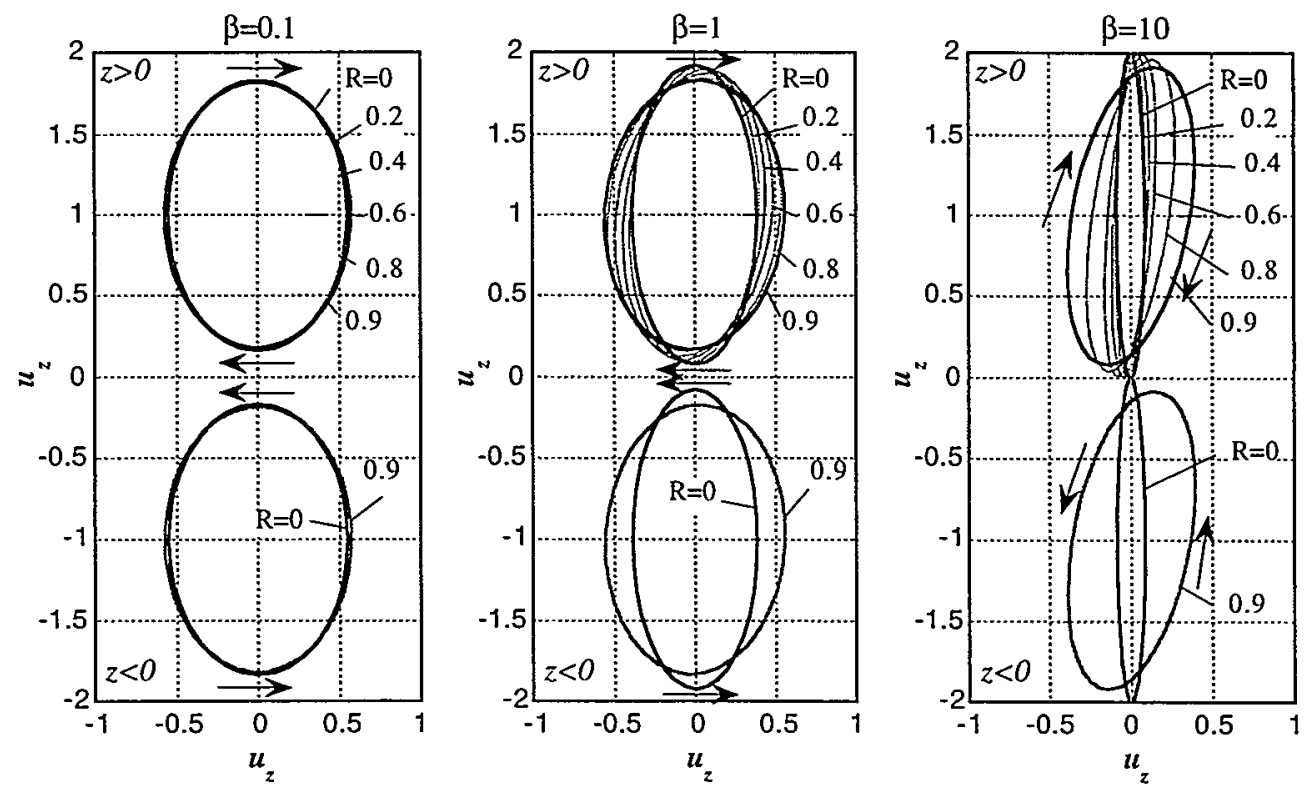

(b) Quasi-antisymmetric mode

Figure 9.4 Particle motion of a single point on the surface of a fracture. In each plot, elliptic trajectories at the top are for the upper surface $(\mathrm{z}>0)$ and at the bottom are for the lower surface $(\mathrm{z}<0)$. All the particle motions except for the high-stiffness $(\beta=10)$ quasi-symmetric case are retrograde. The wave that shows prograde motion is not truly an interface wave since the wave is attenuative. As the relative coupling fracture stiffness (R) increases, elliptic trajectories are skewed and their symmetry across the interface is broken. 


\section{Particle Motions of (Quasi-)Symmetric Rayleigh Interface Wave}
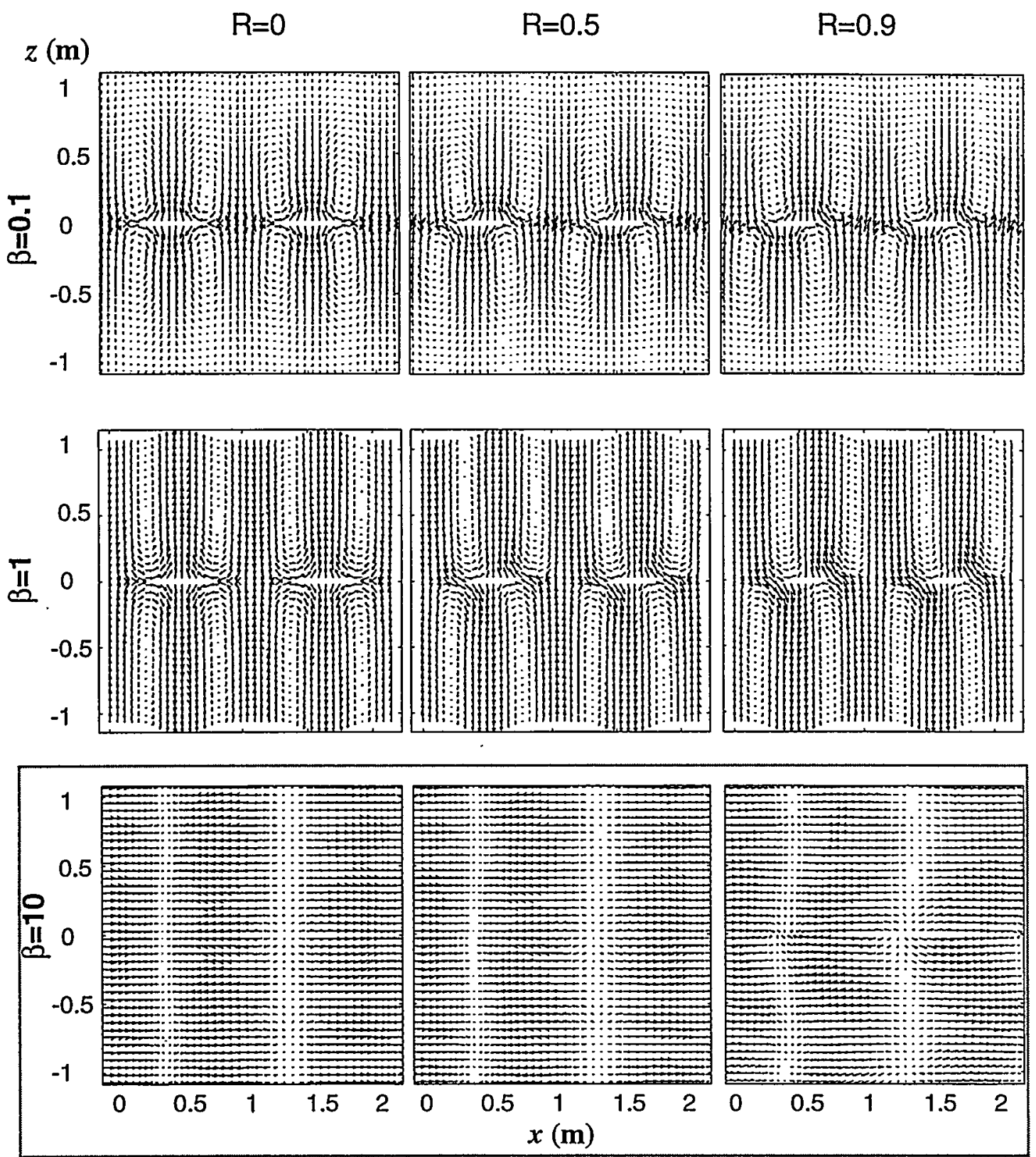

Figure 9.5(a) Particle motions of (quasi-) symmetric Rayleigh interface waves. An increase in the relative coupling fracture stiffness $(R)$ breaks the symmetry in the particle motions across the interface. A phase lag between upper and lower half spaces becomes apparent with increasing coupling fracture stiffness, . In the above plot, the case $\beta=10$ is a slightly leaky surface P-wave. 


\section{Particle Motions of (Quasi-)Anti-symmetric Rayleigh Interface Wave}
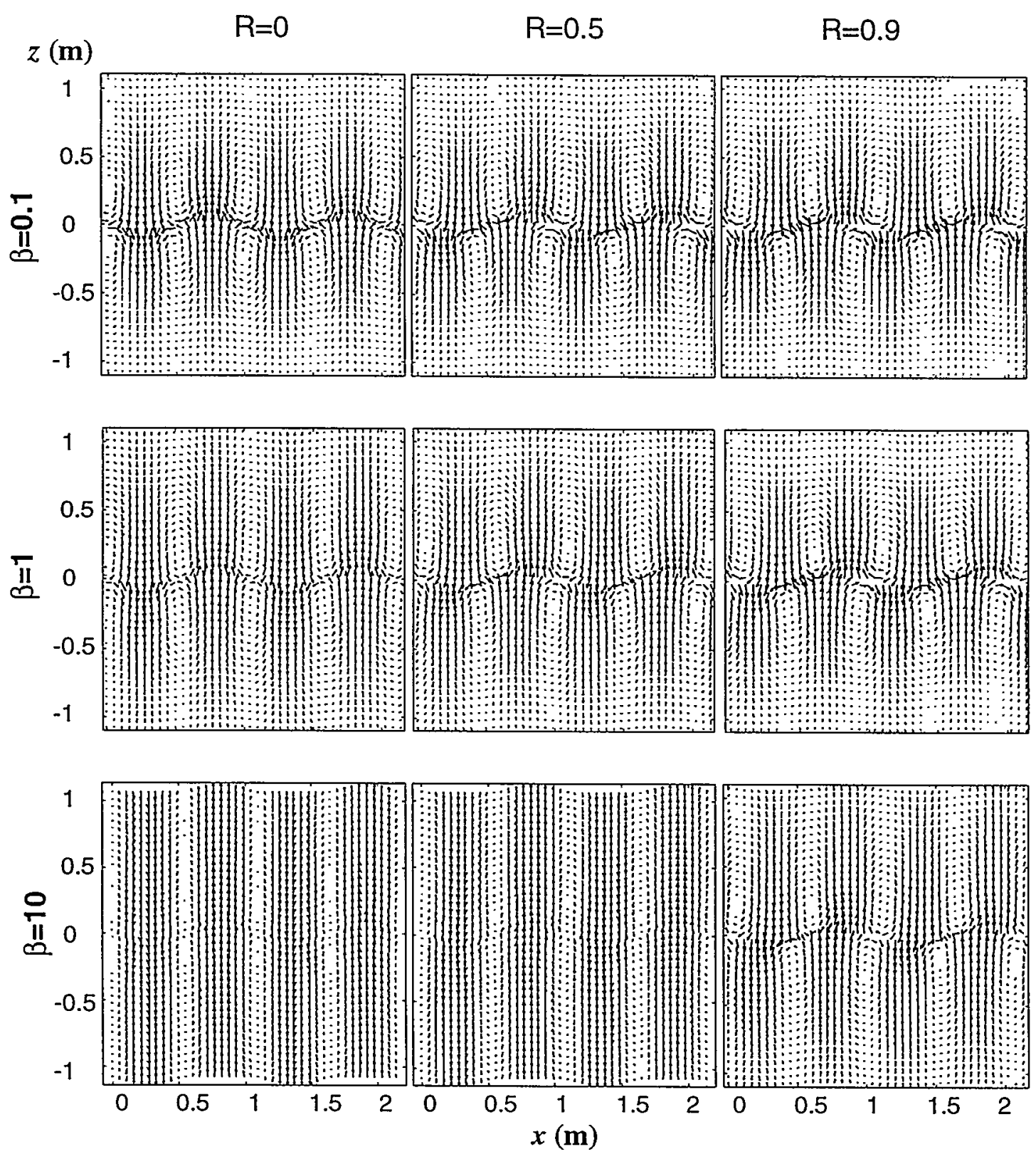

Figure 9.5(b) Particle motions of (quasi-) antisymmetric Rayleigh interface waves. An increase in the relative coupling fracture stiffness $(R)$ breaks the anti-symmetry in the particle motions across the interface. A phase lag between upper and lower halfspaces becomes apparent with increasing coupling fracture stiffness. 


\subsection{Numerical Simulation Using Dynamic Boundary Element Method}

\subsubsection{Numerical Model}

Interface waves propagating along a sheared fracture were simulated using a frequencydomain dynamic boundary element method for elastic plain strain problems. The model used for the simulation is identical to the model used in the previous chapter (Chapter 8). A fracture was modeled by an array of constant element pairs located along the fracture embedded in an infinite medium. The length of the fracture was $12 \mathrm{~m}$ and a pair of directional point sources (force sources) were located $0.086 \mathrm{~m}$ above and below one end of the fracture (Figure 9.6). The source generated a 3-lobe Ricker wavelet with central frequency of $2.65 \mathrm{kHz}$. The two sources were oriented so that they introduced either symmetric or anti-symmetric source displacement. The number of the constant elements used for the simulations was 280 (140 pairs). For a P-wave velocity of $4623 \mathrm{~m} / \mathrm{sec}$ and an S-wave velocity of $2831 \mathrm{~m} / \mathrm{sec}$, the number of the nodes per element was approximately 20 and 12 , respectively. The density of the material used for the simulaitons was $2600 \mathrm{~kg} / \mathrm{cm}^{3}$ and Poisson's ratio was 0.2 .

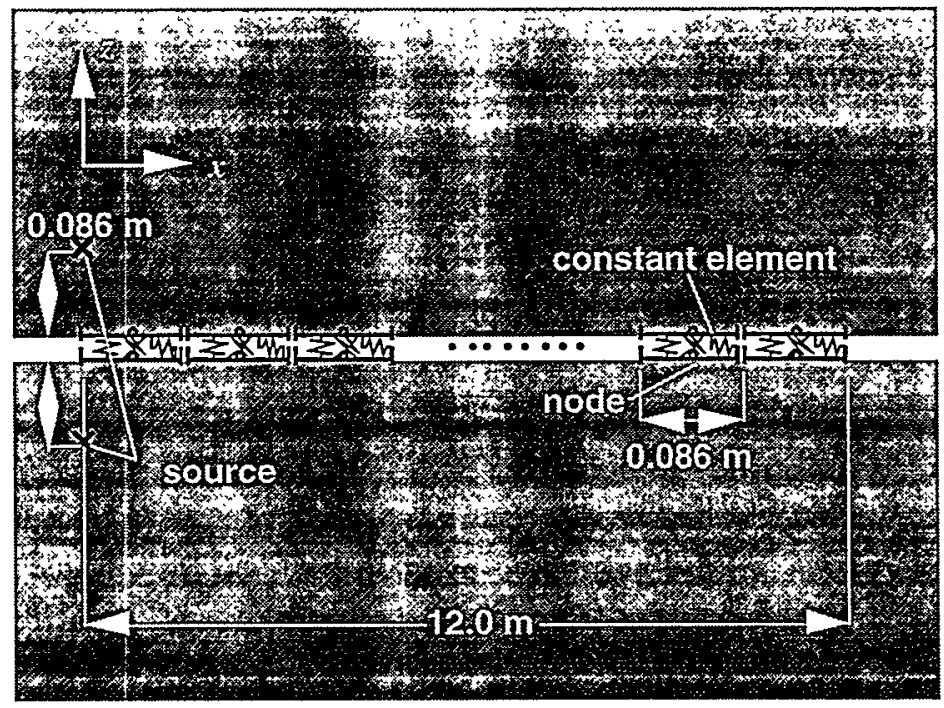

Figure 9.6 A numerical model for simulating a fracture interface wave along a fracture with coupling fracture stiffness. Constant boundary element pair are placed along an interface between two half spaces. Each element pairs is connected by normal, tangential, and coupling displacement-discontinuity fracture stiffness. Directional force is applied at the end of the fracture. To introduce quasi-symmetric waves, two directional forces are applied in the same direction along the $\mathrm{x}$-axis. For quasi-antisymmetric waves, the forces are applied in the same direction along the $y$-axis. 


\subsubsection{Simulated interface waves}

To best see the effect of the coupling stiffness on the interface waves, an intermediate value of $10^{11} \mathrm{~Pa} / \mathrm{m}$ is selected for both normal and tangential fracture stiffnesses. For the central frequency of the source $(2.65 \mathrm{kHz})$ and the velocity of the S-wave $(2831 \mathrm{~m} / \mathrm{sec})$, this stiffness yields an impedance ratio $\beta \sim 1.63$ and a transmission coefficient $\mathrm{T}=0.85$ for normal incident $S$-waves. Figure 9.7(a) shows snapshots of the wavefield generated by a symmetric source (a pair of point sources moving along the fracture). The absolute amplitude of the wave $\left(u=\sqrt{u_{x}^{2}+u_{z}^{2}}\right)$ is shown. Along the fracture, most of the energy is carried by the $\mathrm{P}$-wave and the amplitude of the interface wave is small. The P-wave wavefront generates a strong head wave. An increase in the coupling fracture stiffness (or shear on the fracture) disturbs the symmetry of the wavefield. From the plot, it can be seen

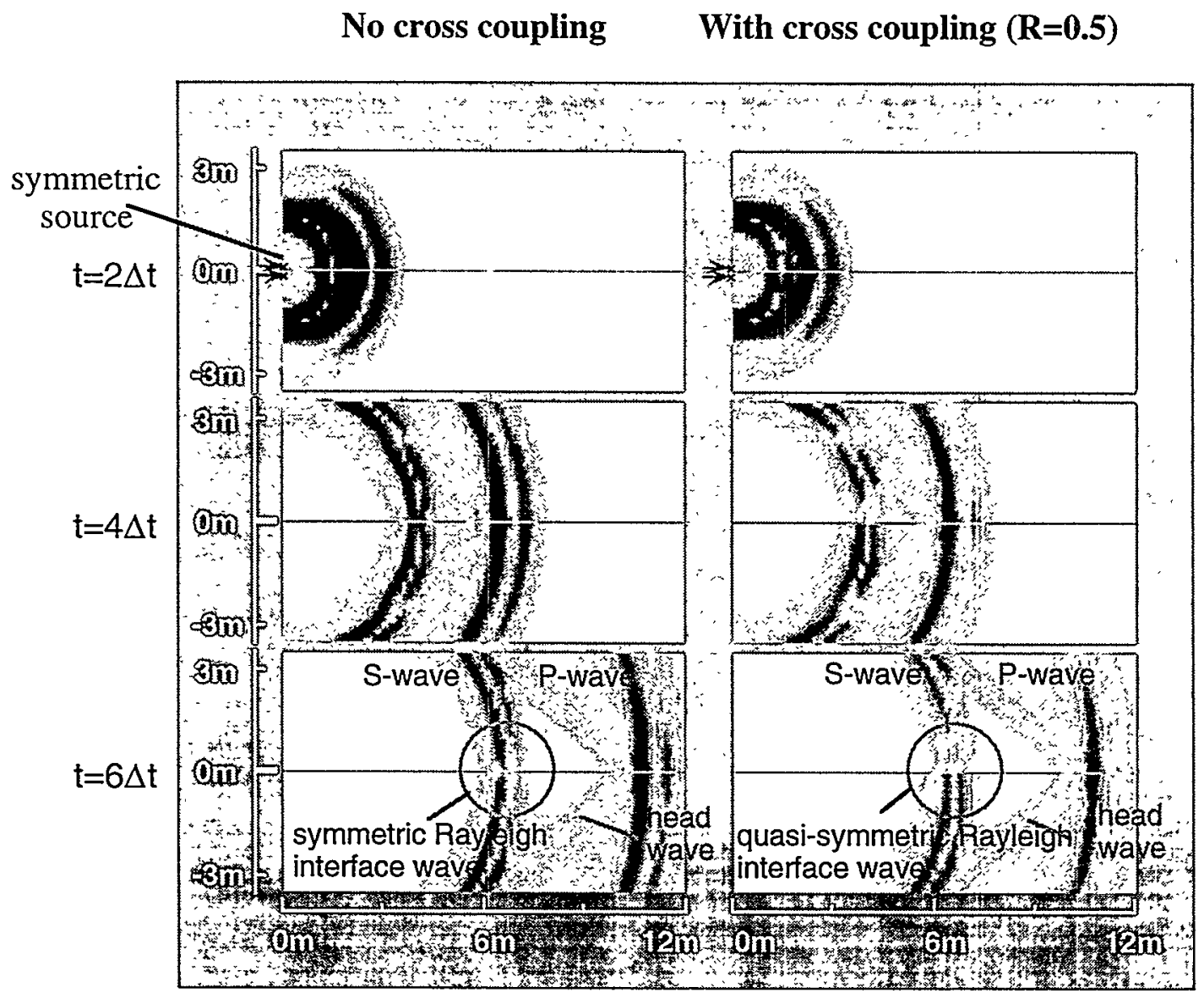

Figure 9.7(a) Snapshots of symmetric and quasi-symmetric fracture interface waves with and without coupling fracture stiffness $\left(\mathrm{R}=\kappa_{z x} / \kappa_{x x}=0\right.$ or 0.5$)$. Normal and tangential fracture stiffness is $10^{11} \mathrm{~Pa} / \mathrm{m}$ $(\beta \sim 1.63, \mathrm{~T} \sim 0.85)$. Absolute displacement amplitude is shown. A symmetric source is used to generate the interface waves. 
that the amplitude of the interface wave in the upper halfspace $(z \geq 0)$ has a slightly advanced phase than the lower halfspace.

The snapshots of the wavefield generated by an anti-symmetric source (a pair of point sources moving perpendicular to the fracture) are shown in Figure 9.7(b). The source generates only a small P-wave along the fracture. Compared with the symmetric source, the amplitude of the interface wave is significantly larger and its velocity slower than the $S$ wave (the S-wave/interface wave wavefront becomes concave on the fracture). Similar to the symmetric source, the interface wave for a sheared fracture shows an advanced phase in the upper halfspace $(\mathrm{z} \geq 0)$.

In Figure 9.8, $x$ - and z- displacements of the waves generated by the symmetric source are shown. An array of receivers is located $7.0 \mathrm{~m}$ from the source parallel to the $\mathrm{z}$-axis.

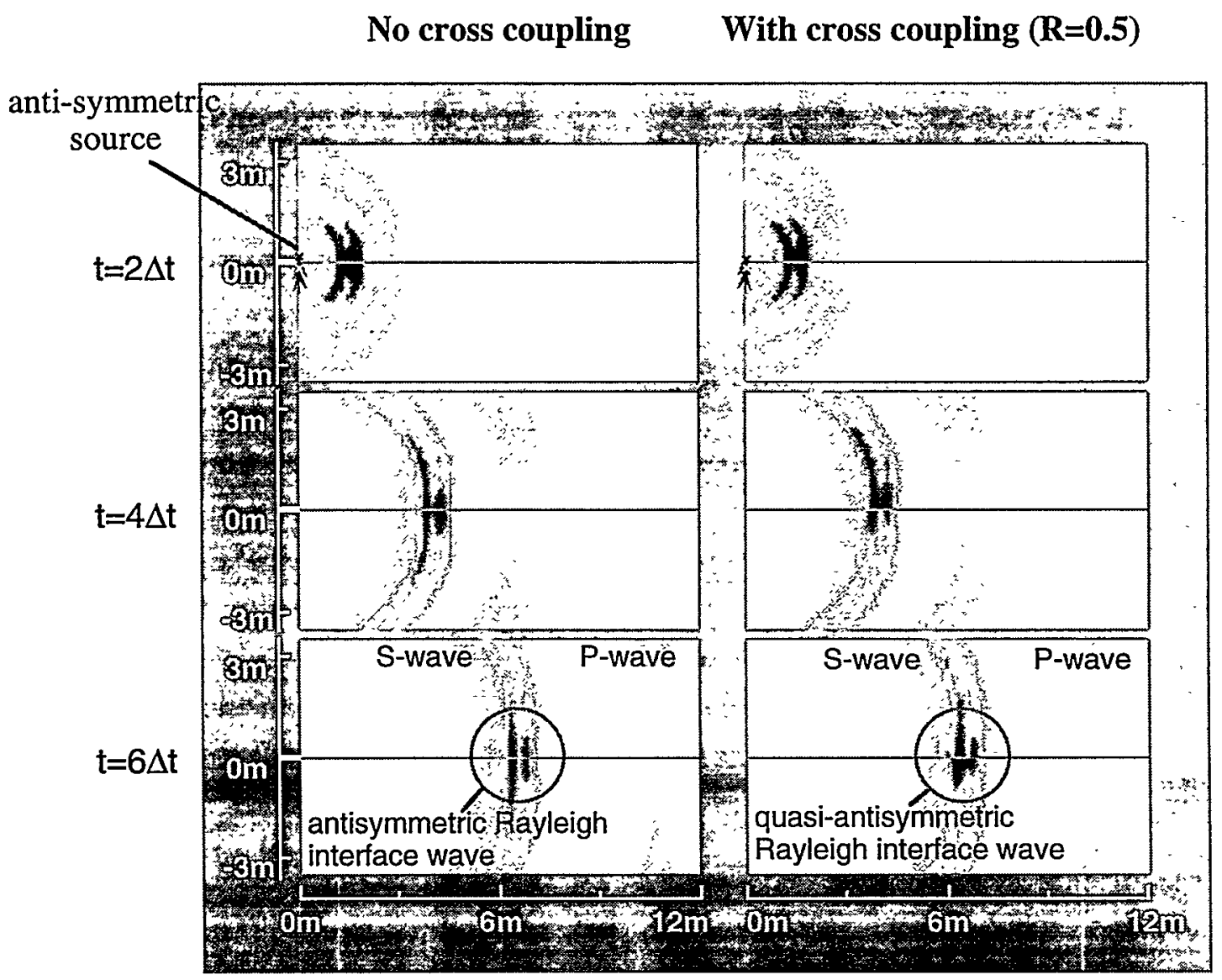

Figure 9.7(b) Snapshots of antisymmetric and quasi-antisymmetric fracture interface waves with and without coupling fracture stiffness $\left(\mathrm{R}=\kappa_{\mathrm{zx}} / \kappa_{\mathrm{xx}}=0\right.$ or 0.5$)$. Normal and tangential fracture stiffness is $10^{11} \mathrm{~Pa} / \mathrm{m}(\beta \sim 1.63, \mathrm{~T} \sim 0.85)$. Absolute displacement amplitude is shown. An antisymmetric source is used to generate the interface waves. 


\section{(Quasi-)Symmetric Interface Wave}

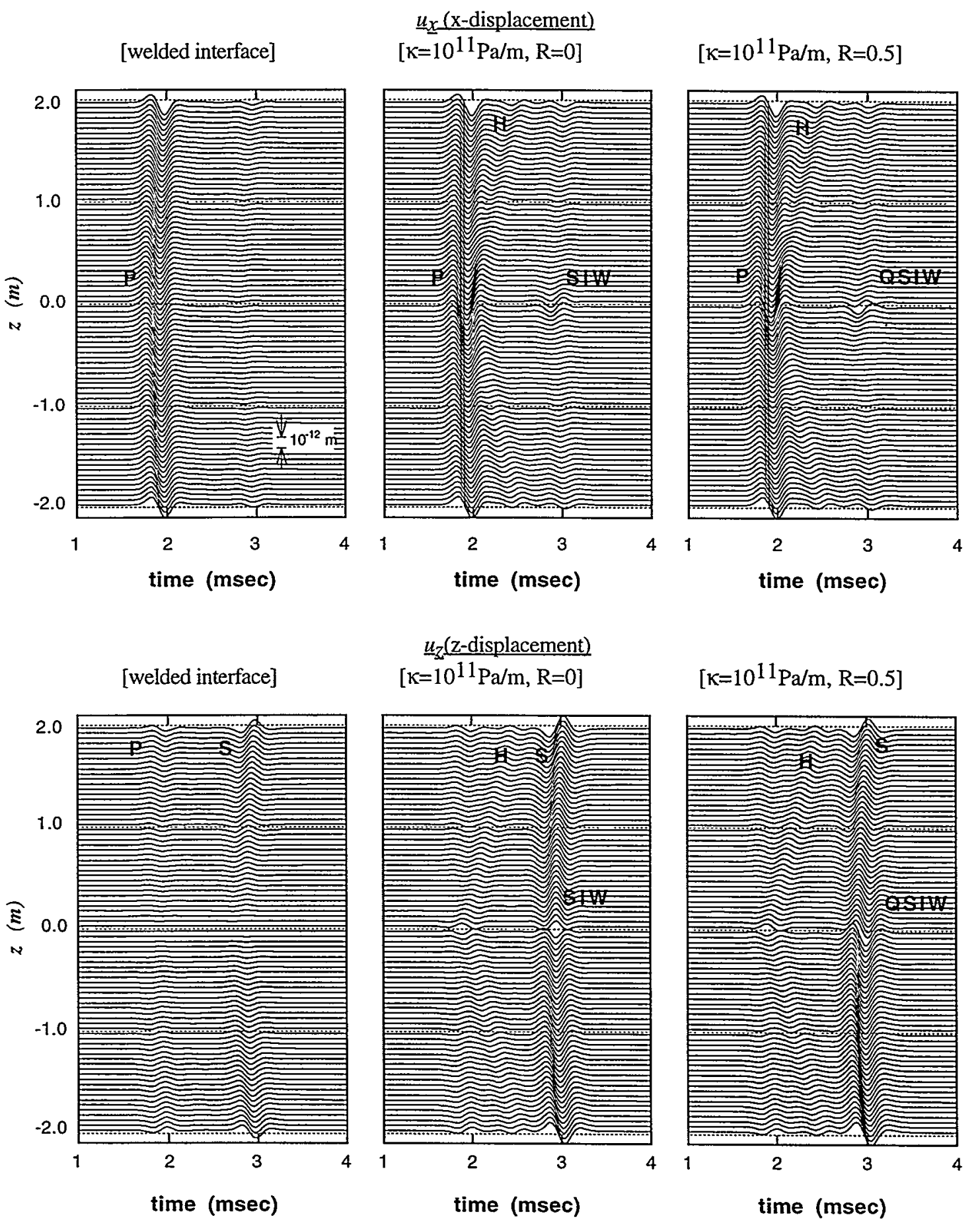

Figure 9.8 Seismograms for waves generated by a symmetric source. The fracture is located at $z=0$. Labels in the plots are, P: P-wave, S: S-wave, H: head-wave, SIW: symmetric interface wave, and QSIW: quasisymmetric interface wave. Introduction of coupling fracture stiffness breaks the symmetry of the waves across the fracture and advances the phase of the quasi-symmetric interface wave in the upper halfspace. 


\title{
(Quasi-)Antisymmetric Interface Wave
}

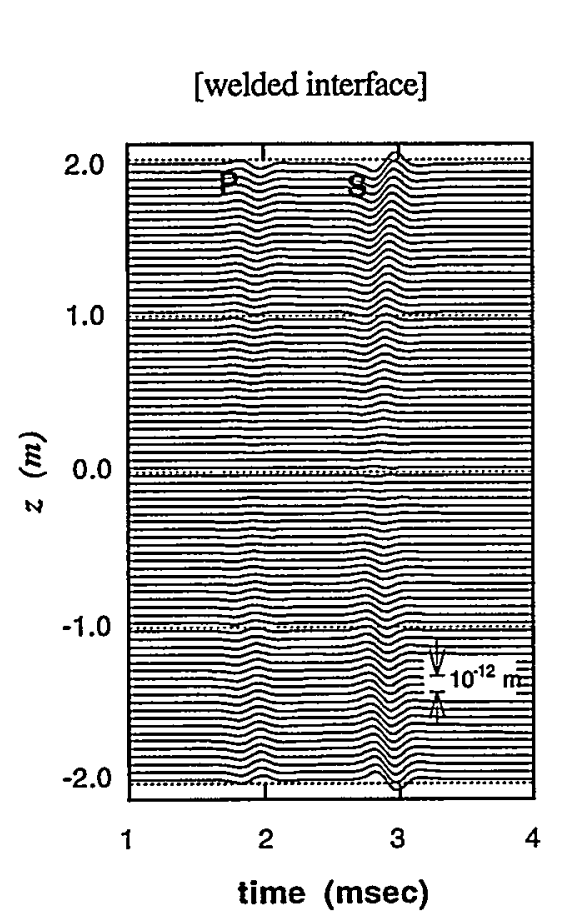

\author{
$\underline{u_{x}}(\mathrm{x}$-displacement) \\ $\left[\kappa=10^{11} \mathrm{~Pa} / \mathrm{m}, \mathrm{R}=0\right]$
}

$\left[\mathrm{K}=10^{11} \mathrm{~Pa} / \mathrm{m}, \mathrm{R}=0.5\right]$
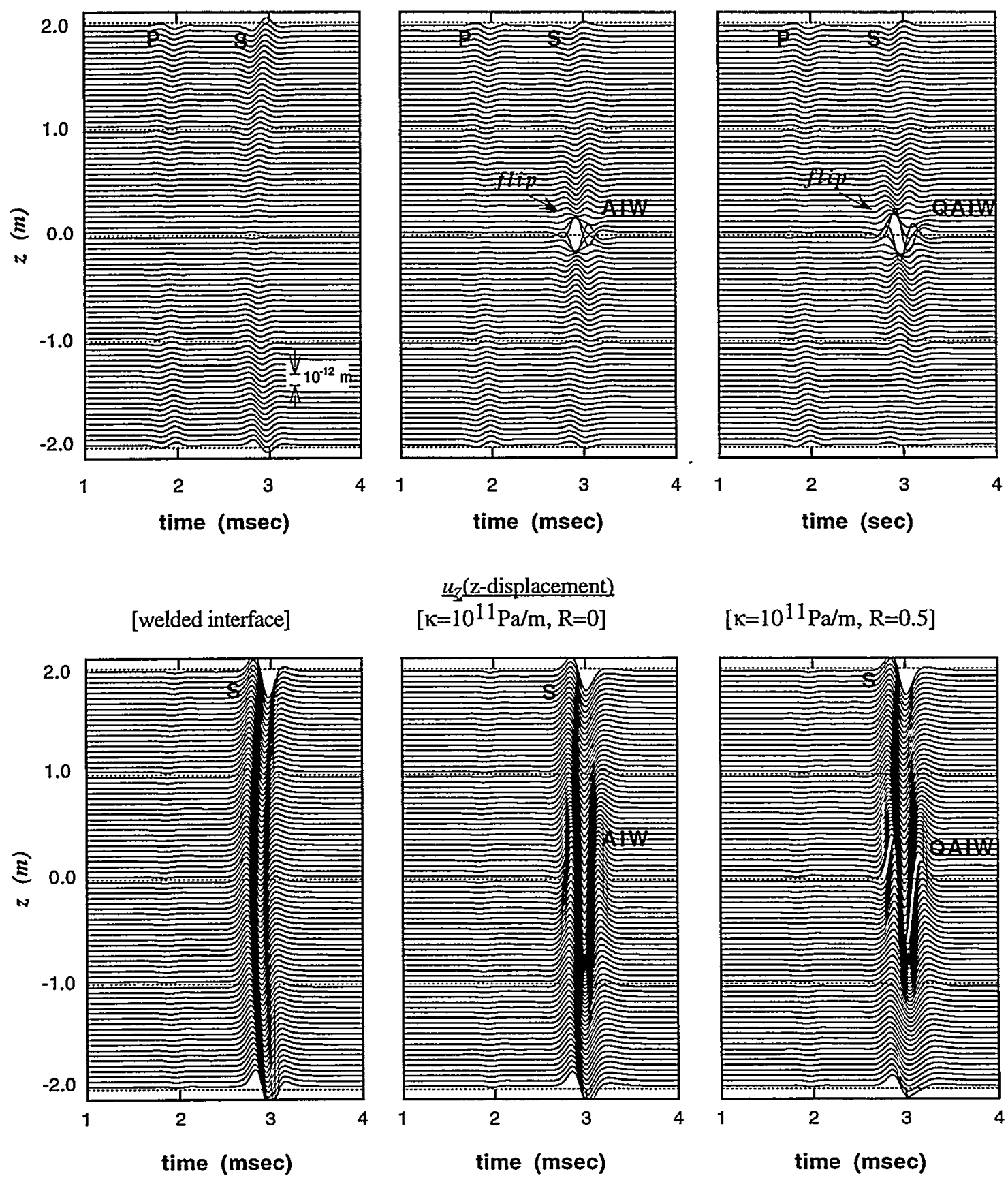

$$
\underset{\left[\kappa=10^{11} \mathrm{~Pa} / \mathrm{m}, \mathrm{R}=0\right]}{\underline{u}_{z}(\mathrm{z} \text {-displacement })}
$$

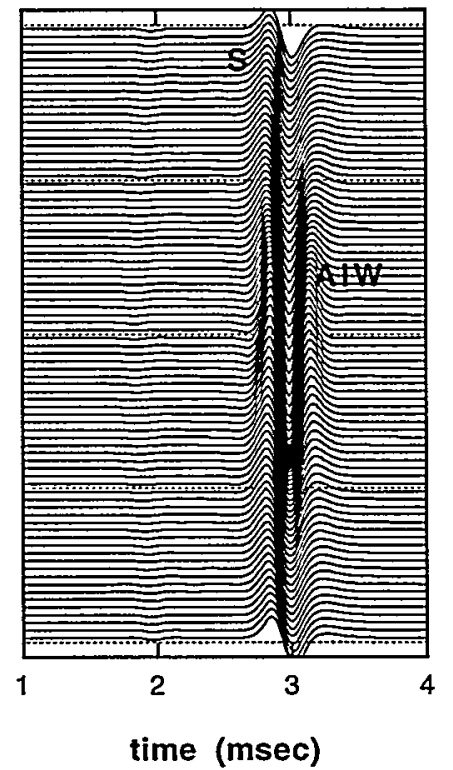

$\left[\kappa=10^{11} \mathrm{~Pa} / \mathrm{m}, \mathrm{R}=0.5\right]$

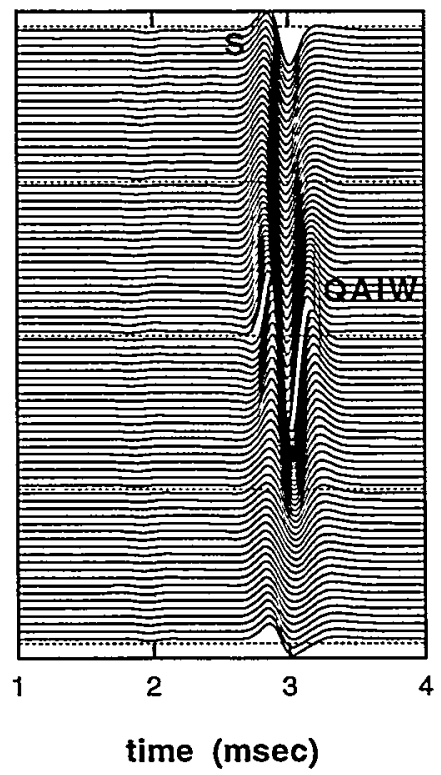

Figure 9.9 Seismograms for waves generated by an anti-symmetric source. Labels in the plots are, P: Pwave, S: S-wave, H: head-wave, AIW: anti-symmetric interface wave, and QAIW: quasi-antisymmetric interface wave. Introduction of coupling fracture stiffness breaks the anti-symmetry of the waves across the fracture and advances the phase of the quasi-antisymmetric interface wave in the upper halfspace. 
For a welded fracture, strong $\mathrm{P}$-wave motion in the $\mathrm{x}$-direction and a small S-wave motion due to the circular wavefront are observed (left column). A decrease in thefracture stiffness introduces a head wave and a symmetric interface wave (middle column). The velocity of the symmetric interface wave is very close to the $S$-wave velocity as the non-dimensional impedance ratio $\beta$ is close to the cut-off impedance ratio given by Eq.(9.21) that yields identical S-wave and interface wave velocities (see Figure 9.3). When the coupling fracture stiffness is introduced, the quasi-symmetric wave exhibits advanced phase for the upper halfspace and retarded phase in the lower half-space, breaking the symmetry in the wavefield (right column).

The waves generated by an anti-symmetric source are shown in Figure 9.9. For a welded fracture, strong z-direction motion due to the S-wave is seen (left). A compliant fracture exhibits an anti-symmetric interface wave along the fracture. The head wave does not have significant amplitude as the P-wave motion along the fracture is very small (middle). The anti-symmetric wave shows a slower velocity than the $\mathrm{S}$-wave and the waveform for the $x$-displacement flips at a certain depth $(z \approx \pm 0.2 \mathrm{~m})$. At this depth, the particle motion changes from retrograde motion at the near surface to a prograde motion. Such a flip is not observed for the symmetric interface waves and the assumed fracture stiffness $(\beta=1.63)$. This is because the velocity of the symmetric interface wave is close to the S-wave velocity. A small $q$ for the velocity of the interface wave given by Eq.(9.3) yields a small second term of the x-direction displacement in Eq.(9.4). As a result, the amplitude of the $x$-displacement decreases monotonically with distance from the fracture. Introduction of the coupling fracture stiffness has a similar effect on the anti-symmetric interface wave as the symmetric wave, changing the phase lag across the fracture and breaking the symmetry of the wavefield.

Numerically simulated particle motions on the surface of the fracture are shown in Figure 9.10 for symmetric and anti-symmetric sources. Displacements for welded and compliant fractures $(\beta=1.63$ ) with and without coupling fracture stiffness $(\mathrm{R}=0$ or 0.5$)$ are compared. In each plot, the top half is for the upper surface and the bottom half is for the lower surface. From the plots, it can be seen that the both (quasi-)symmetric and (quasi-) anti-symmetric waves show elliptic retrograde particle motions. An increase in the coupling stiffness causes elongation of the elliptic trajectories for the symmetric interface wave. Tilting of the elliptic trajectories is observed for both types of interface waves and their direction is consistent with the theoretical prediction (Figure 9.4, middle column). 
Symmetric Source
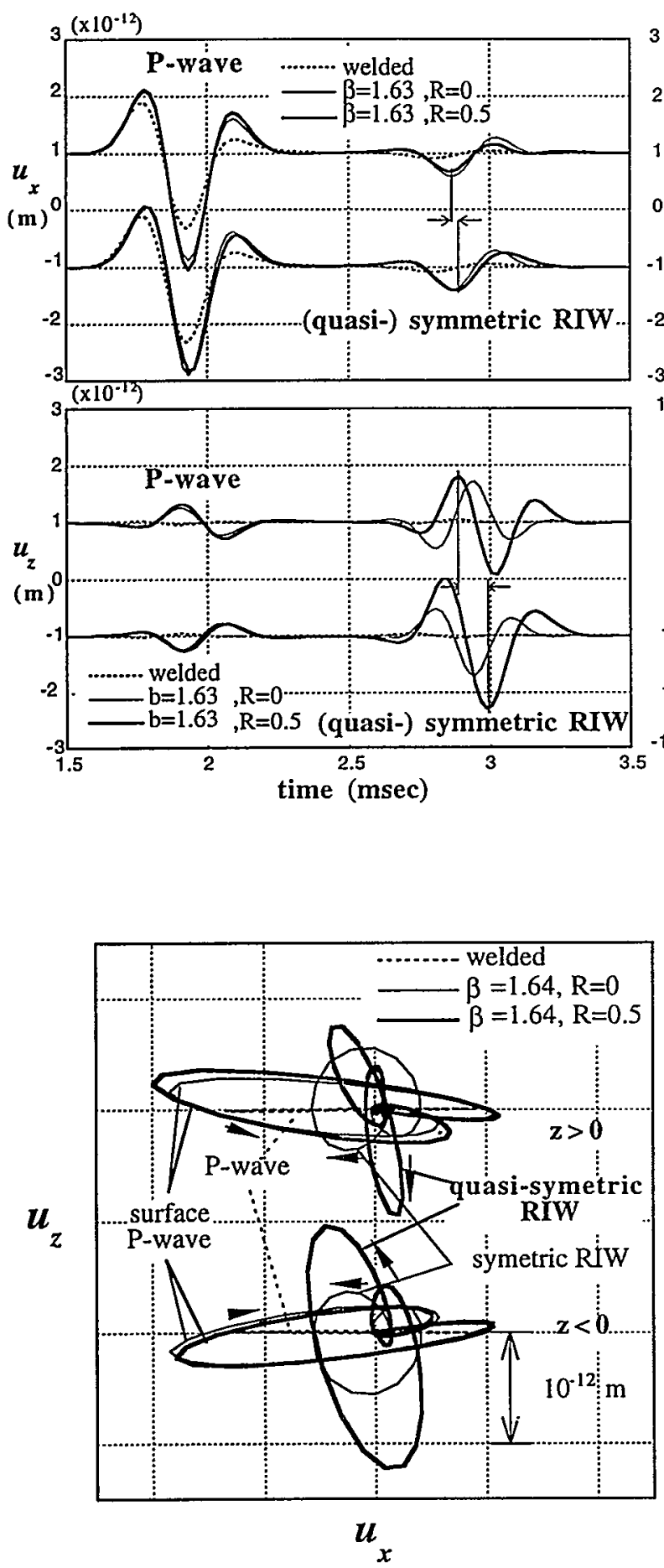

$\underline{\text { Antisymmetric Source }}$
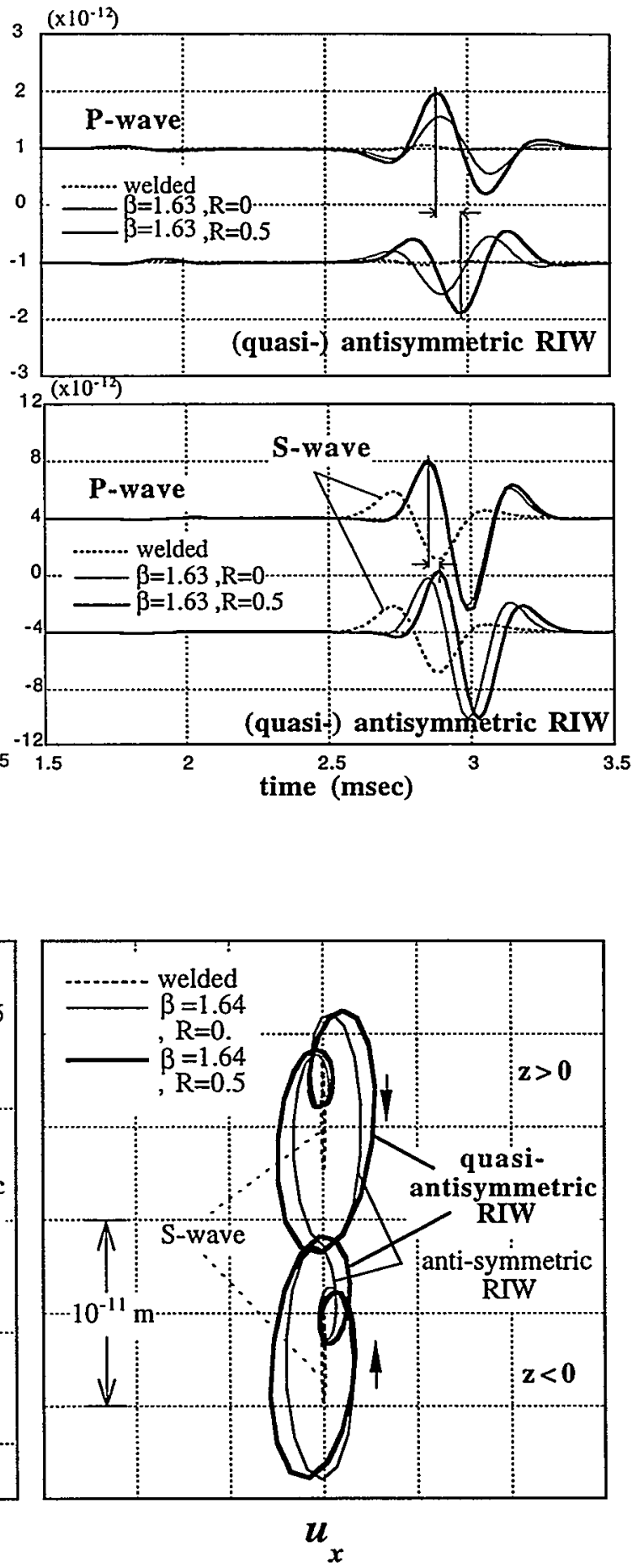

Figure 9.10 Particle motions of interface wave on the fracture surface. Both (quasi-) symmetric and (quasi-)antisymmetric interface waves show elliptic retrograde motion. Increase in the coupling fracture stiffness (R) introduces a phase shift of the waves (waveforms at the top) and tilting of the ellipses. 


\subsection{Summary}

In this chapter, the property of fracture interface waves propagating along a sheared fracture is examined by modeling the effect of the shear using the displacementdiscontinuity boundary conditions with a coupling fracture stiffness. A dispersion equation is derived using a plane wave solution for waves propagating along the fracture. The coupling fracture stiffness relates two independent dispersion equations for symmetric and anti-symmetric interface waves for a fracture without shear. The analysis shows that the resulting particle motions for the interface waves are no longer purely symmetric or antisymmetric. As can be seen from the dispersion equation, both symmetric and antisymmetric motions are coupled through the coupling fracture stiffness. Velocities of the two types of waves and phase lag between displacements on upper and lower surface of the fracture are observed as well. The introduction of the coupling fracture stiffness increases the velocity of the (quasi-) symmetric Rayleigh interface wave and decreases that for the (quasi-) antisymmetric wave. Elliptic particle motions show tilts in the opposite directions for the two waves. For both waves, phase lags are introduced between the opposite sides of the fracture.

The observed changes in the characteristics of the fracture interface wave can potentially be used as a diagnostic tool for detecting and measuring shear stress on a fracture. Such a measurements would be useful for crosshole seismic measurements along a fracture subjected to shear. However, as the effect of shear stress on the characteristics of a fracture interface wave is rather small, experimental confirmation of the results obtained in this chapter is necessary to confirm if the effect is significant enough to be used in reality. 


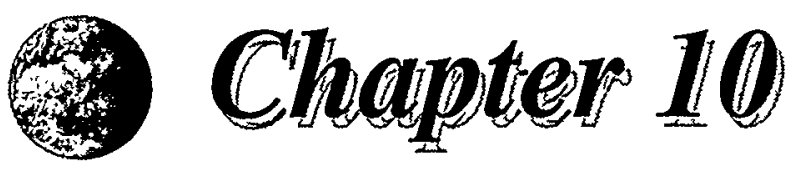

\section{Wave Propagation and Resonance in Media Containing Regularly Spaced Parallel Fractures}

\subsection{Introduction}

In recent years, fractures in reservoir rock have been recognized as important couduits for hydrocarbon production (Nelson, 1987). For fractures to serve as conduits for fluid and gas transport, they must be partially open. This imperfect contact can result in large dynamic normal and tangential compliances when filled with gas and large tangential compliance when filled with fluid. As was discussed in Chapter 8, fractures may also dilate when-subjected to shear stress. In this case, fractures also have a cross-coupling compliance. Compliant fractures are seismically detectable by the changes they impart to wave velocities, amplitudes, and spectral content. By examining these characteristics, reservoir properties that arise from fractures can be investigated.

One of the common geologic structures encountered in reservoir rock is that of closelyspaced parallel fractures (Laubach, 1991; Lorenz and Finley, 1991). Such structures are possibly formed by large regional compressive stresses (Lorenz et al., 1991). Gas flow, 
fluid flow and seismic wave propagation in rock with a single set of parallel fractures can exhibit strong transverse isotropy. When the seismic wavelength is much longer than the fracture spacing, the medium can be replaced by an equivalent transversely isotropic medium (Schoenberg and Douma, 1988; Schoenberg and Muir, 1989; Hood and Schoenberg, 1989). The elastic properties of the equivalent anisotropic medium are then made equivalent to the static properties of the fractured medium. Such an approximation is possible because the stress distribution in a representative volume containing fractures is nearly uniform because of the small spatial variations in stress. Similar approaches have been used by many researchers including Bruggeman (1937) Riznichenko $(1948,1949)$, Postma (1955), Helbig (1958), and Backus (1963) for examining the long-wavelength wave propagation in finely layered geologic structures with welded interfaces. Wave propagation in an equivalent homogeneous medium is frequency-independent, as it is a zero-frequency approximation.

The dynamic behavior of a single fracture, however, is frequency-dependent and its filtering effect on propagating waves is a function of the impedance ratio of the fracture that is a function of fracture stiffness, wave frequency, and the material properties of the medium (Kendall and Tabor, 1971; Schoenberg, 1980). Therefore, even when the wavelength is much longer than the fracture spacing, wave propagation in a fractured medium can exhibit frequency-dependent behavior if the fracture stiffness is sufficiently small. Such a factor is not considered with the static approximation. Furthermore, the static approximation ignores the attenuation of waves due to scattering off the fractures.

If the wavelength is smaller than or comparable to the fracture spacing, the stress variation in a fractured medium is no longer smooth across each fracture. For such a case, the static approximation may no longer be valid. Waves propagating in such media are multiply scattered and the resulting wave velocity and amplitude can be quite different from those for homogeneous media. In Chapter 2, the frequency-dependent filtering characteristics of one-dimensional systems with a zone of regularly and irregularly spaced fractures were examined. It has been shown that the transmission and reflection coefficients of the fractures have a strong correlation with the resonance of the fractured zone that is taken out of the system. Using an exact analytic solution for the dispersion of waves propagating through an infinite number of periodically spaced fractures, it was shown that a spectrum of the wave is characterized by alternating stop and pass bands that correspond to groups of resonance peaks in an equivalent finite system. Such wave behavior was also observed by Helbig (1984) for periodically layered structures with 
welded interfaces and Schoenberg (1983) for reflection of SH-waves obliquely incident on regularly spaced parallel non-welded interfaces (fractures).

In this chapter, a general form of the dispersion equation for plane wave propagation in media containing multiple parallel fractures is derived. The stiffness matrix of the fractures is kept general, i.e., all cross-coupling terms are included. The dispersion equation is derived by imposing periodic boundary conditons on a plane wave solution using Floquet theory (Floquet, 1883; Bedford and Drumheller, 1994). To model the dynamic behavior of the fractures, the displacement-discontinuity boundary conditions are used (Schoenberg, 1980; Pyrak-Nolte et al., 1990a). The resulting dispersion relation is exact, i.e., no longwavelength approximation was used, and can describe full coupling among P-, SV-, and $\mathrm{SH}$-waves. It is then shown that when the effective wave propagation in the $\mathrm{z}$-direction (normal to the fractures) is zero, the dispersion equation degenerates to a set of generalized Rayleigh-Lamb plate wave equations for the case of P-SV waves and a generalized SHplate wave equations for SH-waves. If the thickness of individual layers is large compared with the wavelength, the equation degenerates to the dispersion equation for the fracture interface wave.

\subsection{Derivation of General Dispersion Equation}

A general dispersion equation in a three-dimensional medium including regularly spaced multiple parallel fractures will now be derived. The derivation is similar to that followed for the dispersion equation of the fracture interface wave in Chapter 9.

The displacement of a wave propagating in an isotropic intact medium can be expressed in terms of Helmholtz potentials as

$$
\underline{u}=\underline{\nabla} \cdot \phi+\underline{\nabla} \times \underline{\psi}
$$

where $\phi$ and $\underline{\psi}$ are scalar and vector potentials for P- and S-wave type motions, respectively. If the coordinate system is oriented such that the fractures are parallel to the zplane and the wave is propagating parallel to the $x-z$ plane (Figure 10.1), the components of the displacement are expressed as

$$
\left\{\begin{array}{l}
u_{x} \\
u_{y} \\
u_{z}
\end{array}\right\}=\left\{\begin{array}{l}
\phi_{, x} \\
\phi_{, y} \\
\phi_{, z}
\end{array}\right\}+\left\{\begin{array}{l}
\psi_{z, y}-\psi_{y, z} \\
\psi_{x, z}-\psi_{z, x} \\
\psi_{y, x}-\psi_{x, y}
\end{array}\right\} \Rightarrow\left\{\begin{array}{c}
\phi_{, x}-\psi_{, z} \\
v \\
\phi_{, z}+\psi_{, x}
\end{array}\right\} .
$$




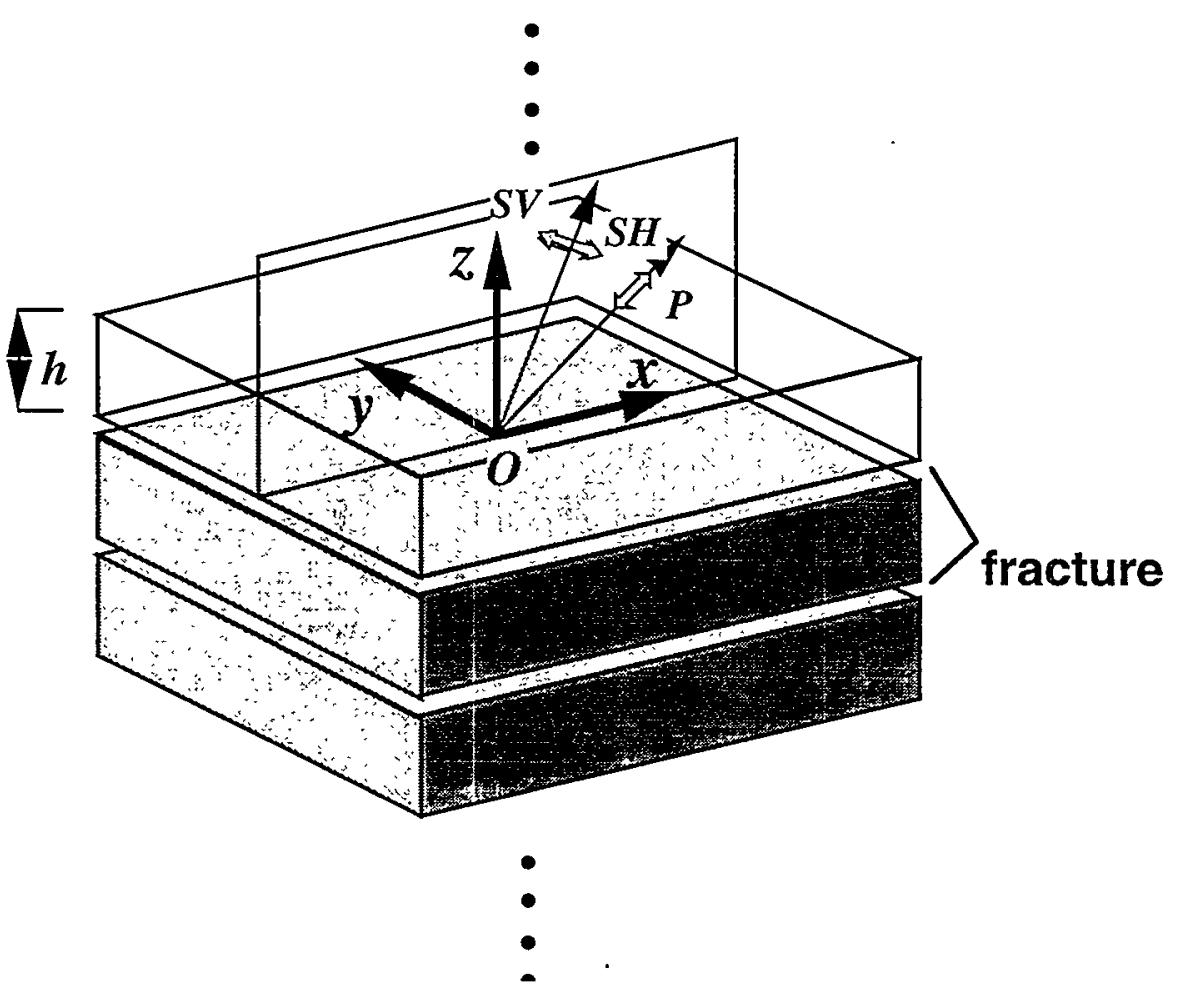

Figure 10.1 P-SV-SH wave propagation in a medium containing regularly spaced parallel fractures. Fracture spacing is h. A general fracture stiffness matrix is used (i.e., the stiffness matrix includes normal, tangential, and coupling fracture stiffnesses).

Note that $\psi$ is redefined as a scalar potential in the above equation. For plane waves, explicit forms of the potentials are given by

$$
\begin{aligned}
& \phi=\left(A e^{i k_{z}^{P} z}+\bar{A} e^{-i k_{z}^{P} z}\right) e^{i\left(k_{x} x-\omega t\right)}, \\
& \psi=\left(B e^{i k_{z}^{S} z}+\bar{B} e^{-i k_{z}^{S} z}\right) e^{i\left(k_{x} x-\omega t\right)}, \\
& v=\left(C e^{i k_{z}^{S} z}+\bar{C} e^{-i k_{z}^{S} z}\right) e^{i\left(k_{x} x-\omega t\right)},
\end{aligned}
$$

where $k_{x}$ is the horizontal wavenumber; $k_{z}^{P}$ and $k_{z}^{S}$ are the vertical wavenumbers for Pand S-waves. An identical horizontal wavenumber is assumed for all the potentials. It is noted that the first terms in the potentials represent "up-going" waves and the second terms are for "down-going" waves in the layered system. By introducing Eq.(10.3) into Eq.(10.2), the components of the displacement are 


$$
\begin{aligned}
& \left\{\begin{array}{l}
u_{x} \\
u_{z} \\
u_{y}
\end{array}\right\}=\left[\begin{array}{cccccc}
i k_{x} & i k_{x} & -i k_{z}^{s} & i k_{z}^{s} & 0 & 0 \\
i k_{z}^{P} & -i k_{z}^{P} & i k_{x} & i k_{x} & 0 & 0 \\
0 & 0 & 0 & 0 & 1 & 1
\end{array}\right] \\
& \times\left[\begin{array}{llllll}
A e^{i k_{z}^{P} z} & \bar{A} e^{-i k_{z}^{P} z} & B e^{i k_{z}^{S} z} & \bar{B} e^{-i k_{z}^{S} z} & C e^{i k z_{z}^{s}} & \bar{C} e^{-i k_{z}^{S} z}
\end{array}\right]^{T} \cdot e^{i\left(k_{x} x-\omega t\right)}
\end{aligned}
$$

The displacement gradients are then given by

$$
\begin{aligned}
& \left\{\begin{array}{l}
u_{x, x} \\
u_{z, x} \\
u_{y, x}
\end{array}\right\}=\left[\begin{array}{cccccc}
-k_{x}^{2} & -k_{x}^{2} & k_{x} k_{z}^{S} & -k_{x} k_{z}^{S} & 0 & 0 \\
-k_{x} k_{z}^{P} & k_{x} k_{z}^{P} & -k_{x}^{2} & -k_{x}^{2} & 0 & 0 \\
0 & 0 & 0 & 0 & i k_{x} & i k_{x}
\end{array}\right] \text {, }
\end{aligned}
$$

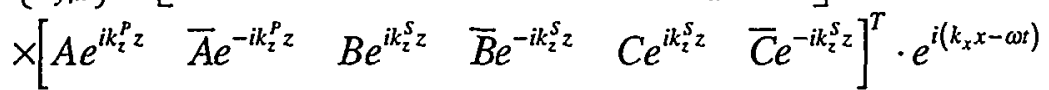

and

$$
\begin{aligned}
& \left\{\begin{array}{l}
u_{x, z} \\
u_{z, z} \\
u_{y, z}
\end{array}\right\}=\left[\begin{array}{cccccc}
-k_{x} k_{z}^{P} & k_{x} k_{z}^{P} & k_{z}^{s^{2}} & k_{z}^{s^{2}} & 0 & 0 \\
-k_{z}^{P 2} & -k_{z}^{P^{2}} & -k_{x} k_{z}^{s} & k_{x} k_{z}^{s} & 0 & 0 \\
0 & 0 & 0 & 0 & i k_{z}^{s} & -i k_{z}^{s}
\end{array}\right]
\end{aligned}
$$

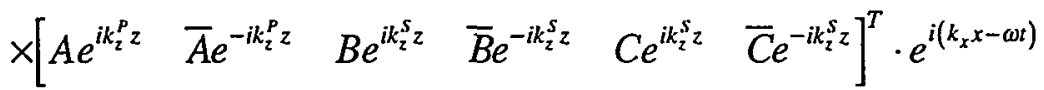

The stress components in a layer are computed as linear combinations of $\mathrm{Eq}(10.5 \mathrm{a})$ and (10.5b). In a medium containing an infinite series of regular periodic fractures, Floquet's theory (Floquet, 1883; Bedford and Drumheller, 1994) can be used to impose periodic boundary conditions on the displacement and stress fields. The theory states that, in a periodic structure, a stationary wavefield must also be periodic. To apply this condition to the waves scattered by the periodic fractures, the effective wave propagation in the $\mathrm{z}$ direction is defined using a wave number $\hat{k}_{z}$. Using $\hat{k}_{z}$, the displacement and stress are expressed as

$$
\begin{aligned}
& \underline{u}=\underline{U}(z) \cdot e^{i\left(\hat{k}_{z} z+k_{x} x-\omega t\right)}, \\
& \underline{\sigma}=\underline{T}(z) \cdot e^{i\left(\hat{k}_{z} z+k_{x} x-\omega t\right)} .
\end{aligned}
$$

Effective wavenumbers are defined following a procedure used by Bedford and Drumheller (1994)

$$
K_{z}^{P \pm}=k_{z}^{P} \pm \hat{k}_{z}, K_{z}^{S \pm}=k_{z}^{S} \pm \hat{k}_{z} .
$$


The components of $\underline{U}(z)$ are rewritten as

$$
\begin{aligned}
& \left\{\begin{array}{l}
u_{x} \\
u_{z} \\
u_{y}
\end{array}\right\}=\left[\begin{array}{cccccc}
i k_{x} & i k_{x} & -i k_{z}^{S} & i k_{z}^{S} & 0 & 0 \\
i k_{z}^{P} & -i k_{z}^{P} & i k_{x} & i k_{x} & 0 & 0 \\
0 & 0 & 0 & 0 & 1 & 1
\end{array}\right\}
\end{aligned}
$$

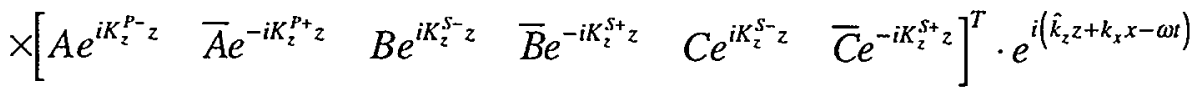

$$
\begin{aligned}
& =\underline{U}(z) \cdot e^{i\left(\hat{k}_{z} z+k_{x} x-\omega t\right)} .
\end{aligned}
$$

The components of $\underline{T}(z)$ are computed from linear combinations of displacement gradients computed by

$$
\begin{aligned}
& \left\{\begin{array}{l}
u_{x, x} \\
u_{z, x} \\
u_{y, x}
\end{array}\right\}=\left[\begin{array}{cccccc}
-k_{x}{ }^{2} & -k_{x}{ }^{2} & k_{x} k_{z}^{S} & -k_{x} k_{z}^{S} & 0 & 0 \\
-k_{x} k_{z}^{P} & k_{x} k_{z}^{P} & -k_{x}^{2} & -k_{x}^{2} & 0 & 0 \\
0 & 0 & 0 & 0 & i k_{x} & i k_{x}
\end{array}\right]
\end{aligned}
$$

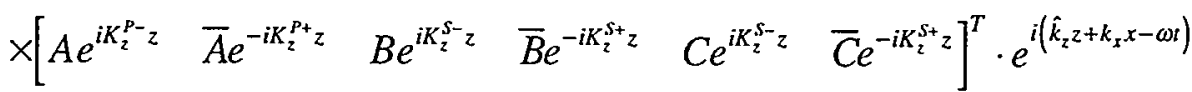

$$
\begin{aligned}
& =\nabla_{x} \underline{U}(z) \cdot e^{i\left(\hat{k}_{z} z+k_{x} x-\omega t\right)}, \\
& \left\{\begin{array}{l}
u_{x, z} \\
u_{z, z} \\
u_{y, z}
\end{array}\right\}=\left[\begin{array}{cccccc}
-k_{x} k_{z}^{P} & k_{x} k_{z}^{P} & k_{z}^{S^{2}} & k_{z}^{S^{2}} & 0 & 0 \\
-k_{z}^{P^{2}} & -k_{z}^{P^{2}} & -k_{x} k_{z}^{S} & k_{x} k_{z}^{S} & 0 & 0 \\
0 & 0 & 0 & 0 & i k_{z}^{S} & -i k_{z}^{S}
\end{array}\right]
\end{aligned}
$$

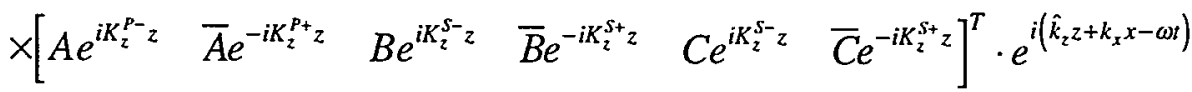

$$
\begin{aligned}
& =\nabla_{z} \underline{U}(z) \cdot e^{i\left(\hat{k}_{z} z+k_{x} x-\omega t\right)} \text {. }
\end{aligned}
$$

Periodic boundary conditions are imposed on the stationary part of the displacement and stress field as

$$
\begin{aligned}
& \underline{U}(0-)=\underline{U}(h+0-), \\
& \underline{T}(0-)=\underline{T}(h+0-),
\end{aligned}
$$

where $h$ is the thickness of a single layer and the origin $(\mathrm{z}=0)$ is taken on the surface of the reference layer. Displacement-discontinuity boundary conditions for a compliant interface are expressed as 


$$
\begin{aligned}
& \underline{T}(0-)=\underline{T}(0+), \\
& \underline{T}(0+)=\underline{\underline{\kappa}}[\underline{U}(0+)-\underline{U}(0-)],
\end{aligned}
$$

where $\underline{\underline{\kappa}}\left(=\left[\kappa_{i j}\right]\right)$ is the general fracture stiffness matrix. By introducing Eq.(10.10a) and (10.10b) into Eq.(10.11a) and (10.11b),

$$
\begin{aligned}
& \underline{T}(h+0-)=\underline{T}(0+), \\
& \underline{T}(0+)=\underline{\underline{\kappa}}[\underline{U}(0+)-\underline{U}(h+0-)] .
\end{aligned}
$$

Note that the above boundary conditions include only the quantities for the reference layer $(0<z<h)$. Eq.(10.12a) and (10.12b) provide six independent equations for the six unknown coefficients of the Helmholtz potentials. The equations can be written in the form of a $6 \times 6$ matrix equation.

To express the matrix in a dimensionless form, the following dimensionless slowness and impedance parameters are introduced

$$
\begin{aligned}
& \xi=\frac{k_{x}}{k^{S}}\left(=\frac{C_{S}}{C_{x}}\right), \zeta=\frac{k_{P}}{k_{S}}\left(=\frac{C_{S}}{C_{P}}\right), \Theta=\sqrt{\xi^{2}-1}, \Phi=\sqrt{\xi^{2}-\zeta^{2}}, \\
& \beta_{i j}^{S}=\frac{2 \kappa_{i j} / \omega}{Z_{S}}(i, j=x, y, z),
\end{aligned}
$$

where $C_{x}$ is the phase velocity along fractures, $C_{S}$ and $C_{P}$ are the $\mathrm{S}$ - and $\mathrm{P}$-wave velocities in an intact medium, and $Z_{s}$ is the S-wave acoustic impedance of the intact medium. It should be noted that the following relations hold for the wavenumbers and slowness parameters

$$
k_{x}^{2}+k_{z}^{P 2}=k^{P 2}=\frac{\omega^{2}}{C_{P}{ }^{2}}, k_{x}^{2}+k_{z}^{s^{2}}=k^{S^{2}}=\frac{\omega^{2}}{C_{S}{ }^{2}}, \quad \xi=k_{x} \frac{C_{S}}{\omega}, i \Theta=k_{z}^{s} \frac{C_{S}}{\omega}, i \Phi=k_{z}^{P} \frac{C_{S}}{\omega} .
$$

To obtain a matrix equation of simplified form, the coefficients of the potentials are redefined as

$$
\begin{aligned}
& A^{\prime}=A\left(1-e^{i K_{z}^{P-h}}\right), B^{\prime}=B\left(1-e^{i K_{2}^{S-h}}\right), C^{\prime}=C\left(1-e^{i K_{z}^{S-h}}\right), \\
& \vec{A}=\bar{A}\left(1-e^{-i K_{z}^{P+h}}\right), \vec{B}=\bar{B}\left(1-e^{-i K_{2}^{S+h}}\right), \vec{C}=\bar{C}\left(1-e^{-i K_{2}^{S+h}}\right) .
\end{aligned}
$$


Using the redefined parameters, the six displacement-discontinuity boundary conditions are expressed as a matrix equation in Figure 10.2.

The matrix equation is simplified further by introducing the following coefficients

$$
\begin{aligned}
& A^{a s y m}=\frac{\vec{A}+A^{\prime}}{2}, B^{a s y m}=\frac{\vec{B}^{\prime}-B^{\prime}}{2}, C^{a s y m}=\frac{\vec{C}^{\prime}+C^{\prime}}{2}, \\
& A^{s y m}=\frac{\vec{A}^{\prime}-A^{\prime}}{2}, B^{s y m}=\frac{\vec{B}+B^{\prime}}{2}, C^{s y m}=\frac{\vec{C}^{\prime}-C^{\prime}}{2} .
\end{aligned}
$$

The resulting matrix equation is shown in Figure 10.3. In this expression, the following functions are used

$$
\begin{aligned}
& a_{-}=\frac{\sin \left(k_{z}^{P} h\right)}{\cos \left(\hat{k}_{z} h\right)-\cos \left(k_{z}^{P} h\right)}=\frac{i \sinh (\alpha \Phi)}{\cos \left(\alpha \hat{\xi}_{z}\right)-\cosh (\alpha \Phi)}, \\
& a_{+}=\frac{e^{i \hat{k}_{z} h}-\cos \left(k_{z}^{P} h\right)}{\cos \left(\hat{k}_{z} h\right)-\cos \left(k_{z}^{P} h\right)}=\frac{e^{i \alpha \hat{\xi}_{z}}-\cosh (\alpha \Phi)}{\cos \left(\alpha \hat{\xi}_{z}\right)-\cosh (\alpha \Phi)}, \\
& b_{-}=\frac{\sin \left(k_{z}^{S} h\right)}{\cos \left(\hat{k}_{z} h\right)-\cos \left(k_{z}^{S} h\right)}=\frac{i \sinh (\alpha \Theta)}{\cos \left(\alpha \hat{\xi}_{z}\right)-\cosh (\alpha \Theta)}, \\
& b_{+}=\frac{e^{i \hat{k}_{z} h}-\cos \left(k_{z}^{S} h\right)}{\cos \left(\hat{k}_{z} h\right)-\cos \left(k_{z}^{S} h\right)}=\frac{e^{i \alpha \hat{\xi}_{z}}-\cosh (\alpha \Theta)}{\cos \left(\alpha \hat{\xi}_{z}\right)-\cosh (\alpha \Theta)}, \\
& \text { and } b_{+}-a_{+}=\frac{i \sin \left(\alpha \hat{\xi}_{z}\right)\{\cosh (\alpha \Theta)-\cosh (\alpha \Phi)\}}{\left\{\cos \left(\alpha \hat{\xi}_{z}\right)-\cosh (\alpha \Theta)\right\}\left\{\cos \left(\alpha \hat{\xi}_{z}\right)-\cosh (\alpha \Phi)\right\}}
\end{aligned}
$$

where

$$
\hat{\xi}_{z}=\hat{k}_{z} / k^{s}, \alpha=k^{s} h=\frac{\omega h}{c_{s}} .
$$

From the first three rows in the matrix of Figure 10.3, the following relations can be found

$$
\left\{\begin{array}{c}
A^{\text {sym }} \\
B^{\text {sym }}
\end{array}\right\}=U^{\text {sym }}\left\{\begin{array}{c}
2 \xi^{2}-1 \\
2 i \xi \Phi
\end{array}\right\},\left\{\begin{array}{c}
A^{\text {asym }} \\
B^{\text {asym }}
\end{array}\right\}=U^{\text {asym }}\left\{\begin{array}{c}
2 i \xi \Theta \\
2 \xi^{2}-1
\end{array}\right\},\left\{\begin{array}{c}
C^{\text {sym }} \\
C^{\text {asym }}
\end{array}\right\}=V^{\text {asym }}\left\{\begin{array}{l}
0 \\
1
\end{array}\right\} \cdot(10
$$




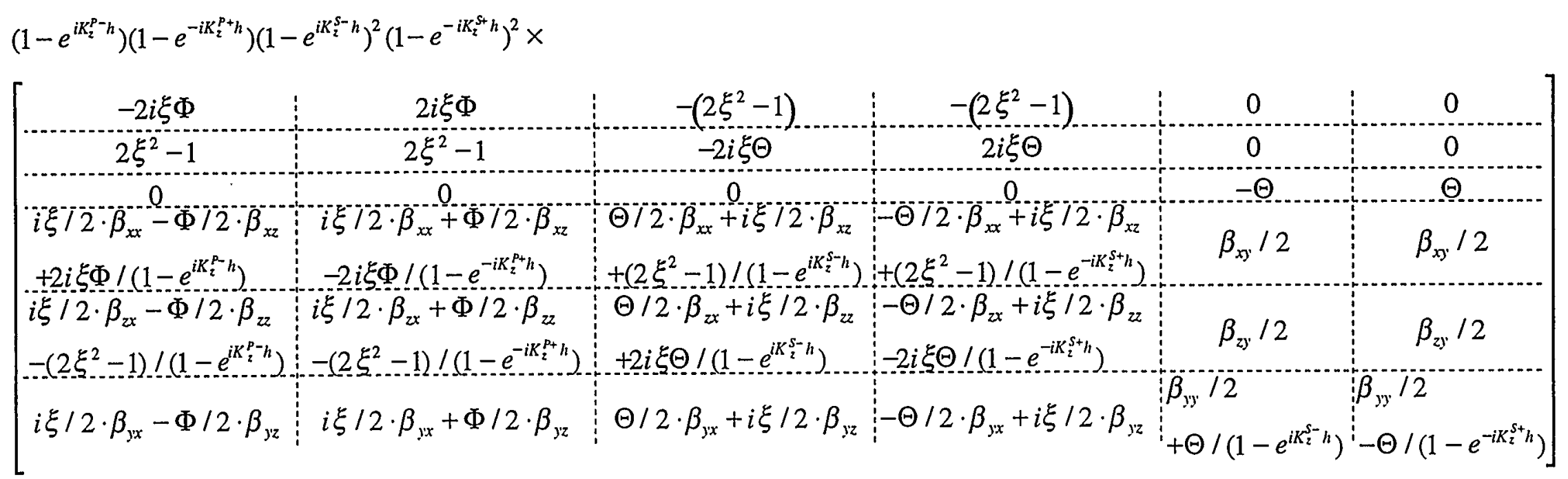

$$
\times\left\{\begin{array}{l}
\frac{A^{\prime}}{A^{\prime}} \\
\frac{B^{\prime}}{B^{\prime}} \\
\frac{C^{\prime}}{C^{\prime}}
\end{array}\right\}=\mathbf{0}
$$

Figure10.2 Matrix equation with dimensionless parameters. 


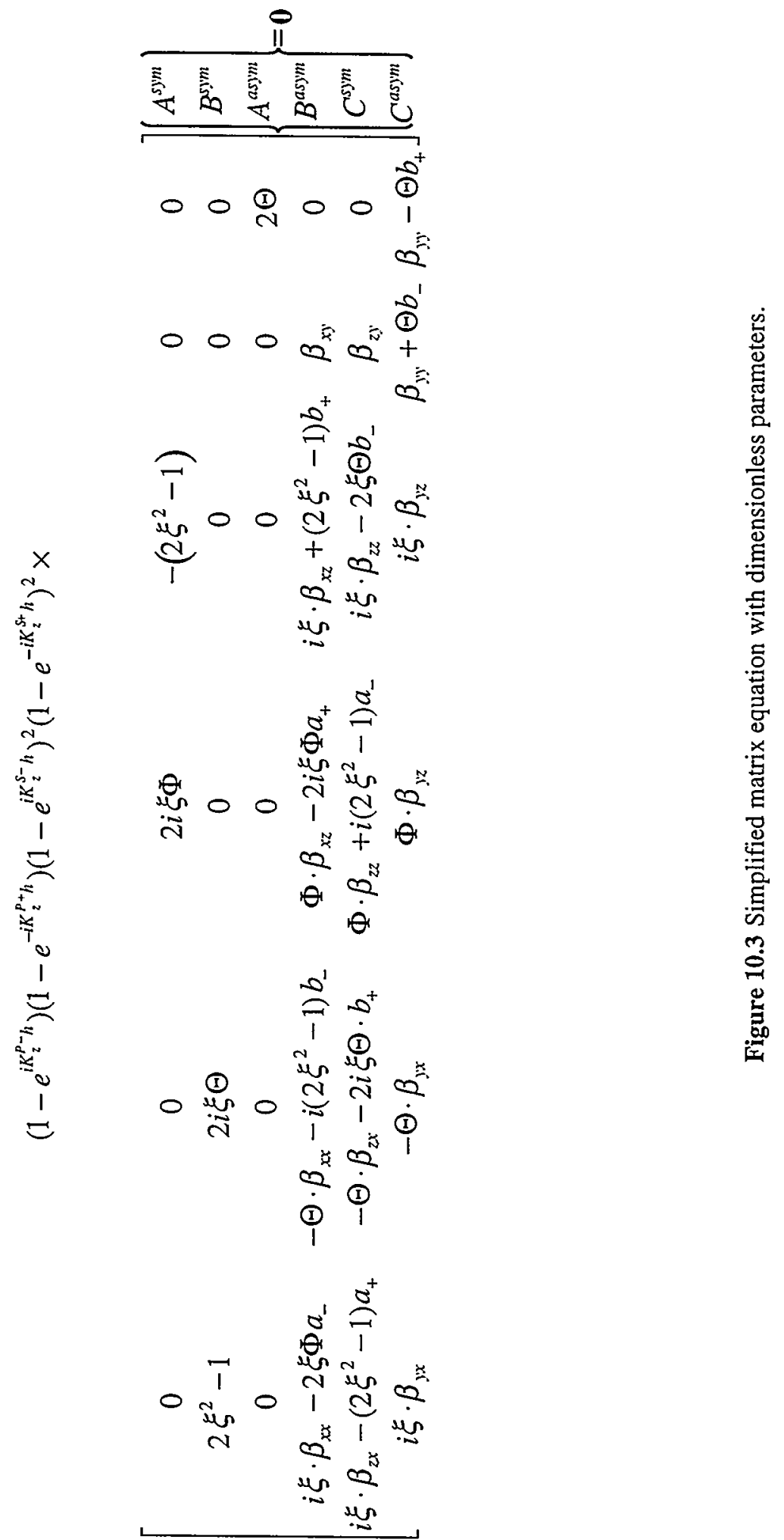


Introduction of Eq.(10.19) reduces the size of the matrix in Figure 10.3 from a $6 \times 6$ to a $3 \times 3$. The resulting matrix equation is

$$
\begin{gathered}
\left(1-e^{i K_{z}^{P-h}}\right)\left(1-e^{-i K_{z}^{P+} h}\right)\left(1-e^{i K_{z}^{S-h}}\right)^{2}\left(1-e^{-i K_{z}^{S+h}}\right)^{2} \times \\
\Theta\left[\begin{array}{ccc}
\beta_{x x}^{S} \Theta-f_{Q} & \beta_{x y}^{S} & \beta_{x z}^{S} \Phi-g_{P} \\
\beta_{y x}^{S} \Theta & \beta_{y y}^{S}+\Theta \cdot f_{R} & \beta_{y z}^{S} \Phi \\
\beta_{z x}^{S} \Theta-g_{Q} & \beta_{z y}^{S} & \beta_{z z}^{S} \Phi-f_{P}
\end{array}\right]\left[\begin{array}{l}
U^{a s y m} \\
V^{a s y m} \\
U^{s y m}
\end{array}\right\}=\mathbf{0}
\end{gathered}
$$

where

$$
\begin{aligned}
& f_{P}=i\left[\left(2 \xi^{2}-1\right)^{2} a_{-}-4 \xi^{2} \Theta \Phi b_{-}\right] \\
& f_{Q}=i\left[\left(2 \xi^{2}-1\right)^{2} b_{-}-4 \xi^{2} \Theta \Phi a_{-}\right] \\
& f_{R}=i b_{-} \\
& g_{P}=2 i \xi \Phi\left(2 \xi^{2}-1\right)\left(b_{+}-a_{+}\right) \\
& g_{Q}=2 i \xi \Theta\left(2 \xi^{2}-1\right)\left(b_{+}-a_{+}\right) .
\end{aligned}
$$

Therefore, a dispersion equation for the general P-SV-SH wave propagation in a medium containing a regularly spaced, infinite series of parallel fractures is obtained as

$$
\begin{aligned}
P\left(\xi^{2}\right) \equiv & \Theta \cdot\left(1-e^{i K_{z}^{P-h}}\right)\left(1-e^{-i K_{z}^{P+} h}\right)\left(1-e^{i K_{z}^{S-h}}\right)^{2}\left(1-e^{-i K_{z}^{S+h}}\right)^{2} \\
& \times \operatorname{det}\left[\begin{array}{ccc}
\beta_{x x}^{S} \Theta-f_{Q} & \beta_{x y}^{S} & \beta_{x z}^{S} \Phi-g_{P} \\
\beta_{y x}^{S} \Theta & \beta_{y y}^{S}+\Theta \cdot f_{R} & \beta_{y z}^{S} \Phi \\
\beta_{z x}^{S} \Theta-g_{Q} & \beta_{z y}^{S} & \beta_{z z}^{S} \Phi-f_{P}
\end{array}\right]=0
\end{aligned}
$$

Except for an exponential factor $e^{-3 i \hat{k}_{z} z}$, the above dispersion equation is either a real function $(\operatorname{Imag}\{P\}=0$ ) or a pure imaginary function $(\operatorname{Real}\{P\}=0)$ for real-valued fracture stiffnesses and wavenumbers. Therefore, standard root finding algorithms can be used.to find real solutions for the slowness $\left(\xi\right.$ or $\hat{\xi}_{z}$ ). 


\subsection{Group Velocity}

When a dispersion relation is expressed in the form $P=P\left(\xi_{x}, \xi_{y}, \xi_{z}, \omega\right)$, the group velocity can be computed by

$$
C_{g_{i}}=\frac{\partial \omega}{\partial k_{i}}=-\frac{P_{,_{i}}}{P_{,_{\omega}}}=\frac{P_{,_{i}}}{\xi_{j} P_{\xi_{j}}-\omega P,_{\omega}} \quad(i=x, y, z)
$$

The summation rule applies for repeated indices. If the fracture stiffness is not azimuthally isotropic, group velocity is a function of azimuthal angle $\phi$ for the source slowness vector. A general fracture impedance ratio matrix $\left[\beta_{i j}^{s}\right]$ can always be obtained by rotating a semidiagonal matrix without coupling between the $\mathrm{x}$ and $\mathrm{y}$-directions around the $\mathrm{z}$-axis as follows

$$
\left[\beta_{i j}^{S}\right]=Q^{T} \cdot\left[\begin{array}{ccc}
\beta_{1} & 0 & \beta_{13} \\
0 & \beta_{2} & \beta_{23} \\
\beta_{31} & \beta_{32} & \beta_{33}
\end{array}\right] \cdot Q=\left[\begin{array}{ccc}
\beta_{1} c^{2} \phi+\beta_{2} s^{2} \phi & \left(\beta_{1}-\beta_{2}\right) s \phi c \phi & \beta_{13} c \phi-\beta_{23} s \phi \\
\left(\beta_{1}-\beta_{2}\right) s \phi c \phi & \beta_{1} s^{2} \phi+\beta_{2} c^{2} \phi & \beta_{13} s \phi+\beta_{23} c \phi \\
\beta_{13} c \phi-\beta_{23} s \phi & \beta_{13} s \phi+\beta_{23} c \phi & \beta_{33}
\end{array}\right]
$$

where $c \phi=\cos \phi, s \phi=\sin \phi$. Therefore, the reference direction for the azimuthal angle can be defined as the first principal axis for the upper $2 \times 2$ matrix of the fracture impedance ratio matrix. The matrix $Q$ that rotates the coordinate system to an arbitrary direction is

$$
Q=\left[\begin{array}{ccc}
c \phi & s \phi & 0 \\
-s \phi & c \phi & 0 \\
0 & 0 & 1
\end{array}\right]
$$

Therefore the general dispersion equation Eq.(10.23) is expressed in cylindrical coordinates as $P=P\left(\xi, \phi, \xi_{z}, \omega\right)$ where $\xi=\sqrt{\xi_{x}{ }^{2}+\xi_{y}{ }^{2}}$ and $\phi=\tan ^{-1}\left(\xi_{y} / \xi_{x}\right)$. The slowness derivatives in Eq.(10.23) are computed by

$$
\begin{aligned}
& P_{,_{i}}=P,_{\xi} \partial \xi / \partial \xi_{i}+P_{,_{\phi}} \cdot \partial \phi / \partial \xi_{i}(i=x, y), \\
& P_{,_{\xi_{z}}}=P_{\hat{\xi}_{z}},
\end{aligned}
$$

or,

$$
\left\{\begin{array}{l}
P_{,_{\xi_{x}}} \\
P_{,_{\xi_{y}}} \\
P_{,_{\xi_{z}}}
\end{array}\right\}=P_{,_{\xi}}\left\{\begin{array}{c}
c \phi \\
s \phi \\
0
\end{array}\right\}+\frac{P,_{\phi}}{\xi}\left\{\begin{array}{c}
-s \phi \\
c \phi \\
0
\end{array}\right\}+P,_{\hat{\xi}_{z}}\left\{\begin{array}{l}
0 \\
0 \\
1
\end{array}\right\}
$$


Therefore, for a given combination of horizontal slownesses $\xi_{x}$ and $\xi_{y}$, the resulting group velocity of the wave can be computed.

\subsection{Decoupled SH-wave in Infinite Series of Regular Fractures}

\subsubsection{Decoupled SH-dispersion equation}

When shear fracture stiffness is azimuthally isotropic and there are no cross-coupling stiffnesses $\left(\beta_{x y}^{S}=\beta_{y x}^{S}=\beta_{z y}^{S}=\beta_{y z}^{S}=\beta_{z x}^{S}=\beta_{x z}^{S}=0\right)$, the dispersion equation (10.22) is decoupled as

$$
\begin{aligned}
& \Theta=0 \\
& \left(1-e^{i K_{z}^{S-h}}\right)\left(1-e^{-i K_{z}^{S+} h}\right)\left(\beta_{y y}^{S}+\Theta \cdot f_{R}\right)=0 \\
& \left(1-e^{i K_{z}^{P-} h}\right)\left(1-e^{-i K_{z}^{P+} h}\right)\left(1-e^{i K_{z}^{S-} h}\right)\left(1-e^{-i K_{z}^{S+} h}\right) \\
& \times\left\{\left(f_{P}-\beta_{z z}^{S} \Phi\right)\left(f_{Q}-\beta_{x x}^{S} \Theta\right)-g_{P} g_{Q}\right\}=0
\end{aligned} .
$$

Eq.(10:28a) is satisfied only when $\xi=k_{x} / k^{s}=1$. This is an SH-wave propagating parallel to the fractures. The second and the third equations are for decoupled SH- and PSV waves, respectively.

The dispersion equation Eq.(10.28b) can be written more explicitly as

$$
\cos \left(\hat{k}_{z} h\right)=\cos \left(k_{z}^{s} h\right)\left\{1-\gamma \cdot \frac{\tan \left(k_{z}^{s} h\right)}{\beta_{y y}^{s}}\right\}, \gamma \equiv \frac{k_{z}^{s}}{k^{s}} .
$$

In the following discussion, the behavior of the SH-wave described by Eq.(10.29) is first examined followed by a special case of $\mathrm{SH}$-wave propagation parallel to the fractures. To perform a parametric study to examine the effect of wave frequency and fracture stiffness, the following two dimensionless parameters are introduced

$$
\begin{aligned}
& \alpha=\frac{\omega h}{c_{s}}, \\
& b=\frac{\kappa_{y y} h}{\mu},
\end{aligned}
$$


where $\mu$ is the shear modulus of a single layer. Parameter $\alpha$ is a normalized frequency and $b$ is a normalized fracture stiffness. Using these parameters, the wave frequency and fracture stiffness can be altered independently.

\subsubsection{SH-Wave with oblique incidence}

By applying Eq.(10.23) to the dispersion equation (10.29), the group velocity of the $\mathrm{SH}$-wave for a specified combination of frequency and fracture stiffness is computed. A velocity surface for the SH-wave is obtained by sweeping over a range of normalized horizontal slownesses $\xi(0<\xi<1)$. Figure 10.4 shows computed group velocities for decoupled $\mathrm{SH}$-wave propagation over a range of frequencies. Poisson's ratio of the intact medium is assumed to be 0.2 . Velocities are computed only for real solutions of the dispersion equation. When the wavelength is relatively long compared with the fracture spacing, the velocity surface has an elliptic shape. Velocity surfaces computed using the static approximation are also shown for comparison. The aspect ratio of the ellipse increases as the fracture stiffness decreases. As the wave frequency increases, the elliptic wavefront increases the aspect ratio, indicating that the velocity anisotropy is frequencydependent. For wavelengths comparable to, or shorter than, the fracture spacing, the velocity surface is distorted due to strong multiple scattering and a filtering effect of the fractures. For certain ranges of the horizontal wave number $\xi$, no real solutions exist for vertical effective slowness or group velocity. This leads to multiplication of a velocity surface with gaps in horizontal group velocity. The width of the gaps increases with decreasing fracture stiffness.

In Chapter 2, it was observed that the spectrum of waves propagating in a periodically fractured one-dimensional system (see Figure $2.24 \mathrm{~b}$ ) is characterized by pass bands that permit non-attenuative propagation of waves and stop bands that prohibit wave propagation. The relation between the multiplied velocity surfaces and the spectra with alternating pass and stop bands can be seen by plotting velocity surfaces as a function of wave frequency (Figure 10.5). A vertical cross-section of the plot along the frequency axis at $C_{g x}=0$ yields the spectrum in Figure $2.24 \mathrm{~b}$. Each pass band in the Figure $2.24 \mathrm{~b}$ is a part of the lobes for the multiplied velocity surface. The plots in Figure 10.4 are vertical cross sections of the velocity surfaces along the horizontal group velocity axis for a frequency $\alpha$. It is noted that the lower frequency limits and higher horizontal group velocity limits of the pass bands are independent of fracture stiffness. From this observation, it can be seen that the velocity surface has only a single lobe if the normalized wave frequency is $\alpha / 2 \pi<0.5$. 

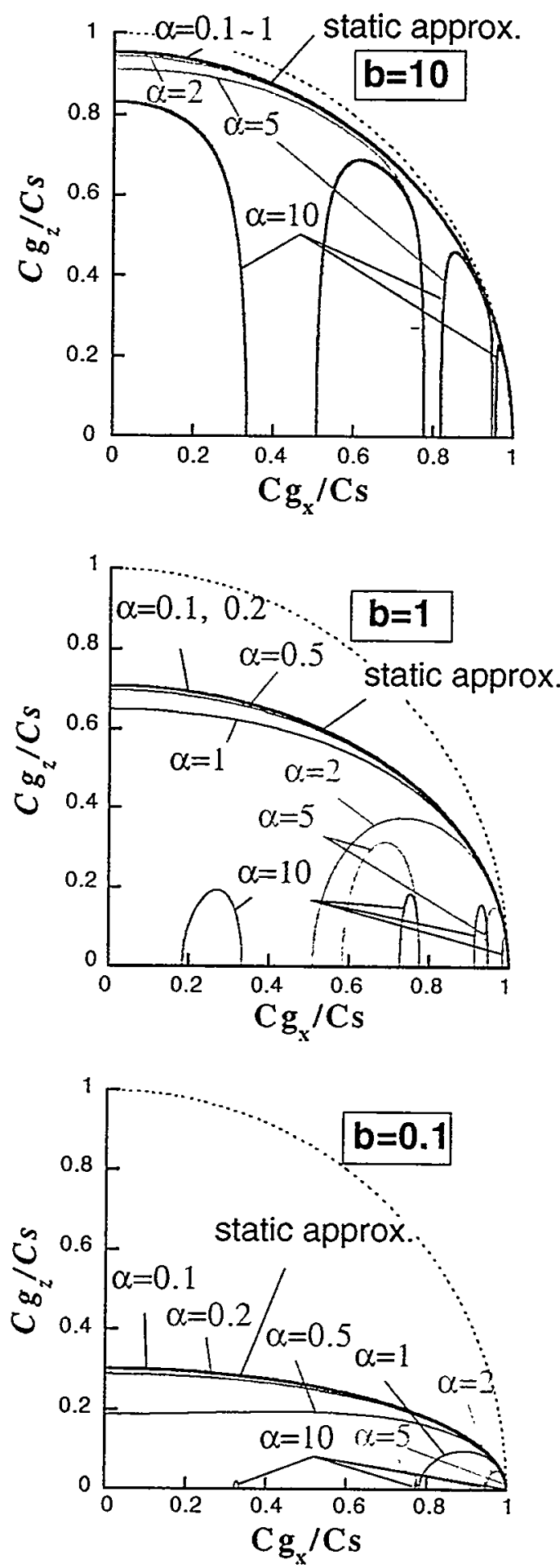

Figure 10.4 Velocity surfaces (group velocity) for decoupled SH-waves. The aspect ratio of the elliptic wavefront for low frequencies $(\alpha)$ increases with decreasing fracture stiffness (b). For high frequencies, the wavefront exhibits multiple lobes and regions of no vertical wave propagation. 


\subsubsection{SH-Wave propagation along fractures}

In a stop band of the frequency dependent velocity surface, waves propagate horizontally through the fractured medium (parallel to the fractures). Particularly at boundaries between pass bands and stop bands, the vertical group velocity becomes zero. Such waves can be seen as generalized cases of SH-plate waves in multiple plates coupled by the displacement-discontinuity boundary conditions. Conditions for such waves to exist are found by applying Eq.(10.23) to Eq.(10.28) or (10.29). The expression for the zdirection group velocity has a numerator

$$
P_{\hat{\xi}_{z}}=\sin \left(\hat{k}_{z} h\right)=\sin \left(\alpha \hat{\xi}_{z}\right)
$$

Therefore, a condition for vertical group velocity to vanish is

$$
\sin \left(\hat{k}_{z} h\right)=0 \text { or } \hat{k}_{z} h=n \pi \quad(\mathrm{n}=0,1,2, \ldots) .
$$

From Eq.(10.29), $\hat{k}_{z} h$ becomes a purely imaginary number if the right hand side of the equation is larger than unity

$$
\hat{k}_{z} h=i \cdot \hat{k}_{l z} h, \hat{k}_{l z} \in R \text {. }
$$

On the other hand, if the equation is smaller than -1 , a wavenumber that satisfies the equation is

$$
\hat{k}_{z} h=\pi+i \cdot \hat{k}_{l z} h, \hat{k}_{l z} \in R .
$$

Regions where real, imaginary, and complex solutions result are graphically represented for the case $\alpha=2 \pi \times 3.5$ (Figure 10.6(a)) and $\xi=0.707$ (incident angle $\theta=45^{\circ}$ ) (Figure $10.6(b))$. Oscillation of the equation is due to the $\cos \left(k_{z}^{s} h\right)$ term in Eq.(10.29). The amplitude of the oscillation increases monotonically with increasing wave frequency, resulting in reduction of the width of pass bands

When Eq.(10.32) is satisfied, the SH wave propagates parallel to the fractures without attenuating in the vertical direction. The condition can be classified into two subconditions

$$
\begin{array}{ll}
\hat{k}_{z} h=2 m \pi & (\mathrm{m}=0,1,2, \ldots), \\
\hat{k}_{z} h=(2 m+1) \pi & (\mathrm{m}=0,1,2, \ldots) .
\end{array}
$$




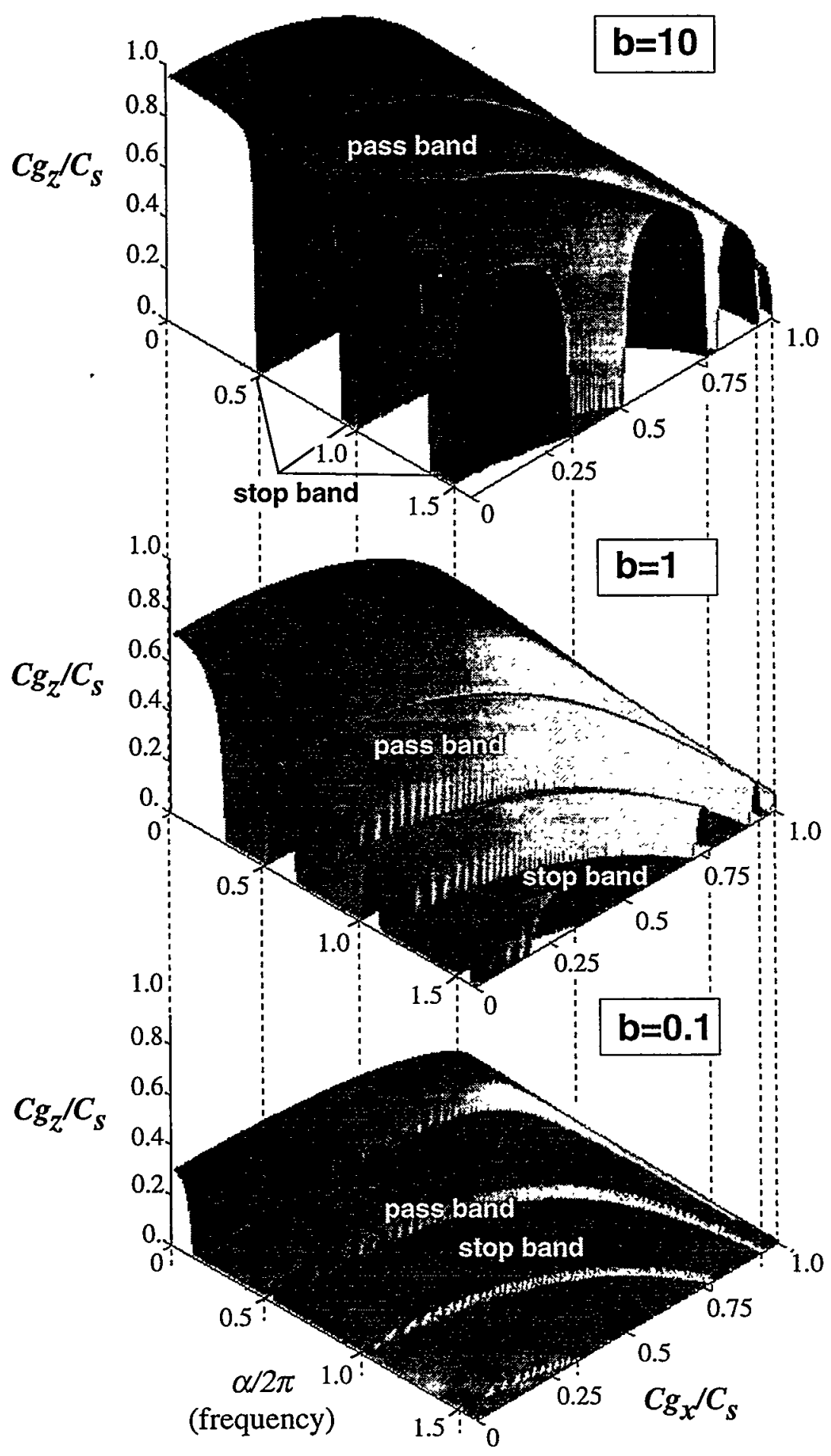

Figure 10.5 Velocity surfaces for SH-wave propagation in an infinite series of regularly spaced parallel fractures. A cross section of the surface along the frequency axis is a spectrum. A cross section along a horizontal group velocity axis is an anisotropic velocity surface for the selected frequency. Upper and lower frequency limits of each pass band are the waves propagating parallel to the fractures. Waves for the lower limits are insensitive to the change in fracture stiffness. 


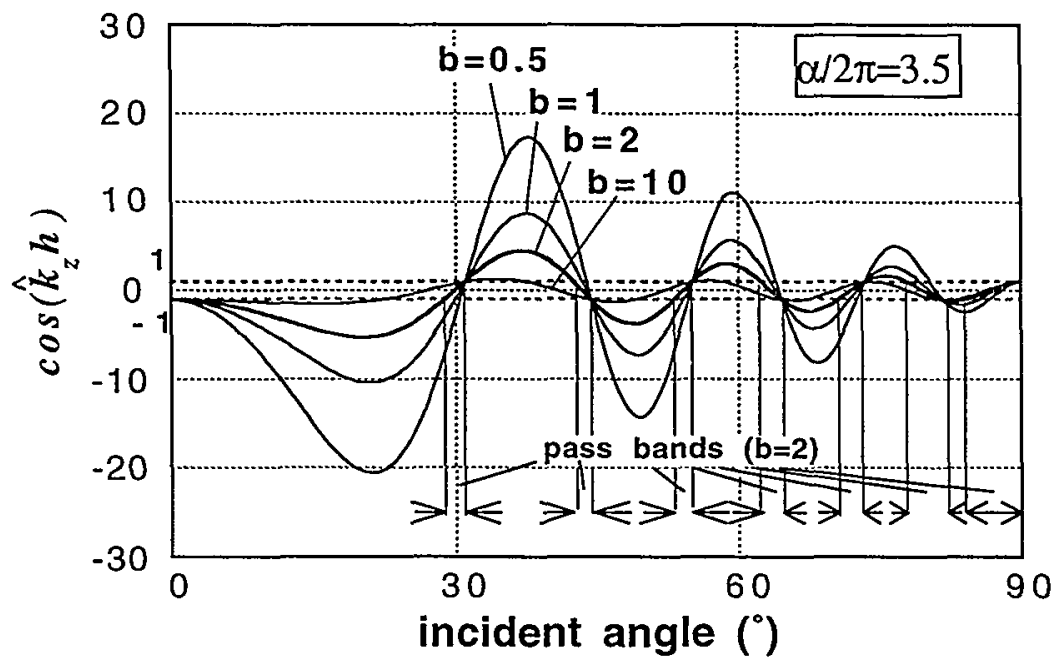

(a) constant wave frequency $(\alpha / 2 \pi=3.5)$.

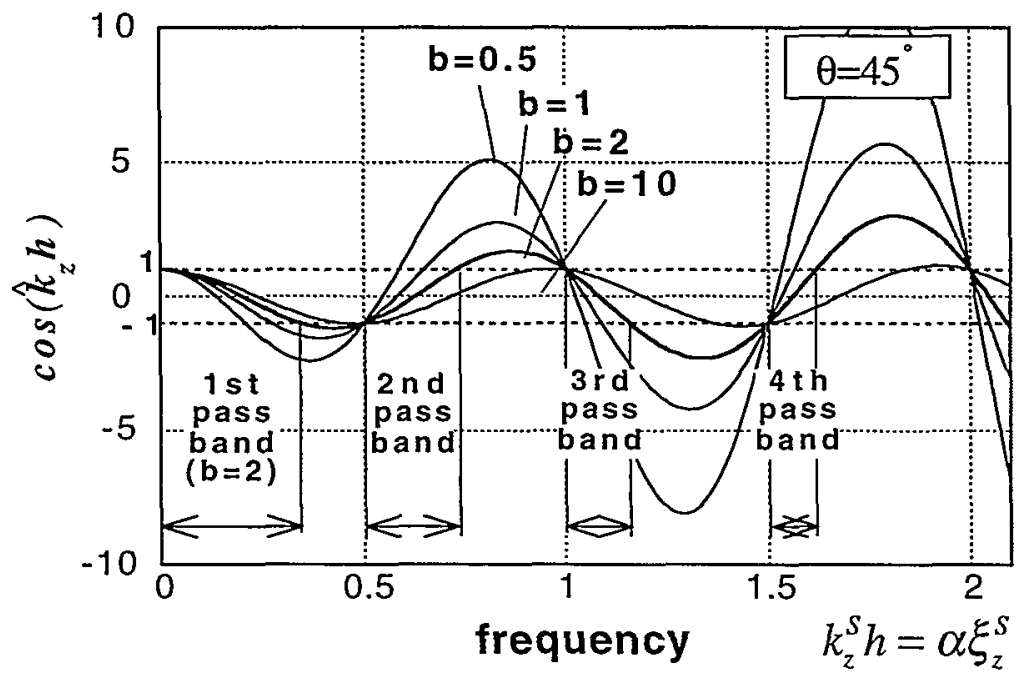

(b) constant horizontal wave number $(\xi=0.707)$.

Figure 10.6 Behavior of the SH-dispersion equation. The value of the equation oscillates around zero. Where the value is between -1 to 1 , a real-valued solution for $z$-direction slowness (or wavenumber) results. If the value is larger than 1 or smaller than -1 , a pure imaginary solution or a complex solution (of the form $\hat{k}_{z} h=\pi+i \hat{k}_{z I}, \hat{k}_{z I}, \in R$ ) is obtained. Regions with real solutions are the pass bands whose width decreases with increasing wave frequency. Note that each pass band for different fracture stiffnesses has a shared point at the boundary between a pass and a stop band.

By introducing Eq.(10.34a) into Eq.(10.6a) and (10.6b), it can be seen that the first set of conditions produces no phase shift in the vertical direction over layer thickness $h$. Therefore, wave motions in adjacent layers are identical. In contrast, Eq.(10.34b) leads to $180^{\circ}$ of phase shift across a layer. The corresponding dispersion equations for both conditions are 


$$
\begin{aligned}
& \sin \left(\frac{k_{z}^{s} h}{2}\right)\left\{b+\left(\frac{k_{z}^{s} h}{2}\right) \cdot \cot \left(\frac{k_{z}^{s} h}{2}\right)\right\}=0, \\
& \cos \left(\frac{k_{z}^{s} h}{2}\right)\left\{b+\left(\frac{k_{z}^{s} h}{2}\right) \cdot \tan \left(\frac{k_{z}^{s} h}{2}\right)\right\}=0,
\end{aligned}
$$

respectively. It is noted that the first terms in Eq.(10.35a) and (10.35b) yield the following conditions, independent of fracture stiffness

$$
\begin{array}{ll}
\sin \left(\frac{k_{z}^{s} h}{2}\right)=0, \text { or } k_{z}^{s} h=2 m \pi & (\mathrm{m}=0,1,2, \ldots), \\
\cos \left(\frac{k_{z}^{s} h}{2}\right)=0, \text { or } k_{z}^{s} h=(2 m+1) \pi \quad(\mathrm{m}=0,1,2, \ldots) .
\end{array}
$$

The second terms in Eq.(10.35a) and (10.35b) depend on the stiffness of the fracture. When the fracture stiffness approaches zero $(b \rightarrow 0)$, these terms yield conditions

$$
\begin{array}{ll}
\cos \left(\frac{k_{z}^{s} h}{2}\right)=0, \text { or } k_{z}^{s} h=(2 m+1) \pi & (\mathrm{m}=0,1,2, \ldots), \\
\sin \left(\frac{k_{z}^{s} h}{2}\right)=0, \text { or } k_{z}^{s} h=2 m \pi & (\mathrm{m}=0,1,2, \ldots) .
\end{array}
$$

For fractures with very high stiffness, $b \rightarrow \infty$ and

$$
\begin{array}{ll}
\sin \left(\frac{k_{z}^{s} h}{2}\right)=0, \text { or } k_{z}^{s} h=2 m \pi & (\mathrm{m}=0,1,2, \ldots) . \\
\cos \left(\frac{k_{z}^{s} h}{2}\right)=0, \text { or } k_{z}^{s} h=(2 m+1) \pi & (\mathrm{m}=0,1,2, \ldots) .
\end{array}
$$

These results show that the solutions of the dispersion equations as a function of fracture stiffness move between the stiffness-independent solutions of Eq.(10.36a) and (10.36b). The dispersion behavior of the generalized SH-plate wave is shown in Figure 10.7 for selected values of fracture stiffness $(b=0.1,1,10)$. Velocities shown in the plot are the group velocity parallel to the fractures. As has been discussed, both stiffness-independent and stiffness-dependent modes exist. Essentially, the plot is a cross section of the velocity surface shown in Figure 10.5 at the zero vertical group velocity. 

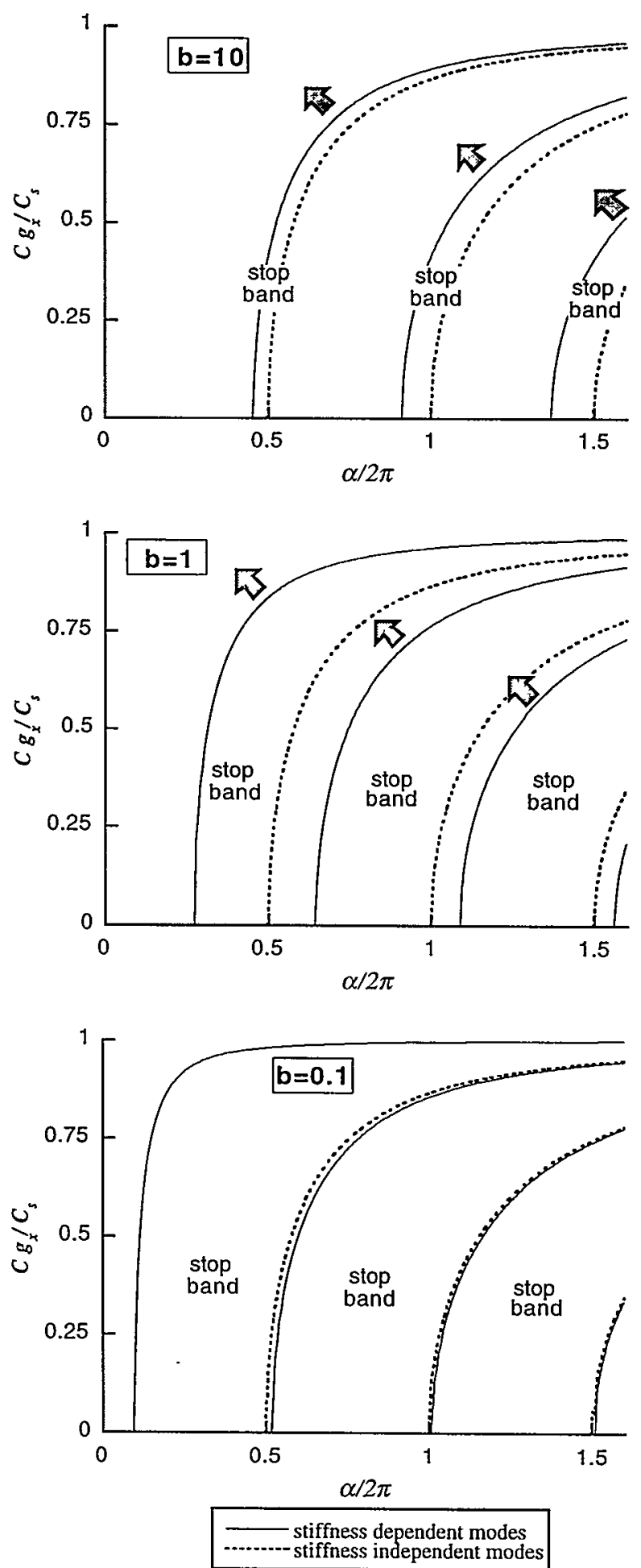

Figure 10.7 Group-velocity dispersion curves for generalized SH-plate waves. Each mode is either dependent on or independent of the fracture stiffness. Each stiffness-dependent mode shifts between two stiffness-independent modes for varying fracture stiffness. 
When Eq.(10.33a) or (10.33b) is satisfied, the amplitude of the SH-wave in the layers decays exponentially away from the source. Therefore the wave motion is localized in the vertical direction without energy dissipation or radiation. Such behavior can be examined quantitatively by computing the particle motion within the layers.

\subsubsection{Particle motions of SH-waves.}

Particle motions in the fractured medium are plotted as a function of horizontal slowness in Figure 10.8(a) and (b). The assumed fracture stiffness is $b=1$ and a normalized wave frequency is $\alpha / 2 \pi=0.5$. For this wave frequency, the changes in the behavior of SH-waves for a single pass band and stop band can be examined by sweeping over a range of horizontal slownesses $(0 \leq \xi \leq 1)$. The fracture spacing $h=0.1 \mathrm{~m}$. It should be noted that for the SH-case, a normalized horizontal group velocity computed from Eq.(10.23) is always identical to a normalized horizontal slowness (or wavenumber).

When $\xi=0$, the wavefield is at a boundary between a stop band and a pass band. From the discussion in the previous subsection, there is no vertical wave propagation for this state and the $\mathrm{SH}$-wave propagates parallel to fractures. From Figure 10.7, this is an SH-plate wave mode independent of fracture stiffness. The particle motion of the mode has the anti-nodes corresponding exactly to the location of fractures (Figure 10.8(b)). As examined analytically in Chapter 2 and experimentally in Chapter 3, fracture stiffness has no effect on the behavior of the mode because stress on the anti-nodes is always zero.

With increasing horizontal slowness (or increasing angle of incidence), the state of the wave passes through a stop band. Both Figure 10.8(a) and (b) show wave motions localized near the source, exponentially decaying in vertical direction. It is noted that the decay in amplitude occurs only across fractures and no amplitude decay occurs within individual layers. The degree of localization is stronger for the center of the stop band and weaker for the edges of the band.

At the other boundary between a stop band and a pass band ( $\xi=0.837)$, once again, the wave motion exists ubiquitously in the fractured medium. Unlike on the other boundary, however, the mode shape in the vertical direction is discontinuous across the fractures. As the discontinuous displacement across a fracture generates stress for finite fracture stiffnesses, the behavior of the mode depends on the fracture stiffness. Within the pass band, there is no decay of wave amplitude in the vertical direction. From Figure 10.8(b) it can be seen that waves with coherent phase are regenerated for high angles of incidence. 

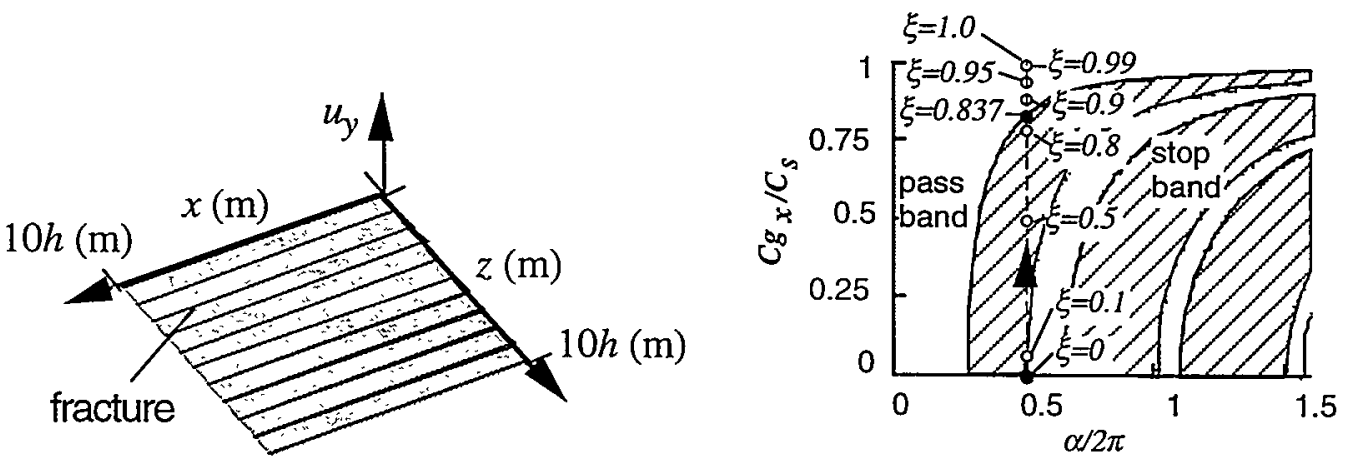

$\xi\left(=C g_{x} / C_{s}\right)=0$

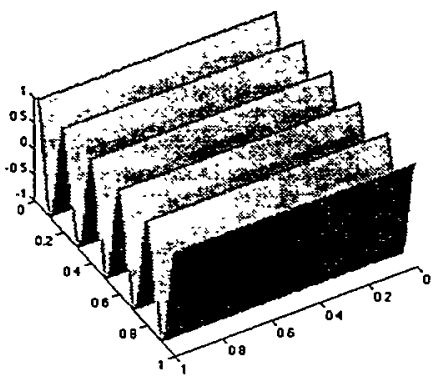

$\xi\left(=C g_{x} / C_{s}\right)=0.1$

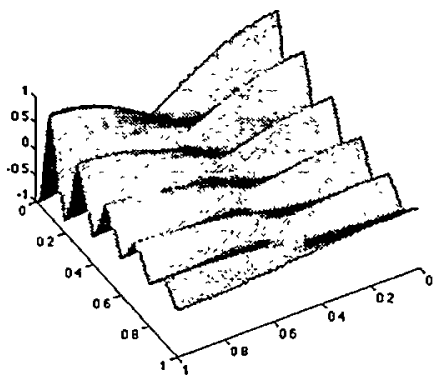

$\xi\left(=C g_{x} / C_{s}\right)=0.5$

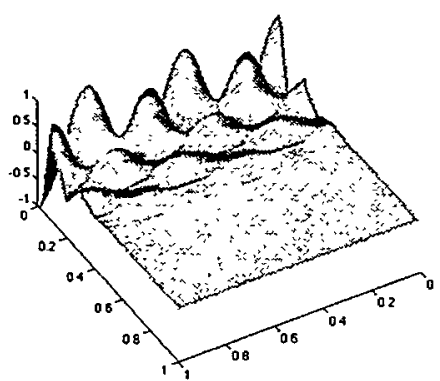

$\xi\left(=C g_{x} / C_{s}\right)=0.8$

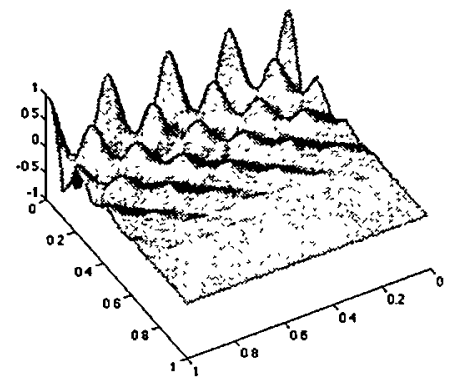

$\xi\left(=C g_{x} / C_{s}\right)=0.837$

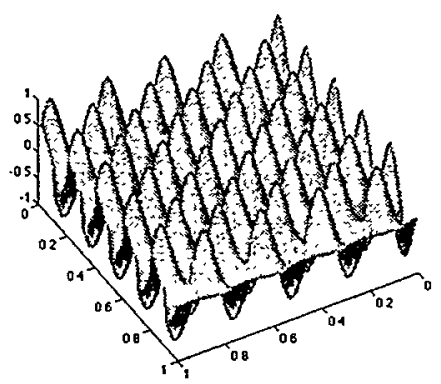

$\xi\left(=C g_{x} / C_{s}\right)=0.9$

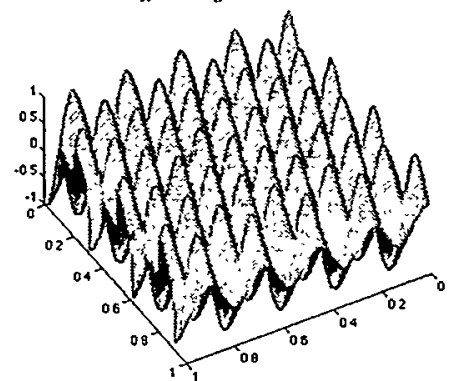

$\xi\left(=C g_{x} / C_{s}\right)=0.95$

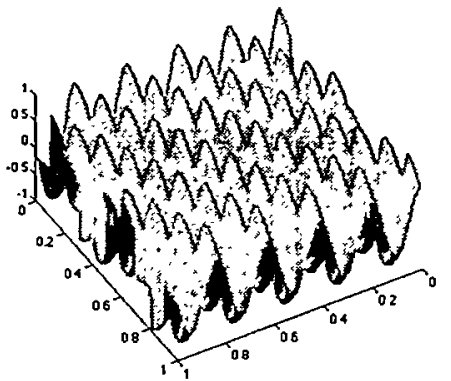

$\xi\left(=C g_{x} / C_{s}\right)=0.99$

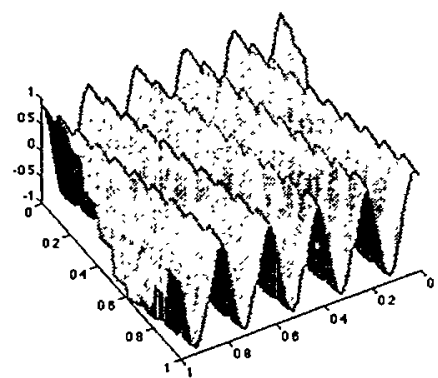

$\xi\left(=C g_{x} / C_{s}\right)=1.0$

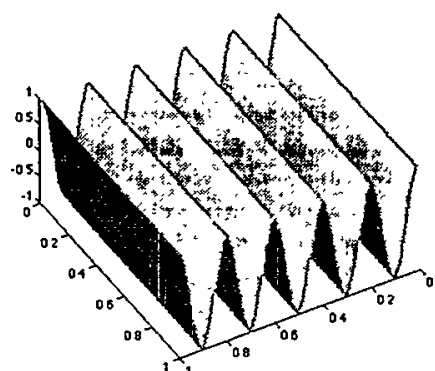

Figure 10.8 (a) Snapshots for obliquely incident SH-waves in a medium containing periodic parallel fractures. Normalized frequency is $\alpha / 2 \pi=0.5$. In the stop band $(0<\xi<0.837)$, wave motion localizes near the source $(\mathrm{z}=0)$. Within a pass band, wave motion is present ubiquitously. 

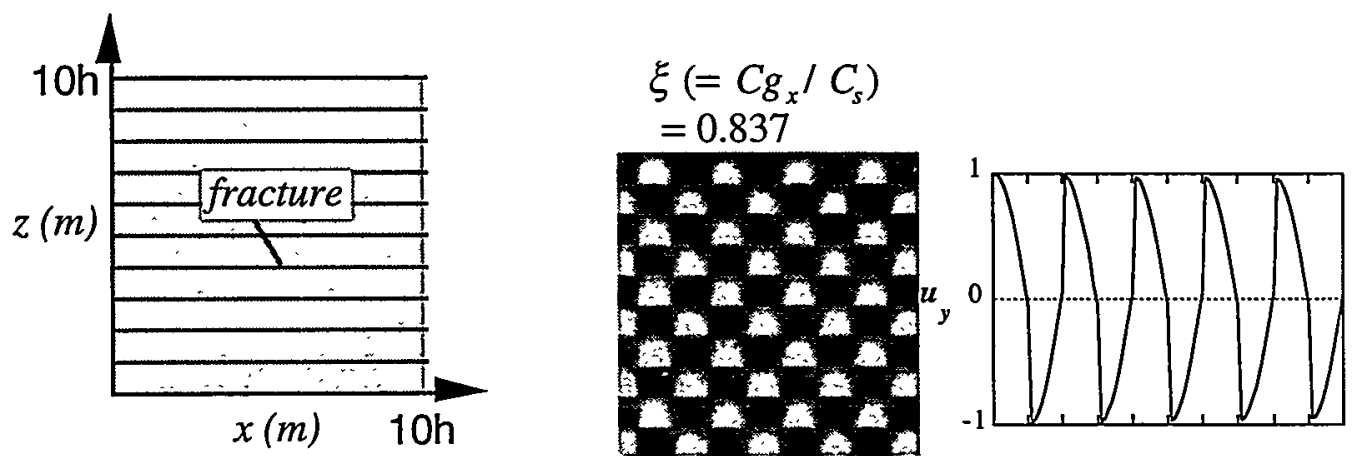

$\xi\left(=C g_{x} / C_{s}\right)=0$
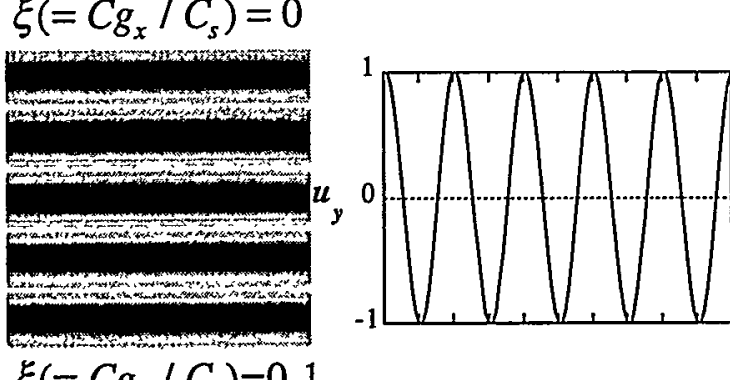

$$
\xi\left(=C g_{x} / C_{s}\right)=0.9
$$

$\xi\left(=C g_{x} / C_{s}\right)=0.1$
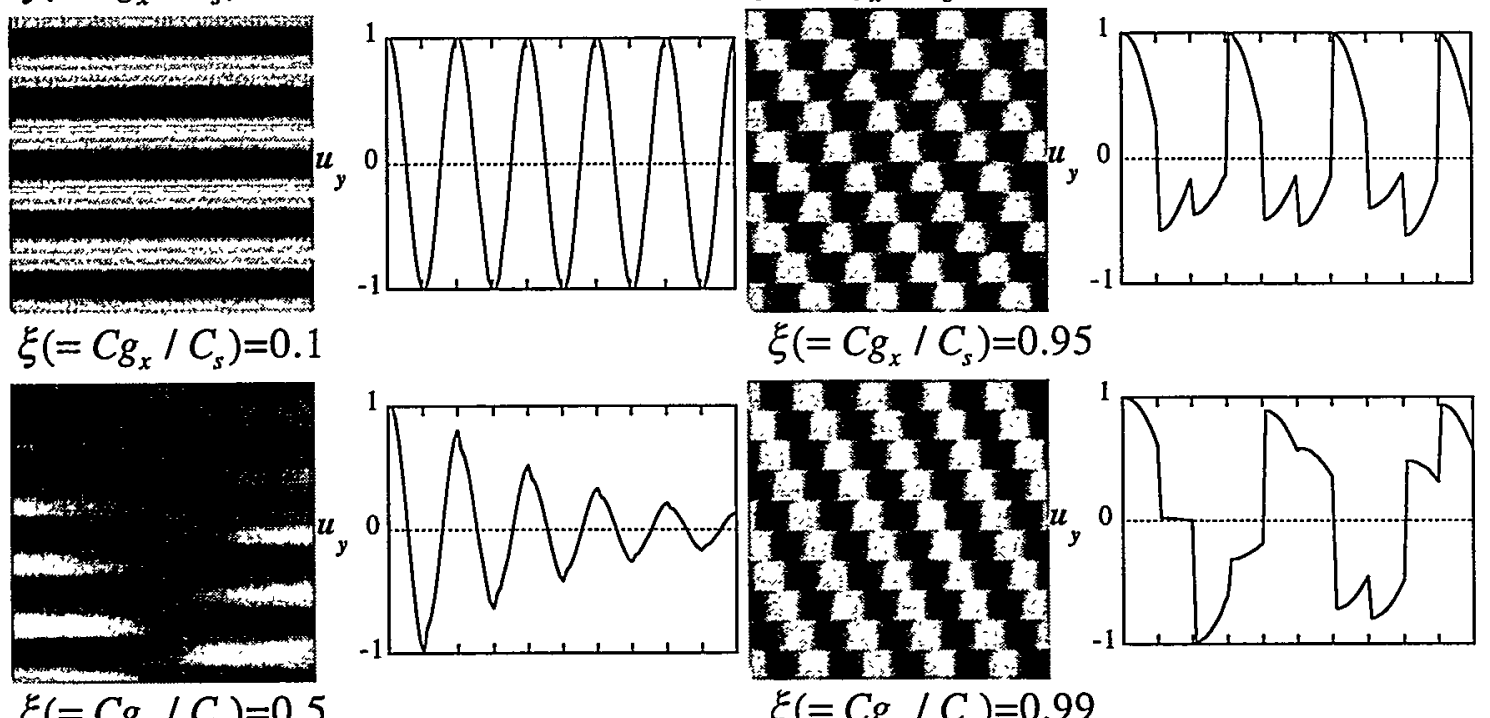

$\xi\left(=C g_{x} / C_{s}\right)=0.5$
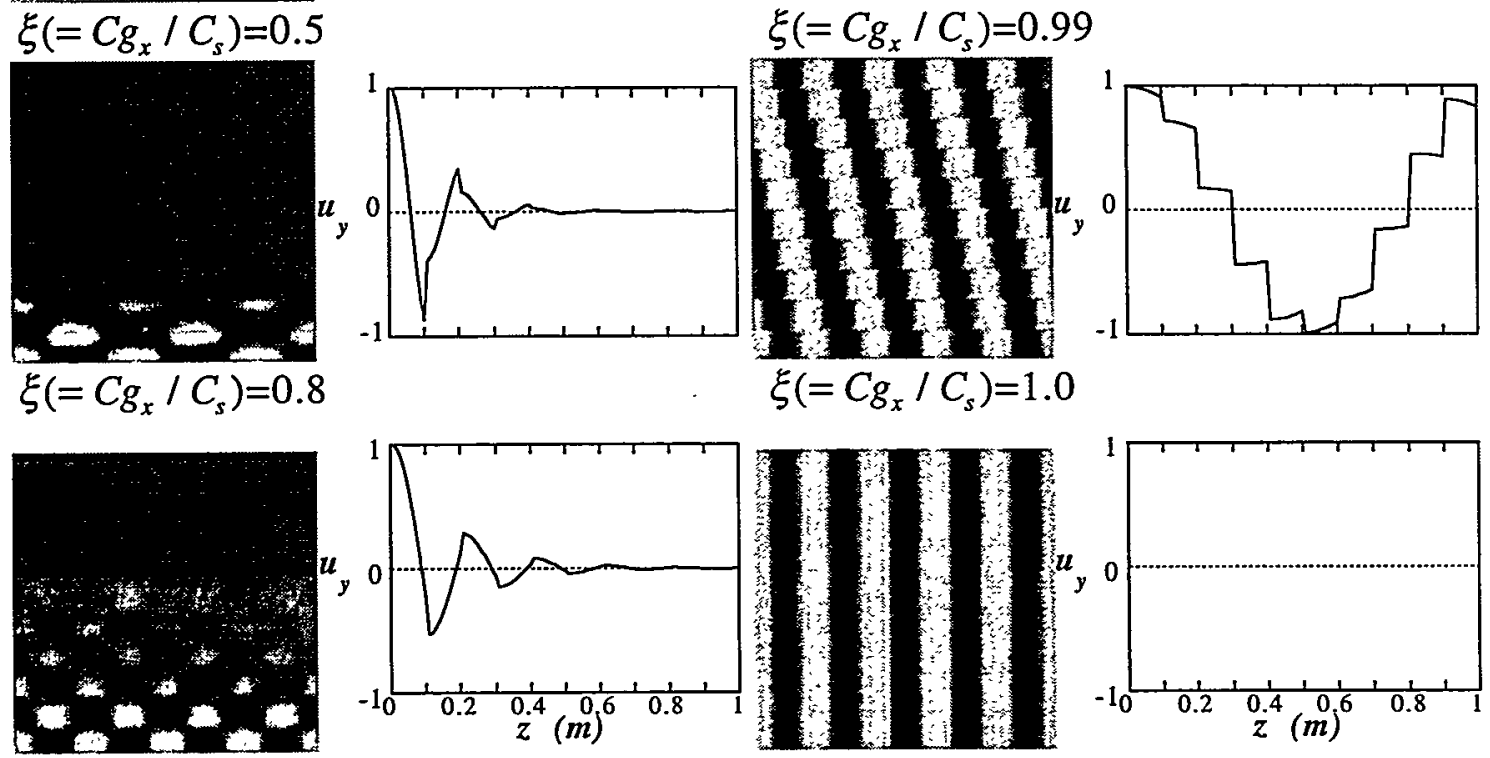

Figure 10.8 (b) Views from the top of Figure 10.8(a). High and low amplitudes are shown in light and dark grays, respectively. Line diagrams to the left of each density diagram show amplitude of the wave measured at $\mathrm{x}=0(\mathrm{~m})$ along the $\mathrm{z}$-direction. 


\subsection{Decoupled P-SV Waves in Infinite Series of Regularly Spaced Parallel Fractures}

\subsubsection{Obliquely incident P-SV wave}

A decoupled dispersion equation for P-SV wave propagation in a periodically fractured system is given by Eq.(10.28c). Solutions of the equation are examined using the dimensionless parameters defined by Eq.(10.30a) and (10.30b).

Velocity surfaces (group velocity) for a range of fracture stiffnesses are shown in Figure 10.9. A constant normalized wave frequency $(\alpha=1)$ and an isotropic fracture stiffness $\left(b_{i j}=b\right.$ for $\mathrm{i}=\mathrm{j}$ and $b_{i j}=0$ for $i \neq j$ ) are assumed. For comparison, velocity surfaces that are computed from a static equivalent medium model for fractured media (Schoenberg and Douma, 1988; Schoenberg and Muir, 1989) are also shown. Dotted quarter circles with normalized group velocities of 1 and 1.62 are the reference velocities for $\mathrm{S}$ - and $\mathrm{P}$-waves in an intact medium, respectively. As the wavelength is much longer than the fracture spacing $\left(h / \lambda_{P}=0.097, h / \lambda_{s}=0.16, \lambda_{P}\right.$ and $\lambda_{S}$ are $\mathrm{P}$ and $S$ - wave wavelength, respectively), both the effective medium (static) approximation and the exact solution yield similar results for relatively high fracture stiffnesses (Figure 10.9(a)). However, for medium to low fracture stiffnesses ( $b \sim 1$ or smaller), the difference between the two solutions becomes significant (Figure 10.9(b)). For very low fracture stiffnesses, the velocity surface for the exact solution exhibits the multiplication, while the effective medium model always yields continuous elliptic wavefronts .

The dotted sections of the SV-wave velocity surfaces for the exact solutions are computed for horizontal slowness $\xi>1$. Although a plane wave source cannot generate such waves, a wavefront often has a finite curvature in real situations that leads to a normalized slowness greater than unity. For a wave incident on a single fracture, scattered waves with a horizontal slowness $\xi>1$ become heterogeneous, yielding a complex vertical slowness. It is interesting that a real effective vertical slowness $\hat{\xi}_{z}$ can result for the case of an infinite series of fractures.

Changes in the velocity surface when the wave frequency is increased and the fracture stiffness is held constant are shown in Figure 10.10. As is observed for the SH-wave, the multiple velocity surfaces appear as the wavelength approaches the fracture spacing. 
[Static approximation]

[Exact solution]
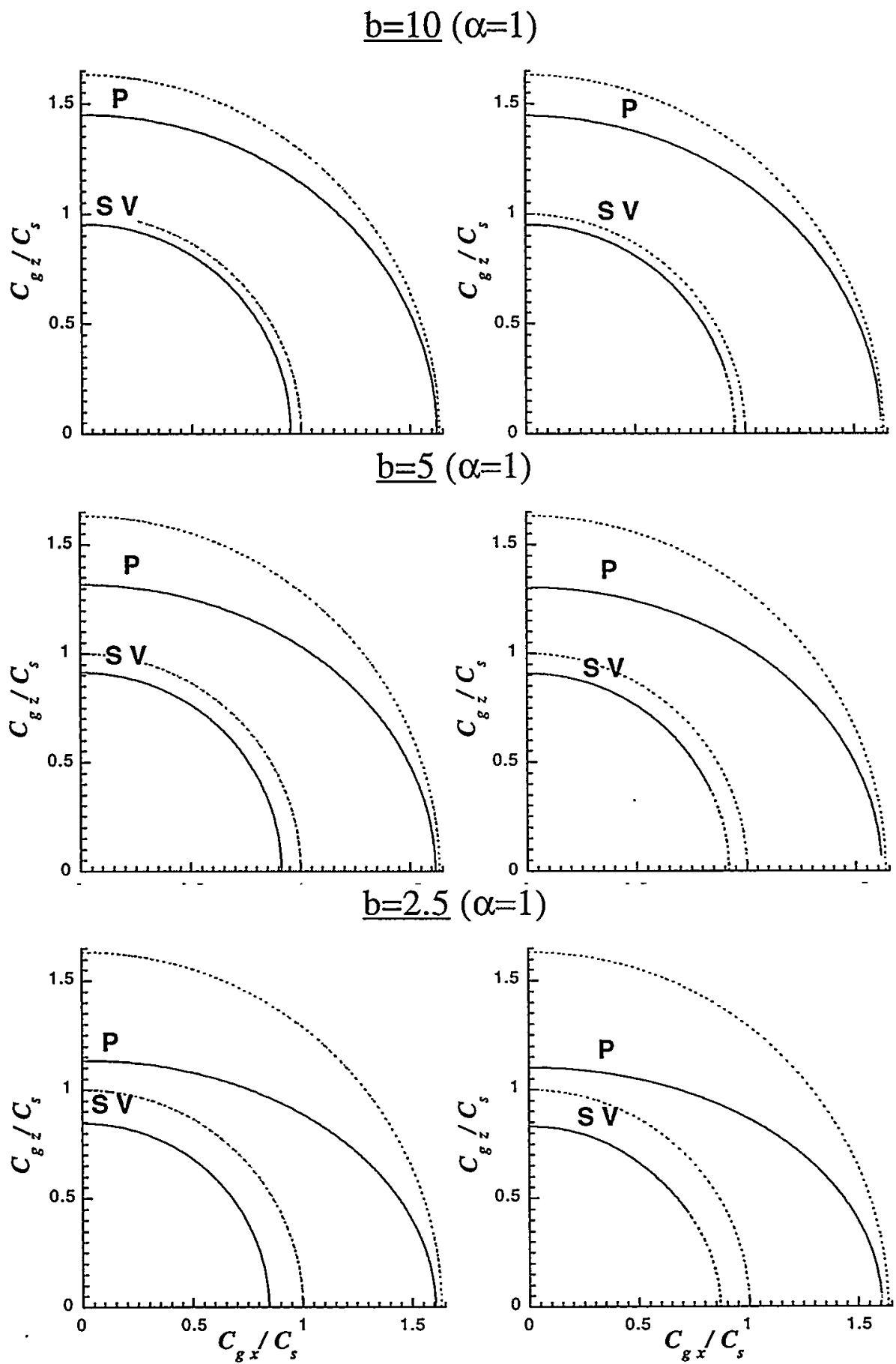

Figure 10.9 (a) Velocity surfaces of $P$ and SV-waves computed for static effective medium models (left) and exact solutions of the dispersion equation for fractured media (right). Dotted quarter circles are reference velocities for $\mathrm{P}$ and $\mathrm{S}$ - waves in an intact medium. For a long wavelength $(\alpha=1)$ and high fracture stiffnesses, both results are almost identical. The dotted sections of the SV-wave velocity surfaces for the exact solutions are computed for a horizontal slowness of $\xi 1$. 
[Static approximation]

[Exact solution]
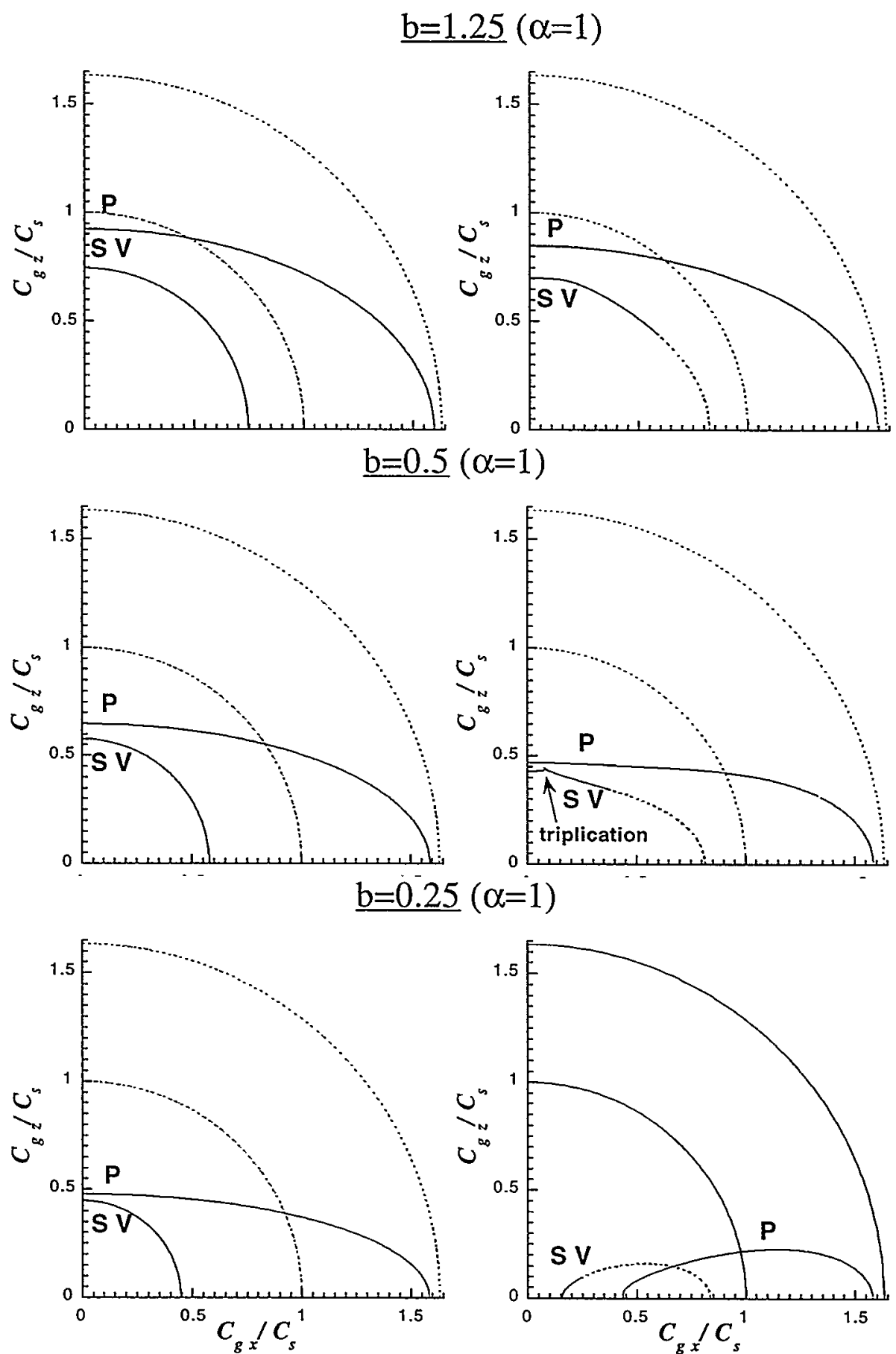

Figure 10.9 (b) Velocity surfaces of $\mathrm{P}$ and SV-waves computed for static effective medium models (left) and exact solutions of the dispersion equation for fractured media (right). Dotted quarter circles are reference velocities for $\mathrm{P}$ and $\mathrm{S}$ - waves in an intact medium. For low fracture stiffnesses, the difference between the results becomes significant. The exact solution shows triplication (case $b=0.5$ ) and multiple group velocities in single directions (case $b=0.25$ ) for very low fracture stiffnesses. 


\section{[Exact solution]}
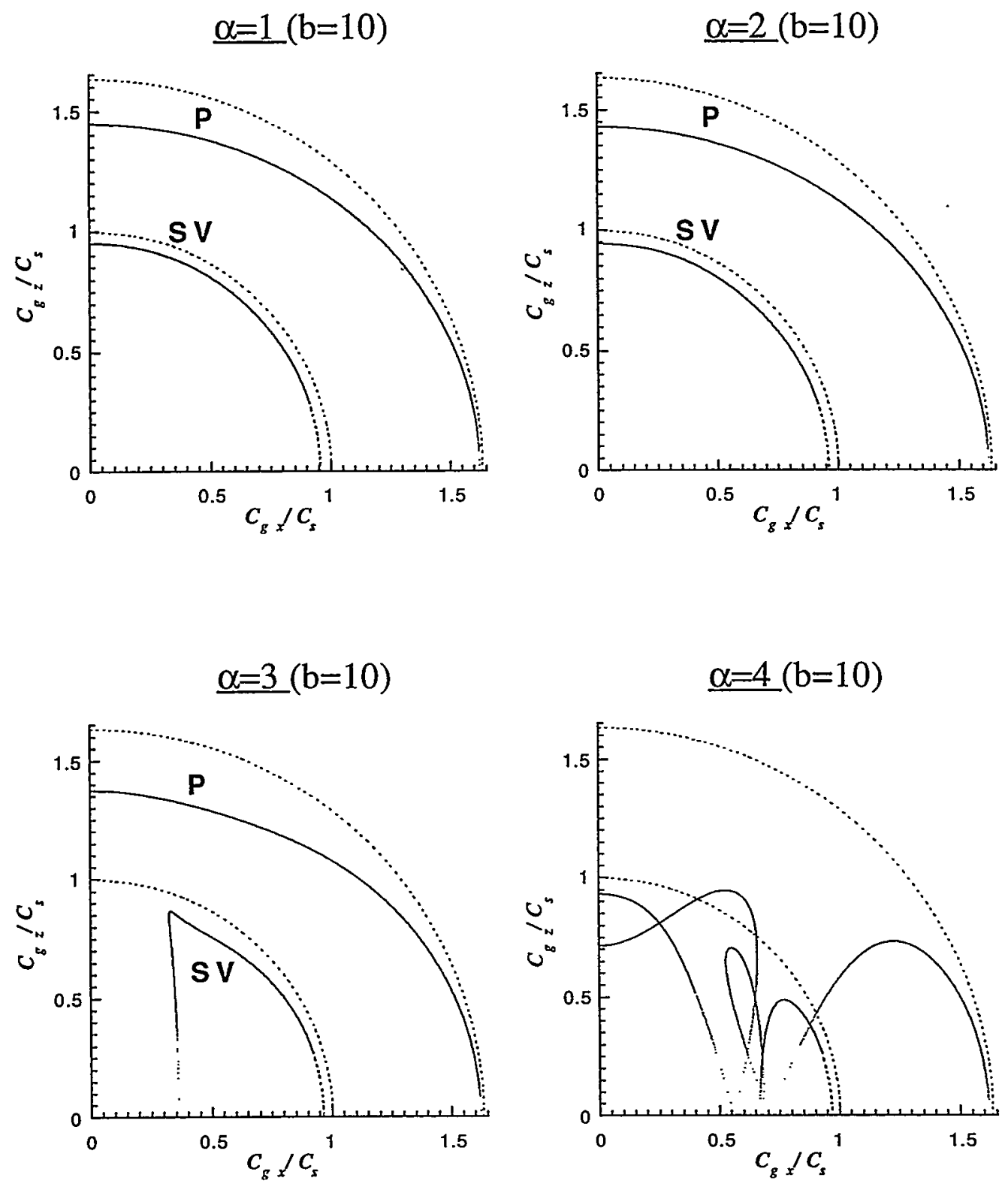

Figure 10.10 Velocity surfaces of $P$ and $S V$-waves computed for a constant fracture stiffness $(b=10)$ and a range of wave frequencies. As the wavelength approaches the fracture spacing, stop bands are formed, separating the velocity surface to multiple segments. 
However, in contrast to the SH-wave case, the velocity surfaces show much more complicated behavior, possibly due to coupling between $\mathrm{P}$ and SV- waves. Each velocity surface has single or multiple intercepts with the zero vertical group velocity axis. At these points, waves in the system are propagating parallel to the fractures. The behavior of the waves propagating parallel to the fractures is examined in the following subsection.

\subsubsection{Generalized Rayleigh-Lamb plate waves}

A condition equivalent to the generalized SH-plate wave (Eq.(10.32)) can be obtained for the P-SV wave propagating parallel to the fractures. In the absence of coupling fracture stiffnesses, the numerator of the vertical group velocity for the P-SV wave can be written in the form of

$$
P_{\hat{\xi}_{z}}=\sin \left(\hat{k}_{z} h\right) \cdot \text { func. }\left(k_{x}\right)=\sin \left(\alpha \hat{\xi}_{z}\right) \cdot \text { func. }(\xi) .
$$

Therefore, a condition for the group velocity to vanish is

$$
\sin \left(\hat{k}_{z} h\right)=0 \text { or } \hat{k}_{z} h=n \pi \quad(\mathrm{n}=0,1,2, \ldots) .
$$

or

$$
\text { func. }\left(k_{x}\right)=0 \text {. }
$$

The condition given by Eq.(10.40a) yields two subconditions

$$
\begin{array}{ll}
\hat{k}_{2} h=2 m \pi & (\mathrm{m}=0,1,2, \ldots), \\
\hat{k}_{z} h=(2 m+1) \pi & (\mathrm{m}=0,1,2, \ldots) .
\end{array}
$$

For Eq.(10.41a) the dispersion equation becomes

$$
\begin{aligned}
& \left(1-e^{i k_{2}^{p} h}\right)\left(1-e^{-i k_{2}^{p} h}\right)\left(1-e^{i k_{2}^{S h}}\right)\left(1-e^{-i k_{2}^{S h}}\right), \\
& \times\left(\beta_{x x}^{S} \Theta-f_{q}\right) \cdot\left(\beta_{x x}^{S} \Phi-f_{p}\right)=0
\end{aligned}
$$

where,

$$
\begin{aligned}
& f_{P}=f_{p} \equiv \operatorname{coth}\left(\frac{\alpha \Phi}{2}\right) \cdot\left(2 \xi^{2}-1\right)^{2}-\operatorname{coth}\left(\frac{\alpha \Theta}{2}\right) \cdot 4 \xi^{2} \Phi \Theta, \\
& f_{Q}=f_{q} \equiv \operatorname{coth}\left(\frac{\alpha \Theta}{2}\right) \cdot\left(2 \xi^{2}-1\right)^{2}-\operatorname{coth}\left(\frac{\alpha \Phi}{2}\right) \cdot 4 \xi^{2} \Phi \Theta,
\end{aligned}
$$


and for Eq.(10.41b),

$$
\begin{aligned}
& \left(1+e^{i k_{2}^{p} h}\right)\left(1+e^{-i k_{2}^{p} h}\right)\left(1+e^{i k_{2}^{S} h}\right)\left(1+e^{-i k_{2}^{S} h}\right) \\
& \times\left(\beta_{x x}^{S} \Theta-f_{q}\right) \cdot\left(\beta_{x x}^{S} \Phi-f_{p}\right)=0
\end{aligned}
$$

where,

$$
\begin{aligned}
& f_{P}=f_{p} \equiv \tanh \left(\frac{\alpha \Phi}{2}\right) \cdot\left(2 \xi^{2}-1\right)^{2}-\tanh \left(\frac{\alpha \Theta}{2}\right) \cdot 4 \xi^{2} \Phi \Theta \\
& f_{Q}=f_{q} \equiv \tanh \left(\frac{\alpha \Theta}{2}\right) \cdot\left(2 \xi^{2}-1\right)^{2}-\tanh \left(\frac{\alpha \Phi}{2}\right) \cdot 4 \xi^{2} \Phi \Theta
\end{aligned}
$$

Together, Eq.(10.43a), (10.43b) and (10.45a), (10.45b) are the Rayleigh-Lamb plate wave equations (Graff, 1975). For zero fracture stiffness, the dispersion equations Eq.(10.42) and (10.44) provide solutions for P-SV wave propagation in a single plate. It should be noted that Eq.(10.43a) and Eq.(10.45b) yield identical symmetric motions while Eq.(10.43b) and Eq.(10.45a) yield identical antisymmetric motions

Dispersion relations for the generalized Rayleigh-Lamb plate wave over a range of fracture stiffnesses are shown in Figure 10.11 (a) and (b). When the fracture stiffness is zero, the dispersion relations for the classical Rayleigh-Lamb plate waves are obtained. As indicated by Eq. $(10.43 a, b)$ and $(10.43 a, b)$, each mode is doubly degenerate. As the fracture stiffness increases, each mode splits to form a mode pair. It can be shown that one out of each mode pair has identical motions in neighboring layers ( 0 mode) while the other shows a motion with a phase $180^{\circ}$ different from the neighboring layers ( $\pi$ mode). The difference in the dispersion behaviors of the modes increase with increasing fracture stiffness. For high fracture stiffnesses, the generalized Rayleigh-Lamb modes once again form pairs but with different partners. The first two modes that correspond to the symmetric and antisymmetric Rayleigh-Lamb plate waves asymptote to bulk P and Swaves, respectively.

Although the behavior of the P-SV wave modes in the multiply fractured system is similar to the SH-wave modes, unlike the SH-case, all modes change their behavior with fracture stiffness. This is because stationary $\mathrm{P}$ and SV-waves with identical horizontal slowness cannot have their anti-nodes on the fractures simultaneously. As either P or SVwaves always generate stress across fractures, the behavior of the waves becomes stiffness 

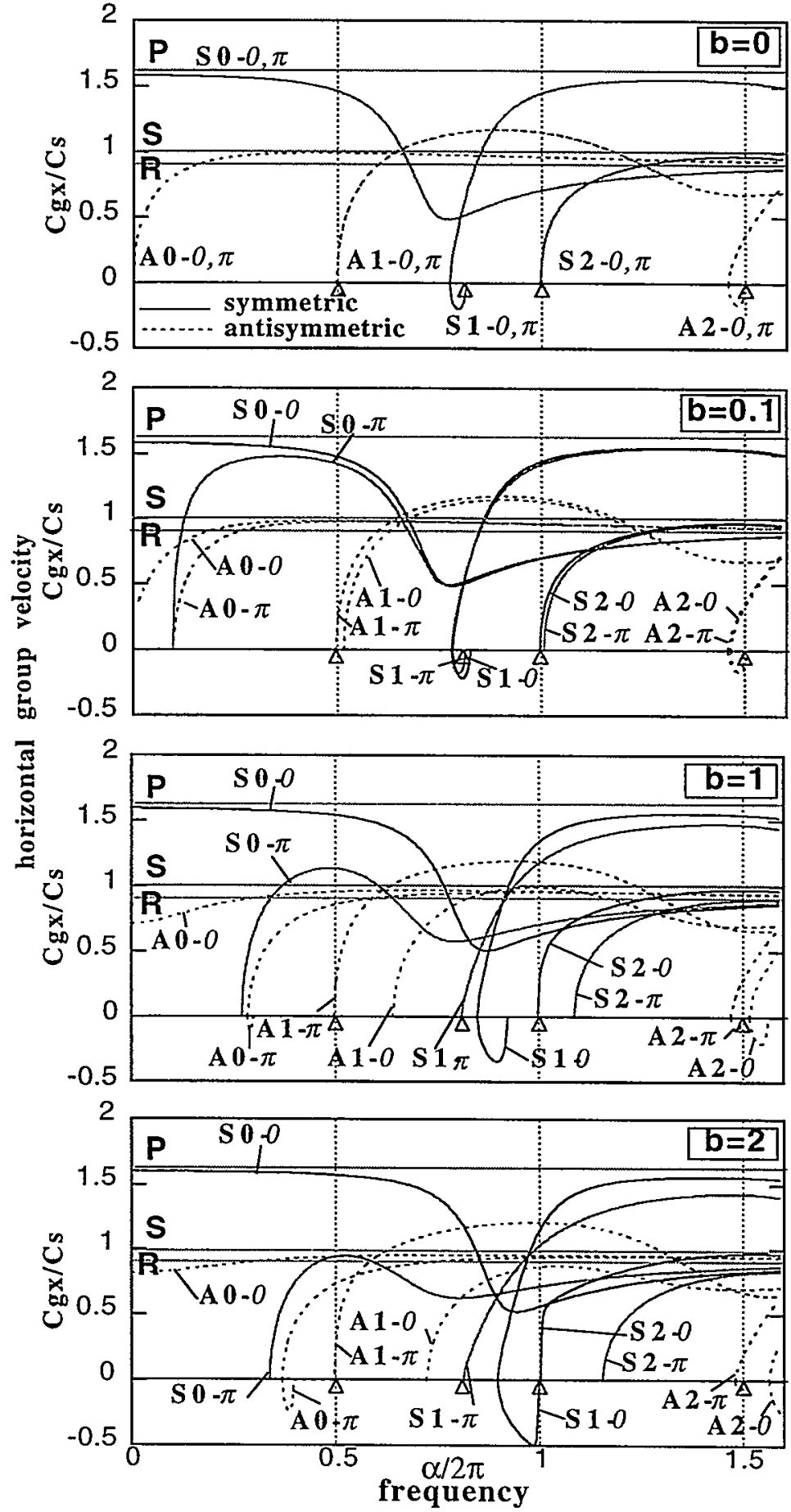

Figure 10.11 (a) Group velocities of generalized Rayleigh-Lamb waves for low to medium fracture stiffnesses. $\mathrm{S}$ and $\mathrm{A}$ indicate symmetric and anti-symmetric particle motion in a single layer, and 0 and $\pi$ after the dash "-" indicates phase difference between motions in neighboring layers. 0 - and $\pi$ - modes split with increasing fracture stiffness. One of the split modes has an intercept with the zero group-velocity axis that is independent of fracture stiffness. It should be noted that some of the modes exhibit negative group velocities. 

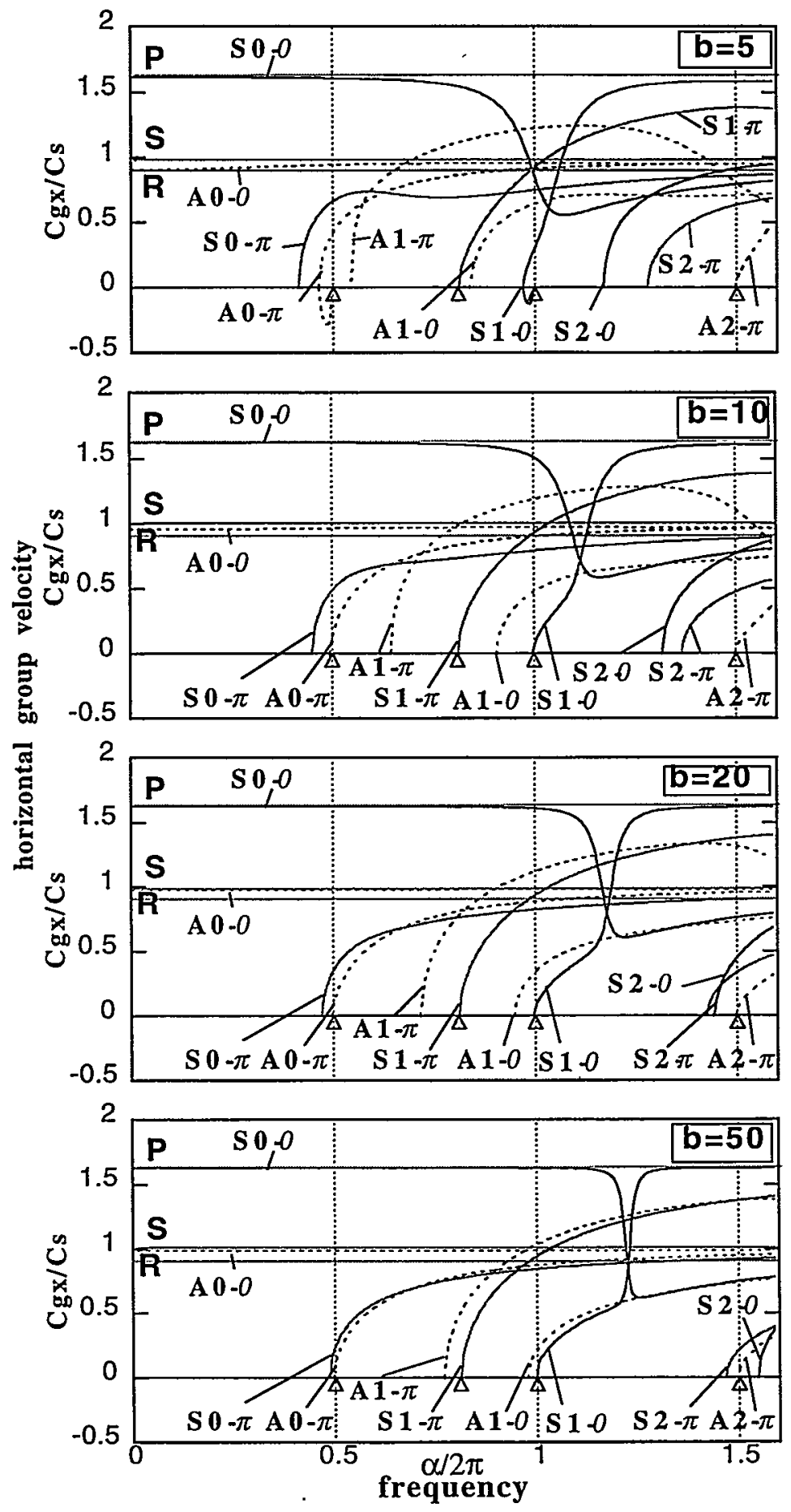

Figure 10.11 (b) Group velocities of generalized Rayleigh-Lamb waves for medium to high fracture stiffnesses. The S0-0 mode and A0-0 mode asymptote to non-dispersive $\mathrm{P}$ and $\mathrm{S}$-waves, respectively. Other modes make pairs between symmetric and antisymmetric modes with identical mode and phase number. Negative group velocity does not occur for high fracture stiffness. 
dependent. However, intercepts for some of the modes with the zero group velocity axis do not change with fracture stiffness (indicated by open triangles in Figure 10.11). This is because the $\mathrm{P}$ and SV-waves are decoupled at these points as the waves are vertically incident on the fracture (horizontal slowness becomes zero). It is noted that the mode that has the immobile intercept is not always the same. Instead, another mode can replace it once its intercept with the horizontal axis catches up with the former immobile intercept. For example, the intercept of the S2-0 mode does not change with increasing fracture stiffness up to $b=2$ but it is replaced by the intercept of the S1-0 mode for higher fracture stiffnesses.

It should be noted that some of the modes show negative group velocities for certain ranges of wave frequency. The negative group velocities occur when the slope of the wavenumber-frequency curve becomes negative as shown in Figure 10.12. Some of the curves in the plots show inflection points below which the group velocity becomes negative. Such wave behavior was investigated by many researchers including Folk (1958), Mindlin (1960), and Graff (1975). Mindlin (1960) showed that another branch that provides complex wavenumbers stems from the inflection point. As the resulting group velocity is complex, waves corresponding to this branch are attenuative. Folk (1958) showed that in order to realize a physical wave that is composed of Fourier components (sine waves with different frequencies), the slope of the curve has to maintain the same sign. This leads to selection of the complex branch below the inflection point (Graff, 1975). Therefore even though negative group velocities result from real solutions of the dispersion equation, they are unphysical.

\subsubsection{Particle motions of P-SV waves}

Particle motions of the decoupled P-SV waves are plotted as a function of horizontal slowness in Figure 10.13 (a) to (c). Arrows showing the particle motions in one of the layers $(0.1<z<0.2 \mathrm{~m})$ are drawn in slightly thicker lines to emphasize the deformation of single layers. The assumed fracture stiffness is $b=10$ and a normalized wave frequency is $\alpha=4$. A range of horizontal slowness $\xi$ is swept to examine the behavior of the waves. Corresponding group velocities have been computed in Section 10.5.1 (see also Figure 10.10 for the case $\alpha=4$ and $b=10$ ).

For most cases, two independent modes ( $\mathrm{P}$ and SV-type modes) result for a single horizontal slowness. When $\xi=0$, waves are propagating vertically with horizontal particle 

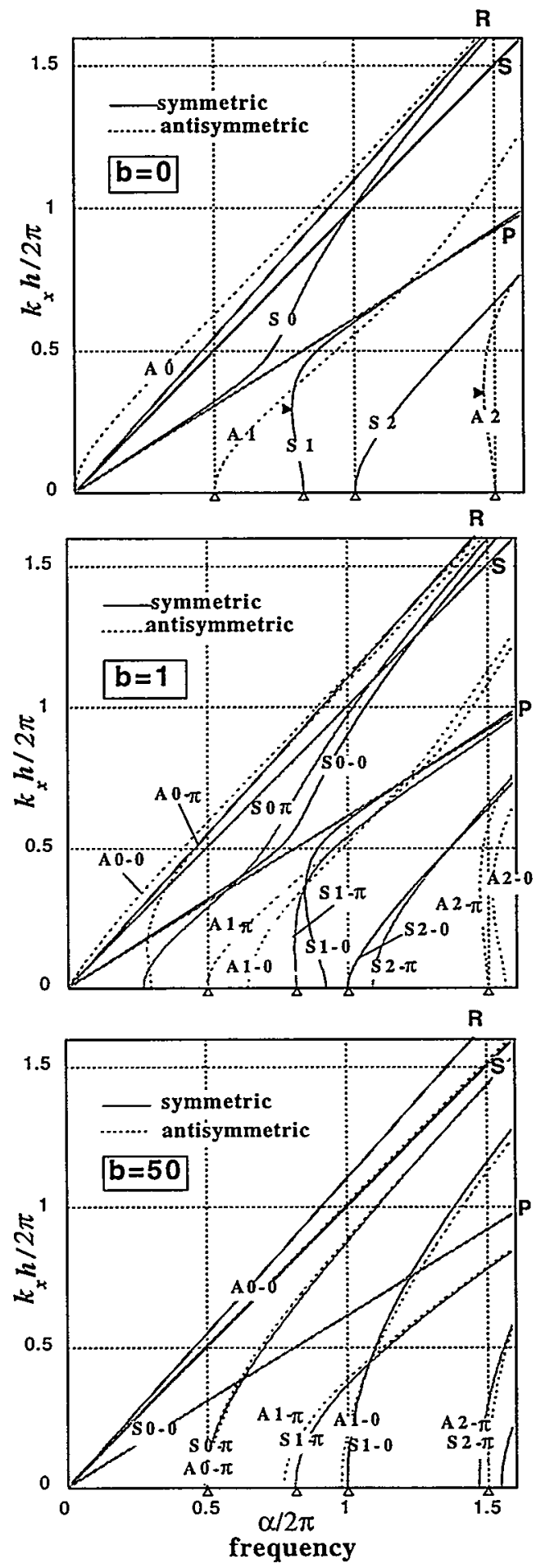

Figure 10.12 Wavenumber (horizontal)-frequency dispersion curves for the generalized Rayleigh-Lamb plate waves. Dispersion curves for $\mathrm{P}$ and $\mathrm{S}$-waves in an intact medium and Rayleigh surface wave for a halfspace are also shown. For low stiffness fractures, some of the dispersion curves have inflection points below which the group velocity becomes negative. 

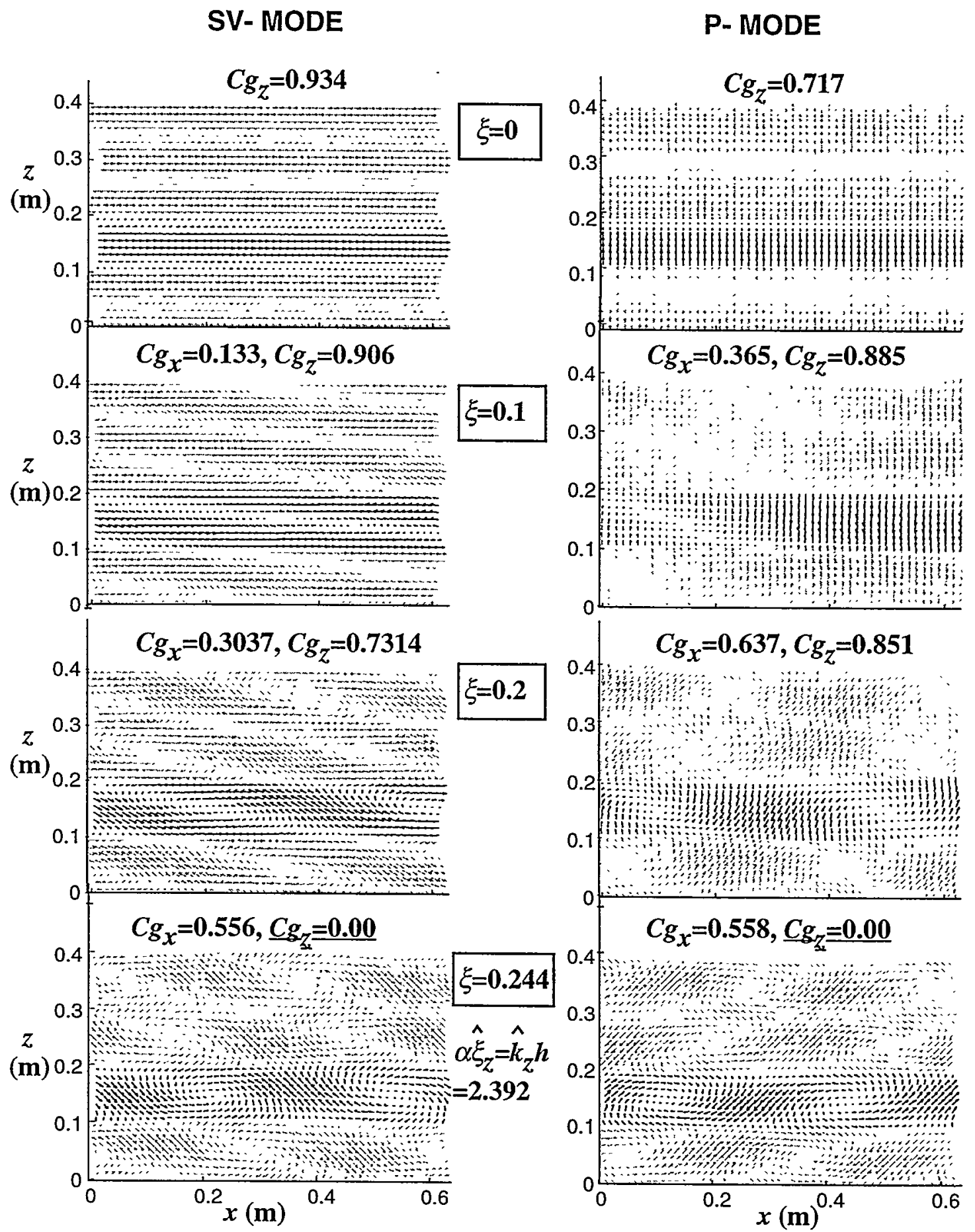

Figure10.13 (a) Particle motions in the first pass band for a static fracture stiffness of $b=10$ and wave frequency of $\alpha=4$. For a horizontal slowness (normalized) of $\xi=0.244$, vertical group velocities vanish to zero. For this slowness, both P- and S- type mode yield identical horizontal group velocities and particle motions that are mirror images of each other. It is noted that the effective vertical slowness does not become zero or the value that yields a resonant condition for a single layer (i.e., $\alpha \xi_{z}$ is not a multiple of $\pi$ ). 


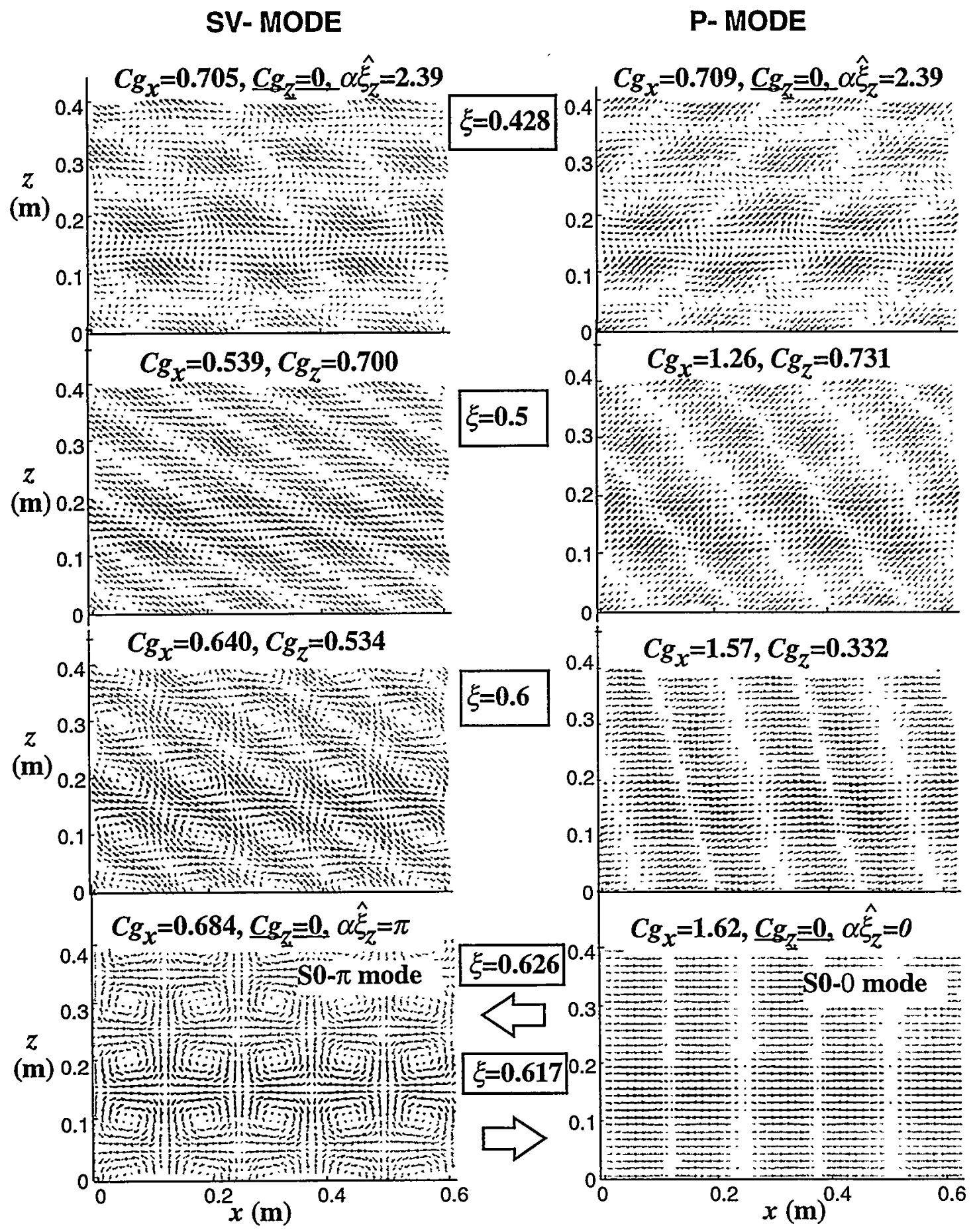

Figure 10.13 (b) Particle motions in the second pass band for a static fracture stiffness of $b=10$ and a wave frequency of $\alpha=4$. At the end of each pass band, group velocity becomes zero again with phase shift across a single layer equal to zero or $\pi$. These are the generalized RayleighLamb plate waves. 


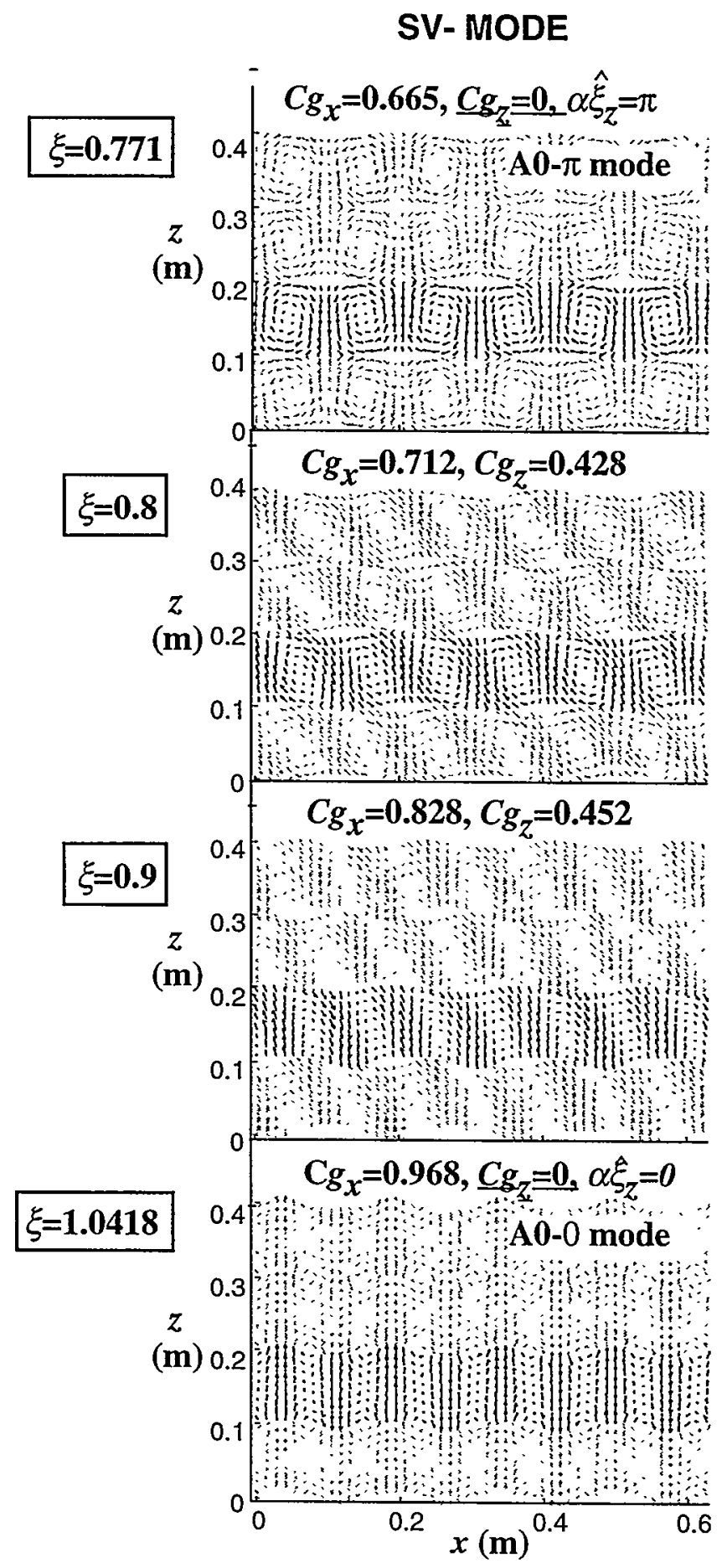

Figure 10.13 (c) Particle motions in the third pass band for the SV-type mode. The static fracture stiffness is $b=10$ and the wave frequency is $\alpha=4$. This band is bound by two modes of the generalized Rayleigh-Lamb plate waves. 
motions in the SV-mode and vertical particle motions in the P-mode (Figure 10.13(a)). As the horizontal slowness increases (or the angle of incidence increases), the wavefield is distorted due to an interaction between the fractures and waves obliquely incident on the fractures. It can be seen that both modes generate elliptic particle motions within single layers. For $\xi=0.244$, the vertical group velocity becomes zero. This is a solution satisfying Eq.(10.40b). The modes of the wave do not belong to the generalized RayleighLamb plate waves as they do not have symmetric or antisymmetric particle motions in a single layer. In fact, both $\mathrm{P}$ and SV- type modes have an identical horizontal group velocity and particle motions that are mirror images of each other. For this reason, the corresponding mode cannot be found in the dispersion curves of the generalized RayleighLamb plate waves shown in Figure 10.11(b).

Between $\xi=0.244$ and 0.428 , no real solutions can be found for the effective vertical slowness. This is a stop band for P-SV wave propagation and the waves attenuate exponentially away from the source. At $\xi=0.428$, another solution for Eq.(10.40b) is obtained. For the SV-mode, real solutions exist up to $\xi=0.684$ for which one of the generalized Rayleigh Lamb modes (mode S0- $\pi$ ) is generated. For the P-mode, the pass band extends up to $\xi=0.617$ for which the S0-0 mode is generated. This is essentially the decelerated bulk $\mathrm{P}$-wave and no P-mode faster than this wave exists.

The third pass band for the SV-mode begins at $\xi=0.244$ for which the A0- $\pi$ mode is generated. This is the last pass band that is terminated by the A0-0 mode, which is essentially the bulk SV-wave. As pointed out previously, the normalized horizontal slowness of the wave can be larger than unity for waves with a large angle of incidence. 


\subsection{Coupled P-SV-SH Wave Propagation}

\subsubsection{General fracture stiffness matrix}

When coupling fracture stiffness is present, the behavior of a medium containing horizontal multiple parallel fractures is no longer transversely isotropic. The dynamic effect of the coupling fracture stiffness has been shown for single fractures in Chapter 8 using $P$ and SV-waves obliquely incident on a fracture and for a fracture interface wave propagating along a fracture.

Group velocities of $\mathrm{P}, \mathrm{SV}$, and SH-waves are computed for a range of source horizontal slowness. To illustrate the effect of coupling fracture stiffness, the following fracture stiffness matrix is assumed:

$$
\left[\kappa_{i j}\right]=\kappa \cdot\left[\begin{array}{ccc}
1 & 0 & R \\
0 & 1-v & 0 \\
R & 0 & 1
\end{array}\right],
$$

where $v$ is the Poisson's ratio of the intact medium $(v=0.2)$ and $R$ is the coupling fracture stiffness ratio between the $\mathrm{x}$ and $\mathrm{z}$-directions. The above matrix can be rotated around the $\mathrm{z}$-axis to obtain a fully populated fracture stiffness matrix.

\subsubsection{Wave propagation along $\mathrm{x}-\mathrm{z}$ plane}

To examine the effect of coupling fracture stiffness between the $\mathrm{x}$ and $\mathrm{z}$-directions, the velocity surfaces for $\mathrm{P}-, \mathrm{SV}-$, and $\mathrm{SH}-$ waves are first computed using the static equivalent medium model (Figure 10.14, left column). The stiffness of the fracture is $b(=\kappa h / \mu)=5$. The coupling fracture stiffness ratio $\mathrm{R}$ is varied as indicated in the plots. It can be seen that an increase in coupling fracture stiffness introduces asymmetry and triplications in the velocity surface. The degree of the asymmetry and triplication increases with increasing coupling fracture stiffness ratio R. From the plots, the SH-wave is not affected by the coupling fracture stiffness. This is because the direction of particle motion for the SHwave is still decoupled from the other directions. The results obtained for the exact dispersion equation for multiply fractured media are shown to the right of the static results for comparison. As the assumed wave frequency is relatively low $(\alpha=1)$, both results are 
[static approximation]
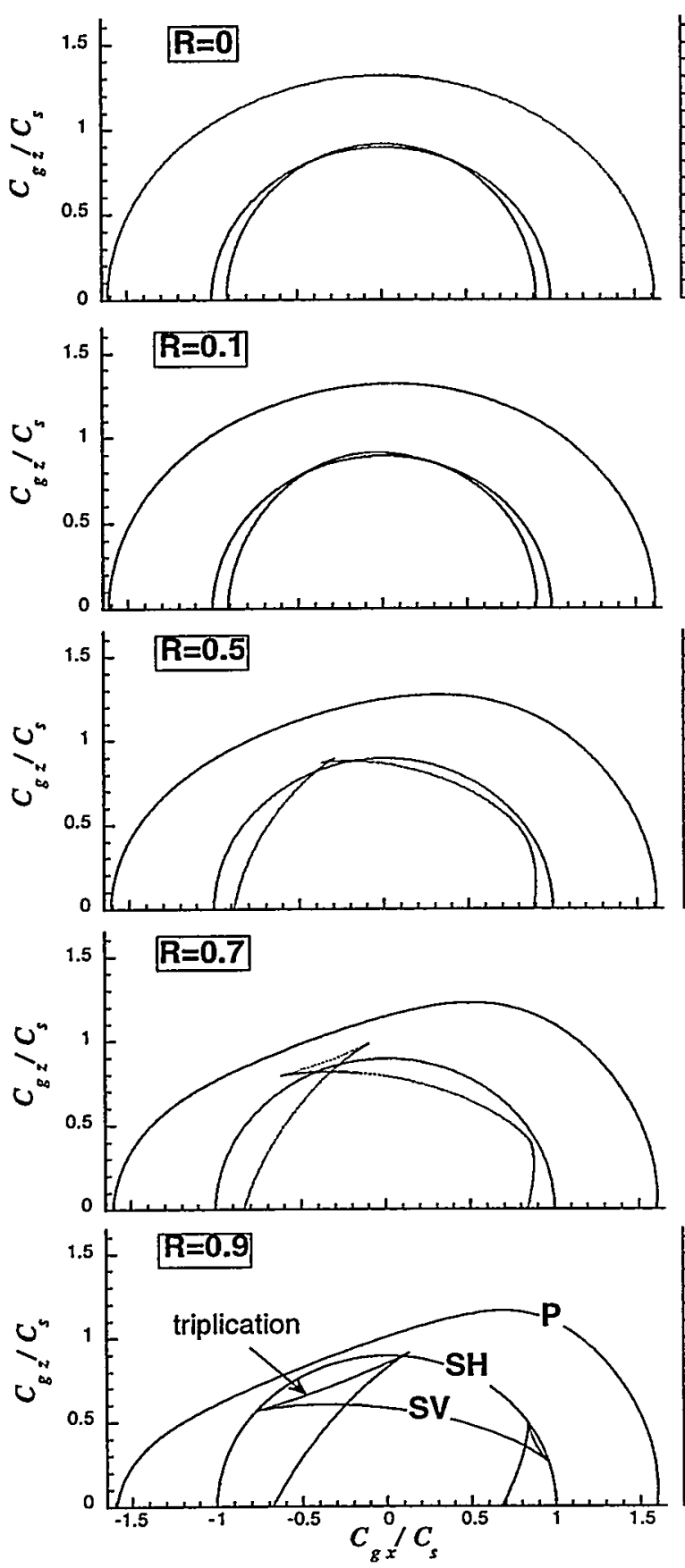

[exact solution]
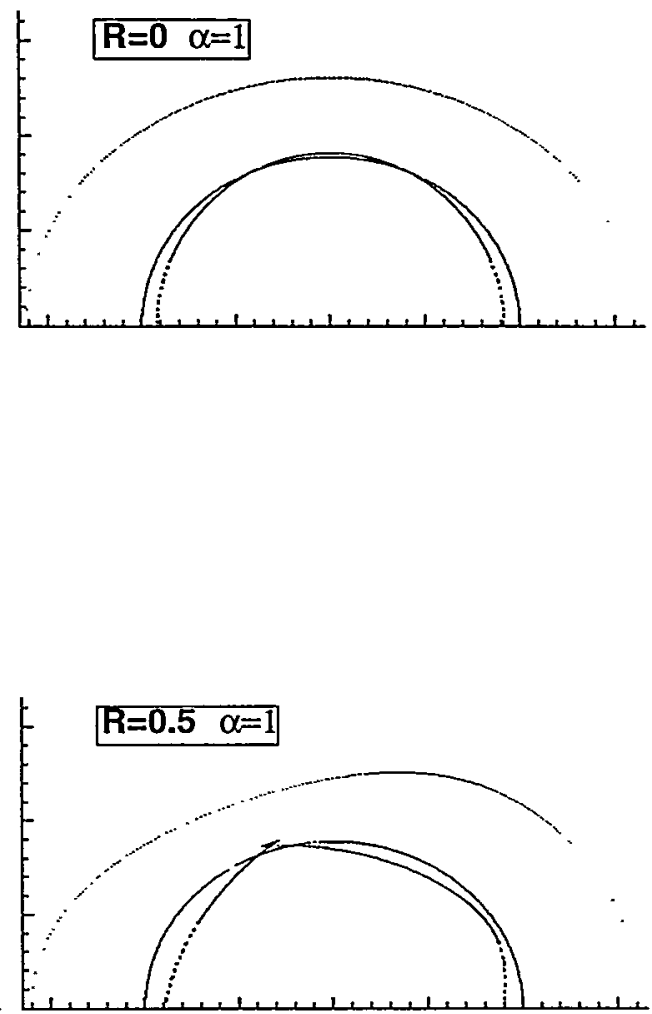

$R=0.9 \alpha=1$

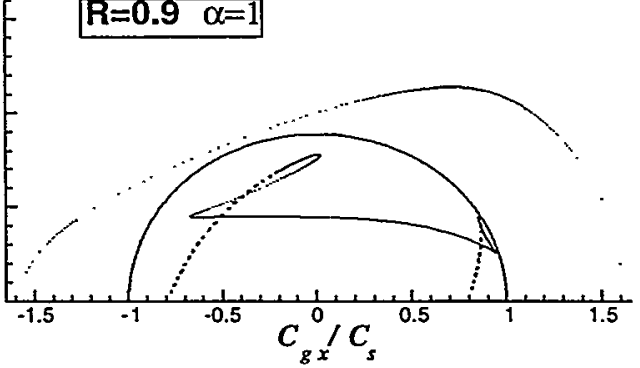

Figure 10.14 Velocity surfaces computed from static equivalent medium model (left) and exact dispersion equation (right) for a fractured medium. Normalized static fracture stiffnesses are $b_{x x}=b_{z z}=5$, $b_{y y}=4, b_{x y}=b_{y x}=0$, and $b_{x z}=b_{z x}=R \times 5$. The effect of a coupling fracture stiffness between the $\mathrm{x}$ and $\mathrm{z}$ directions is examined by varying the coupling parameter $R$. The coupling fracture stiffness introduces asymmetry and triplications in the velocity surfaces. 
[without coupling]
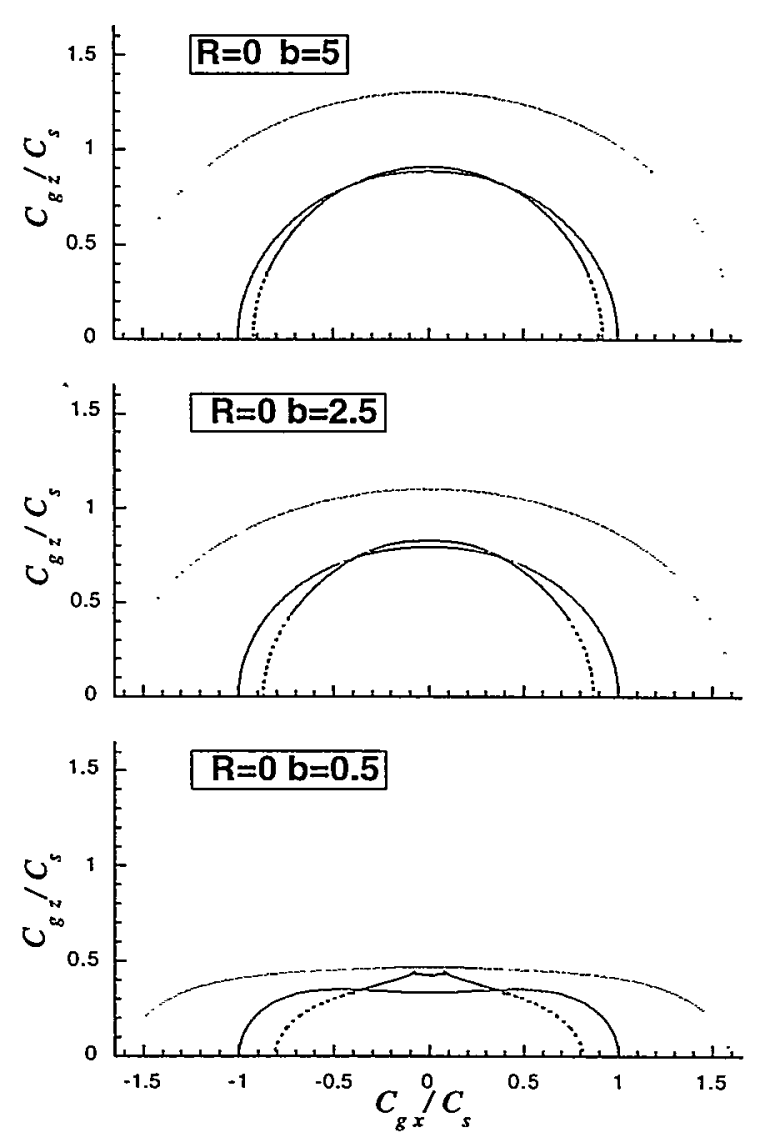

[with $\mathrm{x}-\mathrm{z}$ coupling]
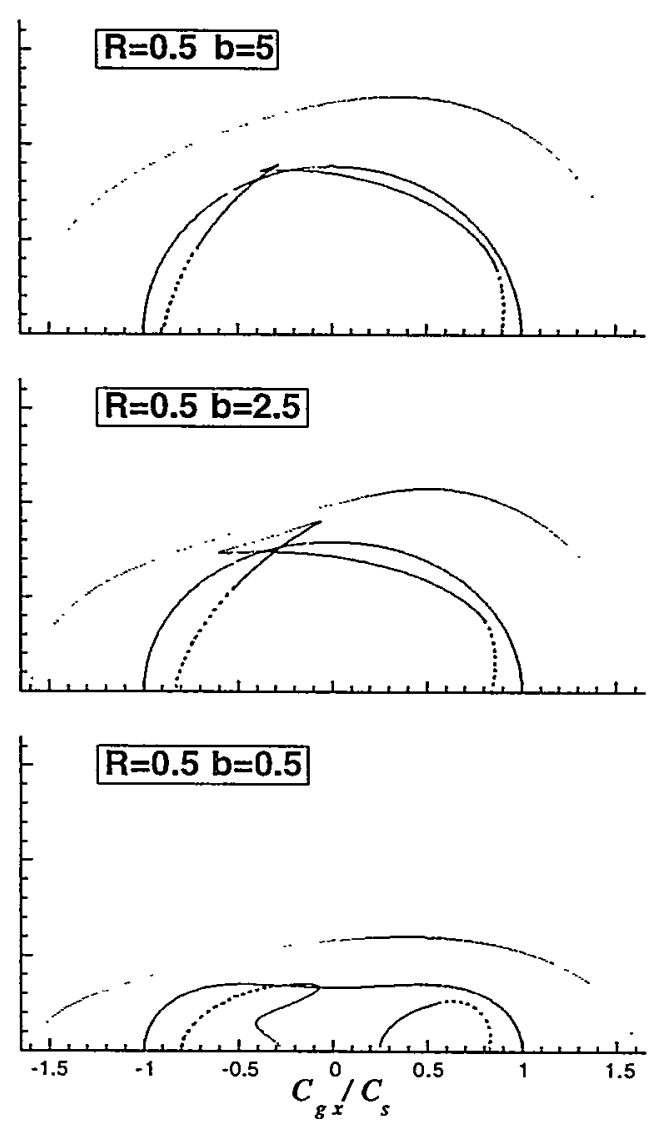

Figure 10.15 Velocity surfaces computed from an exact dispersion equation for fractured medium. Normalized static fracture stiffnesses are $b_{x x}=b_{z z}=b, b_{y y}=b(1-v), b_{x y}=0, b_{x z}=R \times b$, where $v=0.2, b$ $=5$. It can be seen that the coupling affects the shape of the velocity surface greater for lower fracture stiffnesses.

very similar for low-to-intermediate degrees of coupling. For the case of very strong coupling $(R=0.9)$, a significant difference between the exact solution and static approximation can be seen.

Velocity surfaces for lower fracture stiffnesses $(b=0.5$ and 2.5$)$ are also computed using the exact dispersion equation (Figure 10.15). For a low fracture stiffness, the previous analysis shows significant differences between velocity surfaces (see Figure 10.9b). From a comparison between the case $b=5 / R=0.5$ and $b=2.5 / R=0.5$, it can be seen that the degree of asymmetry and triplication increases with decreasing fracture stiffness. For an even smaller fracture stiffness, the SV-wave surface exhibits a stop band that is not observed for the case without coupling fracture stiffness. 


\subsubsection{Fully coupled P-SV-SH wave propagation}

When the fractures in a medium have anisotropic fracture stiffnesses as shown in Eq.(10.46), the group velocity for propagating waves exhibits azimuthal anisotropy. This anisotropy is caused by an azimuthal rotation of the fracture stiffness matrix that introduces a coupling fracture stiffnesses between the $\mathrm{x}$ and $\mathrm{y}$-directions. The velocities are computed using $\mathrm{Eq}(10.23)$ and (10.27).

A single quadrant $(x, y>0)$ of three-dimensional group velocity surfaces for P-, SV-, and SH-waves is shown in Figure 10.16. The dimensionless frequency was $\alpha=1$, and the fracture stiffness used in Eq.(10.46) was $\mathrm{b}=0.5$, yielding an impedance ratio $\beta=1$. The top plot is for the case without a coupling fracture stiffness between the $\mathrm{x}$ and $\mathrm{z}$-directions and the bottom is for the case with a coupling stiffness given by $R=0.5$. Cross-sections of the velocity surfaces along the $\mathrm{x}-\mathrm{z}$ plane are the plots that are shown in Figure 10.15. From the plots, the highly complex geometry of the velocity surfaces can be seen. Due to the coupling fracture stiffness introduced between the $y$ - and other directions, SH- surfaces also exhibits azimuthal anisotropy. 


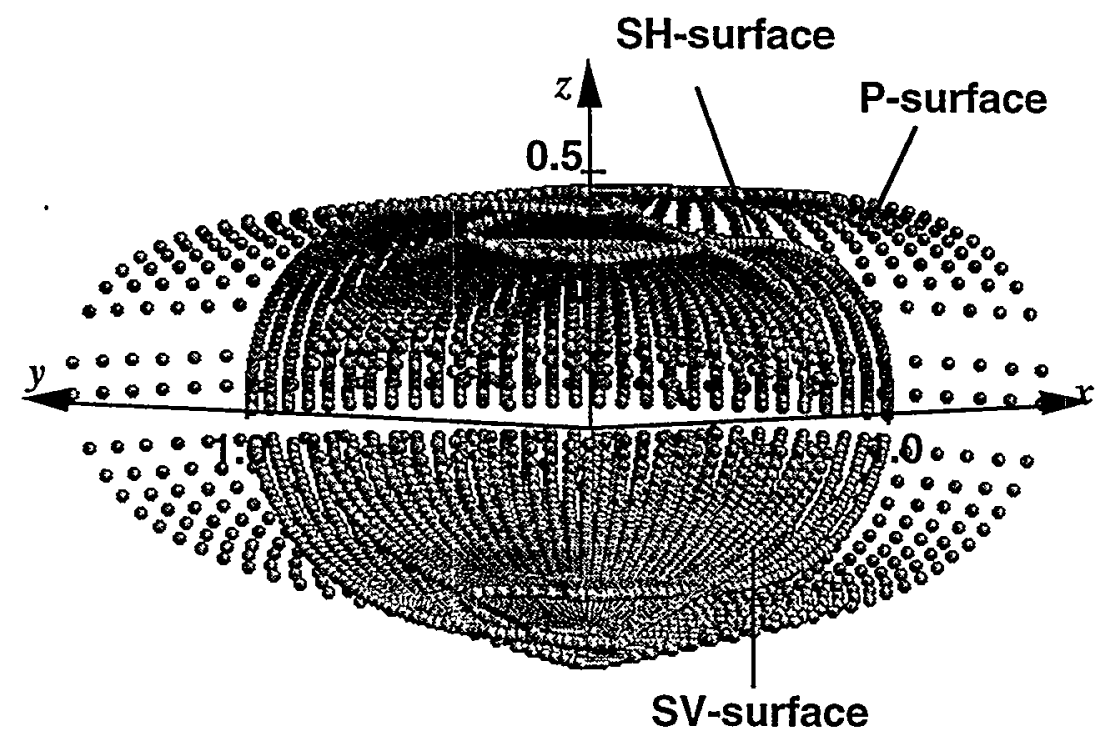

(a) without $x-z$ coupling $\left(b_{x z}=0\right)$

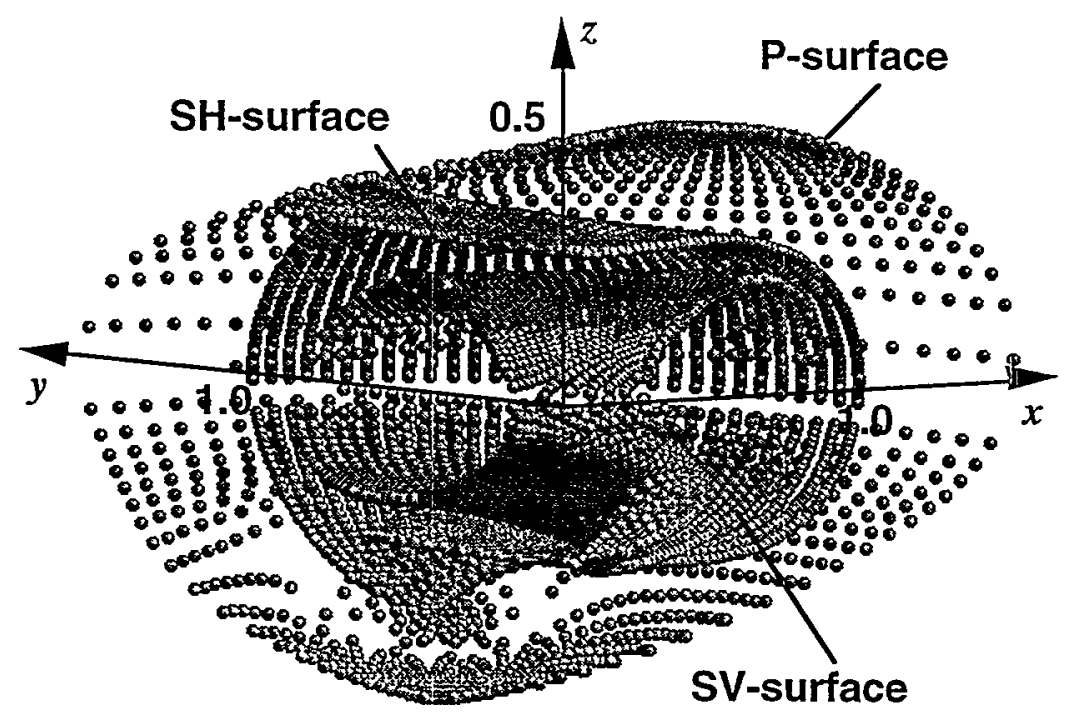

(b) with x-z coupling $\left(b_{x z}=0.25\right)$

Figure 10.16 Velocity surfaces for media containing an infinite series of regularly spaced parallel fractures. For both cases, the frequency parameter is $\alpha=1$, the static tangential stiffnesses are $b_{x x}=0.5$, $b_{y y}=0.4$, the static normal stiffness is $b_{z z}=0.5$. The top plot (a) is computed for $b_{x z}=0$ and the bottom plot (b) is for $b_{x z}=0.25$. 


\subsection{Generalized Fracture Interface Wave}

\subsubsection{Dispersion equation}

If the thickness of the layers is large compared with the wavelength, i.e. $\alpha \rightarrow \infty$, the dispersion equation (10.22) degenerates into that for the fracture interface wave. For this case, $\hat{\xi}_{z}=0$ because no effective vertical wave propagation is present. If the normalized horizontal slowness of the interface wave $\xi$ is such that both $\Theta$ and $\Phi$ are real and positive or, if either one of them is complex, the real components are positive and the dispersion equation becomes

$$
P \equiv \Theta \operatorname{det}\left[\begin{array}{ccc}
\beta_{x x}^{s} \Theta-f & \beta_{x y}^{s} & \beta_{x z}^{s} \Phi \\
\beta_{y x}^{s} \Theta & \beta_{y y}^{s}+\Theta & \beta_{y z}^{s} \Phi \\
\beta_{z x}^{s} \Theta & \beta_{z y}^{s} & \beta_{z z}^{s} \Phi-f
\end{array}\right]=0
$$

where

$$
f_{p}=f_{q}=\left(2 \xi^{2}-1\right)^{2}-4 \xi^{2} \Theta \Phi \equiv f
$$

It is noted that a scalar term multiplied to the matrix in Eq.(10.22) has been dropped. Equation (10.47) is a dispersion equation for interface waves propagating along a fracture. This class of waves can be seen as a pair of Rayleigh surface waves coupled across a single fracture.

If no coupling fracture stiffness is present, Eq.(10.47) becomes

$$
P \equiv \Theta \cdot\left(\beta_{x x}^{s} \Theta-f\right) \cdot\left(\beta_{y y}^{s}+\Theta\right) \cdot\left(\beta_{z z}^{s} \Phi-f\right)=0
$$

Therefore, SV, SH, and P-type waves become decoupled as

$$
\begin{aligned}
& P_{1} \equiv \beta_{x x}^{s} \Theta-f=0 \\
& P_{2} \equiv \beta_{z z}^{s} \Phi-f=0, \\
& P_{3} \equiv \beta_{y y}^{s}+\Theta=0 \\
& P_{4} \equiv \Theta=0
\end{aligned}
$$

It is noted that real-valued slowness $\xi$ cannot satisfy Eq.(10.50c) as the impedance parameter is always real and positive or its real part takes on a positive value. This is because the elastic stiffness of the fracture has to be real and positive. Eq.(10.50d) is equivalent to $\xi=1$. It can be shown that this is a $\mathrm{SH}$-wave propagating parallel to the 
fracture. Therefore, two types of Rayleigh interface wave exist (Eq.(10.50a) and $(10.50 b)$ ), as shown in the previous chapter.

\subsubsection{Anisotropic group velocity}

As was discussed in Section 10.6 for general wave propagation in an infinite series of parallel fractures, the group velocity of the fracture interface wave exhibits azimuthal anisotropy if the fracture stiffness matrix (or impedance ratio matrix) is azimuthally anisotropic. Such fracture stiffness arises when the two principal tangential stiffnesses are not identical or if any coupling fracture stiffness between $z$ - and other directions is present. To see the effect of anisotropic fracture stiffness on the velocities of the fracture interface waves, the following fracture impedance ratio matrix (proportional to the fracture stiffness matrix) is assumed

$$
\left[\beta_{i j}^{S}\right]=\beta \cdot\left[\begin{array}{ccc}
1 & 0 & R \\
0 & 1-\nu & 0 \\
R & 0 & 1
\end{array}\right]
$$

where $\beta=2 \kappa / \omega Z_{s}=1, v=0.2$. The coupling fracture stiffness ratio $\mathrm{R}$ between the $\mathrm{x}$ and $\mathrm{z}$-directions is varied between 0 and 0.9 . The components of group velocity are computed by applying $\mathrm{Eq}(10.23)$ and (10.27) to the dispersion equation Eq.(10.47).

Group velocities plotted as a function of the azimuthal angle are shown in Figure 10.17. The angle is defined for the propagation direction (group velocity) relative to the $x$ axis. As discussed in the previous chapter, the resulting two modes are characterized by symmetric and anti-symmetric particle motions across the fracture in the absence of any coupling fracture stiffnesses. The modes whose particle motions are neither symmetric or antisymmetric due to the introduced coupling stiffness are called quasi-symmetric and quasi-antisymmetric modes.

The quasi-symmetric mode does not show large azimuthal anisotropy for $\mathrm{R}=0$ as an azimuthal rotation of the fracture impedance ratio matrix Eq.(10.51) does not introduce coupling between the $\mathrm{z}$ - and other directions. The quasi-antisymmetric mode, on the other hand, exhibits anisotropy as the rotation of the matrix introduces coupling between the $x$ and $\mathrm{y}$-directions. Along the direction of the $\mathrm{x}$-axis $\left(\theta=0^{\circ}\right)$, an increase in $\mathrm{R}$ results in an increase in group velocity of the quasi-symmetric Rayleigh interface wave and a decrease in the velocity of the quasi-antisymmetric wave. In contrast, along the $y$-axis $\left(\theta=90^{\circ}\right)$, group 


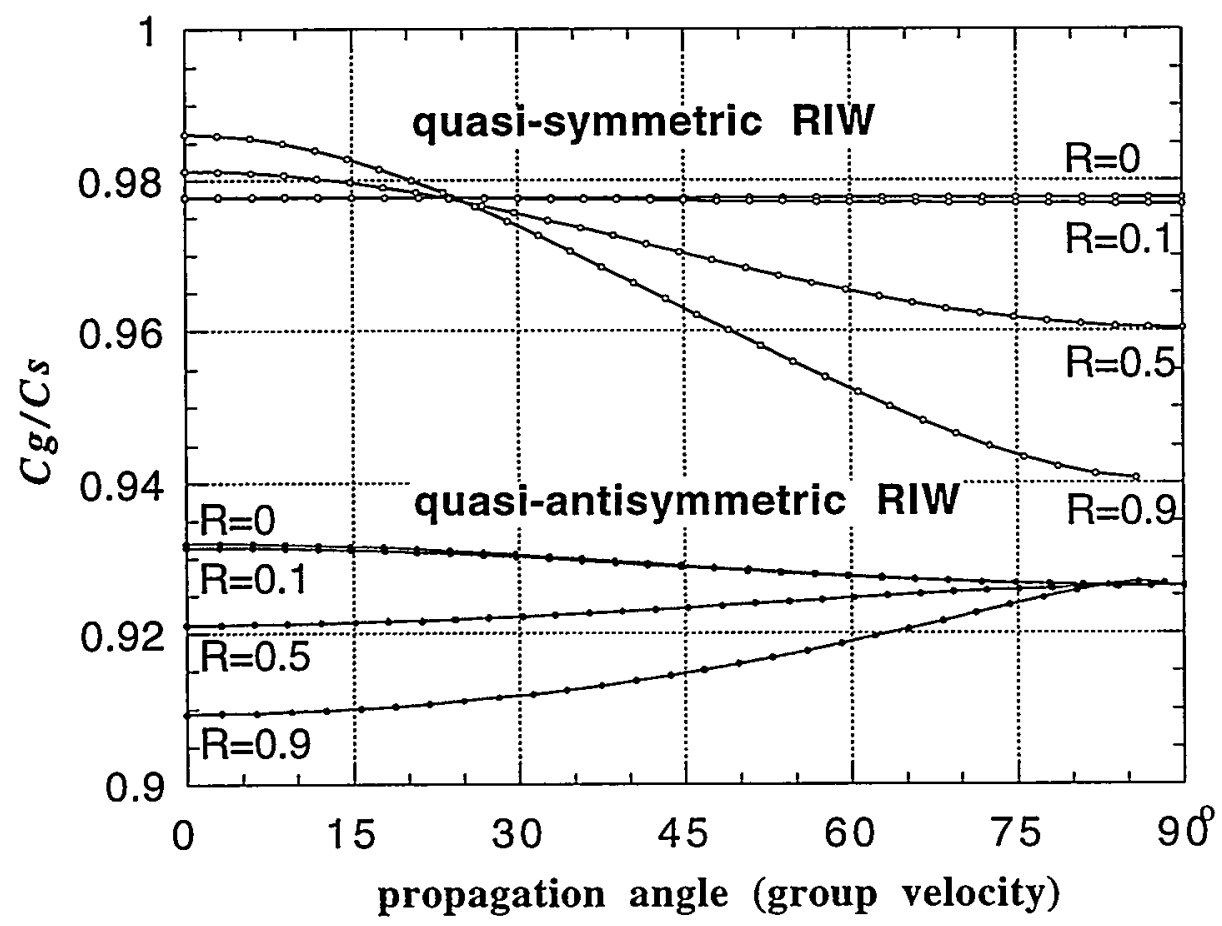

Figure 10.17 Anisotropic group velocities of fracture interface wave with a range of $x-z$ coupling fracture stiffness. Impedance ratios are $\beta_{x x}=\beta_{z z}=1, \beta_{y y}=0.8, \beta_{x y}=0$, and $\beta=\mathrm{R}$. Along the direction of the $\mathrm{x}-$ axis $\left(\theta=0^{\circ}\right)$, an increase in $R$ results in increase in group velocity of the quasi-symmetric Rayleigh interface wave and a decrease in the velocity of the quasi-antisymmetric wave. In contrast, in the y-axis direction $\left(\theta=90^{\circ}\right)$, group velocities show opposite behavior with very small change in the velocity of quasiantisymmetric mode.

velocities show opposite behavior with very small change in the velocity of the quasiantisymmetric mode.

\subsubsection{Particle motion}

In the previous chapter, it has been shown that introduction of a coupling fracture stiffness between the $\mathrm{x}$ and $\mathrm{z}$ - directions changes the particle motions of the fracture interface wave propagating along the $\mathrm{x}$-axis. The elliptic particle motion changes its aspect ratio and tilts its principal axes within the $\mathrm{x}-\mathrm{z}$ plane. Coupling introduced between the $\mathrm{x}$ and $y$-directions due to the azimuthal rotation of the source causes particle motions normal to the direction of wave propagation as well as the tilting of the elliptic particle motions within the $x-y$ plane. 
Particle motions of the fracture interface wave on the surface of the lower halfspace are shown in Figure 10.18. For this example, the impedance Eq.(10.51) with $\beta=1$ is used. The direction of the phase propagation is $45^{\circ}$ (azimuthal angle) from the $x$-axis. A local Cartesian coordinate system (shown as $x^{\prime}-, y^{\prime}-$, and $z^{\prime}$ - axes) is defined by rotating the original system around the $z$-axis. When the shear stiffness is isotropic ( $\beta_{x x} \equiv \beta_{y y}$ for $v=0$ ) and no coupling between the $\mathrm{x}$ and $\mathrm{z}$ - directions is present, both modes exhibit elliptic particle motions parallel to the $x^{\prime}-z^{\prime}$ plane. For anisotropic shear stiffness $\left(\beta_{x x} \neq \beta_{y y}\right)$, the ellipse of the particle motion of the quasi-antisymmetric mode tilts against the $x^{\prime}$-axis. However, the particle motion is still normal to the fracture. When coupling between the $x$ and $z$ - directions is introduced $(R=0.5)$, particle motions of both modes have all components ( $x^{\prime}, y^{\prime}$, and $z$ - components) and the ellipses are tilted against the local coordinate axes. 

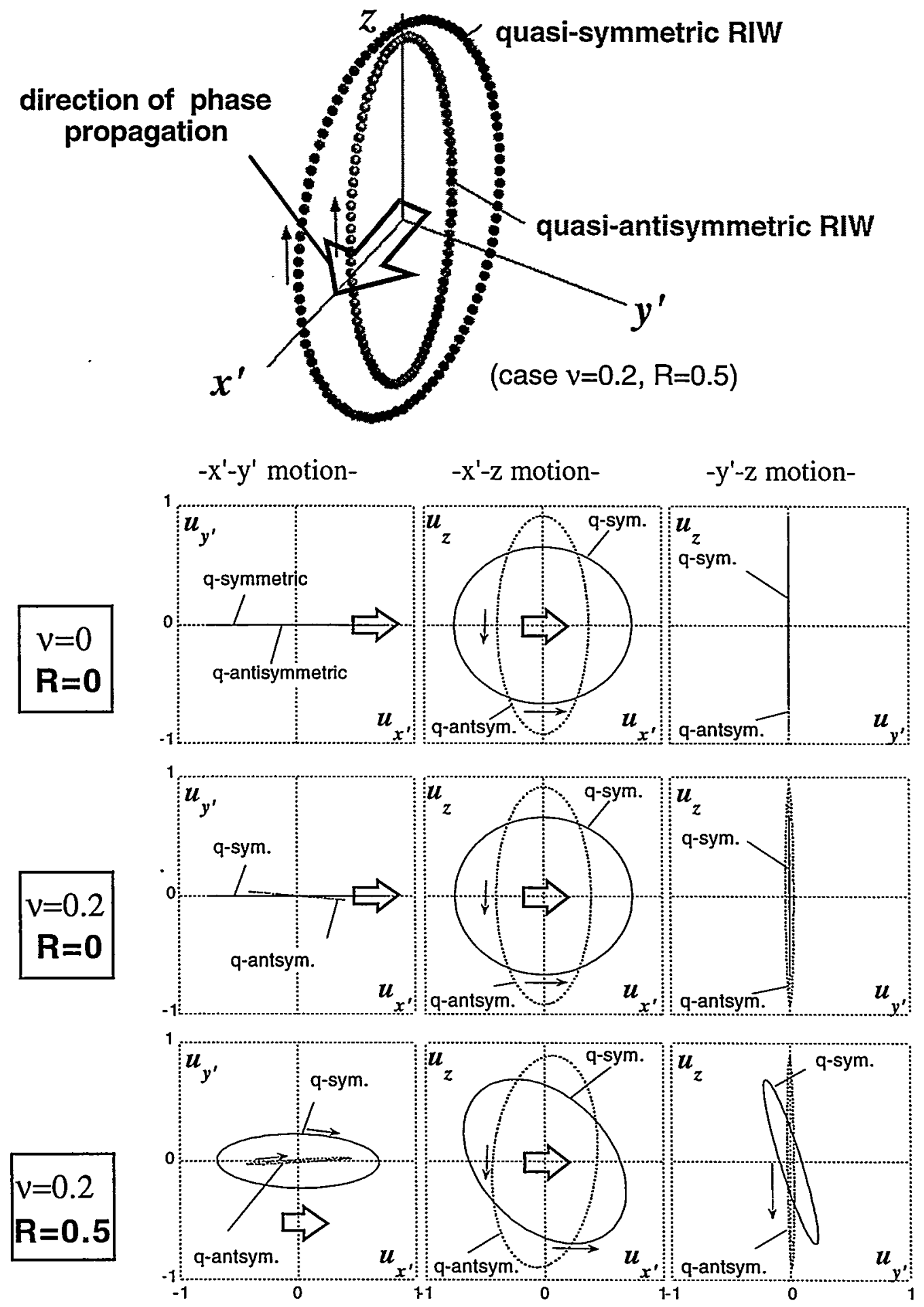

Figure 10.18 Particle motions of fracture interface waves on the surface of the lower halfspace. The direction of propagation (phase) is $45^{\circ}$ (azimuthal angle) from the $x$-axis. The local Cartesian coordinate system rotated around the $z$-axis is shown as $x^{\prime}-y^{\prime}-z$. Coupling among all directions ( $x^{\prime}, y^{\prime}$, and $z$-directions) causes tilting of elliptic trajectories. 


\subsection{Summary}

In this chapter, wave propagation in media containing an infinite series of regularly spaced multiple parallel fractures is examined analytically. An exact dispersion equation for plane waves is derived using Floquet theory to impose periodic boundary conditions on the displacement and stress across fractures whose behavior is modeled by the displacementdiscontinuity boundary conditions.

The derived dispersion equation can describe the group velocity and particle motion of waves propagating at any oblique angle of incidence, including waves propagating parallel to multiple parallel fractures or a single fracture. In some cases, waves propagating parallel to the fractures are shown to be a generalized case of the Rayleigh-Lamb plate waves. The dispersion equation of the Rayleigh-Lamb plate waves is derived by considering the special cases of $0^{\circ}$ and $180^{\circ}$ phase changes across single layers. The dispersion equation of the fracture interface waves with general anisotropic fracture stiffness is also derived by considering the special case of infinitely large fracture spacing.

For a wavelength much longer than the fracture spacing and a relatively high fracture stiffness, wave propagation in a multiply fractured system can be described by a homogeneous equivalent medium model using the static approximation. However, for wavelength comparable to or shorter than the fracture spacing, or for low fracture stiffnesses, the behavior of the wave can no longer be explained by an equivalent medium model. The group velocity exhibits a frequency dependent behavior that increases the degree of anisotropy with increasing wave frequency. For certain combinations of wave frequency and fracture stiffness, the wave propagation in a direction normal to the fractures is prohibited. Such a result is similar to those shown in Chapter 2 for one-dimensional wave propagation, describing alternating regions of wave frequencies where waves can propagate (pass band) or cannot propagate (stop band) across the wave spectrum. At a boundary between a pass and a stop band, the vertical group velocity becomes zero, producing waves propagating parallel to the fractures. As the energy is trapped within individual layers, the system is resonating in the direction normal to the fractures.

The results obtained in this chapter are significant for examining the anisotropic frequency-dependent characteristics of wave propagation in a multiply fractured medium. It has been shown that, for relatively high frequencies and low fracture stiffnesses, the behavior of waves can be quite different from what is predicted from the static effective 
medium approximation. Although the current research is limited to systems with regular fracture spacing and constant fracture stiffness, the general dispersion equation for regulary fractured media has not been derived before, and its results should provide a foundation for investigating wave propagation in more general fractured media.

Future research should include a study of wave propagation in media containing fractures with irregular spacing and fracture stiffness. The spacing and the fracture stiffness may be correlated each other. To tackle such problems, simulations using numerical methods such as the finite difference method and the propagator matrix method may be used. Future research should also examine the amplitude anisotropy in wave propagation as it is not included here. Since the wave amplitudes are commonly known to be more sensitive to the stiffness of a fracture than velocities, they should provide additional and possibly more accurate information about the characteristics of fractured reservoir rocks. 


\section{Chapter 11}

\section{General Summary and Conclusions}

In this thesis, the effects of fractures on the acoustic resonance of rock and concrete have been examined for a variety of fractured systems, including finite one-dimensional and three-dimensional bodies, and an infinite three-dimensional body with regularly-spaced parallel fractures. The purpose of this chapter is to reemphasize the principal findings and conclusions of the preceding chapters and to provide further suggestions for future research.

\subsection{Summary and Conclusions}

A medium containing compliant fractures exhibits acoustic resonance characteristics different from an intact medium, such as decreased acoustical resonance frequencies, an abrupt change in the resonance mode shapes across the fractures, and an increased attenuation when fluid or an attenuative material is present in the fracture. These effects are caused by fractures acting as internal boundaries that interact with propagating waves. A fracture with finite compliance (or stiffness) partially transmits and reflects propagating waves. Such behavior of the wave can be properly modeled by a special boundary condition called the displacement-discontinuity boundary condition (Schoenberg, 1980; 
Pyrak-Nolte et al., 1990a,b). Throughout this thesis, this model has been used extensively to examine the behavior of both propagating waves and standing waves (resonance).

Analytical work in Chapter 2 and experimental work in Chapter 3 demonstrate the significant effects of single and multiple fractures on the resonance of one-dimensional systems. Unlike simple transmission or reflection of a wave from a single fracture, the resonance characteristics of a fractured medium such as resonance frequency and attenuation are found to be very specific to the location of the fracture in the medium with respect to the relevant mode shape. Modes that lead to large strain amplitude at the location of the fracture exhibit large sensitivity to changes in the fracture stiffness and attenuation due to energy dissipation within the fracture.

If the location of the fracture is known, the resonance frequency shift of a medium containing the fracture can be used to determine the dynamic stiffness of the fracture quantitatively. Using a numerical model introduced in Chapter 4, dynamic stiffness of a single fracture in a concrete specimen (Chapter 6) was determined from experimentally measured resonance frequencies. The same technique and code were used to determine the anisotropic elastic moduli of rocks containing compliant microcracks and grain contacts (Chapter 5). Such a technique can be a powerful laboratory tool for determining the material properties of anisotropic rocks and a diagnostic tool for detecting and characterizing defects in any structure built of rock and/or concrete.

The applicability of these resonance techniques to characterize fracture-introduced defects is also demonstrated by a series of semi-site scale tests on concrete bridge columns with artificially introduced damage (Chapter 7). Changes in the dynamic bending stiffness determined from resonance frequencies before and after the destruction and repair of the specimens show excellent agreement with the static stiffness. It is found that the stiffness of the columns corresponds better with the result from the resonance test than the highfrequency wave propagation test indicating that the resonance test is more appropriate for assessing the global integrity of the structure. Also, considering the reduced amount of time and work required for the resonance test when compared to the static loading test and the wave transmission test that requires multiple scans, the resonance test has a significant advantage as a technique to assess the integrity of damaged structures.

In a system containing multiple fractures, the relative locations of the fractures play an important role in determining the behavior of resonance as shown in Chapter 2. For 
fractures with a large compliance, a system with regularly spaced fractures exhibits groups of resonance peaks for which the amplitude of the relevant mode shape is more or less uniformly distributed within the medium. In contrast, a system with irregularly spaced fractures does not show formation of the mode groups. However, the mode shape of the resonances is strongly localized in limited regions within the medium. Such behavior is also observed experimentally in Chapter 6 during the resonance measurements on a fractured concrete cylinder. The localization of resonance in a fractured system is particularly interesting because it can be a diagnostic tool for detecting fractures. For example, it may be applied for detecting a loose key block on a mining roof or an unstable boulder that may trigger a dangerous rock fall.

During the analysis performed in Chapter 2, the resonance of a fractured medium embedded in a one-dimensional infinite medium is examined. It has been shown that the resonance characteristics of the embedded part cut out of the system bears close relation to the spectrum computed for the entire wave train transmitted through the fractured zone. The groups of resonances observed for the fractured part correspond to a spectral band with large transmission coefficients for the propagating wave (pass band) and a spectral band with small transmission coefficients (stop band). Therefore, by examining the spectrum of the propagating wave, a rough estimate of the resonance characteristics of the fractured zone can be obtained. For an infinite series of regularly spaced fractures, a dispersion equation of the wave can be derived using Floquet's theory to examine the behavior of the wave propagating in the medium. For this system, the group velocity approaches zero for frequencies at boundaries between the pass bands and stop bands, indicating that the system is resonating. These frequencies correspond to the peaks in the coda spectrum of the waves transmitted and reflected by the finite fractured zone embedded in an infinite medium.

If a medium contains uniformly distributed microcracks with any preferred orientation, the effect of the additional compliance introduced by a fracture can be analyzed by treating the medium containing the microcracks as an anisotropic homogenous material. In Chapter 5 , wave propagation and resonance in such medium is examined using granite specimens that exhibit approximate transverse isotropy due to oriented microcracks. Elastic moduli of the transversely isotropic effective medium model for the granite are determined from static, ultrasonic, and resonance tests. Measured frequency responses and mode shapes of the granite specimen show good agreement with the behavior of the model using inverted anisotropic elastic moduli. Although the moduli determined from the static, ultrasonic, and 
resonance tests show a consistent trend in the magnitude of their components, results from the ultrasonic tests show the highest moduli while those from the static test show the lowest moduli. This result may reveal a frequency-dependent dynamic behavior of cracks contained in the rock.

To examine the frequency-dependent behavior of multiple fractures, a plane wave analysis is performed to derive a general dispersion equation for wave propagation in a medium containing an infinite series of regularly spaced multiple parallel fractures (Chapter 10). A periodic boundary condition is imposed using Floquet's theory and the behavior of the fracture is modeled under the displacement-discontinuity boundary conditions as in Chapter 2. The dispersion equation describes the behavior of waves incident on the fractures at any angle in the layered three-dimensional medium. For low wave frequencies and high fracture stiffnesses, the resulting group velocities of the waves are close to the velocities computed for an effective medium model using the static approximation for the behavior of the medium containing fractures. Such an approximation is essentially the same as treating a rock containing oriented microcracks as a homogeneous anisotropic rock using an effective medium (Chapter 5). However, for low fracture stiffnesses and wave frequencies that result in wavelengths comparable or shorter than the fracture spacing, the spectrum and the vertical group velocity of the waves exhibit stop and pass bands similar to those seen in the one-dimensional system in Chapter 2.

At boundaries between a pass band and a stop band and within a stop band, waves cannot propagate across fractures, but they can propagate along fractures. Particularly at boundaries between a stop and a pass band, wave motion is present everywhere in the system but there is no vertical propagation of wave energy (vertical group velocity becomes zero.) This can be seen as a resonance of the system in the direction normal to the fractures. Some of these modes have been shown to be a family of the Rayleigh-Lamb plate waves generalized in the sense that an infinite number of parallel layers are coupled by fractures with finite stiffness. The general dispersion equation is also shown to degenerate into a dispersion equation for the fracture interface wave along a fracture in threedimensional space. This dispersion equation is more general than the one derived in Chapter 9 because the fracture stiffness matrix can include all components $(9$ components in a $3 \times 3$ matrix).

The stiffness of the fracture is generalized to include coupling terms between normal and tangential components of stress and displacement in Chapter 8. Such coupling is 
shown to be caused by a shear stress applied to the fracture. Results from experiments and numerical analyses show that a sheared fracture converts part of a P-wave normally incident on a fracture to a polarized S-wave and vise versa. The physical mechanism that leads to this behavior is the elastic dilation of the fracture under the seismically applied shear or normal load. The dilation is due to the coupling fracture stiffness that results from a redistribution of local contact stiffness on the fracture surface caused by the shear. This mechanism is supported by experimental evidence obtained from steel blocks with a regular saw-tooth fracture. Wave transmission through an array of inclined flat slits shows similar behavior to that of a sheared fracture in rock. The same configuration of a fractured steel block tested with fine glass beads in the fracture zone reveals that conversion of waves can occur for a fracture filled with materials such as rock fragments.

An example of the effect of a sheared fracture on the propagation of waves is given in Chapter 9 focusing specifically on the fracture interface wave. A dispersion equation is derived for the fracture interface wave including the shear-induced coupling fracture stiffness. Velocities and particle motions computed from the dispersion equation show a significant effect from the coupling fracture stiffness.

The observed behaviors of waves propagating normal to and parallel to fractures can be used to remotely detect and measure the active shear stress on a fracture. The potential field of application of these methods can be quite broad from stress measurement on an interface within manufactured parts to stress monitoring for geological structures such as seismically active faults.

\subsection{Remaining Problems and Future Research}

Before acoustic resonance can be generally applied as a quantitative tool for material characterization and defect diagnosis, there are still many issues to be resolved.

For example, in reality, most fractures have a finite size. The resonance of three dimensional media including a finite fracture is difficult to tackle due to diffraction of waves by the crack tip. Such a problem is not very tractable for the numerical technique introduced in Chapter 4 due to the singular stress field. Extensive computation using the finite element method or boundary element method may provide solutions for relatively simple problems but the use of such methods becomes impractically expensive (computationally) when the dynamic interaction among multiple fractures is to be solved. 
Attenuation of resonance is also an issue that requires further research. Although simple one dimensional cases are treated in Chapter 2, the effect of attenuation on multidimensional resonance should be further investigated. Attenuation in a structure is conventionally treated using Rayleigh damping that enables the involved eigenvalue problem related to the resonance of the system tractable (for example, Petyt, 1990). However, such an approach is hardly physical and the measured attenuation cannot be related to any mechanical parameters such as viscous and frictional energy loss in a fracture or material. Explicit modeling of the loss mechanism can be computationally expensive. Another type of attenuation is radiation damping due to radiation away from a resonating structure as a propagating wave. Radiation damping can be a key issue when the resonating structure is acoustically coupled to an infinite or semi-infinite space where the propagating wave can escape. This is always the case when the resonance of geological structures is to be examined. Numerical methods such as the finite difference method that can handle a large problem with relatively small computational cost, or the boundary element method that can solve problems with infinite boundaries have been used with limited success. However, for inverting for the location and properties of inherent fractures in a structure from its measured resonance, these numerical techniques have to solve the relevant equations fast enough to be able to allow for multiple iterations.

In inverting the properties of materials and fractures such as elastic moduli and fracture stiffness from measured resonance, identification of individual mode type can be a bottle neck for their accurate determination. In this thesis, the mode shapes associated with an isotropic glass cube and a transversely isotropic granite cube are measured using a laserDoppler vibrometer and show good agreement with the simulated mode shapes. These results indicate that mode shape can be used to identify each measured resonance. Such a technique can be essential if the number of parameters to be determined is large. Particularly if the principal axes of the stiffness parameters are not known, a very large number (up to 21 for elastic moduli and 6 for fracture stiffness of a single fracture) of resonance frequencies are needed to be measured. The feasibility of such a technique should be assessed by laboratory experiments.

Another issue with resonance inversion is the heterogeneity of material properties and the fracture stiffness. As indicated by the resonance of a fractured dolomite specimen (Chapter 5) and the resonance of a fractured concrete cylinder under low axial stress (Chapter 6), heterogeneity in the elastic moduli and fracture stiffness can lead to resonance behaviors that are not expected for uniform moduli and stiffness. Through numerical 
simulations and experiments, the effect of heterogeneity on the resonance should be further investigated.

The cross coupling behavior of waves also needs to be verified quantitatively in laboratory, including its relation to static fracture stiffness. For this purpose, seismic transducers with matched source and receiver characteristics for both P- and S- waves should be used. The current research uses transducers with piezoelectric crystals that have different resonance frequencies for $\mathrm{P}$ - and $\mathrm{S}$-waves $(850 \mathrm{kHz}$ and $500 \mathrm{kHz}$, respectively). Due to the relatively small overlap between the transducers' spectral ranges, the relative magnitude of the shear-induced conversions could not be measured quantitatively. Experimental verification is also needed for the effect of shear on the propagation of the fracture interface wave. Furthermore, it is important to identify the geometry of the fracture interface that leads to the elastic dilation of the fracture. To this end, the relation between three-dimensional contact geometries and their mechanical behavior under a variety of loading conditions should be investigated.

In the final chapter, wave propagation and resonance in media containing regularly spaced parallel fractures was examined. The pass-and-stop band behavior observed in such systems is the most pronounced for fractures with regular spacing and identical fracture stiffness. Wave behavior is expected to be quite different if irregularity is introduced. As speculated from the results in Chapter 2, pass bands for an irregular system would be much narrower and aperiodic, allowing less transmission of energy over a range of frequencies. An approach that incorporates the statistics of fracture spacings and stiffnesses is difficult to execute for analyzing the frequency-dependent wave propagation in an irregular system; consequently, research should be conducted using numerical techniques such as the finite difference method or propagator matrix method.

Finally, it is again emphasized that the research topics covered in this thesis are only a part of the rich and profound physical phenomenon associated with the acoustic resonance of fractured media. It is the author's hope that this work has shed a little more light on the unveiled part of this field. Research on the fundamental physics behind acoustic resonance should continue to establish a more complete body of knowledge on the subject, and that knowledge should be put to practical use for further technological progress in the pertinent fields of engineering. 


\section{References}

Aki, K. and P. G. Richards. 1980. Quantitative Seismology, Vol. I, W. H. Freeman and Co., New York.

Anderson, P. W. 1958. Absence of diffusion in certain random lattices, Physical Review, 109, 1492-1505.

Angel, Y. C. and J. D. Achenbach. 1985. Reflection and transmission of elastic waves by a periodic array of cracks, J. Appl. Mech., 52, 33-41.

Baik, J. and R. B. Thompson. 1984. Ultrasonic scattering from imperfect interfaces: a quasi-static model, J. Nondesruct. Eval., 4, 177-196.

Backus, G. E.. 1962. Long-wave elastic anisotropy produced by horizontal layering, $J$. Geophys. Res., 67, 4427-4440.

Bamnios, G. and A. Trochidis. 1995. Mechanical impedance of a cracked cantilever beam, J. Acoust. Soc. Am. , 97(6), 3625-3635.

Bancroft, D. 1941. The velocity of longitudinal waves in cylindrical bars, Physical Review, 59, 588-593.

Banik, N. C., I. Lerche, J. R. Resnick, and R. T. Shuey. 1985a. Stratigraphic filtering, part I: Derivation of the O'Doherty-Anstey formula, Geophysics, 50(12), 2768-2774.

Banik, N. C., I. Lerche, and R. T. Shuey. 1985b. Stratigraphic filtering, part II: Model spectra: Geophysics, 50(12), 2775-2783. 
Bedford, A. and D. S. Drumheller. 1994. Introduction to Elastic Wave Propagation, John Wiley \& Sons, 141-151.

Birch, F. and D. Bancroft. 1938. Elasticity and internal friction in a column of granite, Bull. Seismol. Soc. Amer., 28, 243-254.

Birch, F. 1975. Velocity and attenuation from resonant vibrations of spheres of rock, glass, and steel, J. Geopys. Res., 80(5), 756-764.

Blandford, G. E., A. R. Ingraffea, and J. A. Liggett. 1981. Two-dimensional stress intensity factor computations using the boundary element method, Int. J. Num. Meth. Engng., 17, 387-404.

Boubié, T., O. Coussy, and B. Zinszner. 1987. Acoustics of Porous Media, Gulf Publishing Co., Houston.

Brebbia, C. A., and J. Dominguez. 1989. Boundary Elements - An Introductory Course, Computational Mechanics Publications, Southampton, U. K.

Brillouin, L. 1953. Wave Propagation in Periodic Structures, Dover Publications, New York.

Brown, S. R., R. L. Kranz, and B. P. Bonner. 1986. Correlation between the surfaces of natural rock joints, Geophys. Res. Lett., 13, 1430-1433.

Brown, S. R. and C. H. Scholz. 1985. Closure of random elastic surfaces in contact, $J$. Geophys. Res., 90, 5531-5545.

Brown, S. R. and C. H. Scholz. 1986. Closure of rock joints, J. Geophys. Res., 91, 4939-4948.

Bruggeman, D. A. G.. 1937. Berechnung der verschiedenen physikalischen Konstanten von heterogenen Substanzen, Annal. der Physik, 29, 160.

Buck, O., R. B. Thompson and D. K. Rehbein. 1984. The interaction of ultrasound with contacting asperities: Application to crack closure and fatigue crack growth, J. Nondestr. Eval., 4, 203-212. 
Budiansky, B. and R. J. O'Connell. 1976. Elastic moduli of a cracked solid, Int. J. Solids Structures, 12, 81-97.

Burridge, R., G. Papanicolau, and B. White, 1988. One-dimensional wave propagation in a highly discontinuous medium, Wave Motion, 10, 19-44.

Byerlee, J. D. 1967a. Theory of friction based on brittle fracture, J. Appl. Phys., 38, 2928-2934.

Byerlee, J. D. 1967b. Frictional characteristics of granite under high confining pressure, $J$. Geophys. Res., 72, 3639-3648.

Byerlee, J. D. and W. F. Brace. 1968. Stick-slip, stable sliding and earthquakes - effect of rock type, pressure, strain rate and stiffness, J. Geophys. Res. 73, 6031-6037.

Carino, N. J., M. Sansalone, N. N. Hsu. 1986. Flaw detection in concrete by frequency spectrum analysis of impact-echo waveforms, Int. Adv. Nondestr. Test., 12, 117-146.

Cawley, P. and R. D. Adams. 1979. The location of defects in structures from measurements of natural frequencies, J. Strain Analysis, 14(2), 49-57.

Chernyshev, S. N. and W. R. Dearman. 1991. Rock Fractures, Butterworths advanced geotechnical engineering, Butterworth-Heinemann, Boston.

Cook, N. G. W. and K. Hodgson. 1965. Some detailed stress-strain curves for rock, J. Geophys. Res., 70(12), 2883-2893.

Davis, A. G. and B. H. Hertlein. 1987. Nondestructive testing of concrete pavement slabs and floors with the transient dynamic response method, Proc. Int. Conf. Structural Faults and Repair, London, 2, 429-433.

Demarest, H. H., Jr.. 1969., Cube resonance method to determine the elastic constants of solids, J. Acoust. Soc. Am., 49, 768-775.

Dieterich, J. H. 1978. Time-dependent friction and the mechanics of stick-slip, PAGEOPH, 116, 790-806.

Dvorkin, J., G. Mavko, and A. Nur 1995. Squirt flow in fully saturated rocks, Geophysics, 60(1), 97-107. 
Ekstein, H. and T. Schiffman. 1956. Free vibration of isotropic cubes and nearly cubic parallelpipeds, J. Appl. Phys., 27(4), 405-412.

Elkin, S. J, A. M. Nacamuli, D. E. Lehman, and J. P. Moehle. Repair of moderately and severely damaged bridge columns, To be published.

Floquet, G. 1883, Sur les équations differentielles linéaires à coefficients periodiques, Annal. Ecole Normale (ser.2), 12, 47-89.

Folk, R. T. 1958. Time dependent boundary value problems in elasticity, $\mathrm{Ph}$. D. Thesis, Lehigh University, Pennsylvania.

Gilbert, F. and G. E. Bacus. 1966. Propagator matrices in elastic wave and vibration problems, Geophysics, 31, 326-332.

Goodman, R. E., 1976. Methods of Geological Engineering in Discontinuous Rocks, West New York.

Graff, K. F. 1975. Wave Motion in Elastic Solids, Dover, New York.

Greenwood, J. A. and J. B. P. Williamson, 1966. Contact of nominally flat surfaces, Proc. Roy. Soc. London, Ser. A, 295, 300.

Gu, B., N. G. W. Cook, K. T. Nihei, and L. R. Myer. 1994. Modeling elastic wave propagation in a medium with non-welded interfaces using the boundary integral equation method, Computer Methods and Advances in Geomechanics, ed. H.J. Siriwardane and M. M. Zaman, A. A. Balkema, Brookfield, Vt., 387-392.

Gu, B., K. T. Nihei, and L. R. Myer. 1996a. Fracture interface waves, J. Geophys. Res., 101, 827-835.

Gu, B., K. T. Nihei, and L. R. Myer. 1996b. Numerical investigation of fracture interface waves, J. Acoust. Soc. Am., 102(1), 120-127.

Gu, B., R. Suarez-Rivera, K. T. Nihei, and L. R. Myer. 1996. Incidence of plane waves upon a fracture, J. Geophys. Res., 101, 25337-25346.

Gudmundson, P. 1982. Eigenfrequency changes of structures due to cracks, notches or other geometrical changes, J. Mech. Phys. Solids, 30(5), 339-353. 
Haines, N. F. 1980. The theory of sound transmission and reflection at contacting surfaces, Report RD/B/N4744, Central Electricity Generating Board, Berkeley Nuclear Laboratories, Bristol, U. K.

Helbig, K.. 1958. Elastiche Wellen in anisotropen Medien, Gerlands Beeitrage zur Geophysik, 67, 177-211, 256-288.

Helbig, K.. 1984. Anisotropy and dispersion in periodically layered media, Geophysics, 49(4), 364-373.

Hesler, G. J. 1995.The Effects of Discontinuities on the Seismic Properties of Rock, $\mathrm{PhD}$ Thesis, University of California at Berkeley.

Hilbert, L. B., T. K. Hwong, N. G. W. Cook, K. T. Nihei, and L. R. Myer. 1994. Effects of strain amplitude on the static and dynamic nonlinear deformation of Berea sandstone, Nelson and Laubach eds., Proc. 1st North Amer. Rock Mech. Symp, Balkema, 497-502.

Hodges, C. H. and J. Woodhouse, 1983. Vibration isolation from irregularity in a nearly periodic structure: Theory and measurements, J. Acoust. Soc. Am., 74(3), 894-905.

Hoek, E. 1970. Influence of structure upon the stability of rock slopes, Proceedings of Symposium on Stability in Open Pit Mining, Vancouver.

Holland, R.. 1968. Resonant properties of piezoelectric ceramic rectangular parallelpipeds, J. Acoust. Soc. Am., 43(5), 988-997.

Hood, J. A. and M. Schoenberg. 1989. Estimation of vertical fracturing from measured elastic moduli, J. Geophys. Res., 94, 15611-15618.

Hopkins, D. L., N. G. W. Cook, and L. R. Myer. 1987. Fracture stiffness and aperture as a function of applied stress and contact geometry, Proc. 28th US Symp. Rock Mech., Tucson, 673-680.

Hopkins, D. L., N. G. W. Cook, and L. R. Myer. 1990. Normal joint stiffness as a function of spatial geometry and surface roughness, Rock Joints, ed. N. Barton and O. Stephansson, Pub. A. A. Balkema, Rotterdam, 203-210. 
Jaeger, J. C. 1959. The frictional properties of joints in rock, Geofis. pura appl., 43, 148158.

Jaeger, J. C. and Rosengren, K. J. 1969. Friction and sliding of joints, Proc. Aust. Inst. Min. Metall., 229, 93-104.

Jaeger, J. C. 1971. Friction of rocks and stability of rock slopes, Geotechnique, 21, $97-$ 134.

Kam, T. Y. and T. Y. Lee. 1992. Detection of cracks in structures using modal test data, Engin. Fract. Mech., 42(2), 381-387.

Kendall, K. and D. Tabor. 1971. An ultrasonic study of the area of contact between stationary and sliding surfaces, Proc. roy. Soc. Lond. A, 323, 321-340.

Kennet, B. L. N.. 1983. Seismic Wave Propagation in Staratified Media, Cambridge University Press, New York, 1983.

King, M. S. 1969. Static and dynamic elastic moduli of rocks under pressure, Rock Mechanics - Theory and practice, W. H. Somerton Ed., Proc. 11 th Symposium on Rock Mechanics, Univ. of Calif. Berkeley, 329-351.

Lamé, M. G.. 1866, Leçons sur la Théorie mathématique de l'Élasticité des Corps Solides, Gauthier-Villars, Paris.

Laubach, S. E. 1991. Fracture patterns in low-permeability-sandstone gas reservoir rocks in the Rocky Mountain region, Proceedings of Joint Society of Petroleum Engineers Rocky Mountain Regional Meeting/Low-Permeability Reservoir Symposium, SPE Paper 21853, Society of Petroleum Engineers, 501-510.

Lehman, D. E., D. J. Moehle, and S. Mahin. 1996. Design of an experimental study on the influence of aspect ratio and longitudinal reinforcement ratio on column response, Fourth Caltrans Seismic Research Workshop, July.

Lin, J.-M., and M. Sansalone. 1994. Impact-echo response of hollow cylindrical concrete structures surrounded by soil and rock: part I-numerical studies, Geotech. Test. J., 17(2), 207-219. 
Lo, T., K. B. Coyner, and M. N. Toksöz. 1985. Experimental determination of elastic anisotropy of Berea sandstone, Chicopee shale, and Chelmsford granite, Geophysics, 51, 164-171.

Lorenz, J. C. and S. J. Finley. 1991. Regional fractures II: Fracturing of Mesaverde Resorvoirs in the Piceance Basin, Colorado, the American Association of Petroleum Geophysics Bulletin, 75(11), 1738-1757.

Lorenz, J. C., L. W. Teufel, N. R. Warpinski. 1991. Regional fractures I: A mechanism for the formation of regional fractures at depth in flat-lying reservoirs, the American Association of Petroleum Geophysics Bulletin, 75(11), 1714-1737.

Love, A. E. H. 1944. A treatise on the mathematical theory of elasticity, Dover, New York.

Lucet, N., P. N. J. Rasolofosaon, and B. Zinszner. 1991. Sonic properties of rocks under confining pressure using the resonant bar technique, J. Acoust. Soc. Am., 89(3), 980990.

Luongo, A. 1992. Mode localization by structural imperfections in one-dimensional continuous systems, J. Sound and Vibration, 155(2), 249-271.

Man, X. C., L. M. McClure, Z. Wang, and R. D. Finch. 1994. Slot depth resolution in vibration signature of beams using frequency shift, J. Acoust. Soc. Am., 95(4), 20292037.

Mavko, G. and A. Nur 1979. Wave attenuation in partially saturated rocks, Geophysics, 44, 161-178.

Maynard, J. D.. 1992. The use of piezoelectric film and ultrasound resonance to determine the complete elastic tensor in one measurement, J. Acoust. Soc. Am., 91(3), 1754-1762.

Mazurek. D. F. and J. T. DeWolf. 1990. Experimental study of bridge monitoring technique, J. Structural Engineering, 116(9), 2532-2549.

Migliori, A., J. L. Sarrao, W. M. Visscher, T. M. Bell, Ming Lei, Z. Fisk, and R. G. Leisure. 1993. Resonant ultrasound spectroscopic techniques for measurement of the elastic moduli of solids, Physica B, 183, 1-24. 
Mikata, Y. and J. D. Achenbach. 1988. Interaction of harmonic waves with a periodic array of inclined cracks, Wave Motion, 10, 59-72.

Mindlin, R. D.. 1949. Compliance of elastic bodies in contact, J. Appl. Mech., 16, Trans. ASME, 71, 259-268.

Mindlin, R. D.. 1956. Simple modes of vibration of crystals, J. Appl. Phys., 27(12), $1462-1466$.

Mindlin, R. D.. 1960. Waves and vibrations in isotropic, elastic plates, In Structural Mechanics, eds. J. N. Goodier and N. Hoff, 199-232.

Murty, G. S. 1975. A theoretical model for the attenuation and dispersion of Stoneley waves at the loosely bonded interface of elastic half-space, Phys. Earth Planet Inter., 11, 65-79.

Nakagawa, S., N. G. W. Cook, G. J. Hesler, K. T. Nihei, and L. R. Myer. 1996. Effects of fracture stiffness on the resonance of a fractured three dimensional elastic body, submitted to $J$. Acoust. Soc. Am.

Nakagawa, S., K. T. Nihei, R. Suárez-Rivera, L. R. Myer, and N. G. W. Cook. 1997. Shear-induced conversions of seismic waves normally incident on a fracture, presented at annual winter meeting of American Geophysical Union, San Francisco.

Nelson, R. A. 1987. Fractured reservoirs: turning knwledge into practice, J. Petroleum Technology, 39, 407-414.

Newland, D. E.. 1989. Mechanical vibration analysis and computation, Longman Scientific \& Technical, John Wiley \& Sons, New York.

Nihei, K. T. 1992. , Micromechanics of seismic wave propagation in granular rocks, $\mathrm{Ph} \mathrm{D}$ Thesis, University of California at Berkeley.

Nihei, K. T., W. Yi, L. R. Myer, and N. G. W. Cook. 1998. Fracture channel waves, submitted to J. Geophys. Res.

Ohno, I.. 1976. Free vibration of a rectangular parallelepiped crystal and its application to determination of orthorhombic crystals, J. Phys. Earth, 24, 355-379,. 
Olson, L. D., C. Wright, and K. H. Stokoe, II. 1990. Strides in nondestructive testing, Civil Engineering, 60(5), 52-55.

Peterson, J. E., Jr., D. Hopkins, and L. Myer. 1993. Application of seismic displacement discontinuity theory to Hanford data, 63rd Annual Meeting and International Exhibition, Society of Exploration Geophysicists, 1006-1009.

Petyt, M. 1990. Introduction to finite element vibration analysis, Cambridge, New York.

Plona, T. J., and J. M. Cook. 1995. Effects of stress cycles on static and dynamic Young's moduli in Castlegate sandstone, Daemen and Schultz eds., Proc. 35th U. S. Symp. on Rock Mech., Balkema, 155-160.

Pochhammer, L. 1876. Uber die Fortpflanzungsgeschwindigkeiten kleiner Schwingungen in einem unbegrenzten istropen Kreiszylinder, J. reine angew. Math., 81, 324-36.

Portela, A., M. H. Aliabadi, and D. P. Rooke. 1992. The dual boundary element method: effective implementation for crack problems, Int. J. Num. Method in Engng., 33, 12691287.

Postma, G. W.. 1955. Wave propagation in a stratified medium, Geophysics, 20, 780806.

Powers, T. C.. 1938. Measuring Young's modulus of elasticity by means of sonic vibrations, Proc. ASTM, 46(1), 460-467.

Press, W. H., S. A. Teukolsky, W. T. Vetterling, and B. P. Flannery. 1988. Numerical Recipies in C - The Art of Scientific Computing, Cambridge.

Pyrak-Nolte, L. J. and N. G. W. Cook. 1987. Elastic interface waves along fracture, Geophys. Res. Lett., 14, 1107-1110.

Pyrak-Nolte, L. J., L. R. Myer, and N. G. W. Cook. 1990a. Transmission of seismic waves across single natural fractures, J. Geophys. Res., 95, 8617-8638.

Pyrak-Notle, L. J., L. R. Myer, and N. G. W. Cook. 1990b. Anisotropy in seismic velocities and amplitudes from multiple parallel fractures, J. Geophys. Res., 95, 1134511358. 
Pyrak-Nolte. L. J., J. Xu, and G. M. Haley. 1992. Elastic interface waves propagating in a fracture, Phys. Rev. Lett., 68, 3650-3653.

Rayleigh, J. W. S. 1945. The theory of sound, Vol. I and II., Dover Publications, New York.

Rehbein, D. K., B. J. Skillings, J. F. Smith, and D. O. Thompson. 1984. Ultrasonic reflection from a stressed interface, J. Nondesruct. Eval., 4, 3-12.

Riznichenko, Iu. V.. 1948. The propagation of seismic waves in discrete and heterogeneous media, Bull. Acad. Sci. USSR, Geograhical and Geophysical Series, 13, 115-128.

Riznichenko, Iu. V.. 1949. Seismic quasi-anisotropy, Bull. Acad. Sci. USSR, Geographical and Geophysical Series, 13, 518-544.

Rokhlin, S. I. and Y. J. Wang. 1991. Analysis of boundary conditions for elastic wave interaction with an interface between two solids, J. Acoust. Soc. Am., 89, 503-515.

Rytov, S. M.. 1956. Acoustical properties of a thinly laminated medium, Soviet Physical Acoustics, 2, 67-80.

Sakata, Y. and M. Ohtsu. 1995, Crack evaluation in concrete members based on ultrasonic spectroscopy, Am. Concr. Inst. Materials J., 92(6), 686-698.

Schoenberg, M. 1980. Elastic wave behavior across linear slip interfaces, J. Acoust. Soc. Am., 68(5), 1516-1521.

Schoenberg, M. 1983. Reflection of elastic waves from periodically stratified media with interfacial slip, Geophysical Prospecting, 31, 265-292.

Schoenberg, M. and J. Douma. 1988. Elastic wave propagation in media with parallel fractures and aligned cracks, Geophys. Prospect., 36, 571-590.

Schoenberg, M. and F. Muir. 1989. A calculus for finely layered anisotropic media, Geophys., 54(5), 581-589.

Soga, N. and O. L. Anderson. 1967. Elastic properties of tektites measured by resonant sphere technique, J. Geophys. Res., 72, 1733-1739. 
Spinner, S., T. W. Reichard, and W. E. Tefft. 1960. A comparison of experimental and theoretical relations between Young's modulus and the flexural and longitudinal resonance frequencies of uniform bars, Journal of Research, Nat. Bureau of Standards, 64A(2), 147-155.

Spinner, S. and W. E. Tefft. 1960. A method for determining mechanical resonance frequencies and for calculating elastic moduli from these frequencies, Am. Soc. Test. mater. Proc., 61, 1221-1238.

Spinner, S. and W. E. Tefft. 1961. A method for determining mechanical resonance frequencies and for calculating elastic moduli from these frequencies, Am. Soc. Test. mater. Proc., 61, 1221-1238.

Suárez-Rivera, R. 1992. The influence of thin clay layers containing liquids on the propagation of shear waves, $\mathrm{Ph} \mathrm{D}$ Thesis, University of California at Berkeley.

Tarricone, P. 1990. Bridges under surveillance, Civil Engineering, 60(5), 48-51.

Visscher, W. M., Migliori, A., Bell, T. M., and Reinert, R. A., 1991. On the normal modes of free vibration on inhomogenous and anisotropic elastic objects, J. Acoust. Soc. Am., 90(4), 2154-2162.

Walsh, J. B. 1965a. The effect of cracks on the compressibility of rock, J. Geophys. Res., 70, 5249-5257.

Walsh, J. B. 1965b. The effect of cracks on the uniaxial elastic compression of rocks, $J$. Geophys. Res., 70, 399-411.

Walsh, J .B. and M. A. Grosenbaugh. 1979. A new model for analyzing the effect of fractures on compressibility, J. Geophys. Res., 84, 3532-3536.

Westergaard, H. M. 1939. Bearing pressures and cracks, J. Appl. Mech., 6, 49-53.

Yoshioka, N. and C. H. Scholz. 1989a. Elastic properties of contacting surfaces under normal and shear loads 1. Theory, J. Geophys. Res., 94, 17681-17690.

Yoshioka, N. and C. H. Scholz. 1989b. Elastic properties of contacting surfaces under normal and shear loads 2. Comparison of theroy with experiment, J. Geophys. Res., 94, 17691-17700. 
Yi, W., S. Nakagawa, K. T. Nihei, J. W. Rector, L. R. Myer, and N. G. W. Cook. 1997. Numerical investigation of fracture induced seismic anisotropy, Extended Abstract, 67th annual meeting of Society of Exploration Geophysics, 960-963.

Ziman, J. M., 1964. Principles of the Theory of Solids, Cambridge U. P., Cambridge 


\section{Appendix A}

\section{Displacement Propagator Matrix Method for a Fractued 1-D System}

Wave propagation and resonance in a one dimensional system containing multiple fractures can be examined analytically or numerically using the propagator matrix method (Kennett, 1983; Aki and Richard, 1980). This appendix shows how fractures are incorporated in a propagator method formulated for the displacement field.

The method is developed for a single Fourier component of the wavefield given by $u(t)=u \cdot e^{-i \omega t}$. As the time-dependent term is common for the entire wavefield, it can be omitted. The basic idea of the displacement propagator matrix method is to relate source displacement on a boundary to receiver displacement on another boundary by incrementally predicting the displacement on the boundaries between two points.

For a one-dimensional system, a wave propagating in the system is decomposed to a down-going wave $u_{d}$ and an up-going wave $u_{u}$. The resulting displacement at any location is obtained by adding these two components. At each interface (fracture) between layers, the displacement-discontinuity boundary conditions shown below are applied to model the transmission and reflection of the waves

$$
\begin{aligned}
& \sigma^{-}=\kappa\left(u^{+}-u^{-}\right) \equiv \kappa[u] \\
& \sigma^{-}=\sigma^{+}
\end{aligned}
$$


where $u^{+}, \sigma^{+}$and $u^{-}, \sigma^{-}$are displacement and stress at opposite sides of a fracture. $\kappa$ is the specific fracture stiffness. At any location in the system, displacement is given by

$$
u=u_{d}+u_{u}
$$

In a continuous part of the system, up-going and down-going waves are expressed as follows

$$
\begin{aligned}
& u_{d}(z)=u_{d}\left(z_{0}\right) \exp \left(\frac{i \omega}{c}\left(z-z_{0}\right)\right) \\
& u_{u}(z)=u_{u}\left(z_{0}\right) \exp \left(-\frac{i \omega}{c}\left(z-z_{0}\right)\right),
\end{aligned}
$$

where $\omega$ is the angular frequency and $c$ is the velocity of the propagating wave. The stress induced by a passage of wave is given by

$$
\sigma=E^{*} \varepsilon=E^{*} \frac{d u}{d z}
$$

The elastic moduli $E^{*}$ is related to the wave velocity in the continuous part of the system by

$$
E^{*}=\rho c^{2}
$$

From Eq.(A.3) (A.5), the stress is obtained as

$$
\sigma=i \omega \rho c\left(u_{d}-u_{u}\right)=i \omega Z\left(u_{d}-u_{u}\right)
$$

By introducing Eq.(A.2) and (A.6) into Eq.(A.1), the displacement-discontinuity boundary conditions are rewritten as

$$
\begin{aligned}
& i \omega Z^{-}\left(\overline{u_{d}^{-}}-u_{u}^{-}\right)=\kappa\left(u_{d}^{+}+u_{u}^{+}-\overline{u_{d}^{-}}-u_{u}^{-}\right) \\
& Z^{+}\left(u_{d}^{+}-u_{u}^{+}\right)=Z^{-}\left(u_{d}^{-}-u_{u}^{-}\right) .
\end{aligned}
$$

Assuming identical material properties for both sides of the fracture $\left(Z^{+}=Z^{-}\right)$, Eq.(A.7a,b) are expressed in a matrix form

$$
\left[\begin{array}{c}
u_{d}^{+} \\
u_{u}^{+}
\end{array}\right]=\left[\begin{array}{cc}
1+i / \beta & -i / \beta \\
i / \beta & 1-i / \beta
\end{array}\right]\left[\begin{array}{l}
u_{d}^{-} \\
u_{u}^{-}
\end{array}\right] \equiv \mathbf{P}\left[\begin{array}{c}
u_{d}^{-} \\
u_{u}^{-}
\end{array}\right],
$$

where the non-dimensional impedance parameter $\beta$ is defined by 


$$
\beta=\frac{2 \kappa / \omega}{Z}
$$

Equation (A.8) provides the phase and amplitude change of the wave propagating across a fracture.

From Eq.(A.3), components of the displacement between two boundaries of a continuous part of the system are

$$
\left[\begin{array}{c}
u_{d}^{-}\left(z_{0}+L\right) \\
u_{u}^{-}\left(z_{0}+L\right)
\end{array}\right]=\left[\begin{array}{cc}
e^{i \phi} & 0 \\
0 & e^{-i \phi}
\end{array}\right]\left[\begin{array}{l}
u_{d}^{+}\left(z_{0}\right) \\
u_{u}^{+}\left(z_{0}\right)
\end{array}\right] \equiv \mathbf{E}\left[\begin{array}{l}
u_{d}^{-} \\
u_{u}^{-}
\end{array}\right]
$$

where $\mathrm{L}$ is the distance between the boundaries. $\phi$ is the phase change over the distance $\mathrm{L}$ given by

$$
\phi=\frac{\omega L}{c} .
$$

A pair of displacements for the up-going and down-going waves on the boundary can be related to another pair of the displacements on a remote boundary by cyclicly applying the matrix equations (A.8) and (A.10) as follows

$$
\begin{aligned}
{\left[\begin{array}{l}
u_{d}^{-} \\
u_{u}^{-}
\end{array}\right]^{(n+m)} } & =\mathbf{E}\left(z_{n+m}, z_{n+m-1}\right) \mathbf{P}^{(n+m-1)} \cdots \mathbf{E}\left(z_{n+2}, z_{n+1}\right) \mathbf{P}^{(n+1)} \mathbf{E}\left(z_{n+1}, z_{n}\right)\left[\begin{array}{l}
u_{d}^{+} \\
u_{u}^{+}
\end{array}\right]^{(n)} \\
& =\mathbf{Q}\left[\begin{array}{l}
u_{d}^{+} \\
u_{u}^{+}
\end{array}\right]^{(n)}
\end{aligned}
$$

Tge displacement within an intact section of the system is obtained by first computing the displacements on a boundary of the section and then applying Eq.(A.3).

Equation (A.12) needs two boundary conditons to be solved. For example, for the forced vibration of a free-end system, the stress at the driving point (driving function) and the free-end (equal to zero) are the necessary and satisfactory conditions. A frequency response (dynamic compliance) of the system can be obtained by setting the driving force (stress) amplitude to unity. For a wave incident on a series of fractures in an infinite medium, the displacement (or stress) of the incident wave is given. An additional condition is provided by setting the zero amplitude of the wave that is reflecting back from infinite distance (Sommerfeld radiation condition). 


\section{Appendix B}

\section{Derivation of Mobilities for a Fractured 1-D System}

In this appendix, the mobilities of fractured one-dimensional systems discussed in Chapter 2 are derived using the propagater matrix method described in Appendix A.

Resonance of a bar containing a single fracture

For a finite system containing a single fracture as discussed in Section 2.2.2, the propagator matrix that relates displacement at the top and the bottom of the system is

$$
\left[\begin{array}{l}
u_{d}^{-} \\
u_{u}^{-}
\end{array}\right]^{(1)}=\mathrm{Q}\left[\begin{array}{l}
u_{d}^{+} \\
u_{u}^{+}
\end{array}\right]^{(0)},
$$

where

$$
\begin{aligned}
\mathbf{Q} & =\left[\begin{array}{cc}
e^{i \phi_{1}} & 0 \\
0 & e^{-i \phi_{1}}
\end{array}\right]\left[\begin{array}{cc}
1+i / \beta & -i / \beta \\
i / \beta & 1-i / \beta
\end{array}\right]\left[\begin{array}{cc}
e^{i \phi_{0}} & 0 \\
0 & e^{-i \phi_{0}}
\end{array}\right] . \\
& =\left[\begin{array}{cc}
(1+i / \beta) e^{i\left(\phi_{1}+\phi_{0}\right)} & -i / \beta e^{i\left(\phi_{1}-\phi_{0}\right)} \\
i / \beta e^{-i\left(\phi_{1}-\phi_{0}\right)} & (1-i / \beta) e^{-i\left(\phi_{1}+\phi_{0}\right)}
\end{array}\right]
\end{aligned}
$$

The symbols and notations in the above equations are adopted from Appendix A. As the system has a stress-free boundary at the end of the second segment, 


$$
\sigma^{-(1)}=\frac{\partial u^{-(1)}}{\partial z}=i \frac{\omega}{c}\left(u_{d}^{-}-u_{u}^{-}\right)^{(1)}=0 .
$$

Therefore,

$$
u_{d}^{-(1)}=u_{u}^{-(1)} .
$$

By introducing Eq.(B.4) into Eq.(B.1),

$$
r \equiv \frac{u_{u}^{+(0)}}{u_{d}^{+(0)}}=\frac{(1+i / \beta) e^{i\left(\phi_{1}+\phi_{0}\right)}-i / \beta e^{-i\left(\phi_{1}-\phi_{0}\right)}}{(1-i / \beta) e^{-i\left(\phi_{1}+\phi_{0}\right)}+i / \beta e^{i\left(\phi_{1}-\phi_{0}\right)}} .
$$

The mobility at the source is

$$
\frac{v^{(0)}}{\sigma^{(0)}}=\frac{\partial u^{(0)}}{\partial t} / E^{*} \frac{\partial u^{(0)}}{\partial z}=\frac{-i \omega \cdot\left(u_{d}^{+}+u_{u}^{+}\right)^{(0)}}{i \omega Z \cdot\left(u_{d}^{+}-u_{u}^{+}\right)^{(0)}}=\frac{(-1)}{Z} \frac{1+r}{1-r}
$$

By introducing the Eq.(B.R) into (B.6), the mobility is

$$
\frac{v^{(0)}}{\sigma^{(0)}}=\frac{1}{i Z} \cdot \frac{2 \cos \phi_{0} \sin \phi_{1}-\beta \cos \left(\phi_{0}+\phi_{1}\right)}{2 \sin \phi_{0} \sin \phi_{1}-\beta \sin \left(\phi_{0}+\phi_{1}\right)}=\frac{1}{Z} \cdot M .
$$

$\mathrm{M}$ is a dimensionless mobility. Therefore, the power of the dimensionless mobility at the source is

$$
M M^{*}=\frac{\left[2 \cos \phi_{0} \sin \phi_{1}-\beta \cos \left(\phi_{0}+\phi_{1}\right)\right]^{2}}{\left[2 \sin \phi_{0} \sin \phi_{1}-\beta \sin \left(\phi_{0}+\phi_{1}\right)\right]^{2}} .
$$

The mobility at the free-end is

$$
\frac{v^{(1)}}{\sigma^{(0)}}=\frac{\partial u^{(1)}}{\partial t} / E^{*} \frac{\partial u^{(0)}}{\partial z}=\frac{-i \omega \cdot\left(u_{d}^{-}+u_{u}^{-}\right)^{(1)}}{i \omega Z \cdot\left(u_{d}^{+}-u_{u}^{+}\right)^{(0)}}=\frac{-\left(u_{d}^{-}+u_{u}^{-}\right)^{(1)}}{Z \cdot(1-r) u_{d}^{+(0)}}
$$

The displacement components at the free-end $\left(u_{d}^{-(1)}\right.$ and $\left.u_{u}^{-(1)}\right)$ are related to $u_{u}^{+(0)}$ through Eq.(B.1). Resulting mobiliy is

$$
\frac{v^{(1)}}{\sigma^{(0)}}=\frac{1}{i Z} \cdot \frac{\beta}{2 \sin \phi_{0} \cdot \sin \phi_{1}-\beta \sin \left(\phi_{0}+\phi_{1}\right)}=\frac{1}{Z} \cdot M .
$$

The power of the dimensionless mobility is 


$$
M M^{*}=\frac{\beta^{2}}{\left[2 \sin \phi_{0} \cdot \sin \phi_{1}-\beta \sin \left(\phi_{0}+\phi_{1}\right)\right]^{2}} .
$$

Resonance of a delaminated halfspace

For a delaminated halfspace discussed in Section 2.4, the propagator matrix equation becomes

$$
\left[\begin{array}{l}
u_{d}^{+} \\
u_{u}^{+}
\end{array}\right]^{(1)}=\mathrm{Q}\left[\begin{array}{l}
u_{d}^{+} \\
u_{u}^{+}
\end{array}\right]^{(0)},
$$

where

$$
\mathbf{Q}=\left[\begin{array}{cc}
1+i / \beta & -i / \beta \\
i / \beta & 1-i / \beta
\end{array}\right]\left[\begin{array}{cc}
e^{i \phi_{0}} & 0 \\
0 & e^{-i \phi_{0}}
\end{array}\right]=\left[\begin{array}{cc}
(1+i / \beta) e^{i \phi_{0}} & -i / \beta e^{-i \phi_{0}} \\
i / \beta e^{i \phi_{0}} & (1-i / \beta) e^{-i \phi_{0}}
\end{array}\right]
$$

Due to the radiation condition (i.e., no wave is propagating from an infinite distance), $u_{u}^{+(1)}=0$. This leads to a relation

$$
r \equiv \frac{u_{u}^{+(0)}}{u_{d}^{+(0)}}=\frac{1}{1+i \beta} e^{2 i \phi_{0}} .
$$

Therefore, the source mobility is computed from

$$
\frac{v^{(0)}}{\sigma^{(0)}}=\frac{(-1)}{Z} \frac{1+r}{1-r}=\frac{(-1)}{Z} \cdot \frac{1+\cos (2 \phi)+i(\beta+\sin (2 \phi))}{1-\cos (2 \phi)+i(\beta-\sin (2 \phi))}=\frac{1}{Z} \cdot M
$$

The power of the dimensionless mobility is

$$
M M^{*}=\frac{2(1+\cos (2 \phi))+\beta^{2}+2 \beta \sin (2 \phi)}{2(1-\cos (2 \phi))+\beta^{2}-2 \beta \sin (2 \phi)} .
$$

